\title{
Dietary Patterns and Growth, Size, Body Composition, and/or Risk of Overweight or Obesity: A Systematic Review
}

2020 Dietary Guidelines Advisory Committee, Dietary Patterns Subcommittee

Published date: July 15, 2020

Nutrition Evidence Systematic Review

Center for Nutrition Policy and Promotion

Food and Nutrition Service

U.S. Department of Agriculture

Braddock Metro Center II

1320 Braddock Place

Alexandria, Virginia 22314 
This systematic review was conducted by the 2020 Dietary Guidelines Advisory Committee in collaboration with the Nutrition Evidence Systematic Review (NESR) team at the Center for Nutrition Policy and Promotion, Food and Nutrition Service, U.S. Department of Agriculture (USDA). All systematic reviews from the 2020

Advisory Committee Project are available on the NESR website: https://nesr.usda.gov/2020-dietaryguidelines-advisory-committee-systematic-reviews.

Conclusion statements drawn as part of this systematic review describe the state of science related to the specific question examined. Conclusion statements do not draw implications, and should not be interpreted as dietary guidance. This portfolio provides the complete documentation for this systematic review. A summary of this review is included in the 2020 Advisory Committee's Scientific Report available at www.DietaryGuidelines.gov.

The contents of this document may be used and reprinted without permission. Endorsements by NESR, the Center for Nutrition Policy and Promotion, the Food and Nutrition Service, or the USDA of derivative products developed from this work may not be stated or implied.

Suggested citation for this systematic review: 2020 Dietary Guidelines Advisory Committee and Nutrition Evidence Systematic Review Team. Dietary Patterns and Growth, Size, Body Composition, and/or Risk of Overweight or Obesity: A Systematic Review. 2020 Dietary Guidelines Advisory Committee Project. Alexandria, VA: U.S. Department of Agriculture, Food and Nutrition Service, Center for Nutrition Policy and Promotion, July 2020. Available at: https://nesr.usda.gov/2020-dietary-guidelines-advisory-committeesystematic-reviews.

Related citation: Dietary Guidelines Advisory Committee. 2020. Scientific Report of the 2020 Dietary Guidelines Advisory Committee: Advisory Report to the Secretary of Agriculture and the Secretary of Health and Human Services. U.S. Department of Agriculture, Agricultural Research Service, Washington, DC.

In accordance with Federal civil rights law and USDA civil rights regulations and policies, the USDA, its Agencies, offices, and employees, and institutions participating in or administering USDA programs are prohibited from discriminating based on race, color, national origin, religion, sex, gender identity (including gender expression), sexual orientation, disability, age, marital status, family/parental status, income derived from a public assistance program, political beliefs, or reprisal or retaliation for prior civil rights activity, in any program or activity conducted or funded by USDA (not all bases apply to all programs). Remedies and complaint filing deadlines vary by program or incident.

Persons with disabilities who require alternative means of communication for program information (e.g., Braille, large print, audiotape, American Sign Language, etc.) should contact the responsible Agency or USDA's TARGET Center at (202) 720-2600 (voice and TTY) or contact USDA through the Federal Relay Service at (800) 877-8339. Additionally, program information may be made available in languages other than English.

To file a program discrimination complaint, complete the USDA Program Discrimination Complaint Form, AD3027, found online at How to File a Program Discrimination Complaint and at any USDA office or write a letter addressed to USDA and provide in the letter all of the information requested in the form. To request a copy of the complaint form, call (866) 632-9992. Submit your completed form or letter to USDA by: (1) mail: U.S. Department of Agriculture, Office of the Assistant Secretary for Civil Rights, 1400 Independence Avenue, SW, Washington, D.C. 20250-9410; (2) fax: (202) 690-7442; or (3) email: program.intake@usda.gov.

USDA is an equal opportunity provider, employer, and lender. 


\section{ACKNOWLEDGEMENTS}

Dietary Patterns Subcommittee:

- Carol Boushey, PhD, MPH, RD, University of Hawaii, Subcommittee Chair

- Jamy Ard, MD, Wake Forest School of Medicine

- Lydia Bazzano, MD, PhD, Tulane University and Ochsner Health System

- Steven Heymsfield, MD, Louisiana State University, Pennington Biomedical Research Center

- Elizabeth Mayer-Davis, PhD, RD, University of North Carolina at Chapel Hill

- Joan Sabaté, MD, DrPH, Loma Linda University

- Linda Snetselaar, PhD, RDN, University of lowa

- Linda Van Horn, PhD, RDN, LD, Northwestern University

- Barbara Schneeman, PhD, Chair of the 2020 Dietary Guidelines Advisory Committee, University of California, Davis

\section{Nutrition Evidence Systematic Review (NESR) Team:}

- Laural Kelly English, PhD, Analyst, Panum Groupi

- Marlana Bates, MPH, RD, Analyst, Panum Group ${ }^{i}$

- Emily Callahan, MS, Analyst, Office of Nutrition Guidance and Analysis (ONGA), Center for Nutrition Policy and Promotion (CNPP), Food and Nutrition Service (FNS), U.S. Department of Agriculture (USDA)

- Gisela Butera, MLIS, MEd, Systematic Review Librarian, Panum Groupi

- Nancy Terry, MS, MLS, Biomedical Librarian, National Institutes of Health Library, U.S. Department of Health and Human Services (HHS)

- Julie Obbagy, PhD, RD, Project Lead, ONGA, CNPP, FNS, USDA

\section{Federal Liaisons:}

- Elizabeth Rahavi, RD, ONGA, CNPP, FNS, USDA

- Clarissa (Claire) Brown, MS, MPH, RD, ONGA, CNPP, FNS, USDA

\section{Project Leadership:}

- Eve Essery Stoody, PhD, Designated Federal Officer and Director, ONGA, CNPP, FNS, USDA

- Janet de Jesus, MS, RD, Nutrition Advisor, Office of Disease Prevention and Health Promotion, Office of the Assistant Secretary for Health, HHS

USDA and HHS implemented a process to identify topics and scientific questions to be examined by the 2020 Dietary Guidelines Advisory Committee. The Committee conducted its review of evidence in subcommittees for discussion by the full Committee during its public meetings. The role of the Committee members involved establishing all aspects of the protocol, which presented the plan for how they would examine the scientific evidence, including the inclusion and exclusion criteria;

\footnotetext{
' Under contract with the Food and Nutrition Service, United States Department of Agriculture.
} 
reviewing all studies that met the criteria they set; deliberating on the body of evidence for each question; and writing and grading the conclusion statements to be included in the scientific report the 2020 Committee submitted to USDA and HHS. The NESR team with assistance from Federal Liaisons and Project Leadership, supported the Committee by facilitating, executing, and documenting the work necessary to ensure the reviews were completed in accordance with NESR methodology. More information about the 2020 Dietary Guidelines Advisory Committee, including the process used to identify topics and questions, can be found at www.DietaryGuidelines.gov. More information about NESR can be found at NESR.usda.gov.

The Committee and NESR staff thank USDA's Agricultural Research Service for coordinating the peer review of this systematic review, and the Federal scientist peer reviewers for their time and expertise.

FUNDING SOURCE: United States Department of Agriculture, Food and Nutrition Service, Center for Nutrition Policy and Promotion, Alexandria, VA 


\section{TABLE OF CONTENTS}

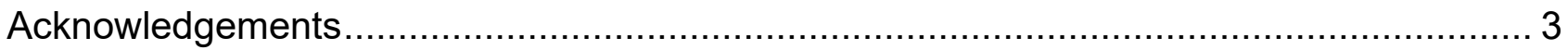

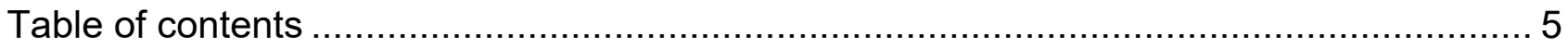

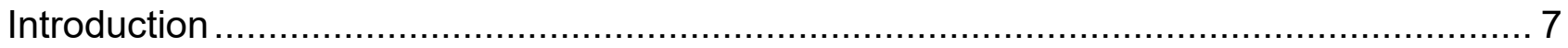

What is the relationship between dietary patterns consumed and growth, size, body composition, and/or risk of overweight or obesity? ................................................ 9

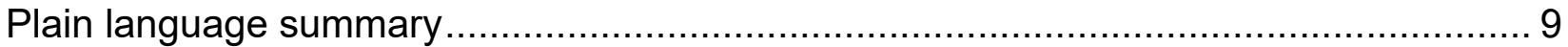

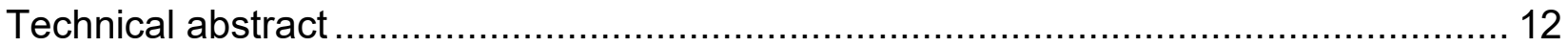

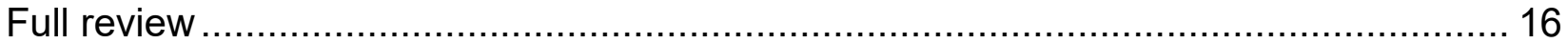

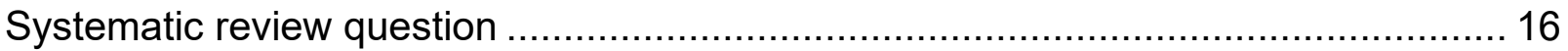

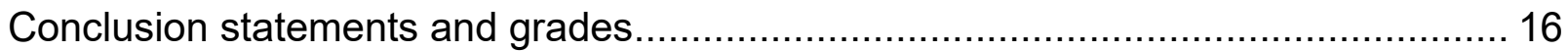

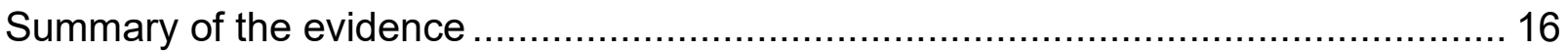

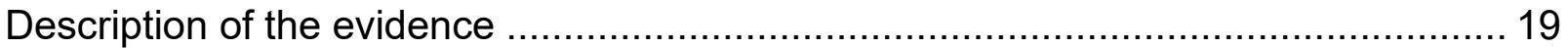

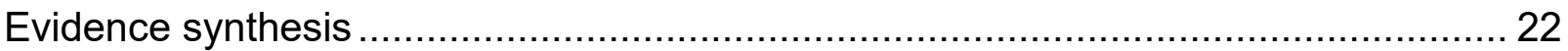

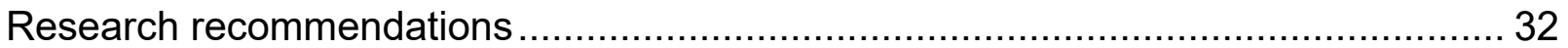

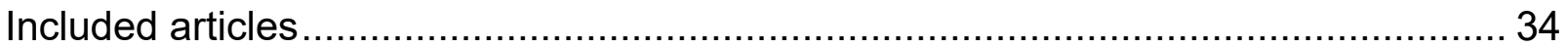

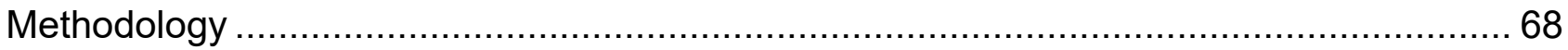

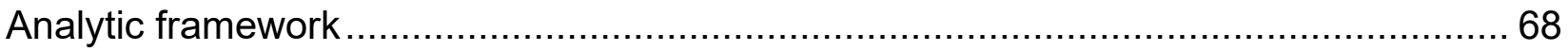

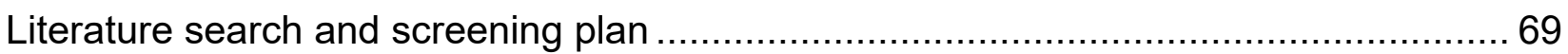

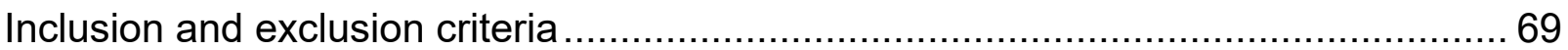

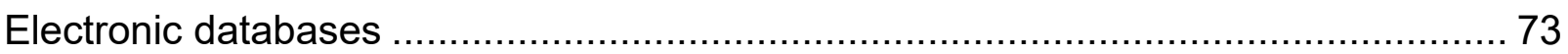

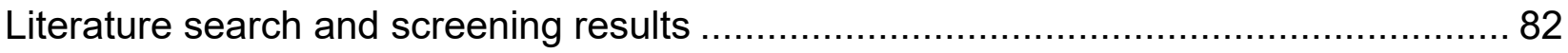

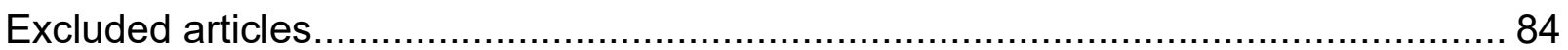

Table 1: Studies that examined the relationship between dietary patterns in children and growth size, body composition, and risk of overweight or obesity.................................4 41 Table 2. Randomized controlled trials that examined diets based on macronutrient proportion and risk of growth size, body composition, and risk of overweight or obesity ... 48 Table 3. Observational studies that examined diets based on macronutrient proportion and risk of growth size, body composition, and risk of overweight or obesity ..........................58 Table 4. Risk of bias for observational studies examining dietary patterns in children and growth size, body composition, and risk of overweight or obesity $4 \mathrm{~F}$

Table 5. Risk of bias for randomized controlled trials examining diets based on macronutrient distribution and growth size, body composition, and risk of overweight or

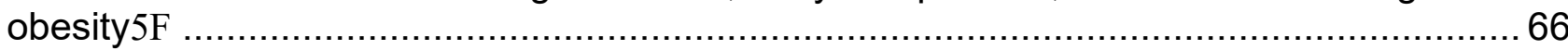

Table 6. Risk of bias in observational studies examining diets based on macronutrient distribution and growth size, body composition, and risk of overweight or obesity $6 \mathrm{~F} \ldots \ldots \ldots .67$

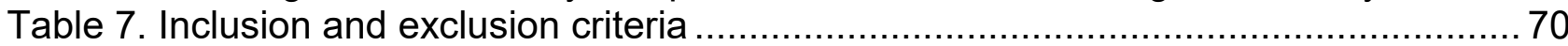


Table 8. Articles excluded after full text screening with rationale for exclusion.................. 84

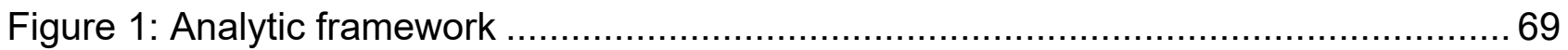

Figure 2: Flow chart of literature search and screening results $12 \mathrm{~F} \ldots \ldots \ldots \ldots \ldots \ldots \ldots \ldots \ldots \ldots . . . . \ldots 3$ 


\section{INTRODUCTION}

This document describes a systematic review conducted to answer the following question: What is the relationship between dietary patterns consumed and growth, size, body composition, and/or risk of overweight or obesity? This systematic review was conducted by the 2020 Dietary Guidelines Advisory Committee, supported by USDA's Nutrition Evidence Systematic Review (NESR).

More information about the 2020 Dietary Guidelines Advisory Committee is available at the following website: www.DietaryGuidelines.gov.

NESR specializes in conducting food- and nutrition-related systematic reviews using a rigorous, protocol-driven methodology. More information about NESR is available at the following website: NESR.usda.gov.

NESR's systematic review methodology involves developing a protocol, searching for and selecting studies, extracting data from and assessing the risk of bias of each included study, synthesizing the evidence, developing conclusion statements, grading the evidence underlying the conclusion statements, and recommending future research. A detailed description of the systematic reviews conducted for the 2020 Dietary Guidelines Advisory Committee, including information about methodology, is available on the NESR website: https://nesr.usda.gov/2020-dietary-guidelines-advisory-committee-systematic-reviews. In addition, starting on page 66, this document describes the final protocol as it was applied in the systematic review. A description of and rationale for modifications made to the protocol are described in the 2020 Dietary Guidelines Advisory Committee Report, Part D: Chapter 8. Dietary Patterns. 


\section{List of abbreviations}

\begin{tabular}{ll}
\hline Abbreviation & Full name \\
\hline AMDR & Acceptable macronutrient distribution range \\
\hline BMI & Body mass index \\
\hline CNPP & Center for Nutrition Policy and Promotion \\
\hline CVD & Cardiovascular disease \\
\hline$\%$ E & \% energy \\
\hline EVOO & Extra virgin olive oil \\
\hline FFMI & Fat-free mass index \\
\hline FMI & Fat mass index \\
\hline FNS & Food and nutrition service \\
\hline f/u & Follow-up \\
\hline HHS & Health and Human Services \\
\hline mo & Month(s) \\
\hline NESR & Nutrition Evidence Systematic Review team \\
\hline ONGA & Office of Nutrition Guidance and Analysis \\
\hline PCS & Prospective cohort study design \\
\hline PREDIMED & Prevención con Dieta Mediterránea \\
\hline RRR & Reduced rank regression \\
\hline USDA & United States Department of Agriculture \\
\hline wk & Week(s) \\
\hline y & Year(s) \\
\hline
\end{tabular}




\section{WHAT IS THE RELATIONSHIP BETWEEN DIETARY PATTERNS CONSUMED AND GROWTH, SIZE, BODY COMPOSITION, AND/OR RISK OF OVERWEIGHT OR OBESITY?}

\section{PLAIN LANGUAGE SUMMARY}

What is the question?

- The question is: What is the relationship between dietary patterns consumed and growth, size, body composition, and/or risk of overweight or obesity?

What is the answer to the question?

Dietary patterns: Children

- Limited evidence suggests that dietary patterns consumed by children or adolescents that are lower in fruits, vegetables, whole grains, and low-fat dairy while being higher in added sugars, refined grains, fried potatoes, and processed meats are associated with higher fat-mass index and BMI later in adolescence.

\section{Dietary patterns: Adults}

- The 2020 Dietary Guidelines Advisory Committee reviewed recently published evidence using a systematic evidence scan and determined that the conclusion drawn by the 2015 Dietary Guidelines Advisory Committee generally reflects the current state of science: Moderate evidence indicates dietary patterns emphasizing vegetables, fruits, and whole grains; seafood and legumes; moderate in dairy products (particularly low and non-fat dairy) and alcohol; lower in meats (including red and processed meats), and low in sugar-sweetened foods and beverages, and refined grains are associated with favorable outcomes related to body weight, (including lower BMI, waist circumference, or percent body fat) or risk of obesity. Components of the dietary patterns associated with these favorable outcomes include higher intakes of unsaturated fats and lower intakes of saturated fats, cholesterol, and sodium.

\section{Diets based on macronutrient distribution: Children}

- No evidence is available to determine a relationship between diets based on macronutrient distribution consumed during childhood and growth, size, body composition, and risk of overweight/obesity.

\section{Diets based on macronutrient distribution: Adults}

- Insufficient evidence is available to determine the relationship between macronutrient distributions with proportions of energy falling outside of the AMDR for at least one macronutrient due to methodological limitations and inconsistent results.

\section{Why was this question asked?}

- This important public health question was identified by the U.S. Departments of Agriculture (USDA) and Health and Human Services (HHS) to be examined by the 2020 Dietary Guidelines Advisory Committee. 


\section{How was this question answered?}

- The 2020 Dietary Guidelines Advisory Committee, Dietary Patterns Subcommittee conducted a systematic review and a systematic evidence scan to answer this question with support from the Nutrition Evidence Systematic Review (NESR) team.

- Dietary patterns were defined as the quantities, proportions, variety, or combination of different foods, drinks, and nutrients (when available) in diets, and the frequency with which they are habitually consumed.

- Diets based on macronutrient distribution were examined when at least one macronutrient proportion outside of the acceptable macronutrient distribution range (AMDR) for carbohydrate, fat, and/or protein, whether or not the foods/food groups consumed were provided.

\section{What is the population of interest?}

- Children and adults, ages 2 years and older

\section{What evidence was found?}

- This review identified 88 articles that met inclusion criteria.

- Twelve articles examined dietary patterns consumed by children or adolescents and growth, size, body composition, and/or risk of overweight or obesity.

- Dietary patterns that were lower in fruits, vegetables, whole grains, and lowfat dairy, and higher in added sugars, refined grains, fried potatoes, and processed meat were related to higher body fat mass and body mass index (BMI).

- The 2020 Committee determined the strength of this evidence was limited.

- Fifty-four articles examined dietary patterns consumed by adults and growth, size, body composition, and/or risk of overweight or obesity.

- The 2020 Committee determined these articles were generally consistent with those from an existing NESR systematic review. Therefore, the 2020 Committee carried forward the conclusion from the 2015 Committee.

- No articles met inclusion criteria that examined diets based on macronutrient distribution consumed by children or adolescents and growth, size, body composition, and/or risk of overweight or obesity.

o The 2020 Committee could not draw conclusions because there were no studies.

- Thirty-one articles examined diets based on macronutrient distribution consumed by adults and growth, size, body composition, and/or risk of overweight or obesity.

- Many limitations in the study design and conduct of included articles were identified. This includes a lack of accounting for key confounders or possible changes in dietary intake over follow-up.

- The 2020 Committee could not draw conclusions because the included studies had several limitations.

How up-to-date is this systematic review? 
- This review searched for studies from January, 2000 to October, 2019; and updated existing systematic reviews that included evidence from January 2000 to January 2014. 


\section{TECHNICAL ABSTRACT}

\section{Background}

- This important public health question was identified by the U.S. Departments of Agriculture (USDA) and Health and Human Services (HHS) to be examined by the 2020 Dietary Guidelines Advisory Committee.

- The 2020 Dietary Guidelines Advisory Committee, Dietary Patterns Subcommittee conducted a systematic review to answer this question with support from the Nutrition Evidence Systematic Review (NESR) team.

- The goal of this systematic review was to examine the following question: What is the relationship between dietary patterns consumed and growth, size, body composition, and/or risk of overweight or obesity?

\section{Conclusion statements and grades}

\section{Dietary patterns: Children}

- Limited evidence suggests that dietary patterns consumed by children or adolescents that are lower in fruits, vegetables, whole grains, and low-fat dairy while being higher in added sugars, refined grains, fried potatoes, and processed meats are associated with higher fat-mass index and BMI later in adolescence. (Grade: Limited)

\section{Dietary patterns: Adults}

- The 2020 Dietary Guidelines Advisory Committee reviewed newly published evidence using a systematic evidence scan and determined that the conclusion drawn by the 2015 Dietary Guidelines Advisory Committee generally reflects the current state of science. Moderate evidence indicates dietary patterns emphasizing vegetables, fruits, and whole grains; seafood and legumes; moderate in dairy products (particularly low and non-fat dairy) and alcohol; lower in meats (including red and processed meats), and low in sugar-sweetened foods and beverages, and refined grains are associated with favorable outcomes related to body weight, (including lower BMI, waist circumference, or percent body fat) or risk of obesity. Components of the dietary patterns associated with these favorable outcomes include higher intakes of unsaturated fats and lower intakes of saturated fats, cholesterol, and sodium. (2015 Dietary Guidelines Advisory Committee Grade: Moderate)

\section{Diets based on macronutrient distribution: Children}

- No evidence is available to determine a relationship between diets based on macronutrient distribution consumed during childhood and growth, size, body composition, and risk of overweight/obesity. (Grade: Grade not assignable)

\section{Diets Based on macronutrient distribution: Adults}

- Insufficient evidence is available to determine the relationship between macronutrient distributions with proportions of energy falling outside of the AMDR for at least one macronutrient and growth, size, body composition, and risk of overweight/obesity, due to methodological limitations and inconsistent results. (Grade: Grade not assignable) 


\section{Methods}

- Two literature searches were conducted using 3 databases (PubMed, Cochrane, and Embase) to identify articles that evaluated the intervention or exposure of dietary patterns consumed and the outcomes of type 2 diabetes. A manual search was conducted to identify articles that may not have been included in the electronic databases searched. Articles were screened by two NESR analysts independently for inclusion based on pre-determined criteria

- Data extraction and risk of bias assessment were conducted for each included study, and both were checked for accuracy. The Committee qualitatively synthesized the body of evidence to inform development of a conclusion statement(s), and graded the strength of evidence using pre-established criteria for risk of bias, consistency, directness, precision, and generalizability.

- Dietary patterns were defined as the quantities, proportions, variety, or combination of different foods, drinks, and nutrients in diets, and the frequency with which they are habitually consumed.

- Diets based on macronutrient distribution were considered to be those with at least one macronutrient proportion outside of the acceptable macronutrient distribution range (AMDR) for carbohydrate, fat, and/or protein, whether or not the foods/food groups consumed were provided.

- Studies examining energy-restricted diets that induce weight loss or treat overweight and obesity for the purposes of treating additional or other medical conditions were excluded.

\section{Summary of the evidence}

- Eighty-eight articles were identified that met inclusion criteria and examined the relationship between dietary patterns and/or diets based on macronutrient proportion and growth, size, body composition, and/or risk of overweight or obesity.

- Twelve articles from prospective cohort studies were identified that examined dietary patterns consumed by children and growth, size, body composition, and/or risk of overweight or obesity, met inclusion criteria, and were published between January 2014 and October 2019.

- Dietary patterns were assessed using a variety of methods including factor/cluster analysis, indices or scores, latent class analysis, and reduced rank regression

- Outcome measures varied across studies and included incidence of overweight/obesity, fat mass, lean mass, body mass index (BMI), central adiposity, and weight/height.

- Despite variability in methods, dietary patterns in childhood or adolescence that generally reflect poorer diet quality tended to associate with higher fatmass index and BMI later in adolescence. However, the findings should be interpreted with caution due to several limitations.

- Across the body of evidence, the direction of significant findings were mixed with relatively small and inconsistent magnitude.

- Most of the studies assessed diet once at baseline with methods that were not necessarily valid, reliable, or applicable in children. 
- Fifty-four articles examined dietary patterns consumed by adults and growth, size, body composition, and/or risk of overweight or obesity. The 2020 Committee determined with a systematic evidence scan that the recently published evidence was generally consistent with the body of evidence from the existing review, and a full systematic review update was not needed at this time. Therefore, the conclusion statement and grade from the existing review were carried forward.

- No studies identified met inclusion criteria that examined diets based on macronutrient distribution consumed by children or adolescents and growth, size, body composition, and/or risk of overweight or obesity.

- Thirty-one articles examined diets based on macronutrient distribution consumed by adults and growth, size, body composition, and/or risk of overweight or obesity, met inclusion criteria, and were published between January 2000 and October 2019

- Twenty-two articles came from randomized controlled trials (RCTs) and nine articles came from prospective cohort studies

- When describing and categorizing studies included in this review, the Committee did not label the diets examined as "low" or "high," because no standard definition is currently available for, for example, "low-carbohydrate" or "high-fat" diets. Instead, the Committee focused on whether, and the extent to which, the proportions of the macronutrients were below or above the AMDR.

- Most of the articles examined distributions in which the proportion of energy from carbohydrate was below the AMDR; fat was above the AMDR; and protein was within the AMDR in at least one of the exposure groups compared.

- Foods or food groups consumed as part of the diet were not reported consistently.

- Results across studies were inconsistent, with the majority of studies reporting no significant association between diets based on macronutrient distribution and growth, size, body composition, and/or risk of overweight or obesity.

- Some studies reported diets based on macronutrient distributions with proportions of energy from carbohydrate and/or fat within or close to AMDRs compared to those that were outside AMDRs significantly associated with favorable fat-mass, BMI, waist circumference, and/or change in weight. Notably, these results were closely linked to better quality of the diet where reported, typically consisting of vegetables, fruit, whole-grains, vegetable oils, nuts, pulses, and/or lean meat or fish

- Numerous limitations that prevent adequate assessment across this body of evidence were identified:

- Several studies did not directly test the difference in macronutrient proportions in the context of various dietary patterns during energy balance.

- Although statistically significant relationships were reported, the gradient between macronutrient distributions was relatively narrow within studies (e.g., $45.3 \%$ vs. $43.8 \%$ carbohydrate) and between studies

- Due to the variety of methods used to estimate macronutrient intake 
and adjust intake for total energy, the confidence in the reported proportions of energy falling outside the AMDR is low.

- Several studies reported to be examining one particular macronutrient of interest, such as "high-protein" or "low-carbohydrate" intake, but the proportion for that nutrient was within the AMDR. 


\section{FULL REVIEW}

\section{Systematic review question}

What is the relationship between dietary patterns consumed and growth, size, body composition, and/or risk of overweight or obesity?

\section{Conclusion statements and grades}

\section{Dietary patterns: Children}

Limited evidence suggests that dietary patterns consumed by children or adolescents that are lower in fruits, vegetables, whole grains, and low-fat dairy while being higher in added sugars, refined grains, fried potatoes, and processed meats are associated with higher fat-mass index and BMI later in adolescence. (Grade: Limited)

\section{Dietary patterns: Adults}

The 2020 Dietary Guidelines Advisory Committee reviewed recently published evidence using a systematic evidence scan and determined that the conclusion drawn by the 2015 Dietary Guidelines Advisory Committee generally reflects the current state of science. Moderate evidence indicates dietary patterns emphasizing vegetables, fruits, and whole grains; seafood and legumes; moderate in dairy products (particularly low and non-fat dairy) and alcohol; lower in meats (including red and processed meats), and low in sugar-sweetened foods and beverages, and refined grains are associated with favorable outcomes related to body weight, (including lower BMI, waist circumference, or percent body fat) or risk of obesity. Components of the dietary patterns associated with these favorable outcomes include higher intakes of unsaturated fats and lower intakes of saturated fats, cholesterol, and sodium. (2015 Dietary Guidelines Advisory Committee Grade: Moderate)

\section{Diets based on macronutrient distribution: Children}

No evidence is available to determine a relationship between diets based on macronutrient distribution consumed by children or adolescents and growth, size, body composition, and risk of overweight/obesity. (Grade: Grade not assignable)

\section{Diets based on macronutrient distribution: Adults}

Insufficient evidence is available to determine the relationship between macronutrient distributions with proportions of energy falling outside of the AMDR for at least one macronutrient and growth, size, body composition, and/or risk of overweight or obesity, due to methodological limitations and inconsistent results. (Grade: Grade not assignable)

\section{Summary of the evidence}

- Eighty-eight articles were identified that met inclusion criteria and examined the relationship between dietary patterns and/or diets based on macronutrient proportion and growth, size, body composition, and/or risk of overweight or obesity.

○ Dietary patterns were defined as the quantities, proportions, variety, or combination of different foods, drinks, and nutrients (when available) in 
diets, and the frequency with which they are habitually consumed.

- Diets based on macronutrient distribution were considered to be those with at least one macronutrient proportion outside of the acceptable macronutrient distribution range (AMDR ii) for carbohydrate, fat, and/or protein, whether or not the foods/food groups consumed were provided.

- When describing and categorizing studies included in this review, the Committee did not label the diets examined as "low" or "high," because no universally accepted, standard definition is currently available, for example, for "low-carbohydrate" or "high-fat" diets. Instead, the Committee focused on whether, and the extent to which, the proportions of the macronutrients were below or above the AMDR.

- Studies examining energy-restricted diets that induce weight loss or treat overweight and obesity for the purposes of treating additional or other medical conditions were excluded.

\section{Dietary patterns: Children}

- Twelve articles examined dietary patterns consumed by children and growth, size, body composition, and/or risk of overweight or obesity, met inclusion criteria, and were published between January 2013 and October 2019. ${ }^{1-12}$

- All 12 articles were from prospective cohort studies.

- Dietary patterns were assessed using a variety of methods including factor/cluster analysis, indices or scores, latent class analysis, and reduced rank regression

- Outcome measures varied across studies and included incidence of overweight/obesity, fat mass, lean mass, body mass index (BMI), central adiposity, and weight/height.

- Despite variability in methods, dietary patterns in childhood or adolescence that generally reflect poorer diet quality tended to associate with higher fat-mass index and BMI later in adolescence. However, the findings should be interpreted with caution due to several limitations.

- Across the body of evidence, the direction of significant findings were mixed with relatively small and inconsistent magnitude.

- Most of the studies assessed diet once at baseline with methods that were not necessarily validated, reliable, or applicable for children

\section{Dietary patterns: Adults}

- Fifty-four articles were identified by a systematic evidence scan examining

\footnotetext{
ii Briefly, the AMDR in adults are as follows: Carbohydrate: 45 to $65 \%$ of energy; Fat: 25 to $35 \%$ of energy; Protein: 10 to $35 \%$ of energy. The AMDR for children are as follows: Carbohydrate: 45 to $65 \%$ of energy; Protein, ages 1-3y: 5 to $20 \%$ of energy, ages 4-18y: 10 to $30 \%$ of energy; Fat, ages 1-3y: 30 to $40 \%$ of energy, ages 4-18y: 25 to $35 \%$ of energy. Source: Institute of Medicine. Dietary Reference Intakes for Energy, Carbohydrate, Fiber, Fat, Fatty Acids, Cholesterol, Protein, and Amino Acids. Washington (DC): The National Academies Press; 2002. For more details on the AMDR, see the Intervention/Exposure Inclusion and Exclusion criteria.
} 
dietary patterns consumed by adults and growth, size, body composition, and/or risk of overweight or obesity. ${ }^{13-60,61,62-66}$

- These articles represent new evidence published since a review done by the 2015 Dietary Guidelines Advisory Committee. iii

- A systematic evidence scan was conducted to identify and examine these articles, and determine whether a full systematic review update was warranted.

- Based on results from the systematic evidence scan, the 2020 Committee determined that the newly published evidence was generally consistent with the body of evidence from the existing review, and a full systematic review update was not needed at this time. Therefore, the conclusion statement and grade from the existing review were carried forward.

\section{Diets based on macronutrient distribution: Children}

- No studies identified met inclusion criteria that examined diets based on macronutrient distribution consumed by children or adolescents and growth, size, body composition, and/or risk of overweight or obesity.

\section{Diets based on macronutrient distribution: Adults}

- Thirty-one articles examined diets based on macronutrient distribution consumed by adults and growth, size, body composition, and/or risk of overweight or obesity, met inclusion criteria, and were published between January 2000 and October 2019. ${ }^{14,23,24,28,33,37,41,55,61,67-88}$

- Twenty-two articles came from randomized controlled trials (RCTs) and nine articles came from prospective cohort studies

- Most of the articles examined distributions in which the proportion of energy from carbohydrate as below the AMDR; fat was above the AMDR; and protein was within the AMDR in at least one of the exposure groups compared.

- Foods or food groups consumed as part of the diet were not reported consistently.

- Results across studies were inconsistent, with the majority of studies reporting no significant association between diets based on macronutrient distribution and growth, size, body composition, and/or risk of overweight or obesity.

- Some studies reported diets based on macronutrient distributions with proportions of energy from carbohydrate and/or fat within or close to AMDRs compared to those that were outside AMDRs significantly associated with favorable fat-mass, BMI, waist circumference, and/or change in weight. Notably, these results were closely linked to better quality of the diet, which typically

\footnotetext{
iii Dietary Guidelines Advisory Committee. Scientific Report of the 2015 Dietary Guidelines Advisory Committee: Advisory Report to the Secretary of Health and Human Services and the Secretary of Agriculture. US Department of Agriculture, Agricultural Research Service. https://health.gov/ourwork/food-nutrition/2015-2020-dietary-guidelines/advisory-report. Published 2015. Accessed April 30, 2020.
} 
consisted of vegetables, fruit, whole-grains, unsaturated vegetable oils, nuts, pulses, and/or lean meat or fish.

- Numerous limitations that prevent adequate assessment across this body of evidence were identified:

- Several studies did not directly test the difference in macronutrient proportions in the context of various dietary patterns during energy balance.

- Although statistically significant relationships were reported, the gradient between macronutrient distributions was relatively narrow within studies (e.g., $45.3 \%$ vs. $43.8 \%$ carbohydrate) and between studies

- Due to the variety of methods used to estimate macronutrient intake and adjust intake for total energy, the confidence in the reported proportions of energy falling outside the AMDR is low.

- Several studies reported to be examining one particular macronutrient of interest, such as "high-protein" or "lowcarbohydrate" intake, but the proportion for that nutrient was within the AMDR.

\section{Description of the evidence}

\section{Dietary patterns: Children}

\section{Population/participant characteristics}

The 12 studies examining dietary patterns in children that are included in this systematic review were conducted in the following countries:

- Australia $^{3}$

- Portugal ${ }^{4}$

- The Netherlands ${ }^{8}$

- United Kingdom ${ }^{1,2,9,11}$

- United States 6,7

One multi-country study from Italy, Estonia, Cyprus, Belgium, Sweden, Hungary, Germany, and Spain was included in 3 articles. ${ }^{5,10,12}$ Multiple articles from the same study were included, but reported unique data on dietary patterns, outcomes, and ages of participants examined. Data from the following cohorts were represented in the evidence:

- Avon Longitudinal Study of Parents and Children (ALSPAC) cohort ${ }^{2,11}$

- Generation R Study ${ }^{8}$

- Generation XXI ${ }^{4}$

- Growing Up Today Study $2^{7}$

- Identification and prevention of Dietary- and lifestyle-induced health EFfects In Children and infantS (IDEFICS) study ${ }^{5,12}$

- Project EAT 6

- Southampton Women's Survey ${ }^{9}$ 
- Western Australian Pregnancy Cohort ${ }^{3}$

Sample size in the included articles ranged from 1,018 to 10,918 participants. Studies included participants who were healthy and/or at risk of chronic disease, or diagnosed with a chronic disease. Studies enrolled both boys and girls in relatively equal amounts. Participants with missing data were excluded from analyses in all studies, and those with diseases that influence diet were typically excluded.

\section{Intervention/exposure}

Dietary patterns were assessed at a mean age of participants, ranging from 2 to 15 years, using a variety of methods.

- Five studies examined adherence to dietary patterns using 5 different indices or scores, $, 3,6-8,12$ and

- Five studies used factor/cluster analysis $2,5,9-11$

- One study each used latent class analysis, ${ }^{4}$ or reduced rank regression. ${ }^{1}$

Dietary intake was assessed using a variety of methods in which the validity and reliability were inconsistent or unknown, or only validated in adults. Most studies assessed diet once at baseline. Three articles reported diet assessments at each age/timepoint examined.

\section{Outcome assessment}

Included studies examined outcomes over follow-up at a range of ages between $4 \mathrm{y}$ to $25 y$. Growth, size, body composition, and/or risk of overweight or obesity outcomes were reported as follows:

- Overweight/obesity incidence ${ }^{10}$

- Fat-mass, fat-mass index (FMI), FMI z-score (FMIz), or change in \% fat mass $1,4,5,8,11,12$

- Lean mass or fat-free mass, including fat-free mass index (FFMI) $)^{5,8,11}$

- Body mass index (BMI) or BMI-z-score ${ }^{2-10,12}$

- Central adiposity or waist circumference ${ }^{3-5,12}$

- Absolute or change in (excess) weight and/or height ${ }^{6,8}$

\section{Dietary patterns: Adults}

Fifty-four articles met inclusion criteria, were published between January 2013 and October 2019, and examined dietary patterns in adults and growth, size, body composition, and/or risk of overweight or obesity. ${ }^{13-60} 61$ 62-66

\section{Diets based on macronutrient distribution: Children}

No studies identified that met inclusion criteria examined diets based on macronutrient proportion in children.

\section{Diets based on macronutrient distribution: Adults}

\section{Population/participant characteristics}

Studies were conducted in populations from the following countries:

- Australia $23,24,70,82$

- China ${ }^{81}$ 
- Denmark ${ }^{83}$

- France ${ }^{75,80}$

- Korea 88

- New Zealand 68,69

- Spain ${ }^{14,76,84,86}$

- Sweden 28

- United Kingdom 55,77

- United States $33,37,41,61,67,71-73,85,87$

- Multiple countries within Europe, ${ }^{74,78}$ or Finland, Sweden, Denmark, and Iceland. ${ }^{79}$

Studies enrolled primarily healthy, adults or older adults. Primary results were included from the Prevención con Dieta Mediterránea (PREDIMED) randomized-controlled trial (RCT), which enrolled adults at high-risk for cardiovascular disease across multiple sites. ${ }^{14,76}$ Multiple articles reported results from the same established cohorts, e.g., Framingham Heart Study Offspring, and reported different macronutrient distributions, dietary patterns, and/or outcomes.

\section{Intervention/exposure}

Dietary intake was measured with various approaches including picture-plate waste, food records, 24-h recalls, validated FFQ, or diet history. Most of the included articles came from RCTs that confirmed dietary compliance with 3-4-day food records at baseline, and during follow-up. In the observational studies, diet assessment was primarily assessed once at baseline using validated methods or every $2 y$ during followup.

Diets based on macronutrient distribution varied across studies, with some studies intentionally examining varied levels of specific macronutrient proportions while other studies focused on adherence to different dietary patterns such as a "Mediterranean" diet, published dietary guidelines, regional dietary patterns, or specific markers of diet quality. Twenty-two trials randomized participants to consume specific diets compared to control groups. Among the nine observational studies, several examined categories of total protein intake or adherence scores for a particular dietary pattern, where the macronutrient proportions of intake that were reported fell outside the AMDR.

Among the macronutrient distributions reported, the proportions of energy falling outside of the AMDR were as follows:

- Carbohydrate below the AMDR ranged between $25.3 \%$ and $44.9 \%$

- Carbohydrate above the AMDR ranged between $66 \%$ and $77.5 \%$

- Fat above the AMDR ranged between $35.2 \%$ and $47 \%$

- Fat below the AMDR ranged between $9.9 \%$ and $19.3 \%$

- Protein was above the AMDR in one study at $43.5 \%$

The majority of studies examined macronutrient distributions in which the proportion of energy from carbohydrate was below the AMDR, and fat above the AMDR, in at least one of the exposure groups compared. ${ }^{14,23,24,28,33,37,55,61,67-72,74-80,82-87}$ Few studies examined macronutrient distributions in which the proportion of energy from carbohydrate was above the AMDR, ${ }^{41,81,88}$ with the proportion from fat either below, 
above, or within the AMDR. Only one study examined macronutrient distributions within the AMDR for carbohydrate and protein, and above the AMDR for fat only. ${ }^{73}$

\section{Outcome assessment}

Outcomes were measured using standardized methods by trained staff across most studies. Included studies primarily utilized dual-energy X-ray absorptiometry (DXA) and/or bio-electrical impedance analysis (BIA) to assess body composition, calibrated stadiometers to obtain height, calibrated scales to obtain weight, and tape to measure waist circumference. Few studies used self-reported anthropometric data ${ }^{82,84,86}$ or provided limited information on outcome assessment methods. ${ }^{61,81,83}$ Included studies examined outcomes over follow-up ranging in the RCT's from 12wk to $2 y$, and in observational studies between $2 y$ and $20 y$. The following growth, size, body composition, and/or risk of overweight or obesity outcomes were reported:

- Overweight and/or obesity incidence, range of follow-up $12 \mathrm{wk}$ to $7 \mathrm{y}^{68,82,84-86}$

- Fat-mass and/or lean or fat-free mass $14,23,33,41,67-72,75,78,88$

- Body mass index (BMI) $)^{14,24,33,41,67-73,75,78,80,84,88}$

- Central adiposity or waist circumference $14,33,37,55,67,69,70,72,73,75,76,78,81,83$

- Absolute or change in body weight ${ }^{14,28,33,37,55,67,69-75,77-79,81,83,84,87,88}$

\section{Evidence synthesis}

Results reported in the studies included in this systematic review are summarized below by evidence that examined dietary patterns in children (Table 1), dietary patterns in adults, and diets based on macronutrient distributions in adults (Table 2).

\section{Dietary patterns: Children}

\section{Summary of results}

Index/score analysis

\section{Mediterranean related indices/scores}

Two studies reporting adherence to "Mediterranean" style indices in children were significantly associated with favorable outcomes.

- Martin-Calvo et $\mathrm{al}^{7}$ reported increased KidMed adherence scores were significantly associated with lower BMI after 7y follow-up.

- Tognon et al ${ }^{12}$ reported increased Mediterranean diet score adherence was significantly associated with lower gains in BMlz, waist-circumference, and waist-hip-ratio, and lower \% fat-mass after $2 \mathrm{y}$ follow-up, but no significant association with absolute waist circumference.

Country-specific indices

Chan She Ping-Delfos et al ${ }^{3}$ reported increased adherence to the Australian Dietary Guideline Index for Children and Adolescents (DGI-CA) was significantly associated with higher BMI, but lower waist-hip-ratio at 17y follow-up.

\section{Other indices/scores}

Two studies examined the relationship between dietary patterns in children using diet quality indices. 
- Hu et al ${ }^{6}$ reported increased a Priori Diet Quality Score (APDQS) adherence scores were significantly associated with lower BMI and reduced excess weight gain at age 25y.

- Nguyen et al ${ }^{8}$ reported that increased Diet Quality Score adherence scores at age $8 y$ were significantly associated with higher fat-free mass, BMI, and absolute weight and height at 10y. In stratification analyses, results for BMI and weight/height were only significant in girls. No significant association was observed between Diet Quality Score adherence scores at age 8y and \% body fat at age $10 y$.

Reduced rank regression

Ambrosini et al ${ }^{1}$ used reduced rank regression with predictor variables: \% $\mathrm{E}$ from free sugars, \%E from fat, dietary energy density (energy/g food consumed), dietary fiber density) to examine two dietary patterns at age 7y, "DP1" and "DP2". Greater adherence to DP1 (higher in confectionery and chocolate, cakes and biscuits, sugarsweetened beverages, low-fiber bread, and lower in fruit, vegetables, high-fiber bread, cereals) was significantly associated with higher FMlz, but not excess adiposity, at age 15y. No significant association was reported for adherence to DP2, which was higher in sugar-sweetened beverages, fruit juices, ready-to-eat breakfast cereals, and lower in whole milk, margarines and oils, cheese, crisps, and FMlz or excess adiposity at $15 \mathrm{y}$.

Factor/cluster/latent class analysis

Pala et al ${ }^{10}$ examined the following dietary patterns identified by factor/cluster analysis: "Snacking" [characterized by street foods such as sandwiches, savory pastries, chocolate bars], "Sweet" [characterized by chocolate spreads, biscuits and sweets/candy, fried meat, soft drinks], "Vegetables and Wholemeal" [characterized by vegetables, fruits, wholemeal cereal products, unsweetened milk], or "Protein and Water" [characterized by fish, meat, eggs, water]. Increased adherence to the "Vegetables and Wholemeal" [characterized by vegetables, fruits, wholemeal cereal products, unsweetened milk] was significantly associated with reduced risk of overweight or obesity after $2 y$ f/u ( age 4-12y). However, there was no significant association between any of the patterns and change in BMI after $2 y \mathrm{f} / \mathrm{u}$ ( age 4-12y)

Okubo et al ${ }^{9}$ reported adherence to the "Prudent" dietary pattern score [characterized by fish and shellfish, vegetables (salad, green, root other), other fruits] was significantly associated with lower fat-mass at age $6 \mathrm{y}$, but not significantly associated with $\mathrm{BMI}$ at age $6 \mathrm{y}$.

Smith et al ${ }^{11}$ reported increased adherence to the "Health Aware" dietary pattern, characterized by fruits and vegetables, high-fiber bread, pasta, cheese, and fish, lower loadings for chips (French fries), crisps (potato and corn snacks), processed meat, fizzy (carbonated) drinks] was significantly associated with lower fat-mass and lean mass gain at $11 \mathrm{y}$ in girls (valid and under-reporting), and boys (under-reporting). Adherence to the "Packed Lunch" dietary pattern, with higher loadings for low-fiber bread, margarine, cheese, cold meats, salty flavorings such as yeast extract, diet squash (dilutable soft drink), was significantly associated with lower fat-mass and lean mass gain at $11 \mathrm{y}$ in girls (valid and under-reporting), and under-reporting boys. 
Adherence to the "Traditional" dietary pattern, with higher loadings for meat, roast potatoes, vegetables, batter and pastry products, low energy-density sauces (e.g. gravy, desserts) was not significantly associated with fat-mass or lean mass.

Bull and Northstone ${ }^{2}$ reported that the "Processed" or the "Packed Lunch" vs. "Healthy" dietary patterns at age $7 \mathrm{y}$ were significantly associated with higher BMI at $17 \mathrm{y} f / \mathrm{u}$. However, the "Traditional" vs. Healthy dietary patterns at age 7y were not significantly associated with BMl at $17 \mathrm{y} f / u$. None of the dietary patterns at age $10 \mathrm{y}$ or $13 \mathrm{y}$ were associated with BMI at $17 y$ follow-up. Several key confounders were not adjusted for in analyses, including race/ethnicity, physical activity, and anthropometry.

Durao et $\mathrm{al}^{4}$ found that increased adherence to the energy-dense foods (EDF) pattern, characterized by sweets, soft drinks, salty pastry, processed meat, was significantly associated with higher FMI, BMIz, and waist-to-hip-ratio at 7y in girls, but associations were not significant in boys. There were no significant associations between consumption of either the "Snacking", or "Healthier" dietary patterns and FMI, BMIz, or waist-hip-ratio at $7 y$ in girls or boys.

Fernandez-Alvira et al ${ }^{5}$ reported that consistent consumption of the "Processed" dietary pattern (baseline to follow-up), or changing to a "Processed-Sweet" combination dietary pattern was significantly associated with higher FMI-z, BMIz, and waist-circumference z-score (WC-z) gain at 2y follow-up. Consumption of the "Processed" or "Sweet" compared to "Healthy" dietary pattern consumption at baseline was significantly associated with higher FMI-z, BMIz, and WC-z at follow-up. There were also many non-significant associations reported, such as those between "Processed" or "Sweet" compared to "Healthy" dietary pattern consumption at baseline and change in FFMIz at follow-up.

Overall, five studies reported increased adherence to indices or scores that emphasize vegetables, fruits, "high-fiber" or "wholegrain" bread, or wholemeal cereals were significantly associated with reduced risk of overweight/obesity ${ }^{10}$, lower BMI or reduced-gain in $\mathrm{BMI}, 6,7,12$ or lower fat mass. ${ }^{9}$ Associations with $\mathrm{BMI}$ were not significant in two of these studies. ${ }^{6,9}$ However, the studies also reported significantly lower gain in excess weight ${ }^{6}$, waist circumference, and waist-hip-ratio (WHR). ${ }^{12}$ Three studies reported dietary patterns characterized by sweets and refined grains (confectionary, biscuits, cakes, pastries), sugar-sweetened beverages, processed meats, and/or salty snacks were significantly associated with higher fat-mass index, BMlz, WHR and/or waist circumference-z-score.$^{1,4,5}$ In Duaro et al, ${ }^{4}$ the results were sex-specific (significant in girls but not boys). Additional non-significant associations were reported in these studies when examining different dietary patterns (e.g., "Healthy" vs."Processed"), or other outcomes (e.g., excess adiposity).

\section{Assessment of the evidence ${ }^{i v}$}

\footnotetext{
iv A detailed description of the methodology used for grading the strength of the evidence is available on the NESR website: https://nesr.usda.gov/2020-dietary-guidelines-advisory-committee-systematicreviews and in Part $\mathrm{C}$ of the following reference: Dietary Guidelines Advisory Committee. 2020. Scientific Report of the 2020 Dietary Guidelines Advisory Committee: Advisory Report to the Secretary of Agriculture and the Secretary of Health and Human Services. U.S. Department of Agriculture, Agricultural Research Service, Washington, DC.
} 
This systematic review update included 12 articles that examined dietary patterns in children and growth, size, body composition, and/or risk of overweight or obesity, met inclusion criteria, and were published between January 2014 and October 2019.

Dietary patterns and outcome measures were assessed using a variety of approaches across studies. Despite variability in methods, dietary patterns in childhood or adolescence that generally reflect poorer diet quality tended to associate with higher fat-mass index and BMI later in adolescence. As outlined and described below, the body of evidence examining dietary patterns in children and growth, size, body composition, and/or risk of overweight or obesity was assessed for the following elements used when grading the strength of evidence.

- Risk of bias: There were a number of potential risks of bias, or limitations, across the body of evidence that make interpretation of results difficult (Table 4). Most studies adjusted for a number of potential confounders, but did not account for all key confounders, such as race/ethnicity. Note that alcohol and smoking are listed on the analytic framework but were not treated as key confounders in the evidence with children or adolescence. Diet assessment methods varied in validity and reliability. Most studies assessed dietary patterns once at baseline, though a few assessed at each age/time-point to account for change in dietary intake over follow-up. However, many used methods that were not necessarily valid, reliable, or applicable in children. Therefore, those studies are at higher risk of bias due to classification of exposure and departure from intended exposure

- Consistency: All 12 articles came from observational studies that used different approaches to assess dietary patterns, with about half each using indices/scores or factor analysis. Among those using indices/scores, each used a different indice/score to assess adherence to different dietary patterns. Multiple approaches to examine dietary patterns were used that varied between studies. Several studies examined dietary patterns that emphasize vegetables, fruits, "high-fiber" or "wholegrain" bread, or wholemeal cereals. Several of the dietary patterns emphasized intake of fish and shellfish, legumes and nuts, reduced-fat or low-fat dairy, and olive oil, but these elements were less consistent across the body of evidence. Whereas, other studies examined dietary patterns characterized by sweets and refined grains (confectionary, biscuits, cakes, pastries), sugar-sweetened beverages, processed meats, and/or salty snacks. Across the dietary patterns examined in children, there was also variation in the level of specificity within included components e.g., "unsweetened milk" vs. "dairy" vs. "reduced- or low-fat dairy". Across the body of evidence, the direction of findings was relatively split depending on the dietary pattern examined or weight status/body composition outcome reported. Effect sizes were relatively small across the body of evidence (e.g., 0.04-SD increase in FMI, or -0.04 decrease in BMI).

- Directness: The populations, intervention, comparators, and outcomes of interest in the included studies are directly related to the systematic review question.

- Precision: Most of the studies did not report power analyses or sample size calculations, but had relatively large analytic sample sizes. 
- Generalizability: The study participants, interventions and/or exposures, comparators, and outcomes examined in the body of evidence are applicable to the U.S. population.

\section{Dietary patterns: Adults}

The 2020 Dietary Guidelines Advisory Committee conducted a systematic evidence scan to identify and examine new evidence published since a review done by the 2015 Dietary Guidelines Advisory Committee.. ${ }^{\vee}$

Results across the recently published body of evidence were generally consistent with the conclusion from the 2015 Committee, showing that dietary patterns of higher diet quality were typically associated with favorable weight status outcomes. Across the studies, the results for the Mediterranean and DASH dietary patterns showed the most consistent significant effects towards healthier weight status. Few studies showed no significant association, in particular those examining Nordic dietary patterns and weight status outcomes. The populations, intervention, comparators, and outcomes of interest are directly related to the systematic review question. The magnitude of effect across the body of evidence is relatively small for body weight, BMI, and waist circumference. The evidence is primarily from prospective cohort studies, and the number of RCTs within this newly published set of evidence is limited. Across these studies, there was insufficient evidence to determine a relationship between dietary patterns in adults and other measures of body composition and/or distribution, such as visceral adipose tissue, subcutaneous fat, and ectopic fat. The study participants, interventions and/or exposures, comparators, and outcomes examined in the body of evidence are applicable to the U.S. population.

The existing conclusion statement from the 2015 Committee noted that the included evidence reported favorable outcomes related to body weight with overall dietary patterns that may include moderate alcoholic beverage intake in adults. However, for the newly published evidence, inclusion of moderate alcohol intake within an overall dietary pattern may not necessarily be recommended, particularly with regards to prevention of co-morbid conditions that may be exacerbated with alcoholic beverage intake e.g. hypertension, cancer, etc. Studies that examine overall dietary patterns in adults often vary in how alcohol intake is measured, the thresholds applied for amounts of alcohol consumed, and scoring procedures of alcohol as a component (e.g., a positive component, positive in moderation, or negative component). Due to this inconsistency, the inclusion and specification of alcoholic beverages within dietary patterns warrants further investigation.

The 2020 Committee determined that the conclusion drawn by the 2015 Committee still reflects the current state of science on dietary patterns in adults and risk of type 2

${ }^{\vee}$ Dietary Guidelines Advisory Committee. Scientific Report of the 2015 Dietary Guidelines Advisory Committee: Advisory Report to the Secretary of Health and Human Services and the Secretary of Agriculture. US Department of Agriculture, Agricultural Research Service. https://health.gov/our-work/food-nutrition/20152020-dietary-guidelines/advisory-report. Published 2015. Accessed April 30, 2020. 
diabetes. Therefore, a full systematic review was not conducted at this time, and the 2015 Committee's conclusion statement and grade were carried forward. The 2015 Committee concluded that: Moderate evidence indicates dietary patterns emphasizing vegetables, fruits, and whole grains; seafood and legumes; moderate in dairy products (particularly low and non-fat dairy) and alcohol; lower in meats (including red and processed meats), and low in sugar-sweetened foods and beverages, and refined grains are associated with favorable outcomes related to body weight, including lower BMI, waist circumference, or percent body fat, or risk of obesity. Components of the dietary patterns associated with these favorable outcomes include higher intakes of unsaturated fats and lower intakes of saturated fats, cholesterol, and sodium.

\section{Diets based on macronutrient distribution: Children}

No studies identified that met inclusion criteria examined diets based on macronutrient proportion in children.

\section{Diets based on macronutrient distribution: Adults}

\section{Summary of results}

Thirty-one studies examined diets based on macronutrient distributions with proportions of energy falling outside of the AMDR for at least one macronutrient and growth, size, body composition, and/or risk of overweight or obesity outcomes. The 31 articles included 22 articles from RCTs (Table 2) and 9 articles from prospective cohort studies (Table 3).

Results across the body of evidence were mixed, depending on various factors including study design, the macronutrient distribution examined, and/or the outcome reported. Significant associations were reported in 14 of the 31 included articles. ${ }^{23,28,41,55,61,72-75,81,83-85,87}$ However, the majority of these studies also reported null, or mixed associations depending on the intervention/exposure group and/or specific outcome examined. No significant associations between diets based on macronutrient distribution and all outcomes were reported in 17 of the 31 included articles. ${ }^{14,24,33,37,67-71,76-80,82,86,88}$

\section{Randomized controlled trials (RCTs)}

Most of the RCTs did not show significantly meaningful differences between diet groups compared based on variations in macronutrient intakes and body size or composition outcomes. A number of these studies did report significant results, but the relationship between the macronutrient distribution and outcomes were indirect and limited by several methodological considerations.

Due et $\mathrm{al}^{72}$ examined the effects of a "low-fat" diet within AMDRs for all macronutrients (23.6\% Fat) and a "high monounsaturated (MUFA) fat" diet consisting of carbohydrate below and fat above the AMDR with $>20 \%$ from MUFA. Results supported significantly decreased fat mass in participants following either diet relative to a control diet at $32.1 \%$ fat.

Gardner et al $^{33}$ tested 'low-fat' compared to 'low-carbohydrate' diets without energy restriction on weight change in participants with BMI between 28 and $40 \mathrm{~kg} / \mathrm{m}^{2}$, 
reporting no clinically meaningful differences in \% body fat, BMI, or waist circumference after $12 \mathrm{mo}$.

Howard et al $^{73}$ tested diets at varied levels of reduced fat intake within the AMDR compared to control diets with fat intake above the AMDR from the Women's Health Initiative, reporting lower BMI, waist circumference and body weight after 1 year, but ultimately no significant differences between groups at the end of 6 y follow-up.

Kahleova et al ${ }^{41}$ examined the effect of a "low-fat vegan" diet based on different macronutrient distribution with fat below the AMDR at $17.5 \%$ energy, compared to fat intake within the AMDR. Participants consuming the "Low-fat vegan" diet had significantly lower lean mass, fat mass, and BMI at 16 weeks. Notably, the duration of follow-up in this study was relative short.

Turner-McGrievy et al ${ }^{61}$ tested the effects of plant-based based dietary patterns on weight loss without energy restriction and observed significant weight loss in all groups after 6mo. Those assigned to consume a "Vegan" diet lost the most weight compared to either "Semi-vegetarian", "Pesco-vegetarian", or "Omnivore" diets. It should be noted that the study was not powered to detect differences between the "Vegan" compared to "Vegetarian" groups.

Paineau et $\mathrm{al}^{75}$ compared reduced fat intake within the AMDR at $32.4 \%$ and reduced fat intake with reduced sugar intake to a control diet with fat intake above the AMDR at $35.6 \%$ fat. Reducing fat within the AMDR with reduced sugar intake was significantly associated with lower fat mass and BMI at 8mo follow-up, but no significant differences were observed in the group that only reduced fat within the AMDR compared to the control group. It should be noted that participants were parents, with their children concomitantly enrolled in the intervention to improve weight and other health outcomes in the family.

Wan et al ${ }^{81}$ assessed "low", "moderate", and "high" levels of fat and carbohydrate intake, with the proportion of fat above the AMDR at $40 \%$ in the "high-fat" distribution, and proportion of carbohydrate slightly above the AMDR at $66 \%$ of energy in the "highcarbohydrate". All other proportions, including "low-fat" were within the AMDR. Lowerfat intake within the AMDR and carbohydrate near or within the AMDR at $66 \%$ or $56 \%$ energy were significantly associated with lower fat mass, BMI, waist circumference, or weight at 6 mo follow-up.

Larsen et $\mathrm{al}^{74}$ reported significant weight regain in previously weight-reduced participants assigned to the "low protein, high glycemic index" diet within AMDRs for all macronutrients. Individuals in the high protein groups, with either high or low glycemic index levels, had $0.93 \mathrm{~kg}$ less weight regain. In the "high-protein" groups' mean intake from protein was within the AMDR at $21.7 \%-22.6 \%$ of energy and intake from carbohydrate was modestly below the AMDR at $43.0 \%-44.7 \%$ of energy.

Reidlinger et al ${ }^{55}$ reported smaller waist circumference and lower weight after 12 weeks in the "dietary guidelines" diet group compared to a control diet, which had a distribution with proportions of carbohydrate below the AMDR and Fat above the AMDR. While this trial had some small differences between intervention groups in all macronutrients, the overriding difference between arms could be the quality of intake with the "dietary guidelines" group consuming more whole grains and reducing sugar 
and salt intake.

Daly et $\mathrm{al}^{23}$ enrolled all older women engaged in resistance training to determine the effects of consuming lean red meat compared to pasta/rice, with carbohydrate intake below the AMDR in both intervention groups at $41 \%$ and $42 \%$. Results showed a significant impact on lean mass in those consuming lean red meat.

All remaining RCTs included in the body of evidence reported no significant effects between diet groups and reported outcomes, including risk of overweight or obesity, lean mass, fat mass, BMI, waist circumference or waist-to-hip ratio, or body weight. ${ }^{14,24,67-71,76-80}$ Several of these studies specifically examined the effects of "Mediterranean" diets on weight and body composition outcomes. ${ }^{14,24,76,80}$ None of the studies examining "Mediterranean" diets reported significant effects between groups on weight, BMI, \% body fat, fat-free mass, truncal fat-mass, waist circumference over follow-up ranging between $4 \mathrm{mo}$ and $3 \mathrm{y}$. The macronutrient distribution for the "Mediterranean" patterns examined in those studies had proportions of carbohydrate slightly below and fat slightly above the AMDR, but because the trials were testing the different dietary patterns, there was often minor contrast between macronutrient distributions in the groups compared. In some cases, both intervention and control groups had proportions similarly outside of the AMDR in the same direction (e.g., $37.8 \%$ carbohydrate, $38.8 \%$ fat, $19.4 \%$ protein vs. $40.3 \%$ carbohydrate, $35.6 \%$ fat, $19.2 \%$ protein). In other studies, the contrast was substantial (e.g., $37.0 \%$ carbohydrate, $47.0 \%$ fat, $13.8 \%$ protein vs. $69.0 \%$ carbohydrate, $14.0 \%$ fat, $14.0 \%$ protein), yet there were no significant effects detected.

\section{Observational studies}

Five observational studies reported significant results and all examined proportions of carbohydrate below and fat above the AMDR. Beunza et al ${ }^{84}$ reported that high Mediterranean Diet Score (MDS), with proportions of carbohydrate and fat within the AMDR, were significantly associated with lower weight gain over follow-up (at 4y) compared to "low MDS" scores with carbohydrate slightly below and fat above the AMDR. Ericson et $\mathrm{al}^{28}$ reported that higher consumption of the "health-conscious" dietary pattern, with proportions of carbohydrate slightly below and fat above the AMDR, were significantly associated with greater weight change over $10 \mathrm{y}$ in men, but when diet was examined continuously, results were not significant. There were no other significant associations in men or women when examining other dietary patterns. Quatromoni et $\mathrm{al}^{87}$ reported higher adherence to the diet quality index (DQI), with proportions of energy within compared to outside the AMDR, was significantly associated with reduced weight gain during 8 y follow-up in men or women. In the study by Ankarfeldt et al, ${ }^{83}$ results from the MONICA cohort reported a $5 \%$ substitution of protein for carbohydrate was associated with a small but statistically significant weight change of 123.4 g/year over 5 years. However, overall, substituting $5 \%$ of energy from protein for either carbohydrate or fat did not result in significant weight change. There were no significant associations reported in the other cohorts examined in that study. Bujnowski et $\mathrm{al}^{85}$ reported greater weight gain over 7 years in men with higher total- and animal-protein intake, but no significant association when examining 
macronutrient distribution based on vegetable-protein intake.

The remaining studies reported no significant associations between diets based on macronutrient distribution compared, with most examining intake of carbohydrate below the AMDR. ${ }^{37,82,86,88}$ In most, the lowest proportion of carbohydrate was associated with the poorest diet quality, if foods or food groups consumed in the diet were reported.

Determining the influence of macronutrient intake among these results is challenging. Although results show that macronutrient distributions of higher diet quality were generally associated with less weight gain over time, the range of carbohydrate intake was commonly not varied within or across studies, and the proximity to the AMDR limits were generally very close (e.g., 66\% carbohydrate). For example, two of the studies reporting significant results compared diets with macronutrient distributions where all levels of carbohydrate intake compared were below the AMDR, and all levels of fat intake compared were above the AMDR. In addition, the primary focus was protein intake in several of the observational studies, but all proportions of protein were within the AMDR and carbohydrate and fat were varied outside the AMDR. ${ }^{37,85,88}$

\section{Assessment of the evidence ${ }^{v i}$}

This systematic review update included 31 articles that examined examined diets based on macronutrient distribution and growth, size, body composition, and/or risk of overweight or obesity, met inclusion criteria, and were published between January 2000 and October 2019.

Most of the articles examined macronutrient distributions in which the proportion of energy from carbohydrate was below the AMDR; Fat was above the AMDR; and protein was within the AMDR in at least one of the exposure groups compared. From a study design perspective, most of the clinical trials had control or comparator exposures that featured a different dietary pattern, either prescribed as a study intervention or as usual intake. The observational studies used derived food patterns or index scores as comparators, generally comparing poorer quality diets to higher quality alternatives. Therefore, across study designs, the comparisons were primarily of different dietary patterns and food groups that resulted in differing macronutrient proportions rather than comparing a different distribution of macronutrients within the same pattern of food intake. As a function of these study designs, the differences in macronutrient proportions between intervention arms were either limited in magnitude, of a similar direction relative to the AMDR (e.g., all below or above the AMDR), only modestly different from the AMDR, or only relevant for a limited subset of the study population. Several included articles were primary or secondary analyses from the PREDIMED trial, which was subject to randomization issues established after publication of the initial study. However, the

\footnotetext{
vi A detailed description of the methodology used for grading the strength of the evidence is available on the NESR website: https://nesr.usda.gov/2020-dietary-guidelines-advisory-committee-systematicreviews and in Part $C$ of the following reference: Dietary Guidelines Advisory Committee. 2020. Scientific Report of the 2020 Dietary Guidelines Advisory Committee: Advisory Report to the Secretary of Agriculture and the Secretary of Health and Human Services. U.S. Department of Agriculture, Agricultural Research Service, Washington, DC.
} 
republished results confirmed the initial findings, even after accounting for participants that were subject to protocol deviation.

Multiple databases were used to obtain publications from a large, comprehensive search. Although several studies were from large prospective cohorts, studies with smaller sample sizes were also included and reported both significant and nonsignificant results. Therefore, this risk of publication bias is low. As outlined and described below, the body of evidence examining diets based on macronutrient distribution and growth, size, body composition, and/or risk of overweight or obesity was assessed for the following elements used when grading the strength of evidence.

- Risk of bias: There were a number of potential risks of bias, or limitations, across the body of evidence that make interpretation of results difficult (Table 5 and Table 6). While studies adjusted for most potential confounders, they did not adjust for all key confounders, such as race/ethnicity, alcohol intake, and/or physical activity. Most of the observational studies and several RCT's used self-reported methods that varied to obtain dietary intake, derive macronutrient intake, and/or assess diet adherence. Most studies selected participants that were free of chronic disease or other conditions at baseline, and are therefore at a higher risk of selection bias. It is likely that the reported results are reflective of generally healthier populations. Several studies did not assess the impact of missing data, either due to lost to follow-up or criteria used when selecting subjects into the analyses. Although several studies reported using standardized methods to collect or calculate anthropometric measures, several studies are at higher risk of bias due to the use of self-reported data or providing limited information on outcome assessment methods.

- Consistency: Study design varied in the body of evidence, with 22 articles from RCTs and 9 articles from prospective cohort studies. Results across studies were inconsistent in direction and magnitude. There was some evidence to suggest that diets based on macronutrient distributions within or closer to the AMDR compared to those that were outside the AMDR (below or above) are associated with favorable weight status outcomes, such as fat-mass, BMl, waist circumference, and/or change in weight, typically if the diet was reflective of higher quality. Among the macronutrient distributions within the AMDR that suggested favorable outcomes, several were comprised of vegetables, fruit, whole-grains, vegetable oils, nuts, pulses and/or lean meat or fish. However, the foods/food groups were inconsistently reported. Proportions of energy associated with these results varied for carbohydrate below the AMDR between $30.9 \%$ and $44.9 \%$, for carbohydrate above the AMDR between $66 \%$ and $69.6 \%$, and for fat above the AMDR between $35.6 \%$ and $41 \%$. Although statistically significant relationships were reported, the gradient between macronutrient distributions was relatively narrow within studies and between studies (e.g., $45.3 \%$ vs. $43.8 \%$ carbohydrate). Several studies compared sufficiently distinct proportions between groups, whereas other proportions were much closer in proximity relative to one another, or to the actual AMDR cut-off (e.g., $44.9 \%$ vs. $45 \%$ ). Studies varied in terms of the distribution of macronutrients that were examined and the methods used to estimate nutrient intakes, derive the 
proportion of energy from macronutrients, and/or apply substitution modelling (e.g., multivariate-nutrient density methods).

- Directness: Several studies reported the intent to directly examine different diets based on macronutrient distribution and the outcome of interest. However, for this review, an important limitation to highlight is that many of the studies were not designed to intentionally examine macronutrient proportions outside of the AMDR. Additionally, most of the studies assessed different dietary patterns as the primary comparators rather than using a common dietary pattern and adjusting macronutrient proportions within that dietary context. Due to the variety of methods used to estimate macronutrient intake and adjust intake for total energy, the confidence in the estimated proportions falling outside the AMDR is low. The gradient between proportions compared within macronutrients varied and in some cases, may not have been large enough to distinguish differences in the outcomes between intervention or exposure groups compared. Several studies reported to be examining "high" or "low" levels of one particular macronutrient of interest, but the proportion for that nutrient was typically within the AMDR. Due to these issues, directness across the body of evidence could not be adequately assessed.

- Precision: Of the 22 RCTs, all demonstrated adequate power but had generally smaller sample sizes and in some cases, a very small sample size $(n=16)$. Two were insufficiently powered to detect differences either in the outcome of interest or between some exposure groups. The assessment of variance within these studies was relatively narrow, but several indicate wider variance in results. Observational studies did not report sample size or power calculations but did have large analytic sample sizes, ranging from $n=1,107$ to $n=20,487$.

- Generalizability: The study participants, interventions and/or exposures, comparators, and outcomes examined in the body of evidence are applicable to the U.S. population. Results across studies may be less generalizable due to differences between countries in terms of national recommendations and macronutrient distributions compared. For example, several of the macronutrient distributions compared were from populations that consume high amounts of rice with carbohydrate well above the AMDR and fat well below the AMDR, which is not typical of diets in the United States.

\section{Research recommendations}

In order to better assess the relationship between dietary patterns and/or diets based on macronutrient distribution and growth, size, body composition, and/or risk of overweight or obesity, future research may:

1. Determine the impact that dietary patterns, independent of adiposity and/or weight-loss, may have on risk of type 2 diabetes, cardiovascular disease, and/or obesity.

2. Conduct well-designed, sufficiently powered, randomized controlled trials, including controlled feeding studies that examine the relationship between 
dietary patterns and growth, size, body composition, and risk of overweight or obesity; particularly lacking are studies that examine dietary patterns in children and adolescents.

3. Identify dietary patterns in childhood that may confer benefit to health outcomes including favorable growth and/or cardiometabolic health factors, over the lifecourse.

4. Develop a standardized definition for what constitutes a "very-low carbohydrate", "low-carbohydrate", "carbohydrate-restricted", and/or "ketogenic diet" dietary pattern based on macronutrient distribution.

5. Examine the relationship between diets based on macronutrient distributions that test proportions of energy from carbohydrate below $25 \%$, within the context of specific dietary patterns, to determine if and what relationship may exist between macronutrient-based dietary patterns and the prevention of obesity or avoidance of excess weight gain. These types of studies need to adequately control for the quality of food intake.

6. Examine additional body fat distribution outcomes and in particular, ectopic fat.

7. Explore the role that macronutrient quality may have in the relationship between "low-carbohydrate" diets and the prevention of obesity (i.e. effect modification), within the context of a constant dietary pattern.

8. Assess information regarding diet at more than one time-point, preferably during the course of follow-up and with more objective measures from improved technologies, to facilitate understanding the relationship between dietary patterns over time and risk of overweight/obesity.

9. Differentiate specific foods and food groups, in particular, the types and amounts, relevant biomarkers, and repeated measures to facilitate both preventive strategies and overall outcomes

10. Include continual updating of systematic reviews that examine the relationship between dietary patterns and growth, size, body composition, and risk of overweight/obesity to incorporate the rapidly emerging and overwhelming volume of literature on this topic, particularly in recent years. 


\section{Included articles}

1. Ambrosini GL, Johns DJ, Northstone K, Emmett PM, Jebb SA. Free sugars and total fat are important characteristics of a dietary pattern associated with adiposity across childhood and adolescence. J Nutr. 2016;146(4):778-784. doi:10.3945/jn.115.224659.

2. Bull CJ, Northstone K. Childhood dietary patterns and cardiovascular risk factors in adolescence: results from the Avon Longitudinal Study of Parents and Children (ALSPAC) cohort. Public Health Nutr. 2016;19(18):3369-3377. doi:10.1017/s1368980016001592.

3. Chan She Ping-Delfos WL, Beilin LJ, Oddy WH, Burrows S, Mori TA. Use of the Dietary Guideline Index to assess cardiometabolic risk in adolescents. Br J Nutr. 2015;113(11):1741-1752. doi:10.1017/s0007114515001026.

4. Durao C, Severo M, Oliveira A, et al. Association between dietary patterns and adiposity from 4 to 7 years of age. Public Health Nutr. 2017;20(11):1973-1982. doi:10.1017/s1368980017000854.

5. Fernandez-Alvira JM, Bammann K, Eiben G, et al. Prospective associations between dietary patterns and body composition changes in European children: the IDEFICS study. Public Health Nutr. 2017;20(18):3257-3265. doi:10.1017/s1368980017002361.

6. Hu T, Jacobs DR, Jr., Larson NI, Cutler GJ, Laska MN, Neumark-Sztainer D. Higher diet quality in adolescence and dietary improvements are related to less weight gain during the transition from adolescence to adulthood. J Pediatr. 2016;178:188-193.e183. doi:10.1016/j.jpeds.2016.08.026.

7. Martin-Calvo N, Chavarro JE, Falbe J, Hu FB, Field AE. Adherence to the Mediterranean dietary pattern and BMI change among US adolescents. Int $\mathrm{J}$ Obes 2016;40(7):1103-1108. doi:10.1038/ijo.2016.59.

8. Nguyen AN, Jen $\mathrm{V}$, Jaddoe VWV, et al. Diet quality in early and mid-childhood in relation to trajectories of growth and body composition. Clin Nutr. 2020;39(3):845-852. doi:10.1016/j.clnu.2019.03.017.

9. Okubo H, Crozier SR, Harvey NC, et al. Diet quality across early childhood and adiposity at 6 years: the Southampton Women's Survey. Int J Obes. 2015;39(10):14561462. doi:10.1038/ijo.2015.97.

10. Pala V, Lissner L, Hebestreit A, et al. Dietary patterns and longitudinal change in body mass in European children: a follow-up study on the IDEFICS multicenter cohort. Eur J Clin Nutr. 2013;67(10):1042-1049. doi:10.1038/ejcn.2013.145.

11. Smith AD, Emmett PM, Newby PK, Northstone K. Dietary patterns and changes in body composition in children between 9 and 11 years. Food Nutr Res. 2014;58.

doi:10.3402/fnr.v58.22769.

12. Tognon G, Hebestreit A, Lanfer A, et al. Mediterranean diet, overweight and body composition in children from eight European countries: cross-sectional and prospective results from the IDEFICS study. Nutr Metab Cardiovasc Dis. 2014;24(2):205-213. doi:10.1016/j.numecd.2013.04.013.

13. Agnoli C, Sieri S, Ricceri F, et al. Adherence to a Mediterranean diet and long-term changes in weight and waist circumference in the EPIC-Italy cohort. Nutr Diabetes. 2018;8(1):22. doi:10.1038/s41387-018-0023-3. 
14. Alvarez-Perez J, Sanchez-Villegas A, Diaz-Benitez EM, et al. Influence of a Mediterranean dietary pattern on body fat distribution: results of the PREDIMEDCanarias intervention randomized trial. J Am Coll Nutr. 2016;35(6):568-580. doi:10.1080/07315724.2015.1102102.

15. Arabshahi S, Ibiebele TI, Hughes MCB, Lahmann PH, Williams GM, van der Pols JC. Dietary patterns and weight change: 15-year longitudinal study in Australian adults. Eur J Nutr. 2017;56(4):1455-1465. doi:10.1007/s00394-016-1191-3.

16. Babio N, Toledo E, Estruch R, et al. Mediterranean diets and metabolic syndrome status in the PREDIMED randomized trial. CMAJ. 2014;186(17):E649-657. doi:10.1503/cmaj.140764.

17. Canhada SL, Luft VC, Giatti L, et al. Ultra-processed foods, incident overweight and obesity, and longitudinal changes in weight and waist circumference: the Brazilian Longitudinal Study of Adult Health (ELSA-Brasil). Public Health Nutr. 2020;23(6):10761086. doi:10.1017/s1368980019002854.

18. Casas R, Sacanella E, Urpi-Sarda M, et al. The effects of the Mediterranean diet on biomarkers of vascular wall inflammation and plaque vulnerability in subjects with high risk for cardiovascular disease. A randomized trial. PLoS One. 2014;9(6):e100084. doi:10.1371/journal.pone.0100084.

19. Casas R, Sacanella E, Urpi-Sarda M, et al. Long-term immunomodulatory effects of a Mediterranean diet in adults at high risk of cardiovascular disease in the PREvencion con Dleta MEDiterranea (PREDIMED) randomized controlled trial. J Nutr. 2016;146(9):1684-1693. doi:10.3945/jn.115.229476.

20. Cespedes Feliciano EM, Tinker L, Manson JE, et al. Change in dietary patterns and change in waist circumference and DXA trunk fat among postmenopausal women. Obesity. 2016;24(10):2176-2184. doi:10.1002/oby.21589.

21. Chen Z, Schoufour JD, Rivadeneira F, et al. Plant-based diet and adiposity over time in a middle-aged and elderly population: the Rotterdam Study. Epidemiology. 2019;30(2):303-310. doi:10.1097/ede.0000000000000961.

22. Czekajlo A, Rozanska D, Zatonska K, Szuba A, Regulska-llow B. Association between dietary patterns and metabolic syndrome in the selected population of Polish adults-results of the PURE Poland Study. Eur J Public Health. 2019;29(2):335-340. doi:10.1093/eurpub/cky207.

23. Daly RM, O'Connell SL, Mundell NL, Grimes CA, Dunstan DW, Nowson CA. Protein-enriched diet, with the use of lean red meat, combined with progressive resistance training enhances lean tissue mass and muscle strength and reduces circulating IL-6 concentrations in elderly women: a cluster randomized controlled trial. Am J Clin Nutr. 2014;99(4):899-910. doi:10.3945/ajcn.113.064154.

24. Davis CR, Bryan J, Hodgson JM, Woodman R, Murphy KJ. A Mediterranean diet reduces F2-isoprostanes and triglycerides among older Australian men and women after 6 months. J Nutr. 2017;147(7):1348-1355. doi:10.3945/jn.117.248419. 25. Davis CR, Hodgson JM, Woodman R, Bryan J, Wilson C, Murphy KJ. A Mediterranean diet lowers blood pressure and improves endothelial function: results from the MedLey randomized intervention trial. Am J Clin Nutr. 2017;105(6):1305-1313. doi:10.3945/ajcn.116.146803.

26. Eguaras S, Toledo E, Buil-Cosiales P, et al. Does the Mediterranean diet counteract the adverse effects of abdominal adiposity? Nutr Metab Cardiovasc Dis.

2015;25(6):569-574. doi:10.1016/j.numecd.2015.03.001. 
27. Eguaras S, Toledo E, Hernandez-Hernandez A, Cervantes S, Martinez-Gonzalez MA. Better adherence to the Mediterranean diet could mitigate the adverse consequences of obesity on cardiovascular disease: the SUN Prospective Cohort. Nutrients. 2015;7(11):9154-9162. doi:10.3390/nu7115457.

28. Ericson U, Brunkwall L, Alves Dias J, et al. Food patterns in relation to weight change and incidence of type 2 diabetes, coronary events and stroke in the Malmo Diet and Cancer cohort. Eur J Nutr. 2019;58(5):1801-1814. doi:10.1007/s00394-018-17279.

29. Estruch R, Martinez-Gonzalez MA, Corella D, et al. Effect of a high-fat Mediterranean diet on bodyweight and waist circumference: a prespecified secondary outcomes analysis of the PREDIMED randomised controlled trial. Lancet Diabetes Endocrinol. 2019;7(5):e6-e17. doi:10.1016/s2213-8587(19)30074-9.

30. Ford C, Chang S, Vitolins MZ, et al. Evaluation of diet pattern and weight gain in postmenopausal women enrolled in the Women's Health Initiative Observational Study. Br J Nutr. 2017;117(8):1189-1197. doi:10.1017/s0007114517000952.

31. Fung TT, Pan A, Hou T, et al. Long-term change in diet quality Is associated with body weight change in men and women. $J$ Nutr. 2015;145(8):1850-1856.

doi:10.3945/jn.114.208785.

32. Funtikova AN, Benitez-Arciniega AA, Gomez SF, Fito M, Elosua R, Schroder H. Mediterranean diet impact on changes in abdominal fat and 10-year incidence of abdominal obesity in a Spanish population. Br J Nutr. 2014;111(8):1481-1487. doi:10.1017/s0007114513003966.

33. Gardner CD, Trepanowski JF, Del Gobbo LC, et al. Effect of low-fat vs lowcarbohydrate diet on 12-month weight loss in overweight adults and the association with genotype pattern or insulin secretion: the DIETFITS randomized clinical trial. JAMA. 2018;319(7):667-679. doi:10.1001/jama.2018.0245.

34. Gomez-Donoso C, Martinez-Gonzalez MA, Gea A, Murphy KJ, Parletta N, BesRastrollo M. A food-based score and incidence of overweight/obesity: The Dietary Obesity-Prevention Score (DOS). Clin Nutr. 2019;38(6):2607-2615.

doi:10.1016/j.clnu.2018.11.003.

35. Gomez-Donoso C, Martinez-Gonzalez MA, Martinez JA, et al. A provegetarian food pattern emphasizing preference for healthy plant-derived foods reduces the risk of overweight/obesity in the SUN Cohort. Nutrients. 2019;11(7). doi:10.3390/nu11071553. 36. Hennein R, Liu C, McKeown NM, et al. Increased Diet Quality is Associated with Long-Term Reduction of Abdominal and Pericardial Fat. Obesity. 2019;27(4):670-677. doi:10.1002/oby.22427.

37. Hruby A, Jacques PF. Dietary protein and changes in markers of cardiometabolic health across 20 years of follow-up in middle-aged Americans. Public Health Nutr. 2018;21(16):2998-3010. doi:10.1017/s1368980018001854.

38. Jenkins DJA, Boucher BA, Ashbury FD, et al. Effect of current dietary recommendations on weight loss and cardiovascular risk factors. J Am Coll Cardiol. 2017;69(9):1103-1112. doi:10.1016/j.jacc.2016.10.089.

39. Johns DJ, Lindroos AK, Jebb SA, Sjostrom L, Carlsson LM, Ambrosini GL. Dietary patterns, cardiometabolic risk factors, and the incidence of cardiovascular disease in severe obesity. Obesity 2015;23(5):1063-1070. doi:10.1002/oby.20920. 40. Kafeshani M, Janghorbani M, Salehi R, Kazemi M, Entezari MH. Dietary Approaches to Stop Hypertension influence on insulin receptor substrate-1gene 
expression: a randomized controlled clinical trial. J Res Med Sci. 2015;20(9):832-837. doi:10.4103/1735-1995.170596.

41. Kahleova H, Dort S, Holubkov R, Barnard ND. A plant-based high-carbohydrate, low-fat diet in overweight individuals in a 16-week randomized clinical trial: the role of carbohydrates. Nutrients. 2018;10(9). doi:10.3390/nu10091302.

42. Kanerva N, Harald K, Mannisto S, et al. Adherence to the healthy Nordic diet is associated with weight change during 7 years of follow-up. Br J Nutr. 2018;120(1):101110. doi:10.1017/s0007114518001344.

43. Konieczna J, Yanez A, Monino M, et al. Longitudinal changes in Mediterranean diet and transition between different obesity phenotypes. Clin Nutr. 2020;39(3):966-975. doi:10.1016/j.clnu.2019.04.002.

44. Leon-Munoz LM, Garcia-Esquinas E, Lopez-Garcia E, Banegas JR, RodriguezArtalejo F. Major dietary patterns and risk of frailty in older adults: a prospective cohort study. BMC Med. 2015;13:11. doi:10.1186/s12916-014-0255-6.

45. Li Y, Roswall N, Strom P, Sandin S, Adami HO, Weiderpass E. Mediterranean and Nordic diet scores and long-term changes in body weight and waist circumference: results from a large cohort study. Br J Nutr. 2015;114(12):2093-2102.

doi:10.1017/s0007114515003840.

46. Li P, Zhang M, Zhu Y, et al. Dietary patterns and changes in cardiovascular risk factors in apparently healthy Chinese women: a longitudinal study. J Clin Biochem Nutr. 2016;58(3):232-239. doi:10.3164/jcbn.15-78.

47. Lima ST, da Silva Nalin de Souza B, Franca AK, Salgado Filho N, Sichieri R. Dietary approach to hypertension based on low glycaemic index and principles of DASH (Dietary Approaches to Stop Hypertension): a randomised trial in a primary care service. Br J Nutr. 2013;110(8):1472-1479. doi:10.1017/s0007114513000718. 48. Maskarinec G, Lim U, Jacobs S, et al. Diet quality in midadulthood predicts visceral adiposity and liver fatness in older ages: the Multiethnic Cohort Study. Obesity. 2017;25(8):1442-1450. doi:10.1002/oby.21868.

49. Mattei J, Sotos-Prieto M, Bigornia SJ, Noel SE, Tucker KL. The Mediterranean diet score is more strongly associated with favorable cardiometabolic risk factors over 2 years than other diet quality indexes in Puerto Rican adults. $J$ Nutr. 2017;147(4):661669. doi:10.3945/jn.116.245431.

50. Mendonca RD, Pimenta AM, Gea A, et al. Ultraprocessed food consumption and risk of overweight and obesity: the University of Navarra Follow-Up (SUN) cohort study. Am J Clin Nutr. 2016;104(5):1433-1440. doi:10.3945/ajcn.116.135004.

51. Mirmiran P, Moslehi N, Mahmoudof H, Sadeghi M, Azizi F. A longitudinal study of adherence to the Mediterranean dietary pattern and metabolic syndrome in a nonMediterranean population. Int J Endocrinol Metab. 2015;13(3):e26128. doi:10.5812/ijem.26128v2.

52. Olstad DL, Lamb KE, Thornton LE, et al. Prospective associations between diet quality and body mass index in disadvantaged women: the Resilience for Eating and Activity Despite Inequality (READI) study. Int J Epidemiol. 2017;46(5):1433-1443. doi:10.1093/ije/dyx040.

53. Perala MM, von Bonsdorff MB, Mannisto S, et al. The healthy Nordic diet predicts muscle strength 10 years later in old women, but not old men. Age Ageing. 2017;46(4):588-594. doi:10.1093/ageing/afx034. 
54. Poulsen SK, Crone C, Astrup A, Larsen TM. Long-term adherence to the New Nordic Diet and the effects on body weight, anthropometry and blood pressure: a 12month follow-up study. Eur J Nutr. 2015;54(1):67-76. doi:10.1007/s00394-014-0686-z. 55. Reidlinger DP, Darzi J, Hall WL, Seed PT, Chowienczyk PJ, Sanders TA. How effective are current dietary guidelines for cardiovascular disease prevention in healthy middle-aged and older men and women? A randomized controlled trial. Am J Clin Nutr. 2015;101(5):922-930. doi:10.3945/ajcn.114.097352.

56. San-Cristobal R, Navas-Carretero S, Livingstone KM, et al. Mediterranean diet adherence and genetic background roles within a web-based nutritional intervention: the Food4Me Study. Nutrients. 2017;9(10). doi:10.3390/nu9101107.

57. Shakeri Z, Mirmiran P, Khalili-Moghadam S, Hosseini-Esfahani F, Ataie-Jafari A, Azizi F. Empirical dietary inflammatory pattern and risk of metabolic syndrome and its components: Tehran Lipid and Glucose Study. Diabetol Metab Syndr. 2019;11:16. doi:10.1186/s13098-019-0411-4.

58. Sidahmed E, Cornellier ML, Ren J, et al. Development of exchange lists for Mediterranean and Healthy Eating diets: implementation in an intervention trial. $J$ Hum Nutr Diet. 2014;27(5):413-425. doi:10.1111/jhn.12158.

59. Steffen LM, Van Horn L, Daviglus ML, et al. A modified Mediterranean diet score is associated with a lower risk of incident metabolic syndrome over 25 years among young adults: the CARDIA (Coronary Artery Risk Development in Young Adults) study. Br J Nutr. 2014;112(10):1654-1661. doi:10.1017/s0007114514002633.

60. Tabung FK, Satija A, Fung TT, Clinton SK, Giovannucci EL. Long-term change in both dietary insulinemic and inflammatory potential is associated with weight gain in adult women and men. $J$ Nutr. 2019;149(5):804-815. doi:10.1093/jn/nxy319. 61. Turner-McGrievy GM, Davidson CR, Wingard EE, Wilcox S, Frongillo EA. Comparative effectiveness of plant-based diets for weight loss: a randomized controlled trial of five different diets. Nutrition. 2015;31(2):350-358. doi:10.1016/j.nut.2014.09.002. 62. Satija A, Malik V, Rimm EB, Sacks F, Willett W, Hu FB. Changes in intake of plantbased diets and weight change: results from 3 prospective cohort studies. Am J Clin Nutr. 2019;110(3):574-582. doi:10.1093/ajcn/nqz049.

63. Roswall N, Angquist L, Ahluwalia TS, et al. Association between Mediterranean and Nordic diet scores and changes in weight and waist circumference: influence of FTO and TCF7L2 loci. Am J Clin Nutr. 2014;100(4):1188-1197.

doi:10.3945/ajcn.114.089706.

64. Tobias DK, Zhang C, Chavarro J, et al. Healthful dietary patterns and long-term weight change among women with a history of gestational diabetes mellitus. Int $\mathrm{J}$ Obes 2016;40(11):1748-1753. doi:10.1038/ijo.2016.156.

65. Wang $T$, Heianza $Y$, Sun D, et al. Improving adherence to healthy dietary patterns, genetic risk, and long term weight gain: gene-diet interaction analysis in two prospective cohort studies. BMJ. 2018;360:j5644. doi:10.1136/bmj.j5644. 66. Winkvist A, Klingberg S, Nilsson LM, et al. Longitudinal 10-year changes in dietary intake and associations with cardio-metabolic risk factors in the Northern Sweden Health and Disease Study. Nutr J. 2017;16(1):20. doi:10.1186/s12937-017-0241-x. 67. Arciero PJ, Gentile CL, Pressman R, et al. Moderate protein intake improves total and regional body composition and insulin sensitivity in overweight adults. Metabolism. 2008;57(6):757-765. doi:10.1016/j.metabol.2008.01.015. 
68. Brown RC, Cox CM, Goulding A. High-carbohydrate versus high-fat diets: effect on body composition in trained cyclists. Med Sci Sports Exerc. 2000;32(3):690-694.

doi:10.1097/00005768-200003000-00021.

69. Dale KS, McAuley KA, Taylor RW, et al. Determining optimal approaches for weight maintenance: a randomized controlled trial. Cmaj. 2009;180(10):E39-46.

doi:10.1503/cmaj.080974.

70. Delbridge EA, Prendergast LA, Pritchard JE, Proietto J. One-year weight maintenance after significant weight loss in healthy overweight and obese subjects: does diet composition matter? Am J Clin Nutr. 2009;90(5):1203-1214. doi:10.3945/ajcn.2008.27209.

71. Donnelly JE, Sullivan DK, Smith BK, et al. Alteration of dietary fat intake to prevent weight gain: Jayhawk Observed Eating Trial. Obesity. 2008;16(1):107-112. doi:10.1038/oby.2007.33.

72. Due A, Larsen TM, Mu H, Hermansen K, Stender S, Astrup A. Comparison of 3 ad libitum diets for weight-loss maintenance, risk of cardiovascular disease, and diabetes: a 6-mo randomized, controlled trial. Am J Clin Nutr. 2008;88(5):1232-1241.

doi:10.3945/ajcn.2007.25695.

73. Howard BV, Curb JD, Eaton CB, et al. Low-fat dietary pattern and lipoprotein risk factors: the Women's Health Initiative Dietary Modification Trial. Am J Clin Nutr. 2010;91(4):860-874. doi:10.3945/ajcn.2009.28034.

74. Larsen TM, Dalskov SM, van Baak M, et al. Diets with high or low protein content and glycemic index for weight-loss maintenance. N Engl J Med. 2010;363(22):21022113. doi:10.1056/NEJMoa1007137.

75. Paineau DL, Beaufils F, Boulier A, et al. Family dietary coaching to improve nutritional intakes and body weight control: a randomized controlled trial. Arch Pediatr Adolesc Med. 2008;162(1):34-43. doi:10.1001/archpediatrics.2007.2.

76. Razquin C, Martinez JA, Martinez-Gonzalez MA, Mitjavila MT, Estruch R, Marti A. A 3 years follow-up of a Mediterranean diet rich in virgin olive oil is associated with high plasma antioxidant capacity and reduced body weight gain. Eur J Clin Nutr. 2009;63(12):1387-1393. doi:10.1038/ejcn.2009.106.

77. Sanders TA, Lewis FJ, Goff LM, Chowienczyk PJ. SFAs do not impair endothelial function and arterial stiffness. Am J Clin Nutr. 2013;98(3):677-683.

doi:10.3945/ajcn.113.063644.

78. Tierney AC, McMonagle J, Shaw DI, et al. Effects of dietary fat modification on insulin sensitivity and on other risk factors of the metabolic syndrome--LIPGENE: a European randomized dietary intervention study. Int J Obes. 2011;35(6):800-809. doi:10.1038/ijo.2010.209.

79. Uusitupa M, Hermansen K, Savolainen MJ, et al. Effects of an isocaloric healthy Nordic diet on insulin sensitivity, lipid profile and inflammation markers in metabolic syndrome -- a randomized study (SYSDIET). J Intern Med. 2013;274(1):52-66.

doi:10.1111/joim.12044.

80. Vincent-Baudry S, Defoort C, Gerber M, et al. The Medi-RIVAGE study: reduction of cardiovascular disease risk factors after a 3-mo intervention with a Mediterranean-type diet or a low-fat diet. Am J Clin Nutr. 2005;82(5):964-971. doi:10.1093/ajcn/82.5.964. 81. Wan Y, Wang F, Yuan J, et al. Effects of macronutrient distribution on weight and related cardiometabolic profile in healthy non-obese Chinese: a 6-month, randomized 
controlled-feeding trial. EBioMedicine. 2017;22:200-207.

doi:10.1016/j.ebiom.2017.06.017.

82. Aljadani HM, Patterson AJ, Sibbritt D, Collins CE. Diet quality and six-year risk of overweight and obesity among mid-age Australian women who were initially in the healthy weight range. Health Promot J Austr. 2016;27(1):29-35. doi:10.1071/he14070. 83. Ankarfeldt MZ, Larsen SC, Angquist L, et al. Interaction between genetic predisposition to adiposity and dietary protein in relation to subsequent change in body weight and waist circumference. PLoS One. 2014;9(10):e110890.

doi:10.1371/journal.pone.0110890.

84. Beunza JJ, Toledo E, Hu FB, et al. Adherence to the Mediterranean diet, long-term weight change, and incident overweight or obesity: the Seguimiento Universidad de Navarra (SUN) cohort. Am J Clin Nutr. 2010;92(6):1484-1493. doi:10.3945/ajen.2010.29764.

85. Bujnowski D, Xun P, Daviglus ML, Van Horn L, He K, Stamler J. Longitudinal association between animal and vegetable protein intake and obesity among men in the United States: the Chicago Western Electric Study. J Am Diet Assoc.

2011;111(8):1150-1155.e1151. doi:10.1016/j.jada.2011.05.002.

86. de la Fuente-Arrillaga C, Martinez-Gonzalez MA, Zazpe I, Vazquez-Ruiz Z, BenitoCorchon S, Bes-Rastrollo M. Glycemic load, glycemic index, bread and incidence of overweight/obesity in a Mediterranean cohort: the SUN project. BMC Public Health. 2014;14:1091. doi:10.1186/1471-2458-14-1091.

87. Quatromoni PA, Pencina M, Cobain MR, Jacques PF, D'Agostino RB. Dietary quality predicts adult weight gain: findings from the Framingham Offspring Study. Obesity 2006;14(8):1383-1391. doi:10.1038/oby.2006.157.

88. So E, Choi SK, Joung H. Impact of dietary protein intake and obesity on lean mass in middle-aged individuals after a 12-year follow-up: the Korean Genome and Epidemiology Study (KoGES). Br J Nutr. 2019;122(3):322-330.

doi:10.1017/s000711451900117x. 
Table 1: Studies that examined the relationship between dietary patterns in children and growth size, body composition, and risk of overweight or obesity ${ }^{\text {vii }}$

Study and Participant

Characteristics ${ }^{\text {viii }}$

\author{
Intervention/Exposure
}

Reduced rank regression (predictor variables: \%E from free sugars, \%E from fat, dietary energy density (energy/g food consumed), dietary fiber density):

United Kingdom, Avon Longitudinal Study of

Parents and Children (ALSPAC) cohort

Prospective Cohort Study

$\mathrm{N}=4729$

$\sim 56 \%$ female

Excluded those with missing data; other reasons NR

\section{Bull, 2016 ${ }^{2}$}

United Kingdom, Avon Longitudinal Study of

Parents and Children (ALSPAC) cohort

Prospective Cohort Study
- "DP1" (categorical, quintiles, Q1, ref) at age 7y: Higher in confectionery and chocolate, cakes and biscuits, SSBs, low-fiber bread, and lower in fruit, vegetables, high-fiber bread, cereals

- "DP2" (categorical, quintiles, Q1, ref) at age 7y: Higher in sugar-sweetened beverages (SSBs), fruit juices, ready-to-eat breakfast cereals, and lower in whole milk, margarines and oils, cheese, crisps

\section{Factor/cluster analysis:}

Three dietary patterns identified at ages 7,10 , and $13 y$ :

- "Healthy", ref.: Non-white bread, reduced-fat milk, cheese, yoghurt and fromage frais, butter, breakfast cereal, rice, pasta, eggs, fish, vegetable and vegetarian dishes, soup, salad, legumes, fruit, crackers and crispbreads, high-energy-density sauces (e.g. mayonnaise), fruit juice, water
Results $^{\text {ix }}$

DP1: $\uparrow$ FMIz, Q excess adiposiy at $15 y$

DP2: $Q$ FMlz, excess adiposiy at $15 y$

\section{Methodological Considerations}

- Did not account for: Race/ethnicity

- Diet assessed via parenreport at age $7 y$, then parentassisted child at older ages

- Did not describe or fully account for selection into analyses or missing data

Funding: The United Kingdom Medical Research Council; Wellcome Trust; University of Bristol

Dietary patterns at $7 y$ :

"Healthy", ref

- Processed: $\uparrow \mathrm{BMI}$ at $17 \mathrm{y} \mathrm{f} / \mathrm{u}$

- Traditional: $Q$, BMI at $17 \mathrm{y} f / u$, NSA

- Packed lunch: $\uparrow$ BMI at 17 y f/u
- Did not account for: Race/ethnicity, Physical activity, Anthropometry

- Diet assessed once each age time point

- Unclear if diet assessment methods were valid/reliable

vii Abbreviations: BMI, body mass index; BMlz, body mass index z-score; CVD, cardiovascular disease; FM, Fat mass; FFM, Fat-free mass; FFMI, Fat-free mass index; FMI, Fat mass index; FMIz, Fat mass index z-score; LM, Lean mass; N/A, Not applicable; NR, Not reported; SSB, sugar-sweetened beverage; WC, waist circumference; wk, week(s); WHR, waist-to-hip ratio; Wt, body weight; y, year(s)

viii Includes last name of first author, publication year, country, analytic sample size, and select participant characteristics.

${ }^{i x}$ Results use the following symbols:

$\uparrow$, Significant (positive) association between higher adherence to diet and higher incidence/risk/absolute outcomes;

$Q$, Aassociation or difference between diet groups and outcome reported;

$\downarrow$, Significant (inverse) association between higher adherence to diet and lower incidence/risk/absolute outcomes 
Study and Participant

\section{$\mathrm{N}=2311$}

$56 \%$ female

Excluded those with missing data on CVD measures, dietary patterns info at all time points, and covariable data
- "Processed": Processed meat, pies and pasties, coated and fried chicken and white fish, pizza, chips, baked beans and tinned pasta, chocolate, sweets, sugar, diet and regular fizzy drinks

- "Traditional": Red meat, poultry, potatoes, vegetables, starch-based products, low-energy-density sauces, puddings, tea, coffee

- Packed Lunch: White bread, margarine, ham and bacon, sweet spreads, salty flavourings, crisps, biscuits, diet squash, tea, coffee
Dietary patterns at $10 y$ :

"Packed Lunch", "Traditional", and

"Processed" vs. "Healthy": $Q$ BMI

at $17 \mathrm{y} f / \mathrm{u}$

Dietary patterns at 13y:

Packed Lunch, Traditional, and Processed vs. Healthy: $Q \mathrm{BMI}$ at $17 \mathrm{y} f / \mathrm{u}$

\section{Chan She Ping-Delfos, Index/score: Dietary Guideline Index for Children and}

\section{5}

Australia, Western

Australian Pregnancy

Cohort

Prospective Cohort Study

\section{$\mathrm{N}=1419$}

Index/score: Dietary Guideline Index for Children and
Adolescents (DGI-CA) score (continuous) at age 14y

DGI-CA components: Fruit/100\% fruit juice, vegetables and legumes, breads and cereals, wholegrain bread relative to total, meat and alternatives (excluding processed meat); dairy products: reduced- or low-fat dairy, water as a beverage, healthy fats: total fats, $<3 / d$ 'extra foods'

\section{$\uparrow \mathrm{BMI}$ at $17 \mathrm{y} \mathrm{f} / \mathrm{u}$}

Q WC at $17 \mathrm{y} f / \mathrm{u}$

$\downarrow$ Waist:Hip Ratio at 17 y f/u
Funding: The United Kingdom Medical Research Council; Wellcome Trust; University of Bristol
$53 \%$ female

Excluded those with missing data,

implausible energy

intake
Latent class analysis:

Three patterns (continuous) at age $4 y$

Portugal, Generation XXI
- "Energy Dense Foods" (EDF): Sweets, soft drinks, salty pastry, processed meat
In girls,

- EDF:

$\circ \uparrow \mathrm{FMl}$ at $7 \mathrm{y}$, but $\mathrm{Q}$ FM\%
- Did not account for: Race/ethnicity

- Diet assessed once at baseline

- Diet assessment record validated in adults only

Funding: University of Western Australia; the Faculty of

Medicine, Dentistry and Health Sciences at the University of Western Australia; the Telethon Kids Institute; the Women and Infants Research Foundation;

Curtin University; and the Raine Medical Research Foundation;

National Health and Medical

Research Council; Telstra

Research Fund; National Health and Medical Research Council

- Did not account for: Race/ethnicity

- Diet assessed once at baseline 
Study and Participant

Prospective Cohort

Study

$\mathrm{N}=3473$

$50 \%$ female

Excluded those with diseases that influence diet, missing data
- "Snacking": Snack foods, lower in fish, meat, eggs, rice, pasta, potatoes, vegetables

- "Healthier": Higher in vegetables, fish, and lower in energy dense foods $\circ \quad \uparrow$ BMlz at 7y

$\circ \uparrow$ waist-to-hip ratio (WHR) at 7y

- Snacking, Healthier:

○ $Q \mathrm{FMI} \%$, FMI at $7 \mathrm{y}$

Q $Q$ WHR at $7 y$

- Q BMIz at $7 y$

In boys,

- EDF, Snacking, Healthier:

$$
\begin{array}{ll}
\circ & \text { Q WHR at } 7 y \\
\circ & \text { Q FMI\%, FMI at } 7 y \\
\circ & \text { Q BMIz at } 7 y
\end{array}
$$

- Diet assessment record validated in adults only

Funding: Programa Operacional de Saúde (Regional Department of Ministry of Health); Portuguese Foundation for Science and Technology (FCT) and by the Calouste Gulbenkian Foundation, EDER from the Operational

Programme Factors of

Competitiveness-COMPETE and through national funding from the FCT (Portuguese Ministry of

Education and Science)

Italy, Estonia, Cyprus, Belgium, Sweden,

Hungary, Germany, Spain,

Identification and prevention of Dietaryand lifestyle-induced health EFfects In Children and infantS (IDEFICS) study

Prospective Cohort Study

$\mathrm{N}=8341$

$50 \%$ female

Excluded those with missing data
Factor/cluster analysis:

Four patterns examined (continuous; and change from baseline to $\mathrm{f} / \mathrm{u}$ ) at age 2-9y

- "Processed": Higher takeaway and high-fat foods, such as savoury pastries, fritters, pizza as main dish, fried potatoes, hamburger, hot dog, kebab and wraps, and lowest scores for products such as wholemeal bread, cooked vegetables, raw vegetables, and fresh fruits without added sugar

- "Sweet": Higher sugar-rich products, like chocolate- or nut-based spread, sweetened drinks, fruit juices, diet drinks, candies, loose candies, marshmallows, and biscuits, packaged cakes, pastries and puddings, with the lowest scores for water, porridge, oatmeal, gruel, unsweetened cereals, muesli, raw vegetables, plain unsweetened milk and plain unsweetened yoghurt

- "Healthy": Higher low-fat foods, foods rich in vitamins and wholegrain foods, e.g. raw vegetables, fresh fruits without added sugar, porridge, oatmeal, gruel,
"Processed" vs. "Healthy":

- $\quad$ Did not account for: Race/ethnicity

- Did not account for missing data

Funding: European Community's Sixth RTD Framework Programme

"Sweet" vs. "Healthy":

- $\uparrow \mathrm{FMlz}$ at f/u (age 4-11y)

- $\quad$ FFMlz at f/u (age 4-11y)

- $\quad \uparrow$ BMIz at f/u (age 4-11y)

- $\uparrow W C z$ at f/u (age 4-11y) 
unsweetened cereals, muesli and plain unsweetened milk, and the lowest values for high-fat, high-sugar products, such as fried potatoes, sweetened drinks, sweetened milk, mayonnaise and mayonnaise-based products, chocolate- or nut-based spread, crisps, corn crisps, popcorn, and biscuits, packaged cakes pastries and puddings

"Processed-Processed" (baseline-

f/u) and "Processed-Sweet" vs.

"Healthy-"Healthy":

- $\quad \uparrow$ FMlz at f/u (age 4-11y)

- $\uparrow$ BMlz at f/u (age 4-11y)

"Processed-Processed" vs. "Healthy-Healthy":

- $\uparrow W C$ at f/u (age 4-11y)

"Processed-Sweet" vs. "HealthyHealthy":

- $\quad \uparrow W C$ at f/u (age 4-11y)

"Sweet-Sweet" vs. "Healthy-

"Healthy":

- $\uparrow W C$ at f/u (age 4-11y)

\section{$\mathrm{Hu}, 2016^{6}$}

United States,

Project EAT

Prospective Cohort Study

\section{$\mathrm{N}=2656$}

$\sim 50 \%$ female

Excluded those with missing data

\section{Martin-Calvo, 2016}

United States, Growing

Up Today Study 2
Index/score: A Priori Diet Quality Score (APDQS, continuous) at age $15 \mathrm{y}$

- Positively rated foods, serving/d: Beans, Chocolate Coffee, Fish, Fruit, Green vegetables, Low-fat dairy, Nuts, Poultry, Tea, Tomato, Whole grain, Yellow vegetables

- Neutral foods, serving/d: Diet soft drink, Eggs, Fruit juice, Margarine, Other vegetables, Potatoes, Refined grains, Seafood, Soup, Beer, Liquor, Wine

- Negatively rated foods, serving/d: Butter, Fried foods, Fried potato, Grain dessert, Organ meat, Processed meat, Red meat, Salty snacks, Sauces, Soft drinks, Sweet bread, Whole fat dairy

Index/score: modified KidMed Index (categorical, quartiles of 2y change, Q1, ref; continuously per-2-pt increase) at age $8-15 y$ $\downarrow$ gain in $\mathrm{BMl}$ after $7 \mathrm{y} \mathrm{f} / \mathrm{u}$

$\downarrow$ weight, excess weight gain at $25 y$
- Diet assessed once at baseline

- Self-reported outcome data

- Did not account for missing data

Funding: National Heart, Lung, and Blood Institute; National Institute on Aging; $\mathrm{NIH}$
- Did not account for: Race/ethnicity

- Self-reported outcome data 
Study and Participant

Prospective Cohort

Study

$\mathrm{N}=10,918$

$55 \%$ female

Excluded those with missing data

\section{Nguyen, 2019}

The Netherlands,

Generation R Study

Prospective Cohort

\section{Study}

$\mathrm{N}=3950$

$51 \%$ female

Excluded those with

missing data
- Positive components: Fruit or fruit juice, vegetables, fish, legumes, modified cereals/bread, nuts, olive oil, reduced- or low-fat dairy, yogurt/cheese; specified frequency/amounts per day or wk.

- $\quad$ Negative components: fast food $>1 / w k$, skipping breakfast, commercial bakery as breakfast, candies/sweets $\geq 1 / d$
- $\quad$ Did not account for missing data

Funding: Breast Cancer

Research Foundation; NIH
Index/score: Diet Quality Score (based on age-specific dietary guidelines), continuous at age $8 y$

- Positive components: intake of fruit $(150 \mathrm{~g} / \mathrm{d})$; vegetables $(150 \mathrm{~g} / \mathrm{d})$; whole grains $(90 \mathrm{~g} / \mathrm{d})$; fish $(60$ $\mathrm{g} / \mathrm{w})$; legumes (84 g/w); nuts $(15 \mathrm{~g} / \mathrm{d})$; dairy $(300 \mathrm{~g} / \mathrm{d})$; oils and fats $(30 \mathrm{~g} / \mathrm{d})$;

- Negative components: sugar-containing beverages $(150 \mathrm{~g} / \mathrm{d})$; and meat $(250 \mathrm{~g} / \mathrm{w})$

\section{Okubo, $2015^{9}$}

United Kingdom, Southampton Women's Survey

Prospective Cohort Study

\section{$\mathrm{N}=1018$}

$\sim 48 \%$ female

Excluded those with missing data

\section{Factor/cluster analysis:}

"Prudent" dietary pattern score (categorical, tertiles, T3, ref; continuous per 1unit increase) at age 3y

"Prudent" dietary pattern characterized by fish and shellfish, vegetables (salad, green, root other), other fruits
Diet Quality Score at 8y:

- $\uparrow$ FFMI at $10 \mathrm{y}$

- Q FMI \% body fat at $10 y$ (results were similar when stratified by sex)

- $\quad \uparrow \mathrm{BMI}$ at $10 \mathrm{y}$ (when stratified by sex, results were only significant in girls)

- $\quad \uparrow$ weight, height at 10y (when stratified by sex, results were only significant in girls)

\section{$\downarrow$ FM at $6 y$}

Q BMI at 6y
- Did not account for: Race/ethnicity

- Diet measured at 4 time points

- Did not account for missing data

Funding: Medical Research Council, the British Heart Foundation, Arthritis Research United Kingdom, the National Osteoporosis Society, the International Osteoporosis Foundation, the Cohen Trust, the 
Biomedical Research Centre,

University of Southampton and

University Hospital Southampton

National Health Service

Foundation Trust, and the

National Institute for Health

Research Musculoskeletal

Biomedical Research Unit,

University of Oxford.

\section{Pala, 2013 ${ }^{10}$}

Italy, Estonia, Cyprus,

Belgium, Sweden,

Hungary, Germany,

Spain,

Identification and

prevention of Dietary-

and lifestyle-induced

health EFfects In

Children and infantS

(IDEFICS) study

Prospective Cohort

Study

$\mathrm{N}=9427$

$49 \%$ female

Excluded those with

missing data

\section{Smith, 2014}

United Kingdom, Avon

Longitudinal Study of

Parents and Children

(ALSPAC) cohort

\section{Factor/cluster analysis:}

"Snacking" (categorical, tertiles, T1, ref; continuous per 1unit increase) at age 2-10y: Street foods (e.g., sandwiches), savory pastries, chocolate bars

"Sweet" (categorical, tertiles, T1, ref; continuous per 1-unit increase) at age 2-10y: Chocolate spreads, biscuits and sweets/candy, fried meat, soft drinks

"Vegetables and Wholemeal" (categorical, tertiles, T1, ref; continuous per 1-unit increase): Vegetables, fruits, wholemeal cereal products, unsweetened milk

"Protein and Water": Fish, meat, eggs, water
Vegetables and Wholemeal:

$\downarrow$ overweight/obesity after $2 \mathrm{y} f / \mathrm{u}$

"Snacking", "Sweet", "Protein and Water": $Q$ overweight/obesity after $2 y f / u$

"Snacking", "Sweet", "Vegetables and Wholemeal", "Protein and Water": $Q$ change in BMI after $2 y$ f/u
- Did not account for: Race/ethnicity

- Diet measured once at baseline

- Water intake was imputed

- Limited generalizability of sample tested

Funding: European Community's

Sixth RTD Framework

Programme

\section{Factor/cluster analysis:}

- "Health Aware" (continuous, per 1 SD increase) at 9y: Higher loadings for fruits and vegetables, high-fiber bread, pasta, cheese, and fish, lower loadings for
"Health Aware": $\downarrow$ FM and lean mass gain at 11y in girls (valid and under-reporting), and underreporting boys
- Did not account for: Race/ethnicity

- Diet assessed once each age time point; unclear timing of diet measurement in relation to outcomes 
Study and Participant

Characteristics $^{\text {viii }}$

Intervention/Exposure

Results $^{\text {ix }}$

Methodological Considerations

\section{Prospective Cohort}

Study

$\mathrm{N}=3911$

$\sim 56 \%$ female

Excluded those with missing data; other reasons NR processed meat, fizzy (carbonated) drinks

- "Traditional" (continuous, per 1 SD increase) at 9y: Higher loadings for meat, roast potatoes, vegetables, batter and pastry products, low energy-density sauces (e.g. gravy, desserts)

- "Packed Lunch" (continuous, per 1 SD increase) at 9y: Higher loadings for low-fiber bread, margarine, cheese, cold meats, salty flavorings such as yeast extract, diet squash (dilutable soft drink)

\section{Tognon, $2014^{12}$} Belgium, Sweden,

Hungary, Germany,

Spain, Identification and prevention of Dietary and lifestyle-induced

health EFfects In

Children and infantS

(IDEFICS) study

Prospective Cohort Study

$\mathrm{N}=9114$

$49 \%$ female missing data

\section{Index/score}

Mediterranean diet score, (high adherence levels, >3pts, vs. low) at 2-9y

- Points for intake higher than median for: vegetables and legumes, fruit and nuts (fruit added or not with sugar, nuts, seeds, dried fruit), cereals (breakfast cereals, white and wholemeal bread, rolls and crispbread, pasta, noodles and rice), fish (fresh or frozen fish, and fried fish and fish fingers)

- Points for intake below median for: dairy (milk, yoghurt, cheese including spreadable cheese), and meat products (fried and non-fried meat, hamburgers, falafel, kebab, etc.) chips (French fries), crisps (potato and corn snacks),

Italy, Estonia, Cyprus,

Excluded those with
"Traditional": $Q$ M gain at 11y in girls and boys (valid- and underreporting)

"Packed Lunch": $\downarrow$ FM gain at 11y in "valid-reporting" girls, $\downarrow$ lean mas
- Unclear if diet assessment methods were valid/reliable

- Did not account for missing data

Funding: The UK Medical

Research Council, the Wellcome

Trust and the University of

Bristol; World Cancer Research

Fund
- Did not account for: Race/ethnicity

- Diet comparison groups not clearly described

- Diet measured once at baseline

- $\quad$ Did not account for missing data

Funding: European Union within the Sixth RTD Framework Program; Swedish Council on Working Life and Social Research (FAS) EpiLife Center and the Swedish Research Council 
Table 2. Randomized controlled trials that examined diets based on macronutrient proportion and risk of growth size, body composition, and risk of overweight or obesity ${ }^{x}$

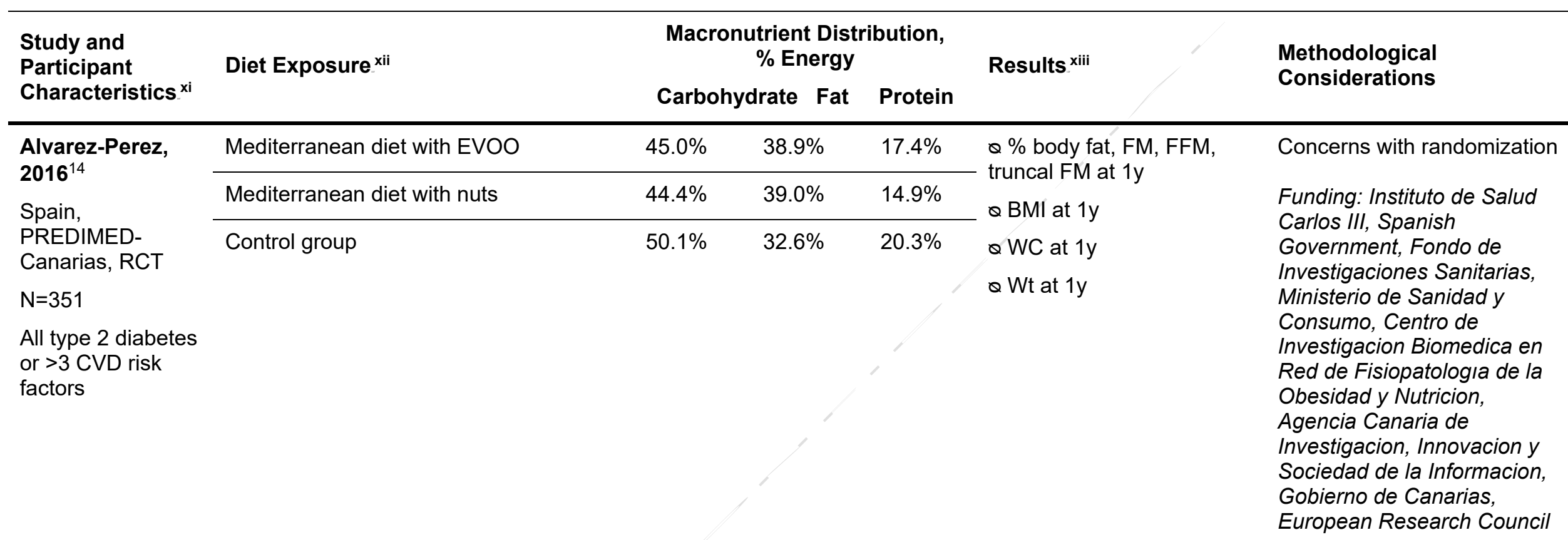

× Abbreviations: \% E, Percent energy; BMI, body mass index; BMlz, body mass index z-score; CVD, cardiovascular disease; EVOO, extra virgin olive oil; FM, Fat mass; FFM, Fat-free mass; FFMI, Fat-free mass index; FMI, Fat mass index; FMIz, Fat mass index z-score; f/u, follow-up; LM, Lean mass; Med,

Mediterranean; MUFA, monounsaturated fat/fatty acid; N/A, Not applicable; NIH, National Institutes of Health; NR, Not reported; PUFA, polyunsaturated fat/fatty acids; RCT, randomized controlled trial; WC, waist circumference; wk, week(s); WHR, waist-to-hip ratio; Wt, body weight; y, year(s)

${ }^{x i}$ Includes last name of first author, publication year, country, analytic sample size, and select participant characteristics.

xii Unless otherwise noted, the first category listed was used as the referent group.

xiii Relevant results for the systematic review question are summarized using the following symbols:

$\uparrow$, Significant (positive) association between higher adherence to diet and higher incidence/risk/absolute outcomes;

$Q$, Association or difference between diet groups and outcome was not statistically signficant;

$\downarrow$, Significant (inverse) association between higher adherence to diet and lower incidence/risk/absolute outcomes 


\begin{tabular}{|c|c|c|c|c|c|c|}
\hline \multirow{2}{*}{$\begin{array}{l}\text { Study and } \\
\text { Participant } \\
\text { Characteristics }^{\mathrm{xi}}\end{array}$} & \multirow{2}{*}{ Diet Exposure } & \multicolumn{3}{|c|}{$\begin{array}{c}\text { Macronutrient Distribution, } \\
\text { \% Energy }\end{array}$} & \multirow{2}{*}{ Results } & \multirow{2}{*}{$\begin{array}{l}\text { Methodological } \\
\text { Considerations }\end{array}$} \\
\hline & & Carbo & Irate Fat & Protein & & \\
\hline \multirow{4}{*}{$\begin{array}{l}\text { Arciero, } \mathbf{2 0 0 8}^{\mathbf{6 7}} \\
\text { United States, } \\
\text { RCT } \\
\mathrm{N}=16 \\
\text { All overweight or } \\
\text { obese }\end{array}$} & $\%$ E in "high-protein" (HP) diet & $25.3 \%$ & $31.3 \%$ & $43.5 \%$ & \multirow{4}{*}{$\begin{array}{l}\text { Q body fat } \%, \text { FM, LM, or } \\
\text { LM/Wt } \% \text { at } 3 \mathrm{mo} \\
\text { Q BMI at } 3 \mathrm{mo} \\
\text { Q WC, abdominal fat }(\% \text {, } \\
\mathrm{kg}) \text { at } 3 \mathrm{mo} \\
\propto \text { Wt at } 3 \mathrm{mo}\end{array}$} & \multirow{4}{*}{$\begin{array}{l}\text { Funding: Abbott } \\
\text { Laboratories, EAS division }\end{array}$} \\
\hline & \multirow[t]{3}{*}{$\%$ E in "moderate-protein" diet } & \multirow[t]{3}{*}{$41.9 \%$} & \multirow[t]{3}{*}{$28.8 \%$} & \multirow[t]{3}{*}{$26.9 \%$} & & \\
\hline & & & & & & \\
\hline & & & & & & \\
\hline \multirow{2}{*}{$\begin{array}{l}\text { Brown, } 2000^{68} \\
\text { New Zealand, } \\
\text { RCT }\end{array}$} & "High Fat" diet & $37.0 \%$ & $47.0 \%$ & $13.8 \%$ & \multirow{4}{*}{$\begin{array}{l}\text { Q Wt status at } 12 \mathrm{wk} \\
\text { Q LM, FM, \% body fat at } \\
12 \mathrm{wk} \\
\text { Q BMl at } 12 \mathrm{wk}\end{array}$} & \multirow{4}{*}{$\begin{array}{l}\text { Funding: Kiwi Co-operative } \\
\text { Dairies, New Zealand }\end{array}$} \\
\hline & \multirow[t]{3}{*}{ "High Carbohydrate" diet } & \multirow[t]{3}{*}{$69.0 \%$} & \multirow[t]{2}{*}{$14.0 \%$} & \multirow{2}{*}{$14.0 \%$} & & \\
\hline $\mathrm{N}=32$ & & & & & & \\
\hline $\begin{array}{l}\text { All competitive } \\
\text { cyclists in good } \\
\text { health }\end{array}$ & & & & & & \\
\hline \multirow{4}{*}{$\begin{array}{l}\text { Dale, } \mathbf{2 0 0 9}^{69} \\
\text { New Zealand, } \\
\text { RCT } \\
\mathrm{N}=174 \\
\text { All women, without } \\
\text { chronic disease }\end{array}$} & $\begin{array}{l}\text { "High Monounsatured Fat" (MUFA) Diet, } \\
\text { year 1, year } 2\end{array}$ & $\begin{array}{l}43 \% \\
50 \%\end{array}$ & $\begin{array}{l}30 \% \\
24 \%\end{array}$ & $\begin{array}{l}23 \% \\
21 \%\end{array}$ & \multirow{3}{*}{$\begin{array}{l}\text { Q FFM, FM at } 1 \mathrm{y}, 2 \mathrm{y} \\
\text { Q BMI at } 1 \mathrm{y}, 2 \mathrm{y} \\
\propto \mathrm{WC} \text { at } 1 \mathrm{y}, 2 \mathrm{y} \\
\propto \mathrm{Wt} \text { at } 1 \mathrm{y}, 2 \mathrm{y}\end{array}$} & \multirow[t]{3}{*}{$\begin{array}{l}\text { Funding: Health Research } \\
\text { Council of New Zealand }\end{array}$} \\
\hline & \multirow[t]{2}{*}{ "High Carbohydrate" Diet, year 1, year 2} & \multirow[t]{2}{*}{$\begin{array}{l}43 \% \\
47 \%\end{array}$} & \multirow[t]{2}{*}{$\begin{array}{l}31 \% \\
27 \%\end{array}$} & \multirow[t]{2}{*}{$\begin{array}{l}22 \% \\
22 \%\end{array}$} & & \\
\hline & & & & & & \\
\hline & Lean meat group & $41 \%$ & $30 \%$ & $24 \%$ & $\uparrow \mathrm{LM}$ at $16 \mathrm{wk}$ & \\
\hline
\end{tabular}




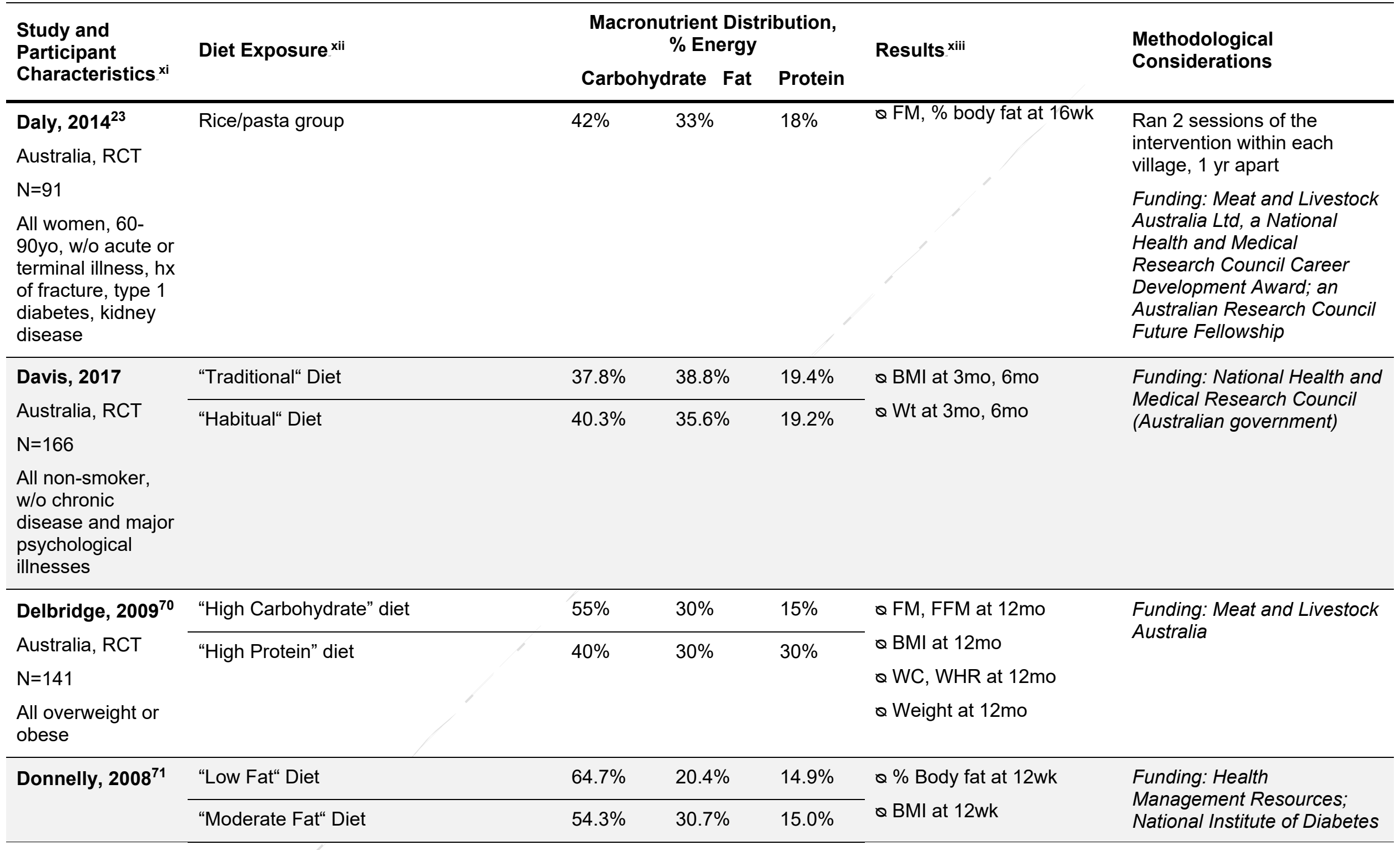




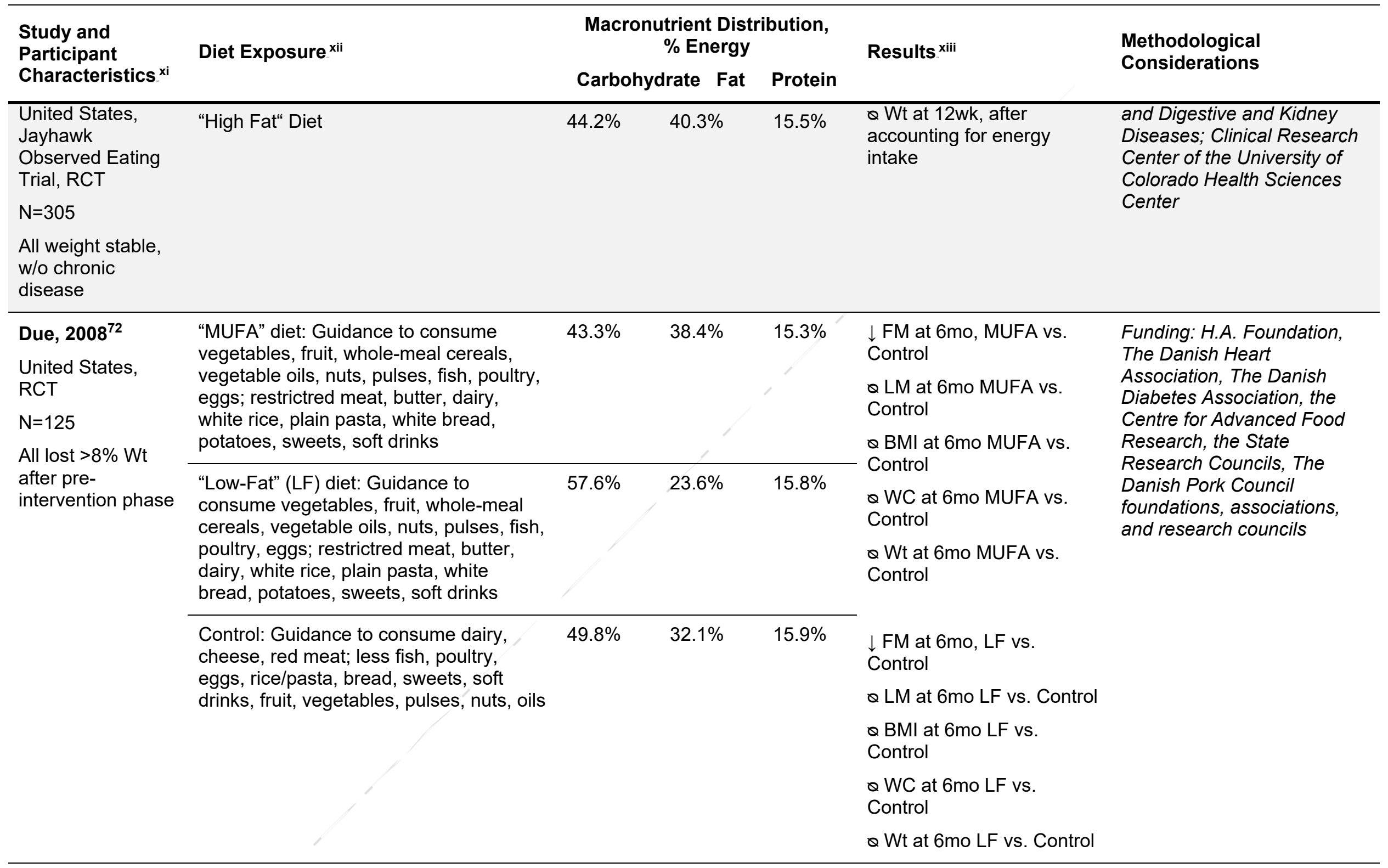




\begin{tabular}{|c|c|c|c|c|c|c|}
\hline \multirow{2}{*}{$\begin{array}{l}\text { Study and } \\
\text { Participant } \\
\text { Characteristics }^{\mathbf{x i}}\end{array}$} & \multirow{2}{*}{ Diet Exposure } & \multicolumn{3}{|c|}{$\begin{array}{c}\text { Macronutrient Distribution, } \\
\% \text { Energy }\end{array}$} & \multirow{2}{*}{ Results $^{\text {xiii }}$} & \multirow{2}{*}{$\begin{array}{l}\text { Methodological } \\
\text { Considerations }\end{array}$} \\
\hline & & Carbo & Irate Fat & Protein & & \\
\hline $\begin{array}{l}\text { Gardner, } \mathbf{2 0 1 8}^{\mathbf{3 3}} \\
\text { United States, } \\
\text { DIETFITS, RCT } \\
\mathrm{N}=609 \\
\text { All overweight or } \\
\text { obese }\end{array}$ & $\begin{array}{l}\text { "Healthy Low Fat" diet: Guidance to } \\
\text { consume oils, fatty meats, whole-fat } \\
\text { dairy, nuts }\end{array}$ & $48.4 \%$ & $28.7 \%$ & $20.6 \%$ & $\begin{array}{l}\text { Q } \% \text { Body fat at } 12 \mathrm{mo} \\
\text { Q BMI at } 12 \mathrm{mo} \\
\text { Q WC at } 12 \mathrm{mo} \\
\otimes \text { Wt at } 12 \mathrm{mo}\end{array}$ & $\begin{array}{l}\text { Study did not restrict energy } \\
\text { but intended for participants } \\
\text { to lose weight } \\
\text { Funding: National Institute of } \\
\text { Diabetes and Digestive and } \\
\text { Kidney Diseases; Nutrition } \\
\text { Science Initiative; National } \\
\text { Heart, Lung, and Blood } \\
\text { Institute, and the Stanford } \\
\text { Clinical and Translational } \\
\text { Science Award }\end{array}$ \\
\hline $\begin{array}{l}\text { Howard, } \mathbf{2 0 1 0}^{\mathbf{7 3}} \\
\text { United States, } \\
\text { Women's Health } \\
\text { Initiative Dietary } \\
\text { Modification Trial, } \\
\text { RCT }\end{array}$ & $\begin{array}{l}\text { "Low Fat Diet" year } 1 \\
\text { year } 3 \\
\text { year } 6 \\
\text { Guidance to reduce fat from } \sim 35 \% \mathrm{E} \text { to } \\
20 \% \mathrm{E} \text { of energy, } 5 \text { servings/d fruits and } \\
\text { vegetables, } 6 \text { serving/d grains }\end{array}$ & $\begin{array}{l}58.0 \% \\
56.4 \% \\
53.9 \%\end{array}$ & $\begin{array}{l}25.3 \% \\
26.5 \% \\
29.3 \%\end{array}$ & $\begin{array}{l}17.5 \% \\
17.6 \% \\
17.7 \%\end{array}$ & \multirow{2}{*}{$\begin{array}{l}\text { Low Fat vs. Control: } \\
\downarrow \text { BMI at } 1 \mathrm{y}, \\
\text { Q BMl at } 3 y, 6 y \\
\downarrow W C \text { at } 1 y, 3 y \\
\text { QWC at } 6 y \\
\downarrow W t \text { at } 1 y \\
\text { Q Wt at } 3 y, 6 y\end{array}$} & \multirow[t]{2}{*}{$\begin{array}{l}\text { Some missing outcome data } \\
\text { not accounted for } \\
\text { Funding: National Heart, } \\
\text { Lung, and Blood Institute, } \\
\text { NIH, US Department of } \\
\text { Health and Human Services }\end{array}$} \\
\hline $\begin{array}{l}\mathrm{N}=2730 \\
\text { All } \\
\text { postmenopausal } \\
\text { women, w/o hx of } \\
\text { cancer, }>32 \% \mathrm{E} \text { fat } \\
\text { intake }\end{array}$ & $\begin{array}{l}\text { Control Diet, year } 1 \\
\text { year } 3 \\
\text { year } 6 \\
\text { Received print-only materials }\end{array}$ & $\begin{array}{l}47.9 \% \\
47.0 \% \\
46.3 \%\end{array}$ & $\begin{array}{l}35.7 \% \\
36.0 \% \\
37 \%\end{array}$ & $\begin{array}{l}16.5 \% \\
17 \% \\
17 \%\end{array}$ & & \\
\hline $\begin{array}{l}\text { Kahleova, } \mathbf{2 0 1 8 ^ { 4 1 }} \\
\text { United States, } \\
\text { RCT }\end{array}$ & $\begin{array}{l}\text { "Low Fat, Vegan" Diet: } \\
\text { Guidance to consume vegetables, } \\
\text { grains, legumes, and fruits, and avoid } \\
\text { animal products and added fats }\end{array}$ & $69.6 \%$ & $17.5 \%$ & $12.3 \%$ & $\begin{array}{l}\downarrow \text { LM, FM, visceral FM at } \\
16 \mathrm{wk} \\
\downarrow \mathrm{BMI} \text { at } 16 \mathrm{wk}\end{array}$ & $\begin{array}{l}\text { Adherence not fully } \\
\text { described }\end{array}$ \\
\hline
\end{tabular}




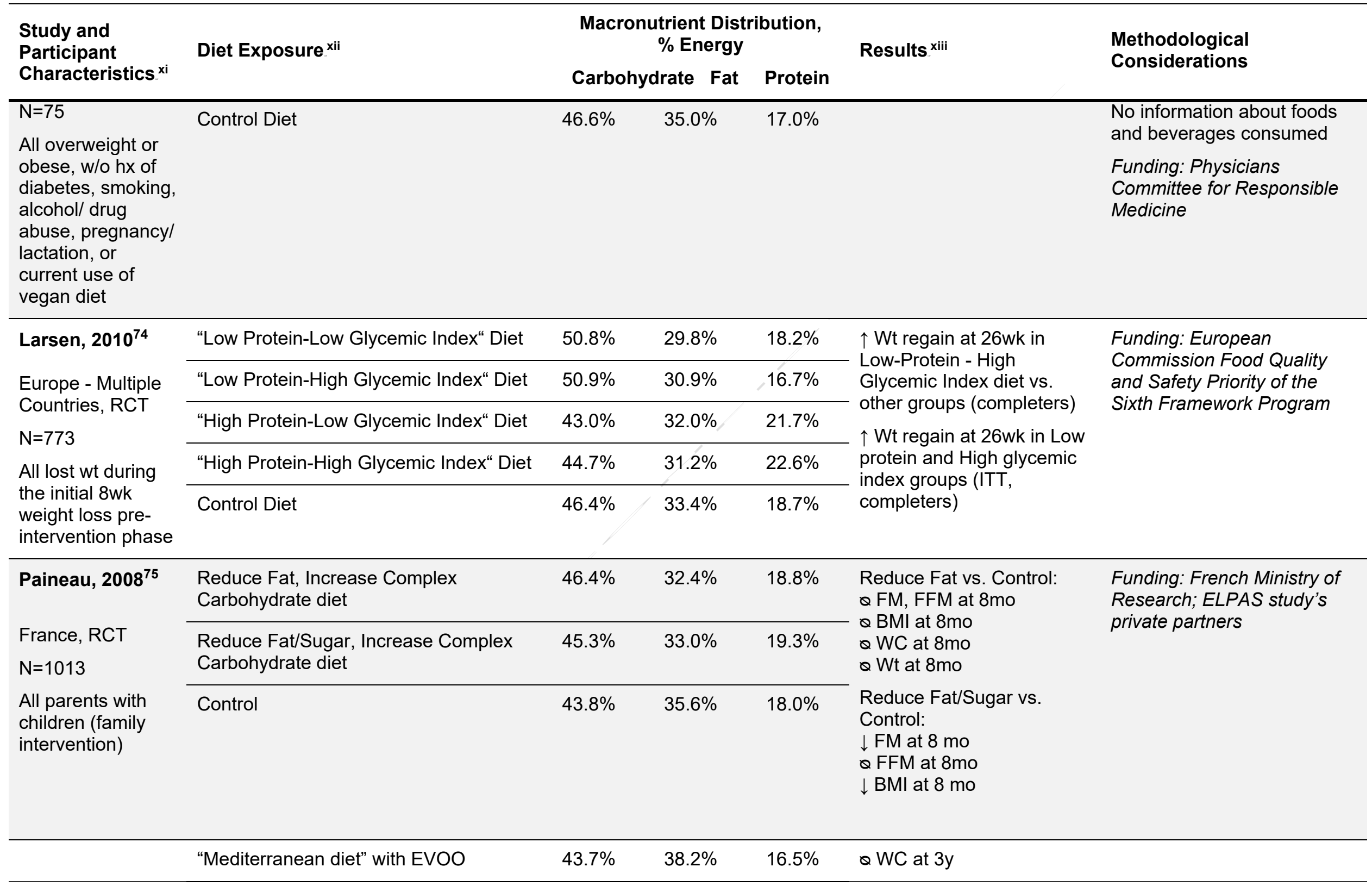




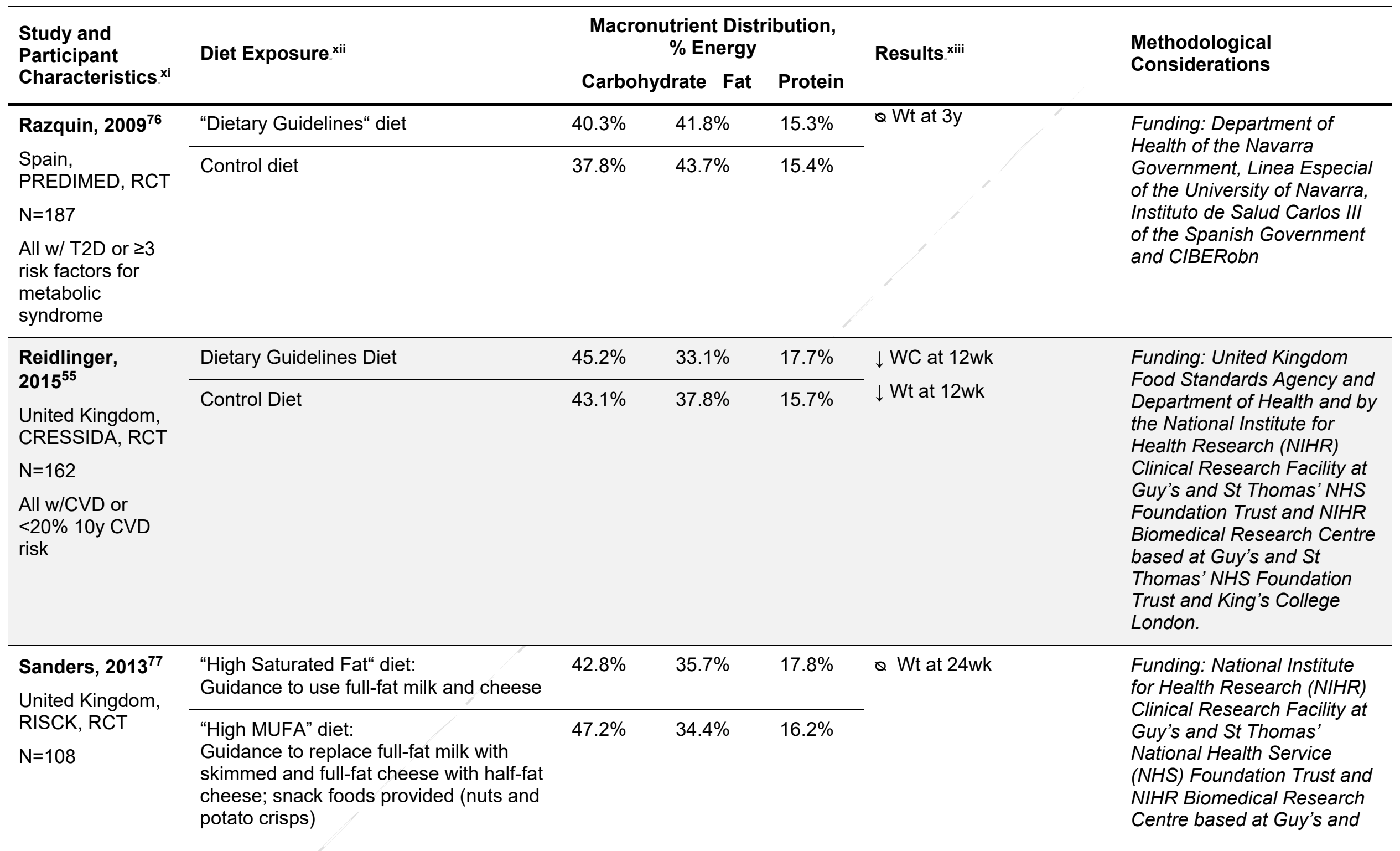




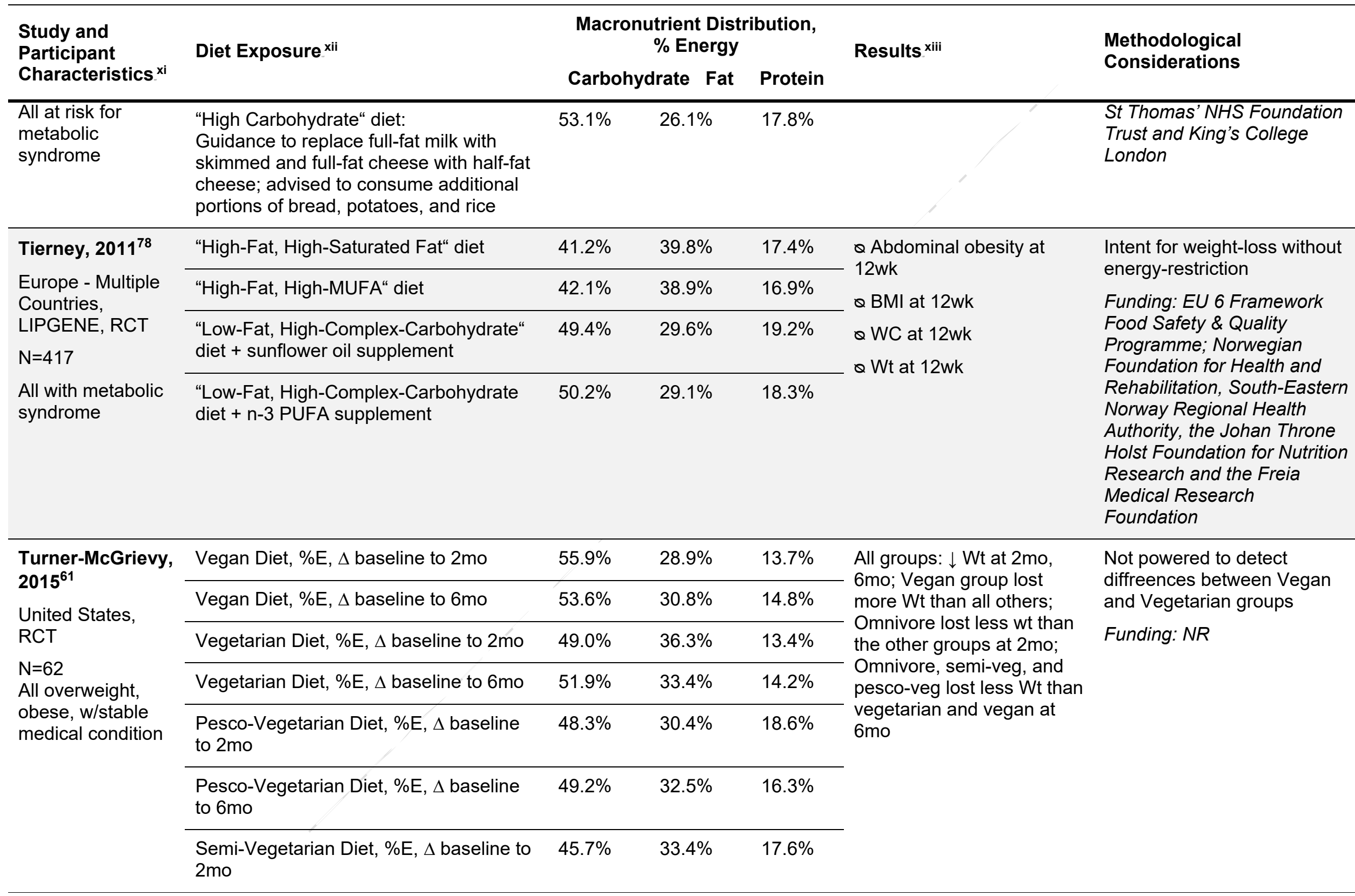




\begin{tabular}{|c|c|c|c|c|c|c|}
\hline \multirow{4}{*}{$\begin{array}{l}\text { Study and } \\
\text { Participant } \\
\text { Characteristics }^{\mathbf{x}}\end{array}$} & \multirow{3}{*}{$\begin{array}{l}\text { Diet Exposure }^{\text {xii }} \\
\text { Semi-Vegetarian Diet, \%E, } \Delta \text { baseline to } \\
6 \mathrm{mo}\end{array}$} & \multicolumn{3}{|c|}{$\begin{array}{c}\text { Macronutrient Distribution, } \\
\% \text { Energy }\end{array}$} & \multirow[t]{4}{*}{ Results $^{\text {xiii }}$} & \multirow{4}{*}{$\begin{array}{l}\text { Methodological } \\
\text { Considerations }\end{array}$} \\
\hline & & \multicolumn{2}{|c|}{ Carbohydrate Fat } & \multirow{2}{*}{$\frac{\text { Protein }}{17.3 \%}$} & & \\
\hline & & $43.1 \%$ & $36.6 \%$ & & & \\
\hline & Omnivorous Diet, \%E, $\Delta$ baseline to $2 \mathrm{mo}$ & $42.6 \%$ & $39.5 \%$ & $19.4 \%$ & & \\
\hline \multirow{4}{*}{$\begin{array}{l}\text { Uusitupa, } 2013^{79} \\
\text { Finland, Sweden, } \\
\text { Denmark, Iceland, } \\
\text { SYSDIET, RCT } \\
\mathrm{N}=166 \\
\text { All with features of } \\
\text { metabolic } \\
\text { syndrome }\end{array}$} & Healthy Nordic Diet & $46.1 \%$ & $32.9 \%$ & $16.9 \%$ & \multirow[t]{4}{*}{$Q$ Wt at $18 / 24 w k$} & \multirow{4}{*}{$\begin{array}{l}\text { Funding: NordForsk; } \\
\text { Academy of Finland, Finnish } \\
\text { Diabetes Research } \\
\text { Foundation }\end{array}$} \\
\hline & \multirow[t]{3}{*}{ Control Diet } & \multirow[t]{3}{*}{$44.6 \%$} & \multirow[t]{3}{*}{$35.2 \%$} & \multirow[t]{3}{*}{$16.2 \%$} & & \\
\hline & & & & & & \\
\hline & & & & & & \\
\hline $\begin{array}{l}\text { Vincent-Baudry, } \\
2005^{80}\end{array}$ & \multirow{3}{*}{$\begin{array}{l}\text { "Mediterranean“ diet: Consume nuts, } \\
\text { whole-meal bread, cereals, fruit, } \\
\text { vegetables , legumes, olive oil; fish 4/wk, } \\
\text { red meat 1/wk; sheep, poultry; oat-brain } \\
\text { enriched pasta, tomato sauce, olive oil } \\
\text { provided }\end{array}$} & \multirow[t]{3}{*}{$45.9 \%$} & \multirow[t]{3}{*}{$34.6 \%$} & \multirow[t]{3}{*}{$19.6 \%$} & \multirow[t]{4}{*}{ Q BMI at $3 \mathrm{mo}$} & \multirow[t]{4}{*}{$\begin{array}{l}\text { Funding: French Research } \\
\text { Ministry; INSERM }\end{array}$} \\
\hline $\begin{array}{l}\text { France, Medi- } \\
\text { RIVAGE, RCT }\end{array}$ & & & & & & \\
\hline $\mathrm{N}=212$ & & & & & & \\
\hline $\begin{array}{l}\text { All at high CVD } \\
\text { risk }\end{array}$ & $\begin{array}{l}\text { "Low-Fat" diet: Consume more poultry } \\
\text { than mammal meat, avoid offal and } \\
\text { saturated-fat-rich animal products, fish } \\
\text { 2-3x/wk; fruit, vegetables, low-fat dairy } \\
\text { products, vegetable oils }\end{array}$ & $44.8 \%$ & $33.9 \%$ & $21.3 \%$ & & \\
\hline
\end{tabular}

xiv Additional funding sources reported in Uusitupa, 2013 were: Finnish Foundation for Cardiovascular Research, The Sigfrid Juselius Foundation, Kuopio University Hospital; the Druvan Foundation, ESPEN, Skane County Council Research and Development Foundation, The Heart-Lung Foundation,

Diabetesfonden and Foundation Cerealia; The Danish Obesity Research Centre, The Danish Council for Strategic Research, The Agricultural Productivity

Fund, The Research Fund of the University of Iceland.

${ }^{x v}$ Additional funding sources reported in Vincent-Baudry, 2005 were: the Provence-Alpes-Côte d'Azur Regional Council, the Bouches du Rhône General

Council, the CRITT-PACA; Rivoire \& Carret Lustucru, Jean Martin, Le Cabanon, Boulangerie Coagulation Surgelés, Distplack Mariani, and Minoterie Giraud. 


\begin{tabular}{|c|c|c|c|c|c|c|c|}
\hline \multirow{2}{*}{$\begin{array}{l}\text { Study and } \\
\text { Participant } \\
\text { Characteristics }^{x i}\end{array}$} & \multirow{2}{*}{ Diet Exposure ${ }^{\text {xii }}$} & \multicolumn{4}{|c|}{$\begin{array}{l}\text { Macronutrient Distribution, } \\
\% \text { Energy }\end{array}$} & \multirow{2}{*}{ Results ${ }^{\text {xiii }}$} & \multirow{2}{*}{$\begin{array}{l}\text { Methodological } \\
\text { Considerations }\end{array}$} \\
\hline & & \multicolumn{3}{|c|}{ Carbohydrate Fat } & Protein & & \\
\hline \multirow{2}{*}{$\begin{array}{l}\text { Wan, } \mathbf{2 0 1 7 ^ { 8 1 }} \\
\text { China, RCT } \\
\mathrm{N}=307\end{array}$} & "Low-Fat, High-Carbohydrate" (LF-HC) & $66.0 \%$ & 20.0 & & $14.0 \%$ & \multirow{2}{*}{$\begin{array}{l}\downarrow W C \text { at } 6 \mathrm{mo} \text { in LF-HC vs. } \\
\text { MF-MC, HF-LC } \\
\text { Q WC at } 6 \mathrm{mo} \text { in HF-LC vs. } \\
\text { MF-MC }\end{array}$} & \multirow{4}{*}{$\begin{array}{l}\text { Funding: National Basic } \\
\text { Research Program of China }\end{array}$} \\
\hline & $\begin{array}{l}\text { "Moderate-Fat, Moderate-Carbohydrate" } \\
\text { (MF-MC) }\end{array}$ & $56.0 \%$ & \multicolumn{2}{|c|}{$30.0 \%$} & $14.0 \%$ & & \\
\hline \multirow[t]{2}{*}{ All BMI $<28$ kg/m2 } & \multirow[t]{2}{*}{ "High-Fat, Low-Carbohydrate" (HF-LC) } & \multirow[t]{2}{*}{$46.0 \%$} & \multirow{2}{*}{\multicolumn{2}{|c|}{$40.0 \%$}} & \multirow[t]{2}{*}{$14.0 \%$} & $\begin{array}{l}\downarrow \text { Wt at } 6 \mathrm{mo} \text { in LF-HC vs. } \\
\text { MF-MC, HF-LC }\end{array}$ & \\
\hline & & & & & & $\begin{array}{l}Q \mathrm{Wt} \text { at } 6 \mathrm{mo} \text { in HF-LC vs. } \\
\text { MF-MC }\end{array}$ & \\
\hline
\end{tabular}


Table 3. Observational studies that examined diets based on macronutrient proportion and risk of growth size, body composition, and risk of overweight or obesity ${ }^{x i}$

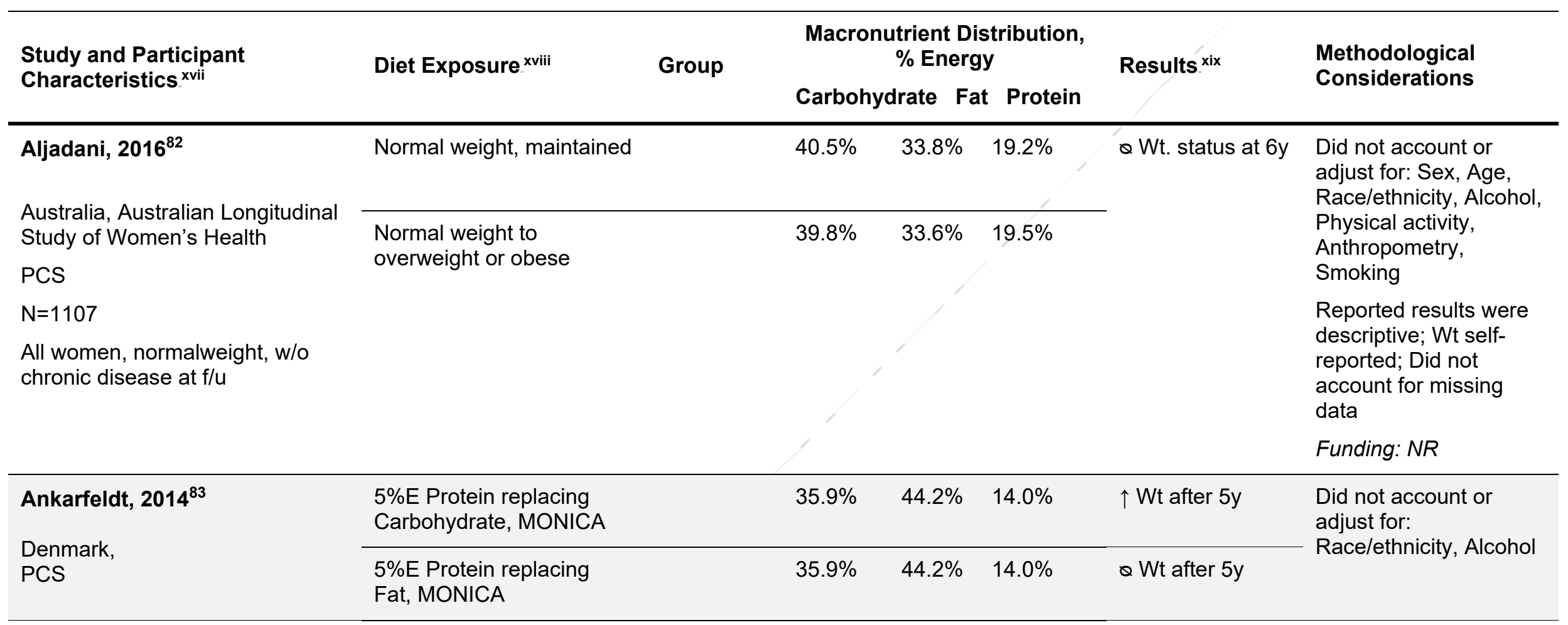

xvi Abbreviations: \% E, Percent energy; BMI, body mass index; BMlz, body mass index z-score; CVD, cardiovascular disease; EVOO, extra virgin olive oil; FM, Fat mass; FFM, Fat-free mass; FFMI, Fat-free mass index; FMI, Fat mass index; f/u, follow-up; LM, Lean mass; Med, Mediterranean; N/A, Not applicable; NHLBI, National Heart, Lung, and Blood Institute; NIH, National Institutes of Health; NR, Not reported; PCS, Prospective cohort study; USDA, US Department of Agriculture; WC, waist circumference; wk, week(s); WHR, waist-to-hip ratio; Wt, body weight; y, year(s)

xvii Includes last name of first author, publication year, country, analytic sample size, and select participant characteristics.

xviii Unless otherwise noted, the first category listed was used as the referent group.

${ }^{x i x}$ Relevant results for the systematic review question are summarized using the following symbols:

$\uparrow$, Significant (positive) association between higher adherence to diet and higher incidence/risk/absolute outcomes;

$Q$, Association or difference between diet groups and outcome was not statistically signficant;

$\downarrow$, Significant (inverse) association between higher adherence to diet and lower incidence/risk/absolute outcomes 


\begin{tabular}{|c|c|c|c|c|c|c|c|}
\hline \multirow{2}{*}{$\begin{array}{l}\text { Study and Participant } \\
\text { Characteristics }\end{array}$} & \multirow[t]{2}{*}{ Diet Exposure } & \multirow[t]{2}{*}{ Group } & \multicolumn{3}{|c|}{$\begin{array}{c}\text { Macronutrient Distribution, } \\
\% \text { Energy }\end{array}$} & \multirow[t]{2}{*}{ Results $^{x i x}$} & \multirow{2}{*}{$\begin{array}{l}\text { Methodological } \\
\text { Considerations }\end{array}$} \\
\hline & & & Carbor & Irate $\mathbf{F}$ & t Protein & & \\
\hline $\begin{array}{l}\text { MONICA, N=1257 } \\
\text { All w/o cancer, CVD, and } \\
\text { diabetes }\end{array}$ & \multicolumn{2}{|l|}{$\begin{array}{l}\text { 5\%E Protein replacing } \\
\text { Carbohydrate, } \mathrm{DCH}\end{array}$} & $41.6 \%$ & $34.2 \%$ & $17.7 \%$ & $\begin{array}{l}\text { Q WC after } 5 y \\
\text { Q Wt after } 5 y\end{array}$ & \multirow[t]{4}{*}{$\begin{array}{l}\text { Funding: Danish } \\
\text { Council for Strategic } \\
\text { Research }\end{array}$} \\
\hline $\begin{array}{l}\text { diabetes } \\
\text { DCH Study, N=2167 }\end{array}$ & \multicolumn{2}{|l|}{$\begin{array}{l}\text { 5\%E Protein replacing } \\
\text { Fat, DCH }\end{array}$} & $41.6 \%$ & $34.2 \%$ & $17.7 \%$ & $\begin{array}{l}\text { Q WC after } 5 y \\
\text { \& Wt after } 5 y\end{array}$ & \\
\hline \multirow{2}{*}{$\begin{array}{l}\text { All w/o cancer, CVD, and } \\
\text { diabetes, stable smoking habits } \\
\text { or weight } \\
\text { Inter } 99, N=3630 \\
\text { All w/o cancer, CVD, diabetes }\end{array}$} & \multicolumn{2}{|l|}{$\begin{array}{l}\text { 5\%E Protein replacing } \\
\text { Carbohydrate, Inter99 }\end{array}$} & $47.5 \%$ & $32.4 \%$ & $14.8 \%$ & $\begin{array}{l}\text { a WC after } 5 y \\
\text { Q Wt after } 5 y\end{array}$ & \\
\hline & \multicolumn{2}{|l|}{$\begin{array}{l}\text { 5\%E Protein replacing } \\
\text { Fat, Inter99 }\end{array}$} & $47.5 \%$ & $32.4 \%$ & $14.8 \%$ & $\begin{array}{l}\text { Q WC after } 5 y \\
\text { \& Wt after } 5 y\end{array}$ & \\
\hline \multirow[t]{2}{*}{ Beunza, $2010^{84}$} & \multirow{4}{*}{$\begin{array}{l}\text { Mediterranean Diet Score } \\
\text { (Trichopoulou, 2003) }\end{array}$} & MDS 0-3 & $41 \%$ & $39 \%$ & $18 \%$ & \multirow{4}{*}{$\begin{array}{l}\text { Q Overweight or } \\
\text { Obesity at } 2 \mathrm{y} \text { or } \\
4 \mathrm{y} \text {; no interaction } \\
\text { with previous } \\
\text { weight gain in } 5 \mathrm{y} \\
\text { pre-baseline } \\
\text { Q BMl after } 2 \mathrm{y} \\
\downarrow \text { absolute } \mathrm{Wt} \\
\text { gain after } 2 \mathrm{y} \text { or } \\
4 \mathrm{y} \text { of } \geq 3 \text { or } 5 \mathrm{~kg} \\
\text { and } \downarrow \text { averge } \mathrm{Wt} \\
\text { gain of } \geq 3 \mathrm{~kg} \text { in } \\
\text { those with MDS } \\
6-9 \text { vs. MDS } 0-3\end{array}$} & \multirow{4}{*}{$\begin{array}{l}\text { Did not account or } \\
\text { adjust for: } \\
\text { Race/ethnicity } \\
\text { Funding: Spanish } \\
\text { Government (Instituto } \\
\text { de Salud Carlos III, } \\
\text { Fondo de } \\
\text { Investigaciones } \\
\text { Sanitarias), Navarra } \\
\text { Regional Government, } \\
\text { University of Navarra, } \\
\text { La Caixa Foundation }\end{array}$} \\
\hline & & MDS 4-6 & $44 \%$ & $36 \%$ & $18 \%$ & & \\
\hline \multirow{2}{*}{$\begin{array}{l}\text { Spain, SUN Cohort } \\
\text { PCS } \\
\text { N=10,376 }\end{array}$} & & MDS, 7-9 & $46 \%$ & $33 \%$ & $18 \%$ & & \\
\hline & & & & & & & \\
\hline \multirow[t]{3}{*}{ Bujnowski, $2011^{85}$} & \multirow[t]{2}{*}{$\% \mathrm{E}$, Total protein } & Q1 & $40.6 \%$ & $41.6 \%$ & $12.8 \%$ & \multirow{4}{*}{$\begin{array}{l}\uparrow \text { Overweight, } \\
\text { Obesity after 7y, } \\
\text { Q4 vs. Q1 for } \\
\text { Total and Animal } \\
\text { protein }\end{array}$} & \multirow{4}{*}{$\begin{array}{l}\text { Did not account or } \\
\text { adjust for } \\
\text { Race/ethnicity, physical } \\
\text { activity } \\
\text { Funding: NIH }\end{array}$} \\
\hline & & Q4 & $35.4 \%$ & $43.4 \%$ & $17.5 \%$ & & \\
\hline & \multirow[t]{2}{*}{$\%$ E, Animal protein } & Q1 & $40.8 \%$ & $41.3 \%$ & $12.9 \%$ & & \\
\hline PCS & & Q4 & $35.0 \%$ & $44.0 \%$ & $17.3 \%$ & & \\
\hline
\end{tabular}




\begin{tabular}{|c|c|c|c|c|c|c|c|}
\hline \multirow{2}{*}{$\begin{array}{l}\text { Study and Participant } \\
\text { Characteristics }\end{array}$} & \multirow[t]{2}{*}{ Diet Exposure ${ }^{x v i i i}$} & \multirow[t]{2}{*}{ Group } & \multicolumn{3}{|c|}{$\begin{array}{c}\text { Macronutrient Distribution, } \\
\% \text { Energy }\end{array}$} & \multirow[t]{2}{*}{ Results $^{x i x}$} & \multirow{2}{*}{$\begin{array}{l}\text { Methodological } \\
\text { Considerations }\end{array}$} \\
\hline & & & Carboh & drate Fat & Protein & & \\
\hline \multirow{2}{*}{$\begin{array}{l}\mathrm{N}=1730 \\
\text { All men }\end{array}$} & \multirow[t]{2}{*}{$\% \mathrm{E}$, Vegetable protein } & Q1 & $35.8 \%$ & $44.9 \%$ & $15.6 \%$ & \multirow{2}{*}{$\begin{array}{l}\text { Q Overweight, } \\
\text { Obesity after } 7 \mathrm{y}, \\
\text { Q4 vs. Q1 for } \\
\text { Vegetable protein }\end{array}$} & \\
\hline & & Q4 & $39.5 \%$ & $41.0 \%$ & $14.8 \%$ & & \\
\hline \multirow[t]{2}{*}{ de la Fuente-Arrillaga, $2014^{86}$} & \multirow[t]{5}{*}{ Glycemic Index } & Q1 & $39.0 \%$ & $38.0 \%$ & $20.0 \%$ & \multirow{5}{*}{$\begin{array}{l}\text { Q Overweight } \\
\text { and obesity after } \\
5 y\end{array}$} & \multirow{6}{*}{$\begin{array}{l}\text { Did not account or } \\
\text { adjust for: } \\
\text { Race/ethnicity, Alcohol } \\
\text { Diet assessed once } \\
\text { Funding: Spanish } \\
\text { Government; the } \\
\text { Navarra Regional } \\
\text { Government; the } \\
\text { University of Navarra }\end{array}$} \\
\hline & & Q2 & $42.0 \%$ & $37.0 \%$ & $18.0 \%$ & & \\
\hline PCS & & Q3 & $43.0 \%$ & $37.0 \%$ & $17.0 \%$ & & \\
\hline $\mathrm{N}=9267$ & & Q4 & $44.0 \%$ & $36.0 \%$ & $16.0 \%$ & & \\
\hline \multirow{6}{*}{$\begin{array}{l}\text { All w/o chronic disease or } \\
\text { following a special diet }\end{array}$} & & Q5 & $47.0 \%$ & $33.0 \%$ & $16.0 \%$ & & \\
\hline & \multirow[t]{5}{*}{ Glycemic Load } & Q1 & $37.0 \%$ & $39.0 \%$ & $20.0 \%$ & \multirow{5}{*}{$\begin{array}{l}\text { Q Overweight } \\
\text { and obesity after } \\
5 y\end{array}$} & \\
\hline & & Q2 & $41.0 \%$ & $37.0 \%$ & $18.0 \%$ & & \\
\hline & & Q3 & $43.0 \%$ & $36.0 \%$ & $17.0 \%$ & & \\
\hline & & Q4 & $45.0 \%$ & $35.0 \%$ & $17.0 \%$ & & \\
\hline & & Q5 & $49.0 \%$ & $32.0 \%$ & $15.0 \%$ & & \\
\hline Ericson, $2019^{28}$ & \multirow{2}{*}{$\begin{array}{l}\text { \%E in women for "Health- } \\
\text { Conscious" Dietary } \\
\text { Pattern }\end{array}$} & Q1 & $44.9 \%$ & $40.2 \%$ & $14.9 \%$ & \multirow{4}{*}{$\begin{array}{l}\text { Q Wt change } \\
\text { after } 10 y\end{array}$} & Did not account or \\
\hline Sweden, Malmo" Diet and Cancer & & Q2 & $45.6 \%$ & $39.2 \%$ & $15.2 \%$ & & Race/ethnicity \\
\hline PCS & \multirow{2}{*}{$\begin{array}{l}\text { Higher loadings for } \\
\text { cottage cheese, fibre-rich } \\
\text { bread, fruits, vegetables, }\end{array}$} & Q3 & $45.8 \%$ & $38.8 \%$ & $14.4 \%$ & & \multirow[t]{2}{*}{$\begin{array}{l}\text { Measured diet once at } \\
\text { baseline using }\end{array}$} \\
\hline $\mathrm{N}=20,487$ & & Q4 & $46.6 \%$ & $37.7 \%$ & $15.6 \%$ & & \\
\hline
\end{tabular}


Macronutrient Distribution,

\% Energy

Methodological

Carbohydrate Fat Protein

breakfast cereals, fish and

low-fat yoghurt; lower

$47.7 \% \quad 36.1 \% \quad 16.2 \%$

loadings for low-fibre

bread, red and processed

meat, sugar-sweetened

beverages

\begin{tabular}{|c|c|c|c|c|c|c|}
\hline \%E in women for "Low-Fat & Q1 & $43.3 \%$ & $42.3 \%$ & $14.4 \%$ & \multirow{5}{*}{$\begin{array}{l}\text { Q Wt change } \\
\text { after } 10 y\end{array}$} & $\begin{array}{l}\text { meal", "tea-breakfast", } \\
\text { and "sugar-rich", which }\end{array}$ \\
\hline \multirow{4}{*}{$\begin{array}{l}\text { Higher loadings for low-fat } \\
\text { margarines, low-fat milk, } \\
\text { low-fat yoghurt; lower } \\
\text { loadings for butter }\end{array}$} & Q2 & $45.3 \%$ & $39.4 \%$ & $15.3 \%$ & & \multirow{13}{*}{$\begin{array}{l}\text { Funding: The Swedish } \\
\text { Research Council, } \\
\text { Region Skåne, Skåne } \\
\text { University Hospital, } \\
\text { Novo Nordic } \\
\text { Foundation, Albert } \\
\text { Påhlsson Research } \\
\text { Foundation }\end{array}$} \\
\hline & Q3 & $46.6 \%$ & $37.8 \%$ & $15.6 \%$ & & \\
\hline & Q4 & $47.1 \%$ & $37.1 \%$ & $15.8 \%$ & & \\
\hline & Q5 & $48.3 \%$ & $35.4 \%$ & $16.2 \%$ & & \\
\hline \multirow{2}{*}{$\begin{array}{l}\text { \%E in women for } \\
\text { "Dressing-Vegetables" } \\
\text { Dietary Pattern }\end{array}$} & Q1 & $47.4 \%$ & $37.4 \%$ & $15.2 \%$ & \multirow{5}{*}{$\begin{array}{l}\text { Q Wt change } \\
\text { after } 10 y\end{array}$} & \\
\hline & Q2 & $46.8 \%$ & $37.9 \%$ & $15.2 \%$ & & \\
\hline \multirow{3}{*}{$\begin{array}{l}\text { Higher loadings for } \\
\text { dressing/oils, vegetables, } \\
\text { poultry, salty snacks, } \\
\text { rice/pasta, fried potatoes, } \\
\text { cheese; lower loadings for } \\
\text { boiled potatoes, jam/sugar }\end{array}$} & Q3 & $46.1 \%$ & $38.5 \%$ & $15.4 \%$ & & \\
\hline & Q4 & $45.3 \%$ & $39.1 \%$ & $15.6 \%$ & & \\
\hline & Q5 & $44.9 \%$ & $39.1 \%$ & $16.0 \%$ & & \\
\hline \multirow{2}{*}{$\begin{array}{l}\text { \%E in men for "Health- } \\
\text { Conscious" Dietary } \\
\text { Pattern }\end{array}$} & Q1 & $44.9 \%$ & $40.5 \%$ & $14.6 \%$ & \multirow{4}{*}{$\begin{array}{l}\downarrow \text { Wt change after } \\
10-y, \text { categorical; } \\
Q \text { continuous }\end{array}$} & \\
\hline & Q2 & $44.5 \%$ & $40.7 \%$ & $14.8 \%$ & & \\
\hline \multirow{2}{*}{$\begin{array}{l}\text { Higher loadings for cream, } \\
\text { fibre-rich bread, fruits, } \\
\text { vegetables, breakfast }\end{array}$} & Q3 & $45.0 \%$ & $40.1 \%$ & $14.9 \%$ & & \\
\hline & Q4 & $45.4 \%$ & $39.4 \%$ & $15.2 \%$ & & \\
\hline
\end{tabular}




\begin{tabular}{|c|c|c|c|c|c|c|c|}
\hline \multirow{2}{*}{$\begin{array}{l}\text { Study and Participant } \\
\text { Characteristics }^{\text {xvii }}\end{array}$} & \multirow[t]{2}{*}{ Diet Exposure ${ }^{\text {xiii }}$} & \multirow{2}{*}{ Group } & \multicolumn{3}{|c|}{$\begin{array}{c}\text { Macronutrient Distribution, } \\
\% \text { Energy }\end{array}$} & \multirow{2}{*}{ Results $^{x i x}$} & \multirow{2}{*}{$\begin{array}{l}\text { Methodological } \\
\text { Considerations }\end{array}$} \\
\hline & & & \multicolumn{3}{|c|}{ Carbohydrate Fat Protein } & & \\
\hline & $\begin{array}{l}\text { cereals, fish, low-fat } \\
\text { yoghurt; lower loadings for } \\
\text { low-fibre bread, red and } \\
\text { processed meat, sugar- } \\
\text { sweetened beverages }\end{array}$ & Q5 & $46.2 \%$ & $38.0 \%$ & $15.7 \%$ & & \\
\hline & \multirow{2}{*}{$\begin{array}{l}\text { \%E in men for "Low-Fat } \\
\text { Products" Dietary Pattern, } \\
\text { Q1 }\end{array}$} & Q1 & $42.2 \%$ & $43.8 \%$ & $14.0 \%$ & \multirow{5}{*}{$\begin{array}{l}\text { Q Wt change } \\
\text { after } 10 \mathrm{y}\end{array}$} & \\
\hline & & Q2 & $44.2 \%$ & $41.0 \%$ & $14.8 \%$ & & \\
\hline & \multirow{3}{*}{$\begin{array}{l}\text { Higher loadings for low-fat } \\
\text { margarines, low-fat milk, } \\
\text { low-fat yoghurt; lower } \\
\text { loadings for butter }\end{array}$} & Q3 & $45.7 \%$ & $39.1 \%$ & $15.2 \%$ & & \\
\hline & & Q4 & $46.3 \%$ & $38.3 \%$ & $15.3 \%$ & & \\
\hline & & Q5 & $47.6 \%$ & $36.6 \%$ & $15.8 \%$ & & \\
\hline & \multirow{2}{*}{$\begin{array}{l}\text { \%E in men for "Dressing- } \\
\text { Vegetables" Dietary } \\
\text { Pattern, Q1 }\end{array}$} & Q1 & $46.6 \%$ & $38.9 \%$ & $14.5 \%$ & \multirow{5}{*}{$\begin{array}{l}\text { Q Wt change } \\
\text { after } 10 \mathrm{y}\end{array}$} & \\
\hline & & Q2 & $45.7 \%$ & $39.5 \%$ & $14.8 \%$ & & \\
\hline & \multirow{3}{*}{$\begin{array}{l}\text { Higher loadings for } \\
\text { dressing/oils, vegetables, } \\
\text { poultry, salty snacks, } \\
\text { rice/pasta, fried potatoes, } \\
\text { cheese; lower loadings for } \\
\text { boiled potatoes, jam/sugar }\end{array}$} & Q3 & $45.0 \%$ & $39.9 \%$ & $15.1 \%$ & & \\
\hline & & Q4 & $44.6 \%$ & $40.2 \%$ & $15.2 \%$ & & \\
\hline & & Q5 & $44.1 \%$ & $40.3 \%$ & $15.6 \%$ & & \\
\hline \multirow{3}{*}{$\begin{array}{l}\text { Hruby, } \mathbf{2 0 1 8 ^ { 3 7 }} \\
\text { United States, Framingham Heart } \\
\text { Study Offspring cohort }\end{array}$} & \multirow[t]{3}{*}{$\%$ E Protein $(\mathrm{g} / \mathrm{d})$} & Q1 & $52.2 \%$ & $27.7 \%$ & $13.1 \%$ & \multirow{3}{*}{$\begin{array}{l}\text { Q WC after } 20 y \\
Q W t \text { after } 20 y\end{array}$} & \multirow{3}{*}{$\begin{array}{l}\text { Did not account or } \\
\text { adjust for: } \\
\text { Race/ethnicity, Physical } \\
\text { activity }\end{array}$} \\
\hline & & Q2 & $55.0 \%$ & $32.1 \%$ & $15.9 \%$ & & \\
\hline & & Q3 & $52.6 \%$ & $32.4 \%$ & $18.1 \%$ & & \\
\hline
\end{tabular}




\begin{tabular}{|c|c|c|c|c|c|c|c|}
\hline \multirow{2}{*}{$\begin{array}{l}\text { Study and Participant } \\
\text { Characteristics }\end{array}$} & \multirow[t]{2}{*}{ Diet Exposure } & \multirow[t]{2}{*}{ Group } & \multicolumn{3}{|c|}{$\begin{array}{c}\text { Macronutrient Distribution, } \\
\% \text { Energy }\end{array}$} & \multirow[t]{2}{*}{ Results } & \multirow{2}{*}{$\begin{array}{l}\text { Methodological } \\
\text { Considerations }\end{array}$} \\
\hline & & & Carbol & Irate $\mathbf{F}$ & Protein & & \\
\hline \multirow[t]{3}{*}{$\begin{array}{l}\text { PCS } \\
N=3066\end{array}$} & & Q4 & $43.1 \%$ & $28.8 \%$ & $21.0 \%$ & \multirow{3}{*}{, } & $\begin{array}{l}\text { Diet assessed once at } \\
\text { baseline }\end{array}$ \\
\hline & & & & & & & $\begin{array}{l}\text { Did not account for } \\
\text { missing data }\end{array}$ \\
\hline & & & & & & & $\begin{array}{l}\text { Funding: USDA - } \\
\text { Agricultural Research } \\
\text { Service; North } \\
\text { American Branch of the } \\
\text { International Life } \\
\text { Sciences Institute; } \\
\text { NHLBl; Boston } \\
\text { University School of } \\
\text { Medicine }\end{array}$ \\
\hline \multirow[t]{2}{*}{ Quatromoni, $2006^{87}$} & \multirow{6}{*}{$\begin{array}{l}\text { Diet Quality Index (DQI) } \\
\text { score, in men }\end{array}$} & 0 & $40.2 \%$ & $39.7 \%$ & $16.7 \%$ & \multirow[t]{6}{*}{$\downarrow$ Wt gain after 8y } & Did not account or \\
\hline & & 1 & $42.7 \%$ & $37.7 \%$ & $16.5 \%$ & & Race/ethnicity \\
\hline \multirow{9}{*}{$\begin{array}{l}\text { United States, Framingham } \\
\text { Offspring Study } \\
\text { PCS } \\
\mathrm{N}=2245 \\
\text { All w/o Hx of cancer }\end{array}$} & & 2 & $45.2 \%$ & $34.0 \%$ & $16.6 \%$ & & \multirow{9}{*}{$\begin{array}{l}\text { Funding: NHLBI; } \\
\text { USDA; Unilever } \\
\text { Research (Colworth, } \\
\text { United Kingdom) }\end{array}$} \\
\hline & & 3 & $50.0 \%$ & $28.8 \%$ & $16.9 \%$ & & \\
\hline & & 4 & $56.5 \%$ & $25.0 \%$ & $16.9 \%$ & & \\
\hline & & 5 & $59.5 \%$ & $23.1 \%$ & $17.3 \%$ & & \\
\hline & \multirow[t]{5}{*}{ DQI score, in women } & 0 & $40.3 \%$ & $41.0 \%$ & $17.1 \%$ & \multirow[t]{5}{*}{$\downarrow$ Wt gain after 8y } & \\
\hline & & 1 & $42.5 \%$ & $38.9 \%$ & $17.1 \%$ & & \\
\hline & & 2 & $44.6 \%$ & $36.6 \%$ & $17.3 \%$ & & \\
\hline & & 3 & $50.1 \%$ & $31.5 \%$ & $17.0 \%$ & & \\
\hline & & 4 & $55.3 \%$ & $26.2 \%$ & $18.0 \%$ & & \\
\hline
\end{tabular}




\begin{tabular}{|c|c|c|c|c|c|c|c|}
\hline \multirow{2}{*}{$\begin{array}{l}\text { Study and Participant } \\
\text { Characteristics }\end{array}$} & \multirow[t]{2}{*}{ Diet Exposure ${ }^{x v i i i}$} & \multirow[t]{2}{*}{ Group } & \multicolumn{3}{|c|}{$\begin{array}{c}\text { Macronutrient Distribution, } \\
\% \text { Energy }\end{array}$} & \multirow[t]{2}{*}{ Results $^{\mathrm{xix}}$} & \multirow{2}{*}{$\begin{array}{l}\text { Methodological } \\
\text { Considerations }\end{array}$} \\
\hline & & & Carboh & Irate Fat & Protein & & \\
\hline & & 5 & $59.6 \%$ & $23.1 \%$ & $18.3 \%$ & & \\
\hline \multirow[t]{2}{*}{ So, $\mathbf{2 0 1 9 ^ { 8 8 }}$} & \multirow[t]{3}{*}{$\% \mathrm{E}$ Protein, in men } & $\mathrm{T} 1$ & $75.4 \%$ & $11.8 \%$ & $11.3 \%$ & \multirow{3}{*}{$\begin{array}{l}\text { Q LM after } 12 y \\
Q \text { BMI after } 12 y \\
\propto W t \text { after } 12 y\end{array}$} & \multirow{3}{*}{$\begin{array}{l}\text { Did not account or } \\
\text { adjust for: } \\
\text { Race/ethnicity } \\
\text { Funding: None }\end{array}$} \\
\hline & & $\mathrm{T} 2$ & $69.9 \%$ & $15.5 \%$ & $13.5 \%$ & & \\
\hline $\begin{array}{l}\text { Korea, KoGES } \\
\text { PCS }\end{array}$ & & T3 & $63.7 \%$ & $19.3 \%$ & $16.0 \%$ & & \\
\hline $\mathrm{N}=4412$ & \multirow[t]{3}{*}{$\%$ E Protein, in women } & $\mathrm{T} 1$ & $77.5 \%$ & $9.9 \%$ & $11.2 \%$ & \multirow{3}{*}{$\begin{array}{l}\text { Q LM after } 12 y \\
\text { Q BMI after } 12 y \\
\text { Q Wt after } 12 y\end{array}$} & \\
\hline \multirow{2}{*}{$\begin{array}{l}\text { Excluded those with low skeletal } \\
\text { muscle mass }\end{array}$} & & $\mathrm{T} 2$ & $71.8 \%$ & $13.9 \%$ & $13.4 \%$ & & \\
\hline & & T3 & $65.2 \%$ & $18.2 \%$ & $15.9 \%$ & & \\
\hline
\end{tabular}


Table 4. Risk of bias for observational studies examining dietary patterns in children and growth size, body composition, and risk of overweight or obesity

\begin{tabular}{|c|c|c|c|c|c|c|c|}
\hline Article & Confounding & $\begin{array}{l}\text { Selection of } \\
\text { participants }\end{array}$ & $\begin{array}{c}\text { Classification of } \\
\text { exposures }\end{array}$ & $\begin{array}{c}\text { Deviations } \\
\text { from } \\
\text { intended } \\
\text { exposures }\end{array}$ & $\begin{array}{c}\text { Missing } \\
\text { data }\end{array}$ & $\begin{array}{c}\text { Outcome } \\
\text { measurement }\end{array}$ & $\begin{array}{l}\text { Selection of } \\
\text { the reported } \\
\text { result }\end{array}$ \\
\hline Ambrosini, 2016 ${ }^{1}$ & Serious & Moderate & Moderate & Serious & Serious & Low & Moderate \\
\hline Bull, 2016² & Serious & Low & Moderate & Serious & Serious & Low & Serious \\
\hline $\begin{array}{c}\text { Chan She Ping-Delfos, } \\
2015^{3}\end{array}$ & Serious & Low & Low & Serious & Moderate & Low & Moderate \\
\hline Durao, $2017^{4}$ & Serious & Moderate & Low & Moderate & Serious & Low & Moderate \\
\hline Fernandez-Alvira, $2017^{5}$ & Serious & Low & Low & Moderate & Serious & Low & Moderate \\
\hline $\mathrm{Hu}, 2016^{6}$ & Moderate & Low & Low & Serious & Serious & Serious & Moderate \\
\hline Martin-Calvo, $2016^{7}$ & Serious & Low & Low & Low & Moderate & Serious & Moderate \\
\hline Nguyen, $2019^{8}$ & Moderate & Low & Low & Moderate & Serious & Low & Moderate \\
\hline Okubo, $2015^{9}$ & Serious & Low & Low & Moderate & Serious & Low & Moderate \\
\hline Pala, 2013 ${ }^{10}$ & Serious & Low & Low & Serious & Serious & Low & Moderate \\
\hline Smith, 2014'11 & Serious & Moderate & Moderate & Moderate & Serious & Low & Moderate \\
\hline Tognon, $2014^{12}$ & Serious & Low & Moderate & Serious & Serious & Low & Moderate \\
\hline
\end{tabular}

${ }^{x x} A$ detailed description of the methodology used for assessing risk of bias is available on the NESR website: https://nesr.usda.gov/2020-dietaryquidelines-advisory-committee-systematic-reviews and in Part C of the following reference: Dietary Guidelines Advisory Committee. 2020. Scientific Report of the 2020 Dietary Guidelines Advisory Committee: Advisory Report to the Secretary of Agriculture and the Secretary of Health and Human Services. U.S. Department of Agriculture, Agricultural Research Service, Washington, DC.

Possible ratings of low, moderate, serious, critical, or no information determined using the "Risk of Bias for Nutrition Observational Studies" tool (RoBNObs) (Dietary Guidelines Advisory Committee. 2020. Scientific Report of the 2020 Dietary Guidelines Advisory Committee: Advisory Report to the Secretary of Agriculture and the Secretary of Health and Human Services. U.S. Department of Agriculture, Agricultural Research Service, Washington, DC.) 
Table 5. Risk of bias for randomized controlled trials examining diets based on macronutrient distribution and growth size, body composition, and risk of overweight or obesity ${ }^{\mathrm{xxi}}$

\begin{tabular}{|c|c|c|c|c|c|c|}
\hline Article & Randomization & $\begin{array}{c}\text { Deviations from } \\
\text { intended } \\
\text { interventions - } \\
\text { effect assignment }\end{array}$ & $\begin{array}{l}\text { Deviations from } \\
\text { intended } \\
\text { interventions - } \\
\text { per protocol }\end{array}$ & $\begin{array}{c}\text { Missing } \\
\text { outcome data }\end{array}$ & $\begin{array}{c}\text { Outcome } \\
\text { measurement }\end{array}$ & $\begin{array}{l}\text { Selection of the } \\
\text { reported result }\end{array}$ \\
\hline Alvarez-Perez, $2016^{14}$ & Some concerns & Low & Low & High & Low & Low \\
\hline Arciero, $2008^{67}$ & Low & Low & Low & Low & Low & High \\
\hline Brown, $2000^{68}$ & Some concerns & Low & Low & Low & Low & Low \\
\hline Dale, $2009^{69}$ & Low & Low & Low & Low & Low & Low \\
\hline Daly, $2014^{23}$ & Low & Some concerns & Low & Low & Low & Some concerns \\
\hline Davis, 2017 J Nutr 24 & Low & Low & Low & Low & Low & Some concerns \\
\hline Delbridge, $2009^{70}$ & Low & Low & Some concerns & Low & Low & Some concerns \\
\hline Donnelly, $2008^{71}$ & Low & Low & Low & Low & Low & Low \\
\hline Due, $2008^{72}$ & Some concerns & Some concerns & Some concerns & Low & Low & Low \\
\hline Garnder, 2018 ${ }^{33}$ & Low & Low & Low & Low & Low & High \\
\hline Howard, $2010^{73}$ & Low & Low & Some concerns & Some concerns & Low & Low \\
\hline Kahleova, 201841 & Low & Low & Low & Low & Low & Low \\
\hline Larsen, $2010^{74}$ & Low & Low & Some concerns & High & Low & Some concerns \\
\hline Paineau, 2008 & Some concerns & Low & Low & Some concerns & Low & Low \\
\hline Razquin, $2009^{76}$ & Some concerns & Low & Low & Low & Low & High \\
\hline Reidlinger, $2015^{55}$ & Low & Low & Low & Low & Low & Low \\
\hline Sanders, $2013^{77}$ & Low & Low & Some concerns & Some concerns & Low & Some concerns \\
\hline Tierney, $2011^{78}$ & Low & Low & Not Applicable & Some concerns & Low & High \\
\hline Turner-McGrievy, $2015^{61}$ & Some concerns & Not Applicable & Low & Low & Low & Low \\
\hline Uusitupa, $2013^{79}$ & Low & Low & Some concerns & High & Low & Some concerns \\
\hline Vincent-Baudry, $2005^{80}$ & Some concerns & Low & Low & Low & Low & Low \\
\hline Wan, $2017^{81}$ & Low & Low & Low & Low & Low & Low \\
\hline
\end{tabular}

xxi Possible ratings of low, some concerns, or high determined using the "Cochrane Risk-of-bias 2.0" (RoB 2.0) (August 2016 version)" (Higgins JPT, Sterne JAC, Savović J, Page MJ, Hróbjartsson A, Boutron I, Reeves B, Eldridge S. A revised tool for assessing risk of bias in randomized trials In: Chandler J, McKenzie J, Boutron I, Welch V (editors). Cochrane Methods. Cochrane Database of Systematic Reviews 2016, Issue 10 (Suppl 1). dx.doi.org/10.1002/14651858.CD201601.) 
Table 6. Risk of bias in observational studies examining diets based on macronutrient distribution and growth size, body composition, and risk of overweight or obesity ${ }^{x i i}$

\begin{tabular}{|c|c|c|c|c|c|c|c|}
\hline Article & Confounding & $\begin{array}{l}\text { Selection of } \\
\text { participants }\end{array}$ & $\begin{array}{l}\text { Classification } \\
\text { of exposures }\end{array}$ & $\begin{array}{c}\text { Deviations from } \\
\text { intended } \\
\text { exposures }\end{array}$ & Missing data & $\begin{array}{c}\text { Outcome } \\
\text { measurement }\end{array}$ & $\begin{array}{c}\text { Selection of } \\
\text { the reported } \\
\text { result }\end{array}$ \\
\hline Aljadani, 2016 & Critical & Serious & Moderate & Serious & Serious & Serious & Serious \\
\hline Ankarfeldt, $2014^{83}$ & Serious & Serious & Moderate & Serious & Serious & Moderate & Moderate \\
\hline Beunza, $2010^{84}$ & Serious & Serious & Moderate & Moderate & Moderate & Moderate & Serious \\
\hline Bujnowski, $2011^{85}$ & Serious & Moderate & Moderate & Serious & Serious & Low & Moderate \\
\hline de la Fuente-Arrillaga, $2014^{86}$ & Serious & Serious & Low & Serious & Moderate & Serious & Moderate \\
\hline Ericson, 201928 & Serious & Serious & Low & Serious & Serious & Moderate & Serious \\
\hline Hruby, $2018^{37}$ & Serious & Low & Moderate & Serious & Serious & Low & Moderate \\
\hline Quatromoni, $2006^{87}$ & Serious & Moderate & Low & Moderate & Moderate & Low & Moderate \\
\hline So, $2019^{88}$ & Serious & Serious & Low & Serious & Moderate & Low & Moderate \\
\hline
\end{tabular}

xxii Possible ratings of low, moderate, serious, critical, or no information determined using the "Risk of Bias for Nutrition Observational Studies" tool (RoBNObs) (Dietary Guidelines Advisory Committee. 2020. Scientific Report of the 2020 Dietary Guidelines Advisory Committee: Advisory Report to the Secretary of Agriculture and the Secretary of Health and Human Services. U.S. Department of Agriculture, Agricultural Research Service, Washington, DC.) 
The NESR team used its rigorous, protocol-driven methodology to support the 2020 Dietary Guidelines Advisory Committee in updating and using an existing systematic review and conducting a new systematic review for the question: What is the relationship between dietary patterns consumed and growth, size, body composition, and/or risk of overweight or obesity?

NESR's systematic review methodology involves:

- Developing a protocol,

- Searching for and selecting studies,

- Extracting data from and assessing the risk of bias of each included study,

- Synthesizing the evidence,

- Developing conclusion statements,

- Grading the evidence underlying the conclusion statements, and

- Recommending future research.

A detailed description of the methodology used in answering this systematic review question is available on the NESR website: https://nesr.usda.gov/2020-dietary-guidelines-advisory-committeesystematic-reviews, and can be found in 2020 Dietary Guidelines Advisory Committee Report, Part C: Methodology. xxiii This systematic review was peer reviewed by Federal scientists, and information about the peer review process can also be found in the Committee's Report, Part C: Methodology. Additional information, including a description of and rationale for any modifications made to the protocol over the course of the Committee's work, are described in the 2020 Dietary Guidelines Advisory Committee Report, Chapter 8: Dietary Patterns.

This document includes a systematic evidence scan and update to an existing systematic review based on conclusions drawn by the 2015 Dietary Guidelines Advisory Committee with support from USDA's Nutrition Evidence Systematic Review (NESR) team. Information about the 2015 Dietary Guidelines Advisory Committee's review of the evidence on dietary patterns and growth, size, body composition, and/or risk of overweight or obesity can be found in their report, which is available at the following website: https://www.dietaryguidelines.gov/current-dietary-guidelines/process-develop-20152020-dg/advisory-committee

Below are details of the final protocol, as it was applied in the full systematic review described herein, including the:

- Analytic framework

- Literature search and screening plan

- Literature search and screening results

\section{ANALYTIC FRAMEWORK}

The analytic framework (Figure 1) illustrates the overall scope of the systematic review question, including the population, the interventions and/or exposures, comparators, and outcomes of interest. It also includes definitions of key terms and identifies key confounders and other factors to be considered in the systematic review. The inclusion and exclusion criteria that follow provide additional information about how parts of the analytic framework were defined and operationalized for the review.

xxiii Dietary Guidelines Advisory Committee. 2020. Scientific Report of the 2020 Dietary Guidelines Advisory Committee: Advisory Report to the Secretary of Agriculture and the Secretary of Health and Human Services. U.S. Department of Agriculture, Agricultural Research Service, Washington, DC. 
Figure 1: Analytic framework

Systematic review question: What is the relationship between dietary patterns consumed and growth, size, body composition, and risk of overweight and obesity?

\begin{tabular}{|lrl}
\hline \multicolumn{1}{|c}{ Intervention/exposure } & vs & \multicolumn{1}{c}{ Comparator } \\
\hline $\begin{array}{l}\text { Consumption of and/or } \\
\text { adherence to a dietary } \\
\text { pattern }\end{array}$ & - $\begin{array}{l}\text { Consumption of and/or } \\
\text { adherence to a different } \\
\end{array}$ \\
& dietary pattern \\
& $\begin{array}{l}\text { Different levels of } \\
\text { consumption of and/or } \\
\text { adherence to a dietary } \\
\text { pattern }\end{array}$
\end{tabular}

Population: Children through older adults; healthy and/or at risk of chronic disease
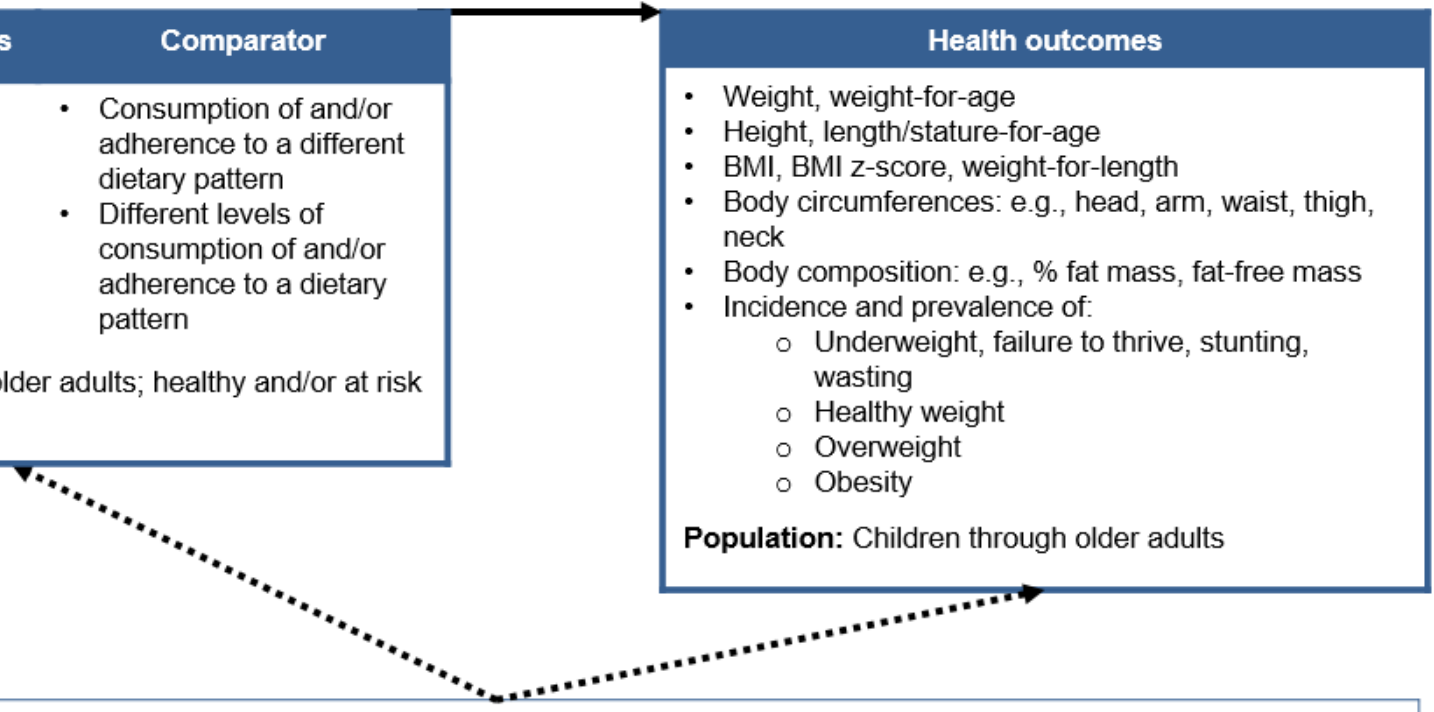

Key Confounders: Sex, Age, Race/Ethnicity, Physical activity, Anthropometry at baseline, Smoking

Potential Confounders: Total energy intake, Medications, Socioeconomic status, Alcohol intake (in adults), Family history of obesity, Supplement usage

\section{Key definitions}

Dietary patterns - The quantities, proportions, variety, or combination of different foods, drinks, and nutrients (when available) in diets, and the frequency with which they are habitually consumed.

\section{Legend}

$\longrightarrow$ The relationship of interest in the systematic review in the systematic review

\section{LITERATURE SEARCH AND SCREENING PLAN}

\section{Inclusion and exclusion criteria}

This table provides the inclusion and exclusion criteria for the systematic review. The inclusion and exclusion criteria are a set of characteristics used to determine which articles identified in the literature search were included in or excluded from the systematic review. 


\begin{tabular}{lll}
\hline Category & Inclusion Criteria & Exclusion Criteria \\
\hline Study design & - Randomized controlled trials & $\bullet$ Uncontrolled trials \\
& - $\begin{array}{l}\text { Non-randomized controlled trials, including } \\
\text { quasi-experimental and controlled before and } \\
\text { after studies }\end{array}$ & $\bullet$ Case-control studies \\
& - Prospective cohort studies & $\bullet$ Uncontrolled before-and-after studies \\
& - Retrospective cohort studies & $\bullet$ Narrative reviews \\
& - Nested case-control studies & $\bullet$ Systematic reviews
\end{tabular}

\section{Intervention/ exposure}

Studies that examine consumption of and/or adherence to a

1. Dietary pattern [i.e., the quantities, proportions, variety, or combination of different foods, drinks, and nutrients (when available) in diets, and the frequency with which they are habitually consumed] including, at a minimum, a description of the foods and beverages in the pattern

- Dietary patterns may be measured or derived using a variety of approaches, such as adherence to a priori patterns (indices/scores), data driven patterns (factor or cluster analysis), reduced rank regression, or other methods, including clinical trials

and/or

2. Diet based on macronutrient distribution outside of the acceptable macronutrient distribution range (AMDR) ${ }^{x \times i v,} \times{ }^{x \times v}$ and

- Include the macronutrient distribution of carbohydrate, fat, and protein of the diet, and

- Include at least one macronutrient outside of the AMDR
- Studies that

1a. Do not provide a description of the dietary pattern, which at minimum, must include the foods and beverages in the pattern (i.e., studies that examine a labeled dietary pattern, but do not describe the foods and beverages consumed) 2a. Examine consumption of and/or adherence to a diet based on macronutrient proportion in which all macronutrients are within the AMDR

$2 \mathrm{~b}$. Do not describe the entire macronutrient distribution of the diet (i.e., studies that only examine a single macronutrient in relation to outcomes)

xxiv Macronutrient percent of energy outside of the AMDR are as follows:

- Carbohydrate for all age groups: $<45$ or $>65$ percent of energy;

- Protein for children, $1-3 y$ : $<5$ or $>20$ percent of energy, Protein for children, $4-18 y$ : $<10$ or $>30$ percent of energy, Protein for adults, age $19 y$ and older: $<10$ or $>35$ percent of energy;

- Fat for children, 1-3y: $<30$ or $>40$ percent of energy, Fat for children, 4-18y: $<25$ or $>35$ percent of energy, Fat for adults, age $19 y$ and older: $<20$ or $>35$ percent of energy.

${ }^{x x v}$ Institute of Medicine. Dietary Reference Intakes for Energy, Carbohydrate, Fiber, Fat, Fatty Acids, Cholesterol, Protein, and Amino Acids. Washington (DC): The National Academies Press; 2002. 


\begin{tabular}{|c|c|c|}
\hline Category & Inclusion Criteria & Exclusion Criteria \\
\hline \multirow[t]{4}{*}{ Comparator } & $\begin{array}{l}\text { - Dietary patterns described by foods and } \\
\text { beverages consumed: }\end{array}$ & $\mathrm{N} / \mathrm{A}$ \\
\hline & $\begin{array}{l}\text { Consumption of and/or adherence to a } \\
\text { different dietary pattern }\end{array}$ & \\
\hline & $\begin{array}{l}\text { Different levels of consumption of and/or } \\
\text { adherence to a dietary pattern }\end{array}$ & \\
\hline & $\begin{array}{l}\text { - Diets described by macronutrient distribution: } \\
\circ \text { Different macronutrient distributions of } \\
\text { carbohydrate, fat, and protein }\end{array}$ & \\
\hline \multirow[t]{10}{*}{ Outcomes ${ }^{x x v i}$} & - Weight, weight-for-age & - Gestational weight gain \\
\hline & - Height, length/stature-for-age & \\
\hline & - $\quad$ BMI, BMI z-score, weight-for-length & \\
\hline & $\begin{array}{l}\text { Body circumferences e.g., head, arm, waist, } \\
\text { thigh, neck }\end{array}$ & \\
\hline & $\begin{array}{l}\text { - Body composition and distribution (e.g., \% fat } \\
\text { mass, fat-free mass) }\end{array}$ & \\
\hline & - Incidence and prevalence of: & \\
\hline & $\begin{array}{l}\text { Underweight, failure to thrive, stunting, } \\
\text { wasting }\end{array}$ & \\
\hline & $\circ$ Healthy weight & \\
\hline & $\circ$ Overweight & \\
\hline & $\circ$ Obesity & \\
\hline \multirow[t]{3}{*}{$\begin{array}{l}\text { Date of } \\
\text { publication }\end{array}$} & $\begin{array}{l}\text { January } 2014 \text { - October } 2019 \text { (this date range is } \\
\text { in addition to the original systematic review, } \\
\text { which included articles published from January } \\
\text { 1980-July 2013) }\end{array}$ & $\begin{array}{l}\text { Articles published prior to January } 1980 \text { or after } \\
\text { October } 2019\end{array}$ \\
\hline & $\begin{array}{l}\text { - Additional search to cover macronutrient } \\
\text { proportion diets: }\end{array}$ & $\begin{array}{l}\text { - Additional search to cover macronutrient } \\
\text { proportion diets: }\end{array}$ \\
\hline & ○ January 2000- December 2013 & $\circ$ Articles published prior to 2000 \\
\hline $\begin{array}{l}\text { Publication } \\
\text { status }\end{array}$ & Articles that have been peer-reviewed & $\begin{array}{l}\text { Articles that have not been peer-reviewed and are } \\
\text { not published in peer-reviewed journals (e.g., } \\
\text { unpublished data, manuscripts, reports, abstracts, } \\
\text { pre-prints, and conference proceedings) }\end{array}$ \\
\hline $\begin{array}{l}\text { Language of } \\
\text { publication }\end{array}$ & Articles published in English & Articles published in languages other than English \\
\hline Country & $\begin{array}{l}\text { Studies conducted in countries ranked as high or } \\
\text { higher human development }\end{array}$ & $\begin{array}{l}\text { Studies conducted in countries ranked as medium } \\
\text { or lower human development }\end{array}$ \\
\hline
\end{tabular}

xxvi The Pregnancy and Lactation Subcommittee of the 2020 Dietary Guidelines Advisory Committee addressed dietary patterns during pregnancy and lactation in relation to gestational weight gain and postpartum weight loss (respectively). xxvii The Human Development classification was based on the Human Development Index (HDI) ranking from the year the study intervention occurred or data were collected (UN Development Program. HDI 1990-2017 HDRO calculations based on data from UNDESA (2017a), UNESCO Institute for Statistics (2018), United Nations Statistics Division (2018b), World Bank (2018b), Barro and Lee (2016) and IMF (2018). Available from: http://hdr.undp.org/en/data). If the study did not report the year in which the intervention occurred or data were collected, the HDI classification for the year of publication 


\begin{tabular}{|c|c|c|}
\hline Category & Inclusion Criteria & Exclusion Criteria \\
\hline $\begin{array}{l}\text { Study } \\
\text { participants }\end{array}$ & $\begin{array}{ll}\text { - } & \text { Human participants } \\
\text { - } & \text { Females } \\
\text { - } & \text { Women during pregnancy and lactation }\end{array}$ & - Non-human participants (i.e., animals) \\
\hline $\begin{array}{l}\text { Age of study } \\
\text { participants }\end{array}$ & $\begin{array}{l}\text { - Age at intervention or exposure: } \\
\quad \begin{aligned} \text { Children and adolescents (ages 2-18 } \\
\text { years) }\end{aligned} \\
\circ \quad \text { Adults (ages 19-64 years) } \\
\circ \quad \text { Older adults (ages } 65 \text { years and } \\
\text { older) } \\
\text { - Age at outcome: } \\
\circ \quad \text { Children and adolescents (ages 2-18 } \\
\quad \text { years) } \\
\circ \quad \begin{array}{l}\text { Adults (ages 19-64 years) } \\
\circ \quad \text { Older adults (ages } 65 \text { years and } \\
\text { older) }\end{array} \\
\end{array}$ & $\begin{array}{l}\text { - Age at intervention or exposure: } \\
\circ \quad \begin{array}{l}\text { Infants and toddlers (birth to } 24 \\
\text { months) }\end{array} \\
\text { - Age at outcome } \\
\circ \quad \begin{array}{l}\text { Infants and toddlers (birth to } 24 \\
\text { months) }\end{array}\end{array}$ \\
\hline $\begin{array}{l}\text { Health status } \\
\text { of study } \\
\text { participants }\end{array}$ & $\begin{array}{l}\text { - Studies that enroll participants who are } \\
\text { healthy and/or at risk for chronic disease } \\
\text { - Studies that enroll some participants } \\
\text { diagnosed with a disease } \\
\text { - Studies that enroll some participants who are } \\
\text { classified with severe undernutrition, or as } \\
\text { underweight, stunted, or obese }\end{array}$ & $\begin{array}{l}\text { - Studies that exclusively enroll participants } \\
\text { diagnosed with a disease, or hospitalized with } \\
\text { an illness or injury } \\
\text { - Studies that exclusively enroll participants } \\
\text { classified as obese (i.e., studies that aim to } \\
\text { treat participants who have already been } \\
\text { classified as obese) or who are post-bariatric } \\
\text { surgery } \\
\text { - Addendum: } \\
\text { o Interventions designed to induce } \\
\text { weight loss or treat overweight and } \\
\text { obesity through energy- } \\
\text { restriction/hypocaloric diets for the } \\
\text { purposes of treating additional or } \\
\text { other medical conditions. }\end{array}$ \\
\hline $\begin{array}{l}\text { Study } \\
\text { duration }\end{array}$ & - Minimum length of intervention of 12 weeks & - Interventions $<12$ weeks \\
\hline $\begin{array}{l}\text { Size of study } \\
\text { groups }\end{array}$ & $\begin{array}{l}\text { - } 30 \text { participants per-arm for interventions, or } \\
\text { - } \quad \text { p power calculation included for interventions } \\
\text { - } \mathrm{n} \geq 1,000 \text { for observational studies }\end{array}$ & $\begin{array}{l}\text { - Fewer than } 30 \text { participants per arm for } \\
\text { interventions, or } \\
\text { - No power calculation reported for } \\
\text { interventions } \\
\text { - Fewer than } 1000 \text { participants for } \\
\text { observational studies }\end{array}$ \\
\hline
\end{tabular}

was applied. HDI values are available from 1980, and then from 1990 to present. If a study was conducted prior to 1990 , the HDI classification from 1990 was applied. If a study was conducted in 2018 or 2019, the most current HDI classification was applied. When a country was not included in the HDI ranking, the current country classification from the World Bank was used instead (The World Bank. World Bank country and lending groups. Available from: https://datahelpdesk.worldbank.org/knowledgebase/articles/906519-world- country-and-lending-groups). 


\section{Electronic databases}

Listed below are the databases searched to identify all potentially relevant articles that have been published to address the systematic review question. Two search strategies were developed and implemented each in three databases (PubMed, Cochrane Central Register of Controlled Trials (CENTRAL), and Embase) to update this systematic review.

\section{Search 1: Literature search to update the existing systematic review}

\section{PubMed}

- Provider: U.S. National Library of Medicine

- Date(s) Searched: October 21, 2019

- Date range searched: January 1, 2014-October 21, 2019

- Search Terms:

\#1 - "dietary pattern*" OR "diet pattern*" OR "eating pattern*" OR "food pattern*" OR "diet quality" OR "eating habit"” OR "dietary habit"” OR "diet habit" OR "food habit" OR "beverage habit" OR "Feeding Behavior"[Mesh:NoExp] OR "dietary profile*" OR "food profile*" OR "diet profile*" OR "eating profile*" OR "dietary guideline* OR "dietary recommendation*" OR "dietary intake*" OR "eating style*" OR "Diet, Mediterranean"[Mesh] OR Mediterranean Diet*[tiab] OR "Dietary Approaches To Stop Hypertension"[Mesh] OR "Dietary Approaches To Stop Hypertension Diet*" OR "DASH diet*" OR "Diet, Gluten-Free"[Mesh] OR "Gluten Free diet"” OR "prudent

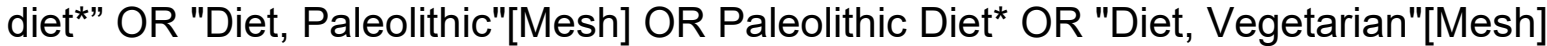
OR vegetarian diet*[tiab] OR vegan diet*$^{*}$ OR "Diet, Healthy"[Mesh] OR "plant based diet $^{* "}$ OR "Diet, Western"[Mesh] OR "western diet*" OR "Diet, CarbohydrateRestricted"[Mesh] OR "low-carbohydrate diet" OR "high carbohydrate diet" OR "Ketogenic Diet"” OR "Nordic Diet"” OR "Diet, Fat-Restricted"[Mesh] OR "Diet, HighFat"[Mesh] OR "Diet, High-Protein"[Mesh] OR high protein diet*[tiab] OR protein intake* OR high-fat diet* OR low fat diet* OR "Diet, Protein-Restricted"[Mesh] OR

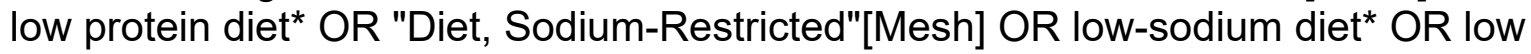
salt diet* OR (("Dietary Proteins"[Mesh] OR dietary protein*[tiab] OR "Dietary Carbohydrates"[Mesh] OR dietary carbohydrate*[tiab] OR "Dietary Fats"[Mesh] OR dietary fat*[tiab] OR hypocaloric OR hypo-caloric) AND (diet[tiab] OR diets[tiab] OR consumption[tiab] OR intake[tiab] OR supplement*[tiab])) OR ((“Guideline Adherence"[Mesh] OR guideline adherence*)AND (diet[tiab] OR dietary[tiab] OR food[tiab] OR beverage*[tiab] OR nutrition*[tiab])) OR diet score* OR diet quality score* OR diet quality index* OR kidmed OR diet index* OR dietary index* OR food score* OR MedDietScore OR healthy eating index[tiab] OR ((pattern[tiab] OR patterns[tiab] OR consumption[tiab] OR habit*[tiab]) AND ("Diet"[Mesh:NoExp] OR diet[tiab] OR diets[tiab] OR dietary[tiab] OR "Food"[Mesh] OR food[tiab] OR foods[tiab] OR "Beverages"[Mesh] OR beverage[tiab] OR beverages[tiab]))

\#2 - "Cardiovascular Diseases"[Mesh:NoExp] OR cardiovascular disease*[tiab] OR coronary artery disease[tiab] OR heart disease*[tiab] OR "Heart Failure"[Mesh] OR heart failure[tiab] OR "Myocardial Infarction"[Mesh] OR myocardial infarction*[tiab] OR "Myocardial Ischemia"[Mesh] OR Myocardial Ischemia*[tiab] OR "Stroke"[Mesh] OR stroke[tiab] OR angina[tiab] OR heart attack[tiab] OR "Venous Thrombosis"[Mesh] OR venous thrombosis[tiab] OR hypertension[tiab] OR high 
blood pressure[tiab] OR "Lipids/blood"[Mesh] OR "Cholesterol, HDL"[Mesh] OR HDL cholesterol[tiab] OR "Cholesterol, LDL"[Mesh] OR LDL cholesterol[tiab] OR total cholesterol[tiab] OR "Triglycerides"[Mesh] OR triglycerides[tiab]

\#3 - "Diabetes Mellitus, Type 2"[Mesh] OR Type 2 diabetes[tiab] OR T2D[tiab] OR adult onset diabetes[tiab] OR "Prediabetic State"[Mesh] OR prediabet*[tiab] OR pre

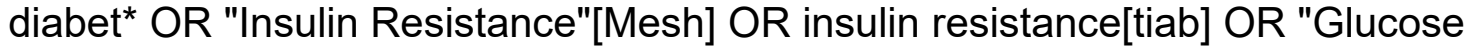
Intolerance"[Mesh] OR glucose intolerance[tiab] OR glucose tolerance[tiab] OR "Glycated Hemoglobin A"[Mesh] OR hemoglobin A1c[ti] OR "Hyperglycemia"[Mesh] OR "Hypoglycemia"[Mesh] OR ((impaired fasting[tiab] OR "Diabetes Mellitus"[Mesh:NoExp]) AND (glucose[tiab] OR glycemi*[tiab] OR high blood sugar[tiab] OR low blood sugar[tiab]))

\#4 - "Body Weights and Measures"[Mesh] OR "Body Weight"[Mesh] OR obesity[tiab] OR obese[tiab] OR overweight[tiab] OR body mass index[tiab] OR BMI[tiab] OR underweight[tiab] OR wasting[tiab] OR healthy weight[tiab] OR "Body Composition"[Mesh] OR body composition[tiab] OR body fat[tiab] OR fat mass[tiab] OR fat free mass[tiab] OR body height[tiab] OR stunting[tiab] OR stunted[tiab] OR "Growth Charts"[Mesh] OR growth chart*[tiab] OR "Growth"[Mesh:NoExp] OR waist circumference[tiab] OR head circumference[tiab] OR arm circumference[tiab] OR thigh circumference[tiab] OR neck circumference[tiab] OR

"Anthropometry"[Mesh:NoExp] OR "Overnutrition"[Mesh] OR anthropometr*[tiab] OR adiposity[tiab] OR calf circumference[tiab] OR skin fold*[tiab] OR healthy weight[tiab] OR weight for height[tiab] OR stature for age[tiab] OR weight for age[tiab] OR height for age[tiab] OR length for age[tiab] OR weight for length[tiab]

\#5 - (\#2 OR \#3 OR \#4)

\#6 - (\#1 AND \#5) NOT ("Animals"[Mesh] NOT ("Animals"[Mesh] AND "Humans"[Mesh])) NOT (editorial[ptyp] OR comment[ptyp] OR news[ptyp] OR letter[ptyp] OR review[ptyp] OR systematic review[ptyp] OR systematic review[ti] OR meta-analysis[ptyp] OR meta-analysis[ti] OR meta-analyses[ti] OR retracted publication[ptyp] OR retraction of publication[ptyp] OR retraction of publication[tiab] OR retraction notice[ti])

Filters: Publication date from 2014/01/01 to 2019/10/21; English

\section{Cochrane Central Register of Controlled Trials (CENTRAL)}

- Provider: John Wiley \& Sons

- Date(s) Searched: October 21, 2019

- Date range searched: January 1, 2014-October 21, 2019

- Search Terms:

\#1 - ("dietary pattern"” OR "diet pattern"” OR "eating pattern*" OR "food pattern*" OR "diet quality" OR "eating habit"” OR "dietary habit" OR "diet habit" OR "food habit" OR "beverage habit"” OR [mh ^"Feeding Behavior"] OR "dietary profile*" OR "food profile*" OR "diet profile*" OR "eating profile*" OR "dietary guideline*" OR "dietary recommendation" OR "dietary intake *" OR "eating style*" OR [mh "Diet, Mediterranean"] OR "Mediterranean Diet*" OR [mh "Dietary Approaches To Stop Hypertension"] OR "Dietary Approaches To Stop Hypertension Diet" OR "DASH diet $^{\star \prime}$ OR [mh "Diet, Gluten-Free"] OR "Gluten Free diet"” OR "prudent diet"” OR [mh "Diet, Paleolithic"] OR "Paleolithic Diet*" OR [mh "Diet, Vegetarian"] OR 
"vegetarian diet"” OR "vegan diet*" OR [mh "Diet, Healthy"] OR "plant based diet" OR [mh "Diet, Western"] OR "western diet" OR [mh "Diet, CarbohydrateRestricted"] OR "low-carbohydrate diet" OR "high carbohydrate diet" OR "Ketogenic Diet"” OR "Nordic Diet"” OR [mh "Diet, Fat-Restricted"] OR [mh "Diet, High-Fat"] OR [mh "Diet, High-Protein"] OR "high protein diet*" OR "protein intake" OR "high-fat diet" OR "low fat diet*" OR [mh "Diet, Protein-Restricted"] OR "low protein diet"” OR [mh "Diet, Sodium-Restricted"] OR "low-sodium diet"” OR "low salt diet $\left.^{* \prime}\right): \mathrm{ti}, \mathrm{ab}, \mathrm{kw}$

\#2 - (([mh "Dietary Proteins"] OR "dietary protein*” OR [mh "Dietary Carbohydrates"] OR "dietary carbohydrate*" OR [mh "Dietary Fats"] OR "dietary fat*" OR hypocaloric OR hypo-caloric) NEAR/6 (diet OR diets OR consumption OR intake OR supplement $\left.{ }^{\star}\right)$ )

\#3 - ([mh "Guideline Adherence"] OR guideline adherence*) NEAR/6 (diet OR dietary OR food OR beverage* OR nutrition*))

\#4 - ("diet score*" OR "diet quality score*" OR "diet quality index*" OR kidmed OR "diet index*" OR "dietary index*" OR "food score*" OR MedDietScore OR "healthy eating index*"):ti,ab,kw

\#5 - (((pattern OR patterns OR consumption OR habit $\left.{ }^{\star}\right)$ NEAR/6 ([mh ^ Diet] OR diet OR diets OR dietary OR [mh Food] OR food OR foods OR [mh Beverages] OR beverage OR beverages))):ti,ab,kw

\#6 - \#1 OR \#2 OR \#3 OR \#4 OR \#5

\#7 - [mh ^"Cardiovascular Diseases"] OR [mh "Heart Failure"] OR [mh "Myocardial Infarction"] OR [mh "Myocardial Ischemia"] OR [mh Stroke] OR [mh "Venous Thrombosis"] OR [mh Lipids/BL] OR [mh "Cholesterol, HDL"] OR [mh "Cholesterol, LDL"] OR [mh Triglycerides]"

\#8 - ("cardiovascular disease *" OR "coronary artery disease" OR "heart disease*" OR "heart failure*" OR "myocardial infarction*" OR "myocardial ischemia*” OR stroke OR angina OR "heart attack" OR "venous thrombosis" OR hypertension OR "high blood pressure" OR "HDL cholesterol" OR "LDL cholesterol" OR "total cholesterol" OR triglycerides):ti,ab,kw

\#9 - \#7 OR \#8

\#10 - [mh "Diabetes Mellitus, Type 2"] OR [mh "Prediabetic State"] OR [mh "Insulin Resistance"] OR [mh "Glucose Intolerance"] OR [mh "Glycated Hemoglobin A"] OR [mh Hyperglycemia] OR [mh Hypoglycemia]

\#11 - ("Type 2 diabetes" OR T2D OR "adult onset diabetes" OR prediabet* OR pre diabet* $^{*}$ OR "insulin resistance" OR "glucose intolerance" OR "glucose tolerance" OR "hemoglobin A1c"):ti,ab,kw

\#12 - ((“impaired fasting" OR [mh ^"Diabetes Mellitus"]) NEAR/6 (glucose OR glycemi* OR "high blood sugar" OR "low blood sugar"))):ti,ab,kw

\#13 - \#10 OR \#11 OR \#12

\#14 - [mh "Body Weights and Measures"] OR [mh "Body Weight"] OR [mh "Body Composition"] OR [mh "Growth Charts"] OR [mh ^"Growth"] OR [mh ^"Anthropometry"] OR [mh "Overnutrition"] 
\#15 - (obesity OR obese OR overweight OR "body mass index" OR BMI OR underweight OR wasting OR "healthy weight" OR "body composition" OR " body fat" OR "fat mass" OR "fat free mass" OR "body height" OR stunting OR stunted OR "growth chart" OR "waist circumference" OR "head circumference" OR "arm circumference" OR "thigh circumference" OR "neck circumference" OR "calf circumference" OR anthropometr* OR adiposity OR "skin fold" OR "healthy weight" OR "weight for height" OR "stature for age" OR "weight for age" OR "height for age" OR "length for age" OR "weight for length"):ti,ab,kw

\section{\#16 - \#14 OR \#15}

\#17 - \#9 OR \#13 OR \#16

\#18 - \#6 AND \#17" with Publication Year from 2014 to 2019, in Trials (Word variations have been searched)

\section{Embase}

- Provider: Elsevier

- Date(s) Searched: October 21, 2019

- Date range searched: January 1, 2014-October 21, 2019

- Search Terms:

\#1 - 'feeding behavior'/de OR 'mediterranean diet'/exp OR 'dash diet'/exp OR 'gluten free diet'/exp OR 'paleolithic diet'/de OR 'vegetarian diet'/exp OR 'healthy diet'/de OR 'western diet'/de OR 'low carbohydrate diet'/exp OR 'low fat diet'/de OR 'lipid diet'/exp OR 'protein diet'/exp OR 'protein restriction'/de OR 'sodium restriction'/de

\#2 - 'dietary pattern"':ab,ti OR 'diet pattern': ab,ti OR 'eating pattern':ab,ti OR 'food pattern':ab,ti OR 'diet quality':ab,ti OR 'eating habit"':ab,ti OR 'dietary habit':ab,ti OR 'diet habit":ab,ti OR 'food habit': ab,ti OR 'beverage habit': ab,ti OR 'dietary profile ${ }^{* 1}: a b, t i$ OR 'food profile ${ }^{* 1}:$ ab,ti OR 'diet profile ${ }^{* 1}: a b, t i$ OR 'eating profile ${ }^{* 1}: a b, t i$ OR 'dietary guideline ${ }^{* 1}: a b, t i$ OR 'dietary recommendation"':ab,ti OR 'dietary intake ${ }^{* \prime}: a b, t i$ OR 'eating style*':ab,ti OR 'mediterranean diet*':ab,ti OR 'dietary approaches to stop hypertension $\operatorname{diet}^{* \prime}: a b, t i$ OR 'dash $\operatorname{diet}^{* \prime}: a b$, ti OR 'gluten free $\operatorname{diet}^{* \prime}: a b, t i$ OR 'prudent $\operatorname{diet}^{* \prime}: a b, t i$ OR 'paleolithic $\operatorname{diet}^{* \prime}: a b, t i$ OR 'vegetarian $\operatorname{diet}^{\star \prime}: a b$, ti OR 'vegan $\operatorname{diet}^{* \prime}: a b, t i$ OR 'plant based $\operatorname{diet}^{* 1}:$ ab,ti OR 'western $\operatorname{diet}^{\star \prime}:$ ab,ti OR 'low-carbohydrate diet"':ab,ti OR 'high carbohydrate diet': ab,ti OR 'ketogenic $\operatorname{diet}^{* \prime}: a b, t i$ OR 'nordic diet"':ab,ti OR 'high protein $\operatorname{diet}^{* \prime}: a b, t i$ OR 'protein intake ${ }^{*}: a b, t i$ OR 'high-fat diet"':ab,ti OR 'low fat diet"':ab,ti OR 'low protein diet"':ab,ti OR 'lowsodium $\operatorname{diet}^{* \prime}: a b, t i$ OR 'low salt diet ${ }^{* \prime}: a b, t i$

\#3 - (('dietary protein*' OR 'dietary carbohydrate*' OR 'dietary fat*' OR hypocaloric OR hypo-caloric) NEAR/6 (diet OR diets OR consumption OR intake OR supplement)):ab,ti

\#4 - ('guideline adherence' NEAR/6 (diet OR dietary OR food OR beverage OR nutrition*)):ab,ti

\#5 - 'diet score':ab,ti OR 'diet quality score':ab,ti OR kidmed:ab,ti OR 'diet index':ab,ti OR 'dietary index':ab,ti OR 'diet quality index':ab,ti OR 'food score':ab,ti OR meddietscore:ab,ti OR 'healthy eating index':ab,ti

\#6 - ((pattern OR patterns OR consumption OR habit*) NEAR/6 (diet OR diets OR 
dietary OR food OR foods OR beverage OR beverages)):ab,ti

\#7 \#1 OR \#2 OR \#3 OR \#4 OR \#5 OR \#6

\#8 - 'cardiovascular disease'/de OR 'heart failure'/exp OR 'heart infarction'/exp OR 'heart muscle ischemia'/exp OR 'cerebrovascular accident'/exp OR 'vein thrombosis'/exp OR 'high density lipoprotein cholesterol'/de OR 'low density lipoprotein cholesterol'/de OR 'triacylglycerol'/exp

\#9 - 'cardiovascular disease ${ }^{* \prime}: a b$, ti OR 'coronary artery disease':ab,ti OR 'heart disease':ab,ti OR 'heart failure':ab,ti OR 'myocardial infarction*':ab,ti OR 'myocardial ischemia*':ab,ti OR stroke:ab,ti OR angina:ab,ti OR 'heart attack':ab,ti OR 'venous thrombosis':ab,ti OR 'hypertension':ab,ti OR 'high blood pressure':ab,ti OR 'hdl cholesterol':ab,ti OR 'Idl cholesterol':ab,ti OR 'total cholesterol':ab,ti OR triglycerides:ab,ti

\section{\#10 - \#8 OR \#9}

\#11 - 'non insulin dependent diabetes mellitus'/exp OR 'impaired glucose tolerance'/exp OR 'insulin resistance'/de OR 'glucose intolerance'/de OR 'glycosylated hemoglobin'/exp OR 'hyperglycemia'/de OR 'hypoglycemia'/exp

\#12 - 'type 2 diabetes':ab,ti OR t2d:ab,ti OR 'adult onset diabetes':ab,ti OR

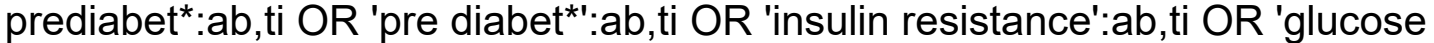
intolerance':ab,ti OR 'glucose tolerance':ab,ti OR 'hemoglobin a1c':ab,ti

\#13 - ('impaired fasting' OR 'diabetes mellitus]') NEAR/6 (glucose OR glycemi* OR 'high blood sugar' OR 'low blood sugar')):ab,ti

\section{\#14 - \#11 OR \#12 OR \#13}

\#15 - 'weight, mass and size'/exp OR 'body weight'/exp OR 'body composition'/exp OR 'growth chart'/de OR 'growth'/de OR 'anthropometry'/de OR 'overnutrition'/exp

\#16 - obesity:ab,ti OR obese:ab,ti OR overweight:ab,ti OR 'body mass index':ab,ti OR bmi:ab,ti OR underweight:ab,ti OR wasting:ab,ti OR 'body composition':ab,ti OR 'body fat':ab,ti OR 'fat mass':ab,ti OR 'fat free mass':ab,ti OR 'body height':ab,ti OR stunting:ab,ti OR stunted:ab,ti OR 'growth chart' $: a b, t i$ OR 'waist circumference':ab,ti OR 'head circumference':ab,ti OR 'arm circumference':ab,ti OR 'thigh circumference':ab,ti OR 'neck circumference':ab,ti OR 'calf circumference':ab,ti OR anthropometr*:ab,ti OR adiposity:ab,ti OR 'skin fold*':ab,ti OR 'healthy weight':ab,ti OR 'weight for height':ab,ti OR 'stature for age':ab,ti OR 'weight for age':ab,ti OR 'height for age':ab,ti OR 'length for age':ab,ti OR 'weight for length':ab,ti

\#17 - \#15 OR \#16

\#18 - \#10 OR \#14 OR \#17

\#19 - \#7 AND \#18

\#20 - \#7 AND \#18 AND ([article]/lim OR [article in press]/lim) AND [humans]/lim AND [english]/lim AND [2014-2019]/py NOT ([conference abstract]/lim OR [conference review]/lim OR [conference paper]/lim OR [editorial]/lim OR [erratum]/lim OR [letter]/lim OR [note]/lim OR [review]/lim OR [systematic review]/lim OR [meta analysis]/lim)

Search 2: Additional literature search to cover diets based on macronutrient 


\section{distribution}

\section{PubMed}

- Provider: U.S. National Library of Medicine

- Date(s) Searched: November 8, 2019

- Date range searched: January 1, 2000-December 31, 2013

- Search Terms:

\#1 - Macronutrient* OR Macro-nutrient* OR "Dietary Carbohydrates"[Mesh] OR dietary carbohydrate* OR carbohydrate diet* OR "Diet, Carbohydrate-

Restricted"[Mesh] OR carbohydrate-restrict* OR low carbohydrate* OR high carbohydrate* OR carbohydrate intake* OR "Dietary Proteins"[Mesh] OR protein

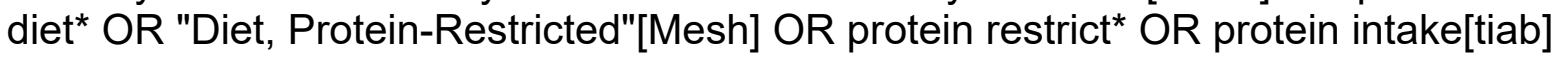
OR low protein* OR "Diet, High-Protein"[Mesh] OR high protein* OR Ketogenic Diet* OR "Dietary Fats"[Mesh] OR dietary fat* OR fat intake* OR "Diet, HighFat"[Mesh] OR high fat* OR "Diet, Fat-Restricted"[Mesh] OR fat-restricted* OR low fat $^{*}$

\#2 - "Cardiovascular Diseases"[Mesh:NoExp] OR cardiovascular disease*[tiab] OR coronary artery disease[tiab] OR heart disease*[tiab] OR "Heart Failure"[Mesh] OR heart failure[tiab] OR "Myocardial Infarction"[Mesh] OR myocardial infarction*[tiab] OR "Myocardial Ischemia"[Mesh] OR Myocardial Ischemia*[tiab] OR "Stroke"[Mesh] OR stroke[tiab] OR angina[tiab] OR heart attack[tiab] OR "Venous Thrombosis"[Mesh] OR venous thrombosis[tiab] OR hypertension[tiab] OR high blood pressure[tiab] OR "Lipids/blood"[Mesh] OR "Cholesterol, HDL"[Mesh] OR HDL cholesterol[tiab] OR "Cholesterol, LDL"[Mesh] OR LDL cholesterol[tiab]

\#3 - "Diabetes Mellitus, Type 2"[Mesh] OR Type 2 diabetes[tiab] OR T2D[tiab] OR adult onset diabetes[tiab] OR "Prediabetic State"[Mesh] OR prediabet*[tiab] OR pre diabet* OR "Insulin Resistance"[Mesh] OR insulin resistance[tiab] OR "Glucose Intolerance"[Mesh] OR glucose intolerance[tiab] OR glucose tolerance[tiab] OR "Glycated Hemoglobin A"[Mesh] OR hemoglobin A1c[ti] OR "Hyperglycemia"[Mesh] OR "Hypoglycemia"[Mesh] OR ((impaired fasting[tiab] OR "Diabetes Mellitus"[Mesh:NoExp]) AND (glucose[tiab] OR glycemi[tiab] OR high blood sugar[tiab] OR low blood sugar[tiab]))

\#4 - "Body Weights and Measures"[Mesh] OR "Body Weight"[Mesh] OR obesity[tiab] OR obese[tiab] OR overweight[tiab] OR body mass index[tiab] OR BMI[tiab] OR underweight[tiab] OR wasting[tiab] OR healthy weight[tiab] OR "Body Composition"[Mesh] OR body composition[tiab] OR body fat[tiab] OR fat mass[tiab] OR fat free mass[tiab] OR body height[tiab] OR stunting[tiab] OR stunted[tiab] OR "Growth Charts"[Mesh] OR growth chart*[tiab] OR "Growth"[Mesh:NoExp] OR waist circumference[tiab] OR head circumference[tiab] OR arm circumference[tiab] OR thigh circumference[tiab] OR neck circumference[tiab] OR "Anthropometry"[Mesh:NoExp] OR "Overnutrition"[Mesh] OR anthropometr*[tiab] OR adiposity[tiab] OR calf circumference[tiab] OR skin fold*[tiab] OR healthy weight[tiab] OR weight for height[tiab] OR stature for age[tiab] OR weight for age[tiab] OR height for age[tiab] OR length for age[tiab] OR weight for length[tiab]

\#5 - (\#2 OR \#3 OR \#4)

\#6 - (\#1 AND \#5) NOT ("Animals"[Mesh] NOT ("Animals"[Mesh] AND 
"Humans"[Mesh])) NOT (editorial[ptyp] OR comment[ptyp] OR news[ptyp] OR letter[ptyp] OR review[ptyp] OR systematic review[ptyp] OR systematic review[ti] OR meta-analysis[ptyp] OR meta-analysis[ti] OR meta-analyses[ti] OR retracted publication[ptyp] OR retraction of publication[ptyp] OR retraction of publication[tiab] OR retraction notice[ti])

Filters: Publication date from 2000/01/01 to 2013/12/31; English

\section{Cochrane Central Register of Controlled Trials (CENTRAL)}

- Provider: John Wiley \& Sons

- Date(s) Searched: November 8, 2019

- Date range searched: January 1, 2000-December 31, 2013

- Search Terms:

\#1 - [mh "Dietary Carbohydrates"] OR [mh "Diet, Carbohydrate-Restricted"] OR [mh "Dietary Proteins"] OR [mh "Diet, Protein-Restricted"] OR [mh "Diet, High-Protein"] OR [mh "Dietary Fats"] OR [mh "Diet, High-Fat"] OR [mh "Diet, Fat-Restricted"]

\#2 - (macronutrient* OR macro-nutrient* OR "dietary carbohydrate*" OR "carbohydrate diet"” OR "carbohydrate-restrict"” OR "low carbohydrate*" OR "high carbohydrate*" OR "carbohydrate intake*" OR "protein diet" OR "protein restrict" OR "protein intake" OR "low protein"” OR "high protein*" OR "Ketogenic Diet"” OR "dietary fat" OR "fatty diet*" OR "high fat" OR "fat-restricted" OR "low fat"):ti, ab, kw

\section{\#3 - \#1 OR \#2}

\#4 - [mh ^"Cardiovascular Diseases"] OR [mh "Heart Failure"] OR [mh "Myocardial Infarction"] OR [mh "Myocardial Ischemia"] OR [mh Stroke] OR [mh "Venous Thrombosis"] OR [mh Lipids/BL] OR [mh "Cholesterol, HDL"] OR [mh "Cholesterol, LDL"] OR [mh Triglycerides]

\#5 - ("cardiovascular disease*" OR "coronary artery disease *" OR "heart disease*" OR "heart failure*" OR "myocardial infarction" OR "myocardial ischemia*" OR stroke OR angina OR "heart attack" OR "venous thrombosis" OR hypertension OR "high blood pressure" OR "HDL cholesterol" OR "LDL cholesterol" OR "total cholesterol" OR triglycerides):ti,ab,kw

\section{\#6 - \#4 OR \#5}

\#7 - [mh "Diabetes Mellitus, Type 2"] OR [mh "Prediabetic State"] OR [mh "Insulin Resistance"] OR [mh "Glucose Intolerance"] OR [mh "Glycated Hemoglobin A"] OR [mh Hyperglycemia] OR [mh Hypoglycemia]

\#8 - ("Type 2 diabetes" OR T2D OR "adult onset diabetes" OR prediabet* OR pre diabet* OR "insulin resistance" OR "glucose intolerance" OR "glucose tolerance" OR "hemoglobin A1c"):ti,ab,kw"

\#9 - (("impaired fasting” OR [mh ^"Diabetes Mellitus"]) NEAR/6 (glucose OR glycemi* OR "high blood sugar" OR "low blood sugar")):ti,ab,kw

\section{\#10 - \#7 OR \#8 OR \#9}

\#11 - [mh "Body Weights and Measures"] OR [mh "Body Weight"] OR [mh "Body Composition"] OR [mh "Growth Charts"] OR [mh ^"Growth"] OR [mh ^"Anthropometry"] OR [mh "Overnutrition"] 
\#12 - (obesity OR obese OR overweight OR "body mass index" OR BMI OR underweight OR wasting OR "healthy weight" OR "body composition" OR " body fat" OR "fat mass" OR "fat free mass" OR "body height" OR stunting OR stunted OR "growth chart" OR "waist circumference" OR "head circumference" OR "arm circumference" OR "thigh circumference" OR "neck circumference" OR "calf circumference" OR anthropometr* OR adiposity OR "skin fold" OR "healthy weight" OR "weight for height" OR "stature for age" OR "weight for age" OR "height for age" OR "length for age" OR "weight for length"):ti,ab,kw

\section{\#13 - \#11 OR \#12}

\#14 - \#6 OR \#10 OR \#13

\#15 - \#3 AND \#14" with Publication Year from 2000 to 2013, in Trials (Word variations have been searched)

\section{Embase}

- Provider: Elsevier

- Date(s) Searched: November 8, 2019

- Date range searched: January 1, 2000-December 31, 2013

- Search Terms:

\#1 - 'macronutrient'/exp OR 'carbohydrate diet'/exp OR 'low carbohydrate diet'/exp OR 'protein intake'/exp OR 'protein restriction'/exp OR 'protein diet'/exp OR 'fat intake'/exp OR 'lipid diet'/exp OR 'low fat diet'/exp

\#2 - macronutrient*:ab,ti OR 'macro nutrient*':ab,ti OR 'dietary carbohydrate*':ab,ti OR 'carbohydrate diet' ${ }^{* 1}$ ab,ti OR 'carbohydrate-restrict"':ab,ti OR 'low carbohydrate ${ }^{* \prime}: a b, t i$ OR 'high carbohydrate ${ }^{* \prime}: a b, t i$ OR 'carbohydrate intake ${ }^{* \prime}: a b, t i$ OR 'protein diet ${ }^{* \prime}: a b, t i$ OR 'protein restrict*':ab,ti OR 'protein intake':ab,ti OR 'low

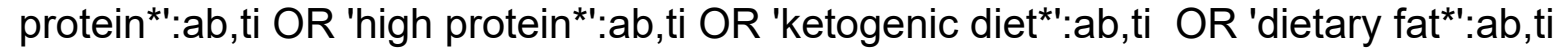
OR 'fat intake ${ }^{* \prime}: a b, t i$ OR 'high fat"':ab,ti OR 'fat-restricted ${ }^{* \prime}: a b, t i$ OR 'low fat ${ }^{* \prime}: a b, t i$

\section{\#3 - \#1 OR \#2}

\#4 - 'cardiovascular disease'/de OR 'heart failure'/exp OR 'heart infarction'/exp OR 'heart muscle ischemia'/exp OR 'cerebrovascular accident'/exp OR 'vein thrombosis'/exp OR 'high density lipoprotein cholesterol'/de OR 'low density lipoprotein cholesterol'/de OR 'triacylglycerol'/exp

\#5 - 'cardiovascular disease ${ }^{*}: a b, t i$ OR 'coronary artery disease':ab,ti OR 'heart disease':ab,ti OR 'heart failure':ab,ti OR 'myocardial infarction*':ab,ti OR 'myocardial ischemia*':ab,ti OR stroke:ab,ti OR angina:ab,ti OR 'heart attack':ab,ti OR 'venous thrombosis':ab,ti OR 'hypertension':ab,ti OR 'high blood pressure':ab,ti OR 'hdl cholesterol':ab,ti OR 'Idl cholesterol':ab,ti OR 'total cholesterol':ab,ti OR triglycerides:ab,ti

\section{\#6 - \#4 OR \#5}

\#7 - 'non insulin dependent diabetes mellitus'/exp OR 'impaired glucose tolerance'/exp OR 'insulin resistance'/de OR 'glucose intolerance'/de OR 'glycosylated hemoglobin'/exp OR 'hyperglycemia'/de OR 'hypoglycemia'/exp

\#8 - 'type 2 diabetes':ab,ti OR t2d:ab,ti OR 'adult onset diabetes':ab,ti OR prediabet*:ab,ti OR 'pre diabet ${ }^{* \prime}: a b, t i$ OR 'insulin resistance':ab,ti OR 'glucose 
intolerance':ab,ti OR 'glucose tolerance':ab,ti OR 'hemoglobin a1c':ab,ti

\#9 - (''impaired fasting' OR 'diabetes mellitus]') NEAR/6 (glucose OR glycemi OR 'high blood sugar' OR 'low blood sugar')):ab,ti

\section{\#10 - \#7 OR \#8 OR \#9}

\#11 - 'weight, mass and size'/exp OR 'body weight'/exp OR 'body composition'/exp OR 'growth chart'/de OR 'growth'/de OR 'anthropometry'/de OR 'overnutrition'/exp

\#12 - obesity:ab,ti OR obese:ab,ti OR overweight:ab,ti OR 'body mass index':ab,ti OR bmi:ab,ti OR underweight:ab,ti OR wasting:ab,ti OR 'body composition':ab,ti OR 'body fat':ab,ti OR 'fat mass':ab,ti OR 'fat free mass':ab,ti OR 'body height':ab,ti OR stunting:ab,ti OR stunted:ab,ti OR 'growth chart':ab,ti OR 'waist circumference':ab,ti OR 'head circumference':ab,ti OR 'arm circumference':ab,ti OR 'thigh circumference':ab,ti OR 'neck circumference':ab,ti OR 'calf circumference':ab,ti OR anthropometr*:ab,ti OR adiposity:ab,ti OR 'skin fold*':ab,ti OR 'healthy weight':ab,ti OR 'weight for height':ab,ti OR 'stature for age':ab,ti OR 'weight for age':ab,ti OR 'height for age':ab,ti OR 'length for age':ab,ti OR 'weight for length':ab,ti

\#13 - \#11 OR \#12

\#14 - \#6 OR \#10 OR \#13

\#15 - \#3 AND \#14

\#16 - \#3 AND \#14 AND ([article]/lim OR [article in press]/lim) AND [humans]/lim AND [english]/lim AND [2000-2013]/py NOT ([conference abstract]/lim OR [conference review]/lim OR [conference paper]/lim OR [editorial]/lim OR [erratum]/lim OR [letter]/lim OR [note]/lim OR [review]/lim OR [systematic review]/lim OR [meta analysis]/lim) 


\section{LITERATURE SEARCH AND SCREENING RESULTS}

The flow chart (Figure 2) below illustrates the literature search and screening results for articles to answer this systematic review question, which were searched together with similar systematic review questions for screening efficiency. The results of two electronic database searches, after removal of duplicates, were combined for efficiency and screened by two NESR analysts independently using a step-wise process by reviewing titles, abstracts, and full-texts to determine which articles met the inclusion criteria (Table 7) for each systematic review question depicted in the flow chart. Refer to Table 8 for the rationale for exclusion for each excluded full-text article. A manual search was done to find articles that were not identified when searching the electronic databases; all manually identified articles are also screened to determine whether they meet criteria for inclusion. 
Figure 2: Flow chart of literature search and screening results ${ }^{\text {xxviii }}$

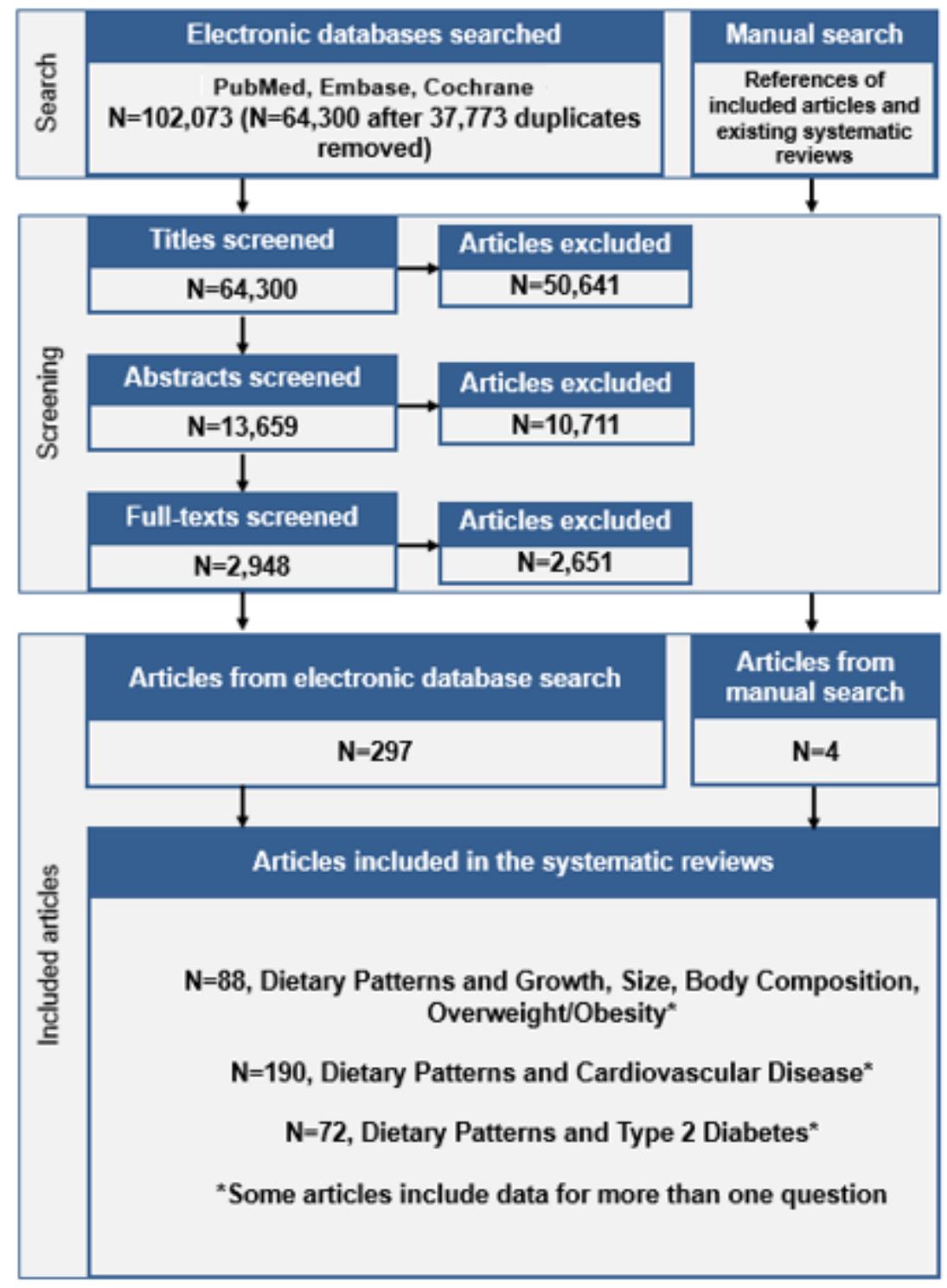

xxviii The flow chart depicts the combined raw search totals from the electronic databases that were searched from both searches that included a total of 102,073 raw results, which were comprised of 63,384 raw results from the first search strategy and 38,689 raw results from the second search strategy as described in the Electronic databases section. That section also provides the date range searched. Search results were combined for efficiency. Results were screened together in screening software by two analysts independently. Due to topical overlap, both of the searches were designed to comprehensively identify relevant literature in this systematic review question as well as two additional systematic reviews questions to avoid screening the same results multiple times. 


\section{Excluded articles}

The table below lists the articles excluded after full-text screening from two literature searches for this systematic review question, as well as two additional systematic review questions. At least one reason for exclusion is provided for each article, which may not reflect all possible reasons. Information about articles excluded after title and abstract screening is available upon request.

\section{Table 8. Articles excluded after full text screening with rationale for exclusion}

\begin{tabular}{|c|c|c|}
\hline No. & Citation & Rationale \\
\hline 1 & $\begin{array}{l}\text { Al Wattar B H, Dodds, J, Placzek, A, Beresford, L, Spyreli, E, Moore, A, Gonzalez Carreras, FJ, Austin, F, Murugesu, N, } \\
\text { Roseboom, TJ, Bes-Rastrollo, M, Hitman, GA, Hooper, R, Khan, KS, Thangaratinam, S. Mediterranean-style diet in } \\
\text { pregnant women with metabolic risk factors (ESTEEM): A pragmatic multicentre randomised trial. PLoS Med. } 2019 . \\
\text { 16:e1002857. doi:10.1371/journal.pmed.1002857 }\end{array}$ & Outcome; Participants \\
\hline 2 & $\begin{array}{l}\text { A low-carbohydrate diet reduces obesity and may improve dyslipidaemia compared with a low-fat diet. Evidence-Based } \\
\text { Healthcare and Public Health. 2004. 8:370-372. doi:10.1016/j.ehbc.2004.09.006 }\end{array}$ & $\begin{array}{l}\text { Study Design; } \\
\text { Publication Status }\end{array}$ \\
\hline 3 & $\begin{array}{l}\text { Assessment of control of cardiovascular risk factors in obese posmenopausal women after monitoring a structured dietary } \\
\text { education and exercise program. (SISIFO Program). Hipertension y riesgo vascular. } 33 \text { (3) (pp 103-110), 2016. Date of } \\
\text { publication: } 01 \text { jul 2016. 2016. . doi:10.1016/j.hipert.2016.02.002 }\end{array}$ & Language \\
\hline 4 & $\begin{array}{l}\text { Brazil nut consumption improves postprandial satiety, blood glucose and insulin responses in healthy adults. Circulation. } \\
\text { 2018. 138:. doi:unavailable }\end{array}$ & Publication Status \\
\hline 5 & $\begin{array}{l}\text { Compliance, palatability and feasibility of paleolithic and Australian guide to healthy eating diets in healthy women: a } 4 \text {-week } \\
\text { dietary intervention. Nutrients. } 8 \text { (8) (no pagination), 2016. Article number: } 481 \text {. Date of publication: } 06 \text { aug 2016.. 2016. : } \\
\text { doi:10.3390/nu8080481 }\end{array}$ & $\begin{array}{l}\text { Publication Status; } \\
\text { Study duration }\end{array}$ \\
\hline 6 & Diets that work. The Journal of clinical endocrinology and metabolism. 2014. 99:31A-32A. doi:unavailable & Publication Status \\
\hline 7 & Effective dietary interventions for managing overweight and obesity in children. Nurs N Z. 2007. 13:30-1. doi:unavailable & $\begin{array}{l}\text { Study Design; } \\
\text { Publication Status }\end{array}$ \\
\hline 8 & $\begin{array}{l}\text { Healthier protein sources may cut risks of coronary heart disease. Substituting fish, poultry and low-fat dairy for red meat } \\
\text { makes a difference. Heart Advis. 2010. 13:7. doi:unavailable }\end{array}$ & $\begin{array}{l}\text { Intervention/Exposure; } \\
\text { Publication Status }\end{array}$ \\
\hline 9 & $\begin{array}{l}\text { High fat diet more than a waistline worry. American journal of Alzheimer's disease and other dementias. 2004. 19:330-331. } \\
\text { doi:unavailable }\end{array}$ & $\begin{array}{l}\text { Study Design; } \\
\text { Publication Status }\end{array}$ \\
\hline 10 & $\begin{array}{l}\text { Mediterranean diets and metabolic syndrome status in the PREDIMED randomized trial. CMAJ : Canadian Medical } \\
\text { Association journal = journal de l'Association medicale canadienne. 2018. 190:E808. doi:10.1503/cmaj.180791 }\end{array}$ & Publication Status \\
\hline
\end{tabular}




\begin{tabular}{|c|c|c|}
\hline No. & Citation & Rationale \\
\hline 11 & Mediterranean eating linked to lower diabetes risk. Harvard men's health watch. 2014. 18:8. doi:unavailable & $\begin{array}{l}\text { Study Design; } \\
\text { Publication Status }\end{array}$ \\
\hline 12 & $\begin{array}{l}\text { Nutritional intervention based on mediterranean diet reduces blood pressure and immune cells response after immune } \\
\text { stimulation. Annals of nutrition \& metabolism. 2017. Conference: } 10 \text { th Annual Conference of the International Symposium on } \\
\text { Immunonutrition. Spain. } 71: 70-71 \text {. doi:unavailable }\end{array}$ & Publication Status \\
\hline 13 & $\begin{array}{l}\text { Physical activity rather than food knowledge/preferences underlie waist circumference improvements in early preventive } \\
\text { programs. Digestive and liver disease. 2017. Conference: 24th National SIGENP Congress. Italy. 49:e284. } \\
\text { doi:10.1016/j.dld.2017.09.115 }\end{array}$ & Publication Status \\
\hline 14 & $\begin{array}{l}\text { Short-term effects of different dietary approaches on body weight and body composition among obese female. Obesity facts. } \\
\text { 2018. Conference: 25th European Congress on Obesity, ECO 2018. Austria. 11:135-136. doi:10.1159/000489691 }\end{array}$ & Publication Status \\
\hline 15 & $\begin{array}{l}\text { Summaries for Patients. Effect of Allowing Choice of Diet on Weight Loss. Ann Intern Med. 2015. 162:I-22. doi:10.7326/p15- } \\
9021\end{array}$ & Publication Status \\
\hline 16 & $\begin{array}{l}\text { Aadland, EK, Lavigne, C, Graff, IE, Eng, O, Paquette, M, Holthe, A, Mellgren, G, Jacques, H, Liaset, B. Lean seafood } \\
\text { intake reduces cardiovascular risk factors in healthy subjects. Annals of nutrition and metabolism. 2015. 67:335-336. } \\
\text { doi:10.1159/000440895 }\end{array}$ & $\begin{array}{l}\text { Intervention/Exposure; } \\
\text { Study duration }\end{array}$ \\
\hline 17 & $\begin{array}{l}\text { Aadland, EK, Lavigne, C, Graff, IE, Eng, O, Paquette, M, Holthe, A, Mellgren, G, Jacques, H, Liaset, B. Lean-seafood } \\
\text { intake reduces cardiovascular lipid risk factors in healthy subjects: results from a randomized controlled trial with a crossover } \\
\text { design. Am J Clin Nutr. } 2015.102: 582-92 \text {. doi:10.3945/ajcn.115.112086 }\end{array}$ & $\begin{array}{l}\text { Intervention/Exposure; } \\
\text { Comparator }\end{array}$ \\
\hline 18 & $\begin{array}{l}\text { Aballay, LR, Osella, AR, De La Quintana, AG, Diaz, MDP. Nutritional profile and obesity: results from a random-sample } \\
\text { population-based study in Cordoba, Argentina. Eur J Nutr. 2016. 55:675-685. doi:10.1007/s00394-015-0887-0 }\end{array}$ & Study Design \\
\hline 19 & $\begin{array}{l}\text { Abbasi, F, McLaughlin, T, Lamendola, C, Kim, HS, Tanaka, A, Wang, T, Nakajima, K, Reaven, GM. High carbohydrate } \\
\text { diets, triglyceride-rich lipoproteins, and coronary heart disease risk. Am J Cardiol. 2000. 85:45-8. doi:10.1016/s0002- } \\
\text { 9149(99)00604-9 }\end{array}$ & Study duration \\
\hline 20 & $\begin{array}{l}\text { Abdel-Megeid, FY, Abdelkarem, HM, El-Fetouh, AM. Unhealthy nutritional habits in university students are a risk factor for } \\
\text { cardiovascular diseases. Saudi Medical Journal. 2011. 32:621-627. doi:unavailable }\end{array}$ & Study Design \\
\hline 21 & $\begin{array}{l}\text { Aberg, G, Edman, G, Rossner, S. Perceived hunger, palatability, and adherence: A comparison of high- and low-fat diets. } \\
\text { Obes Res Clin Pract. 2008. 2:71-142. doi:10.1016/j.orcp.2008.03.001 }\end{array}$ & $\begin{array}{l}\text { Study duration ; } \\
\text { Health Status }\end{array}$ \\
\hline 22 & $\begin{array}{l}\text { Abete, I, Parra, D, De Morentin, BM, Alfredo Martinez, J. Effects of two energy-restricted diets differing in the } \\
\text { carbohydrate/protein ratio on weight loss and oxidative changes of obese men. Int J Food Sci Nutr. 2009. } 60 \text { Suppl 3:1-13. } \\
\text { doi:10.1080/09637480802232625 }\end{array}$ & Study duration \\
\hline 23 & $\begin{array}{l}\text { Abete, I, Parra, D, Martinez, JA. Legume-, fish-, or high-protein-based hypocaloric diets: effects on weight loss and } \\
\text { mitochondrial oxidation in obese men. J Med Food. 2009. 12:100-8. doi:10.1089/jmf.2007.0700 }\end{array}$ & Study duration \\
\hline
\end{tabular}


No.

Citation

24 Abu-Saad, K, Novikov, I, Gimpelevitz, I, Benderly, M, Alpert, G, Goldbourt, U, Kalter-Leibovici, O. Micronutrient intake and adherence to DASH diet are associated with incident major adverse cardiovascular events and all-cause mortality in a bi-ethnic population. European heart journal. 2017. 38:1120-. doi:10.1093/eurheartj/ehx502.P5321

25 Acosta-Navarro, JC, Oki, AM, Antoniazzi, L, Bonfim, MAC, Hong, V, Gaspar, MCA, Sandrim, VC, Nogueira, A. Consumption of animal-based and processed food associated with cardiovascular risk factors and subclinical atherosclerosis biomarkers in men. Rev Assoc Med Bras (1992). 2019. 65:43-50. doi:10.1590/1806-9282.65.1.43

26 Adachi, H, Hino, A. Trends in nutritional intake and serum cholesterol levels over 40 years in Tanushimaru, Japanese men. J Epidemiol. 2005. 15:85-9. doi:10.2188/jea.15.85

27 Adamsson, V, Cederholm, T, Vessby, B, Risérus, U. Influence of a healthy Nordic diet on serum fatty acid composition and associations with blood lipoproteins - Results from the NORDIET study. Food and Nutrition Research. 2014. 58:. doi:10.3402/fnr.v58.24114

28 Adamsson, V, Reumark, A, Fredriksson, IB, Hammarstrom, E, Vessby, B, Johansson, G, Riserus, U. Effects of a healthy Nordic diet on cardiovascular risk factors in hypercholesterolaemic subjects: a randomized controlled trial (NORDIET). J Intern Med. 2011. 269:150-9. doi:10.1111/j.1365-2796.2010.02290.x

29 Adechian, S, Balage, M, Remond, D, Migne, C, Quignard-Boulange, A, Marset-Baglieri, A, Rousset, S, Boirie, Y, Gaudichon, C, Dardevet, D, Mosoni, L. Protein feeding pattern, casein feeding, or milk-soluble protein feeding did not change the evolution of body composition during a short-term weight loss program. Am J Physiol Endocrinol Metab. 2012. 303:E973-82. doi:10.1152/ajpendo.00285.2012

30 Adelina, R. BMI correlated to dietary pattern of Indonesian college students lives in Taipei city, Taiwan. Pakistan Journal of Medical and Health Sciences. 2018. 12:1308-1311. doi:unavailable

31 Adochio, RL, Leitner, JW, Gray, K, Draznin, B, Cornier, MA. Early responses of insulin signaling to high-carbohydrate and high-fat overfeeding. Nutr Metab (Lond). 2009. 6:37. doi:10.1186/1743-7075-6-37

32 Adriouch, S, Julia, C, Kesse-Guyot, E, Mejean, C, Ducrot, P, Peneau, S, Donnenfeld, M, Deschasaux, M, Menai, M, Hercberg, S, Touvier, M, Fezeu, LK. Prospective association between a dietary quality index based on a nutrient profiling system and cardiovascular disease risk. Eur J Prev Cardiol. 2016. 23:1669-76. doi:10.1177/2047487316640659

33 Adriouch, S, Lelong, H, Kesse-Guyot, E, Baudry, J, Lampuré, A, Galan, P, Hercberg, S, Touvier, M, Fezeu, LK. Compliance with nutritional and lifestyle recommendations in 13,000 patients with a cardiometabolic disease from the NutrinetSanté study. Nutrients. 2017. 9:. doi:10.3390/nu9060546

34 Aeberli, I, Kaspar, M, Zimmermann, MB. Dietary intake and physical activity of normal weight and overweight 6 to 14 year old Swiss children. Swiss Med Wkly. 2007. 137:424-30. doi:2007/29/smw-11696

35 Aeberli, I, Spinas, GA, Lehmann, R, I'Allemand, D, Molinari, L, Zimmermann, MB. Diet determines features of the metabolic syndrome in 6- to 14-year-old children. Int J Vitam Nutr Res. 2009. 79:14-23. doi:10.1024/0300-9831.79.1.14

\section{Rationale}

Publication Status

Study Design

Study Design

Intervention/Exposure; Outcome

Intervention/Exposure; Publication Date

Overlaps with Existing Review

Intervention/Exposure; Study duration

Study Design; Intervention/Exposure

Study duration

Intervention/Exposure

Study Design

Study Design

Study Design 
No.

Citation

36 Aguiar-Bloemer, AC, Japur, CC, Francisco, LV, Diez-Garcia, RW. Dietary quality differences between women with and without weight loss in nutritional treatment. Clinical Nutrition ESPEN. 2019. doi:10.1016/j.clnesp.2019.08.003

37 Ahadi, Z, Qorbani, M, Kelishadi, R, Ardalan, G, Motlagh, ME, Asayesh, H, Zeynali, M, Chinian, M, Larijani, B, Shafiee, G, Heshmat, R. Association between breakfast intake with anthropometric measurements, blood pressure and food consumption behaviors among Iranian children and adolescents: the CASPIAN-IV study. Public Health. 2015. 129:740-7. doi:10.1016/j.puhe.2015.03.019

Ahluwalia, N, Ferrieres, J, Dallongeville, J, Simon, C, Ducimetiere, P, Amouyel, P, Arveiler, D, Ruidavets, JB. Association of macronutrient intake patterns with being overweight in a population-based random sample of men in France. Diabetes Metab. 2009. 35:129-36. doi:10.1016/j.diabet.2008.09.006

39 Ahmadi-Abhari, S, Luben, RN, Powell, N, Bhaniani, A, Chowdhury, R, Wareham, NJ, Forouhi, NG, Khaw, KT. Dietary intake of carbohydrates and risk of type 2 diabetes: the European Prospective Investigation into Cancer-Norfolk study. Br J Nutr 2014. 111:342-52. doi:10.1017/s0007114513002298

40 Ahn, Y, Park, SJ, Kwack, HK, Kim, MK, Ko, KP, Kim, SS. Rice-eating pattern and the risk of metabolic syndrome especially waist circumference in Korean Genome and Epidemiology Study (KoGES). BMC Public Health. 2013. 13:61. doi:10.1186/14712458-13-61

41 Ahuja, KD, Ashton, EL, Ball, MJ. Effects of two lipid-lowering, carotenoid-controlled diets on the oxidative modification of lowdensity lipoproteins in free-living humans. Clin Sci (Lond). 2003. 105:355-61. doi:10.1042/cs20030050

42 Akbaraly, T, Wurtz, P, Singh-Manoux, A, Shipley, MJ, Haapakoski, R, Lehto, M, Desrumaux, C, Kahonen, M, Lehtimaki, T, Mikkila, V, Hingorani, A, Humphries, SE, Kangas, AJ, Soininen, P, Raitakari, O, Ala-Korpela, M, Kivimaki, M. Association of circulating metabolites with healthy diet and risk of cardiovascular disease: analysis of two cohort studies. Sci Rep. 2018. 8:8620. doi:10.1038/s41598-018-26441-1

43 Akbaraly, TN, Shipley, MJ, Ferrie, JE, Virtanen, M, Lowe, G, Hamer, M, Kivimaki, M. Long-term adherence to healthy dietary guidelines and chronic inflammation in the prospective Whitehall II study. Am J Med. 2015. 128:152-160.e4. doi:10.1016/j.amjmed.2014.10.002

44 Akbari-Sedigh, A, Asghari, G, Yuzbashian, E, Dehghan, P, Imani, H, Mirmiran, P. Association of dietary pattern with carotid intima media thickness among children with overweight or obesity. Diabetol Metab Syndr. 2019. 11:77. doi:10.1186/s13098019-0472-4

45 Akdemir, M, Donmez, L, Polat, H. The effect of nutritional and physical activity interventions on nutritional status and obesity in primary school children: A cluster randomized controlled study. Kuwait Medical Journal. 2017. 49:105-113. doi:unavailable

46 Akinyemi, RO, Allan, L, Owolabi, MO, Akinyemi, JO, Ogbole, G, Ajani, A, Firbank, M, Ogunniyi, A, Kalaria, RN. Profile and determinants of vascular cognitive impairment in African stroke survivors: the CogFAST Nigeria Study. J Neurol Sci. 2014. 346:241-9. doi:10.1016/j.jns.2014.08.042

47 Akita, S, Sacks, FM, Svetkey, LP, Conlin, PR, Kimura, G. Effects of the Dietary Approaches to Stop Hypertension (DASH) diet on the pressure-natriuresis relationship. Hypertension. 2003. 42:8-13. doi:10.1161/01.Hyp.0000074668.08704.6e

\section{Rationale}

Study Design

Study Design;

Intervention/Exposure

Study Design

Intervention/Exposure

Study Design

Study duration

Intervention/Exposure

Outcome

Study Design

Intervention/Exposure

Intervention/Exposure

Outcome 
48 Akter, S, Kurotani, K, Kashino, I, Goto, A, Mizoue, T, Noda, M, Sawada, N, Tsugane, S. High Dietary Acid Load Score Is

Intervention/Exposure Associated with Increased Risk of Type 2 Diabetes in Japanese Men: The Japan Public Health Center-based Prospective Study. J Nutr. 2016. 146:1076-83. doi:10.3945/jn.115.225177

49 Akter, S, Nanri, A, Mizoue, T, Noda, M, Sawada, N, Sasazuki, S, Tsugane, S. Dietary acid load and mortality among Japanese men and women: the Japan Public Health Center-based Prospective Study. Am J Clin Nutr. 2017. 106:146-154. doi:10.3945/ajcn.117.152876

50 Al Hourani, H, Atoum, M, Alboqai, O, Ismail, LC, Al Dhaheri, A, Hijjawi, N. Effectiveness of dietary intervention for obese women in Jordan. International Journal of Food Sciences and Nutrition. 2009. 60:76-82. doi:10.1080/09637480903022727

51 Al Suwaidi, J. Dietary patterns and their association with acute coronary heart disease: Lessons from the REGARDS Study. Glob Cardiol Sci Pract. 2015. 2015:56. doi:10.5339/gcsp.2015.56

52 Al-Delaimy, WK, Natarajan, L, Rock, CL, Sun, S, Flatt, SW, Pierce, JP. Insulin-like growth factor I, insulin-like growth factor I binding protein 1, insulin, glucose, and leptin serum levels are not influenced by a reduced-fat, high-fiber diet intervention. Cancer Epidemiol Biomarkers Prev. 2006. 15:1238-9. doi:10.1158/1055-9965.Epi-06-0160

53 Aldrich, ND, Reicks, MM, Sibley, SD, Redmon, JB, Thomas, W, Raatz, SK. Varying protein source and quantity do not significantly improve weight loss, fat loss, or satiety in reduced energy diets among midlife adults. Nutr Res. 2011. 31:104-12. doi:10.1016/j.nutres.2011.01.004

54 Alemany, JA, Nindl, BC, Kellogg, MD, Tharion, WJ, Young, AJ, Montain, SJ. Effects of dietary protein content on IGF-I, testosterone, and body composition during 8 days of severe energy deficit and arduous physical activity. J Appl Physiol (1985). 2008. 105:58-64. doi:10.1152/japplphysiol.00005.2008

55 Alenazi, SA, Koura, HM, Zaki, SM, Mohamed, AH. Prevalence of Obesity Among Male Adolescents in Arar Saudi Arabia: Future Risk of Cardiovascular Disease. Indian J Community Med. 2015. 40:182-7. doi:10.4103/0970-0218.158864

56 AlEssa, HB, Bhupathiraju, SN, Malik, VS, Wedick, NM, Campos, H, Rosner, B, Willett, WC, Hu, FB. Carbohydrate quality and quantity and risk of type 2 diabetes in US women. Am J Clin Nutr. 2015. 102:1543-53. doi:10.3945/ajcn.115.116558

57 Alexander, S, Ostfeld, RJ, Allen, K, Williams, KA. A plant-based diet and hypertension. J Geriatr Cardiol. 2017. 14:327-330. doi:10.11909/j.issn.1671-5411.2017.05.014

58 Alexy, U, Sichert-Hellert, W, Kersting, M, Schultze-Pawlitschko, V. Pattern of long-term fat intake and BMI during childhood and adolescence--results of the DONALD Study. Int J Obes Relat Metab Disord. 2004. 28:1203-9. doi:10.1038/sj.ijo.0802708

59 Alhassan, S, Kim, S, Bersamin, A, King, AC, Gardner, CD. Dietary adherence and weight loss success among overweight women: results from the A TO Z weight loss study. Int J Obes (Lond). 2008. 32:985-91. doi:10.1038/ijo.2008.8

60 Alhazmi, A, Stojanovski, E, McEvoy, M, Garg, ML. Macronutrient intake and type 2 diabetes risk in middle-aged Australian women. Results from the Australian Longitudinal Study on Women's Health. Public Health Nutr. 2014. 17:1587-94. doi:10.1017/s1368980013001870

Study Design

Study Design

Study Design; Health Status

Power/Size

Study duration

Study Design

Intervention/Exposure

Study Design

Power/Size

Weight

loss/Hypocaloric

Intervention/Exposure 


\section{No. Citation}

61 Alimujiang, A, Colditz, GA, Gardner, JD, Park, Y, Berkey, CS, Sutcliffe, S. Childhood diet and growth in boys in relation to timing of puberty and adult height: the Longitudinal Studies of Child Health and Development. Cancer Causes Control. 2018. 29:915-926. doi:10.1007/s10552-018-1068-2

62 Alissa, EM, Bahjri, SM, Al-Ama, N, Ahmed, WH, Ferns, GA. High cardiovascular risk in young Saudi males: cardiovascular risk factors, diet and inflammatory markers. Clin Chim Acta. 2006. 365:288-96. doi:10.1016/j.cca.2005.09.007

63 Aljuraiban, GS, Gibson, R, Oude Griep, LM, Okuda, N, Steffen, LM, Van Horn, L, Chan, Q. Perspective: The Application of A Priori Diet Quality Scores to Cardiovascular Disease Risk-A Critical Evaluation of Current Scoring Systems. Adv Nutr. 2019. : doi:10.1093/advances/nmz059

64 Allan, GM, Ivers, N, Sharma, AM. Diets for weight loss and prevention of negative health outcomes. Can Fam Physician. 2011. 57:894-5. doi:unavailable

65 Aller, EE, Larsen, TM, Claus, H, Lindroos, AK, Kafatos, A, Pfeiffer, A, Martinez, JA, Handjieva-Darlenska, T, Kunesova, M, Stender, S, Saris, WH, Astrup, A, van Baak, MA. Weight loss maintenance in overweight subjects on ad libitum diets with high or low protein content and glycemic index: the DIOGENES trial 12-month results. Int J Obes (Lond). 2014. 38:1511-7. doi:10.1038/ijo.2014.52

66 Aller, R, De Luis, DA, Izaola, O, La Calle, F, Del Olmo, L, Fernandez, L, Arranz, T, Gonzalez Hernandez, JM. Effect of soluble fiber intake in lipid and glucose leves in healthy subjects: A randomized clinical trial. Diabetes Research and Clinical Practice. 2004. 65:7-11. doi:10.1016/j.diabres.2003.11.005

67 Allison, MA, Aragaki, AK, Ray, RM, Margolis, KL, Beresford, SA, Kuller, L, Jo O'Sullivan, M, Wassertheil-Smoller, S, Van Horn, L. A Randomized Trial of a Low-Fat Diet Intervention on Blood Pressure and Hypertension: Tertiary Analysis of the WHI Dietary Modification Trial. Am J Hypertens. 2016. 29:959-68. doi:10.1093/ajh/hpv196

68 Almohanna, A, Conforti, F, Eigel, W, Barbeau, W. Impact of Dietary Acculturation on the Food Habits, Weight, Blood Pressure, and Fasting Blood Glucose Levels of International College Students. J Am Coll Health. 2015. 63:307-14. doi:10.1080/07448481.2015.1025075

69 Al-Nakeeb, Y, Lyons, M, Dodd, LJ, Al-Nuaim, A. An investigation into the lifestyle, health habits and risk factors of young adults. Int J Environ Res Public Health. 2015. 12:4380-94. doi:10.3390/ijerph120404380

70 Alnasir, FA, Fateha, BE. Low carbohydrate diet. Its effects on selected body parameters of obese patients. Saudi Med J. 2003. 24:949-52. doi:unavailable

71 Alonso, A, Beunza, JJ, Bes-Rastrollo, M, Pajares, RM, Martinez-Gonzalez, MA. Vegetable protein and fiber from cereal are inversely associated with the risk of hypertension in a Spanish cohort. Arch Med Res. 2006. 37:778-86. doi:10.1016/j.arcmed.2006.01.007

72 Al-Sarraj, T, Saadi, H, Calle, MC, Volek, JS, Fernandez, ML. Carbohydrate restriction, as a first-line dietary intervention, effectively reduces biomarkers of metabolic syndrome in Emirati adults. J Nutr. 2009. 139:1667-76. doi:10.3945/jn.109.109603

\section{Rationale}

Study Design;

Intervention/Exposure Outcome

Study Design;

Intervention/Exposure

Study Design

Study Design

Publication Status

Intervention/Exposure

Intervention/Exposure; Comparator

Intervention/Exposure

Intervention/Exposure;

Comparator

Intervention/Exposure

Study Design

Intervention/Exposure; Outcome

Study duration 
73 Al-Sarraj, T, Saadi, H, Volek, JS, Fernandez, ML. Carbohydrate restriction favorably alters lipoprotein metabolism in Emirati subjects classified with the metabolic syndrome. Nutr Metab Cardiovasc Dis. 2010. 20:720-6. doi:10.1016/j.numecd.2009.06.004

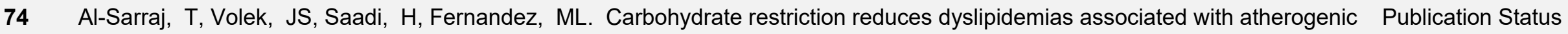
lipoprotein profiles in Emirati men and women with metabolic syndrome. FASEB journal. 2013. 27:. doi:unavailable

75 Alshaikh, MK, Rawaf, S, Quezada-Yamamoto, H. Cardiovascular risk and fruit and vegetable consumption among women in Study Design KSA; A cross-sectional study. J Taibah Univ Med Sci. 2018. 13:444-451. doi:10.1016/j.jtumed.2018.06.001

76 Alsharari, ZD, Leander, K, Sjogren, P, Carlsson, A, Cederholm, T, de Faire, U, Hellenius, ML, Marklund, M, Riserus, U. Association between carbohydrate intake and fatty acids in the de novo lipogenic pathway in serum phospholipids and adipose tissue in a population of Swedish men. Eur J Nutr. 2019. .. doi:10.1007/s00394-019-02058-6

77 Al-Sobayel, H, Al-Hazzaa, HM, Abahussain, NA, Qahwaji, DM, Musaiger, AO. Gender differences in leisure-time versus nonleisure-time physical activity among Saudi adolescents. Ann Agric Environ Med. 2015. 22:344-8. doi:10.5604/12321966.1152091

78 Al-Solaiman, Y, Jesri, A, Mountford, WK, Lackland, DT, Zhao, Y, Egan, BM. DASH lowers blood pressure in obese hypertensives beyond potassium, magnesium and fibre. J Hum Hypertens. 2010. 24:237-46. doi:10.1038/jhh.2009.58

79 Altorf-van der Kuil, W, Engberink, MF, De Neve, M, van Rooij, FJ, Hofman, A, van't Veer, P, Witteman, JC, Franco, OH, Geleijnse, JM. Dietary amino acids and the risk of hypertension in a Dutch older population: the Rotterdam Study. Am J Clin Nutr. 2013. 97:403-10. doi:10.3945/ajcn.112.038737

80 Altorf-van der Kuil, W, Engberink, MF, van Rooij, FJ, Hofman, A, van't Veer, P, Witteman, JC, Geleijnse, JM. Dietary protein and risk of hypertension in a Dutch older population: the Rotterdam study. J Hypertens. 2010. 28:2394-400. doi:10.1097/HJH.0b013e32833eff63

81 Alvarez-Alvarez, I, Toledo, E, Lecea, O, Salas-Salvado, J, Corella, D, Buil-Cosiales, P, Zomeno, MD, Vioque, J, Martinez, JA, Konieczna, J, Baron-Lopez, FJ, Lopez-Miranda, J, Estruch, R, Bueno-Cavanillas, A, Alonso-Gomez, AM, Tur, JA, Tinahones, FJ, Serra-Majem, L, Martin, V, Ortega-Calvo, M, Vazquez, C, Pinto, X, Vidal, J, Daimiel, L, Delgado-Rodriguez, M, Matia, P, Gonzalez, JI, Diaz-Lopez, A, Paz-Graniel, I, Munoz, MA, Fito, M, Pertusa-Martinez, S, Abete, I, Garcia-Rios, A, Ros, E, Ruiz-Canela, M, Martinez-Gonzalez, MA. Adherence to a priori dietary indexes and baseline prevalence of cardiovascular risk factors in the PREDIMED-Plus randomised trial. Eur J Nutr. 2019. :. doi:10.1007/s00394-019-01982-x

82 Ambrosini, GL, Emmett, PM, Northstone, K, Howe, LD, Tilling, K, Jebb, SA. Identification of a dietary pattern prospectively associated with increased adiposity during childhood and adolescence. Int J Obes (Lond). 2012. 36:1299-305 doi:10.1038/ijo.2012.127

83 Ambrosini, GL, Emmett, PM, Northstone, K, Jebb, SA. Tracking a dietary pattern associated with increased adiposity in childhood and adolescence. Obesity (Silver Spring). 2014. 22:458-65. doi:10.1002/oby.20542

84 Ambrosini, GL, Solis-Trapala, I, Ahern, AL, Fuller, NR, Holzapfel, C, Hauner, H, Caterson, ID, Jebb, SA. Greater improvements in diet quality among overweight participants following a group-based commercial weight loss programme than those receiving support to lose weight in primary care. Nutr J. 2018. 17:64. doi:10.1186/s12937-018-0370-X

Study Design;

Outcome

Study Design;

Intervention/Exposure

Study duration

Intervention/Exposure

Intervention/Exposure

Study Design

Publication Date

Overlaps with Existing Review

Outcome

Intervention/Exposure 
85 Ambroszkiewicz, J, Chelchowska, M, Rowicka, G, Klemarczyk, W, Strucinska, M, Gajewska, J. Anti-Inflammatory and ProInflammatory Adipokine Profiles in Children on Vegetarian and Omnivorous Diets. Nutrients. 2018. 10: doi:10.3390/nu10091241

86 Ambroszkiewicz, J, Chelchowska, M, Szamotulska, K, Rowicka, G, Klemarczyk, W, Strucinska, M, Gajewska, J. Bone status and adipokine levels in children on vegetarian and omnivorous diets. Clin Nutr. 2019. 38:730-737.

Study Design doi:10.1016/j.clnu.2018.03.010

87 Ambroszkiewicz, J, Klemarczyk, W, Gajewska, J, Chelchowska, M, Rowicka, G, Oltarzewski, M, Laskowska-Klita, T. Serum Study Design concentration of adipocytokines in prepubertal vegetarian and omnivorous children. Med Wieku Rozwoj. 2011. 15:326-34. doi:unavailable

88 Ambroszkiewicz, J, Laskowska-Klita, T, Klemarczyk, W. Low serum leptin concentration in vegetarian prepubertal children. Rocz Akad Med Bialymst. 2004. 49:103-5. doi:unavailable

89 Amini, P, Maghsoudi, Z, Feizi, A, Ghiasvand, R, Askari, G. Effects of High Protein and Balanced Diets on Lipid Profiles and Inflammation Biomarkers in Obese and Overweight Women at Aerobic Clubs: A Randomized Clinical Trial. Int J Prev Med. 2016. 7:110. doi:10.4103/2008-7802.190608

90 Amor, AJ, Masana, L, Soriguer, F, Goday, A, Calle-Pascual, A, Gaztambide, S, Rojo-Martinez, G, Valdes, S, Gomis, R, Ortega, E. Estimating Cardiovascular Risk in Spain by the European Guidelines on Cardiovascular Disease Prevention in Clinical Practice. Rev Esp Cardiol (Engl Ed). 2015. 68:417-25. doi:10.1016/j.rec.2014.05.023

91 Ampatzoglou, A, Atwal, KK, Maidens, CM, Williams, CL, Ross, AB, Thielecke, F, Jonnalagadda, SS, Kennedy, OB, Yaqoob, P. Increased whole grain consumption does not affect blood biochemistry, body composition, or gut microbiology in healthy, low-habitual whole grain consumers. J Nutr. 2015. 145:215-21. doi:10.3945/jn.114.202176

92 An, R, Burd, NA. Change in daily energy intake associated with pairwise compositional change in carbohydrate, fat and protein Study Design intake among US adults, 1999-2010. Public Health Nutr. 2015. 18:1343-52. doi:10.1017/s1368980014001876

93 Anders, S, Schroeter, C. Diabetes, diet-health behavior, and obesity. Front Endocrinol (Lausanne). 2015. 6:33. doi:10.3389/fendo.2015.00033 94 Andersen, LB, Arnberg, K, Trolle, E, Michaelsen, KF, Bro, R, Pipper, CB, Molgaard, C. The effects of water and dairy drinks
on dietary patterns in overweight adolescents. Int J Food Sci Nutr. 2016. 67:314-24. doi:10.3109/09637486.2016.1150435

95 Andersen, LB, Molgaard, C, Ejlerskov, KT, Trolle, E, Michaelsen, KF, Bro, R, Pipper, CB. Development of Dietary Patterns Spanning Infancy and Toddlerhood: Relation to Body Size, Composition and Metabolic Risk Markers at Three Years. AIMS Public Health. 2015. 2:332-357. doi:10.3934/publichealth.2015.3.332 patterns with body composition in older adults differ by gender and PPAR-gamma Pro12Ala genotype. Eur J Nutr. 2010. 49:385-94. doi:10.1007/s00394-010-0096-9

Study Design

Intervention/Exposure

Study Design

Intervention/Exposure

Study Design

Outcome

Study Design

Intervention/Exposure; Age at

Intervention/Exposure Study Design 
97 Anderson, AL, Harris, TB, Tylavsky, FA, Perry, SE, Houston, DK, Hue, TF, Strotmeyer, ES, Sahyoun, NR. Dietary patterns

Outcome and survival of older adults. J Am Diet Assoc. 2011. 111:84-91. doi:10.1016/j.jada.2010.10.012

98 Anderson, AL, Harris, TB, Tylavsky, FA, Perry, SE, Houston, DK, Lee, JS, Kanaya, AM, Sahyoun, NR. Dietary patterns, insulin sensitivity and inflammation in older adults. Eur J Clin Nutr. 2012. 66:18-24. doi:10.1038/ejcn.2011.162

99 Anderson, AS, Haynie, KR, McMillan, RP, Osterberg, KL, Boutagy, NE, Frisard, MI, Davy, BM, Davy, KP, Hulver, MW. Early skeletal muscle adaptations to short-term high-fat diet in humans before changes in insulin sensitivity. Obesity (Silver Spring). 2015. 23:720-4. doi:10.1002/oby.21031

100 Anderson-Vasquez, HE, Perez-Martinez, P, Ortega Fernandez, P, Wanden-Berghe, C. Impact of the consumption of a rich diet in butter and it replacement for a rich diet in extra virgin olive oil on anthropometric, metabolic and lipid profile in postmenopausal women. Nutr Hosp. 2015. 31:2561-70. doi:10.3305/nh.2015.31.6.8732

101 Andersson, J, Mellberg, C, Otten, J, Ryberg, M, Rinnstrom, D, Larsson, C, Lindahl, B, Hauksson, J, Johansson, B, Olsson, $\mathrm{T}$. Left ventricular remodelling changes without concomitant loss of myocardial fat after long-term dietary intervention. Int $\mathrm{J}$ Cardiol. 2016. 216:92-6. doi:10.1016/j.ijcard.2016.04.050

102 Andrikopoulos, S. The Paleo diet and diabetes. Med J Aust. 2016. 205:151-2. doi:10.5694/mja16.00347

103 Angelis, A, loakeimidis, N, Aznaouridis, K, Georgakopoulos, C, Gourgouli, I, Rokas, K, Terentes, D, Zisimos, K, Chrysohoou, C, Aggeli, K, etal, . Mediterranean diet adherence in essential hypertension middle aged men, outcome in endogenous testosterone left ventricular geometry and central haemodynamics. European heart journal. Conference: european society of cardiology, ESC congress 2017. Spain. 2017. 38:1054. doi:10.1093/eurheartj/ehx502.5027

104 Anglin, JC, Borchardt, N, Ramos, E, Mhoon, K. Diet quality of adults using intuitive eating for weight loss - pilot study. Nutr Health. 2013. 22:255-64. doi:10.1177/0260106015601943

105 Anil, S, Charlton, KE, Tapsell, LC, Probst, Y, Ndanuko, R, Batterham, MJ. Identification of dietary patterns associated with blood pressure in a sample of overweight Australian adults. J Hum Hypertens. 2016. 30:672-678. doi:10.1038/jhh.2016.10

106 Ankarfeldt, MZ, Angquist, L, Jakobsen, MU, Overvad, K, Tjonneland, A, Halkjaer, J, Astrup, A, Sorensen, TI. Interactions of dietary protein and adiposity measures in relation to subsequent changes in body weight and waist circumference. Obesity (Silver Spring). 2014. 22:2097-103. doi:10.1002/oby.20812

107 Ankarfeldt, MZ, Ängquist, L, Stocks, T, Jakobsen, MU, Overvad, K, Halkjær, J, Saris, WHM, Astrup, A, Sørensen, TIA. Body composition, dietary protein and body weight regulation. Reconciling conflicting results from intervention and observational studies?. PLoS ONE. 2014. 9:. doi:10.1371/journal.pone.0101134

108 Ankarfeldt, MZ, Gottliebsen, K, Angquist, L, Astrup, A, Heitmann, BL, Sorensen, TI. Dietary protein and urinary nitrogen in relation to 6-year changes in fat mass and fat-free mass. Int J Obes (Lond). 2015. 39:162-8. doi:10.1038/ijo.2014.80

109 Annuzzi, G, Bozzetto, L, Costabile, G, Della Corte, G, Anniballi, G, Vitale, M, Cocozza, S, Giacco, R, Vetrani, C, Rivieccio, A, etal, . Effects of dietary polyphenols and/or n-3 fatty acids on postprandial lipaemia in people at high cardiovascular risk: the Etherpaths project. Diabetologia. 2012. 55:S362. doi:10.1007/s00125-012-2688-9

110 Anonymous, . Popular diets equally effective for losing weight. Journal of family practice. 2005. 54:306. doi:unavailable

Outcome

Power/Size

Study duration

Study Design;

Intervention/Exposure; Outcome

Publication Status

Publication Status

Intervention/Exposure; Study duration

Study Design

Intervention/Exposure Study Design;

Intervention/Exposure

Power/Size

Publication Status Publication Status 
111 Antonio de Luis, D, Izaola, O, Primo, D, Aller, R. Modifications of serum levels of omentin-1 and other cardiovascular risk

Study Design; factors following weight loss secondary to a Mediterranean hypocaloric diet. Clin Nutr. 2018. 37:2280-2283 doi:10.1016/j.clnu.2017.11.009

112 Antonio, J, Ellerbroek, A, Evans, C, Silver, T, Peacock, CA. High protein consumption in trained women: bad to the bone?. J Power/Size Int Soc Sports Nutr. 2018. 15:6. doi:10.1186/s12970-018-0210-6

113 Antonio, J, Ellerbroek, A, Silver, T, Vargas, L, Peacock, C. The effects of a high protein diet on indices of health and body composition--a crossover trial in resistance-trained men. J Int Soc Sports Nutr. 2016. 13:3. doi:10.1186/s12970-016-0114-2

114 Antonio, J, Ellerbroek, A, Silver, T, Vargas, L, Tamayo, A, Buehn, R, Peacock, CA. A High Protein Diet Has No Harmful Effects: A One-Year Crossover Study in Resistance-Trained Males. J Nutr Metab. 2016. 2016:9104792. doi:10.1155/2016/9104792

115 Antonio, J, Peacock, CA, Ellerbroek, A, Fromhoff, B, Silver, T. The effects of consuming a high protein diet (4.4 g/kg/d) on body composition in resistance-trained individuals. J Int Soc Sports Nutr. 2014. 11:19. doi:10.1186/1550-2783-11-19

116 Anwar, A, Aslam, S, Akram, A. A comparative study of dietary patterns in the patients with coronary artery disease and control. Pakistan Journal of Medical and Health Sciences. 2017. 11:1541-1542. doi:unavailable

117 Apolzan, JW, Hsia, DS, Martin, CK. Effects of a higher vs. lower protein diet on weight loss in teenagers: results from a randomized controlled trial. FASEB journal. 2016. 30:. doi:unavailable

118 Apolzan, JW, Hsia, DS, Martin, CK. Effects of a higher vs. Lower protein energy restricted diet on parameters of the metabolic syndrome in adolescents: a randomized, controlled trial. Diabetes. 2016. 65:A336-. doi:10.2337/db16-861-1374

119 Appannah, G, Pot, GK, Huang, RC, Oddy, WH, Beilin, LJ, Mori, TA, Jebb, SA, Ambrosini, GL. Identification of a dietary pattern associated with greater cardiometabolic risk in adolescence. Nutr Metab Cardiovasc Dis. 2015. 25:643-50. doi:10.1016/j.numecd.2015.04.007

120 Appannah, G, Pot, GK, Oddy, WH, Jebb, SA, Ambrosini, GL. Determinants of a dietary pattern linked with greater metabolic risk and its tracking during adolescence. J Hum Nutr Diet. 2018. 31:218-227. doi:10.1111/jhn.12519

121 Appel, LJ, Sacks, FM, Carey, VJ, Obarzanek, E, Swain, JF, Miller, ER, 3rd, Conlin, PR, Erlinger, TP, Rosner, BA, Laranjo, NM, Charleston, J, McCarron, P, Bishop, LM. Effects of protein, monounsaturated fat, and carbohydrate intake on blood pressure and serum lipids: results of the OmniHeart randomized trial. Jama. 2005. 294:2455-64. doi:10.1001/jama.294.19.2455

122 Arabshahi, S, Lahmann, PH, Hughes, MC, Williams, GW, van der Pols, JC. Dietary behaviours, weight loss attempts and change in waist circumference: 15-year longitudinal study in Australian adults. Asia Pac J Clin Nutr. 2017. 26:657-664. doi:10.6133/apjcn.062016.04

123 Arabzadegan, N, Daneshzad, E, Fatahi, S, Moosavian, SP, Surkan, PJ, Azadbakht, L. Effects of dietary whole grain, fruit, and vegetables on weight and inflammatory biomarkers in overweight and obese women. Eat Weight Disord. 2019. : doi:10.1007/s40519-019-00757-x 
124 Archer, WR, Lamarche, B, Deriaz, O, Landry, N, Corneau, L, Despres, JP, Bergeron, J, Couture, P, Bergeron, N. Variations in body composition and plasma lipids in response to a high-carbohydrate diet. Obes Res. 2003. 11:978-86. doi:10.1038/oby.2003.135

125 Archer, WR, Lamarche, B, St-Pierre, AC, Mauger, JF, Deriaz, O, Landry, N, Corneau, L, Despres, JP, Bergeron, J, Couture, $\mathrm{P}$, Bergeron, N. High carbohydrate and high monounsaturated fatty acid diets similarly affect LDL electrophoretic characteristics in men who are losing weight. J Nutr. 2003. 133:3124-9. doi:10.1093/jn/133.10.3124

126 Archero, F, Ricotti, R, Solito, A, Carrera, D, Civello, F, Di Bella, R, Bellone, S, Prodam, F. Adherence to the Mediterranean Diet among School Children and Adolescents Living in Northern Italy and Unhealthy Food Behaviors Associated to Overweight. Nutrients. 2018. 10:. doi:10.3390/nu10091322

127 Arciero, PJ, Ormsbee, MJ, Gentile, CL, Nindl, BC, Brestoff, JR, Ruby, M. Increased protein intake and meal frequency reduces abdominal fat during energy balance and energy deficit. Obesity (Silver Spring). 2013. 21:1357-66. doi:10.1002/oby.20296

128 Ard, JD, Lewis, KH, Rothberg, A, Auriemma, A, Coburn, SL, Cohen, SS, Loper, J, Matarese, L, Pories, WJ, Periman, S. Effectiveness of a Total Meal Replacement Program (OPTIFAST Program) on Weight Loss: Results from the OPTIWIN Study. Obesity. 2019. 27:22-29. doi:10.1002/oby.22303

129 Arefhosseini, SR, Edwards, CA, Malkova, D, Higgins, S. Effect of advice to increase carbohydrate and reduce fat intake on dietary profile and plasma lipid concentrations in healthy postmenopausal women. Ann Nutr Metab. 2009. 54:138-44. doi:10.1159/000210435

130 Arguin, H, Tremblay, A, Blundell, JE, Despres, JP, Richard, D, Lamarche, B, Drapeau, V. Impact of a non-restrictive satiating diet on anthropometrics, satiety responsiveness and eating behaviour traits in obese men displaying a high or a low satiety phenotype. Br J Nutr. 2017. 118:750-760. doi:10.1017/s0007114517002549

131 Armeno, M, Verini, A, Del Pino, M, Araujo, MB, Mestre, G, Reyes, G, Caraballo, RH. A Prospective Study on Changes in Nutritional Status and Growth Following Two Years of Ketogenic Diet (KD) Therapy in Children with Refractory Epilepsy. Nutrients. 2019. 11:. doi:10.3390/nu11071596

132 Arnlov, J, Lind, L, Sundstrom, J, Andren, B, Vessby, B, Lithell, H. Insulin resistance, dietary fat intake and blood pressure predict left ventricular diastolic function 20 years later. Nutr Metab Cardiovasc Dis. 2005. 15:242-9. doi:10.1016/j.numecd.2004.10.002

133 Arnold, K, Weinhold, KR, Andridge, R, Johnson, K, Orchard, TS. Improving Diet Quality Is Associated with Decreased Inflammation: Findings from a Pilot Intervention in Postmenopausal Women with Obesity. J Acad Nutr Diet. 2018. 118:21352143. doi:10.1016/j.jand.2018.05.014

134 Arouca, A, Moreno, L, Marcos, A, Kafatos, A, Michels, N, De Henauw, S. Diet as a moderator in the association of adiposity and sedentary behavior with inflammatory biomarkers in european adolescents. Annals of nutrition \& metabolism. 2018. 73:201. doi:10.1159/000493135 
135 Arredondo, A, Torres, C, Orozco, E, Pacheco, S, Huang, F, Zambrano, E, Bolanos-Jimenez, F. Socio-economic indicators, dietary patterns, and physical activity as determinants of maternal obesity in middle-income countries: Evidences from a cohort

Study Design; study in Mexico. Int J Health Plann Manage. 2019. 34:e713-e725. doi:10.1002/hpm.2684

136 Arsiwalla, DD, Arnold, AW, Teel, KP, Ulrich, PV, Gropper, SS. The interactive role of eating regulation and stress in the prediction of weight-related outcomes among college students. Stress Health. 2018. 34:59-71. doi:10.1002/smi.2760

Outcome

Study Design;

Intervention/Exposure

137 Arvidsson, E, Viguerie, N, Andersson, I, Verdich, C, Langin, D, Arner, P. Effects of different hypocaloric diets on protein secretion from adipose tissue of obese women. Diabetes. 2004. 53:1966-71. doi:10.2337/diabetes.53.8.1966

138 Asano, M, Kushida, M, Yamamoto, K, Tomata, Y, Tsuji, I, Tsuduki, T. Abdominal Fat in Individuals with Overweight Reduced by Consumption of a 1975 Japanese Diet: A Randomized Controlled Trial. Obesity (Silver Spring). 2019. 27:899-907. doi:10.1002/oby.22448

139 Asghari, G, Yuzbashian, E, Mirmiran, P, Hooshmand, F, Najafi, R, Azizi, F. Dietary Approaches to Stop Hypertension (DASH) Dietary Pattern Is Associated with Reduced Incidence of Metabolic Syndrome in Children and Adolescents. J Pediatr. 2016. 174:178-184.e1. doi:10.1016/j.jpeds.2016.03.077

140 Asghari, G, Yuzbashian, E, Mirmiran, P, Mahmoodi, B, Azizi, F. Fast Food Intake Increases the Incidence of Metabolic Syndrome in Children and Adolescents: Tehran Lipid and Glucose Study. PLoS One. 2015. 10:e0139641. doi:10.1371/journal.pone.0139641

141 Ashourpour, M, Kaveh, MH, Ta Bata Baee, HR, Ta Ghdir, M, Salehi, M. Dietary pattern of children with malnutrition in a Persian population. Gazzetta Medica Italiana Archivio per le Scienze Mediche. 2018. 177:185-192. doi:10.23736/S03933660.17.03577-X

142 Ashton, EL, Pomeroy, S, Foster, JE, Kaye, RS, Nestel, PJ, Ball, M. Diet high in monounsaturated fat does not have a different effect on arterial elasticity than a low-fat, high-carbohydrate diet. J Am Diet Assoc. 2000. 100:537-42. doi:10.1016/s0002-8223(00)00167-x

143 Ashworth, A, Mitchell, K, Blackwell, JR, Vanhatalo, A, Jones, AM. High-nitrate vegetable diet increases plasma nitrate and nitrite concentrations and reduces blood pressure in healthy women. Public Health Nutr. 2015. 18:2669-78. doi:10.1017/s1368980015000038

144 Assmann, KE, Adjibade, M, Andreeva, VA, Hercberg, S, Galan, P, Kesse-Guyot, E. Association Between Adherence to the Mediterranean Diet at Midlife and Healthy Aging in a Cohort of French Adults. J Gerontol A Biol Sci Med Sci. 2018. 73:347-354. doi:10.1093/gerona/glx066

145 Assmann, KE, Adjibade, M, Shivappa, N, Hebert, JR, Wirth, MD, Touvier, M, Akbaraly, T, Hercberg, S, Galan, P, Julia, C, Kesse-Guyot, E. The Inflammatory Potential of the Diet at Midlife Is Associated with Later Healthy Aging in French Adults. J Nutr. 2018. 148:437-444. doi:10.1093/jn/nxx061

146 Assmann, KE, Joslowski, G, Buyken, AE, Cheng, G, Remer, T, Kroke, A, Gunther, AL. Prospective association of protein intake during puberty with body composition in young adulthood. Obesity (Silver Spring). 2013. 21:E782-9. doi:10.1002/oby.20516 
147 Assmann, KE, Lassale, C, Andreeva, VA, Jeandel, C, Hercberg, S, Galan, P, Kesse-Guyot, E. A Healthy Dietary Pattern at Midlife, Combined with a Regulated Energy Intake, Is Related to Increased Odds for Healthy Aging. J Nutr. 2015. 145:2139-45. doi:10.3945/jn.115.210740

148 Assmann, KE, Lassale, C, Galan, P, Hercberg, S, Kesse-Guyot, E. Dietary quality and 6-year anthropometric changes in a sample of French middle-aged overweight and obese adults. PLoS One. 2014. 9:e87083. doi:10.1371/journal.pone.0087083

149 Astrup, A, Damsgaard, CT, Papadaki, A, Jensen, SM, Dalskov, SM, Hlavaty, P, Saris, WHM, Martinez, JA, HandjievaDarlenska, T, Rohr-Andersen, M, etal, . 1 The use of an ad libitum higher-protein, low-glycemic index diet in overweight children: the Diogenes Study. FASEB journal. 2013. 27:. doi:unavailable

150 Astrup, A, Hjorth, MF, Ritz, C, Poulsen, SK, Larsen, TM, Zohar, Y. Pretreatment fasting plasma glucose and insulin as determinants of weight loss success: the new nordic diet study. Diabetes. 2017. 66:A205-. doi:unavailable

151 Astrup, A, Rodrigues, D, Bendtsen, L, Larsen, T, Ritz, C, Handijeva-Darlenska, T, Van Baak, M, Martinez, J, Saris, W. The importance of amounts of protein, and the protein to carbohydrate ratio for weight control: analyses from the DiOGenes study. Obesity reviews. 2014. 15:181. doi:10.1111/obr.12152

152 Astrup, A, Rodrigues, DMT, Bendtsen, LQ, Larsen, TM, Ritz, C. Role of protein and fat content, and protein/carbohydrate ratio in weight maintenance? The DiOGenes Study. Annals of nutrition and metabolism. Conference: 12th european nutrition conference, FENS 2015. Berlin germany. Conference start: 20151020. Conference end: 20151023. Conference publication: (var.pagings). 2015. 67:156-157. doi:10.1159/000440895

153 Astrup, A. Protein, carbs and fats in personalized weight control-efficacy, effectiveness and safety. Obesity facts. 2014. 7:3. doi:10.1159/000363668

154 Astrup, A. The role of protein and carbohydrates for weight loss and maintenance: evidence from the Diogenes study. Australasian medical journal. 2011. 4:717. doi:unavailable

155 Ata, SM, Vaishnav, U, Puglisi, M, Lofgren, IE, Wood, RJ, Volek, JS, Fernandez, ML. Macronutrient composition and increased physical activity modulate plasma adipokines and appetite hormones during a weight loss intervention. $\mathrm{J}$ Womens Health (Larchmt). 2010. 19:139-45. doi:10.1089/jwh.2009.1472

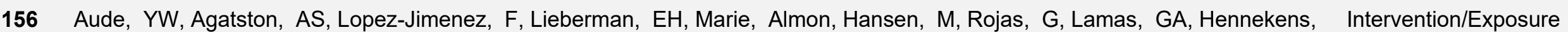
$\mathrm{CH}$. The national cholesterol education program diet vs a diet lower in carbohydrates and higher in protein and monounsaturated fat: a randomized trial. Arch Intern Med. 2004. 164:2141-6. doi:10.1001/archinte.164.19.2141

157 Auslander, W, Haire-Joshu, D, Houston, C, Rhee, CW, Williams, JH. A controlled evaluation of staging dietary patterns to reduce the risk of diabetes in African-American women. Diabetes Care. 2002. 25:809-14. doi:10.2337/diacare.25.5.809

158 Austel, A, Ranke, C, Wagner, N, Gorge, J, Ellrott, T. Weight loss with a modified Mediterranean-type diet using fat modification: a randomized controlled trial. Eur J Clin Nutr. 2015. 69:878-84. doi:10.1038/ejcn.2015.11

159 Avanzini, F, Marzona, I, Baviera, M, Barlera, S, Milani, V, Caimi, V, Longoni, P, Tombesi, M, Silletta, MG, Tognoni, G, Roncaglioni, MC. Improving cardiovascular prevention in general practice: Results of a comprehensive personalized strategy in subjects at high risk. Eur J Prev Cardiol. 2016. 23:947-55. doi:10.1177/2047487315613664 
160 Aynaci, G, Akdemir, O. The Relationship Between Lifestyle, Health Promotion Lifestyle Profile II And High Blood Pressure In

161 Azadbakht, L, Izadi, V, Surkan, PJ, Esmaillzadeh, A. Effect of a High Protein Weight Loss Diet on Weight, High-Sensitivity CReactive Protein, and Cardiovascular Risk among Overweight and Obese Women: A Parallel Clinical Trial. Int J Endocrinol. 2013. 2013:971724. doi:10.1155/2013/971724

162 Azadbakht, L, Kimiagar, M, Mehrabi, Y, Zadeh, AE. Soy inclusion in the diet improves features of the metabolic syndrome: a randomized cross-over study in postmenopausal women. Iranian journal of diabetes and lipid disorders. 2007. 7:E11. doi:unavailable

163 Azadbakht, L, Kimiagar, M, Mehrabi, Y, Zadeh, AE. To determine the effects of soy consumption on markers of inflammation and endothelial function in postmenopausal women with the metabolic syndrome. Iranian journal of diabetes and lipid disorders. 2007. 6:81-92, E46. doi:unavailable

164 Azadbakht, L, Mirmiran, P, Esmaillzadeh, A, Azizi, F. Better dietary adherence and weight maintenance achieved by a longterm moderate-fat diet. Br J Nutr. 2007. 97:399-404. doi:10.1017/s0007114507328602

165 Azadbakht, L, Mirmiran, P, Esmaillzadeh, A, Azizi, T, Azizi, F. Beneficial effects of a Dietary Approaches to Stop Hypertension eating plan on features of the metabolic syndrome. Diabetes Care. 2005. 28:2823-31. doi:10.2337/diacare.28.12.2823

166 Babio, N, Becerra-Tomas, N, Martinez-Gonzalez, MA, Corella, D, Estruch, R, Ros, E, Sayon-Orea, C, Fito, M, SerraMajem, L, Aros, F, Lamuela-Raventos, RM, Lapetra, J, Gomez-Gracia, E, Fiol, M, Diaz-Lopez, A, Sorli, JV, Martinez, JA, Salas-Salvado, J. Consumption of Yogurt, Low-Fat Milk, and Other Low-Fat Dairy Products Is Associated with Lower Risk of Metabolic Syndrome Incidence in an Elderly Mediterranean Population. J Nutr. 2015. 145:2308-16. doi:10.3945/jn.115.214593

167 Backx, EM, Tieland, M, Borgonjen-van den Berg, KJ, Claessen, PR, van Loon, LJ, de Groot, LC. Protein intake and lean body mass preservation during energy intake restriction in overweight older adults. Int J Obes (Lond). 2016. 40:299-304. doi:10.1038/ijo.2015.182

168 Bacopoulou, F, Landis, G, Rentoumis, A, Tsitsika, A, Efthymiou, V. Mediterranean diet decreases adolescent waist circumference. Eur J Clin Invest. 2017. 47:447-455. doi:10.1111/eci.12760

169 Bagherniya, M, Sharma, M, Mostafavi Darani, F, Maracy, MR, Safarian, M, Allipour Birgani, R, Bitarafan, V, Keshavarz, SA. School-Based Nutrition Education Intervention Using Social Cognitive Theory for Overweight and Obese Iranian Adolescent Girls: A Cluster Randomized Controlled Trial. Int Q Community Health Educ. 2017. 38:37-45. doi:10.1177/0272684x17749566

170 Bahadori, B, Yazdani-Biuki, B, Krippl, P, Brath, H, Uitz, E, Wascher, TC. Low-fat, high-carbohydrate (low-glycaemic index) diet induces weight loss and preserves lean body mass in obese healthy subjects: results of a 24-week study. Diabetes Obes Metab. 2005. 7:290-3. doi:10.1111/j.1463-1326.2004.00445.x

171 Bahari, T, Uemura, H, Katsuura-Kamano, S, Yamaguchi, M, Nakamoto, M, Miki, K, Ishizu, M, Arisawa, K. Nutrient-Derived Dietary Patterns and Their Association With Metabolic Syndrome in a Japanese Population. J Epidemiol. 2018. 28:194-201. doi:10.2188/jea.JE20170010 
172 Bai, G, Zhang, J, Zhao, C, Wang, Y, Qi, Y, Zhang, B. Adherence to a healthy lifestyle and a DASH-style diet and risk of hypertension in Chinese individuals. Hypertens Res. 2017. 40:196-202. doi:10.1038/hr.2016.119

173 Bailes, JR, Strow, MT, Werthammer, J, McGinnis, RA, Elitsur, Y. Effect of low-carbohydrate, unlimited calorie diet on the treatment of childhood obesity: a prospective controlled study. Metab Syndr Relat Disord. 2003. 1:221-5. doi:10.1089/154041903322716697

174 Bailey, BW, Perkins, A, Tucker, LA, LeCheminant, JD, Tucker, JM, Moncur, B. Adherence to the 2010 Dietary Guidelines for Americans and the relationship to adiposity in young women. J Nutr Educ Behav. 2015. 47:86-93. doi:10.1016/j.jneb.2014.08.011

175 Bailey, BW, Sullivan, DK, Kirk, EP, Donnelly, JE. Dietary predictors of visceral adiposity in overweight young adults. Br J Nutr. 2010. 103:1702-5. doi:10.1017/s0007114509993771

176 Bajaj, A, Rader, DJ. Can changes in the plasma lipidome help explain the cardiovascular benefits of the Mediterranean diet?. Am J Clin Nutr. 2017. 106:965-966. doi:10.3945/ajcn.117.165886

177 Bajerska, J, Chmurzynska, A, Muzsik, A, Krzyzanowska, P, Madry, E, Malinowska, AM, Walkowiak, J. Weight loss and metabolic health effects from energy-restricted Mediterranean and Central-European diets in postmenopausal women: A randomized controlled trial. Sci Rep. 2018. 8:11170. doi:10.1038/s41598-018-29495-3

178 Bajerska, J, Chmurzynska, A, Muzsik, A, Menclewicz, K, Krzyzanowska, P, Walkowiak, J. Adherence to the mediterranean and the central european diets in relation to weight loss changes in postmenopausal women with diagnosed metabolic syndrome. Annals of nutrition \& metabolism. 2017. 71:994-. doi:10.1159/000480486

179 Bajerska, J, Chmurzynska, A, Muzsik, A, Menclewicz, K, Krzyzanowska, P, Walkowiak, J. Effects of a 16-week dietary intervention with either a mediterranean diet or a central european diet on anthropometric parameters in obese postmenopausal with diagnosed metabolic syndrome. Annals of nutrition \& metabolism. 2017. 71:994-995. doi:10.1159/000480486

180 Bakker, LE, van Schinkel, LD, Guigas, B, Streefland, TC, Jonker, JT, van Klinken, JB, van der Zon, GC, Lamb, HJ, Smit, JW, Pijl, H, Meinders, AE, Jazet, IM. A 5-day high-fat, high-calorie diet impairs insulin sensitivity in healthy, young South Asian men but not in Caucasian men. Diabetes. 2014. 63:248-58. doi:10.2337/db13-0696

181 Bales, CW, Porter Starr, KN, Orenduff, MC, McDonald, SR, Molnar, K, Jarman, AK, Onyenwoke, A, Mulder, H, Payne, ME, Pieper, CF. Influence of Protein Intake, Race, and Age on Responses to a Weight-Reduction Intervention in Obese Women(). Curr Dev Nutr. 2017. 1:. doi:10.3945/cdn.117.000703

182 Ballard, KD, Quann, EE, Kupchak, BR, Volk, BM, Kawiecki, DM, Fernandez, ML, Seip, RL, Maresh, CM, Kraemer, WJ, Volek, JS. Dietary carbohydrate restriction improves insulin sensitivity, blood pressure, microvascular function, and cellular adhesion markers in individuals taking statins. Nutr Res. 2013. 33:905-12. doi:10.1016/j.nutres.2013.07.022

183 Ballesteros-Pomar, MD, Calleja-Fernandez, AR Vidal-Casariego, A Urioste-Fondo, AM, Cano-Rodriguez, I. Effectiveness of energy-restricted diets with different protein:carbohydrate ratios: the relationship to insulin sensitivity. Public Health Nutr. 2010. 13:2119-26. doi:10.1017/s1368980009991881
Outcome; Country

Intervention/Exposure

Study Design

Study Design; Study duration

Study Design

Weight

loss/Hypocaloric

Publication Status

Publication Status

Study duration

Intervention/Exposure; Health Status

Study Design

Power/Size 


\section{No. Citation}

184 Balthazar, EA, de Oliveira, MR. Differences in dietary pattern between obese and eutrophic children. BMC Res Notes. 2011. 4:567. doi:10.1186/1756-0500-4-567

185 Bamberger, C, Rossmeier, A, Lechner, K, Wu, L, Waldmann, E, Stark, RG, Altenhofer, J, Henze, K, Parhofer, KG. A Walnut-Enriched Diet Reduces Lipids in Healthy Caucasian Subjects, Independent of Recommended Macronutrient Replacement and Time Point of Consumption: a Prospective, Randomized, Controlled Trial. Nutrients. 2017. 9:. doi:10.3390/nu9101097

186 Banini, AE, Allen, JC, Allen, HG, Boyd, LC, Lartey, A. Fatty acids, diet, and body indices of type II diabetic American whites and blacks and Ghanaians. Nutrition. 2003. 19:722-6. doi:10.1016/s0899-9007(03)00108-4

187 Bansal, S, Cramp, L, Blalock, D, Zelleke, T, Carpenter, J, Kao, A. The ketogenic diet: Initiation at goal calories versus gradual caloric advancement. Pediatric Neurology. 2014. 50:26-30. doi:10.1016/j.pediatrneurol.2013.08.006

188 Banuls, C, Martinez-Triguero, ML, Lopez-Ruiz, A, Morillas, C, Lacomba, R, Victor, VM, Rocha, M, Hernandez-Mijares, A. Evaluation of cardiovascular risk and oxidative stress parameters in hypercholesterolemic subjects on a standard healthy diet including low-fat milk enriched with plant sterols. J Nutr Biochem. 2010. 21:881-6. doi:10.1016/j.jnutbio.2009.07.001

189 Barchitta, M, Quattrocchi, A, Adornetto, V, Marchese, AE, Agodi, A. Tumor necrosis factor-alpha -308 G>A polymorphism, adherence to Mediterranean diet, and risk of overweight/obesity in young women. Biomed Res Int. 2014. 2014:742620. doi:10.1155/2014/742620

190 Barnard, ND, Scialli, AR, Bertron, P, Hurlock, D, Edmonds, K, Talev, L. Effectiveness of a low-fat vegetarian diet in altering serum lipids in healthy premenopausal women. Am J Cardiol. 2000. 85:969-72. doi:10.1016/s0002-9149(99)00911-x

191 Barnard, ND, Scialli, AR, Turner-McGrievy, G, Lanou, AJ, Glass, J. The effects of a low-fat, plant-based dietary intervention on body weight, metabolism, and insulin sensitivity. Am J Med. 2005. 118:991-7. doi:10.1016/j.amjmed.2005.03.039

192 Barnes, TL, French, SA, Mitchell, NR, Wolfson, J. Fast-food consumption, diet quality and body weight: cross-sectional and prospective associations in a community sample of working adults. Public Health Nutr. 2016. 19:885-92. doi:10.1017/s1368980015001871

193 Barnett, TD, Barnard, ND, Radak, TL. Development of symptomatic cardiovascular disease after self-reported adherence to the Atkins diet. J Am Diet Assoc. 2009. 109:1263-5. doi:10.1016/j.jada.2009.04.003

194 Baron, KG, Reid, KJ, Horn, LV, Zee, PC. Contribution of evening macronutrient intake to total caloric intake and body mass index. Appetite. 2013. 60:246-251. doi:10.1016/j.appet.2012.09.026

195 Barona, J, Jones, JJ, Kopec, RE, Comperatore, M, Andersen, C, Schwartz, SJ, Lerman, RH, Fernandez, ML. A Mediterranean-style low-glycemic-load diet increases plasma carotenoids and decreases LDL oxidation in women with metabolic syndrome. J Nutr Biochem. 2012. 23:609-15. doi:10.1016/j.jnutbio.2011.02.016

196 Barros, L, Lopes, C, Oliveira, A. Child and family characteristics are associated with a dietary variety index in 4-year-old children from the Generation XXI cohort. Nutr Res. 2019. 63:76-85. doi:10.1016/j.nutres.2018.12.001

\section{Rationale}

Study Design

Study duration

Study Design

Study Design

Outcome

Intervention/Exposure;

Comparator

Study Design

Study duration

Power/Size

Comparator; Outcom

Study Design

Study duration

Intervention/Exposure

Study Design 
197 Basciani, S, Costantini, D, Contini, S, Persichetti, A, Watanabe, M, Mariani, S, Lubrano, C, Spera, G, Lenzi, A, Gnessi, L.

Study Design; Study Safety and efficacy of a multiphase dietetic protocol with meal replacements including a step with very low calorie diet. duration Endocrine. 2015. 48:863-70. doi:10.1007/s12020-014-0355-2

198 Basharat, S, Gilani, SA, Burq, Al, Bashir, S. Low glycaemic index diet is effective in managing weight among obese postpartum women. Journal of the Pakistan Medical Association. 2018. 68:548-552. doi:unavailable

199 Baskaran, C, Carson, TL, Campoverde Reyes, KJ, Becker, KR, Slattery, MJ, Tulsiani, S, Eddy, KT, Anderson, EJ, Hubbard, JL, Misra, M, Klibanski, A. Macronutrient intake associated with weight gain in adolescent girls with anorexia nervosa. Int J Eat Disord. 2017. 50:1050-1057. doi:10.1002/eat.22732

200 Bathrellou, E, Kontogianni, MD, Chrysanthopoulou, E, Georgousopoulou, E, Chrysohoou, C, Pitsavos, C, Panagiotakos, D. Adherence to a DASH-style diet and cardiovascular disease risk: The 10-year follow-up of the ATTICA study. Nutr Health. 2019. 25:225-230. doi:10.1177/0260106019862995

201 Batis, C, Mendez, MA, Gordon-Larsen, P, Sotres-Alvarez, D, Adair, L, Popkin, B. Using both principal component analysis and reduced rank regression to study dietary patterns and diabetes in Chinese adults. Public Health Nutr. 2016. 19:195-203. doi:10.1017/s1368980014003103

202 Batis, C, Mendez, MA, Sotres-Alvarez, D, Gordon-Larsen, P, Popkin, B. Dietary pattern trajectories during 15 years of followup and $\mathrm{HbA} 1 \mathrm{c}$, insulin resistance and diabetes prevalence among Chinese adults. J Epidemiol Community Health. 2014 68:773-9. doi:10.1136/jech-2013-203560

203 Baulderstone, L, Yaxley, A, Luszcz, M, Miller, M Diet Liberalisation in Older Australians Decreases Frailty without Increasing the Risk of Developing Chronic Disease J Frailty Aging, 2012 1(4): 174-82.doi 10.14283/jfa.2012.27

204 Baudry, J, Mejean, C, Peneau, S, Galan, P, Hercberg, S, Lairon, D, Kesse-Guyot, E. Health and dietary traits of organic food consumers: results from the NutriNet-Sante study. Br J Nutr. 2015. 114:2064-73. doi:10.1017/s0007114515003761

205 Bauer, LB, Reynolds, LJ, Douglas, SM, Kearney, ML, Hoertel, HA, Shafer, RS, Thyfault, JP, Leidy, HJ. A pilot study examining the effects of consuming a high-protein vs normal-protein breakfast on free-living glycemic control in overweight/obese 'breakfast skipping' adolescents. Int J Obes (Lond). 2015. 39:1421-4. doi:10.1038/ijo.2015.101

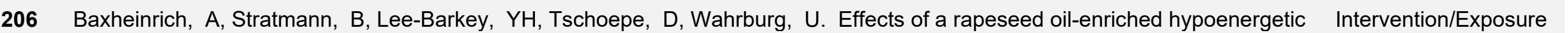
diet with a high content of alpha-linolenic acid on body weight and cardiovascular risk profile in patients with the metabolic syndrome. Br J Nutr. 2012. 108:682-91. doi:10.1017/s0007114512002875

207 Baxter, KA, Ware, RS, Batch, JA, Truby, H. Predicting success: factors associated with weight change in obese youth undertaking a weight management program. Obes Res Clin Pract. 2013. 7:e147-e154. doi:10.1016/j.orcp.2011.09.004

208 Bazzano, LA, He, J, Ogden, LG, Loria, CM, Whelton, PK. Dietary fiber intake and reduced risk of coronary heart disease in US men and women: the National Health and Nutrition Examination Survey I Epidemiologic Follow-up Study. Arch Intern Med. 2003. 163:1897-904. doi:10.1001/archinte.163.16.1897

209 Bazzano, LA, Reynolds, K, Hu, T, Yao, L, Bunol, C, Liu, Y, Chen, CS, He, J. Effect of a low-carbohydrate diet on weight and cardiovascular risk factors: a randomized controlled trial. Circulation. 2012. 125:. doi:unavailable 
210 Bazzocchi, A, Santoro, A, Mercatelli, D, Ponti, F, Diano, D, Zanirato Rambaldi, G, Scurti, M, Berendsen, A, Pietruszka, B, Jennings, A, etal, . New dietary strategies addressing the specific needs of elderly population for a healthy aging in Europe (NU-AGE): the effect of a tailored Mediterranean diet on body composition. Skeletal radiology. 2017. 46:856-. doi:10.1007/s00256-017-2619-4

211 Beasley, J, Viswanathan, S, Wylie-Rosett, J. Associations between change in DASH diet scores and CVD risk factors in the PREMIER Trial. FASEB journal. 2014. 28:. doi:unavailable

212 Beasley, JM, Yi, SS, Ahn, J, Kwon, SC, Wylie-Rosett, J. Dietary Patterns in Chinese Americans are Associated with Cardiovascular Disease Risk Factors, the Chinese American Cardiovascular Health Assessment (CHA CHA). J Immigr Minor Health. 2019. 21:1061-1069. doi:10.1007/s10903-018-0800-z

213 Beauchesne-Rondeau, E, Gascon, A, Bergeron, J, Jacques, H. Plasma lipids and lipoproteins in hypercholesterolemic men fed a lipid-lowering diet containing lean beef, lean fish, or poultry. Am J Clin Nutr. 2003. 77:587-93. doi:10.1093/ajcn/77.3.587

214 Beavers, KM, Gordon, MM, Easter, L, Beavers, DP, Hairston, KG, Nicklas, BJ, Vitolins, MZ. Effect of protein source during weight loss on body composition, cardiometabolic risk and physical performance in abdominally obese, older adults: a pilot feeding study. Journal of nutrition, health \& aging. 2014. 19:87-95. doi:10.1007/s12603-015-0438-7

215 Beavers, KM, Nesbit, BA, Kiel, JR, Sheedy, JL, Arterburn, LM, Collins, AE, Ford, SA, Henderson, RM, Coleman, CD, Beavers, DP. Effect of an Energy-Restricted, Nutritionally Complete, Higher Protein Meal Plan on Body Composition and Mobility in Older Adults With Obesity: A Randomized Controlled Trial. J Gerontol A Biol Sci Med Sci. 2019. 74:929-935. doi:10.1093/gerona/gly146

216 Beck, KL, Jones, B, Ullah, I, McNaughton, SA, Haslett, SJ, Stonehouse, W. Associations between dietary patterns, sociodemographic factors and anthropometric measurements in adult New Zealanders: an analysis of data from the 2008/09 New Zealand Adult Nutrition Survey. Eur J Nutr. 2018. 57:1421-1433. doi:10.1007/s00394-017-1421-3

217 Becker, GF, Passos, EP, Moulin, CC. Short-term effects of a hypocaloric diet with low glycemic index and low glycemic load on body adiposity, metabolic variables, ghrelin, leptin, and pregnancy rate in overweight and obese infertile women: a randomized controlled trial. Am J Clin Nutr. 2015. 102:1365-72. doi:10.3945/ajcn.115.117200

218 Becquey, E, Savy, M, Danel, P, Dabire, HB, Tapsoba, S, Martin-Prevel, Y. Dietary patterns of adults living in Ouagadougou and their association with overweight. Nutr J. 2010. 9:13. doi:10.1186/1475-2891-9-13

219 Bedard, A, Corneau, L, Dodin, S, Lemieux, S. Sex Differences in the Effects of Repeated Taste Exposure to the Mediterranean Diet: A 6-month Follow-up Study. Can J Diet Pract Res. 2016. 77:125-32. doi:10.3148/cjdpr-2015-052

Publication Status

Study Design

Study duration

Intervention/Exposure

Intervention/Exposure

Bedard, A, Corneau, L, Lamarche, B, Dodin, S, Lemieux, S. Sex Differences in the Impact of the Mediterranean Diet on LDL Particle Size Distribution and Oxidation. Nutrients. 2015. 7:3705-23. doi:10.3390/nu7053705

221 Bedard, A, Corneau, L, Vohl, MC, Dodin, S, Lemieux, S. Effect of the Mediterranean diet on the lipid-lipoprotein profile: is it influenced by the family history of dyslipidemia?. J Nutrigenet Nutrigenomics. 2014. 7:177-87. doi:10.1159/000374116

Study Design

Intervention/Exposure

Bedard, A, Dodin, S, Corneau, L, Lemieux, S. Impact of the traditional Mediterranean diet on the Framingham risk score and the metabolic syndrome according to sex. Metab Syndr Relat Disord. 2014. 12:95-101. doi:10.1089/met.2012.0076

Study Design

Study Design; Study duration

Study duration

Study Design; Study duration

Study Design;

Intervention/Exposure 
223 Bedard, A, Garcia-Aymerich, J, Sanchez, M, Le Moual, N, Clavel-Chapelon, F, Boutron-Ruault, MC, Maccario, J, Varraso, R. Confirmatory Factor Analysis Compared with Principal Component Analysis to Derive Dietary Patterns: A Longitudinal Study in Adult Women. J Nutr. 2015. 145:1559-68. doi:10.3945/jn.114.204479

224 Bédard, A, Lamarche, B, Corneau, L, Dodin, S, Lemieux, S. Sex differences in the impact of the Mediterranean diet on systemic inflammation. Nutrition Journal. 2015. 14:. doi:10.1186/s12937-015-0035-y

225 Bekkouche, L, Bouchenak, M, Malaisse, WJ, Yahia, DA. The Mediterranean diet adoption improves metabolic, oxidative, and inflammatory abnormalities in Algerian metabolic syndrome patients. Horm Metab Res. 2014. 46:274-82. doi:10.1055/s-00331363657

226 Belanger, M, Poirier, M, Jbilou, J, Scarborough, P. Modelling the impact of compliance with dietary recommendations on cancer and cardiovascular disease mortality in Canada. Public Health. 2014. 128:222-30. doi:10.1016/j.puhe.2013.11.003

227 Belin, RJ, Greenland, P, Allison, M, Martin, L, Shikany, JM, Larson, J, Tinker, L, Howard, BV, Lloyd-Jones, D, Van Horn, L. Diet quality and the risk of cardiovascular disease: The Women's Health Initiative (WHI). American Journal of Clinical Nutrition. 2011. 94:49-57. doi:10.3945/ajcn.110.011221

Outcome

Comparator

Study Design

Intervention/Exposure; Publication Date

Overlaps with Existing Review

228 Bello, JK, Stulberg, DB, Zhou, Y, Wang, C. Physical Activity and Consumption Patterns of Reproductive-Aged Women by BMI Category. Matern Child Health J. 2018. 22:713-724. doi:10.1007/s10995-018-2440-2

229 Bel-Serrat, S, Mouratidou, T, Bornhorst, C, Peplies, J, De Henauw, S, Marild, S, Molnar, D, Siani, A, Tornaritis, M, Veidebaum, T, Krogh, V, Moreno, LA. Food consumption and cardiovascular risk factors in European children: the IDEFICS study. Pediatr Obes. 2013. 8:225-36. doi:10.1111/j.2047-6310.2012.00107.x

230 Bel-Serrat, S, Ojeda-Rodriguez, A, Heinen, MM, Buoncristiano, M, Abdrakhmanova, S, Duleva, V, Sant'Angelo, VF, Fijalkowska, A, Hejgaard, T, Huidumac, C, Hyska, J, Kujundzic, E, Milanovic, SM, Ovezmyradova, G, Perez-Farinos, N, Petrauskiene, A, Rito, Al, Shengelia, L, Braunerova, RT, Rutter, H, Murrin, CM, Kelleher, CC, Breda, J. Clustering of Multiple Energy Balance-Related Behaviors in School Children and its Association with Overweight and Obesity-WHO European Childhood Obesity Surveillance Initiative (COSI 2015(-)2017). Nutrients. 2019. 11:. doi:10.3390/nu11030511

231 Bemelmans, WJ, Broer, J, de Vries, JH, Hulshof, KF, May, JF, Meyboom-De Jong, B. Impact of Mediterranean diet education versus posted leaflet on dietary habits and serum cholesterol in a high risk population for cardiovascular disease. Public Health Nutr. 2000. 3:273-83. doi:10.1017/s1368980000000318

232 Ben Ayed, H, Yaich, S, Ben Hmida, M, Trigui, M, Ben Jemaa, M, Jedidi, J, Karray, R, Mejdoub, Y, Feki, H, Kassis, M, Damak, J. Prevalence, determinants and outcomes of general and abdominal obesity in medical students. Obesity Medicine. 2019. 13:39-44. doi:10.1016/j.obmed.2018.12.007

233 Berard, E, Bongard, V, Haas, B, Dallongeville, J, Moitry, M, Cottel, D, Ruidavets, JB, Ferrieres, J. Score of Adherence to 2016 European Cardiovascular Prevention Guidelines Predicts Cardiovascular and All-Cause Mortality in the General Population. Can J Cardiol. 2017. 33:1298-1304. doi:10.1016/j.cjca.2017.06.008

Study Design; Intervention/Exposure Study Design

Study Design

Intervention/Exposure; Comparator

Study Design; Intervention/Exposure

Study Design; Intervention/Exposure 

and cardiovascular disease risk factors: the Swedish INTERGENE research program. Am J Clin Nutr. 2008. 88:289-97. doi:10.1093/ajcn/88.2.289

235 Bergeron, N, Chiu, S, Williams, PT, King S M, Krauss, RM. Effects of red meat, white meat, and nonmeat protein sources on atherogenic lipoprotein measures in the context of low compared with high saturated fat intake: a randomized controlled trial. Am J Clin Nutr. 2019. :. doi:10.1093/ajcn/nqz035

236 Berglund, L, Lefevre, M, Ginsberg, HN, Kris-Etherton, PM, Elmer, PJ, Stewart, PW, Ershow, A, Pearson, TA, Dennis, BH, Roheim, PS, Ramakrishnan, R, Reed, R, Stewart, K, Phillips, KM. Comparison of monounsaturated fat with carbohydrates as a replacement for saturated fat in subjects with a high metabolic risk profile: studies in the fasting and postprandial states. Am J Clin Nutr. 2007. 86:1611-20. doi:10.1093/ajcn/86.5.1611

237 Bergouignan, A, Gozansky, WS, Barry, DW, Leitner, W, MacLean, PS, Hill, JO, Draznin, B, Melanson, EL. Increasing dietary fat elicits similar changes in fat oxidation and markers of muscle oxidative capacity in lean and obese humans. PLoS One. 2012. 7:e30164. doi:10.1371/journal.pone.0030164

238 Berkey, CS, Rockett, HR, Field, AE, Gillman, MW, Frazier, AL, Camargo, CA, Jr, Colditz, GA. Activity, dietary intake, and weight changes in a longitudinal study of preadolescent and adolescent boys and girls. Pediatrics. 2000. 105:E56. doi:10.1542/peds.105.4.e56

239 Berlin, KS, Kamody, RC, Thurston, IB, Banks, GG, Rybak, TM, Ferry, RJ, Jr. Physical Activity, Sedentary Behaviors, and Nutritional Risk Profiles and Relations to Body Mass Index, Obesity, and Overweight in Eighth Grade. Behav Med. 2017. 43:3139. doi:10.1080/08964289.2015.1039956

240 Bermudez, OI, Velez-Carrasco, W, Schaefer, EJ, Tucker, KL. Dietary and plasma lipid, lipoprotein, and apolipoprotein profiles among elderly Hispanics and non-Hispanics and their association with diabetes. Am J Clin Nutr. 2002. 76:1214-21. doi:10.1093/ajcn/76.6.1214

241 Bernstein, AM, Sun, Q, Hu, FB, Stampfer, MJ, Manson, JE, Willett, WC. Major dietary protein sources and risk of coronary heart disease in women. Circulation. 2010. 122:876-83. doi:10.1161/circulationaha.109.915165

242 Berryman, CE, Fleming, JA, Kris-Etherton, PM. Inclusion of Almonds in a Cholesterol-Lowering Diet Improves Plasma HDL Subspecies and Cholesterol Efflux to Serum in Normal-Weight Individuals with Elevated LDL Cholesterol. J Nutr. 2017. 147:1517-1523. doi:10.3945/jn.116.245126

243 Bersamin, A, Luick, BR, King, IB, Stern, JS, Zidenberg-Cherr, S. Westernizing diets influence fat intake, red blood cell fatty acid composition, and health in remote Alaskan Native communities in the center for Alaska Native health study. J Am Diet Assoc. 2008. 108:266-73. doi:10.1016/j.jada.2007.10.046

244 Bertoli, S, Neri, IG, Trentani, C, Ferraris, C, De Amicis, R, Battezzati, A, Veggiotti, P, De Giorgis, V, Tagliabue, A. Shortterm effects of ketogenic diet on anthropometric parameters, body fat distribution, and inflammatory cytokine production in GLUT1 deficiency syndrome. Nutrition. 2015. 31:981-7. doi:10.1016/j.nut.2015.02.017

\section{Rationale}

Study Design;

Publication Date

Overlaps with Existing

Review

Study duration

Study duration

Study duration

Intervention/Exposure

Study Design;

Intervention/Exposure

Study Design

Intervention/Exposure

Intervention/Exposure

Study Design

Health Status 


\begin{tabular}{|c|c|c|}
\hline No. & Citation & Rationale \\
\hline 245 & $\begin{array}{l}\text { Bertz, F, Winkvist, A, Brekke, HK. Sustainable weight loss among overweight and obese lactating women is achieved with an } \\
\text { energy-reduced diet in line with dietary recommendations: results from the LEVA randomized controlled trial. J Acad Nutr Diet. } \\
\text { 2015. 115:78-86. doi:10.1016/j.jand.2014.05.017 }\end{array}$ & $\begin{array}{l}\text { Weight } \\
\text { loss/Hypocaloric }\end{array}$ \\
\hline 246 & $\begin{array}{l}\text { Berz, JP, Singer, MR, Guo, X, Daniels, SR, Moore, LL. Use of a DASH food group score to predict excess weight gain in } \\
\text { adolescent girls in the National Growth and Health Study. Arch Pediatr Adolesc Med. 2011. 165:540-6. } \\
\text { doi:10.1001/archpediatrics.2011.71 }\end{array}$ & $\begin{array}{l}\text { Publication Date } \\
\text { Overlaps with Existing } \\
\text { Review }\end{array}$ \\
\hline 247 & $\begin{array}{l}\text { Best, TH, Franz, DN, Gilbert, DL, Nelson, DP, Epstein, MR. Cardiac complications in pediatric patients on the ketogenic diet. } \\
\text { Neurology. 2000. 54:2328-30. doi:10.1212/wnl.54.12.2328 }\end{array}$ & $\begin{array}{l}\text { Study Design; } \\
\text { Comparator }\end{array}$ \\
\hline 248 & $\begin{array}{l}\text { Bethancourt, HJ, Kratz, M, O'Connor, K. A short-term religious "fast" from animal products has a minimal impact on } \\
\text { cardiometabolic health biomarkers irrespective of concurrent shifts in distinct plant-based food groups. Am J Clin Nutr. } 2019 . \\
\text { 110:722-732. doi:10.1093/ajcn/nqz153 }\end{array}$ & Study duration \\
\hline 249 & $\begin{array}{l}\text { Beulen, Y, Martinez-Gonzalez, MA, van de Rest, O, Salas-Salvado, J, Sorli, JV, Gomez-Gracia, E, Fiol, M, Estruch, R, } \\
\text { Santos-Lozano, JM, Schroder, H, Alonso-Gomez, A, Serra-Majem, L, Pinto, X, Ros, E, Becerra-Tomas, N, Gonzalez, JI, } \\
\text { Fito, M, Martinez, JA, Gea, A. Quality of Dietary Fat Intake and Body Weight and Obesity in a Mediterranean Population: } \\
\text { Secondary Analyses within the PREDIMED Trial. Nutrients. 2018. 10:. doi:10.3390/nu10122011 }\end{array}$ & Intervention/Exposure \\
\hline 250 & $\begin{array}{l}\text { Beydoun, HA, Huang, S, Beydoun, MA, Hossain, S, Zonderman, AB. Mediating-Moderating Effect of Allostatic Load on the } \\
\text { Association between Dietary Approaches to Stop Hypertension Diet and All-Cause and Cause-Specific Mortality: 2001-2010 } \\
\text { National Health and Nutrition Examination Surveys. Nutrients. 2019. 11:. doi:10.3390/nu11102311 }\end{array}$ & Intervention/Exposure \\
\hline 251 & $\begin{array}{l}\text { Beyerlein, A, Uusitalo, UM, Virtanen, SM, Vehik, K, Yang, J, Winkler, C, Kersting, M, Koletzko, S, Schatz, D, Aronsson, } \\
\text { CA, Elding Larsson, H, Krischer, JP, Ziegler, AG, Norris, JM, Hummel, S. Intake of Energy and Protein is Associated with } \\
\text { Overweight Risk at Age 5.5 Years: Results from the Prospective TEDDY Study. Obesity (Silver Spring). 2017. 25:1435-1441. } \\
\text { doi:10.1002/oby.21897 }\end{array}$ & $\begin{array}{l}\text { Study Design; } \\
\text { Intervention/Exposure }\end{array}$ \\
\hline 252 & $\begin{array}{l}\text { Bhargava, A, Guthrie, JF. Unhealthy eating habits, physical exercise and macronutrient intakes are predictors of } \\
\text { anthropometric indicators in the Women's Health Trial: Feasibility Study in Minority Populations. Br J Nutr. 2002. 88:719-28. } \\
\text { doi:10.1079/bjn2002739 }\end{array}$ & $\begin{array}{l}\text { Intervention/Exposure; } \\
\text { Comparator }\end{array}$ \\
\hline 253 & $\begin{array}{l}\text { Bhattacharyya, M, Maity, S, Bandyopadhyay, S. Exploring the Missing Links Between Dietary Habits and Diseases. IEEE } \\
\text { Trans Nanobioscience. 2017. 16:226-238. doi:10.1109/tnb.2017.2654121 }\end{array}$ & Study Design \\
\hline 254 & $\begin{array}{l}\text { Bian, JT, Szczurek, M, Ranieri, C, Grizelj, I, Cavka, A, Robinson, A, Marsh, G, Li, K, Sanyaolu, RA, Shinde, S, etal, . } \\
\text { Weight loss with low carbohydrate diets improves flow induced vasodilation in resistance arteries. Circulation. 2014. 130:. } \\
\text { doi:unavailable }\end{array}$ & Publication Status \\
\hline 255 & $\begin{array}{l}\text { Bianco, A, Thomas, E, Bellafiore, M, Martines, F, Messina, G, Battaglia, G, Karsten, B, Sahin, FN, Bielec, G, Paoli, A, } \\
\text { Palma, A. Mediterranean diet and dietary protein supplementation as possible predicting variables of weight management: An } \\
\text { update of the protein project. Acta Medica Mediterranea. } 2015.31: 1265-1270 \text {. doi:unavailable }\end{array}$ & Study Design \\
\hline
\end{tabular}


256 Biazzi Leal, D, Altenburg de Assis, MA, Hinnig, PF, Schmitt, J, Soares Lobo, A, Bellisle, F, Di Pietro, PF, Vieira, FK, de

Moura Araujo, PH, de Andrade, DF. Changes in Dietary Patterns from Childhood to Adolescence and Associated Body Adiposity Status. Nutrients. 2017. 9:. doi:10.3390/nu9101098

257 Bihuniak, JD, Ramos, A, Huedo-Medina, T, Hutchins-Wiese, H, Kerstetter, JE, Kenny, AM. Adherence to a MediterraneanStyle Diet and Its Influence on Cardiovascular Risk Factors in Postmenopausal Women. J Acad Nutr Diet. 2016. 116:17671775. doi:10.1016/j.jand.2016.06.377

258 Bilenko, N, Fraser, D, Vardi, H, Shai, I, Shahar, DR. Mediterranean diet and cardiovascular diseases in an Israeli population. Prev Med. 2005. 40:299-305. doi:10.1016/j.ypmed.2004.06.004

259 Binns, A, Gray, M, Henson, AC, Fort, IL. Changes in Lean Mass and Serum Myostatin with Habitual Protein Intake and HighVelocity Resistance Training. J Nutr Health Aging. 2017. 21:1111-1117. doi:10.1007/s12603-017-0883-6

260 Bisschop, PH, Ackermans, MT, Endert, E, Ruiter, AF, Meijer, AJ, Kuipers, F, Sauerwein, HP, Romijn, JA. The effect of carbohydrate and fat variation in euenergetic diets on postabsorptive free fatty acid release. Br J Nutr. 2002. 87:555-9. doi:10.1079/bjnbjn2002578

261 Bisschop, PH, Bandsma, RH, Stellaard, F, ter Harmsel, A, Meijer, AJ, Sauerwein, HP, Kuipers, F, Romijn, JA. Low-fat, high-carbohydrate and high-fat, low-carbohydrate diets decrease primary bile acid synthesis in humans. Am $\mathrm{J}$ Clin Nutr. 2004 79:570-6. doi:10.1093/ajcn/79.4.570

262 Bisschop, PH, de Metz, J, Ackermans, MT, Endert, E, Pijl, H, Kuipers, F, Meijer, AJ, Sauerwein, HP, Romijn, JA. Dietary fat content alters insulin-mediated glucose metabolism in healthy men. Am J Clin Nutr. 2001. 73:554-9. doi:10.1093/ajcn/73.3.554

263 Bjerregaard, P, Pedersen, HS, Mulvad, G. The associations of a marine diet with plasma lipids, blood glucose, blood pressure and obesity among the inuit in Greenland. Eur J Clin Nutr. 2000. 54:732-7. doi:10.1038/sj.ejcn.1601088

264 Black, MH, Watanabe, RM, Trigo, E, Takayanagi, M, Lawrence, JM, Buchanan, TA, Xiang, AH. High-fat diet is associated with obesity-mediated insulin resistance and beta-cell dysfunction in Mexican Americans. J Nutr. 2013. 143:479-85. doi:10.3945/jn.112.170449

265 Black, MH, Watanabe, RM, Trigo, E, Takayanagi, M, Lawrence, JM, Buchanan, TA, Xiang, AH. High-fat diet is associated with obesity-mediated insulin resistance and $\beta$-cell dysfunction in Mexican Americans. Journal of Nutrition. 2013. 143:479-485. doi:10.3945/jn.112.170449

266 Black, RN, Spence, M, McMahon, RO, Cuskelly, GJ, Ennis, CN, McCance, DR, Young, IS, Bell, PM, Hunter, SJ. Effect of eucaloric high- and low-sucrose diets with identical macronutrient profile on insulin resistance and vascular risk: a randomized controlled trial. Diabetes. 2006. 55:3566-72. doi:10.2337/db06-0220

267 Bladbjerg, EM, Larsen, TM, Due, A, Stender, S, Astrup, A, Jespersen, J. Effects on markers of inflammation and endothelial cell function of three ad libitum diets differing in type and amount of fat and carbohydrate: a 6-month randomised study in obese individuals. Br J Nutr. 2011. 106:123-9. doi:10.1017/s0007114510005829

Study Design;

Intervention/Exposure

Study Design

Intervention/Exposure

Study duration

Study duration

Study duration

Study duration

Intervention/Exposure

Study Design

Power/Size; Study

Design

Intervention/Exposure; Publication Date

Overlaps with Existing Review

Power/Size 


\section{No. Citation}

268 Blesso, CN, Andersen, CJ, Barona, J, Volek, JS, Fernandez, ML. Whole egg consumption improves lipoprotein profiles and insulin sensitivity to a greater extent than yolk-free egg substitute in individuals with metabolic syndrome. Metabolism. 2013. 62:400-10. doi:10.1016/j.metabol.2012.08.014

269 Blesso, CN, Andersen, CJ, Barona, J, Volk, B, Volek, JS, Fernandez, ML. Effects of carbohydrate restriction and dietary cholesterol provided by eggs on clinical risk factors in metabolic syndrome. J Clin Lipidol. 2013. 7:463-71. doi:10.1016/j.jacl.2013.03.008

270 Bligh, HF, Godsland, IF, Frost, G, Hunter, KJ, Murray, P, MacAulay, K, Hyliands, D, Talbot, DC, Casey, J, Mulder, TP, Berry, MJ. Plant-rich mixed meals based on Palaeolithic diet principles have a dramatic impact on incretin, peptide $Y Y$ and satiety response, but show little effect on glucose and insulin homeostasis: an acute-effects randomised study. Br J Nutr. 2015. 113:574-84. doi:10.1017/s0007114514004012

271 Block, JP. Mediterranean diet lowers cardiovascular risk even without weight loss. Journal of clinical outcomes management. 2013. 20:150-152. doi:unavailable

272 Blomfield, RL, Collins, CE, Hutchesson, MJ, Young, MD, Jensen, ME, Callister, R, Morgan, PJ. Impact of self-help weight loss resources with or without online support on the dietary intake of overweight and obese men: the SHED-IT randomised controlled trial. Obes Res Clin Pract. 2014. 8:e476-87. doi:10.1016/j.orcp.2013.09.004

273 Blomquist, C, Chorell, E, Ryberg, M, Mellberg, C, Worrsjo, E, Makoveichuk, E, Larsson, C, Lindahl, B, Olivecrona, G, Olsson, T. Decreased lipogenesis-promoting factors in adipose tissue in postmenopausal women with overweight on a Paleolithic-type diet. Eur J Nutr. 2018. 57:2877-2886. doi:10.1007/s00394-017-1558-0

274 Bloomer, RJ, Gunnels, TA, Schriefer, JM. Comparison of a Restricted and Unrestricted Vegan Diet Plan with a Restricted Omnivorous Diet Plan on Health-Specific Measures. Healthcare (Basel). 2015. 3:544-55. doi:10.3390/healthcare3030544

275 Blumenthal, JA, Sherwood, A, Smith, PJ, Mabe, S, Watkins, L, Lin, PH, Craighead, LW, Babyak, M, Tyson, C, Young, K, Ashworth, M, Kraus, W, Liao, L, Hinderliter, A. Lifestyle modification for resistant hypertension: The TRIUMPH randomized clinical trial. Am Heart J. 2015. 170:986-994.e5. doi:10.1016/j.ahj.2015.08.006

276 Boden-Albala, B, Elkind, MS, White, H, Szumski, A, Paik, MC, Sacco, RL. Dietary total fat intake and ischemic stroke risk: the Northern Manhattan Study. Neuroepidemiology. 2009. 32:296-301. doi:10.1159/000204914

277 Boers, I, Muskiet, FA, Berkelaar, E, Schut, E, Penders, R, Hoenderdos, K, Wichers, HJ, Jong, MC. Favourable effects of consuming a Palaeolithic-type diet on characteristics of the metabolic syndrome: a randomized controlled pilot-study. Lipids Health Dis. 2014. 13:160. doi:10.1186/1476-511x-13-160

278 Bogart, LM, Elliott, MN, Uyeda, K, Hawes-Dawson, J, Klein, DJ, Schuster, MA. Preliminary healthy eating outcomes of SNaX, a pilot community-based intervention for adolescents. Journal of Adolescent Health. 2011. 48:196-202. doi:10.1016/j.jadohealth.2010.06.004

279 Bogl, LH, Pietilainen, KH, Rissanen, A, Kangas, AJ, Soininen, P, Rose, RJ, Ala-Korpela, M, Kaprio, J. Association between habitual dietary intake and lipoprotein subclass profile in healthy young adults. Nutr Metab Cardiovasc Dis. 2013. 23:1071-8. doi:10.1016/j.numecd.2012.11.007

\section{Rationale}

Intervention/Exposure; Comparator

Power/Size

Study duration

Publication Status

Intervention/Exposure

Power/Size

Study Design; Study duration

Study Design;

Intervention/Exposure

Intervention/Exposure

Study duration

Intervention/Exposure

Study Design 
280 Bogomolova, S, Zarnowiecki, D, Wilson, A, Fielder, A, Procter, N, Itsiopoulos, C, O'Dea, K, Strachan, J, Ballestrin, M, Champion, A, Parletta, N. Dietary intervention for people with mental illness in South Australia. Health Promot Int. 2018. 33:71-83. doi:10.1093/heapro/daw055

281 Bonaccio, M, Di Castelnuovo, A, Costanzo, S, Persichillo, M, De Curtis, A, Donati, MB, de Gaetano, G, lacoviello, L. Adherence to the traditional Mediterranean diet and mortality in subjects with diabetes. Prospective results from the MOLI-SANI study. Eur J Prev Cardiol. 2016. 23:400-7. doi:10.1177/2047487315569409

282 Bondia-Pons, I, Martinez, JA, de la Iglesia, R, Lopez-Legarrea, P, Poutanen, K, Hanhineva, K, Zulet Mde, L. Effects of short- and long-term Mediterranean-based dietary treatment on plasma LC-QTOF/MS metabolic profiling of subjects with metabolic syndrome features: The Metabolic Syndrome Reduction in Navarra (RESMENA) randomized controlled trial. Mol Nutr Food Res. 2015. 59:711-28. doi:10.1002/mnfr.201400309

283 Bondonno, NP, Lewis, JR, Blekkenhorst, LC, Shivappa, N, Woodman, RJ, Bondonno, CP, Ward, NC, Hebert, JR, Thompson, PL, Prince, RL, Hodgson, JM. Dietary inflammatory index in relation to sub-clinical atherosclerosis and atherosclerotic vascular disease mortality in older women. Br J Nutr. 2017. 117:1577-1586. doi:10.1017/s0007114517001520

284 Bonfanti, N, Fernández, JM, Gomez-Delgado, F, Pérez-Jiménez, F. Effect of two hypocaloric diets and their combination with physical exercise on basal metabolic rate and body composition. Nutricion hospitalaria. 2014. 29:635-643. doi:10.3305/nh.2014.29.3.7119

285 Booth, A, Nowson, C, Worsley, A, Magerison, C, Jorna, M. Dietary approaches for weight loss with increased intakes of fruit vegetables and dairy products. Nutrition \& dietetics. 2008. 65:115-120. doi:unavailable

286 Bopp, MJ, Houston, DK, Lenchik, L, Easter, L, Kritchevsky, SB, Nicklas, BJ. Lean mass loss is associated with low protein intake during dietary-induced weight loss in postmenopausal women. J Am Diet Assoc. 2008. 108:1216-20. doi:10.1016/j.jada.2008.04.017

287 Borges, CA, Marchioni, DML, Levy, RB, Slater, B. Dietary patterns associated with overweight among Brazilian adolescents. Appetite. 2018. 123:402-409. doi:10.1016/j.appet.2018.01.001

288 Bortolotti, M, Kreis, R, Debard, C, Cariou, B, Faeh, D, Chetiveaux, M, Ith, M, Vermathen, P, Stefanoni, N, Le, KA Schneiter, P, Krempf, M, Vidal, H, Boesch, C, Tappy, L. High protein intake reduces intrahepatocellular lipid deposition in humans. Am J Clin Nutr. 2009. 90:1002-10. doi:10.3945/ajcn.2008.27296

289 Bosco, G, Lodi, A, Cenci, L, Grimaldi, K, Parmagnani, A, Paoli, A. Hunger-related hormones, body composition and metabolism in overweight subjects two months after a mediterranean ketogenetic diet weight loss program. Eating and weight disorders. 2014. 19:423. doi:10.1007/s40519-014-0134-3

290 Bosse, MC, Davis, SC, Puhl, SM, Pedersen, M, Low, V, Reiner, L, Dominguez, T, Seals, N. Effects of Zone diet macronutrient proportions on blood lipids, blood glucose, body composition, and treadmill exercise performance. Nutrition Research. 2004. 24:521-530. doi:10.1016/j.nutres.2004.04.001

Study Design; Health

Status

Health Status

Intervention/Exposure; Outcome

Intervention/Exposure

Language

Intervention/Exposure; Publication Date

Overlaps with Existing Review

Study Design;

Intervention/Exposure

Study Design

Study duration

Publication Status

Study duration 
291 Boucher, AB, Adesanya, EA, Owei, I, Gilles, AK, Ebenibo, S, Wan, J, Edeoga, C, Dagogo-Jack, S. Dietary habits and leisure-time physical activity in relation to adiposity, dyslipidemia, and incident dysglycemia in the pathobiology of prediabetes in a biracial cohort study. Metabolism. 2015. 64:1060-7. doi:10.1016/j.metabol.2015.05.015

292 Bouillon, K, Singh-Manoux, A, Jokela, M, Shipley, MJ, Batty, GD, Brunner, EJ, Sabia, S, Tabák, AG, Akbaraly, T, Ferrie, JE, Kivimäki, M. Decline in low-density lipoprotein cholesterol concentration: Lipid-lowering drugs, diet, or physical activity? Evidence from the Whitehall II study. Heart. 2011. 97:923-930. doi:10.1136/hrt.2010.216309

293 Bounds, RG, Grandjean, PW, O'Brien, BC, Inman, C, Crouse, SF. Diet and short term plasma lipoprotein-lipid changes after exercise in trained Men. Int J Sport Nutr Exerc Metab. 2000. 10:114-27. doi:10.1123/ijsnem.10.2.114

294 Bowen, J, Noakes, M, Clifton, P. High dairy-protein versus high mixed-protein energy restricted diets - the effect on bone turnover and calcium excretion in overweight adults. Asia pacific journal of clinical nutrition. 2003. 12 Suppl:S52. doi:unavailable

295 Bowen, J, Noakes, M, Clifton, PM. A high dairy protein, high-calcium diet minimizes bone turnover in overweight adults during weight loss. J Nutr. 2004. 134:568-73. doi:10.1093/jn/134.3.568

296 Bowen, J, Noakes, M, Clifton, PM. Effect of calcium and dairy foods in high protein, energy-restricted diets on weight loss and metabolic parameters in overweight adults. Int J Obes (Lond). 2005. 29:957-65. doi:10.1038/sj.ijo.0802895

Outcome

Study duration

Intervention/Exposure; Publication Status

Intervention/Exposure; Comparator

Intervention/Exposure; Comparator

297 Bowen, KJ, Kris-Etherton, PM, Jones, PJ, Reddivari, L. Effects of diets that vary in fatty acid composition on fecal short-chain fatty acid levels and their relationship with circulating lipids and lipoproteins. Circulation. 2019. 139:. doi:10.1161/circ.139.suppl_1.P293

298 Bowling, A, Davison, K, Haneuse, S, Beardslee, W, Miller, DP. ADHD Medication, Dietary Patterns, Physical Activity, and BMI in Children: A Longitudinal Analysis of the ECLS-K Study. Obesity (Silver Spring). 2017. 25:1802-1808. doi:10.1002/oby.21949

299 Bowman, SA, Spence, JT. A comparison of low-carbohydrate vs. high-carbohydrate diets: energy restriction, nutrient quality and correlation to body mass index. J Am Coll Nutr. 2002. 21:268-74. doi:10.1080/07315724.2002.10719220

300 Boyle, KE, Canham, JP, Consitt, LA, Zheng, D, Koves, TR, Gavin, TP, Holbert, D, Neufer, PD, Ilkayeva, O, Muoio, DM, Houmard, JA. A high-fat diet elicits differential responses in genes coordinating oxidative metabolism in skeletal muscle of lean and obese individuals. J Clin Endocrinol Metab. 2011. 96:775-81. doi:10.1210/jc.2010-2253

301 Bozzetto, L, Annuzzi, G, Pacini, G, Costabile, G, Vetrani, C, Vitale, M, Griffo, E, Giacco, A, De Natale, C, Cocozza, S, Della Pepa, G, Tura, A, Riccardi, G, Rivellese, AA. Polyphenol-rich diets improve glucose metabolism in people at high cardiometabolic risk: a controlled randomised intervention trial. Diabetologia. 2015. 58:1551-60. doi:10.1007/s00125-015-3592$\mathrm{X}$

302 Brader, L, Uusitupa, M, Dragsted, LO, Hermansen, K. Effects of an isocaloric healthy Nordic diet on ambulatory blood pressure in metabolic syndrome: a randomized SYSDIET sub-study. Eur J Clin Nutr. 2014. 68:57-63.

Publication Status

Intervention/Exposure

Study Design

Study duration

Intervention/Exposure doi:10.1038/ejcn.2013.192

Study Design;

Publication Status 

with the metabolic syndrome: a SYSDIET sub study. Obesity reviews. 2011. 12:230. doi:10.1111/j.1467-789X.2011.00889.x

304 Bradley, U, Spence, M, Courtney, CH, McKinley, MC, Ennis, CN, McCance, DR, McEneny, J, Bell, PM, Young, IS, Hunter SJ. Low-fat versus low-carbohydrate weight reduction diets: effects on weight loss, insulin resistance, and cardiovascular risk: a randomized control trial. Diabetes. 2009. 58:2741-8. doi:10.2337/db09-0098

305 Braha, K, Cupak, A, Pokrivcak, J, Qineti, A, Rizov, M. Economic analysis of the link between diet quality and health: Evidence from Kosovo. Econ Hum Biol. 2017. 27:261-274. doi:10.1016/j.ehb.2017.08.003

306 Brand-Miller, JC, Colagiuri, S, Gan, ST. Insulin sensitivity predicts glycemia after a protein load. Metabolism. 2000. 49:1-5. doi:10.1016/s0026-0495(00)90488-8

307 Branis, NM, Etesami, M, Walker, RW, Berk, ES, Albu, JB. Effect of a 1-week, eucaloric, moderately high-fat diet on peripheral insulin sensitivity in healthy premenopausal women. BMJ Open Diabetes Res Care. 2015. 3:e000100. doi:10.1136/bmjdrc-2015-000100

308 Brassard, D, Tessier-Grenier, M, Allaire, J, Rajendiran, E, She, Y, Ramprasath, V, Gigleux, I, Talbot, D, Levy, E, Tremblay, A, Jones, PJ, Couture, P, Lamarche, B. Comparison of the impact of SFAs from cheese and butter on cardiometabolic risk factors: a randomized controlled trial. Am J Clin Nutr. 2017. 105:800-809. doi:10.3945/ajcn.116.150300

309 Bravo-Herrera, MD, Lopez-Miranda, J, Marin, C, Gomez, P, Gomez, MJ, Moreno, JA, Perez-Martinez, P, Blanco, A, Jimenez-Gomez, Y, Perez-Jimenez, F. Tissue factor expression is decreased in monocytes obtained from blood during Mediterranean or high carbohydrate diets. Nutr Metab Cardiovasc Dis. 2004. 14:128-32. doi:unavailable

310 Bray, GA, Champagne, CM. Dietary Patterns May Modify Central Adiposity. Journal of the American Dietetic Association. 2009. 109:1354-1355. doi:10.1016/j.jada.2009.05.013

311 Bray, GA, Lovejoy, JC, Most-Windhauser, M, Smith, SR, Volaufova, J, Denkins, Y, de Jonge, L, Rood, J, Lefevre, M, Eldridge, AL, Peters, JC. A 9-mo randomized clinical trial comparing fat-substituted and fat-reduced diets in healthy obese men: the Ole Study. Am J Clin Nutr. 2002. 76:928-34. doi:10.1093/ajcn/76.5.928

312 Bray, GA, Redman, LM, de Jonge, L, Rood, J, Smith, SR. Effect of Three Levels of Dietary Protein on Metabolic Phenotype of Healthy Individuals With 8 Weeks of Overfeeding. J Clin Endocrinol Metab. 2016. 101:2836-43. doi:10.1210/jc.2016-1313

313 Bray, GA, Redman, LM, de Jonge, L, Rood, J, Sutton, EF, Smith, SR. Plasma Amino Acids During 8 Weeks of Overfeeding: Relation to Diet Body Composition and Fat Cell Size in the PROOF Study. Obesity (Silver Spring). 2018. 26:324-331. doi:10.1002/oby.22087

314 Bray, GA, Ryan, DH, Johnson, W, Champagne, CM, Johnson, CM, Rood, J, Williamson, DA, Sacks, FM. Markers of dietary protein intake are associated with successful weight loss in the POUNDS Lost trial. Clin Obes. 2017. 7:166-175. doi:10.1111/cob. 12188

\section{Rationale}

Study Design

ntervention/Exposure; Publication Status

Study duration

Study Design Outcome

Study duration

Study duration

Intervention/Exposure; Comparator

Study duration

Publication Status

Intervention/Exposure; Comparator

Power/Size

Intervention/Exposure; Outcome

Weight

loss/Hypocaloric 
Bray, GA, Smith, SR, de Jonge, L, Xie, H, Rood, J, Martin, CK, Most, M, Brock, C, Mancuso, S, Redman, LM. Effect of dietary protein content on weight gain, energy expenditure, and body composition during overeating: a randomized controlled trial. Jama. 2012. 307:47-55. doi:10.1001/jama.2011.1918

316 Bray, GA, Smith, SR, DeJonge, L, de Souza, R, Rood, J, Champagne, CM, Laranjo, N, Carey, V, Obarzanek, E, Loria, CM, Anton, SD, Ryan, DH, Greenway, FL, Williamson, D, Sacks, FM. Effect of diet composition on energy expenditure during weight loss: the POUNDS LOST Study. Int J Obes (Lond). 2012. 36:448-55. doi:10.1038/ijo.2011.173

317 Bray, GA, Vollmer, WM, Sacks, FM, Obarzanek, E, Svetkey, LP, Appel, LJ. A further subgroup analysis of the effects of the DASH diet and three dietary sodium levels on blood pressure: results of the DASH-Sodium Trial. Am J Cardiol. 2004. 94:222-7. doi:10.1016/j.amjcard.2004.03.070

318 Brehm, BJ, Seeley, RJ, Daniels, SR, D'Alessio, DA. A randomized trial comparing a very low carbohydrate diet and a calorierestricted low fat diet on body weight and cardiovascular risk factors in healthy women. J Clin Endocrinol Metab. 2003. 88:161723. doi:10.1210/jc.2002-021480

319 Brehm, BJ, Spang, SE, Lattin, BL, Seeley, RJ, Daniels, SR, D'Alessio, DA. The role of energy expenditure in the differentia weight loss in obese women on low-fat and low-carbohydrate diets. J Clin Endocrinol Metab. 2005. 90:1475-82. doi:10.1210/jc.2004-1540

320 Breitenbach, Z, Raposa, B, Szabó, Z, Polyák, É, Szűcs, Z, Kubányi, J, Figler, M. Examination of Hungarian college students' eating habits, physical activity and body composition. European Journal of Integrative Medicine. 2016. 8:13-17. doi:10.1016/j.eujim.2016.11.007

321 Brennan, IM, Luscombe-Marsh, ND, Seimon, RV, Otto, B, Horowitz, M, Wishart, JM, Feinle-Bisset, C. Effects of fat, protein, and carbohydrate and protein load on appetite, plasma cholecystokinin, peptide YY, and ghrelin, and energy intake in lean and obese men. Am J Physiol Gastrointest Liver Physiol. 2012. 303:G129-40. doi:10.1152/ajpgi.00478.2011

322 Bril, N, Shelef, I, Schwarzfuchs, D, Serfaty, D, Gepner, Y, Lerner, M, Cohen, N, Shemesh, E, Tangi-Rosental, O, Sarusi, B, etal, . Acute diet induced thermogenesis (DIT), specific foods, and visceral adiposity. Clinical nutrition. 2013. 32:S24-S25. doi:unavailable

323 Brinkworth, GD, Buckley, JD, Noakes, M, Clifton, PM. Renal function following long-term weight loss in individuals with abdominal obesity on a very-low-carbohydrate diet vs high-carbohydrate diet. J Am Diet Assoc. 2010. 110:633-8. doi:10.1016/j.jada.2009.12.016

324 Brinkworth, GD, Noakes, M, Buckley, JD, Keogh, JB, Clifton, PM. Long-term effects of a very-low-carbohydrate weight loss diet compared with an isocaloric low-fat diet after 12 mo. Am J Clin Nutr. 2009. 90:23-32. doi:10.3945/ajcn.2008.27326

325 Brinkworth, GD, Noakes, M, Keogh, JB, Luscombe, ND, Wittert, GA, Clifton, PM. Long-term effects of a high-protein, lowcarbohydrate diet on weight control and cardiovascular risk markers in obese hyperinsulinemic subjects. Int J Obes Relat Metab Disord. 2004. 28:661-70. doi:10.1038/sj.ijo.0802617

\section{Rationale}

Study duration

Intervention/Exposure; Weight

loss/Hypocaloric; Study duration

Study Design;

Intervention/Exposure

Power/Size

Power/Size

Study Design

Study duration

Publication Status

Weight

loss/Hypocaloric

Weight loss/Hypocaloric

Power/Size 
326 Brito Beck da Silva, K, Leovigildo Fiaccone, R, Couto, RD, Ribeiro-Silva Rde, C. EVALUATION OF THE EFFECTS OF A PROGRAMME PROMOTING ADEQUATE AND HEALTHY EATING ON ADOLESCENT HEALTH MARKERS: AN INTERVENTIONAL STUDY. Nutr Hosp. 2015. 32:1582-90. doi:10.3305/nh.2015.32.4.9512

327 Brito Beck da Silva, K, Ortelan, N, Giardini Murta, S, Sartori, I, Couto, RD, Leovigildo Fiaccone, R, Lima Barreto, M, Jones Bell, M, Barr Taylor, C, Ribeiro-Silva, RC. Evaluation of the Computer-Based Intervention Program Stayingfit Brazil to Promote Healthy Eating Habits: The Results from a School Cluster-Randomized Controlled Trial. Int J Environ Res Public Health. 2019. 16:. doi:10.3390/ijerph16101674

328 Brixval, CS, Andersen, LB, Heitmann, BL. Fat intake and weight development from 9 to 16 years of age: the European youth heart study - a longitudinal study. Obes Facts. 2009. 2:166-70. doi:10.1159/000219134

329 Brons, C, Jensen, CB, Storgaard, H, Hiscock, NJ, White, A, Appel, JS, Jacobsen, S, Nilsson, E, Larsen, CM, Astrup, A Quistorff, B, Vaag, A. Impact of short-term high-fat feeding on glucose and insulin metabolism in young healthy men. J Physiol. 2009. 587:2387-97. doi:10.1113/jphysiol.2009.169078

330 Brooking, LA, Williams, SM, Mann, JI. Effects of macronutrient composition of the diet on body fat in indigenous people at high risk of type 2 diabetes. Diabetes Res Clin Pract. 2012. 96:40-6. doi:10.1016/j.diabres.2011.11.021

331 Brouwer-Brolsma, EM, van Lee, L, Streppel, MT, Sluik, D, van de Wiel, AM, de Vries, JHM, Geelen, A, Feskens, EJM Nutrition Questionnaires plus (NQplus) study, a prospective study on dietary determinants and cardiometabolic health in Dutch adults. BMJ Open. 2018. 8:e020228. doi:10.1136/bmjopen-2017-020228

332 Brown, DL, Conley, KM, Sanchez, BN, Resnicow, K, Cowdery, JE, Sais, E, Murphy, J, Skolarus, LE, Lisabeth, LD, Morgenstern, LB. A Multicomponent Behavioral Intervention to Reduce Stroke Risk Factor Behaviors: The Stroke Health and Risk Education Cluster-Randomized Controlled Trial. Stroke. 2015. 46:2861-7. doi:10.1161/strokeaha.115.010678

333 Browning, JD, Baker, JA, Rogers, T, Davis, J, Satapati, S, Burgess, SC. Short-term weight loss and hepatic triglyceride reduction: evidence of a metabolic advantage with dietary carbohydrate restriction. Am J Clin Nutr. 2011. 93:1048-52 doi:10.3945/ajcn.110.007674

334 Browning, JD, Davis, J, Saboorian, MH, Burgess, SC. A low-carbohydrate diet rapidly and dramatically reduces intrahepatic triglyceride content. Hepatology. 2006. 44:487-8. doi:10.1002/hep.21264

335 Brunerova, L, Smejkalova, V, Potockova, J, Andel, M. A comparison of the influence of a high-fat diet enriched in monounsaturated fatty acids and conventional diet on weight loss and metabolic parameters in obese non-diabetic and Type 2 diabetic patients. Diabet Med. 2007. 24:533-40. doi:10.1111/j.1464-5491.2007.02104.x

336 Bruno, E, Manoukian, S, Venturelli, E, Oliverio, A, Rovera, F, lula, G, Morelli, D, Peissel, B, Azzolini, J, Roveda, E, Pasanisi, P. Adherence to Mediterranean Diet and Metabolic Syndrome in BRCA Mutation Carriers. Integr Cancer Ther. 2018. 17:153-160. doi:10.1177/1534735417721015

337 Brynes, AE, Adamson, J, Dornhorst, A, Frost, GS. The beneficial effect of a diet with low glycaemic index on 24 h glucose profiles in healthy young people as assessed by continuous glucose monitoring. Br J Nutr. 2005. 93:179-82. doi:10.1079/bjn20041318

Intervention/Exposure; Outcome

Intervention/Exposure

Study duration

Study duration

Study Design;

Outcome

Intervention/Exposure

Study duration

Publication Status

Intervention/Exposure

Outcome

Study duration 
338 Bucher Della Torre, S, Wild, P, Dorribo, V, Amati, F, Danuser, B. Eating Habits of Professional Firefighters: Comparison With National Guidelines and Impact Healthy Eating Promotion Program. J Occup Environ Med. 2019. 61:e183-e190. doi:10.1097/jom.0000000000001565

339 Buckland, NJ, Camidge, D, Croden, F, Lavin, JH, Stubbs, RJ, Hetherington, MM, Blundell, JE, Finlayson, G. A Low Energy-Dense Diet in the Context of a Weight-Management Program Affects Appetite Control in Overweight and Obese Women. J Nutr. 2018. 148:798-806. doi:10.1093/jn/nxy041

340 Buendia, JR, Bradlee, ML, Singer, MR, Moore, LL. Diets higher in protein predict lower high blood pressure risk in Framingham Offspring Study adults. Am J Hypertens. 2015. 28:372-9. doi:10.1093/ajh/hpu157

341 Bui, Q. Dietary fat modification and the risk of future cardiovascular events and mortality. Am Fam Physician. 2013. 87:609-10. doi:unavailable

342 Buijsse, B, Boeing, H, Drogan, D, Schulze, MB, Feskens, EJ, Amiano, P, Barricarte, A, Clavel-Chapelon, F, de LauzonGuillain, B, Fagherazzi, G, Fonseca-Nunes, A, Franks, PW, Huerta, JM, Jakobsen, MU, Kaaks, R, Key, TJ, Khaw, KT, Masala, G, Moskal, A, Nilsson, PM, Overvad, K, Pala, V, Panico, S, Redondo, ML, Ricceri, F, Rolandsson, O, Sanchez, MJ, Sluijs, I, Spijkerman, AM, Tjonneland, A, Tumino, R, van der, DI A, van der Schouw, YT, Langenberg, C, Sharp, SJ, Forouhi, NG, Riboli, E, Wareham, NJ. Consumption of fatty foods and incident type 2 diabetes in populations from eight European countries. Eur J Clin Nutr. 2015. 69:455-61. doi:10.1038/ejcn.2014.249

343 Buijsse, B, Jacobs, DR, Jr, Steffen, LM, Kromhout, D, Gross, MD. Plasma Ascorbic Acid, A Priori Diet Quality Score, and Incident Hypertension: A Prospective Cohort Study. PLoS One. 2015. 10:e0144920. doi:10.1371/journal.pone.0144920

344 Buil-Cosiales, P, Toledo, E, Salas-Salvadó, J, Zazpe, I, Farràs, M, Basterra-Gortari, FJ, Diez-Espino, J, Estruch, R, Corella, D, Ros, E, Marti, A, Gómez-Gracia, E, Ortega-Calvo, M, Arós, F, Moñino, M, Serra-Majem, L, Pintó, X, Lamuela-Raventós, RM, Babio, N, Gonzalez, JI, Fitó, M, Martínez-González, MA. Association between dietary fibre intake and fruit, vegetable or whole-grain consumption and the risk of CVD: Results from the PREvención con Dleta MEDiterránea (PREDIMED) trial. British Journal of Nutrition. 2016. 116:534-546. doi:10.1017/S0007114516002099

345 Buil-Cosiales, P, Zazpe, I, Toledo, E, Corella, D, Salas-Salvado, J, Diez-Espino, J, Ros, E, Fernandez-Creuet Navajas, J, Santos-Lozano, JM, Aros, F, Fiol, M, Castaner, O, Serra-Majem, L, Pinto, X, Lamuela-Raventos, RM, Marti, A, BasterraGortari, FJ, Sorli, JV, Verdu-Rotellar, JM, Basora, J, Ruiz-Gutierrez, V, Estruch, R, Martinez-Gonzalez, MA. Fiber intake and all-cause mortality in the Prevencion con Dieta Mediterranea (PREDIMED) study. Am J Clin Nutr. 2014. 100:1498-507. doi:10.3945/ajcn.114.093757

346 Bullo, M, Casas, R, Portillo, MP, Basora, J, Estruch, R, Garcia-Arellano, A, Lasa, A, Juanola-Falgarona, M, Aros, F, Salas Salvado, J. Dietary glycemic index/load and peripheral adipokines and inflammatory markers in elderly subjects at high cardiovascular risk. Nutr Metab Cardiovasc Dis. 2013. 23:443-50. doi:10.1016/j.numecd.2011.09.009

347 Bunyard, LB, Dennis, KE, Nicklas, BJ. Dietary intake and changes in lipoprotein lipids in obese, postmenopausal women placed on an American Heart Association Step 1 diet. J Am Diet Assoc. 2002. 102:52-7. doi:10.1016/s0002-8223(02)90016-7

Outcome

Study Design;

Publication Status

Intervention/Exposure

Outcome

Intervention/Exposure

Intervention/Exposure

Outcome

Study Design 


\section{No. Citation}

348 Burger, KN, Beulens, JW, Boer, JM, Spijkerman, AM, van der, DI A. Dietary glycemic load and glycemic index and risk of coronary heart disease and stroke in Dutch men and women: the EPIC-MORGEN study. PLoS One. 2011. 6:e25955. doi:10.1371/journal.pone.0025955

349 Burgess, B, Raynor, HA, Tepper, BJ. PROP Nontaster Women Lose More Weight Following a Low-Carbohydrate Versus a Low-Fat Diet in a Randomized Controlled Trial. Obesity (Silver Spring). 2017. 25:1682-1690. doi:10.1002/oby.21951

350 Burke, LE, Hudson, AG, Warziski, MT, Styn, MA, Music, E, Elci, OU, Sereika, SM. Effects of a vegetarian diet and treatment preference on biochemical and dietary variables in overweight and obese adults: a randomized clinical trial. Am $\mathrm{J}$ Clin Nutr. 2007. 86:588-96. doi:10.1093/ajcn/86.3.588

351 Burke, LE, Styn, MA, Steenkiste, AR, Music, E, Warziski, M, Choo, J. A randomized clinical trial testing treatment preference and two dietary options in behavioral weight management: preliminary results of the impact of diet at 6 months-PREFER study. Obesity (Silver Spring). 2006. 14:2007-17. doi:10.1038/oby.2006.235

352 Burke, LE, Warziski, M, Styn, MA, Music, E, Hudson, AG, Sereika, SM. A randomized clinical trial of a standard versus vegetarian diet for weight loss: the impact of treatment preference. Int J Obes (Lond). 2008. 32:166-76. doi:10.1038/sj.ijo.0803706

353 Burke, V, Beilin, LJ, Cutt, HE, Mansour, J, Williams, A, Mori, TA. A lifestyle program for treated hypertensives improved health-related behaviors and cardiovascular risk factors, a randomized controlled trial. J Clin Epidemiol. 2007. 60:133-41. doi:10.1016/j.jclinepi.2006.05.012

354 Burkhart, SJ, Pelly, FE. Dietary intake of athletes seeking nutrition advice at a major international competition. Nutrients. 2016. 8:. doi:10.3390/nu8100638

355 Buscemi, S, Verga, S, Tranchina, MR, Cottone, S, Cerasola, G. Effects of hypocaloric very-low-carbohydrate diet vs. Mediterranean diet on endothelial function in obese women*. Eur J Clin Invest. 2009. 39:339-47. doi:10.1111/j.13652362.2009.02091.x

356 Buschard, K, Thomassen, K, Lynge, E, Vejborg, I, Tjonneland, A, von Euler-Chelpin, M, Andersen, ZJ. Diabetes, diabetes treatment, and mammographic density in Danish Diet, Cancer, and Health cohort. Cancer Causes Control. 2017. 28:13-21. doi:10.1007/s10552-016-0829-z

357 Buteau-Poulin, D, Poirier, P, Despres, JP, Almeras, N. Assessing nutritional quality as a 'vital sign' of cardiometabolic health Br J Nutr. 2019. 122:195-205. doi:10.1017/s0007114519001016

358 Buyken, AE, Flood, V, Empson, M, Rochtchina, E, Barclay, AW, Brand-Miller, J, Mitchell, P. Carbohydrate nutrition and inflammatory disease mortality in older adults. Am J Clin Nutr. 2010. 92:634-43. doi:10.3945/ajcn.2010.29390

359 Byrne, DW, Rolando, LA, Aliyu, MH, McGown, PW, Connor, LR, Awalt, BM, Holmes, MC, Wang, L, Yarbrough, MI. Modifiable Healthy Lifestyle Behaviors: 10-Year Health Outcomes From a Health Promotion Program. Am J Prev Med. 2016. 51:1027-1037. doi:10.1016/j.amepre.2016.09.012

\section{Rationale}

Intervention/Exposure

Intervention/Exposure; Comparator

Intervention/Exposure; Publication Date Overlaps with Existing Review

Intervention/Exposure

Intervention/Exposure; Publication Date Overlaps with Existing Review

Intervention/Exposure

Study Design

Study duration

Intervention/Exposure

Study Design

Intervention/Exposure Intervention/Exposure 
360 Cade, JE, Burley, VJ, Greenwood, DC. The UK Women's Cohort Study: comparison of vegetarians, fish-eaters and meateaters. Public Health Nutr. 2004. 7:871-8. doi:unavailable

361 Cai, J, Nuli, R, Zhang, Y, Zhang, Y, Abudusemaiti, M, Kadeer, A, Tian, X, Xiao, H. Association of Dietary Patterns with Type 2 Diabetes Mellitus among Middle-Aged Adults in Uygur Population of Xinjiang Region. J Nutr Sci Vitaminol (Tokyo). 2019. 65:362-374. doi:10.3177/jnsv.65.362

362 Cai, J, Zhang, Y, Nuli, R, Zhang, Y, Abudusemaiti, M, Kadeer, A, Tian, X, Xiao, H. Interaction between dietary patterns and TCF7L2 polymorphisms on type 2 diabetes mellitus among Uyghur adults in Xinjiang Province, China. Diabetes Metab Syndr Obes. 2019. 12:239-255. doi:10.2147/dmso.S191759

363 Cakir, M, Akbulut, UE, Okten, A. Association between Adherence to the Mediterranean Diet and Presence of Nonalcoholic Fatty Liver Disease in Children. Child Obes. 2016. 12:279-85. doi:10.1089/chi.2015.0197

364 Calleja Fernandez, A, Vidal Casariego, A, Cano Rodriguez, I, Ballesteros Pomar, MD. One-year effectiveness of two hypocaloric diets with different protein/carbohydrate ratios in weight loss and insulin resistance. Nutr Hosp. 2012. 27:2093-101. doi:10.3305/nh.2012.27.6.6133

365 Camargo, A, Meneses, ME, Pérez-Martínez, P, Delgado-Lista, J, Rangel-Zúñiga, OA, Marín, C, Almadén, Y, YuberoSerrano, EM, González-Guardia, L, Fuentes, F, Tinahones, FJ, Roche, HM, Malagón, MM, Pérez-Jiménez, F, LópezMiranda, J. Dietary fat modifies lipid metabolism in the adipose tissue of metabolic syndrome patients. Genes and Nutrition. 2014. 9:. doi:10.1007/s12263-014-0409-3

366 Cameron, JD, Riou, ME, Tesson, F, Goldfield, GS, Rabasa-Lhoret, R, Brochu, M, Doucet, E. The TaqIA RFLP is associated with attenuated intervention-induced body weight loss and increased carbohydrate intake in post-menopausal obese women. Appetite. 2013. 60:111-116. doi:10.1016/j.appet.2012.09.010

367 Camhi, SM, Crouter, SE, Hayman, LL, Must, A, Lichtenstein, AH. Lifestyle Behaviors in Metabolically Healthy and Unhealthy Overweight and Obese Women: A Preliminary Study. PLoS One. 2015. 10:e0138548. doi:10.1371/journal.pone.0138548

368 Camhi, SM, Stefanick, ML, Katzmarzyk, PT, Young, DR. Metabolic syndrome and changes in body fat from a low-fat diet and/or exercise randomized controlled trial. Obesity (Silver Spring). 2010. 18:548-54. doi:10.1038/oby.2009.304

369 Camhi, SM, Whitney Evans, E, Hayman, LL, Lichtenstein, AH, Must, A. Healthy eating index and metabolically healthy obesity in U.S. adolescents and adults. Prev Med. 2015. 77:23-7. doi:10.1016/j.ypmed.2015.04.023

370 Caminhotto Rde, O, Fonseca, FL, Castro, NC, Arantes, JP, Sertie, RA. Atkins diet program rapidly decreases atherogenic index of plasma in trained adapted overweight men. Arch Endocrinol Metab. 2015. 59:568-71. doi:10.1590/23593997000000106

371 Campbell, CP, Raubenheimer, D, Badaloo, AV, Gluckman, PD, Martinez, C, Gosby, A, Simpson, SJ, Osmond, C, Boyne, MS, Forrester, TE. Developmental contributions to macronutrient selection: a randomized controlled trial in adult survivors of malnutrition. Evol Med Public Health. 2016. 2016:158-69. doi:10.1093/emph/eov030

\section{Outcome}

Intervention/Exposure

Study Design;

Intervention/Exposure

Intervention/Exposure; Publication Date

Overlaps with Existing Review

Study Design

Study duration

Study duration ;

Health Status 
372 Campbell, DD, Meckling, KA. Effect of the protein:carbohydrate ratio in hypoenergetic diets on metabolic syndrome risk factors in exercising overweight and obese women. Br J Nutr. 2012. 108:1658-71. doi:10.1017/s0007114511007215

373 Campbell, EK, Fidahusain, M, Campbell li, TM. Evaluation of an Eight-Week Whole-Food Plant-Based Lifestyle Modification Program. Nutrients. 2019. 11:. doi:10.3390/nu11092068

374 Campbell, TC. A plant-based diet and animal protein: questioning dietary fat and considering animal protein as the main cause of heart disease. J Geriatr Cardiol. 2017. 14:331-337. doi:10.11909/j.issn.1671-5411.2017.05.011

375 Campbell, WW, Kim, JE, Amankwaah, AF, Gordon, SL, Weinheimer-Haus, EM. Higher Total Protein Intake and Change in Total Protein Intake Affect Body Composition but Not Metabolic Syndrome Indexes in Middle-Aged Overweight and Obese Adults Who Perform Resistance and Aerobic Exercise for 36 Weeks. J Nutr. 2015. 145:2076-83. doi:10.3945/jn.115.213595

376 Campbell, WW, O'Connor, LE, Li, J, Sayer, RD, Wright, AJ. Adopting, abandoning, and re-adopting healthy eating patterns sends cardiovascular disease risk factors on a rollercoaster ride. FASEB journal. 2017. 31:. doi:unavailable

377 Campbell, WW, Tang, M. Protein intake, weight loss, and bone mineral density in postmenopausal women. J Gerontol A Biol Sci Med Sci. 2010. 65:1115-22. doi:10.1093/gerona/glq083

378 Campos-Nonato, I, Hernandez, L, Barquera, S. Effect of a High-Protein Diet versus Standard-Protein Diet on Weight Loss and Biomarkers of Metabolic Syndrome: A Randomized Clinical Trial. Obes Facts. 2017. 10:238-251. doi:10.1159/000471485

379 Can, AS, Uysal, C, Palaoglu, KE. Short term effects of a low-carbohydrate diet in overweight and obese subjects with low HDL-C levels. BMC Endocr Disord. 2010. 10:18. doi:10.1186/1472-6823-10-18

380 Canfi, A, Gepner, Y, Schwarzfuchs, D, Golan, R, Shahar, DR, Fraser, D, Witkow, S, Greenberg, I, Sarusi, B, Vardi, H, Friger, M, Stampfer, MJ, Shai, I. Effect of changes in the intake of weight of specific food groups on successful body weight loss during a multi-dietary strategy intervention trial. J Am Coll Nutr. 2011. 30:491-501. doi:10.1080/07315724.2011.10719995

381 Cano-lbanez, N, Bueno-Cavanillas, A, Martinez-Gonzalez, MA, Salas-Salvado, J, Corella, D, Freixer, GL, Romaguera, D, Vioque, J, Alonso-Gomez, AM, Warnberg, J, Martinez, JA, Serra-Majem, L, Estruch, R, Tinahones, FJ, Lapetra, J, Pinto, X, Tur, JA, Garcia-Rios, A, Garcia-Molina, L, Delgado-Rodriguez, M, Matia-Martin, P, Daimiel, L, Martin-Sanchez, V, Vidal, J, Vazquez, C, Ros, E, Bartolome-Resano, J, Palau-Galindo, A, Portoles, O, Torres, L, Miquel, Fiol, Sanchez, MTC, SortoSanchez, C, Moreno-Morales, N, Abete, I, Alvarez-Perez, J, Sacanella, E, Bernal-Lopez, MR, Santos-Lozano, JM, FanloMaresma, M, Bouzas, C, Razquin, C, Becerra-Tomas, N, Ortega-Azorin, C, Limona R L, Morey, M, Roman-Macia, J, Goicolea-Guemez, L, Vazquez-Ruiz, Z, Barrubes, L, Fito, M, Gea, A. Effect of changes in adherence to Mediterranean diet on nutrient density after 1-year of follow-up: results from the PREDIMED-Plus Study. Eur J Nutr. 2019. :. doi:10.1007/s00394019-02087-1

382 Carbajo, MA, Castro, MJ, Kleinfinger, S, Gomez-Arenas, S, Ortiz-Solorzano, J, Wellman, R, Garcia-lanza, C, Luque, E. Effects of a balanced energy and high protein formula diet (Vegestart complet(R)) vs. low-calorie regular diet in morbid obese patients prior to bariatric surgery (laparoscopic single anastomosis gastric bypass): a prospective, double-blind randomized Study duration
Health Status

Study Design; Intervention/Exposure Study Design

Intervention/Exposure

Publication Status

Intervention/Exposure

Intervention/Exposure Study Design

Intervention/Exposure; Publication Date

Overlaps with Existing Review

Outcome study. Nutr Hosp. 2010. 25:939-48. doi:unavailable 


\section{No.}

383 Cardel, M, Lemas, DJ, Jackson, KH, Friedman, JE, Fernandez, JR. Higher Intake of PUFAs Is Associated with Lower Total and Visceral Adiposity and Higher Lean Mass in a Racially Diverse Sample of Children. J Nutr. 2015. 145:2146-52. doi:10.3945/jn.115.212365

384 Cardillo, S, Seshadri, P, Iqbal, N. The effects of a low-carbohydrate versus low-fat diet on adipocytokines in severely obese adults: three-year follow-up of a randomized trial. Eur Rev Med Pharmacol Sci. 2006. 10:99-106. doi:unavailable

385 Cardoso, DA, Moreira, AS, de Oliveira, GM, Raggio Luiz, R, Rosa, G. A COCONUT EXTRA VIRGIN OIL-RICH DIET INCREASES HDL CHOLESTEROL AND DECREASES WAIST CIRCUMFERENCE AND BODY MASS IN CORONARY ARTERY DISEASE PATIENTS. Nutr Hosp. 2015. 32:2144-52. doi:10.3305/nh.2015.32.5.9642

386 Carpentier, A, Zinman, B, Leung, N, Giacca, A, Hanley, AJ, Harris, SB, Hegele, RA, Lewis, GF. Free fatty acid-mediated impairment of glucose-stimulated insulin secretion in nondiabetic Oji-Cree individuals from the Sandy Lake community of Ontario, Canada: a population at very high risk for developing type 2 diabetes. Diabetes. 2003. 52:1485-95. doi:10.2337/diabetes.52.6.1485

387 Carstens, MT, Goedecke, JH, Dugas, L, Evans, J, Kroff, J, Levitt, NS, Lambert, EV. Fasting substrate oxidation in relation to habitua dietary fat intake and insulin resistance in non-diabetic women: A case for metabolic flexibility?. Nutrition and Metabolism. 2013. 10:. doi:10.1186/1743-7075-10-8

388 Carty, CL, Kooperberg, C, Neuhouser, ML, Tinker, L, Howard, B, Wactawski-Wende, J, Beresford, SA, Snetselaar, L, Vitolins, M, Allison, M, Budrys, N, Prentice, R, Peters, U. Low-fat dietary pattern and change in body-composition traits in the Women's Health Initiative Dietary Modification Trial. Am J Clin Nutr. 2011. 93:516-24. doi:10.3945/ajcn.110.006395

389 Casas, R, Sacanella, E, Urpí-Sardà, M, Corella, D, Castañer, O, Lamuela-Raventos, RM, Salas-Salvadó, J, MartínezGonzález, MA, Ros, E, Estruch, R. Long-Term Immunomodulatory Effects of a Mediterranean Diet in Adults at High Risk of Cardiovascular Disease in the PREvención con Dleta MEDiterránea (PREDIMED) Randomized Controlled Trial. Journal of nutrition. 2016. 146:1684-1693. doi:10.3945/jn.115.229476

390 Casazza, K, Cardel, M, Dulin-Keita, A, Hanks, LJ, Gower, BA, Newton, AL, Wallace, S. Reduced carbohydrate diet to improve metabolic outcomes and decrease adiposity in obese peripubertal African American girls. J Pediatr Gastroenterol Nutr. 2012. 54:336-42. doi:10.1097/MPG.0b013e31823df207

391 Casazza, K, Dulin-Keita, A, Gower, BA, Fernandez, JR. Relationships between reported macronutrient intake and insulin dynamics in a multi-ethnic cohort of early pubertal children. Int J Pediatr Obes. 2009. 4:249-56. doi:10.3109/17477160902763366

392 Casazza, K. A reduced carbohydrate diet results in loss in lean mass in peripubertal African American girls. FASEB journal. 2010. 24:. doi:unavailable

393 Cases, J, Romain, C, Dallas, C, Gerbi, A, Cloarec, M. Regular consumption of Fiit-ns, a polyphenol extract from fruit and vegetables frequently consumed within the Mediterranean diet, improves metabolic ageing of obese volunteers: a randomized double-blind, parallel trial. Int J Food Sci Nutr. 2015. 66:120-5. doi:10.3109/09637486.2014.971229

\section{Rationale}

Study Design

Study duration ;

Health Status

Health Status

Study duration

Study Design

Publication Date

Overlaps with Existing Review

Study Design;

Outcome

Study duration

Study Design

Publication Status

Intervention/Exposure 
394 Cassady, BA, Charboneau, NL, Brys, EE, Crouse, KA, Beitz, DC, Wilson, T. Effects of low carbohydrate diets high in red meats or poultry, fish and shellfish on plasma lipids and weight loss. Nutr Metab (Lond). 2007. 4:23. doi:10.1186/1743-7075-423

395 Cassani, RS, Fassini, PG, Silvah, JH, Lima, CM, Marchini, JS. Impact of weight loss diet associated with flaxseed on inflammatory markers in men with cardiovascular risk factors: a clinical study. Nutrition journal. 2015. 14:5. doi:10.1186/14752891-14-5

Intervention/Exposure

396 Castaldo, G, Monaco, L, Castaldo, L, Galdo, G, Cereda, E. An observational study of sequential protein-sparing, very lowcalorie ketogenic diet (Oloproteic diet) and hypocaloric Mediterranean-like diet for the treatment of obesity. Int J Food Sci Nutr. 2016. 67:696-706. doi:10.1080/09637486.2016.1186157

397 Castro, Mbtd, Araujo, MC, Barbosa Cunha, D, Bezerra, IN, Adegboye, ARA, Kac, G, Sichieri, R. Effect of high protein intake and nutritional advice on body weight maintainance among overweight and obese postpartum women. Annals of nutrition \& metabolism. 2017. 71:845-846. doi:10.1159/000480486

398 Cataife, G. Small area estimation of obesity prevalence and dietary patterns: a model applied to Rio de Janeiro city, Brazil. Health Place. 2014. 26:47-52. doi:10.1016/j.healthplace.2013.12.004

399 Catenacci, VA, Odgen, L, Phelan, S, Thomas, JG, Hill, J, Wing, RR, Wyatt, H. Dietary habits and weight maintenance success in high versus low exercisers in the National Weight Control Registry. J Phys Act Health. 2014. 11:1540-8. doi:10.1123/jpah.2012-0250

400 Cayanan, EA, Hoyos, CM, Djavadkhani, Y, Wong, KKW, Yee, BY, Phillips, C, Marshall, NS, Grunstein, RR. Effectiveness of two maintenance diets following a very vow energy diet to reduce cardio-metabolic risk in obese sleep apnea patients: a randomised controlled trial. Obesity reviews. 2016. 17:38-. doi:10.1111/obr.12400

401 Cayanan, EA, Marshall, NS, Hoyos, CM, Djavadkhani, Y, Yee, B, Wong, KK, Grunstein, RR. Effectiveness of two maintenance diets following a very low energy diet to reduce cardiometabolic risk in obese sleep apnea patients: a randomised controlled trial. Journal of sleep research. 2014. 23:68-. doi:10.1111/jsr.12213

402 Celis-Morales, C, Livingstone, KM, Affleck, A, Navas-Carretero, S, San-Cristobal, R, Martinez, JA, Marsaux, CFM, Saris, WHM, O'Donovan, CB, Forster, H, Woolhead, C, Gibney, ER, Walsh, MC, Brennan, L, Gibney, M, Moschonis, G, Lambrinou, CP, Mavrogianni, C, Manios, Y, Macready, AL, Fallaize, R, Lovegrove, JA, Kolossa, S, Daniel, H, Traczyk, I, Drevon, CA, Mathers, JC. Correlates of overall and central obesity in adults from seven European countries: findings from the Food4Me Study. Eur J Clin Nutr. 2018. 72:207-219. doi:10.1038/s41430-017-0004-y

403 Cespedes, EM, Hu, FB, Redline, S, Rosner, B, Gillman, MW, Rifas-Shiman, SL, Taveras, EM. Chronic insufficient sleep and diet quality: Contributors to childhood obesity. Obesity (Silver Spring). 2016. 24:184-90. doi:10.1002/oby.21196

Chai, L, Collins, C, May, C, Holder, C, Brown, LJ, Burrows, T. An online telehealth nutrition intervention to support parents in child weight management - A randomised feasibility controlled trial. Obesity facts. 2019. 12:111-112. doi:10.1159/000489691

405 Champagne, CM, Bray, G, Sacks, F, Miketinas, D. Fiber intake, dietary energy density, and diet-type predict 6-month weightloss in free-living adults who adhered to prescribed macronutrient and energy composition of varying diets. FASEB journal. 2017. 31:. doi:unavailable

Study Design;

Publication Status

Publication Status

Study Design

Intervention/Exposure

Publication Status

Publication Status

Study Design

Study Design;

Outcome

Publication Status

Publication Status 
406 Champagne, CM, Broyles, ST, Moran, LD, Cash, KC, Levy, EJ, Lin, PH, Batch, BC, Lien, LF, Funk, KL, Dalcin, A, Loria trial. J Am Diet Assoc. 2011. 111:1826-35. doi:10.1016/j.jada.2011.09.014

407 Chan, R, Leung, J, Woo, J, Kwok, T. Associations of dietary protein intake on subsequent decline in muscle mass and physical functions over four years in ambulant older Chinese people. J Nutr Health Aging. 2014. 18:171-7. doi:10.1007/s12603013-0379-y

408 Chang, JJ, Bena, J, Kannan, S, Kim, J, Burguera, B, Kashyap, SR. LIMITED CARBOHYDRATE REFEEDING INSTRUCTION FOR LONG-TERM WEIGHT MAINTENANCE FOLLOWING A KETOGENIC, VERY-LOW-CALORIE MEAL

Intervention/Exposure; Country PLAN. Endocr Pract. 2017. 23:649-656. doi:10.4158/ep161383.Or

409 Chang, L, Vethakkan, S, Nesaretnam, K, Teng, K. Effects of exchanging dietary saturated fatty acids or carbohydrate for monounsaturated fatty acids on inflammatory responses in abdominally obese Malaysians: a randomized controlled trial. Obesity reviews. 2014. 15:94-. doi:10.1111/obr.12149

410 Chang, LF, Vethakkan, SR, Nesaretnam, K, Sanders, TA, Teng, KT. Adverse effects on insulin secretion of replacing saturated fat with refined carbohydrate but not with monounsaturated fat: A randomized controlled trial in centrally obese subjects. J Clin Lipidol. 2016. 10:1431-1441.e1. doi:10.1016/j.jacl.2016.09.006

411 Chaparro, MP, Crespi, CM, Anderson, CE, Wang, MC, Whaley, SE. The 2009 Special Supplemental Nutrition Program for Women, Infants, and Children (WIC) food package change and children's growth trajectories and obesity in Los Angeles County. Am J Clin Nutr. 2019. 109:1414-1421. doi:10.1093/ajcn/nqy347

412 Chaput, JP, Tremblay, A, Rimm, EB, Bouchard, C, Ludwig, DS. A novel interaction between dietary composition and insulin secretion: effects on weight gain in the Quebec Family Study. Am J Clin Nutr. 2008. 87:303-9. doi:10.1093/ajcn/87.2.303

413 Charkiewicz, AE, Jamiolkowski, J, Pedzinski, B, Krzyzak, M, Maslach, D, Szpak, A, Omeljaniuk, WJ. Changes in Dietary Patterns and the Nutritional Status in Men in the Metallurgical Industry in Poland Over A 21-Year Period. Ann Nutr Metab. 2018. 72:161-171. doi:10.1159/000485389

414 Chauveau, P, Vendrely, B, El Haggan, W, Barthe, N, Rigalleau, V, Combe, C, Aparicio, M. Body composition of patients on Health Status a very low-protein diet: a two-year survey with DEXA. J Ren Nutr. 2003. 13:282-7. doi:unavailable

415 Chavez Palencia, C, Larrosa Haro, A, Romero Velarde, E, Lopez-Uriarte, PJ. Efficacy of a modified carbohydrate diet in obese women. Annals of nutrition \& metabolism. 2017. 71:1034-1035. doi:10.1159/000480486

416 Chee, C, Mansell, P, Stephens, F, Cordon, S, Kavani, M, Bawden, S, Hoad, C, Gowland, P, Macdonald, I. Differential effects of carbohydrate vs fat overfeeding on liver fat content and lipid metabolism in healthy overweight males. Diabetologia. 2015. 58:S336. doi:10.1007/s00125-015-3687-4

417 Chen, Q, Turban, S, Miller, ER, Appel, LJ. The effects of dietary patterns on plasma renin activity: results from the Dietary Approaches to Stop Hypertension trial. J Hum Hypertens. 2012. 26:664-9. doi:10.1038/jhh.2011.87

Intervention/Exposure

Publication Status

Intervention/Exposure

Intervention/Exposure

Intervention/Exposure

Study Design;

Comparator

Publication Status

Publication Status

Publication Date Overlaps with Existing Review 
418 Chen, ST, Maruthur, NM, Appel, LJ. The effect of dietary patterns on estimated coronary heart disease risk: results from the Dietary Approaches to Stop Hypertension (DASH) trial. Circ Cardiovasc Qual Outcomes. 2010. 3:484-9. doi:10.1161/circoutcomes.109.930685

419 Chen, X, Pang, Z, Li, K. Dietary fat, sedentary behaviors and the prevalence of the metabolic syndrome among Qingdao adults. Nutr Metab Cardiovasc Dis. 2009. 19:27-34. doi:10.1016/j.numecd.2008.01.010

420 Chen, Y, Xiang, J, Wang, Z, Xiao, Y, Zhang, D, Chen, X, Li, H, Liu, M, Zhang, Q. Associations of Bone Mineral Density with Lean Mass, Fat Mass, and Dietary Patterns in Postmenopausal Chinese Women: A 2-Year Prospective Study. PLoS One. 2015. 10:e0137097. doi:10.1371/journal.pone.0137097

421 Cheraghi, Z, Mirmiran, P, Mansournia, MA, Moslehi, N, Khalili, D, Nedjat, S. The association between nutritional exposures and metabolic syndrome in the Tehran Lipid and Glucose Study (TLGS): a cohort study. Public Health. 2016. 140:163-171. doi:10.1016/j.puhe.2016.07.003

422 Cheraghi, Z, Nedjat, S, Mirmiran, P, Moslehi, N, Mansournia, N, Etminan, M, Mansournia, MA, McCandless, LC. Effects of food items and related nutrients on metabolic syndrome using Bayesian multilevel modelling using the Tehran Lipid and Glucose Study (TLGS): A cohort study. BMJ Open. 2018. 8:. doi:10.1136/bmjopen-2017-020642

423 Chiu, S, Bergeron, N, Williams, PT, Bray, GA, Sutherland, B, Krauss, RM. Comparison of the DASH (Dietary Approaches to Stop Hypertension) diet and a higher-fat DASH diet on blood pressure and lipids and lipoproteins: a randomized controlled trial. Am J Clin Nutr. 2016. 103:341-7. doi:10.3945/ajcn.115.123281

424 Chiu, S, Williams, PT, Dawson, T, Bergman, RN, Stefanovski, D, Watkins, SM, Krauss, RM. Diets high in protein or saturated fat do not affect insulin sensitivity or plasma concentrations of lipids and lipoproteins in overweight and obese adults. $\mathrm{J}$ Nutr. 2014. 144:1753-9. doi:10.3945/jn.114.197624

425 Chiu, TH, Huang, HY, Chiu, YF, Pan, WH, Kao, HY, Chiu, JP, Lin, MN, Lin, CL. Taiwanese vegetarians and omnivores: dietary composition, prevalence of diabetes and IFG. PLoS One. 2014. 9:e88547. doi:10.1371/journal.pone.0088547

426 Chiu, THT, Pan, WH, Lin, MN, Lin, CL. Vegetarian diet, change in dietary patterns, and diabetes risk: a prospective study. Nutr Diabetes. 2018. 8:12. doi:10.1038/s41387-018-0022-4

427 Chiu, YF, Hsu, CC, Chiu, TH, Lee, CY, Liu, TT, Tsao, CK, Chuang, SC, Hsiung, CA. Cross-sectional and longitudinal comparisons of metabolic profiles between vegetarian and non-vegetarian subjects: a matched cohort study. Br J Nutr. 2015. 114:1313-20. doi:10.1017/s0007114515002937

428 Chiuve, SE, Sandhu, RK, Moorthy, MV, Glynn, RJ, Albert, CM. Dietary Fat Intake Is Differentially Associated with Risk of Paroxysmal Compared with Sustained Atrial Fibrillation in Women. J Nutr. 2015. 145:2092-101. doi:10.3945/jn.115.212860

429 Choi, H, Song, S, Kim, J, Chung, J, Yoon, J, Paik, HY, Song, Y. High carbohydrate intake was inversely associated with high-density lipoprotein cholesterol among Korean adults. Nutr Res. 2012. 32:100-6. doi:10.1016/j.nutres.2011.12.013

430 Choi, SE, Brandeau, ML, Basu, S. Expansion of the National Salt Reduction Initiative: A Mathematical Model of Benefits and Risks of Population-Level Sodium Reduction. Med Decis Making. 2016. 36:72-85. doi:10.1177/0272989x15583846
Intervention/Exposure; Publication Date

Overlaps with Existing Review

Study Design; Country

Country

Intervention/Exposure; Outcome

Intervention/Exposure

Study duration

Study duration

Study Design; Country

Intervention/Exposure

Country

Intervention/Exposure;

Outcome

Study Design

Study Design;

Intervention/Exposure 
431 Chomentowski, P, Dube, JJ, Amati, F, Stefanovic-Racic, M, Zhu, S, Toledo, FG, Goodpaster, BH. Moderate exercise attenuates the loss of skeletal muscle mass that occurs with intentional caloric restriction-induced weight loss in older, overweight to obese adults. J Gerontol A Biol Sci Med Sci. 2009. 64:575-80. doi:10.1093/gerona/glp007

432 Chong, MF, Fielding, BA, Frayn, KN. Mechanisms for the acute effect of fructose on postprandial lipemia. Am J Clin Nutr 2007. 85:1511-20. doi:10.1093/ajcn/85.6.1511

433 Christie, C. Proposed Dietary Guidelines 2015 and Implications for Cardiovascular Disease and Diabetes. J Cardiovasc Nurs 2015. 30:375-8. doi:10.1097/jcn.0000000000000286

434 Chuang, SY, Chiu, TH, Lee, CY, Liu, TT, Tsao, CK, Hsiung, CA, Chiu, YF. Vegetarian diet reduces the risk of hypertension independent of abdominal obesity and inflammation: a prospective study. J Hypertens. 2016. 34:2164-71. doi:10.1097/hjh.0000000000001068

435 Chun, BO, Yun, ME, Kim, IM, Lee, JK. Effects of short-term training camp including aerobic exercise and vegetarian diet on body composition, physical fitness and blood biochemical parameters in collegians. Gazzetta Medica Italiana Archivio per le Scienze Mediche. 2017. 176:289-298. doi:10.23736/S0393-3660.16.03277-0

436 Chung, GKK, Yu, RHY, Ho, SSY, Woo, J, Ho, SC. Associations of consuming specific fruit and vegetable subgroups with LDL-C status in early postmenopausal Chinese women. Menopause. 2018. 25:436-443. doi:10.1097/gme.0000000000001008

437 Chung, HV, Iversen, CS, Lai, M, Saka, S, Mahabub-ul Anwar, M, Nigg, CR. Omega-3 fatty acids from fish, other nutrient intake, and lifestyle factors: exploring the relationship in children. Asia Pac J Public Health. 2014. 26:517-26. doi:10.1177/1010539513485970

438 Churm, R, Prior, SL, Stephens, JW, Caplin, S, Barry, J, Luzio, SD, Bracken, RM. The hepatic and metabolic impact of a three week hypocaloric diet in overweight patients: a pilot study. Diabetic medicine. 2017. 34:105-106. doi:10.1111/dme.13302

439 Claessens, M, van Baak, MA, Monsheimer, S, Saris, WH. The effect of a low-fat, high-protein or high-carbohydrate ad libitum diet on weight loss maintenance and metabolic risk factors. Int J Obes (Lond). 2009. 33:296-304. doi:10.1038/ijo.2008.278

440 Clamp, LD, Hume, DJ, Lambert, EV, Kroff, J. Successful and unsuccessful weight-loss maintainers: strategies to counteract metabolic compensation following weight loss. J Nutr Sci. 2018. 7:e20. doi:10.1017/jns.2018.11

441 Clark, RL, Famodu, OA, Holaskova, I, Infante, AM, Murray, PJ, Olfert, IM, McFadden, JW, Downes, MT, Chantler, PD, Duespohl, MW, Cuff, CF, Olfert, MD. Educational intervention improves fruit and vegetable intake in young adults with metabolic syndrome components. Nutr Res. 2019. 62:89-100. doi:10.1016/j.nutres.2018.11.010

442 Clarys, P, Deriemaeker, P, Huybrechts, I, Hebbelinck, M, Mullie, P. Dietary pattern analysis: a comparison between matched vegetarian and omnivorous subjects. Nutr J. 2013. 12:82. doi:10.1186/1475-2891-12-82

443 Clemente-Postigo, M, Queipo-OrtunoBoto-Ordonez, MI, Coin-Araguez, L, Roca-Rodriguez, MM, Delgado-Lista, J, Cardona F, Andres-Lacueva, C, Tinahones, FJ. The effect of acute and chronic red wine consumption on lipopolysaccharide concentrations. Journal of diabetes. 2013. 5:73. doi:10.1111/1753-0407.12032

444 Clifton, PM, Keogh, JB, Foster, PR, Noakes, M. Effect of weight loss on inflammatory and endothelial markers and FMD using two low-fat diets. Int J Obes (Lond). 2005. 29:1445-51. doi:10.1038/sj.ijo.0803039
Intervention/Exposure; Comparator

Study duration

Study Design

Intervention/Exposure; Outcome; Country

Study duration

Intervention/Exposure; Outcome; Country

Study Design; Intervention/Exposure

Publication Status

Intervention/Exposure

Intervention/Exposure; Study duration

Study Design

Study Design

Publication Status

Intervention/Exposure 


\begin{tabular}{|c|c|c|}
\hline No. & Citation & Rationale \\
\hline 445 & $\begin{array}{l}\text { Clifton, PM, Keogh, JB, Noakes, M. Long-term effects of a high-protein weight-loss diet. Am J Clin Nutr. 2008. 87:23-9. } \\
\text { doi:10.1093/ajcn/87.1.23 }\end{array}$ & $\begin{array}{l}\text { Weight } \\
\text { loss/Hypocaloric }\end{array}$ \\
\hline 446 & $\begin{array}{l}\text { Clifton, PM, Noakes, M, Keogh, J, Foster, P. Effect of an energy reduced high protein red meat diet on weight loss and } \\
\text { metabolic parameters in obese women. Asia pacific journal of clinical nutrition. 2003. } 12 \text { Suppl:S10. doi:unavailable }\end{array}$ & $\begin{array}{l}\text { Intervention/Exposure; } \\
\text { Publication Status }\end{array}$ \\
\hline 447 & $\begin{array}{l}\text { Clifton, PM, Noakes, M, Keogh, JB. Very low-fat (12\%) and high monounsaturated fat (35\%) diets do not differentially affect } \\
\text { abdominal fat loss in overweight, nondiabetic women. J Nutr. 2004. 134:1741-5. doi:10.1093/jn/134.7.1741 }\end{array}$ & Intervention/Exposure \\
\hline 448 & $\begin{array}{l}\text { Clifton, PM, Turner, K, Keogh, JB. Effect of lean red meat and dairy on insulin sensitivity. Diabetes. 2015. 64:A23. } \\
\text { doi:10.2337/db151385 }\end{array}$ & Publication Status \\
\hline 449 & $\begin{array}{l}\text { Clinton, CM, O'Brien, S, Law, J, Renier, CM, Wendt, MR. Whole-foods, plant-based diet alleviates the symptoms of } \\
\text { osteoarthritis. Arthritis. 2015. 2015:708152. doi:10.1155/2015/708152 }\end{array}$ & Outcome \\
\hline 450 & $\begin{array}{l}\text { Cohen, J, Rimm, EB, Martínez-González, MA, Salas-Salvadó, J, Covas, MI, Corella, D, Lamuela-Raventós, RM, Estruch, } \\
\text { R. The association between obesity status and long-term adherence to mediterranean diet in the predimed trial. Circulation. } \\
\text { 2013. 127:. doi:unavailable }\end{array}$ & Publication Status \\
\hline 451 & $\begin{array}{l}\text { Cohen, JF, Kraak, VI, Choumenkovitch, SF, Hyatt, RR, Economos, CD. The CHANGE study: a healthy-lifestyles intervention } \\
\text { to improve rural children's diet quality. J Acad Nutr Diet. 2014. 114:48-53. doi:10.1016/j.jand.2013.08.014 }\end{array}$ & $\begin{array}{l}\text { Intervention/Exposure; } \\
\text { Outcome }\end{array}$ \\
\hline 452 & $\begin{array}{l}\text { Coleman, MD, Nickols-Richardson, SM. Urinary ketones reflect serum ketone concentration but do not relate to weight loss in } \\
\text { overweight premenopausal women following a low-carbohydrate/high-protein diet. J Am Diet Assoc. 2005. 105:608-11. } \\
\text { doi:10.1016/j.jada.2005.01.004 }\end{array}$ & $\begin{array}{l}\text { Study Design; } \\
\text { Outcome }\end{array}$ \\
\hline 453 & $\begin{array}{l}\text { Coles, LT, Fletcher, EA, Galbraith, CE, Clifton, PM. Patient freedom to choose a weight loss diet in the treatment of } \\
\text { overweight and obesity: a randomized dietary intervention in type } 2 \text { diabetes and pre-diabetes. Int J Behav Nutr Phys Act. } 2014 . \\
\text { 11:64. doi:10.1186/1479-5868-11-64 }\end{array}$ & $\begin{array}{l}\text { Intervention/Exposure; } \\
\text { Health Status }\end{array}$ \\
\hline 454 & $\begin{array}{l}\text { Coletta, AM, Sanchez, B, O'Connor, A, Dalton, R, Springer, S, Koozehchian, MS, Murano, PS, Woodman, CR, } \\
\text { Rasmussen, C, Kreider, RB. Alignment of diet prescription to genotype does not promote greater weight loss success in } \\
\text { women with obesity participating in an exercise and weight loss program. Obes Sci Pract. 2018. 4:554-574. } \\
\text { doi:10.1002/osp4.305 }\end{array}$ & $\begin{array}{l}\text { Study Design; } \\
\text { Intervention/Exposure }\end{array}$ \\
\hline 455 & $\begin{array}{l}\text { Colette, C, Percheron, C, Pares-Herbute, N, Michel, F, Pham, TC, Brillant, L, Descomps, B, Monnier, L. Exchanging } \\
\text { carbohydrates for monounsaturated fats in energy-restricted diets: effects on metabolic profile and other cardiovascular risk } \\
\text { factors. Int J Obes Relat Metab Disord. 2003. 27:648-56. doi:10.1038/sj.ijo.0802299 }\end{array}$ & Study duration \\
\hline 456 & $\begin{array}{l}\text { Colica, C, Merra, G, Gasbarrini, A, De Lorenzo, A, Cioccoloni, G, Gualtieri, P, Perrone, MA, Bernardini, S, Bernardo, V, Di } \\
\text { Renzo, L, Marchetti, M. Efficacy and safety of very-low-calorie ketogenic diet: a double blind randomized crossover study. Eur } \\
\text { Rev Med Pharmacol Sci. 2017. 21:2274-2289. doi:unavailable }\end{array}$ & Study duration \\
\hline 457 & $\begin{array}{l}\text { Colombo, C, Muti, P, Pala, V, Cavalleri, A, Venturelli, E, Locardi, M, Berrino, F, Secreto, G. Plant-based diet, serum fatty } \\
\text { acid profile, and free radicals in postmenopausal women: the diet and androgens (DIANA) randomized trial. Int J Biol Markers. } \\
\text { 2005. 20:169-76. doi:unavailable }\end{array}$ & Intervention/Exposure \\
\hline
\end{tabular}


458 Conlin, PR, Chow, D, Miller, ER, 3rd, Svetkey, LP, Lin, PH, Harsha, DW, Moore, TJ, Sacks, FM, Appel, LJ. The effect of dietary patterns on blood pressure control in hypertensive patients: results from the Dietary Approaches to Stop Hypertension (DASH) trial. Am J Hypertens. 2000. 13:949-55. doi:10.1016/s0895-7061(99)00284-8

459 Cook, CM, McCormick, CN, Knowles, M, Kaden, VN. A Commercially Available Portion-Controlled Diet Program Is More Effective for Weight Loss than a Self-Directed Diet: Results from a Randomized Clinical Trial. Front Nutr. 2017. 4:55. doi:10.3389/fnut.2017.00055

460 Cooper, JA, Watras, AC, Adams, AK, Schoeller, DA. Effects of dietary fatty acid composition on 24-h energy expenditure and chronic disease risk factors in men. Am J Clin Nutr. 2009. 89:1350-6. doi:10.3945/ajcn.2008.27419

461 Cooper, JA, Watras, AC, Shriver, T, Adams, AK, Schoeller, DA. Influence of dietary fatty acid composition and exercise on changes in fat oxidation from a high-fat diet. J Appl Physiol (1985). 2010. 109:1011-8. doi:10.1152/japplphysiol.01025.2009

462 Corella, D, Coltell, O, Ortega-Azorin, C, Portoles, O, Barragan, R, Saez-Tormo, G, Burguete, C, Castello, A, Ordovas, JM, Sorli, JV. FNDC5 polymorphisms and cardiovascular risk factors and disease. Modulation by Mediterranean diet and physical activity. Annals of nutrition and metabolism. 2015. 67:286-287. doi:10.1159/000440895

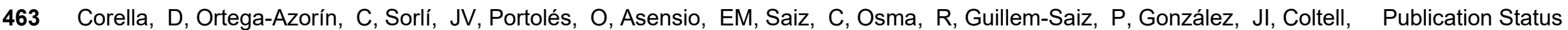
O. Mediterranean diet, adiponectin levels, genetic polymorphisms and incidence of cardiovascular diseases in the predimedvalencia study. Cardiology (switzerland). 2014. 128:64-. doi:10.1159/000365062

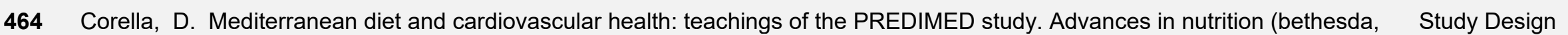
md.). 2014. 5:330S-336S. doi:10.3945/an.113.005389

465 Cormier, H, Thifault, E, Garneau, V, Tremblay, A, Drapeau, V, Perusse, L, Vohl, MC. Association between yogurt consumption, dietary patterns, and cardio-metabolic risk factors. Eur J Nutr. 2016. 55:577-587. doi:10.1007/s00394-015-0878-1

466 Cornier, MA, Bergman, BC, Bessesen, DH. The effects of short-term overfeeding on insulin action in lean and reduced-obese individuals. Metabolism: Clinical and Experimental. 2006. 55:1207-1214. doi:10.1016/j.metabol.2006.05.003

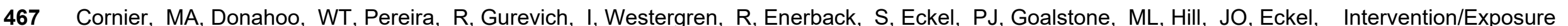
$\mathrm{RH}$, Draznin, B. Insulin sensitivity determines the effectiveness of dietary macronutrient composition on weight loss in obese women. Obes Res. 2005. 13:703-9. doi:10.1038/oby.2005.79

468 Corsino, L, Rocha-Goldberg, MP, Batch, BC, Ortiz-Melo, DI, Bosworth, HB, Svetkey, LP. The Latino Health Project: Pilo testing a culturally adapted behavioral weight loss intervention in obese and overweight Latino adults. Ethnicity and Disease. 2012. 22:51-57. doi:unavailable

469 Cosgrove, K, Johnston, CS. Examining the Impact of Adherence to a Vegan Diet on Acid-Base Balance in Healthy Adults. Plant Foods Hum Nutr. 2017. 72:308-313. doi:10.1007/s11130-017-0620-7

470 Costa, MB, Ferreira, SR, Franco, LJ, Gimeno, SG, lunes, M. Dietary patterns in a high-risk population for glucose intolerance. Japanese-Brazilian Diabetes Study Group. J Epidemiol. 2000. 10:111-7. doi:10.2188/jea.10.111

Intervention/Exposure; Comparator

Study duration

Study Design;

Intervention/Exposure; Outcome

Study duration

Study Design

471 Costa, S, Pinto, A, Santos, AC, Oliveira, A. The association of problematic eating behaviours with food quality and body mass index at 7 years of age. Eur J Clin Nutr. 2019. 73:549-557. doi:10.1038/s41430-018-0169-z

Intervention/Exposure 
472 Couch, SC, Saelens, BE, Hinn, K, Dart, KB, Khoury, P, Mitsnefes, M, Daniels, SR, Urbina, EM. Effects of a clinic-initiated behavioral nutrition intervention emphasizing the dash diet on blood pressure control in adolescents with elevated blood pressure. Journal of the american society of hypertension. 2014. 8:e116. doi:10.1016/j.jash.2014.03.262

473 Couch, SC, Saelens, BE, Levin, L, Dart, K, Falciglia, G, Daniels, SR. The efficacy of a clinic-based behavioral nutrition intervention emphasizing a DASH-type diet for adolescents with elevated blood pressure. J Pediatr. 2008. 152:494-501. doi:10.1016/j.jpeds.2007.09.022

474 Couch, SC, Saelens, BE. Factors Associated with Pediatric Hypertension in Mexico. Journal of the American Dietetic Association. 2009. 109:992-995. doi:10.1016/j.jada.2009.03.015

475 Courie, R, Gaillard, M, Lainas, P, Hansel, B, Naveau, S, Dagher, I, Tranchart, H. Weight outcome after 2 years of a diet that excludes six processed foods: exploratory study of the "1,2,3 diet" in a moderately obese population. Diabetes Metab Syndr Obes. 2018. 11:345-355. doi:10.2147/dmso.S165598

476 Coyle, EF, Jeukendrup, AE, Oseto, MC, Hodgkinson, BJ, Zderic, TW. Low-fat diet alters intramuscular substrates and reduces lipolysis and fat oxidation during exercise. Am J Physiol Endocrinol Metab. 2001. 280:E391-8. doi:10.1152/ajpendo.2001.280.3.E391

477 Craig, LC, McNeill, G, Macdiarmid, JI, Masson, LF, Holmes, BA. Dietary patterns of school-age children in Scotland: association with socio-economic indicators, physical activity and obesity. Br J Nutr. 2010. 103:319-34. doi:10.1017/s0007114509991942

478 Crane, MM, Jeffery, RW, Sherwood, NE. Exploring Gender Differences in a Randomized Trial of Weight Loss Maintenance. Am J Mens Health. 2017. 11:369-375. doi:10.1177/1557988316681221

479 Crichton, GE, Alkerwi, A. Dairy food intake is positively associated with cardiovascular health: findings from Observation of Cardiovascular Risk Factors in Luxembourg study. Nutr Res. 2014. 34:1036-44. doi:10.1016/j.nutres.2014.04.002

480 Crujeiras, A, Bellido, D, Sajoux, I, Moreno, B, Casanueva, F. Comparison of a very low-calorie-ketogenic diet with a standard low-calorie diet in the treatment of obesity: values at 24 months of treatment. Obesity facts. Conference: european obesity summit (EOS): 1st joint congress of EASO and IFSO-EC. Gothenburg sweden. Conference start: 20160601. Conference end: 20160604. Conference publication: (var.pagings). 2016. 9:253. doi:10.1159/000446744

481 Cuenca-Garcia, M, Ortega, FB, Ruiz, JR, Gonzalez-Gross, M, Labayen, I, Jago, R, Martinez-Gomez, D, Dallongeville, J, Bel-Serrat, S, Marcos, A, Manios, Y, Breidenassel, C, Widhalm, K, Gottrand, F, Ferrari, M, Kafatos, A, Molnar, D, Moreno, LA, De Henauw, S, Castillo, MJ, Sjostrom, M. Combined influence of healthy diet and active lifestyle on cardiovascular disease risk factors in adolescents. Scand J Med Sci Sports. 2014. 24:553-62. doi:10.1111/sms.12022

482 Cueto-Galan, R, Baron, FJ, Valdivielso, P, Pinto, X, Corbella, E, Gomez-Gracia, E, Warnberg, J. Changes in fatty liver index after consuming a Mediterranean diet: 6-year follow-up of the PREDIMED-Malaga trial. Med Clin (Barc). 2017. 148:435443. doi:10.1016/j.medcli.2016.11.032

Intervention/Exposure; Publication Date

Overlaps with Existing Review

Study Design; Publication Status

Study Design; Intervention/Exposure

Study duration

Study Design

Intervention/Exposure

Intervention/Exposure

Publication Status

Study Design

Outcome 


\begin{tabular}{|c|c|c|}
\hline No. & Citation & Rationale \\
\hline 483 & $\begin{array}{l}\text { Culling, KS, Neil, HA, Gilbert, M, Frayn, KN. Effects of short-term low- and high-carbohydrate diets on postprandial } \\
\text { metabolism in non-diabetic and diabetic subjects. Nutr Metab Cardiovasc Dis. 2009. 19:345-51. } \\
\text { doi:10.1016/j.numecd.2007.09.003 }\end{array}$ & Study duration \\
\hline 484 & $\begin{array}{l}\text { Cunha, DB, da Costa, THM, da Veiga, GV, Pereira, RA, Sichieri, R. Ultra-processed food consumption and adiposity } \\
\text { trajectories in a Brazilian cohort of adolescents: ELANA study. Nutr Diabetes. 2018. 8:28. doi:10.1038/s41387-018-0043-z }\end{array}$ & Intervention/Exposure \\
\hline 485 & $\begin{array}{l}\text { Czekajlo, A, Rozanska, D, Zatonska, K, Szuba, A, Regulska-llow, B. Association between dietary patterns and } \\
\text { cardiovascular risk factors in a selected population of Lower Silesia (PURE Study Poland). Ann Agric Environ Med. } 2018 . \\
\text { 25:635-641. doi:10.26444/aaem/76321 }\end{array}$ & Study Design \\
\hline 486 & $\begin{array}{l}\text { da Costa, TH, Reis, CE, da Silva, FV, Casulari, LA. Improvement in metabolic parameters in obese subjects after } 16 \text { weeks } \\
\text { on a Brazilian-staple calorie-restricted diet. Nutr Res Pract. 2014. 8:410-6. doi:10.4162/nrp.2014.8.4.410 }\end{array}$ & $\begin{array}{l}\text { Weight } \\
\text { loss/Hypocaloric }\end{array}$ \\
\hline 487 & $\begin{array}{l}\text { da Silva, SM, Luiz, RR, Pereira, RA. Risk and protection factors for cardiovascular diseases among adults of Cuiaba, Mato } \\
\text { Grosso, Brazil. Rev Bras Epidemiol. 2015. 18:425-38. doi:10.1590/1980-5497201500020011 }\end{array}$ & Study Design \\
\hline 488 & $\begin{array}{l}\text { Dabbagh-Moghaddam, A, Kamali, M, Hojjati, A, Foroughi, M, Ghiasvand, R, Askari, G, Hosseinzadeh, J. The Relationship } \\
\text { between Dietary Patterns with Blood Pressure in Iranian Army Staffs. Adv Biomed Res. 2018. 7:127. } \\
\text { doi:10.4103/abr.abr_35_18 }\end{array}$ & Study Design \\
\hline 489 & $\begin{array}{l}\text { Dahlman, I, Linder, K, Arvidsson Nordstrom, E, Andersson, I, Liden, J, Verdich, C. Changes in adipose tissue gene } \\
\text { expression with energy-restricted diets in obese women Am J Clin Nutr. } 2005 \text { Sep;82(3): } 709 \text {. American journal of clinical } \\
\text { nutrition. 2005. 81:1275-1285. doi:unavailable }\end{array}$ & Study duration \\
\hline 490 & $\begin{array}{l}\text { Dai, J, Krasnow, RE, Reed, T. Midlife moderation-quantified healthy diet and 40-year mortality risk from CHD: the prospective } \\
\text { National Heart, Lung, and Blood Institute Twin Study. Br J Nutr. 2016. 116:326-34. doi:10.1017/s0007114516001914 }\end{array}$ & Power/Size \\
\hline 491 & $\begin{array}{l}\text { Dallas Hall, W, Feng, Z, George, VA, Lewis, CE, Oberman, A, Huber, M, Fouad, M, Cutler, JA. Low-fat diet: Effect on } \\
\text { athropometrics, blood pressure, glucose, and insulin in older women. Ethnicity and Disease. 2003. 13:337-343. doi:unavailable }\end{array}$ & Intervention/Exposure \\
\hline 492 & $\begin{array}{l}\text { Dalle Grave, R, Calugi, S, Gavasso, I, El Ghoch, M, Marchesini, G. A randomized trial of energy-restricted high-protein } \\
\text { versus high-carbohydrate, low-fat diet in morbid obesity. Obesity (Silver Spring). 2013. 21:1774-81. doi:10.1002/oby.20320 }\end{array}$ & Intervention/Exposure \\
\hline 493 & $\begin{array}{l}\text { Dalzill, C, Nigam, A, Juneau, M, Guilbeault, V, Latour, E, Mauriege, P, Gayda, M. Intensive lifestyle intervention improves } \\
\text { cardiometabolic and exercise parameters in metabolically healthy obese and metabolically unhealthy obese individuals. Can J } \\
\text { Cardiol. 2014. 30:434-40. doi:10.1016/j.cjca.2013.11.033 }\end{array}$ & $\begin{array}{l}\text { Study Design; } \\
\text { Intervention/Exposure }\end{array}$ \\
\hline 494 & $\begin{array}{l}\text { Damasceno, NR, Sala-Vila, A, Cofan, M, Perez-Heras, AM, Fito, M, Ruiz-Gutierrez, V, Martinez-Gonzalez, MA, Corella, D, } \\
\text { Aros, F, Estruch, R, Ros, E. Mediterranean diet supplemented with nuts reduces waist circumference and shifts lipoprotein } \\
\text { subfractions to a less atherogenic pattern in subjects at high cardiovascular risk. Atherosclerosis. 2013. 230:347-53. } \\
\text { doi:10.1016/j.atherosclerosis.2013.08.014 }\end{array}$ & $\begin{array}{l}\text { Outcome; Publication } \\
\text { Date Overlaps with } \\
\text { Existing Review }\end{array}$ \\
\hline
\end{tabular}


495 Damsgaard, CT, Dalskov, SM, Laursen, RP, Ritz, C, Hjorth, MF, Lauritzen, L, Sorensen, LB, Petersen, RA, Andersen, MR, Stender, S, Andersen, R, Tetens, I, Molgaard, C, Astrup, A, Michaelsen, KF. Provision of healthy school meals does not affect the metabolic syndrome score in 8-11-year-old children, but reduces cardiometabolic risk markers despite increasing waist circumference. Br J Nutr. 2014. 112:1826-36. doi:10.1017/s0007114514003043

496 Damsgaard, CT, Papadaki, A, Jensen, SM, Ritz, C, Dalskov, SM, Hlavaty, P, Saris, WH, Martinez, JA, HandjievaDarlenska, T, Andersen, MR, Stender, S, Larsen, TM, Astrup, A, Molgaard, C, Michaelsen, KF. Higher protein diets consumed ad libitum improve cardiovascular risk markers in children of overweight parents from eight European countries. $J$ Nutr. 2013. 143:810-7. doi:10.3945/jn.112.173427

497 D'Anci, KE, Watts, KL, Kanarek, RB, Taylor, HA. Low-carbohydrate weight-loss diets. Effects on cognition and mood. Appetite. 2009. 52:96-103. doi:10.1016/j.appet.2008.08.009

498 Daniels, SR. DASH to stop metabolic syndrome. Journal of Pediatrics. 2016. 174:1-3. doi:10.1016/j.jpeds.2016.05.040

499 Daniuseviciute-Brazaite, L, Abromaitiene, L. Evaluation of students' dietary behaviours depending on gender. Progress in Nutrition. 2018. 20:21-29. doi:10.23751/pn.v20i1.6247

500 Dansinger, ML, Gleason, JA, Griffith, JL, Selker, HP, Schaefer, EJ. Comparison of the Atkins, Ornish, Weight Watchers, and Zone diets for weight loss and heart disease risk reduction: a randomized trial. Jama. 2005. 293:43-53. doi:10.1001/jama.293.1.43

501 Darakhshan, F, Pelloux, V, Rouault, C, Laromiguiere, M, Debrus, G, Massiera, F, Basdevant, A, Clément, K, Rizkalla, SW. Effect of a high-protein-low-glycaemic-index hypocaloric diet on adiposity markers, cardiovascular and metabolic risk factors: a randomised controlled trial. Diabetes. 2010. :. doi:unavailable

502 Das, SK, Roberts, SB, Bhapkar, MV, Villareal, DT, Fontana, L, Martin, CK, Racette, SB, Fuss, PJ, Kraus, WE, Wong, WW, Saltzman, E, Pieper, CF, Fielding, RA, Schwartz, AV, Ravussin, E, Redman, LM. Body-composition changes in the Comprehensive Assessment of Long-term Effects of Reducing Intake of Energy (CALERIE)-2 study: a 2-y randomized controlled trial of calorie restriction in nonobese humans. Am J Clin Nutr. 2017. 105:913-927. doi:10.3945/ajcn.116.137232

503 Dashti, HM, Al-Zaid, NS, Mathew, TC, Al-Mousawi, M, Talib, H, Asfar, SK, Behbahani, Al. Long term effects of ketogenic diet in obese subjects with high cholesterol level. Mol Cell Biochem. 2006. 286:1-9. doi:10.1007/s11010-005-9001-X

504 Dashti, HM, Bo-Abbas, YY, Asfar, SK, Mathew, TC, Hussein, T, Behbahani, A, Khoursheed, MA, Al-Sayer, HM, Al-Zaid, NS. Ketogenic diet modifies the risk factors of heart disease in obese patients. Nutrition. 2003. 19:901-2. doi:10.1016/s08999007(03)00161-8

505 Dashti, HM, Mathew, TC, Hussein, T, Asfar, SK, Behbahani, A, Khoursheed, MA, Al-Sayer, HM, Bo-Abbas, YY, Al-Zaid, NS. Long-term effects of a ketogenic diet in obese patients. Exp Clin Cardiol. 2004. 9:200-5. doi:unavailable

506 Datta Banik, S, Andrade Olalde, AC, Rodriguez, L, Dickinson, F. The effect of socioeconomic indicators and macronutrient intake rate on body composition in adolescents 12 to 16 years old in Merida, Yucatan. Anthropol Anz. 2014. 71:347-68. doi:10.1127/0003-5548/2014/0402

Outcome; Study duration Publication Status Study Design; Intervention/Exposure

Weight

loss/Hypocaloric

Publication Status

Intervention/Exposure Study Design; Intervention/Exposure Intervention/Exposure

Study Design; Intervention/Exposure Study Design 
507 Dauchet, L, Kesse-Guyot, E, Czernichow, S, Bertrais, S, Estaquio, C, Peneau, S, Vergnaud, AC, Chat-Yung, S, Castetbon, K, Deschamps, V, Brindel, P, Hercberg, S. Dietary patterns and blood pressure change over 5-y follow-up in the SU.VI.MAX cohort. Am J Clin Nutr. 2007. 85:1650-6. doi:10.1093/ajcn/85.6.1650

Publication Date

Overlaps with Existing Review

508 D'Auria, E, Fabiano, V, Bertoli, S, Bedogni, G, Bosetti, A, Pendezza, E, Sartorio, MUA, Leone, A, Spadafranca, A, Borsani, Study Design B, Stucchi, F, Battezzati, A, Zuccotti, GV. Growth Pattern, Resting Energy Expenditure, and Nutrient Intake of Children with Food Allergies. Nutrients. 2019. 11:. doi:10.3390/nu11020212

509 Davis, JN, Kelly, LA, Lane, CJ, Ventura, EE, Byrd-Williams, CE, Alexandar, KA, Azen, SP, Chou, CP, Spruijt-Metz, D, Weigensberg, MJ, Berhane, K, Goran, MI. Randomized control trial to improve adiposity and insulin resistance in overweight Latino adolescents. Obesity (Silver Spring). 2009. 17:1542-8. doi:10.1038/oby.2009.19

510 Davis, JN, Ventura, EE, Alexander, KE, Salguero, LE, Weigensberg, MJ, Crespo, NC, Spruijt-Metz, D, Goran, MI. Feasibility of a home-based versus classroom-based nutrition intervention to reduce obesity and type 2 diabetes in Latino youth Int J Pediatr Obes. 2007. 2:22-30. doi:10.1080/17477160601133077

511 de Castro, MB, Kac, G, de Leon, AP, Sichieri, R. High-protein diet promotes a moderate postpartum weight loss in a prospective cohort of Brazilian women. Nutrition. 2009. 25:1120-8. doi:10.1016/j.nut.2009.02.006

Intervention/Exposure de Castro, MB, Sichieri, R, Barbosa Brito Fdos, S, Nascimento, S, Kac, G. Mixed dietary pattern is associated with a slower decline of body weight change during postpartum in a cohort of Brazilian women. Nutr Hosp. 2014. 29:519-25. doi:10.3305/nh.2014.29.3.7155

513 de Castro, MBT, Cunha, DB, Araujo, MC, Bezerra, IN, Adegboye, ARA, Kac, G, Sichieri, R. High protein diet promotes body weight loss among Brazilian postpartum women. Matern Child Nutr. 2019. 15:e12746. doi:10.1111/mcn.12746

514 de Deus, RM, Mingoti, SA, Jaime, PC, Lopes, AC. The impact of a nutritional intervention on the nutritional status and anthropometric profile of participants in the health Gym Programme in Brazil. Cien Saude Colet. 2015. 20:1937-46. doi:10.1590/1413-81232015206.11882014

515 de Ferranti, SD, Milliren, CE, Denhoff, ER, Quinn, N, Osganian, SK, Feldman, HA, Ebbeling, CB, Ludwig, DS. Providing food to treat adolescents at risk for cardiovascular disease. Obesity (Silver Spring). 2015. 23:2109-17. doi:10.1002/oby.21246

516 De Goede, J, Verschuren, WM, Boer, JM, Kromhout, D, Geleijnse, JM. N-6 and n-3 fatty acid cholesteryl esters in relation to incident stroke in a Dutch adult population: a nested case-control study. Nutr Metab Cardiovasc Dis. 2013. 23:737-43. doi:10.1016/j.numecd.2012.03.001

517 de Haas, SCM, de Jonge, EAL, Voortman, T, Graaff, JSD, Franco, OH, Ikram, MA, Rivadeneira, F, Kiefte-de Jong, JC, Schoufour, JD. Dietary patterns and changes in frailty status: the Rotterdam study. European Journal of Nutrition. 2018. 57:2365-2375. doi:10.1007/s00394-017-1509-9

518 De La Iglesia, R, Lopez-Legarrea, P, Abete, I, Navas-Carrtero, S, Martinez, JA, Zulet, MA. The beneficial effects of the resmena dietary pattern on OxLDL in patients with metabolic syndrome. Annals of nutrition and metabolism. 2013. 63:171. doi:10.1159/000354245

Intervention/Exposure; Country

Power/Size

Intervention/Exposure

Intervention/Exposure

Power/Size

Intervention/Exposure

Outcome

Publication Status 
519 de la Iglesia, R, Lopez-Legarrea, P, Celada, P, Sanchez-Muniz, FJ, Martinez, JA, Zulet, MA. Beneficial effects of the

RESMENA dietary pattern on oxidative stress in patients suffering from metabolic syndrome with hyperglycemia are associated to dietary TAC and fruit consumption. Int J Mol Sci. 2013. 14:6903-19. doi:10.3390/ijms14046903

520 De Lorenzo, A, Petroni, ML, De Luca, PP, Andreoli, A, Morini, P, lacopino, L, Innocente, I, Perriello, G. Use of quality control indices in moderately hypocaloric Mediterranean diet for treatment of obesity. Diabetes Nutr Metab. 2001. 14:181-8. doi:unavailable

521 de Luis, D, Izaola, O, Primo, D, Aller, R. Role of rs670 variant of APOA1 gene on metabolic response after a high fat vs. a low fat hypocaloric diets in obese human subjects. J Diabetes Complications. 2019. 33:249-254. doi:10.1016/j.jdiacomp.2018.10.015

522 de Luis, DA, Aller, R, Izaola, O, Bachiller, R, Pacheco, D. Cardiovascular risk factors and adipocytokines levels after two hypocaloric diets with different fat distribution in obese subjects and rs6923761 gene variant of glucagon-like peptide 1 receptor. J Endocrinol Invest. 2014. 37:853-9. doi:10.1007/s40618-014-0116-3

523 De Luis, DA, Aller, R, Izaola, O, De La Fuente, B, Conde, R, Eiros Bouza, JM. Relation of -55CT polymorphism of UCP3 gene with weight loss and metabolic changes after a high monounsaturated fat diet in obese non diabetic patients. Eur Rev Med Pharmacol Sci. 2013. 17:2810-5. doi:unavailable

524 de Luis, DA, Aller, R, Izaola, O, de la Fuente, B, Conde, R, Sagrado, MG, Primo, D. Evaluation of weight loss and adipocytokines levels after two hypocaloric diets with different macronutrient distribution in obese subjects with rs9939609 gene variant. Diabetes Metab Res Rev. 2012. 28:663-8. doi:10.1002/dmrr.2323

525 De Luis, DA, Aller, R, Izaola, O, De La Fuente, B, Primo, D, Conde, R, Sagrado, MG. Evaluation of weight loss and adipocytokine levels after two hypocaloric diets with different macronutrient distribution in obese subjects with the rs6923761 gene variant of glucagon-like peptide 1 receptor. Annals of Nutrition and Metabolism. 2014. 63:277-282. doi:10.1159/000356710

526 de Luis, DA, Aller, R, Izaola, O, Diaz Soto, G, Lopez Gomez, JJ, Gomez Hoyos, E, Torres, B, Villar, A, Romero, E. Effects of a High-Protein/Low-Carbohydrate versus a Standard Hypocaloric Diet on Weight and Cardiovascular Risk Factors during 9 Months: Role of a Genetic Variation in the Cannabinoid Receptor Gene (CNR1) (G1359A Polymorphism). Ann Nutr Metab. 2015. 66:125-31. doi:10.1159/000375412

527 de Luis, DA, Aller, R, Izaola, O, Gonzalez Sagrado, M, Bellioo, D, Conde, R. Effects of a low-fat versus a low-carbohydrate diet on adipocytokines in obese adults. Horm Res. 2007. 67:296-300. doi:10.1159/000099329

528 de Luis, DA, Aller, R, Izaola, O, Gonzalez Sagrado, M, Conde, R. Effect of two different hypocaloric diets in transaminases and insulin resistance in nonalcoholic fatty liver disease and obese patients. Nutr Hosp. 2010. 25:730-5. doi:unavailable

529 De Luis, DA, Aller, R, Izaola, O, González Sagrado, M, Conde, R. The effects of a low-fat versus a low carbohydrate diet in obese adults. Medicina clinica. 2009. 132:203-207. doi:10.1016/j.medcli.2008.03.003

530 De Luis, DA, Aller, R, Izaola, O, Pacheco, D. ROLE OF RS9939609 FTO GENE VARIANT IN WEIGHT LOSS, INSULIN RESISTANCE AND METABOLIC PARAMETERS AFTER A HIGH MONOUNSATURATED VS A HIGH POLYUNSATURATED FAT HYPOCALORIC DIETS. Nutr Hosp. 2015. 32:175-81. doi:10.3305/nh.2015.32.1.9169

Intervention/Exposure; Comparator

Intervention/Exposure; Comparator

Intervention/Exposure

Intervention/Exposure

Intervention/Exposure

Comparator

Outcome; Health

Status

Power/Size

Language

Intervention/Exposure; Comparator 
531 de Luis, DA, Aller, R, Izaola, O, Primo, D, Urdiales, S, Romero, E. Effects of a High-Protein/Low-Carbohydrate Diet versus a Standard Hypocaloric Diet on Weight and Cardiovascular Risk Factors: Role of a Genetic Variation in the rs9939609 FTO Gene Variant. J Nutrigenet Nutrigenomics. 2015. 8:128-36. doi:10.1159/000441142

532 de Luis, DA, Aller, R, Izaola, O, Romero, E. Effects of a high-protein/low-carbohydrate versus a standard hypocaloric diet on adipocytokine levels and cardiovascular risk factors during 9 months, role of rs6923761 gene variant of glucagon-like peptide 1 receptor. J Endocrinol Invest. 2015. 38:1183-9. doi:10.1007/s40618-015-0304-9

533 de Luis, DA, Izaola, O, Aller, R, de la Fuente, B, Bachiller, R, Romero, E. Effects of a high-protein/low carbohydrate versus a standard hypocaloric diet on adipocytokine levels and insulin resistance in obese patients along 9 months. J Diabetes Complications. 2015. 29:950-4. doi:10.1016/j.jdiacomp.2015.06.002

534 de Luis, DA, Izaola, O, de la Fuente, B, Primo, D, Romero, E. Role of Fatty Acid-Binding Protein 2 Ala54Thr Genotype on Weight Loss and Cardiovascular Risk Factors after a High-Protein/Low-Carbohydrate versus a Standard Hypocaloric Diet during 9 Months. Ann Nutr Metab. 2015. 67:81-6. doi:10.1159/000438947

535 de Luis, DA, Izaola, O, Primo, D, Aller, R. Different effects of high-protein/low-carbohydrate versus standard hypocaloric diet on insulin resistance and lipid profile: Role of rs16147 variant of neuropeptide Y. Diabetes Res Clin Pract. 2019. 156:107825. doi:10.1016/j.diabres.2019.107825

536 de Luis, DA, Izaola, O, Primo, D, Aller, R. Polymorphism rs16147 of the Neuropeptide Y Gene Modifies the Response of Cardiovascular Risk Biomarkers and Adipokines to Two Hypocaloric Diets. J Nutrigenet Nutrigenomics. 2017. 10:63-72. doi:10.1159/000478528

537 de Luis, DA, Izaola, O, Primo, D, de la Fuente, B, Mulero, I, Aller, R. The rs1862513 Variant in Resistin Gene-Modified Insulin Resistance and Insulin Levels after Weight Loss Secondary to Hypocaloric Diet. Ann Nutr Metab. 2016. 69:256-262. doi:10.1159/000453676

538 de Luis, DA, Izaola, O, Primo, D, Ovalle, HF, Lopez, JJ, Gomez, E, Ortola, A, Aller, R. Biochemical, Anthropometric and Lifestyle Factors Related with Weight Maintenance after Weight Loss Secondary to a Hypocaloric Mediterranean Diet. Ann Nutr Metab. 2017. 71:217-223. doi:10.1159/000484446

539 de Luis, DA, Perez Castrillon, JL, Aller, R, Izaola, O, Bachiller, C. Response of osteocalcin and insulin resistance after a hypocaloric diet in obese patients. Eur Rev Med Pharmacol Sci. 2015. 19:2174-9. doi:unavailable

540 de Luis, DA, Romero, E, Izaola, O, Primo, D, Aller, R. Cardiovascular Risk Factors and Insulin Resistance after Two Hypocaloric Diets with Different Fat Distribution in Obese Subjects: Effect of the rs10767664 Gene Variant in Brain-Derived Neurotrophic Factor. J Nutrigenet Nutrigenomics. 2017. 10:163-171. doi:10.1159/000485248

541 de Luis, DA, Sagrado, MG, Conde, R, Aller, R, Izaola, O. The effects of two different hypocaloric diets on glucagon-like peptide 1 in obese adults, relation with insulin response after weight loss. J Diabetes Complications. 2009. 23:239-43. doi:10.1016/j.jdiacomp.2007.12.006

\section{Weight}

loss/Hypocaloric

Weight

loss/Hypocaloric

Weight

loss/Hypocaloric

Weight

loss/Hypocaloric

Intervention/Exposure; Comparator

Intervention/Exposure; Comparator

Study Design;

Intervention/Exposure

Study Design;

Intervention/Exposure

Study Design

Intervention/Exposure; Comparator

Weight loss/Hypocaloric 
542 de Mello, VD, Schwab, U, Kolehmainen, M, Koenig, W, Siloaho, M, Poutanen, K, Mykkanen, H, Uusitupa, M. A diet high in fatty fish, bilberries and wholegrain products improves markers of endothelial function and inflammation in individuals with impaired glucose metabolism in a randomised controlled trial: the Sysdimet study. Diabetologia. 2011. 54:2755-67. doi:10.1007/s00125-011-2285-3

543 De Miguel-Etayo, P, Moreno, LA, Santabarbara, J, Martin-Matillas, M, Azcona-San Julian, MC, Marti Del Moral, A, Campoy, C, Marcos, A, Garagorri, JM. Diet quality index as a predictor of treatment efficacy in overweight and obese adolescents: The EVASYON study. Clin Nutr. 2019. 38:782-790. doi:10.1016/j.clnu.2018.02.032

544 de Paula Franco, E, Moraes de Oliveira, GM, Raggio Luiz, R, Rosa, G. EFFECT OF HYPOENERGETIC DIET COMBINED WITH CONSUMPTION OF COCONUT FLOUR IN OVERWEIGHT WOMEN. Nutr Hosp. 2015. 32:2012-8. doi:10.3305/nh.2015.32.5.9661

545 de Roos, NM, Bots, ML, Siebelink, E, Schouten, E, Katan, MB. Flow-mediated vasodilation is not impaired when HDLcholesterol is lowered by substituting carbohydrates for monounsaturated fat. $\mathrm{Br} \mathrm{J}$ Nutr. 2001. 86:181-8. doi:10.1079/bjn2001365

546 de Rougemont, A, Normand, S, Nazare, JA, Skilton, MR, Sothier, M, Vinoy, S, Laville, M. Beneficial effects of a 5-week low-glycaemic index regimen on weight control and cardiovascular risk factors in overweight non-diabetic subjects. Br $\mathrm{J}$ Nutr. 2007. 98:1288-98. doi:10.1017/s0007114507778674

547 de Souza, RGM, Gomes, AC, de Castro, IA, Mota, JF. A baru almond-enriched diet reduces abdominal adiposity and improves high-density lipoprotein concentrations: a randomized, placebo-controlled trial. Nutrition. 2018. 55-56:154-160. doi:10.1016/j.nut.2018.06.001

548 de Souza, RJ, Bray, GA, Carey, VJ, Hall, KD, LeBoff, MS, Loria, CM, Laranjo, NM, Sacks, FM, Smith, SR. Effects of 4 weight-loss diets differing in fat, protein, and carbohydrate on fat mass, lean mass, visceral adipose tissue, and hepatic fat: results from the POUNDS LOST trial. Am J Clin Nutr. 2012. 95:614-25. doi:10.3945/ajcn.111.026328

549 de Souza, RJ, Swain, JF, Appel, LJ, Sacks, FM. Alternatives for macronutrient intake and chronic disease: a comparison of the OmniHeart diets with popular diets and with dietary recommendations. Am J Clin Nutr. 2008. 88:1-11. doi:10.1093/ajen/88.1.1

550 Dearborn, JL, Qiao, Y, Guallar, E, Steffen, LM, Gottesman, RF, Zhang, Y, Wasserman, BA. Polyunsaturated fats carbohydrates and carotid disease: The Atherosclerosis Risk in Communities (ARIC) Carotid MRI study. Atherosclerosis. 2016. 251:361-366. doi:10.1016/j.atherosclerosis.2016.05.024

551 DeClercq, V, Cui, Y, Forbes, C, Grandy, SA, Keats, M, Parker, L, Sweeney, E, Yu, ZM, Dummer, TJB. Association between Diet Quality and Adiposity in the Atlantic PATH Cohort. Nutrients. 2017. 9:. doi:10.3390/nu9101155

Study Design

Comparator

Intervention/Exposure

\section{Study duration}

Intervention/Exposure; Comparator

Intervention/Exposure

Weight

loss/Hypocaloric

Intervention/Exposure; Outcome

Outcome

Study Design

552 Decombaz, J, Fleith, M, Hoppeler, H, Kreis, R, Boesch, C. Effect of diet on the replenishment of intramyocellular lipids after exercise. Eur J Nutr. 2000. 39:244-7. doi:10.1007/s003940070002

553 Decombaz, J, Schmitt, B, Ith, M, Decarli, B, Diem, P, Kreis, R, Hoppeler, H, Boesch, C. Postexercise fat intake repletes intramyocellular lipids but no faster in trained than in sedentary subjects. Am J Physiol Regul Integr Comp Physiol. 2001. 281:R760-9. doi:10.1152/ajpregu.2001.281.3.R760
Study duration

Intervention/Exposure; Study duration 
554 Defoort, C, Vincent-Baudry, S, Lairon, D. Effects of 3-month Mediterranean-type diet on postprandial TAG and apolipoprotein B48 in the Medi-RIVAGE cohort. Public Health Nutr. 2011. 14:2302-8. doi:10.1017/s1368980011002552

555 Dehghan, M, Mente, A, Zhang, X, Swaminathan, S, Li, W, Mohan, V, Iqbal, R, Kumar, R, Wentzel-Viljoen, E, Rosengren, A, Amma, LI, Avezum, A, Chifamba, J, Diaz, R, Khatib, R, Lear, S, Lopez-Jaramillo, P, Liu, X, Gupta, R, Mohammadifard, N, Gao, N, Oguz, A, Ramli, AS, Seron, P, Sun, Y, Szuba, A, Tsolekile, L, Wielgosz, A, Yusuf, R, Hussein Yusufali, A, Teo, KK, Rangarajan, S, Dagenais, G, Bangdiwala, SI, Islam, S, Anand, SS, Yusuf, S. Associations of fats and carbohydrate intake with cardiovascular disease and mortality in 18 countries from five continents (PURE): a prospective cohort study. Lancet. 2017. 390:2050-2062. doi:10.1016/s0140-6736(17)32252-3

556 Dekker, LH, van Dam, RM, Snijder, MB, Peters, RJ, Dekker, JM, de Vries, JH, de Boer, EJ, Schulze, MB, Stronks, K, Nicolaou, M. Comparable Dietary Patterns Describe Dietary Behavior across Ethnic Groups in the Netherlands, but Different Elements in the Diet Are Associated with Glycated Hemoglobin and Fasting Glucose Concentrations. J Nutr. 2015. 145:188491. doi:10.3945/jn.114.207472

557 Delgado Floody, PA, Caamano Navarrete, F, Jerez Mayorga, D, Cofre-Lizama, A, Guzman-Guzman, IP. The association between children's food habits, anthropometric parameters and health-related quality of life in Chilean school-age children. Nutr Hosp. 2019. 36:1061-1066. doi:10.20960/nh.02643

558 Della Pepa, G, Vetrani, C, Vitale, M, Bozzetto, L, Costabile, G, Cipriano, P, Mangione, A, Patti, L, Riccardi, G, Rivellese, AA, Annuzzi, G. Effects of a diet naturally rich in polyphenols on lipid composition of postprandial lipoproteins in high cardiometabolic risk individuals: an ancillary analysis of a randomized controlled trial. Eur J Clin Nutr. 2019. : doi:10.1038/s41430-019-0459-0

559 Dello Russo, M, Ahrens, W, De Henauw, S, Eiben, G, Hebestreit, A, Kourides, Y, Lissner, L, Molnar, D, Moreno, LA, Pala, V, Veidebaum, T, Siani, A, Russo, P. The Impact of Adding Sugars to Milk and Fruit on Adiposity and Diet Quality in Children: A Cross-Sectional and Longitudinal Analysis of the Identification and Prevention of Dietary- and Lifestyle-Induced Health Effects in Children and Infants (IDEFICS) Study. Nutrients. 2018. 10:. doi:10.3390/nu10101350

560 Demol, S, Yackobovitch-Gavan, M, Shalitin, S, Nagelberg, N, Gillon-Keren, M, Phillip, M. Low-carbohydrate (low \& high-fat) versus high-carbohydrate low-fat diets in the treatment of obesity in adolescents. Acta Paediatr. 2009. 98:346-51. doi:10.1111/j.1651-2227.2008.01051.x

561 Denke, MA. Dietary prescriptions to control dyslipidemias. Circulation. 2002. 105:132-5. doi:10.1161/hc0202.103479 Study Design

562 Denke, MA. Metabolic effects of high-protein, low-carbohydrate diets. American Journal of Cardiology. 2001. 88:59-61. Publication Status doi:10.1016/S0002-9149(01)01586-7

563 Denova-Gutierrez, E, Munoz-Aguirre, P, Shivappa, N, Hebert, JR, Tolentino-Mayo, L, Batis, C, Barquera, S. Dietary Inflammatory Index and Type 2 Diabetes Mellitus in Adults: The Diabetes Mellitus Survey of Mexico City. Nutrients. 2018. 10: doi:10.3390/nu10040385

564 Denova-Gutierrez, E, Tucker, KL, Flores, M, Barquera, S, Salmeron, J. Dietary Patterns Are Associated with Predicted Cardiovascular Disease Risk in an Urban Mexican Adult Population. J Nutr. 2016. 146:90-7. doi:10.3945/jn.115.217539

Study Design;

Intervention/Exposure

Outcome

Intervention/Exposure;

Comparator

Study Design;

Outcome

Intervention/Exposure

Outcome

Intervention/Exposure 
565 Deriemaeker, P, Aerenhouts, D, De Ridder, D, Hebbelinck, M, Clarys, P. Health aspects, nutrition and physical characteristics in matched samples of institutionalized vegetarian and non-vegetarian elderly (> 65yrs). Nutr Metab (Lond). 2011. 8:37. doi:10.1186/1743-7075-8-37

566 Derks, IPM, Bolhuis, K, Sijbrands, EJG, Gaillard, R, Hillegers, MHJ, Jansen, PW. Predictors and patterns of eating behaviors across childhood: Results from The Generation R study. Appetite. 2019. 141:104295. doi:10.1016/j.appet.2019.05.026

567 Detopoulou, P, Fragopoulou, E, Alepoudea, E, Nomikos, T, Kalogeropoulos, N, Antonopoulou, S. Associations between erythrocyte fatty acids and Mediterranean diet in Greek volunteers. Hellenic Journal of Atherosclerosis. 2018. 9:17-31. doi:unavailable

568 Devlin, BL, Leveritt, MD, Kingsley, M, Belski, R. Dietary Intake, Body Composition, and Nutrition Knowledge of Australian Football and Soccer Players: Implications for Sports Nutrition Professionals in Practice. Int J Sport Nutr Exerc Metab. 2017. 27:130-138. doi:10.1123/ijsnem.2016-0191

569 Di Daniele, N, Petramala, L, Di Renzo, L, Sarlo, F, Della Rocca, DG, Rizzo, M, Fondacaro, V, lacopino, L, Pepine, CJ, De Lorenzo, A. Body composition changes and cardiometabolic benefits of a balanced Italian Mediterranean Diet in obese patients with metabolic syndrome. Acta Diabetologica. 2013. 50:409-416. doi:10.1007/s00592-012-0445-7

570 Di Renzo, L, Cioccoloni, G, Falco, S, Abenavoli, L, Moia, A, Sinibaldi Salimei, P, De Lorenzo, A. Influence of FTO rs9939609 and Mediterranean diet on body composition and weight loss: a randomized clinical trial. J Transl Med. 2018. 16:308. doi:10.1186/s12967-018-1680-7

571 Di Renzo, L, Marsella, LT, Sarlo, F, Soldati, L, Gratteri, S, Abenavoli, L, De Lorenzo, A. C677T gene polymorphism of MTHFR and metabolic syndrome: response to dietary intervention. J Transl Med. 2014. 12:329. doi:10.1186/s12967-014-03294

572 Diaf, M, Khaled, MB, Sellam, F. Correlation between dietary fat intake and atherogenic indices in normal, overweight and obese adults with or without type 2 diabetes. Romanian Journal of Diabetes, Nutrition and Metabolic Diseases. 2015. 22:347360. doi:10.1515/rjdnmd-2015-0041

573 Diaz, KM, Booth, JN, 3rd, Calhoun, DA, Irvin, MR, Howard, G, Safford, MM, Muntner, P, Shimbo, D. Healthy lifestyle factors and risk of cardiovascular events and mortality in treatment-resistant hypertension: the Reasons for Geographic and Racial Differences in Stroke study. Hypertension. 2014. 64:465-71. doi:10.1161/hypertensionaha.114.03565

574 Diaz-Gutierrez, J, Ruiz-Canela, M, Gea, A, Fernandez-Montero, A, Martinez-Gonzalez, MA. Association Between a Healthy Lifestyle Score and the Risk of Cardiovascular Disease in the SUN Cohort. Rev Esp Cardiol (Engl Ed). 2018. 71:1001-1009. doi:10.1016/j.rec.2017.10.038

575 Diaz-Gutierrez, J, Ruiz-Estigarribia, L, Bes-Rastrollo, M, Ruiz-Canela, M, Martin-Moreno, JM, Martinez-Gonzalez, MA. The role of lifestyle behaviour on the risk of hypertension in the SUN cohort: The hypertension preventive score. Prev Med. 2019. 123:171-178. doi:10.1016/j.ypmed.2019.03.026

576 DiBello, JR, Kraft, P, McGarvey, ST, Goldberg, R, Campos, H, Baylin, A. Comparison of 3 methods for identifying dietary patterns associated with risk of disease. Am J Epidemiol. 2008. 168:1433-43. doi:10.1093/aje/kwn274

Study Design;

Intervention/Exposure

Study duration

Intervention/Exposure

Study Design; Intervention/Exposure

Health Status

Intervention/Exposure Intervention/Exposure; Outcome

Study Design 
577 Diethelm, K, Gunther, AL, Schulze, MB, Standl, M, Heinrich, J, Buyken, AE. Prospective relevance of dietary patterns at the beginning and during the course of primary school to the development of body composition. Br J Nutr. 2014. 111:1488-98. doi:10.1017/s0007114513004017

578 Diez-Espino, J, Basterra-Gortari, FJ, Salas-Salvado, J, Buil-Cosiales, P, Corella, D, Schroder, H, Estruch, R, Ros, E, Gomez-Gracia, E, Aros, F, Fiol, M, Lapetra, J, Serra-Majem, L, Pinto, X, Babio, N, Quiles, L, Fito, M, Marti, A, Toledo, E. Egg consumption and cardiovascular disease according to diabetic status: The PREDIMED study. Clin Nutr. 2017. 36:10151021. doi:10.1016/j.clnu.2016.06.009

579 Diez-Espino, J, Buil-Cosiales, P, Babio, N, Toledo, E, Corella, D, Ros, E, Fito, M, Gomez-Gracia, E, Estruch, R, Fiol, M, Lapetra, J, Alonso-Gomez, A, Serra-Majem, L, Pinto, X, Sorli, JV, Munoz, MA, Basora, J, Martinez-Gonzalez, MA. Impact of Life's Simple 7 on the incidence of major cardiovascular events in high-risk Spanish adults in the PREDIMED study cohort. Rev Esp Cardiol (Engl Ed). 2019. :. doi:10.1016/j.rec.2019.05.010

580 Dimitriadis, D, Mamplekou, E, Dimitriadis, P, Komessidou, V, Dimitriadis, G, Papageorgiou, C. The Association between Obesity and Symptoms of Psychopathology and its Relationship with Sedentary Behavior and Mediterranean Diet. Isr J Psychiatry Relat Sci. 2016. 53:17-24. doi:unavailable

581 Ding, M, Ellervik, C, Huang, T, Jensen, MK, Curhan, GC, Pasquale, LR, Kang, JH, Wiggs, JL, Hunter, DJ, Willett, WC Rimm, EB, Kraft, P, Chasman, DI, Qi, L, Hu, FB, Qi, Q. Diet quality and genetic association with body mass index: results from 3 observational studies. Am J Clin Nutr. 2018. 108:1291-1300. doi:10.1093/ajcn/nqy203

582 Ding, N, Wang, X, Tucker, KL, Weisskopf, MG, Sparrow, D, Hu, H, Park, SK. Dietary patterns, bone lead and incident coronary heart disease among middle-aged to elderly men. Environ Res. 2019. 168:222-229. doi:10.1016/j.envres.2018.09.035

583 Dinu, M, Colombini, B, Pagliai, G, Cesari, F, Gori, A, Giusti, B, Marcucci, R, Sofi, F. Effects of a dietary intervention with Mediterranean and vegetarian diets on hormones that influence energy balance: results from the CARDIVEG study. Int J Food Sci Nutr. 2019. :1-8. doi:10.1080/09637486.2019.1658723

584 Dinu, M, Pagliai, G, Colombini, B, Sereni, A, Gori, AM, Giusti, B, Marcucci, R, Casini, A, Sofi, F. Dietary intervention with vegetarian and mediterranean diets for cardiovascular prevention: effects on hormones involved in the energy balance. Nutrition, metabolism and cardiovascular diseases. 2019. 29:881-. doi:10.1016/j.numecd.2019.05.038

585 Dipla, K, Makri, M, Zafeiridis, A, Soulas, D, Tsalouhidou, S, Mougios, V, Kellis, S. An isoenergetic high-protein, moderatefat diet does not compromise strength and fatigue during resistance exercise in women. Br J Nutr. 2008. 100:283-6. doi:10.1017/s0007114507898679

586 Dipnall, JF, Pasco, JA, Meyer, D, Berk, M, Williams, LJ, Dodd, S, Jacka, FN. The association between dietary patterns, diabetes and depression. J Affect Disord. 2015. 174:215-24. doi:10.1016/j.jad.2014.11.030

587 Divens, LL, Carter-Holmes, DV. Optimizing Blood Pressure in African-American Women with Dietary Approaches to Stop Hypertension (DASH). J Natl Black Nurses Assoc. 2019. 30:1-6. doi:unavailable

588 Djuric, Z, Ren, J, Blythe, J, VanLoon, G, Sen, A. A Mediterranean dietary intervention in healthy American women changes plasma carotenoids and fatty acids in distinct clusters. Nutr Res. 2009. 29:156-63. doi:10.1016/j.nutres.2009.03.001 
589 Dolman, RC, Wentzel-Viljoen, E, Jerling, JC, Feskens, EJ, Kruger, A, Pieters, M. The use of predefined diet quality scores in the context of CVD risk during urbanization in the South African Prospective Urban and Rural Epidemiological (PURE) study. Public Health Nutr. 2014. 17:1706-16. doi:10.1017/s1368980013002206

590 Domenech, M, Roman, P, Lapetra, J, Garcia de la Corte, FJ, Sala-Vila, A, de la Torre, R, Corella, D, Salas-Salvado, J, Ruiz-Gutierrez, V, Lamuela-Raventos, RM, Toledo, E, Estruch, R, Coca, A, Ros, E. Mediterranean diet reduces 24-hour ambulatory blood pressure, blood glucose, and lipids: one-year randomized, clinical trial. Hypertension. 2014. 64:69-76. doi:10.1161/hypertensionaha.113.03353

591 Dominguez, LJ, Bes-Rastrollo, M, de la Fuente-Arrillaga, C, Toledo, E, Beunza, JJ, Barbagallo, M, Martinez-Gonzalez, MA. Similar prediction of total mortality, diabetes incidence and cardiovascular events using relative- and absolute-component Mediterranean diet score: the SUN cohort. Nutr Metab Cardiovasc Dis. 2013. 23:451-8. doi:10.1016/j.numecd.2011.10.009

592 Dominguez-Reyes, T, Astudillo-Lopez, CC, Salgado-Goytia, L, Munoz-Valle, JF, Salgado-Bernabe, AB, Guzman-Guzman, IP, Castro-Alarcon, N, Moreno-Godinez, ME, Parra-Rojas, I. Interaction of dietary fat intake with APOA2, APOA5 and LEPR polymorphisms and its relationship with obesity and dyslipidemia in young subjects. Lipids Health Dis. $2015.14: 106$. doi:10.1186/s12944-015-0112-4

593 Dominique Ashen, M, Blumenthal, RS. Low HDL cholesterol levels. New England Journal of Medicine. 2005. 353:1252-1260. doi:10.1056/NEJMcp044370

594 Donahoo, W, Wyatt, HR, Kriehn, J, Stuht, J, Dong, F, Hosokawa, P, Grunwald, GK, Johnson, SL, Peters, JC, Hill, JO. Dietary fat increases energy intake across the range of typical consumption in the United States. Obesity (Silver Spring). 2008. 16:64-9. doi:10.1038/oby.2007.31

595 Dong, D, Bilger, M, van Dam, RM, Finkelstein, EA. Consumption Of Specific Foods And Beverages And Excess Weight Gain Among Children And Adolescents. Health Aff (Millwood). 2015. 34:1940-8. doi:10.1377/hlthaff.2015.0434

596 Doo, EY, Jun, DW, Yang, SY, Kim, YS, Lee, SM. The intervention and education of low-sodium diet decreases insulin resistance in obese subjects. Clinical nutrition (edinburgh, scotland). 2015. 34:S210-S211. doi:unavailable

597 Doo, M, Kim, Y. Sleep duration and dietary macronutrient consumption can modify the cardiovascular disease for Korean women but not for men. Lipids Health Dis. 2016. 15:17. doi:10.1186/s12944-015-0170-7

598 Doostvandi, T, Bahadoran, Z, Mozaffari-Khosravi, H, Mirmiran, P, Azizi, F. Food intake patterns are associated with the risk of impaired glucose and insulin homeostasis: a prospective approach in the Tehran Lipid and Glucose Study. Public Health Nutr. 2016. 19:2467-74. doi:10.1017/s1368980016000616

599 Doostvandi, T, Bahadoran, Z, Mozaffari-Khosravi, H, Tahmasebinejad, Z, Mirmiran, P, Azizi, F. The association of dietary patterns and the incidence of insulin resistance after a 3-year follow-up: Tehran Lipid and Glucose Study. Asia Pac J Clin Nutr. 2017. 26:531-538. doi:10.6133/apjcn.032016.12

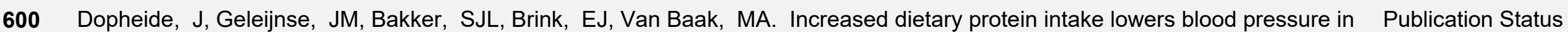
overweight subjects. Obesity reviews. 2011. 12:275-276. doi:10.1111/j.1467-789X.2011.00889.x

Outcome; Publication Date Overlaps with Existing Review

Intervention/Exposure

Study Design Publication Status

Study duration

Intervention/Exposure; Outcome

Publication Status

Study Design

Outcome

Outcome 
Dopheide, J, Teunissen-Beekman, KFM, Geleijnse, JM, Bakker, SJL, Brink, EJ, Van Baak, MA. Increased dietary protein intake lowers blood pressure in overweight subjects. Annals of nutrition \& metabolism. 2013. 62:148. doi:10.1159/000346990

602 Dorenbos, E, Drummen, M, Rijks, J, Adam, T, Stouthart, P, Alfredo Martinez, J, Navas-Carretero, S, Stratton, G, Swindell, N, Fogelholm, M, Raben, A, Westerterp-Plantenga, M, Vreugdenhil, A. PREVIEW (Prevention of Diabetes Through Lifestyle Intervention and Population Studies in Europe and Around the World) study in children aged 10 to 17 years: Design, methods and baseline results. Diabetes Obes Metab. 2018. 20:1096-1101. doi:10.1111/dom.13216

603 Dorgan, JF, Liu, L, Barton, BA, Deshmukh, S, Snetselaar, LG, Van Horn, L, Stevens, VJ, Robson, AM, Lasser, NL, Himes, JH, Shepherd, JA, Pourfarzib, R, Pettee Gabriel, K, Kriska, A, Kwiterovich, PO, Jr. Adolescent diet and metabolic syndrome in young women: results of the Dietary Intervention Study in Children (DISC) follow-up study. J Clin Endocrinol Metab. 2011. 96:E1999-2008. doi:10.1210/jc.2010-2726

604 Dorosty, AR, Emmett, PM, Cowin, IS, Reilly, JJ. Factors associated with early adiposity rebound. Pediatrics. 2000. 105:1115-1118. doi:10.1542/peds.105.5.1115

605 Dow, CA, Stauffer, BL, Greiner, JJ, DeSouza, CA. Influence of habitual high dietary fat intake on endothelium-dependent vasodilation. Appl Physiol Nutr Metab. 2015. 40:711-5. doi:10.1139/apnm-2015-0006

606 Downer, MK, Gea, A, Stampfer, M, Sanchez-Tainta, A, Corella, D, Salas-Salvado, J, Ros, E, Estruch, R, Fito, M, GomezGracia, E, Aros, F, Fiol, M, De-la-Corte, FJ, Serra-Majem, L, Pinto, X, Basora, J, Sorli, JV, Vinyoles, E, Zazpe, I, MartinezGonzalez, MA. Predictors of short- and long-term adherence with a Mediterranean-type diet intervention: the PREDIMED randomized trial. Int J Behav Nutr Phys Act. 2016. 13:67. doi:10.1186/s12966-016-0394-6

607 Drake, I, Sonestedt, E, Ericson, U, Wallstrom, P, Orho-Melander, M. A Western dietary pattern is prospectively associated with cardio-metabolic traits and incidence of the metabolic syndrome. Br J Nutr. 2018. 119:1168-1176. doi:10.1017/s000711451800079x

608 Draper, CF, Vassallo, I, Di Cara, A, Milone, C, Comminetti, O, Monnard, I, Godin, JP, Scherer, M, Su, M, Jia, W, Guiraud, SP, Praplan, F, Guignard, L, Ammon Zufferey, C, Shevlyakova, M, Emami, N, Moco, S, Beaumont, M, Kaput, J, Martin, FP. A 48-Hour Vegan Diet Challenge in Healthy Women and Men Induces a BRANCH-Chain Amino Acid Related, Health Associated, Metabolic Signature. Mol Nutr Food Res. 2018. 62:. doi:10.1002/mnfr.201700703

609 Drehmer, M, Camey, SA, Nunes, MA, Duncan, BB, Lacerda, M, Pinheiro, AP, Schmidt, MI. Fibre intake and evolution of BMI: from pre-pregnancy to postpartum. Public Health Nutr. 2013. 16:1403-13. doi:10.1017/s1368980012003849

610 Drehmer, M, Odegaard, AO, Schmidt, MI, Duncan, BB, Cardoso, LO, Matos, SMA, Molina, Mdcb, Barreto, SM, Pereira, MA. Brazilian dietary patterns and the dietary approaches to stop hypertension (DASH) diet-relationship with metabolic syndrome and newly diagnosed diabetes in the ELSA-Brasil study. Diabetol Metab Syndr. 2017. 9:13. doi:10.1186/s13098-0170211-7

611 Drenowatz, C, Shook, RP, Hand, GA, Hebert, JR, Blair, SN. The independent association between diet quality and body composition. Sci Rep. 2014. 4:4928. doi:10.1038/srep04928

\section{Rationale}

Publication Status

Study Design

Intervention/Exposure

Age at

Intervention/Exposure

Study Design

Outcome

Outcome

Study duration

Intervention/Exposure; Age at

Intervention/Exposure

Study Design

Study Design 
612 Dreon, DM, Fernstrom, HA, Williams, PT, Krauss, RM. Reduced LDL particle size in children consuming a very-low-fat diet is related to parental LDL-subclass patterns. Am J Clin Nutr. 2000. 71:1611-6. doi:10.1093/ajcn/71.6.1611

613 Drogan, D, Hoffmann, K, Schulz, M, Bergmann, MM, Boeing, H, Weikert, C. A food pattern predicting prospective weight change is associated with risk of fatal but not with nonfatal cardiovascular disease. J Nutr. 2007. 137:1961-7. doi:10.1093/jn/137.8.1961

614 Drouin-Chartier, JP, Tremblay, AJ, Lépine, MC, Lemelin, V, Lamarche, B, Couture, P. Substitution of dietary $\omega-6$ polyunsaturated fatty acids for saturated fatty acids decreases LDL apolipoprotein B-100 production rate in men with dyslipidemia associated with insulin resistance: a randomized controlled trial. American journal of clinical nutrition. 2018. 107:26-34. doi:10.1093/ajcn/nqx013

615 Drummen, M, Dorenbos, E, Vreugdenhil, ACE, Raben, A, Fogelholm, M, Westerterp-Plantenga, MS, Adam, TC. Long-term effects of increased protein intake after weight loss on intrahepatic lipid content and implications for insulin sensitivity: a PREVIEW study. Am J Physiol Endocrinol Metab. 2018. 315:E885-e891. doi:10.1152/ajpendo.00162.2018

616 Du, H, Dong, CY, Lin, QY. Risk factors of acute myocardial infarction in middle-aged and adolescent people (< 45 years) in Yantai. BMC Cardiovasc Disord. 2015. 15:106. doi:10.1186/s12872-015-0102-5

617 Dubois, L, Carter, MA, Farmer, A, Girard, M, Burnier, D, Tatone-Tokuda, F, Porcherie, M. Higher intakes of energy and grain products at 4 years of age are associated with being overweight at 6 years of age. Journal of Nutrition. 2011. 141:20242029. doi:10.3945/jn.111.143347

618 Dubois, L, Diasparra, M, Bogl, LH, Fontaine-Bisson, B, Bedard, B, Tremblay, RE, Kaprio, J, Boivin, M. Dietary Intake at 9 Years and Subsequent Body Mass Index in Adolescent Boys and Girls: A Study of Monozygotic Twin Pairs. Twin Res Hum Genet. 2016. 19:47-59. doi:10.1017/thg.2015.97

619 Dubois, L, Farmer, AP, Girard, M, Peterson, K. Preschool children's eating behaviours are related to dietary adequacy and body weight. Eur J Clin Nutr. 2007. 61:846-55. doi:10.1038/sj.ejcn.1602586

620 Duckworth, LC, Gately, PJ, Radley, D, Cooke, CB, King, RF, Hill, AJ. RCT of a high-protein diet on hunger motivation and weight-loss in obese children: an extension and replication. Obesity (Silver Spring). 2009. 17:1808-10. doi:10.1038/oby.2009.95

621 Dudum, R, Juraschek, SP, Appel, LJ. Dose-dependent effects of lifestyle interventions on blood lipid levels: Results from the PREMIER trial. Patient Educ Couns. 2019. 102:1882-1891. doi:10.1016/j.pec.2019.05.005

622 Due, A, Larsen, TM, Hermansen, K, Stender, S, Holst, JJ, Toubro, S, Martinussen, T, Astrup, A. Comparison of the effects on insulin resistance and glucose tolerance of 6-mo high-monounsaturated-fat, low-fat, and control diets. Am J Clin Nutr. 2008. 87:855-62. doi:10.1093/ajcn/87.4.855

623 Due, A, Larsen, TM, Mu, H, Hermansen, K, Stender, S, Toubro, S, Allison, DB, Astrup, A. The effect of three different ad libitum diets for weight loss maintenance: a randomized 18-month trial. Eur J Nutr. 2017. 56:727-738. doi:10.1007/s00394-0151116-6
Study duration

Intervention/Exposure

Intervention/Exposure; Comparator

Intervention/Exposure; Outcome

Study Design; Intervention/Exposure

Intervention/Exposure

Intervention/Exposure

Study Design;

Intervention/Exposure; Outcome

Study duration

Intervention/Exposure; Comparator

Power/Size

Weight

loss/Hypocaloric 
624 Due, A, Toubro, S, Skov, AR, Astrup, A. Effect of normal-fat diets, either medium or high in protein, on body weight in overweight subjects: a randomised 1-year trial. Int J Obes Relat Metab Disord. 2004. 28:1283-90. doi:10.1038/sj.ijo.0802767

625 Dugas, C, Belanger, M, Perron, J, Weisnagel, SJ, Tchernof, A, Marc, I, Robitaille, J. Is A Healthy Diet Associated with Lower Anthropometric and Glycemic Alterations in Predisposed Children Born from Mothers with Gestational Diabetes Mellitus?. Nutrients. 2019. 11:. doi:10.3390/nu11030570

626 Duhita, MR, Schutz, Y, Montani, JP, Dulloo, AG, Miles-Chan, JL. Does gender influence dietary protein-induced thermogenesis? A pilot dose response study. Obesity reviews. 2018. 19:103-. doi:10.1111/(ISSN)1467-789X

627 Duijzer, G, Haveman-Nies, A, Jansen, SC, Beek, JT, van Bruggen, R, Willink, MGJ, Hiddink, GJ, Feskens, EJM. Effect and maintenance of the SLIMMER diabetes prevention lifestyle intervention in Dutch primary healthcare: a randomised controlled trial. Nutr Diabetes. 2017. 7:e268. doi:10.1038/nutd.2017.21

628 Dujmovic, M, Kresic, G, Mandic, ML, Kenjeric, D, Cvijanovic, O. Changes in dietary intake and body weight in lactating and non-lactating women: prospective study in northern coastal Croatia. Coll Antropol. 2014. 38:179-87. doi:unavailable

629 Dulloo, AG, Antic, V, Yang, Z, Montani, JP. Propellers of growth trajectories to obesity and the metabolic syndrome. Int J Obes (Lond). 2006. 30 Suppl 4:S1-3. doi:10.1038/sj.ijo.0803512

630 Dumesnil, JG, Turgeon, J, Tremblay, A, Poirier, P, Gilbert, M, Gagnon, L, St-Pierra, S, Garneau, C, Lemieux, I, Pascot, A, Bergeron, J, Deapés, JP. Effect of a low-glycaemic index-low-fat-high protein diet on the atherogenic metabolic risk profile of abdominally obese men. British Journal of Nutrition. 2001. 86:557-568. doi:unavailable

631 Dumesnil, JG, Turgeon, J, Tremblay, A, Poirier, P, Gilbert, M, Gagnon, L, St-Pierre, S, Garneau, C, Lemieux, I, Pascot, A, Bergeron, J, Despres, JP. Effect of a low-glycaemic index--low-fat--high protein diet on the atherogenic metabolic risk profile of abdominally obese men. Br J Nutr. 2001. 86:557-68. doi:10.1079/bjn2001427

632 Dunlap, BS, Bailes, JR, Jr. Unlimited energy, restricted carbohydrate diet improves lipid parameters in obese children. Metab Syndr Relat Disord. 2008. 6:32-6. doi:10.1089/met.2007.0023

633 Dunn, SL, Siu, W, Freund, J, Boutcher, SH. The effect of a lifestyle intervention on metabolic health in young women. Diabetes Metab Syndr Obes. 2014. 7:437-44. doi:10.2147/dmso.S67845

634 Durao, C, Oliveira, A, Santos, AC, Severo, M, Guerra, A, Barros, H, Lopes, C. Protein intake and dietary glycemic load of 4year-olds and association with adiposity and serum insulin at 7 years of age: sex-nutrient and nutrient-nutrient interactions. Int $\mathrm{J}$ Obes (Lond). 2017. 41:533-541. doi:10.1038/ijo.2016.240

635 Durkalec-Michalski, K, Zawieja, EE, Zawieja, BE, Jurkowska, D, Buchowski, MS, Jeszka, J. Effects of Low Versus Moderate Glycemic Index Diets on Aerobic Capacity in Endurance Runners: Three-Week Randomized Controlled Crossover Trial. Nutrients. 2018. 10:. doi:10.3390/nu10030370

636 Durrer, C, Lewis, N, Wan, Z, Ainslie, PN, Jenkins, NT, Little, JP. Short-Term Low-Carbohydrate High-Fat Diet in Healthy Young Males Renders the Endothelium Susceptible to Hyperglycemia-Induced Damage, An Exploratory Analysis. Nutrients. 2019. 11: doi:10.3390/nu11030489

Intervention/Exposure

Study Design

Publication Status

Intervention/Exposure

Participants

Publication Status

Study duration

Study duration

Study Design

Intervention/Exposure

Intervention/Exposure

Study duration

Study duration 
No.

637 Durward, CM, Zack, MK, Shlisky, JD, Campbell, JK, Jonnalagadda, SS, Nickols-Richardson, SM. Predictors of early weight loss in a diet and exercise weight loss trial. FASEB journal. 2011. 25:. doi:unavailable

638 Dyson, PA, Beatty, S, Matthews, DR. A low-carbohydrate diet is more effective in reducing body weight than healthy eating in both diabetic and non-diabetic subjects. Diabet Med. 2007. 24:1430-5. doi:10.1111/j.1464-5491.2007.02290.x

639 Dyson, PA, Beatty, S, Matthews, DR. An assessment of low-carbohydrate or low-fat diets for weight loss at 2 year's follow-up. Diabetic medicine. 2010. 27:363-364. doi:10.1111/j.1464-5491.2010.02926.x

640 Earnest, CP, Church, TS. Evaluation of a Voluntary Worksite Weight Loss Program on Metabolic Syndrome. Metab Syndr Relat Disord. 2015. 13:406-14. doi:10.1089/met.2015.0075

641 Ebbeling, CB, Feldman, HA, Klein, GL, Wong, JMW, Bielak, L, Steltz, SK, Luoto, PK, Wolfe, RR, Wong, WW, Ludwig, DS. Outcome Effects of a low carbohydrate diet on energy expenditure during weight loss maintenance: randomized trial. Bmj. 2018. 363:k4583. doi:10.1136/bmj.k4583

642 Ebbeling, CB, Klein, GL, Luoto, PK, Wong, JMW, Bielak, L, Eddy, RG, Steltz, SK, Devlin, C, Sandman, M, Hron, B, Shimy, Study Design K, Heymsfield, SB, Wolfe, RR, Wong, WW, Feldman, HA, Ludwig, DS. A randomized study of dietary composition during weight-loss maintenance: Rationale, study design, intervention, and assessment. Contemp Clin Trials. 2018. 65:76-86. doi:10.1016/j.cct.2017.12.004

643 Ebbeling, CB, Leidig, MM, Feldman, HA, Lovesky, MM, Ludwig, DS. Effects of a low-glycemic load vs low-fat diet in obese young adults: a randomized trial. Jama. 2007. 297:2092-102. doi:10.1001/jama.297.19.2092

644 Ebbeling, CB, Leidig, MM, Sinclair, KB, Hangen, JP, Ludwig, DS. A reduced-glycemic load diet in the treatment of adolescent obesity. Arch Pediatr Adolesc Med. 2003. 157:773-9. doi:10.1001/archpedi.157.8.773

645 Ebbeling, CB, Leidig, MM, Sinclair, KB, Seger-Shippee, LG, Feldman, HA, Ludwig, DS. Effects of an ad libitum low-glycemic load diet on cardiovascular disease risk factors in obese young adults. Am J Clin Nutr. 2005. 81:976-82 doi:10.1093/ajcn/81.5.976

646 Ebbeling, CB, Swain, JF, Feldman, HA, Wong, WW, Hachey, DL, Garcia-Lago, E, Ludwig, DS. Effects of dietary composition on energy expenditure during weight-loss maintenance. Jama. 2012. 307:2627-34. doi:10.1001/jama.2012.6607

647 Ebrahimof, S, Hosseini-Esfahani, F, Mirmiran, P, Fallah-Kezabi, M, Azizi, F. Food Patterns and Framingham Risk Score in Iranian Adults: Tehran Lipid and Glucose Study: 2005-2011. Metab Syndr Relat Disord. 2018. 16:64-71. doi:10.1089/met.2017.0125

648 Echeverría, G, Dussaillant, C, McGee, EE, Mena, C, Nitsche, MP, Urquiaga, I, Bitran, M, Pedrals, N, Rigotti, A. Promoting and Implementing the Mediterranean Diet in the Southern Hemisphere: the Chilean Experience. European Journal of Clinica Nutrition. 2018. :. doi:10.1038/s41430-018-0307-7

649 Eckel, RH, Hernandez, TL, Bell, ML, Weil, KM, Shepard, TY, Grunwald, GK, Sharp, TA, Francis, CC, Hill, JO. Carbohydrate balance predicts weight and fat gain in adults. Am J Clin Nutr. 2006. 83:803-8. doi:10.1093/ajcn/83.4.803

\section{Rationale}

Publication Status

Weight loss/Hypocaloric

Publication Status

Study Design

Weight loss/Hypocaloric

Intervention/Exposure

Intervention/Exposure Study duration

Language

Study Design

Intervention/Exposure; Study duration 
650 Eftekhari, MH, Rajaeifard, AR, Ahmadi, A, Kashfi, SM, Khajeh Rahim, AA. Effect of two isocaloric diets, low fat- high calcium and low fat- high fiber on weight reduction, lipid profile, and blood pressure. Iranian Cardiovascular Research Journal. 2009.

Intervention/Exposure; 3:200-206. doi:unavailable

651 Egert, S, Baxheinrich, A, Lee-Barkey, YH, Tschoepe, D, Stehle, P, Stratmann, B, Wahrburg, U. Effects of a hypoenergetic diet rich in alpha-linolenic acid on fatty acid composition of serum phospholipids in overweight and obese patients with metabolic syndrome. Nutrition. 2018. 49:74-80. doi:10.1016/j.nut.2017.11.002

652 Egert, S, Kratz, M, Kannenberg, F, Fobker, M, Wahrburg, U. Effects of high-fat and low-fat diets rich in monounsaturated fatty acids on serum lipids, LDL size and indices of lipid peroxidation in healthy non-obese men and women when consumed under controlled conditions. Eur J Nutr. 2011. 50:71-9. doi:10.1007/s00394-010-0116-9

653 Eilat-Adar, S. Reduce simple carbohydrate and animal protein intake and increase polyunsaturated fatty acid intake in patients with metabolic syndrome and insulin resistance. J Am Diet Assoc. 2008. 108:802-3. doi:10.1016/j.jada.2008.02.013

654 Ejlerskov, KT, Larnkjaer, A, Pedersen, D, Ritz, C, Molgaard, C, Michaelsen, KF. IGF-I at 9 and 36 months of age - relations with body composition and diet at 3 years - the SKOT cohort. Growth Horm IGF Res. 2014. 24:239-44. doi:10.1016/j.ghir.2014.10.005

655 Ek, A, Chamberlain, KL, Ejderhamn, J, Fisher, PA, Marcus, C, Chamberlain, P, Nowicka, P. The More and Less Study: a randomized controlled trial testing different approaches to treat obesity in preschoolers. BMC Public Health. 2015. 15:735. doi:10.1186/s12889-015-1912-1

656 El Ansari, W, Suominen, S, Berg-Beckhoff, G. Mood and food at the University of Turku in Finland: nutritional correlates of perceived stress are most pronounced among overweight students. Int J Public Health. 2015. 60:707-16. doi:10.1007/s00038015-0717-4

657 Elbelt, U, Schuetz, T, Knoll, N, Burkert, S. Self-Directed Weight Loss Strategies: Energy Expenditure Due to Physical Activity Is Not Increased to Achieve Intended Weight Loss. Nutrients. 2015. 7:5868-88. doi:10.3390/nu7075256

658 Ellingsen, I, Hjerkinn, EM, Arnesen, H, Seljeflot, I, Hjermann, I, Tonstad, S. Follow-up of diet and cardiovascular risk factors 20 years after cessation of intervention in the Oslo Diet and Antismoking Study. Eur J Clin Nutr. 2006. 60:378-85. doi:10.1038/sj.ejcn.1602327

659 Elliott, SA, Truby, H, Lee, A, Harper, C, Abbott, RA, Davies, PS. Associations of body mass index and waist circumference with: energy intake and percentage energy from macronutrients, in a cohort of Australian children. Nutr J. 2011. 10:58. doi:10.1186/1475-2891-10-58

660 Ellsworth, DL, Costantino, NS, Blackburn, HL, Engler, RJ, Kashani, M, Vernalis, MN. Lifestyle modification interventions differing in intensity and dietary stringency improve insulin resistance through changes in lipoprotein profiles. Obes Sci Pract. 2016. 2:282-292. doi:10.1002/osp4.54

661 Eloranta, AM, Schwab, U, Venalainen, T, Kiiskinen, S, Lakka, HM, Laaksonen, DE, Lakka, TA, Lindi, V. Dietary quality indices in relation to cardiometabolic risk among Finnish children aged 6-8 years - The PANIC study. Nutr Metab Cardiovasc Dis. 2016. 26:833-41. doi:10.1016/j.numecd.2016.05.005

Intervention/Exposure; Outcome

Study duration

Study Design Publication Status

Study Design;

intervention/Exposure; Outcome; Age at Intervention/Exposure

Study Design;

Intervention/Exposure

Study Design

Study Design; Intervention/Exposure

Intervention/Exposure

Study Design

Intervention/Exposure

Study Design 
662 Eloranta, AM, Schwab, U, Venäläinen, T, Kiiskinen, S, Lakka, HM, Laaksonen, DE, Lakka, TA, Lindi, V. Dietary quality indices in relation to cardiometabolic risk among Finnish children aged 6-8 years - The PANIC study. Nutrition, Metabolism and Cardiovascular Diseases. 2016. 26:833-841. doi:10.1016/j.numecd.2016.05.005

663 Elvevoll, EO, Eilertsen, KE, Brox, J, Dragnes, BT, Falkenberg, P, Olsen, JO, Kirkhus, B, Lamglait, A, Osterud, B. Seafood diets: hypolipidemic and antiatherogenic effects of taurine and n-3 fatty acids. Atherosclerosis. 2008. 200:396-402.

Intervention/Exposure; doi:10.1016/j.atherosclerosis.2007.12.021

664 Emond, A, Emmett, P, Steer, C, Golding, J. Feeding symptoms, dietary patterns, and growth in young children with autism spectrum disorders. Pediatrics. 2010. 126:e337-e342. doi:10.1542/peds.2009-2391

665 Engberink, MF, Geleijnse, JM, Bakker, SJ, Larsen, TM, Handjieva-Darlesnka, T, Kafatos, A, Martinez, JA, Pfeiffer, AF Kunesova, M, Jebb, SA, Holst, C, Astrup, A, Saris, WH, Brink, EJ, van Baak, MA. Effect of a high-protein diet on maintenance of blood pressure levels achieved after initial weight loss: the DiOGenes randomized study. J Hum Hypertens 2015. 29:58-63. doi:10.1038/jhh.2014.30

666 Engel, S, Tholstrup, T. Butter increased total and LDL cholesterol compared with olive oil but resulted in higher HDL cholesterol compared with a habitual diet. Am J Clin Nutr. 2015. 102:309-15. doi:10.3945/ajcn.115.112227

667 Entezari, MH, Salehi, R, Kazemi, M, Janghorbani, M, Kafeshani, M. Comparison of the effect of the Dietary Approaches to Stop Hypertension diet with usual dietary advice on expression of peroxisome proliferators-activated receptor gamma gene in women: A randomized controlled clinical trial. ARYA Atheroscler. 2018. 14:24-31. doi:10.22122/arya.v14i1.1565

668 Erber, E, Hopping, BN, Grandinetti, A, Park, SY, Kolonel, LN, Maskarinec, G. Dietary patterns and risk for diabetes: The multiethnic cohort. Diabetes Care. 2010. 33:532-538. doi:10.2337/dc09-1621

Comparator

Intervention/Exposure; Health Status

Intervention/Exposure

Study duration

Power/Size

Intervention/Exposure Publication Date

Overlaps with Existing Review

669 Ericson, U, Hellstrand, S, Brunkwall, L, Schulz, CA, Sonestedt, E, Wallstrom, P, Gullberg, B, Wirfalt, E, Orho-Melander, M. Food sources of fat may clarify the inconsistent role of dietary fat intake for incidence of type 2 diabetes. Am J Clin Nutr. 2015. 101:1065-80. doi:10.3945/ajcn.114.103010

670 Ericson, U, Rukh, G, Stojkovic, I, Sonestedt, E, Gullberg, B, Wirfalt, E, Wallstrom, P, Orho-Melander, M. Sex-specific interactions between the IRS1 polymorphism and intakes of carbohydrates and fat on incident type 2 diabetes. Am J Clin Nutr. 2013. 97:208-16. doi:10.3945/ajcn.112.046474

671 Erlinger, TP, Miller, ER, 3rd, Charleston, J, Appel, LJ. Inflammation modifies the effects of a reduced-fat low-cholesterol diet

on lipids: results from the DASH-sodium trial. Circulation. 2003. 108:150-4. doi:10.1161/01.Cir.0000080288.30567.86

Intervention/Exposure

Intervention/Exposure obese children: adaptation of the Healthy Lifestyle-Diet Index in Turkey. J Pediatr Endocrinol Metab. 2018. 31:391-398. doi:10.1515/jpem-2017-0271

673 Escurriol, V, Cofan, M, Serra, M, Bullo, M, Basora, J, Salas-Salvado, J, Corella, D, Zazpe, I, Martinez-Gonzalez, MA, RuizGutierrez, V, Estruch, R, Ros, E. Serum sterol responses to increasing plant sterol intake from natural foods in the Mediterranean diet. Eur J Nutr. 2009. 48:373-82. doi:10.1007/s00394-009-0024-z

Study Design; Intervention/Exposure

Study Design

Outcome; Publication Date Overlaps with Existing Review 
674 Esfandiari, S, Bahadoran, Z, Mirmiran, P, Tohidi, M, Azizi, F. Adherence to the dietary approaches to stop hypertension trial

(DASH) diet is inversely associated with incidence of insulin resistance in adults: the Tehran lipid and glucose study. $J$ Clin Biochem Nutr. 2017. 61:123-129. doi:10.3164/jcbn.16-95

675 Eshriqui, I, Vilela, AA, Rebelo, F, Farias, DR, Castro, MB, Kac, G. Gestational dietary patterns are not associated with bloo pressure changes during pregnancy and early postpartum in a Brazilian prospective cohort. Eur J Nutr. 2016. 55:21-32. doi:10.1007/s00394-014-0819-4

676 Eslamian, G, Mirmiran, P, Asghari, G, Hosseini-Esfahani, F, Yuzbashian, E, Azizi, F. Low carbohydrate diet score does not predict metabolic syndrome in children and adolescents: Tehran Lipid and Glucose Study. Arch Iran Med. 2014. 17:417-22. doi:014176/aim.008

677 Esmaeili, SS, Fallahi, F, Gholami Fesharaki, M, Noormohammadi, G. A Randomized Trial on the Effect of Razavi's Dietary Pattern on the Components of Metabolic Syndrome. Iran Red Crescent Med J. 2014. 16:e14601. doi:10.5812/ircmj.14601

678 Esposito, K, Marfella, R, Ciotola, M, Di Palo, C, Giugliano, F, Giugliano, G, D'Armiento, M, D'Andrea, F, Giugliano, D. Effect of a Mediterranean-style diet on endothelial dysfunction and markers of vascular inflammation in the metabolic syndrome: A randomized trial. Journal of the American Medical Association. 2004. 292:1440-1446. doi:10.1001/jama.292.12.1440

679 Essah, PA, Levy, JR, Sistrun, SN, Kelly, SM, Nestler, JE. Effect of weight loss by a low-fat diet and a low-carbohydrate diet on peptide YY levels. Int J Obes (Lond). 2010. 34:1239-42. doi:10.1038/ijo.2010.48

680 Esser, D, van Dijk, SJ, Oosterink, E, Muller, M, Afman, LA. A high-fat SFA, MUFA, or n3 PUFA challenge affects the vascular response and initiates an activated state of cellular adherence in lean and obese middle-aged men. $\mathrm{J}$ Nutr. 2013 143:843-51. doi:10.3945/jn.113.174540

681 Estrada-Reyes, C, Tlatempa-Sotelo, P, Valdes-Ramos, R, Cabanas-Armesilla, M, Manjarrez-Montes-de-Oca, R. Dietary Patterns and Fitness Level in Mexican Teenagers. J Nutr Metab. 2018. 2018:7159216. doi:10.1155/2018/7159216

682 Estruch, R, Casas, R, Sacanella, E, Urpi-Sarda, M, Corella, C, Cataner, O, Salas-Salvado, J, Martinez-Gonzalez, MA, Lamuela-Raventos, RM. Mediterranean diet and inflammatory biomarkers. Annals of nutrition and metabolism. 2015. 67:41-42. doi:10.1159/000440895

683 Estruch, R, Martinez-Gonzalez, MA, Corella, D, Basora-Gallisa, J, Ruiz-Gutierrez, V, Covas, MI, Fiol, M, Gomez-Gracia, E Lopez-Sabater, MC, Escoda, R, Pena, MA, Diez-Espino, J, Lahoz, C, Lapetra, J, Saez, G, Ros, E. Effects of dietary fibre intake on risk factors for cardiovascular disease in subjects at high risk. J Epidemiol Community Health. 2009. 63:582-8. doi:10.1136/jech.2008.082214

684 Estruch, R, Martinez-Gonzalez, MA, Corella, D, Salas-Salvado, J, Fito, M, Chiva-Blanch, G, Fiol, M, Gomez-Gracia, E, Aros, F, Lapetra, J, Serra-Majem, L, Pinto, X, Buil-Cosiales, P, Sorli, JV, Munoz, MA, Basora-Gallisa, J, Lamuela-Raventos, RM, Serra-Mir, M, Ros, E. Retracted: Effect of a high-fat Mediterranean diet on bodyweight and waist circumference: a prespecified secondary outcomes analysis of the PREDIMED randomised controlled trial. Lancet Diabetes Endocrinol. 2016. 4:666-676. doi:10.1016/s2213-8587(16)30085-7 
685 Estruch, R, Martínez-González, MA, Corella, D, Salas-Salvadó, J, Fitó, M, Chiva-Blanch, G, Fiol, M, Gómez-Gracia, E,

Arós, F, Lapetra, J, Serra-Majem, L, Pintó, X, Buil-Cosiales, P, Sorlí, JV, Muñoz, MA, Basora-Gallisá, J, Lamuela-

Raventós, RM, Serra-Mir, M, Ros, E. Effect of a high-fat Mediterranean diet on bodyweight and waist circumference: a prespecified secondary outcomes analysis of the PREDIMED randomised controlled trial. The Lancet Diabetes and Endocrinology. 2016. 4:666-676. doi:10.1016/S2213-8587(16)30085-7

686 Estruch, R, Martinez-Gonzalez, MA, Corella, D, Salas-Salvado, J, Ruiz-Gutierrez, V, Covas, MI, Fiol, M, Gomez-Gracia, E, Lopez-Sabater, MC, Vinyoles, E, Aros, F, Conde, M, Lahoz, C, Lapetra, J, Saez, G, Ros, E. Effects of a Mediterraneanstyle diet on cardiovascular risk factors: a randomized trial. Ann Intern Med. 2006. 145:1-11. doi:10.7326/0003-4819-145-1200607040-00004

687 Estruch, R, Martínez-González, MA. Mediterranean diets reduced cardiovascular events more than a low-fat diet in high-risk persons. Annals of Internal Medicine. 2013. 158:JC3. doi:10.7326/0003-4819-158-12-201306180-02003

688 Estruch, R, Ros, E, Salas-Salvadó, J, Covas, MI, Corella, D, Arós, F, Gómez-Gracia, E, Ruiz-Gutiérrez, V, Fiol, M, Lapetra, J, Lamuela-Raventos, RM, Serra-Majem, L, Pintó, X, Basora, J, Muñoz, MA, Sorlí, JV, Martínez, JA, MartínezGonzález, MA. Primary prevention of cardiovascular disease with a Mediterranean diet. New England Journal of Medicine. 2013. 368:1279-1290. doi:10.1056/NEJMoa1200303

689 Estruch, R. Anti-inflammatory effects of the Mediterranean diet: the experience of the PREDIMED study. Proc Nutr Soc. 2010. 69:333-40. doi:10.1017/s0029665110001539

690 Estruch, R. Cardiovascular mortality: how can it be prevented?. Nefrologia. 2014. 34:561-9. doi:10.3265/Nefrologia.pre2014.Apr.12481

691 Evans, EM, Mojtahedi, MC, Thorpe, MP, Valentine, RJ, Kris-Etherton, PM, Layman, DK. Effects of protein intake and gender on body composition changes: a randomized clinical weight loss trial. Nutr Metab (Lond). 2012. 9:55. doi:10.1186/17437075-9-55

692 Fagherazzi, G, Gusto, G, Mancini, FR, Dow, C, Rajaobelina, K, Balkau, B, Boutron-Ruault, MC, Bonnet, F. Determinants of 20-year non-progression to Type 2 diabetes in women at very high risk: the E3N cohort study. Diabet Med. 2018. 35:1716-1721. doi:10.1111/dme.13774

693 Faghihnia, N, Tsimikas, S, Miller, ER, Witztum, JL, Krauss, RM. Changes in lipoprotein(a), oxidized phospholipids, and LDL subclasses with a low-fat high-carbohydrate diet. J Lipid Res. 2010. 51:3324-30. doi:10.1194/jlr.M005769

694 Fajcsak, Z, Gabor, A, Kovacs, V, Martos, E. The effects of 6-week low glycemic load diet based on low glycemic index foods in overweight/obese children--pilot study. J Am Coll Nutr. 2008. 27:12-21. doi:10.1080/07315724.2008.10719670

695 Fallaize, R, Livingstone, KM, Celis-Morales, C, Macready, AL, San-Cristobal, R, Navas-Carretero, S, Marsaux, CFM, O'Donovan, CB, Kolossa, S, Moschonis, G, Walsh, MC, Gibney, ER, Brennan, L, Bouwman, J, Manios, Y, Jarosz, M, Martinez, JA, Daniel, H, Saris, WHM, Gundersen, TE, Drevon, CA, Gibney, MJ, Mathers, JC, Lovegrove, JA. Association between Diet-Quality Scores, Adiposity, Total Cholesterol and Markers of Nutritional Status in European Adults: Findings from the Food4Me Study. Nutrients. 2018. 10:. doi:10.3390/nu10010049

Intervention/Exposure; Publication Date

Overlaps with Existing Review

Publication Status

Study Design;

Publication Status

Publication Status

Study Design;

Publication Status

Weight

oss/Hypocaloric

Power/Size

Intervention/Exposure

Study Design

Study Design 
696 Farhadnejad, H, Asghari, G, Mirmiran, P, Azizi, F. Dietary approach to stop hypertension diet and cardiovascular risk factors

Power/Size among 10- to 18-year-old individuals. Pediatr Obes. 2018. 13:185-194. doi:10.1111/ijpo.12268

697 Farhangi, MA, Jahangiry, L. Dietary diversity score is associated with cardiovascular risk factors and serum adiponectin concentrations in patients with metabolic syndrome. BMC Cardiovasc Disord. 2018. 18:68. doi:10.1186/s12872-018-0807-3

698 Farnsworth, E, Luscombe, ND, Noakes, M, Wittert, G, Argyiou, E, Clifton, PM. Effect of a high-protein, energy-restricted diet on body composition, glycemic control, and lipid concentrations in overweight and obese hyperinsulinemic men and women. Am J Clin Nutr. 2003. 78:31-9. doi:10.1093/ajcn/78.1.31

699 Farrell, P, Negin, J, Awoke, M, Thow, AM, Taua, M, Faumuina, T, Mihrshahi, S, Vizintin, P, Richards, J. Associations between sociodemographic and behaviour factors, and dietary risk factors for overweight and obesity, in Samoan women Appetite. 2019. 134:155-161. doi:10.1016/j.appet.2018.12.037

700 Farvid, MS, Malekshah, AF, Pourshams, A, Poustchi, H, Sepanlou, SG, Sharafkhah, M, Khoshnia, M, Farvid, M, Abnet, CC, Kamangar, F, Dawsey, SM, Brennan, P, Pharoah, PD, Boffetta, P, Willett, WC, Malekzadeh, R. Dietary Protein Sources and All-Cause and Cause-Specific Mortality: The Golestan Cohort Study in Iran. Am J Prev Med. 2017. 52:237-248. doi:10.1016/j.amepre.2016.10.041

701 Fatemi, MJ, Fararouei, M, Moravej, H, Dianatinasab, M. Stunting and its associated factors among 6-7-year-old children in southern Iran: a nested case-control study. Public Health Nutr. 2018. :1-8. doi:10.1017/s136898001800263x

702 Fava, F, Gitau, R, Griffin, BA, Gibson, GR, Tuohy, KM, Lovegrove, JA. The type and quantity of dietary fat and carbohydrate alter faecal microbiome and short-chain fatty acid excretion in a metabolic syndrome 'at-risk' population. Int $\mathrm{J}$ Obes (Lond). 2013. 37:216-23. doi:10.1038/ijo.2012.33

703 Fearnbach, SN, Masterson, TD, Schlechter, HA, Ross, AJ, Rykaczewski, MJ, Loken, E, Downs, DS, Thivel, D, Keller, KL. Impact of imposed exercise on energy intake in children at risk for overweight. Nutrition Journal. 2016. 15:. doi:10.1186/s12937016-0206-5

704 Feart, C, Jutand, MA, Larrieu, S, Letenneur, L, Delcourt, C, Combe, N, Barberger-Gateau, P. Energy, macronutrient and fatty acid intake of French elderly community dwellers and association with socio-demographic characteristics: data from the Bordeaux sample of the Three-City Study. Br J Nutr. 2007. 98:1046-57. doi:10.1017/s0007114507756520

705 Fechner, E, Bilet, L, Peters, HPF, Hiemstra, H, Schrauwen, P, Mensink, RP. Investigating The Effectiveness Of A Healthy Diet On Cardiovascular Risk Markers - A Challenge-Based Human Trial. Atherosclerosis. 2019. 287:e148-. doi:10.1016/j.atherosclerosis.2019.06.441

706 Feng, R, Du, S, Chen, Y, Zheng, S, Zhang, W, Na, G, Li, Y, Sun, C. High carbohydrate intake from starchy foods is positively associated with metabolic disorders: a Cohort Study from a Chinese population. Sci Rep. 2015. 5:16919. doi:10.1038/srep16919

707 Feng, R, Sun, G, Zhang, Y, Sun, Q, Ju, L, Sun, C, Wang, C. Short-term high-fat diet exacerbates insulin resistance and glycolipid metabolism disorders in young obese men with hyperlipidemia, as determined by metabolomics analysis using ultraHPLC-quadrupole time-of-flight mass spectrometry. J Diabetes. 2019. 11:148-160. doi:10.1111/1753-0407.12828

Power/Size

Power/Size

Study Design

ntervention/Exposure; Country

Study Design; Intervention/Exposure

Power/Size

Intervention/Exposure; Study duration

Study Design

Publication Status

Country

Study duration 
Ferdowsian, HR, Barnard, ND, Hoover, VJ, Katcher, HI, Levin, SM, Green, AA, Cohen, JL. A multicomponent intervention reduces body weight and cardiovascular risk at a GEICO corporate site. Am J Health Promot. 2010. 24:384-7. doi:10.4278/ajhp.081027-QUAN-255

709 Ferland, A, Chateau-Degat, ML, Hernandez, TL, Eckel, RH. Tissue-specific responses of lipoprotein lipase to dietary macronutrient composition as a predictor of weight gain over 4 years. Obesity (Silver Spring). 2012. 20:1006-11. doi:10.1038/oby.2011.372

710 Fernandez de la Puebla, RA, Fuentes, F, Perez-Martinez, P, Sanchez, E, Paniagua, JA, Lopez-Miranda, J, Perez-Jimenez, $F$. A reduction in dietary saturated fat decreases body fat content in overweight, hypercholesterolemic males. Nutr Metab Cardiovasc Dis. 2003. 13:273-7. doi:unavailable

711 Fernandez, C, Kasper, NM, Miller, AL, Lumeng, JC, Peterson, KE. Association of Dietary Variety and Diversity With Body Mass Index in US Preschool Children. Pediatrics. 2016. 137:e20152307. doi:10.1542/peds.2015-2307

712 Fernandez, ML. Plant-Based Diet Quality is Associated with Changes in Plasma Adiposity Biomarker Concentrations in Women. J Nutr. 2019. 149:551-552. doi:10.1093/jn/nxy317

713 Fernandez-Lazaro, Cl, Toledo, E, Salas-Salvado, J, Corella, D, Fito, M, Martinez, JA, Buil-Cosiales, P. PREDIMED-Plus trial: one-year changes in the quality of dietary carbohydrate intake and concurrent changes in cardiovascular risk factors. Annals of nutrition \& metabolism. 2019. 75:20-21. doi:10.1159/000501441

714 Ferranti, R, Marventano, S, Castellano, S, Giogianni, G, Nolfo, F, Rametta, S, Matalone, M, Mistretta, A. Sleep quality and Study Design duration is related with diet and obesity in young adolescent living in Sicily, Southern Italy. Sleep Sci. 2016. 9:117-22. doi:10.1016/j.slsci.2016.04.003

715 Ferrara, LA, Innelli, P, Palmieri, V, Limauro, S, De Luca, G, Ferrara, F, Liccardo, E, Celentano, A. Effects of different dietary protein intakes on body composition and vascular reactivity. Eur J Clin Nutr. 2006. 60:643-9. doi:10.1038/sj.ejcn.1602363

716 Ferreira, AA, Souza-Filho, ZA, Goncalves, MJF, Santos, J, Pierin, AMG. Relationship between alcohol drinking and arteria hypertension in indigenous people of the Mura ethnics, Brazil. PLoS One. 2017. 12:e0182352. doi:10.1371/journal.pone.0182352

717 Feuerstein, JS, Bautista, LT, Bjerke, WS. A dietary approach for treating dyslipidemia and hyperglycemia. Current Nutrition and Food Science. 2011. 7:271-274. doi:unavailable

718 Field, AE, Willett, WC, Lissner, L, Colditz, GA. Dietary fat and weight gain among women in the Nurses' Health Study. Obesity (Silver Spring). 2007. 15:967-76. doi:10.1038/oby.2007.616

719 Filaire, E, Maso, F, Degoutte, F, Jouanel, P, Lac, G. Food restriction, performance, psychological state and lipid values in judo athletes. Int J Sports Med. 2001. 22:454-9. doi:10.1055/s-2001-16244

720 Finelli, C, Crispino, P, Gioia, S, La Sala, N, D'amico, L, La Grotta, M, Miro, O, Colarusso, D. The improvement of large high-density lipoprotein (HDL) particle levels, and presumably HDL metabolism, depend on effects of low-carbohydrate diet and weight loss. EXCLI Journal. 2016. 15:166-176. doi:10.17179/excli2015-642

\section{Rationale}

Publication Date

Overlaps with Existing Review

Study duration

Study duration

Intervention/Exposure

Study Design;

Publication Status

Publication Status

Intervention/Exposure

Study Design

Study Design

Intervention/Exposure

Study duration

Publication Status 
721 Fito, M, Estruch, R, Salas-Salvado, J, Martinez-Gonzalez, MA, Aros, F, Vila, J, Corella, D, Diaz, O, Saez, G, de la Torre,

R, Mitjavila, MT, Munoz, MA, Lamuela-Raventos, RM, Ruiz-Gutierrez, V, Fiol, M, Gomez-Gracia, E, Lapetra, J, Ros, E, Serra-Majem, L, Covas, MI. Effect of the Mediterranean diet on heart failure biomarkers: a randomized sample from the PREDIMED trial. Eur J Heart Fail. 2014. 16:543-50. doi:10.1002/ejhf.61

722 Fito, M, Hernaez, A, Olga, C, Pinto, X, Estruch, R, Salas-Salvado, J, Corella, D, Blanchart, G, Gaixas, S, Aros, F, etal, . Effects of the mediterranean diet on the lipid profile and lipoprotein-related markers. Annals of nutrition \& metabolism. 2017. 71:193-194. doi:10.1159/000480486

723 Fitzpatrick, SL, Coughlin, JW, Appel, LJ, Tyson, C, Stevens, VJ, Jerome, GJ, Dalcin, A, Brantley, PJ, Hill-Briggs, F. Application of Latent Class Analysis to Identify Behavioral Patterns of Response to Behavioral Lifestyle Interventions in Overweight and Obese Adults. Int J Behav Med. 2015. 22:471-80. doi:10.1007/s12529-014-9446-y

724 Fleming, J, Kris-Etherton, P, Petersen, K, Baer, D. Effect of mediterranean diet with varying amounts of lean beef on measures of arterial stiffness and central blood pressure. Circulation. 2018. 138:. doi:unavailable

725 Fleming, J, Kris-Etherton, P, Petersen, K, Baer, D. The dose-response effect of a mediterranean style diet with lean beef on lipids and lipoproteins. Circulation. 2019. 139:. doi:10.1161/circ.139.suppl_1.045

726 Fleming, RM. The effect of high-, moderate-, and low-fat diets on weight loss and cardiovascular disease risk factors. Prev Cardiol. 2002. 5:110-8. doi:10.1111/j.1520-037x.2002.01231.x

727 Fletcher, EA, Lamb, KE, McNaughton, SA, Garnett, SP, Dunstan, DW, Baur, LA, Salmon, J. Cross-sectional and prospective mediating effects of dietary intake on the relationship between sedentary behaviour and body mass index in adolescents. BMC Public Health. 2017. 17:751. doi:10.1186/s12889-017-4771-0

728 Fletcher, ES, Rugg-Gunn, AJ, Matthews, JN, Hackett, A, Moynihan, PJ, Mathers, JC, Adamson, AJ. Changes over 20 years in macronutrient intake and body mass index in 11- to 12-year-old adolescents living in Northumberland. Br $\mathrm{J} \mathrm{Nutr.} 2004$. 92:321-33. doi:10.1079/bjn20041199

729 Fletcher, S, Wright, C, Jones, A, Parkinson, K, Adamson, A. Tracking of toddler fruit and vegetable preferences to intake and adiposity later in childhood. Matern Child Nutr. 2017. 13:. doi:10.1111/mcn.12290

730 Flock, MR, Kris-Etherton, PM. Dietary Guidelines for Americans 2010: implications for cardiovascular disease. Curr Atheroscler Rep. 2011. 13:499-507. doi:10.1007/s11883-011-0205-0

731 Flood, A, Mai, V, Pfeiffer, R, Kahle, L, Remaley, AT, Rosen, CJ, Lanza, E, Schatzkin, A. The effects of a high-fruit and vegetable, high-fiber, low-fat dietary intervention on serum concentrations of insulin, glucose, IGF-I and IGFBP-3. Eur J Clin Nutr. 2008. 62:186-96. doi:10.1038/sj.ejcn.1602726

732 Florencio, TM, Bueno, NB, Clemente, AP, Albuquerque, FC, Britto, RP, Ferriolli, E, Sawaya, AL. Weight gain and reduced energy expenditure in low-income Brazilian women living in slums: a 4-year follow-up study. Br J Nutr. 2015. 114:462-71. doi: $10.1017 / \mathrm{s} 0007114515001816$

733 Flores, M, Macias, N, Rivera, M, Lozada, A, Barquera, S, Rivera-Dommarco, J, Tucker, KL. Dietary patterns in Mexican adults are associated with risk of being overweight or obese. J Nutr. 2010. 140:1869-73. doi:10.3945/jn.110.121533 
734 Fonseca, MJ, Durao, C, Lopes, C, Santos, AC. Weight following birth and childhood dietary intake: A prospective cohort study. Nutrition. 2017. 33:58-64. doi:10.1016/j.nut.2016.08.008

735 Foraker, RE, Pennell, M, Sprangers, P, Vitolins, MZ, DeGraffinreid, C, Paskett, ED. Effect of a low-fat or low-carbohydrate weight-loss diet on markers of cardiovascular risk among premenopausal women: a randomized trial. J Womens Health (Larchmt). 2014. 23:675-80. doi:10.1089/jwh.2013.4638

736 Ford, CN, Weber, MB, Staimez, LR, Anjana, RM, Lakshmi, K, Mohan, V, Narayan, KMV, Harish, R. Dietary changes in a diabetes prevention intervention among people with prediabetes: the Diabetes Community Lifestyle Improvement Program trial. Acta Diabetologica. 2019. 56:197-209. doi:10.1007/s00592-018-1249-1

737 Ford, DW, Hartman, TJ, Still, C, Wood, C, Mitchell, DC, Erickson, P, Bailey, R, Smiciklas-Wright, H, Coffman, DL, Jensen, GL. Body mass index, poor diet quality, and health-related quality of life are associated with mortality in rural older adults. J Nut Gerontol Geriatr. 2014. 33:23-34. doi:10.1080/21551197.2014.875819

738 Forouhi, NG, Sharp, SJ, Du, H, van der, DI A, Halkjaer, J, Schulze, MB, Tjonneland, A, Overvad, K, Jakobsen, MU, Boeing, H, Buijsse, B, Palli, D, Masala, G, Feskens, EJ, Sorensen, TI, Wareham, NJ. Dietary fat intake and subsequent weight change in adults: results from the European Prospective Investigation into Cancer and Nutrition cohorts. Am J Clin Nutr. 2009. 90:1632-41. doi:10.3945/ajcn.2009.27828

739 Forsythe, CE, French, MA, Goh, YK, Clandinin, MT. Cholesterolaemic influence of palmitic acid in the sn-1, 3 v. the sn-2 position with high or low dietary linoleic acid in healthy young men. Br J Nutr. 2007. 98:337-44. doi:10.1017/s0007114507704993

740 Forsythe, CE, Phinney, SD, Feinman, RD, Volk, BM, Freidenreich, D, Quann, E, Ballard, K, Puglisi, MJ, Maresh, CM, Kraemer, WJ, Bibus, DM, Fernandez, ML, Volek, JS. Limited effect of dietary saturated fat on plasma saturated fat in the context of a low carbohydrate diet. Lipids. 2010. 45:947-62. doi:10.1007/s11745-010-3467-3

741 Fortes, C, Forastiere, F, Farchi, S, Rapiti, E, Pastori, G, Perucci, CA. Diet and overall survival in a cohort of very elderly people. Epidemiology. 2000. 11:440-5. doi:10.1097/00001648-200007000-00013

742 Foscolou, A, Georgousopoulou, E, Magriplis, E, Naumovski, N, Rallidis, L, Matalas, AL, Chrysohoou, C, Tousoulis, D, Pitsavos, C, Panagiotakos, D. The mediating role of Mediterranean diet on the association between Lp(a) levels and cardiovascular disease risk: A 10-year follow-up of the ATTICA study. Clin Biochem. 2018. 60:33-37. doi:10.1016/j.clinbiochem.2018.07.011

743 Foscolou, A, Magriplis, E, Tyrovolas, S, Soulis, G, Bountziouka, V, Mariolis, A, Piscopo, S, Valacchi, G, Anastasiou, F, Gotsis, E, Metallinos, G, Tyrovola, D, Polystipioti, A, Polychronopoulos, E, Matalas, AL, Lionis, C, Zeimbekis, A, Tur, JA, Sidossis, LS, Panagiotakos, D. Lifestyle determinants of healthy ageing in a Mediterranean population: The multinational MEDIS study. Exp Gerontol. 2018. 110:35-41. doi:10.1016/j.exger.2018.05.008

744 Foscolou, A, Polychronopoulos, E, Paka, E, Tyrovolas, S, Bountziouka, V, Zeimbekis, A, Tyrovola, D, Ural, D, Panagiotakos, D. Lifestyle and health determinants of cardiovascular disease among Greek older adults living in Eastern Aegean Islands: An adventure within the MEDIS study. Hellenic J Cardiol. 2016. 57:407-414. doi:10.1016/j.hjc.2016.11.021
Intervention/Exposure; Outcome

Intervention/Exposure

Intervention/Exposure

Outcome

Intervention/Exposure

Study duration

Intervention/Exposure; Comparator

Intervention/Exposure; Comparator

Intervention/Exposure

Study Design;

Outcome

Study Design 
745 Foster, GD, Wyatt, HR, Hill, JO, Makris, AP, Rosenbaum, DL, Brill, C, Stein, RI, Mohammed, BS, Miller, B, Rader, DJ, Zemel, B, Wadden, TA, Tenhave, T, Newcomb, CW, Klein, S. Weight and metabolic outcomes after 2 years on a lowcarbohydrate versus low-fat diet: a randomized trial. Ann Intern Med. 2010. 153:147-57. doi:10.7326/0003-4819-153-3201008030-00005

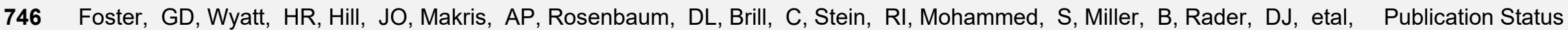
Weight and metabolic outcomes after 2 years on a low-carbohydrate versus low-fat diet. Obstetrical \& gynecological survey. 2010. 65:769-770. doi:10.1097/OGX.0b013e31821342ba

747 Foster, GD, Wyatt, HR, Hill, JO, McGuckin, BG, Brill, C, Mohammed, BS, Szapary, PO, Rader, DJ, Edman, JS, Klein, S. A randomized trial of a low-carbohydrate diet for obesity. N Engl J Med. 2003. 348:2082-90. doi:10.1056/NEJMoa022207

748 Franklin, KA, Eriksson, M, Larsson, C, Lindahl, B, Mellberg, C, Sahlin, C, Olsson, T, Ryberg, M. Palaeolithic diet and obstructive sleep apnoea in overweight females: a randomised controlled trial. European respiratory journal. 2016. 48: doi:10.1183/13993003.congress-2016.PA2376

749 Frankwich, KA, Egnatios, J, Kenyon, ML, Rutledge, TR, Liao, PS, Gupta, S, Herbst, KL, Zarrinpar, A. Differences in Weight Power/Size Loss Between Persons on Standard Balanced vs Nutrigenetic Diets in a Randomized Controlled Trial. Clin Gastroenterol Hepatol. 2015. 13:1625-1632.e1. doi:10.1016/j.cgh.2015.02.044

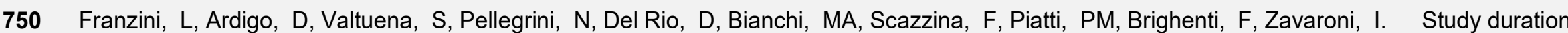
Food selection based on high total antioxidant capacity improves endothelial function in a low cardiovascular risk population. Nutr Metab Cardiovasc Dis. 2012. 22:50-7. doi:10.1016/j.numecd.2010.04.001

751 Franzon, K, Byberg, L, Sjogren, P, Zethelius, B, Cederholm, T, Kilander, L. Predictors of Independent Aging and Survival: A Outcome 16-Year Follow-Up Report in Octogenarian Men. J Am Geriatr Soc. 2017. 65:1953-1960. doi:10.1111/jgs.14971

752 Fraser, D, Bilenko, N, Vardy, H, Abu-Saad, K, Shai, I, Abu-Shareb, H, Shahar, DR. Differences in food intake and disparity in obesity rates between adult Jews and Bedouins in Southern Israel. Ethnicity and Disease. 2008. 18:13-18. doi:unavailable

753 Freire, RD, Cardoso, MA, Gimeno, SG, Ferreira, SR. Dietary fat is associated with metabolic syndrome in Japanese Brazilians. Diabetes Care. 2005. 28:1779-85. doi:10.2337/diacare.28.7.1779

754 Freisling, H, Pisa, PT, Ferrari, P, Byrnes, G, Moskal, A, Dahm, CC, Vergnaud, AC, Boutron-Ruault, MC, Fagherazzi, G, Cadeau, C, Kuhn, T, Neamat-Allah, J, Buijsse, B, Boeing, H, Halkjaer, J, Tjonneland, A, Hansen, CP, Quiros, JR, Travier, N, Molina-Montes, E, Amiano, P, Huerta, JM, Barricarte, A, Khaw, KT, Wareham, N, Key, TJ, Romaguera, D, Lu, Y, Lassale, CM, Naska, A, Orfanos, P, Trichopoulou, A, Masala, G, Pala, V, Berrino, F, Tumino, R, Ricceri, F, de Magistris, MS, Bueno-de-Mesquita, HB, Ocke, MC, Sonestedt, E, Ericson, U, Johansson, M, Skeie, G, Weiderpass, E, Braaten, T, Peeters, PH, Slimani, N. Main nutrient patterns are associated with prospective weight change in adults from 10 European countries. Eur J Nutr. 2016. 55:2093-104. doi:10.1007/s00394-015-1023-X

755 Freitas-Vilela, AA, Smith, AD, Kac, G, Pearson, RM, Heron, J, Emond, A, Hibbeln, JR, Castro, MB, Emmett, PM. Dietary patterns by cluster analysis in pregnant women: relationship with nutrient intakes and dietary patterns in 7-year-old offspring. Matern Child Nutr. 2017. 13:. doi:10.1111/mcn.12353

Study Design

Study Design

Study Design;

Intervention/Exposure

\section{Study Design}


756 French, SA, Harnack, L, Jeffery, RW. Fast food restaurant use among women in the Pound of Prevention study: dietary,

Intervention/Exposure behavioral and demographic correlates. Int J Obes Relat Metab Disord. 2000. 24:1353-9. doi:10.1038/sj.ijo.0801429

757 Frisch, S, Zittermann, A, Berthold, HK, Gotting, C, Kuhn, J, Kleesiek, K, Stehle, P, Kortke, H. A randomized controlled trial on the efficacy of carbohydrate-reduced or fat-reduced diets in patients attending a telemedically guided weight loss program. Cardiovasc Diabetol. 2009. 8:36. doi:10.1186/1475-2840-8-36

758 Fritzen, AM, Lundsgaard, AM, Jordy, AB, Poulsen, SK, Stender, S, Pilegaard, H, Astrup, A, Larsen, TM, Wojtaszewski, JF, Richter, EA, Kiens, B. New Nordic Diet-Induced Weight Loss Is Accompanied by Changes in Metabolism and AMPK Signaling in Adipose Tissue. J Clin Endocrinol Metab. 2015. 100:3509-19. doi:10.1210/jc.2015-2079

759 Frolich, S, Lehmann, N, Weyers, S, Wahl, S, Dragano, N, Budde, T, Kalsch, H, Mahabadi, AA, Erbel, R, Moebus, S, Jockel, $\mathrm{KH}$, Schmidt, B. Association of dietary patterns with five-year degree and progression of coronary artery calcification in the Heinz Nixdorf Recall study. Nutr Metab Cardiovasc Dis. 2017. 27:999-1007. doi:10.1016/j.numecd.2017.09.002

760 Fuehrlein, BS, Rutenberg, MS, Silver, JN, Warren, MW, Theriaque, DW, Duncan, GE, Stacpoole, PW, Brantly, ML. Differential metabolic effects of saturated versus polyunsaturated fats in ketogenic diets. J Clin Endocrinol Metab. 2004. 89:1641-5. doi:10.1210/jc.2003-031796

761 Fuentes, F, Lopez-Miranda, J, Perez-Martinez, P, Jimenez, Y, Marin, C, Gomez, P, Fernandez, JM, Caballero, J, DelgadoLista, J, Perez-Jimenez, F. Chronic effects of a high-fat diet enriched with virgin olive oil and a low-fat diet enriched with alphalinolenic acid on postprandial endothelial function in healthy men. Br J Nutr. 2008. 100:159-65. doi:10.1017/s0007114508888708

762 Fuentes, F, Lopez-Miranda, J, Sanchez, E, Sanchez, F, Paez, J, Paz-Rojas, E, Marin, C, Gomez, P, Jimenez-Pereperez, J, Ordovas, JM, Perez-Jimenez, F. Mediterranean and low-fat diets improve endothelial function in hypercholesterolemic men. Ann Intern Med. 2001. 134:1115-9. doi:10.7326/0003-4819-134-12-200106190-00011

763 Fukuda, Y, Umeno, Y, Taniguchi, Y, Marukawa, S, Kurihara, H, Nakajima, H, Yamasaki, T. Analysis of Dietary Factors Affecting Body Mass Index in Elderly Patients With Type 2 Diabetes Mellitus. J Clin Med Res. 2019. 11:563-571. doi:10.14740/jocmr3893

764 Fung, TT, Chiuve, SE, McCullough, ML, Rexrode, KM, Logroscino, G, Hu, FB. Adherence to a DASH-style diet and risk of coronary heart disease and stroke in women. Arch Intern Med. 2008. 168:713-20. doi:10.1001/archinte.168.7.713

765 Fung, TT, Feskanich, D. Dietary patterns and risk of hip fractures in postmenopausal women and men over 50 years. Osteoporos Int. 2015. 26:1825-30. doi:10.1007/s00198-015-3081-6

766 Fung, TT, Rimm, EB, Spiegelman, D, Rifai, N, Tofler, GH, Willett, WC, Hu, FB. Association between dietary patterns and plasma biomarkers of obesity and cardiovascular disease risk. Am J Clin Nutr. 2001. 73:61-7. doi:10.1093/ajcn/73.1.61

767 Fung, TT, Schulze, M, Manson, JE, Willett, WC, Hu, FB. Dietary patterns, meat intake, and the risk of type 2 diabetes in women. Arch Intern Med. 2004. 164:2235-40. doi:10.1001/archinte.164.20.2235

Weight

loss/Hypocaloric

Power/Size

Outcome

Study duration

Intervention/Exposure; Outcome

Intervention/Exposure

Study Design; Health Status

Publication Date Overlaps with Existing Review

Outcome

Intervention/Exposure

Publication Date

Overlaps with Existing Review 

stroke risk in women. Stroke. 2004. 35:2014-9. doi:10.1161/01.Str.0000135762.89154.92

\section{Rationale}

Publication Date

Overlaps with Existing Review

Study duration

Study duration doi:10.1016/j.yclnex.2017.05.002

770 Furtado, JD, Campos, H, Appel, LJ, Miller, ER, Laranjo, N, Carey, VJ, Sacks, FM. Effect of protein, unsaturated fat, and carbohydrate intakes on plasma apolipoprotein B and VLDL and LDL containing apolipoprotein C-III: results from the OmniHeart Trial. Am J Clin Nutr. 2008. 87:1623-30. doi:10.1093/ajcn/87.6.1623

771 Furtado, JD, Campos, H, Sumner, AE, Appel, LJ, Carey, VJ, Sacks, FM. Dietary interventions that lower lipoproteins containing apolipoprotein C-III are more effective in whites than in blacks: results of the OmniHeart trial. Am J Clin Nutr. 2010. 92:714-22. doi:10.3945/ajcn.2009.28532

772 Gadelha, Pcfp, de Arruda, IKG, Coelho, PBP, Queiroz, PMA, Maio, R, da Silva Diniz, A. Consumption of ultraprocessed foods, nutritional status, and dyslipidemia in schoolchildren: a cohort study. Eur J Clin Nutr. 2019. 73:1194-1199. doi:10.1038/s41430-019-0404-2

773 Gadgil, MD, Anderson, CA, Kandula, NR, Kanaya, AM. Dietary patterns in Asian Indians in the United States: an analysis of the metabolic syndrome and atherosclerosis in South Asians Living in America study. J Acad Nutr Diet. 2014. 114:238-43. doi:10.1016/j.jand.2013.09.021

774 Gadgil, MD, Appel, LJ, Yeung, E, Anderson, CA, Sacks, FM, Miller, ER, 3rd. The effects of carbohydrate, unsaturated fat, and protein intake on measures of insulin sensitivity: results from the OmniHeart trial. Diabetes Care. 2013. 36:1132-7. doi:10.2337/dc12-0869

775 Gaeini, Z, Bahadoran, Z, Mirmiran, P, Djazayery, A. The Association between Dietary Fat Pattern and the Risk of Type 2 Diabetes. Prev Nutr Food Sci. 2019. 24:1-7. doi:10.3746/pnf.2019.24.1.1

776 Gajda, K, Sulich, A, Hamulka, J, Bialkowska, A. Comparing diabetic with non-diabetic overweight subjects through assessing Study Design dietary intakes and key parameters of blood biochemistry and haematology. Rocz Panstw Zakl Hig. 2014. 65:133-8. doi:unavailable

777 Galan-Lopez, P, Ries, F, Gisladottir, T, Dominguez, R, Sanchez-Oliver, AJ. Healthy Lifestyle: Relationship between Mediterranean Diet, Body Composition and Physical Fitness in 13 to 16-Years Old Icelandic Students. Int J Environ Res Public Health. 2018. 15:. doi:10.3390/ijerph15122632

778 Galan-Lopez, P, Sanchez-Oliver, AJ, Ries, F, Gonzalez-Jurado, JA. Mediterranean Diet, Physical Fitness and Body Composition in Sevillian Adolescents: A Healthy Lifestyle. Nutrients. 2019. 11:. doi:10.3390/nu11092009

779 Galbreath, M, Campbell, B, LaBounty, P, Bunn, J, Dove, J, Harvey, T, Hudson, G, Gutierrez, JL, Levers, K, Galvan, E, Jagim, A, Greenwood, L, Cooke, MB, Greenwood, M, Rasmussen, C, Kreider, RB. Effects of Adherence to a Higher Protein Diet on Weight Loss, Markers of Health, and Functional Capacity in Older Women Participating in a Resistance-Based Exercise Program. Nutrients. 2018. 10:. doi:10.3390/nu10081070
Intervention/Exposure

Study Design

Study Design

Outcome; Study

duration

Intervention/Exposure

Study Design

Study Design

Intervention/Exposure 
780 Gao, J, Sun, H, Liang, X, Gao, M, Zhao, H, Qi, Y, Wang, Y, Liu, Y, Li, J, Zhu, Y, Zhao, Y, Wang, W, Ma, L, Wu, S. Ideal cardiovascular health behaviors and factors prevent the development of hypertension in prehypertensive subjects. Clin Exp Interventior Hypertens. 2015. 37:650-5. doi:10.3109/10641963.2015.1047938

781 Garaulet, M, Martinez, A, Victoria, F, Perez-Llamas, F, Ortega, RM, Zamora, S. Difference in dietary intake and activity level between normal-weight and overweight or obese adolescents. J Pediatr Gastroenterol Nutr. 2000. 30:253-8. doi:10.1097/00005176-200003000-00008

782 Garcia Hermoso, A, Saavedra Garcia, JM, Escalante Gonzalez, Y, Dominguez Pachon, AM. Effect of long-term physical exercise program and/or diet on metabolic syndrome in obese boys. Nutr Hosp. 2014. 30:94-103.

Country doi:10.3305/nh.2014.30.1.7448

783 Garcia-Arellano, A, Ramallal, R, Ruiz-Canela, M, Salas-Salvado, J, Corella, D, Shivappa, N, Schroder, H, Hebert, JR, Ros E, Gomez-Garcia, E, Estruch, R, Lapetra, J, Aros, F, Fiol, M, Serra-Majem, L, Pinto, X, Babio, N, Gonzalez, JI, Fito, M, Martinez, JA, Martinez-Gonzalez, MA. Dietary Inflammatory Index and Incidence of Cardiovascular Disease in the PREDIMED Study. Nutrients. 2015. 7:4124-38. doi:10.3390/nu7064124

784 Garcia-Calzon, S, Gea, A, Razquin, C, Corella, D, Lamuela-Raventos, RM, Martinez, JA, Martinez-Gonzalez, MA, Zalba, G Marti, A. Longitudinal association of telomere length and obesity indices in an intervention study with a Mediterranean diet: the PREDIMED-NAVARRA trial. Int J Obes (Lond). 2014. 38:177-82. doi:10.1038/ijo.2013.68

785 Garcia-Calzon, S, Martinez-Gonzalez, MA, Razquin, C, Corella, D, Salas-Salvado, J, Martinez, JA, Zalba, G, Marti, A. Pro12Ala polymorphism of the PPARgamma2 gene interacts with a mediterranean diet to prevent telomere shortening in the PREDIMED-NAVARRA randomized trial. Circ Cardiovasc Genet. 2015. 8:91-9. doi:10.1161/circgenetics.114.000635

786 Garcia-Calzon, S, Zalba, G, Ruiz-Canela, M, Shivappa, N, Hebert, JR, Martinez, JA, Fito, M, Gomez-Gracia, E, MartinezGonzalez, MA, Marti, A. Dietary inflammatory index and telomere length in subjects with a high cardiovascular disease risk from the PREDIMED-NAVARRA study: cross-sectional and longitudinal analyses over 5 y. Am J Clin Nutr. 2015. 102:897-904. doi:10.3945/ajen.115.116863

787 García-López, M, Toledo, E, Beunza, JJ, Aros, F, Estruch, R, Salas-Salvadó, J, Corella, D, Ros, E, Covas, MI, GómezGracia, E, etal, . Mediterranean diet and heart rate: the PREDIMED randomised trial. International journal of cardiology. 2014 171:299-301. doi:10.1016/j.ijcard.2013.11.074

788 Garcia-Perez, I, Posma, JM, Gibson, R, Chambers, ES, Hansen, TH, Vestergaard, H, Hansen, T, Beckmann, M, Pedersen, O, Elliott, P, Stamler, J, Nicholson, JK, Draper, J, Mathers, JC, Holmes, E, Frost, G. Objective assessment of dietary patterns by use of metabolic phenotyping: a randomised, controlled, crossover trial. Lancet Diabetes Endocrinol. 2017. 5:184195. doi:10.1016/s2213-8587(16)30419-3

789 Garcia-Rios, A, Alcala-Diaz, JF, Gomez-Delgado, F, Delgado-Lista, J, Marin, C, Leon-Acuna, A, Camargo, A, RodriguezCantalejo, F, Blanco-Rojo, R, Quintana-Navarro, G, etal, . Beneficial effect of CETP gene polymorphism in combination with a Mediterranean diet influencing lipid metabolism in metabolic syndrome patients: CORDIOPREV study. Clinical nutrition. (no pagination), 2016. 2016. Date of Publication: February 15:. doi:10.1016/j.clnu.2016.12.011

Study Design;

Intervention/Exposure

Intervention/Exposure; Comparator

Intervention/Exposure

Intervention/Exposure Intervention/Exposure; Outcome

Intervention/Exposure

Publication Status

Study duration

Intervention/Exposure; Health Status 
790 Garcia-Silva, J, Navarrete N N, Peralta-Ramirez, MI, Garcia-Sanchez, A, Ferrer-Gonzalez, MA, Caballo, VE. Efficacy of Cognitive Behavioral Therapy in Adherence to the Mediterranean Diet in Metabolic Syndrome Patients: A Randomized Controlled Trial. J Nutr Educ Behav. 2018. 50:896-904. doi:10.1016/j.jneb.2018.06.003

791 Garcia-Unciti, M, Martinez, JA, Izquierdo, M, Gorostiaga, EM, Grijalba, A, Ibanez, J. Effect of resistance training and hypocaloric diets with different protein content on body composition and lipid profile in hypercholesterolemic obese women. Nutr Hosp. 2012. 27:1511-20. doi:10.3305/nh.2012.27.5.5921

792 Gardener, H, Wright, CB, Cabral, D, Scarmeas, N, Gu, Y, Cheung, K, Elkind, MS, Sacco, RL, Rundek, T. Mediterranean diet and carotid atherosclerosis in the Northern Manhattan Study. Atherosclerosis. 2014. 234:303-10. doi:10.1016/j.atherosclerosis.2014.03.011

793 Gardner, CD, Coulston, A, Chatterjee, L, Rigby, A, Spiller, G, Farquhar, JW. The effect of a plant-based diet on plasma lipids in hypercholesterolemic adults: a randomized trial. Ann Intern Med. 2005. 142:725-33. doi:10.7326/0003-4819-142-9200505030-00007

794 Gardner, CD, Hauser, M, Gobbo, LD, Trepanowski, J, Rigdon, J, loannidis, J, King, A, Desai, M. Neither insulin secretion nor genotype pattern modify 12-month weight loss effects of healthy low-fat vs. healthy low-carbohydrate diets among adults with obesity. Circulation. 2017. 135:. doi:unavailable

795 Gardner, CD, Kiazand, A, Alhassan, S, Kim, S, Stafford, RS, Balise, RR, Kraemer, HC, King, AC. Comparison of the Atkins, Zone, Ornish, and LEARN diets for change in weight and related risk factors among overweight premenopausal women: the A TO Z Weight Loss Study: a randomized trial. Jama. 2007. 297:969-77. doi:10.1001/jama.297.9.969

796 Gardner, CD, Offringa, LC, Hartle, JC, Kapphahn, K, Cherin, R. Weight loss on low-fat vs. low-carbohydrate diets by insulin resistance status among overweight adults and adults with obesity: A randomized pilot trial. Obesity (Silver Spring). 2016. 24:79-86. doi:10.1002/oby.21331

797 Garemo, M, Elamin, A, Gardner, A. Weight status and food habits of preschool children in Abu Dhabi, United Arab Emirates: NOPLAS project. Asia Pac J Clin Nutr. 2018. 27:1302-1314. doi:10.6133/apjcn.201811_27(6).0018

798 Garg, R, Sun, B, Williams, J. Effect of low salt diet on insulin resistance in salt-sensitive versus salt-resistant hypertension. Hypertension. 2014. 64:1384-7. doi:10.1161/hypertensionaha.114.03880

Power/Size

Outcome

Intervention/Exposur

Publication Status

Weight

loss/Hypocaloric

Weight

loss/Hypocaloric

Study Design

Intervention/Exposure; Study duration

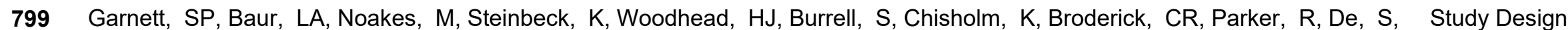
Shrinivasan, S, Hopley, L, Hendrie, G, Ambler, GR, Kohn, MR, Cowell, CT. Researching Effective Strategies to Improve Insulin Sensitivity in Children and Teenagers - RESIST. A randomised control trial investigating the effects of two different diets on insulin sensitivity in young people with insulin resistance and/or pre-diabetes. BMC Public Health. $2010.10: 575$. doi:10.1186/1471-2458-10-575

800 Garnett, SP, Gow, M, Ho, M, Baur, LA, Noakes, M, Woodhead, HJ, Broderick, CR, Burrell, S, Chisholm, K, Halim, J, De, S, Steinbeck, K, Srinivasan, S, Ambler, GR, Kohn, MR, Cowell, CT. Optimal macronutrient content of the diet for adolescents with prediabetes; RESIST a randomised control trial. J Clin Endocrinol Metab. 2013. 98:2116-25. doi:10.1210/jc.2012-4251 
801 Garnett, SP, Gow, M, Ho, M, Baur, LA, Noakes, M, Woodhead, HJ, Broderick, CR, Chisholm, K, Briody, J, De, S, Steinbeck, K, Srinivasan, S, Ambler, GR, Cowell, CT. Improved insulin sensitivity and body composition, irrespective of macronutrient intake, after a 12 month intervention in adolescents with pre-diabetes; RESIST a randomised control trial. BMC Pediatr. 2014. 14:289. doi:10.1186/s12887-014-0289-0

802 Garralda-Del-Villar, M, Carlos-Chilleron, S, Diaz-Gutierrez, J, Ruiz-Canela, M, Gea, A, Martinez-Gonzalez, MA, BesRastrollo, M, Ruiz-Estigarribia, L, Kales, SN, Fernandez-Montero, A. Healthy Lifestyle and Incidence of Metabolic Syndrome in the SUN Cohort. Nutrients. 2018. 11:. doi:10.3390/nu11010065

803 Gately, PJ, King, NA, Greatwood, HC, Humphrey, LC, Radley, D, Cooke, CB, Hill, AJ. Does a high-protein diet improve weight loss in overweight and obese children?. Obesity (Silver Spring). 2007. 15:1527-34. doi:10.1038/oby.2007.181

804 Gawęcka, A, Ryterska, K, Wysokiński, P, Ficek, K, Wilk, K, Banaszczak, M, Jakubczak, K, Piotrowki, J, Czerwińska, M, Hołowko, J, etal, . CHANGES IN BODY MASS AND BLOOD LIPID LEVELS IN PATIENTS UNDERGOING CALORIC RESTRICTION. Annales academiae medicae stetinensis. 2014. 60:29-33. doi:unavailable

805 Geiker, NRW, Toennesen, LL, Astrup, A, Backer, V. The efficacy of a high protein/low glycemic index diet intervention in nonobese patients with asthma. Eur J Clin Nutr. 2018. 72:511-516. doi:10.1038/s41430-018-0092-3

806 Gemmink, A, Bakker, LE, Guigas, B, Kornips, E, Schaart, G, Meinders, AE, Jazet, IM, Hesselink, MK. Lipid droplet dynamics and insulin sensitivity upon a 5-day high-fat diet in Caucasians and South Asians. Sci Rep. $2017.7: 42393$. doi:10.1038/srep42393

807 Genaro Pde, S, Pinheiro Mde, M, Szejnfeld, VL, Martini, LA. Dietary protein intake in elderly women: association with muscle and bone mass. Nutr Clin Pract. 2015. 30:283-9. doi:10.1177/0884533614545404

808 Genoni, A, Lyons-Wall, P, Lo, J, Devine, A. Cardiovascular, Metabolic Effects and Dietary Composition of Ad-Libitum Paleolithic vs. Australian Guide to Healthy Eating Diets: A 4-Week Randomised Trial. Nutrients. 2016. 8:. doi:10.3390/nu8050314

809 Georgiopoulos, G, Karatzi, K, Euthimiou, E, Laina, A, Kontogiannis, C, Mareti, A, Mavroeidis, I, Kouzoupis, A, Mitrakou, A, Papamichael, C, Stamatelopoulos, K. Association of macronutrient consumption with arterial aging in adults without clinically overt cardiovascular disease: a 5-year prospective cohort study. Eur J Nutr. 2019. 58:2305-2314. doi:10.1007/s00394-0181781-3

810 Georgoulis, M, Labrou, K, Yiannakouris, N, Mourati, I, Vagiakis, E, Kechribari, I, Kokkinos, A, Kontogianni, M. Effects of a weight-loss Mediterranean lifestyle intervention on obstructive sleep apnea: preliminary results of a randomized controlled clinical trial. Journal of sleep research. 2018. 27:149-150. doi:10.1111/jsr.12751

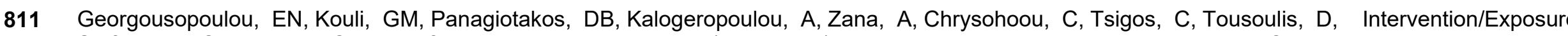
Stefanadis, C, Pitsavos, C. Anti-inflammatory diet and 10-year (2002-2012) cardiovascular disease incidence: The ATTICA study. Int J Cardiol. 2016. 222:473-478. doi:10.1016/j.ijcard.2016.08.007 
812 Georgousopoulou, EN, Mellor, DD, Naumovski, N, Polychronopoulos, E, Tyrovolas, S, Piscopo, S, Valacchi, G, Anastasiou,

F, Zeimbekis, A, Bountziouka, V, Gotsis, E, Metallinos, G, Tyrovola, D, Foscolou, A, Tur, JA, Matalas, AL, Lionis, C,

Sidossis, L, Panagiotakos, D. Mediterranean lifestyle and cardiovascular disease prevention. Cardiovasc Diagn Ther. 2017.

7:S39-s47. doi:10.21037/cdt.2017.03.11

813 Gepner, Y, Shelef, I, Komy, O, Cohen, N, Schwarzfuchs, D, Bril, N, Rein, M, Serfaty, D, Kenigsbuch, S, Zelicha, H, Yaskolka Meir, A, Tene, L, Bilitzky, A, Tsaban, G, Chassidim, Y, Sarusy, B, Ceglarek, U, Thiery, J, Stumvoll, M, Bluher, M, Stampfer, MJ, Rudich, A, Shai, I. The beneficial effects of Mediterranean diet over low-fat diet may be mediated by decreasing hepatic fat content. J Hepatol. 2019. 71:379-388. doi:10.1016/j.jhep.2019.04.013

814 Gerhard, GT, Connor, SL, Wander, RC, Connor, WE. Plasma lipid and lipoprotein responsiveness to dietary fat and cholesterol in premenopausal African American and white women. Am J Clin Nutr. 2000. 72:56-63. doi:10.1093/ajcn/72.1.56

815 Giacco, R, Lappi, J, Costabile, G, Kolehmainen, M, Schwab, U, Landberg, R, Uusitupa, M, Poutanen, K, Pacini, G, Rivellese, AA, Riccardi, G, Mykkanen, H. Effects of rye and whole wheat versus refined cereal foods on metabolic risk factors: a randomised controlled two-centre intervention study. Clin Nutr. 2013. 32:941-9. doi:10.1016/j.clnu.2013.01.016

816 Giardina, S, Hernandez-Alonso, P, Diaz-Lopez, A, Salas-Huetos, A, Salas-Salvado, J, Bullo, M. Changes in circulating miRNAs in healthy overweight and obese subjects: Effect of diet composition and weight loss. Clin Nutr. 2019. 38:438-443. doi:10.1016/j.clnu.2017.11.014

817 Gibas, MK, Gibas, KJ. Induced and controlled dietary ketosis as a regulator of obesity and metabolic syndrome pathologies. Diabetes Metab Syndr. 2017. 11 Suppl 1:S385-s390. doi:10.1016/j.dsx.2017.03.022

818 Gibbs, BB, Tudorascu, D, Bryce, C, Comer, D, Fischer, G, Hess, R, Huber, K, McTigue, K, Simkin-Silverman, L, Conroy, MB. Dietary habits associated with 6-and 24-month weight loss maintenance in primary care patients. Circulation. 2018. 137: doi:unavailable

819 Gilardini, L, Croci, M, Pasqualinotto, L, Caffetto, K, Invitti, C. Dietary Habits and Cardiometabolic Health in Obese Children Obes Facts. 2015. 8:101-9. doi:10.1159/000381157

820 Gilis-Januszewska, A, Barengo, NC, Lindstrom, J, Wojtowicz, E, Acosta, T, Tuomilehto, J, Schwarz, PEH, Piwonska-Solska B, Szybinski, Z, Windak, A, Hubalewska-Dydejczyk, A. Predictors of long term weight loss maintenance in patients at high risk of type 2 diabetes participating in a lifestyle intervention program in primary health care: The DE-PLAN study. PLoS One. 2018. 13:e0194589. doi:10.1371/journal.pone.0194589

821 Gillingham, MB, Purnell, JQ, Jordan, J, Stadler, D, Haqq, AM, Harding, CO. Effects of higher dietary protein intake on energy balance and metabolic control in children with long-chain 3-hydroxy acyl-CoA dehydrogenase (LCHAD) or trifunctional protein (TFP) deficiency. Mol Genet Metab. 2007. 90:64-9. doi:10.1016/j.ymgme.2006.08.002

822 Gingras, V, Rifas-Shiman, SL, Taveras, EM, Oken, E, Hivert, MF. Dietary behaviors throughout childhood are associated with adiposity and estimated insulin resistance in early adolescence: a longitudinal study. Int J Behav Nutr Phys Act. 2018. 15:129. doi:10.1186/s12966-018-0759-0 
823 Ginos, BNR, Navarro, SL, Schwarz, Y, Gu, H, Wang, D, Randolph, TW, Shojaie, A, Hullar, MAJ, Lampe, PD, Kratz, M,

Neuhouser, ML, Raftery, D, Lampe, JW. Circulating bile acids in healthy adults respond differently to a dietary pattern characterized by whole grains, legumes and fruits and vegetables compared to a diet high in refined grains and added sugars: $A$ randomized, controlled, crossover feeding study. Metabolism. 2018. 83:197-204. doi:10.1016/j.metabol.2018.02.006

824 Giontella, A, Bonafini, S, Tagetti, A, Bresadola, I, Minuz, P, Gaudino, R, Cavarzere, P, Ramaroli, DA, Marcon, D, Branz, L, Study Design Nicolussi Principe, L, Antoniazzi, F, Maffeis, C, Fava, C. Relation between Dietary Habits, Physical Activity, and Anthropometric and Vascular Parameters in Children Attending the Primary School in the Verona South District. Nutrients. 2019. 11: doi:10.3390/nu11051070

825 Glabska, D, Cackowska, K, Guzek, D. Comparison of the Body Composition of Caucasian Young Normal Body Mass Women Measured in the Follicular Phase, Depending on the Carbohydrate Diet Level. Medicina (Kaunas). 2018. 54:. doi:10.3390/medicina54060104

826 Goff, LM, Frost, GS, Hamilton, G, Thomas, EL, Dhillo, WS, Dornhorst, A, Bell, JD. Carbohydrate-induced manipulation of insulin sensitivity independently of intramyocellular lipids. Br J Nutr. 2003. 89:365-75. doi:10.1079/bjn2002789

827 Gogebakan, O, Kohl, A, Osterhoff, MA, van Baak, MA, Jebb, SA, Papadaki, A, Martinez, JA, Handjieva-Darlenska, T, Hlavaty, P, Weickert, MO, Holst, C, Saris, WH, Astrup, A, Pfeiffer, AF. Effects of weight loss and long-term weight maintenance with diets varying in protein and glycemic index on cardiovascular risk factors: the diet, obesity, and genes (DiOGenes) study: a randomized, controlled trial. Circulation. 2011. 124:2829-38. doi:10.1161/circulationaha.111.033274

828 Gokmen-Ozel, H, Ferguson, C, Evans, S, Daly, A, MacDonald, A. Does a lower carbohydrate protein substitute impact on blood phenylalanine control, growth and appetite in children with PKU?. Mol Genet Metab. 2011. 104 Suppl:S64-7. doi:10.1016/j.ymgme.2011.09.014

829 Goldberg, JM, O'Mara, K. Metabolic and anthropometric changes in obese subjects from an unrestricted calorie, high monounsaturated fat, very low carbohydrate diet. Journal of Clinical Ligand Assay. 2000. 23:97-103. doi:unavailable

830 Goldberg, R, Temprosa, M, Otvos, J, Brunzell, J, Marcovina, S, Mather, K, Arakaki, R, Watson, K, Horton, E, BarrettConnor, E. Lifestyle and metformin treatment favorably influence lipoprotein subfraction distribution in the Diabetes Prevention Program. J Clin Endocrinol Metab. 2013. 98:3989-98. doi:10.1210/jc.2013-1452

831 Goletzke, J, Herder, C, Joslowski, G, Bolzenius, K, Remer, T, Wudy, SA, Roden, M, Rathmann, W, Buyken, AE. Habitually higher dietary glycemic index during puberty is prospectively related to increased risk markers of type 2 diabetes in younger adulthood. Diabetes Care. 2013. 36:1870-1876. doi:10.2337/dc12-2063

832 Golley, RK, Hendrie, GA. Evaluation of the relative concentration of serum fatty acids C14:0, C15:0 and C17:0 as markers of children's dairy fat intake. Ann Nutr Metab. 2014. 65:310-6. doi:10.1159/000368325

833 Golzarand, M, Bahadoran, Z, Mirmiran, P, Azizi, F. Protein Foods Group and 3-Year Incidence of Hypertension: A Prospective Study From Tehran Lipid and Glucose Study. J Ren Nutr. 2016. 26:219-25. doi:10.1053/j.jrn.2016.01.017

834 Golzarand, M, Bahadoran, Z, Mirmiran, P, Sadeghian-Sharif, S, Azizi, F. Dietary phytochemical index is inversely associated with the occurrence of hypertension in adults: a 3-year follow-up (the Tehran Lipid and Glucose Study). Eur J Clin Nutr. 2015. 69:392-8. doi:10.1038/ejcn.2014.233

Study Design

Intervention/Exposure

Intervention/Exposure; Study duration

Intervention/Exposure

Health Status

Study Design

Intervention/Exposure

Intervention/Exposure; Comparator

Intervention/Exposure; Outcome

Intervention/Exposure

Intervention/Exposure; Outcome 
835 Gomez-Arbelaez, D, Bellido, D, Castro, Al, Ordonez-Mayan, L, Carreira, J, Galban, C, Martinez-Olmos, MA, Crujeiras, AB,

Sajoux, I, Casanueva, FF. Body Composition Changes After Very-Low-Calorie Ketogenic Diet in Obesity Evaluated by 3 Standardized Methods. J Clin Endocrinol Metab. 2017. 102:488-498. doi:10.1210/jc.2016-2385

836 Gomez-Arbelaez, D, Crujeiras, AB, Castro, Al, Martinez-Olmos, MA, Canton, A, Ordonez-Mayan, L, Sajoux, I, Galban, C, Bellido, D, Casanueva, FF. Resting metabolic rate of obese patients under very low calorie ketogenic diet. Nutr Metab (Lond). 2018. 15:18. doi:10.1186/s12986-018-0249-z

837 Gomez-Delgado, F, Alcala-Diaz, JF, Garcia-Rios, A, Delgado-Lista, J, Ortiz-Morales, A, Rangel-Zuniga, O, Tinahones, FJ, Gonzalez-Guardia, L, Malagon, MM, Bellido-Munoz, E, Ordovas, JM, Perez-Jimenez, F, Lopez-Miranda, J, Perez-Martinez, P. Polymorphism at the TNF-alpha gene interacts with Mediterranean diet to influence triglyceride metabolism and inflammation status in metabolic syndrome patients: From the CORDIOPREV clinical trial. Mol Nutr Food Res. 2014. 58:1519-27. doi:10.1002/mnfr.201300723

838 Gomez-Huelgas, R, Jansen-Chaparro, S, Baca-Osorio, AJ, Mancera-Romero, J, Tinahones, FJ, Bernal-Lopez, MR. Effects of a long-term lifestyle intervention program with Mediterranean diet and exercise for the management of patients with metabolic syndrome in a primary care setting. Eur J Intern Med. 2015. 26:317-23. doi:10.1016/j.ejim.2015.04.007

839 Gomez-Marin, B, Gomez-Delgado, F, Lopez-Moreno, J, Alcala-Diaz, JF, Jimenez-Lucena, R, Torres-Pena, JD, Garcia-Rios, A, Ortiz-Morales, AM, Yubero-Serrano, EM, Del Mar Malagon, M, Lai, CQ, Delgado-Lista, J, Ordovas, JM, Lopez-Miranda, J, Perez-Martinez, P. Long-term consumption of a Mediterranean diet improves postprandial lipemia in patients with type 2 diabetes: the Cordioprev randomized trial. Am J Clin Nutr. 2018. 108:963-970. doi:10.1093/ajcn/nqy144

840 Goni, L, Riezu-Boj, JI, Milagro, FI, Corrales, FJ, Ortiz, L, Cuervo, M, Martinez, JA. Interaction between an ADCY3 Genetic Variant and Two Weight-Lowering Diets Affecting Body Fatness and Body Composition Outcomes Depending on Macronutrient Distribution: A Randomized Trial. Nutrients. 2018. 10:. doi:10.3390/nu10060789

841 Goni, L, Sun, D, Heianza, Y, Wang, T, Huang, T, Cuervo, M, Martinez, JA, Shang, X, Bray, GA, Sacks, FM, Qi, L. Macronutrient-specific effect of the MTNR1B genotype on lipid levels in response to 2 year weight-loss diets. J Lipid Res. 2018. 59:155-161. doi:10.1194/jlr.P078634

842 Goni, L, Sun, D, Heianza, Y, Wang, T, Huang, T, Martinez, JA, Shang, X, Bray, GA, Smith, SR, Sacks, FM, Qi, L. A circadian rhythm-related MTNR1B genetic variant modulates the effect of weight-loss diets on changes in adiposity and body composition: the POUNDS Lost trial. Eur J Nutr. 2019. 58:1381-1389. doi:10.1007/s00394-018-1660-y

843 González Devia, LJ, Monroy Romero, PA, Almonacid Urrego, CC, Orjuela, OL, Huérfano, MJ, Mendieta Zerón, H. Comparative study of risk factors related to cardiovascular disease in children from Bogotá, Colombia and Toluca, Mexico. Revista de la Facultad de Ciencias Médicas (Córdoba, Argentina). 2014. 71:98-105. doi:unavailable

844 Gooding, HC, Shay, CM, Ning, H, Gillman, MW, Chiuve, SE, Reis, JP, Allen, NB, Lloyd-Jones, DM. Optimal Lifestyle Components in Young Adulthood Are Associated With Maintaining the Ideal Cardiovascular Health Profile Into Middle Age. J Am Heart Assoc. 2015. 4:. doi:10.1161/jaha.115.002048

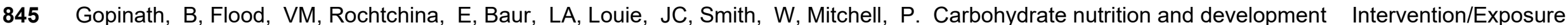
of adiposity during adolescence. Obesity (Silver Spring). 2013. 21:1884-90. doi:10.1002/oby.20405

Study Design;

Intervention/Exposure

Intervention/Exposure; Comparator

Intervention/Exposure

Health Status

Weight

loss/Hypocaloric

Intervention/Exposure

Intervention/Exposure

Study Design

Intervention/Exposure; Outcome 


\begin{tabular}{|c|c|c|}
\hline No. & Citation & Rationale \\
\hline 846 & $\begin{array}{l}\text { Gopinath, B, Russell, J, Kifley, A, Flood, VM, Mitchell, P. Adherence to Dietary Guidelines and Successful Aging Over } 10 \\
\text { Years. J Gerontol A Biol Sci Med Sci. 2016. 71:349-55. doi:10.1093/gerona/glv189 }\end{array}$ & Outcome \\
\hline 847 & $\begin{array}{l}\text { Gorczyca, D, Pasciak, M, Szponar, B, Gamian, A, Jankowski, A. An impact of the diet on serum fatty acid and lipid profiles in } \\
\text { Polish vegetarian children and children with allergy. Eur J Clin Nutr. 2011. 65:191-5. doi:10.1038/ejcn.2010.231 }\end{array}$ & Study Design \\
\hline 848 & $\begin{array}{l}\text { Gordon, MM, Bopp, MJ, Easter, L, Miller, GD, Lyles, MF, Houston, DK, Nicklas, BJ, Kritchevsky, SB. Effects of dietary } \\
\text { protein on the composition of weight loss in post-menopausal women. J Nutr Health Aging. 2008. 12:505-9. doi:unavailable }\end{array}$ & $\begin{array}{l}\text { Weight } \\
\text { loss/Hypocaloric }\end{array}$ \\
\hline 849 & $\begin{array}{l}\text { Goree, LL, Chandler-Laney, P, Ellis, AC, Casazza, K, Granger, WM, Gower, BA. Dietary macronutrient composition affects } \\
\text { beta cell responsiveness but not insulin sensitivity. Am J Clin Nutr. 2011. 94:120-7. doi:10.3945/ajcn.110.002162 }\end{array}$ & $\begin{array}{l}\text { Outcome; Study } \\
\text { duration }\end{array}$ \\
\hline 850 & $\begin{array}{l}\text { Gorna, I, Kowalowka, M, Morawska, A, Kosewski, G, Boleslawska, I, Przyslawskia, J. Influence of the frequency of } \\
\text { consumption of foodstuffs on the risk of overweight and obesity in a group of post-menopausal women. Prz Menopauzalny. } \\
\text { 2019. 18:39-45. doi:10.5114/pm.2019.84156 }\end{array}$ & $\begin{array}{l}\text { Study Design; } \\
\text { Intervention/Exposure }\end{array}$ \\
\hline 851 & $\begin{array}{l}\text { Gorostegi-Anduaga, I, Corres, P, Jurio-Iriarte, B, Martinez-Aguirre, A, Perez-Asenjo, J, Aispuru, GR, Arenaza, L, } \\
\text { Romaratezabala, E, Arratibel-Imaz, I, Mujika, I, Francisco-Terreros, S, Maldonado-Martin, S. Clinical, physical, physiological, } \\
\text { and dietary patterns of obese and sedentary adults with primary hypertension characterized by sex and cardiorespiratory fitness: } \\
\text { EXERDIET-HTA study. Clin Exp Hypertens. 2018. 40:141-149. doi:10.1080/10641963.2017.1346111 }\end{array}$ & $\begin{array}{l}\text { Study Design; } \\
\text { Outcome }\end{array}$ \\
\hline 852 & $\begin{array}{l}\text { Goss, AM, Goree, LL, Ellis, AC, Chandler-Laney, PC, Casazza, K, Lockhart, ME, Gower, BA. Effects of diet macronutrient } \\
\text { composition on body composition and fat distribution during weight maintenance and weight loss. Obesity (Silver Spring). } 2013 . \\
21: 1139-42 \text {. doi:10.1002/oby. } 20191\end{array}$ & Study \\
\hline 853 & $\begin{array}{l}\text { Goss, AM, Gower, BA, Soleymani, T, Stewart, M, Fontaine, K. Effects of an egg-based, carbohydraterestricted diet on body } \\
\text { composition, fat distribution, and metabolic health in older adults with obesity: preliminary results from a randomized controlled } \\
\text { trial. FASEB journal. 2017. 31:. doi:unavailable }\end{array}$ & Publication Status \\
\hline 854 & $\begin{array}{l}\text { Goulet, J, Lamarche, B, Nadeau, G, Lemieux, S. Effect of a nutritional intervention promoting the Mediterranean food pattern } \\
\text { on plasma lipids, lipoproteins and body weight in healthy French-Canadian women. Atherosclerosis. 2003. 170:115-24. } \\
\text { doi:10.1016/s0021-9150(03)00243-0 }\end{array}$ & Study Design \\
\hline 855 & $\begin{array}{l}\text { Gower, B, Goss, A, Soleymani, T. Metabolically healthy obese individuals lose more visceral and total body fat with a low- } \\
\text { glycemic diet under controlled feeding conditions. Diabetes. 2016. 65:A70-. doi:10.2337/db16-1-381 }\end{array}$ & Publication Status \\
\hline 856 & $\begin{array}{l}\text { Gower, BA, Goree, LL, Chandler-Laney, PC, Ellis, AC, Casazza, K, Granger, WM. A higher-carbohydrate, lower-fat diet } \\
\text { reduces fasting glucose concentration and improves beta-cell function in individuals with impaired fasting glucose. Metabolism. } \\
\text { 2012. 61:358-65. doi:10.1016/j.metabol.2011.07.011 }\end{array}$ & Study duration \\
\hline 857 & $\begin{array}{l}\text { Gower, BA, Goss, AM. A lower-carbohydrate, higher-fat diet reduces abdominal and intermuscular fat and increases insulin } \\
\text { sensitivity in adults at risk of type } 2 \text { diabetes. J Nutr. 2015. 145:177s-83s. doi:10.3945/jn.114.195065 }\end{array}$ & $\begin{array}{l}\text { Intervention/Expo } \\
\text { Study duration }\end{array}$ \\
\hline
\end{tabular}


858 Goyenechea, E, Holst, C, van Baak, MA, Saris, WH, Jebb, S, Kafatos, A, Pfeiffer, A, Handjiev, S, Hlavaty, P, Stender, S Larsen, TM, Astrup, A, Martinez, JA. Effects of different protein content and glycaemic index of ad libitum diets on diabetes risk factors in overweight adults: the DIOGenes multicentre, randomized, dietary intervention trial. Diabetes Metab Res Rev. 2011. 27:705-16. doi:10.1002/dmrr.1218

859 Grafenauer, SJ, Tapsell, LC, Beck, EJ, Batterham, MJ. Baseline dietary patterns are a significant consideration in correcting dietary exposure for weight loss. Eur J Clin Nutr. 2013. 67:330-6. doi:10.1038/ejcn.2013.26

860 Graffouillere, L, Deschasaux, M, Mariotti, F, Neufcourt, L, Shivappa, N, Hebert, JR, Wirth, MD, Latino-Martel, P, Hercberg, S, Galan, P, Julia, C, Kesse-Guyot, E, Touvier, M. Prospective association between the Dietary Inflammatory Index and mortality: modulation by antioxidant supplementation in the SU.VI.MAX randomized controlled trial. Am J Clin Nutr. 2016. 103:878-85. doi:10.3945/ajcn.115.126243

861 Grandl, G, Straub, L, Rudigier, C, Arnold, M, Wueest, S, Konrad, D, Wolfrum, C. Short-term feeding of a ketogenic diet induces more severe hepatic insulin resistance than an obesogenic high-fat diet. J Physiol. 2018. 596:4597-4609. doi:10.1113/jp275173

862 Granic, A, Mendonca, N, Sayer, AA, Hill, TR, Davies, K, Siervo, M, Mathers, JC, Jagger, C. Effects of dietary patterns and low protein intake on sarcopenia risk in the very old: The Newcastle 85+ study. Clin Nutr. 2019. : doi:10.1016/j.clnu.2019.01.009

863 Grau, K, Tetens, I, Bjornsbo, KS, Heitman, BL. Overall glycaemic index and glycaemic load of habitual diet and risk of heart disease. Public Health Nutr. 2011. 14:109-18. doi:10.1017/s136898001000176x

864 Gray, A, Smith, C. Fitness, dietary intake, and body mass index in urban Native American youth. J Am Diet Assoc. 2003. 103:1187-91. doi:10.1016/s0002-8223(03)00979-9

865 Gray, DL, O'Brien, KD, D'Alessio, DA, Brehm, BJ, Deeg, MA. Plasma glycosylphosphatidylinositol-specific phospholipase D predicts the change in insulin sensitivity in response to a low-fat but not a low-carbohydrate diet in obese women. Metabolism. 2008. 57:473-8. doi:10.1016/j.metabol.2007.11.007

866 Gray-Donald, K, St-Arnaud-McKenzie, D, Gaudreau, P, Morais, JA, Shatenstein, B, Payette, H. Protein intake protects against weight loss in healthy community-dwelling older adults. J Nutr. 2014. 144:321-6. doi:10.3945/jn.113.184705

867 Greco, M, Chiefari, E, Montalcini, T, Accattato, F, Costanzo, FS, Pujia, A, Foti, D, Brunetti, A, Gulletta, E. Early effects of a hypocaloric, Mediterranean diet on laboratory parameters in obese individuals. Mediators Inflamm. 2014. 2014:750860. doi:10.1155/2014/750860

868 Green, CA, Yarborough, BJ, Leo, MC, Yarborough, MT, Stumbo, SP, Janoff, SL, Perrin, NA, Nichols, GA, Stevens, VJ. The STRIDE weight loss and lifestyle intervention for individuals taking antipsychotic medications: a randomized trial. Am J Psychiatry. 2015. 172:71-81. doi:10.1176/appi.ajp.2014.14020173

869 Green, JG, Johnson, NA, Sachinwalla, T, Cunningham, CW, Thompson, MW, Stannard, SR. Low-carbohydrate diet does not affect intramyocellular lipid concentration or insulin sensitivity in lean, physically fit men when protein intake is elevated. Metabolism. 2010. 59:1633-41. doi:10.1016/j.metabol.2010.03.013

\section{Power/Size}

Intervention/Exposure; Outcome

Participants

\section{Outcome}

Intervention/Exposure Study Design

Intervention/Exposure; Outcome

Intervention/Exposure

Intervention/Exposure Intervention/Exposure Study duration 


\section{No. Citation}

870 Green, KK, Shea, JL, Vasdev, S, Randell, E, Gulliver, W, Sun, G. Higher Dietary Protein Intake is Associated with Lower Body Fat in the Newfoundland Population. Clin Med Insights Endocrinol Diabetes. 2010. 3:25-35. doi:10.4137/cmed.s4619

871 Greenberg, I, Stampfer, MJ, Schwarzfuchs, D, Shai, I. Adherence and success in long-term weight loss diets: the dietary intervention randomized controlled trial (DIRECT). J Am Coll Nutr. 2009. 28:159-68. doi:10.1080/07315724.2009.10719767

872 Greenlee, H, Strizich, G, Lovasi, GS, Kaplan, RC, Biggs, ML, Li, Cl, Richardson, J, Burke, GL, Fitzpatrick, AL, Fretts, AM Psaty, BM, Fried, LP. Concordance With Prevention Guidelines and Subsequent Cancer, Cardiovascular Disease, and Mortality: A Longitudinal Study of Older Adults. Am J Epidemiol. 2017. 186:1168-1179. doi:10.1093/aje/kwx150

873 Greer, BK, Edsall, KM, Greer, AE. Reliability of BOD POD Measurements Remains High After a Short-Duration LowCarbohydrate Diet. Int J Sport Nutr Exerc Metab. 2016. 26:145-9. doi:10.1123/ijsnem.2015-0184

874 Grieb, P, Klapcinska, B, Smol, E, Pilis, T, Pilis, W, Sadowska-Krepa, E, Sobczak, A, Bartoszewicz, Z, Nauman, J, Stanczak, K, Langfort, J. Long-term consumption of a carbohydrate-restricted diet does not induce deleterious metabolic effects. Nutr Res. 2008. 28:825-33. doi:10.1016/j.nutres.2008.09.011

875 Griffin, BA, Walker, CG, Jebb, SA, Moore, C, Frost, GS, Goff, L, Sanders, TAB, Lewis, F, Griffin, M, Gitau, R, Lovegrove JA. APOE4 Genotype Exerts Greater Benefit in Lowering Plasma Cholesterol and Apolipoprotein B than Wild Type (E3/E3), after Replacement of Dietary Saturated Fats with Low Glycaemic Index Carbohydrates. Nutrients. 2018. 10: doi:10.3390/nu10101524

876 Griffin, HJ, Cheng, HL, O'Connor, HT, Rooney, KB, Petocz, P, Steinbeck, KS. Higher protein diet for weight management in young overweight women: a 12-month randomized controlled trial. Diabetes Obes Metab. 2013. 15:572-5. doi:10.1111/dom.12056

877 Griffin, HJ, O'Connor, HT, Rooney, KB, Steinbeck, KS, Cheng, HL, Petocz, P. Comparison of higher-protein and highercarbohydrate diets in overweight and obese young women. Obesity reviews. 2010. 11:823-. doi:10.1111/j.1467789X.2010.00822.x

878 Griffin, LE, Djuric, Z, Angiletta, CJ, Mitchell, CM, Baugh, ME, Davy, KP, Neilson, AP. A Mediterranean diet does not alter plasma trimethylamine N-oxide concentrations in healthy adults at risk for colon cancer. Food Funct. 2019. 10:2138-2147. doi:10.1039/c9fo00333a

879 Grimaldi, M, Ciano, O, Manzo, M, Rispoli, M, Guglielmi, M, Limardi, A, Calatola, P, Lucibello, M, Pardo, S, Capaldo, B, Riccardi, G. Intensive dietary intervention promoting the Mediterranean diet in people with high cardiometabolic risk: a nonrandomized study. Acta Diabetol. 2018. 55:219-226. doi:10.1007/s00592-017-1078-7

$880 \mathrm{Gu}, \mathrm{Y}, \mathrm{Yu}, \mathrm{H}, \mathrm{Li}, \mathrm{Y}, \mathrm{Ma}, \mathrm{X}, \mathrm{Lu}, \mathrm{J}, \mathrm{Yu}, \mathrm{W}$, Xiao, Y, Bao, Y, Jia, W. Beneficial effects of an 8-week, very low carbohydrate diet intervention on obese subjects. Evid Based Complement Alternat Med. 2013. 2013:760804. doi:10.1155/2013/760804

$881 \mathrm{Gu}, \mathrm{Y}$, Zhao, A, Huang, F, Zhang, Y, Liu, J, Wang, C, Jia, W, Xie, G, Jia, W. Very low carbohydrate diet significantly alters the serum metabolic profiles in obese subjects. J Proteome Res. 2013. 12:5801-11. doi:10.1021/pr4008199

882 Guasch-Ferre, M, Hruby, A, Salas-Salvado, J, Martinez-Gonzalez, MA, Sun, Q, Willett, WC, Hu, FB. Olive oil consumption and risk of type 2 diabetes in US women. Am J Clin Nutr. 2015. 102:479-86. doi:10.3945/ajcn.115.112029

\section{Rationale}

Study Design;

Intervention/Exposure

Weight

loss/Hypocaloric

Intervention/Exposure

Study duration

Study Design

Power/Size

Publication Status

Outcome

Comparator

Study Design

Study Design

Intervention/Exposure 
883 Guasch-Ferre, M, Hu, FB, Martinez-Gonzalez, MA, Fito, M, Bullo, M, Estruch, R, Ros, E, Corella, D, Recondo, J, GomezGracia, E, Fiol, M, Lapetra, J, Serra-Majem, L, Munoz, MA, Pinto, X, Lamuela-Raventos, RM, Basora, J, Buil-Cosiales, P Sorli, JV, Ruiz-Gutierrez, V, Martinez, JA, Salas-Salvado, J. Olive oil intake and risk of cardiovascular disease and mortality in the PREDIMED Study. BMC Med. 2014. 12:78. doi:10.1186/1741-7015-12-78

884 Guay, V, Lamarche, B, Charest, A, Tremblay, AJ, Couture, P. Effect of short-term low- and high-fat diets on low-density lipoprotein particle size in normolipidemic subjects. Metabolism. 2012. 61:76-83. doi:10.1016/j.metabol.2011.06.002

885 Guevara-Cruz, M, Tovar, AR, Aguilar-Salinas, CA, Medina-Vera, I, Gil-Zenteno, L, Hernandez-Viveros, I, Lopez-Romero, P, Ordaz-Nava, G, Canizales-Quinteros, S, Guillen Pineda, LE, Torres, N. A dietary pattern including nopal, chia seed, soy protein, and oat reduces serum triglycerides and glucose intolerance in patients with metabolic syndrome. J Nutr. 2012. 142:649. doi:10.3945/jn.111.147447

886 Gulseth, HL, Gjelstad, IM, Tierney, AC, Shaw, DI, Helal, O, Hees, AM, Delgado-Lista, J, Leszczynska-Golabek, I, Karlstrom, B, Lovegrove, J, Defoort, C, Blaak, EE, Lopez-Miranda, J, Dembinska-Kiec, A, Riserus, U, Roche, HM, Birkeland, KI, Drevon, CA. Dietary fat modifications and blood pressure in subjects with the metabolic syndrome in the LIPGENE dietary intervention study. Br J Nutr. 2010. 104:160-3. doi:10.1017/s0007114510000565

887 Gulseth, HL, Gjelstad, IMF, Tiereny, AC, McCarthy, D, Lovegrove, JA, Defoort, C, Blaak, EE, Lopez-Miranda, J, Dembinska-Kiec, A, Riserus, U, Roche, HM, Drevon, CA, Birkeland, KI. Effects of dietary fat on insulin secretion in subjects with the metabolic syndrome. Eur J Endocrinol. 2019. 180:321-328. doi:10.1530/eje-19-0022

888 Gulseth, HL, Gjelstad, IMF, Tierney, AC, Shaw, DI, Helal, O, Hees, AMJV, Delgado-Lista, J, Leszczynska-Golabek, I, Karlström, B, Lovegrove, J, Defoort, C, Blaak, EE, Lopez-Miranda, J, Dembinska-Kiec, A, Risérus, U, Roche, HM, Birkeland, KI, Drevon, CA. Dietary fat modifications and blood pressure in subjects with the metabolic syndrome in the LIPGENE dietary intervention study. British Journal of Nutrition. 2010. 104:160-163. doi:10.1017/S0007114510000565

889 Gungor, S, Celiloglu, OS, Raif, SG, Ozcan, OO, Selimoglu, MA. Malnutrition and Obesity in Children With ADHD. J Atten Disord. 2016. 20:647-52. doi:10.1177/1087054713478465

890 Gunther, AL, Buyken, AE, Kroke, A. The influence of habitual protein intake in early childhood on BMI and age at adiposity rebound: results from the DONALD Study. Int J Obes (Lond). 2006. 30:1072-9. doi:10.1038/sj.ijo.0803288

891 Gunther, AL, Karaolis-Danckert, N, Kroke, A, Remer, T, Buyken, AE. Dietary protein intake throughout childhood is associated with the timing of puberty. J Nutr. 2010. 140:565-71. doi:10.3945/jn.109.114934

892 Gunther, AL, Remer, T, Kroke, A, Buyken, AE. Early protein intake and later obesity risk: which protein sources at which time points throughout infancy and childhood are important for body mass index and body fat percentage at $7 \mathrm{y}$ of age?. Am $\mathrm{J}$ Clin Nutr. 2007. 86:1765-72. doi:10.1093/ajcn/86.5.1765

893 Gunther, AL, Schulze, MB, Kroke, A, Diethelm, K, Joslowski, G, Krupp, D, Wudy, S, Buyken, AE. Early Diet and Later Cancer Risk: Prospective Associations of Dietary Patterns During Critical Periods of Childhood with the GH-IGF Axis, Insulin Resistance and Body Fatness in Younger Adulthood. Nutr Cancer. 2015. 67:877-92. doi:10.1080/01635581.2015.1056313

Intervention/Exposure

Study duration

Intervention/Exposure

\author{
Int
}

Intervention/Exposure

Outcome

Intervention/Exposure

Study Design;

Intervention/Exposure

Age at Intervention/Exposure

Outcome

Intervention/Exposure; Age at Intervention/Exposure

Age at

Intervention/Exposure 
894 Gutierrez-Bedmar, M, Martinez-Gonzalez, MA, Munoz-Bravo, C, Ruiz-Canela, M, Mariscal, A, Salas-Salvado, J, Estruch, R, Corella, D, Aros, F, Fito, M, Lapetra, J, Serra-Majem, L, Pinto, X, Alonso-Gomez, A, Portoles, O, Fiol, M, Bullo, M, Castaner, O, Ros, E, Gomez-Gracia, E. Chromium Exposure and Risk of Cardiovascular Disease in High Cardiovascular Risk Subjects- Nested Case-Control Study in the Prevention With Mediterranean Diet (PREDIMED) Study. Circ J. 2017. 81:11831190. doi:10.1253/circj.CJ-17-0032

895 Gwin, JA, Leidy, HJ. Effects of dietary protein evenly spread throughout the day on sleep and mood in overweight women during sub-chronic energy balance and energy restriction. FASEB journal. 2017. 31:. doi:unavailable

896 Gwynne, M, Mounsey, A. Mediterranean diet: Higher fat but lower risk. Journal of Family Practice. 2013. 62:745-748. doi:unavailable

$897 \mathrm{Ha}$, SA, Lee, SY, Kim, KA, Seo, JS, Sohn, CM, Park, HR, Kim, KW. Eating habits, physical activity, nutrition knowledge and self-efficacy by obesity status in upper-grade elementary school students. Nutr Res Pract. 2016. 10:597-605. doi:10.4162/nrp.2016.10.6.597

898 Haas, MC, Bodner, EV, Brown, CJ, Bryan, D, Buys, DR, Keita, AD, Flagg, LA, Goss, A, Gower, B, Hovater, M, Hunter, G, Ritchie, CS, Roth, DL, Wingo, BC, Ard, J, Locher, JL. Calorie restriction in overweight seniors: response of older adults to a dieting study: the CROSSROADS randomized controlled clinical trial. J Nutr Gerontol Geriatr. 2014. 33:376-400. doi:10.1080/21551197.2014.965993

899 Habowski, S, Ziegenfuss, T, Sandrock, J, Raub, B, Kedia, W, Lopez, H. A prospective evaluation of a commercial weight loss program on body weight and body circumferences in overweight/obese men and women. FASEB journal. 2017. 31: doi:unavailable

900 Haghighatdoost, F, Malekahmadi, M, Onvani, S, Ramezani, N, Azadbakht, L. Macronutrient composition and Body Mass Index vary by season in college students. Progress in Nutrition. 2018. 20:483-490. doi:10.23751/pn.v20i3.5313

Intervention/Exposure

Publication Status

Publication Status

Study Design;

Intervention/Exposure

Intervention/Exposure; Comparator

\section{Publication Status}

Study Design; Comparator

901 Haire-Joshu, DL, Schwarz, CD, Peskoe, SB, Budd, EL, Brownson, RC, Joshu, CE. A group randomized controlled trial integrating obesity prevention and control for postpartum adolescents in a home visiting program. Int $\mathrm{J}$ Behav Nutr Phys Act. 2015. 12:88. doi:10.1186/s12966-015-0247-8

902 Hajiluian, G, Farhangi, MA, Jahangiry, L. Mediterranean dietary pattern and VEGF +405 G/C gene polymorphisms in patients with metabolic syndrome: An aspect of genenutrient interaction. PLoS ONE. 2017. 12:. doi:10.1371/journal.pone.0171637

903 Halbesma, N, Bakker, SJ, Jansen, DF, Stolk, RP, De Zeeuw, D, De Jong, PE, Gansevoort, RT. High protein intake associates with cardiovascular events but not with loss of renal function. J Am Soc Nephrol. 2009. 20:1797-804. doi:10.1681/asn.2008060649

904 Halkjaer, J, Tjonneland, A, Overvad, K, Sorensen, TI. Dietary predictors of 5-year changes in waist circumference J Am Diet Assoc. 2009. 109:1356-66. doi:10.1016/j.jada.2009.05.015

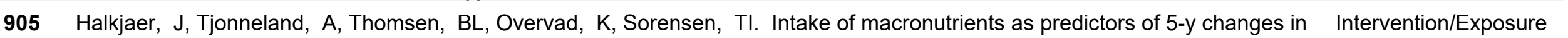
waist circumference. Am J Clin Nutr. 2006. 84:789-97. doi:10.1093/ajcn/84.4.789 
906 Hall, KD, Ayuketah, A, Brychta, R, Cai, H, Cassimatis, T, Chen, KY, Chung, ST, Costa, E, Courville, A, Darcey, V, Fletcher, LA, Forde, CG, Gharib, AM, Guo, J, Howard, R, Joseph, PV, McGehee, S, Ouwerkerk, R, Raisinger, K, Rozga, I, Stagliano, M, Walter, M, Walter, PJ, Yang, S, Zhou, M. Ultra-Processed Diets Cause Excess Calorie Intake and Weight Gain: An Inpatient Randomized Controlled Trial of Ad Libitum Food Intake. Cell Metab. 2019. 30:67-77.e3. doi:10.1016/j.cmet.2019.05.008

907 Hall, KD, Bemis, T, Brychta, R, Chen, KY, Courville, A, Crayner, EJ, Goodwin, S, Guo, J, Howard, L, Knuth, ND, Miller, BV, 3rd, Prado, CM, Siervo, M, Skarulis, MC, Walter, M, Walter, PJ, Yannai, L. Calorie for Calorie, Dietary Fat Restriction Results in More Body Fat Loss than Carbohydrate Restriction in People with Obesity. Cell Metab. 2015. 22:427-36. doi:10.1016/j.cmet.2015.07.021

908 Hall, KD, Chen, KY, Guo, J, Lam, YY, Leibel, RL, Mayer, LE, Reitman, ML, Rosenbaum, M, Smith, SR, Walsh, BT, Ravussin, E. Energy expenditure and body composition changes after an isocaloric ketogenic diet in overweight and obese men. Am J Clin Nutr. 2016. 104:324-33. doi:10.3945/ajcn.116.133561

909 Hall, WD, Feng, Z, George, VA, Lewis, CE, Oberman, A, Huber, M, Fouad, M, Cutler, JA. Low-fat diet: effect on anthropometrics, blood pressure, glucose, and insulin in older women. Ethn Dis. 2003. 13:337-43. doi:unavailable

Hallikainen, M, Toppinen, L, Mykkanen, H, Agren, JJ, Laaksonen, DE, Miettinen, TA, Niskanen, L, Poutanen, KS, Gylling $\mathrm{H}$. Interaction between cholesterol and glucose metabolism during dietary carbohydrate modification in subjects with the metabolic syndrome. Am J Clin Nutr. 2006. 84:1385-92. doi:10.1093/ajcn/84.6.1385

911 Hamideh, S, Behzad, M, Ebrahim, G, Hassan, E, Mojtaba, S. Diet, hypertension, hypercholesterolemia and diabetes in ischemic heart diseases. Pakistan Journal of Medical Sciences. 2007. 23:597-601. doi:unavailable

912 Hammons, AJ, Hannon, BA, Teran-Garcia, M, Barragan, M, Villegas, E, Wiley, A, Fiese, B. Effects of Culturally Tailored Nutrition Education on Dietary Quality of Hispanic Mothers: A Randomized Control Trial. J Nutr Educ Behav. 2019. : doi:10.1016/j.jneb.2019.06.017

913 Hamnvlk, OPR, Saidana, F, Levy, BD, Loscalzo, J. Against the grain. New England Journal of Medicine. 2014. 371:13331338. doi:10.1056/NEJMcps1301321

914 Han, CJ, Korde, L, Reding, S, Reding, K. Impact of a lifestyle intervention on metabolic pathways: results from the diet, exercise, emotional processing, and mindfulness (DEEM) intervention. Molecular cancer research. 2016. 14:. doi:10.1158/15573125.METCA15-A34

915 Han, JS, Kim, AJ. The Effect of Nutrition and Exercise or Exercise Program alone on Macronutrients, Sodium Intake and Physical Strength in Middle Aged Obese Women. Korean j obes. 2014. 23:187-193. doi:10.7570/kjo.2014.23.3.187

916 Han, Y, Kang, D, Lee, SA. Effect of 'rice' pattern on high blood pressure by gender and obesity: using the community-based KoGES cohort. Public Health Nutr. 2019. :1-11. doi:10.1017/s136898001900168x

917 Handjieva-Darlenska, T, Holst, C, Grau, K, Blaak, E, Martinez, JA, Oppert, JM, Taylor, MA, Sørensen, TIA, Astrup, A. Clinical correlates of weight loss and attrition during a 10-week dietary intervention study: Results from the NUGENOB project. Obesity Facts. 2015. 5:928-936. doi:10.1159/000345951

Study Design; Study duration

Intervention/Exposure

Intervention/Exposure

Study Design; Country

Intervention/Exposure

Study Design

Publication Status

Language

Outcome Intervention/Exposure 
918 Hankinson, AL, Daviglus, ML, Van Horn, L, Chan, Q, Brown, I, Holmes, E, Elliott, P, Stamler, J. Diet composition and activity level of at risk and metabolically healthy obese American adults. Obesity (Silver Spring). 2013. 21:637-43. doi:10.1002/oby.20257

919 Hansen-Krone, IJ, Enga, KF, Njolstad, I, Hansen, JB, Braekkan, SK. Heart healthy diet and risk of myocardial infarction and venous thromboembolism. The Tromso Study. Thromb Haemost. 2012. 108:554-60. doi:10.1160/th11-11-0818

Intervention/Exposure; Publication Date

Overlaps with Existing Review

920 Harber, MP, Schenk, S, Barkan, AL, Horowitz, JF. Alterations in carbohydrate metabolism in response to short-term dietary Study duration carbohydrate restriction. Am J Physiol Endocrinol Metab. 2005. 289:E306-12. doi:10.1152/ajpendo.00069.2005

921 Harber, MP, Schenk, S, Barkan, AL, Horowitz, JF. Effects of dietary carbohydrate restriction with high protein intake on protein metabolism and the somatotropic axis. J Clin Endocrinol Metab. 2005. 90:5175-81. doi:10.1210/jc.2005-0559

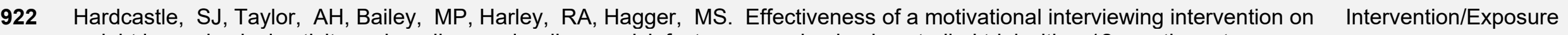
weight loss, physical activity and cardiovascular disease risk factors: a randomised controlled trial with a 12-month postintervention follow-up. Int J Behav Nutr Phys Act. 2013. 10:40. doi:10.1186/1479-5868-10-40

923 Harding, AH, Day, NE, Khaw, KT, Bingham, S, Luben, R, Welsh, A, Wareham, NJ. Dietary fat and the risk of clinical type diabetes: the European prospective investigation of Cancer-Norfolk study. Am J Epidemiol. 2004. 159:73-82. doi:10.1093/aje/kwh004

924 Hardy, LL, Mihrshahi, S, Gale, J, Nguyen, B, Baur, LA, O'Hara, BJ. Translational research: are community-based child obesity treatment programs scalable?. BMC Public Health. 2015. 15:652. doi:10.1186/s12889-015-2031-8

925 Hargrove, RL, Etherton, TD, Pearson, TA, Harrison, EH, Kris-Etherton, PM. Low fat and high monounsaturated fat diets decrease human low density lipoprotein oxidative susceptibility in vitro. J Nutr. 2001. 131:1758-63. doi:10.1093/jn/131.6.1758

926 Haring, B, von Ballmoos, MC, Appel, LJ, Sacks, FM. Healthy dietary interventions and lipoprotein (a) plasma levels: results from the Omni Heart Trial. PLoS One. 2014. 9:e114859. doi:10.1371/journal.pone.0114859

927 Haro, C, Garcia-Carpintero, S, Rangel-Zuniga, OA, Alcala-Diaz, JF, Landa, BB, Clemente, JC, Perez-Martinez, P, LopezMiranda, J, Perez-Jimenez, F, Camargo, A. Consumption of Two Healthy Dietary Patterns Restored Microbiota Dysbiosis in Obese Patients with Metabolic Dysfunction. Mol Nutr Food Res. 2017. 61:. doi:10.1002/mnfr.201700300

928 Harrington, DM, Champagne, CM, Broyles, ST, Johnson, WD, Tudor-Locke, C, Katzmarzyk, PT. Cardiometabolic risk factor response to a lifestyle intervention: a randomized trial. Metab Syndr Relat Disord. 2015. 13:125-31. doi:10.1089/met.2014.0112

929 Harrington, JM, Dahly, DL, Fitzgerald, AP, Gilthorpe, MS, Perry, IJ. Capturing changes in dietary patterns among older adults: a latent class analysis of an ageing Irish cohort. Public Health Nutr. 2014. 17:2674-86. doi:10.1017/s1368980014000111

930 Harris, C, Flexeder, C, Thiering, E, Buyken, A, Berdel, D, Koletzko, S, Bauer, CP, Bruske, I, Koletzko, B, Standl, M. Changes in dietary intake during puberty and their determinants: results from the GINIplus birth cohort study. BMC Public Health. 2015. 15:841. doi:10.1186/s12889-015-2189-0

Study Design; Intervention/Exposure Study duration

Outcome

Outcome

Intervention/Exposure; Comparator

Power/Size

Intervention/Exposure 
931 Harris, KA, West, SG, Vanden Heuvel, JP, Kris-Etherton, PM. A refined carbohydrate diet attenuates weight loss in insulin resistant individuals. Circulation. 2012. 125:. doi:unavailable

932 Harrison, T, McCullough, D, Lane, KE, Boddy, LM, Stewart, CE, Enright, KJ, Amirabdollahian, F, Schmidt, MA, Davies, IG. Dietary carbohydrate intake, visceral adipose tissue and associated markers of cardiometabolic risk. Proceedings of the nutrition society. 2018. 77:. doi:10.1017/S0029665118001751

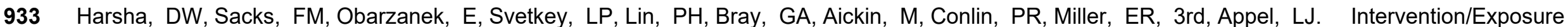
Effect of dietary sodium intake on blood lipids: results from the DASH-sodium trial. Hypertension. 2004. 43:393-8. doi:10.1161/01.HYP.0000113046.83819.a2

934 Hartman, TJ, Albert, PS, Zhang, Z, Bagshaw, D, Kris-Etherton, PM, Ulbrecht, J, Miller, CK, Bobe, G, Colburn, NH, Lanza $\mathrm{E}$. Consumption of a legume-enriched, low-glycemic index diet is associated with biomarkers of insulin resistance and inflammation among men at risk for colorectal cancer. Journal of Nutrition. 2010. 140:60-67. doi:10.3945/jn.109.114249

935 Harvey, Cjdc, Schofield, GM, Zinn, C, Thornley, SJ, Crofts, C, Merien, FLR. Low-carbohydrate diets differing in carbohydrate restriction improve cardiometabolic and anthropometric markers in healthy adults: A randomised clinical trial. PeerJ. 2019. 7:e6273. doi:10.7717/peerj.6273

936 Harvie, M, Wright, C, Pegington, M, McMullan, D, Mitchell, E, Martin, B, Cutler, RG, Evans, G, Whiteside, S, Maudsley, S, Camandola, S, Wang, R, Carlson, OD, Egan, JM, Mattson, MP, Howell, A. The effect of intermittent energy and carbohydrate restriction $\mathrm{v}$. daily energy restriction on weight loss and metabolic disease risk markers in overweight women. Br $\mathrm{J}$ Nutr. 2013. 110:1534-47. doi:10.1017/s0007114513000792

937 Hashemian, M, Farvid, MS, Poustchi, H, Murphy, G, Etemadi, A, Hekmatdoost, A, Kamangar, F, Sheikh, M, Pourshams, A, Country Sepanlou, SG, Fazeltabar Malekshah, A, Khoshnia, M, Gharavi, A, Brennan, PJ, Boffetta, P, Dawsey, SM, Reedy, J, Subar, AF, Abnet, CC, Malekzadeh, R. The application of six dietary scores to a Middle Eastern population: a comparative analysis of mortality in a prospective study. Eur J Epidemiol. 2019. 34:371-382. doi:10.1007/s10654-019-00508-3

938 Hassan, NE, El Shebini, SM, Ahmed, NH. Association between Dietary Patterns, Breakfast Skipping and Familial Obesity among a Sample of Egyptian Families. Open Access Maced J Med Sci. 2016. 4:213-8. doi:10.3889/oamjms.2016.050

939 Hassannejad, R, Kazemi, I, Sadeghi, M, Mohammadifard, N, Roohafza, H, Sarrafzadegan, N, Talaei, M, Mansourian, M. Longitudinal association of metabolic syndrome and dietary patterns: A 13-year prospective population-based cohort study. Nutr Metab Cardiovasc Dis. 2018. 28:352-360. doi:10.1016/j.numecd.2017.10.025

940 Hassapidou, M, Tziomalos, K, Lazaridou, S, Pagkalos, I, Papadimitriou, K, Kokkinopoulou, A, Tzotzas, T. The Nutrition Health Alliance (NutriHeAl) Study: A Randomized, Controlled, Nutritional Intervention Based on Mediterranean Diet in Greek Municipalities. J Am Coll Nutr. 2019. :1-7. doi:10.1080/07315724.2019.1660928

941 Hassapidou, MN, Fotiadou, E. Dietary intakes and food habits of adolescents in northern Greece. Int J Food Sci Nutr. 2001. 52:109-16. doi:10.1080/713671767

942 Haub, MD, Wells, AM, Tarnopolsky, MA, Campbell, WW. Effect of protein source on resistive-training-induced changes in body composition and muscle size in older men. Am J Clin Nutr. 2002. 76:511-7. doi:10.1093/ajcn/76.3.511

Intervention/Exposure

Intervention/Exposure

Intervention/Exposure Publication Status $-$ 
943 Haufe, S, Engeli, S, Kast, P, Bohnke, J, Utz, W, Haas, V, Hermsdorf, M, Mahler, A, Wiesner, S, Birkenfeld, AL, Sell, H, Otto, C, Mehling, H, Luft, FC, Eckel, J, Schulz-Menger, J, Boschmann, M, Jordan, J. Randomized comparison of reduced fat and reduced carbohydrate hypocaloric diets on intrahepatic fat in overweight and obese human subjects. Hepatology. 2011. 53:1504-14. doi:10.1002/hep.24242

944 Haugaard, SB, Madsbad, S, Hoy, CE, Vaag, A. Dietary intervention increases n-3 long-chain polyunsaturated fatty acids in skeletal muscle membrane phospholipids of obese subjects. Implications for insulin sensitivity. Clin Endocrinol (Oxf). 2006. 64:169-78. doi:10.1111/j.1365-2265.2006.02444.x

945 Haulrik, N, Toubro, S, Dyerberg, J, Stender, S, Skov, AR, Astrup, A. Effect of protein and methionine intakes on plasma homocysteine concentrations: a 6-mo randomized controlled trial in overweight subjects. Am J Clin Nutr. 2002. 76:1202-6. doi:10.1093/ajcn/76.6.1202

946 Hauner, H. High-fat mediterranean diet leads to slight weight reduction. Deutsche medizinische wochenschrift (1946). 2016. 141:1278. doi:10.1055/s-0042-111570

947 Hauser, ME, Hartle, J, Qin, F, Rigdon, J, Del Gobbo, L, Shih, C, Gardner, CD. Dietary adherence and dietary quality are associated with weight loss success among those following low-fat and low-carbohydrate diets. Circulation. 2018. 137: doi:unavailable

948 Hays, NP, Starling, RD, Liu, X, Sullivan, DH, Trappe, TA, Fluckey, JD, Evans, WJ. Effects of an ad libitum low-fat, highcarbohydrate diet on body weight, body composition, and fat distribution in older men and women: a randomized controlled trial. Arch Intern Med. 2004. 164:210-7. doi:10.1001/archinte.164.2.210

$949 \mathrm{He}, \mathrm{DH}$, Yang, M, Zhang, RH, Ma, XG, Huang, LC, Huang, ES, Gu, W, Zhu, YB, Zhao, D, Zhu, XH, Ding, GQ, Zhou, B. Dietary Patterns Associated Metabolic Syndrome in Chinese Adults. Biomed Environ Sci. 2015. 28:370-3. doi:10.3967/bes2015.051

950 He, F, Bixler, EO, Liao, J, Berg, A, Imamura Kawasawa, Y, Fernandez-Mendoza, J, Vgontzas, AN, Liao, D. Habitual sleep variability, mediated by nutrition intake, is associated with abdominal obesity in adolescents. Sleep Med. 2015. 16:1489-94. doi:10.1016/j.sleep.2015.07.028

951 Heatherly, AJ, Killen, LG, Smith, AF, Waldman, HS, Seltmann, CL, Hollingsworth, A, O'Neal, EK. Effects of Ad libitum LowCarbohydrate High-Fat Dieting in Middle-Age Male Runners. Med Sci Sports Exerc. 2018. 50:570-579. doi:10.1249/mss.0000000000001477

952 Hebestreit, A, Bornhorst, C, Pala, V, Barba, G, Eiben, G, Veidebaum, T, Hadjigergiou, C, Molnar, D, Claessens, M, Fernandez-Alvira, JM, Pigeot, I. Dietary energy density in young children across Europe. Int J Obes (Lond). 2014. 38 Suppl 2:S124-34. doi:10.1038/ijo.2014.143

953 Heggen, E, Klemsdal, TO, Haugen, F, Holme, I, Tonstad, S. Effect of a low-fat versus a low-gycemic-load diet on inflammatory biomarker and adipokine concentrations. Metab Syndr Relat Disord. 2012. 10:437-42. doi:10.1089/met.2012.0012

954 Heggen, E, Svendsen, M, Klemsdal, TO, Tonstad, S. Low Carbohydrate and Moderately Fat-Reduced Diets Similarly Affected Early Weight Gain in Varenicline-Treated Overweight or Obese Smokers. Nicotine Tob Res. 2016. 18:1440-8. doi:10.1093/ntr/ntv164

\section{Weight}

loss/Hypocaloric

Study Design

Outcome

Publication Status;

Language

Publication Status

Power/Size

Publication Status

Intervention/Exposur

Study duration

Intervention/Exposure

Weight

oss/Hypocaloric

Intervention/Exposure 
955 Heianza, Y, Ma, W, Huang, T, Wang, T, Zheng, Y, Smith, SR, Bray, GA, Sacks, FM, Qi, L. Macronutrient IntakeAssociated FGF21 Genotype Modifies Effects of Weight-Loss Diets on 2-Year Changes of Central Adiposity and Body

Intervention/Exposure; Composition: The POUNDS Lost Trial. Diabetes Care. 2016. 39:1909-1914. doi:10.2337/dc16-1111

956 Heianza, Y, Sun, D, Ma, W, Zheng, Y, Champagne, CM, Bray, GA, Sacks, FM, Qi, L. Gut-microbiome-related LCT genotype and 2-year changes in body composition and fat distribution: the POUNDS Lost Trial. Int J Obes (Lond). 2018. 42:1565-1573. doi:10.1038/s41366-018-0046-9

957 Heikkilä, HM, Krachler, B, Rauramaa, R, Schwab, US. Diet, insulin secretion and insulin sensitivity - The Dose-Responses to Exercise Training (DR's EXTRA) Study (ISRCTN45977199). British Journal of Nutrition. 2014. 112:1530-1541. doi:10.1017/S0007114514002426

958 Heikkila, HM, Krachler, B, Savonen, K, Hassinen, M, Rauramaa, R, Schwab, US. Combined low-saturated fat intake and high fitness may counterbalance diabetogenic effects of obesity: the DR's EXTRA Study. Eur J Clin Nutr. 2013. 67:1000-2. doi:10.1038/ejcn.2013.138

959 Helge, JW. Prolonged adaptation to fat-rich diet and training; effects on body fat stores and insulin resistance in man. Int J Obes Relat Metab Disord. 2002. 26:1118-24. doi:10.1038/sj.ijo.0802058

960 Helms, ER, Zinn, C, Rowlands, DS, Naidoo, R, Cronin, J. High-protein, low-fat, short-term diet results in less stress and fatigue than moderate-protein moderate-fat diet during weight loss in male weightlifters: a pilot study. Int J Sport Nutr Exerc Metab. 2015. 25:163-70. doi:10.1123/ijsnem.2014-0056

961 Henderson, M, Benedetti, A, Gray-Donald, K. Dietary composition and its associations with insulin sensitivity and insulin secretion in youth. Br J Nutr. 2014. 111:527-34. doi:10.1017/s0007114513002572

962 Hengeveld, LM, Wijnhoven, HAH, Olthof, MR, Brouwer, IA, Harris, TB, Kritchevsky, SB, Newman, AB, Visser, M Prospective associations of poor diet quality with long-term incidence of protein-energy malnutrition in community-dwelling older adults: the Health, Aging, and Body Composition (Health ABC) Study. Am J Clin Nutr. 2018. 107:155-164. doi:10.1093/ajcn/nqx020

963 Hermanussen, M. Nutritional protein intake is associated with body mass index in young adolescents. Georgian Med News. 2008. :84-8. doi:unavailable

Comparator

Intervention/Exposure

Study Design

Study Design;

Intervention/Exposure

Study duration

Study duration

Study Design; Intervention/Exposure

Outcome

Publication Status;

Country

964 Hernáez, Á, Castañer, O, Elosua, R, Pintó, X, Estruch, R, Salas-Salvadó, J, Corella, D, Arós, F, Serra-Majem, L, Fiol, M, Ortega-Calvo, M, Ros, E, Martínez-González, Á M, De La Torre, R, López-Sabater, MC, Fitó, M. Mediterranean Diet Improves High-Density Lipoprotein Function in High-Cardiovascular-Risk Individuals. Circulation. 2017. 135:633-643. doi:10.1161/CIRCULATIONAHA.116.023712

965 Hernaez, A, Castaner, O, Elosua, R, Pinto, X, Estruch, R, Salas-Salvado, J, Corella, D, Aros, F, Serra-Majem, L, Fiol, M, Ortega-Calvo, M, Ros, E, Martinez-Gonzalez, MA, de la Torre, R, Lopez-Sabater, MC, Fito, M. Mediterranean Diet Improves High-Density Lipoprotein Function in High-Cardiovascular-Risk Individuals: A Randomized Controlled Trial. Circulation. 2017. 135:633-643. doi:10.1161/circulationaha.116.023712 
966 Hernaez, A, Castaner, O, Goday, A, Ros, E, Pinto, X, Estruch, R, Salas-Salvado, J, Corella, D, Aros, F, Serra-Majem, L, Martinez-Gonzalez, MA, Fiol, M, Lapetra, J, de la Torre, R, Lopez-Sabater, MC, Fito, M. The Mediterranean Diet decreases LDL atherogenicity in high cardiovascular risk individuals: a randomized controlled trial. Mol Nutr Food Res. 2017. 61:. doi:10.1002/mnfr.201601015

967 Hernaez, A, Sanllorente, A, Castaner, O, Martinez-Gonzalez, MA, Ros, E, Pinto, X, Estruch, R, Salas-Salvado, J, Corella, D, Alonso-Gomez, AM, Serra-Majem, L, Fiol, M, Lapetra, J, Gomez-Gracia, E, de la Torre, R, Lamuela-Raventos, RM, Fito, M. Increased Consumption of Virgin Olive Oil, Nuts, Legumes, Whole Grains, and Fish Promotes HDL Functions in Humans. Mol Nutr Food Res. 2019. 63:e1800847. doi:10.1002/mnfr.201800847

968 Hernandez, JA, Del Valle Laveaga, D, Cano, JM. Sub-patterns of food consumption and hyperglycemia in Mexican young people: a study by factor analysis. Food Nutr Res. 2016. 60:30185. doi:10.3402/fnr.v60.30185

969 Hernandez-Mijares, A, Banuls, C, Rocha, M, Morillas, C, Martinez-Triguero, ML, Victor, VM, Lacomba, R, Alegria, A, Barbera, R, Farre, R, Lagarda, MJ. Effects of phytosterol ester-enriched low-fat milk on serum lipoprotein profile in mildly hypercholesterolaemic patients are not related to dietary cholesterol or saturated fat intake. Br J Nutr. 2010. 104:1018-25. doi:10.1017/s0007114510001686

970 Herrmann, TS, Bean, ML, Black, TM, Wang, P, Coleman, RA. High glycemic index carbohydrate diet alters the diurnal rhythm of leptin but not insulin concentrations. Exp Biol Med (Maywood). 2001. 226:1037-44. doi:10.1177/153537020122601111

971 Hess, AL, Carayol, J, Blaedel, T, Hager, J, Di Cara, A, Astrup, A, Saris, WHM, Larsen, LH, Valsesia, A. Analysis of circulating angiopoietin-like protein 3 and genetic variants in lipid metabolism and liver health: the DiOGenes study. Genes Nutr. 2018. 13:7. doi:10.1186/s12263-018-0597-3

972 Hesselink, AE, Bilo, HJ, Jonkers, R, Martens, M, de Weerdt, I, Rutten, GE. A cluster-randomized controlled trial to study the effectiveness of a protocol-based lifestyle program to prevent type 2 diabetes in people with impaired fasting glucose. BMC Fam Pract. 2013. 14:184. doi:10.1186/1471-2296-14-184

973 Hickey, JT, Hickey, L, Yancy, WS, Hepburn, J, Westman, EC. Clinical use of a carbohydrate-restricted diet to treat the dyslipidemia of the metabolic syndrome. Metab Syndr Relat Disord. 2003. 1:227-32. doi:10.1089/154041903322716705

974 Hickling, S, Hung, J, Knuiman, M, Divitini, M, Beilby, J. Are the associations between diet and C-reactive protein independent of obesity?. Prev Med. 2008. 47:71-6. doi:10.1016/j.ypmed.2008.02.007

975 Hickner, RC. Take flight to reduce cardiovascular disease risk in youth. Exerc Sport Sci Rev. 2014. 42:143-4. doi:10.1249/jes.0000000000000028

976 Hikmat, F, Appel, LJ. Effects of the DASH diet on blood pressure in patients with and without metabolic syndrome: results from the DASH trial. J Hum Hypertens. 2014. 28:170-5. doi:10.1038/jhh.2013.52

977 Hill, AM, Harris Jackson, KA, Roussell, MA, West, SG, Kris-Etherton, PM. Type and amount of dietary protein in the treatment of metabolic syndrome: a randomized controlled trial. Am J Clin Nutr. 2015. 102:757-70. doi:10.3945/ajcn.114.104026

Study Design; Intervention/Exposure

Intervention/Exposure

\section{Study duration}

Intervention/Exposure; Outcome

Intervention/Exposure

Intervention/Exposure

Study Design

Intervention/Exposure

Study Design;

Publication Status

Study duration

Power/Size 
978 Hinderliter, A, Smith, P, Sherwood, A, Blumenthal, J. Lifestyle interventions reduce the need for guideline-directed antihypertensive medication. Hypertension. 2018. 72:. doi:10.1161/hyp.72.suppl_1.P388

979 Hinderliter, AL, Sherwood, A, Craighead, LW, Lin, PH, Watkins, L, Babyak, MA, Blumenthal, JA. The long-term effects of lifestyle change on blood pressure: One-year follow-up of the ENCORE study. Am J Hypertens. 2014. 27:734-41. doi:10.1093/ajh/hpt183

980 Hindy, G, Sonestedt, E, Ericson, U, Jing, XJ, Zhou, Y, Hansson, O, Renstrom, E, Wirfalt, E, Orho-Melander, M. Role of TCF7L2 risk variant and dietary fibre intake on incident type 2 diabetes. Diabetologia. 2012. 55:2646-2654. doi:10.1007/s00125012-2634-X

981 Hirsch, O, Kluckner, VJ, Brandt, S, Moss, A, Weck, M, Florath, I, Wabitsch, M, Hebebrand, J, Schimmelmann, BG, Christiansen, H. Restrained and external-emotional eating patterns in young overweight children-results of the Ulm Birth Cohort Study. PLoS One. 2014. 9:e105303. doi:10.1371/journal.pone.0105303

982 Hjartaker, A, Knudsen, MD, Tretli, S, Weiderpass, E. Consumption of berries, fruits and vegetables and mortality among 10,000 Norwegian men followed for four decades. Eur J Nutr. 2015. 54:599-608. doi:10.1007/s00394-014-0741-9

983 Hjerkinn, EM, Sandvik, L, Hjermann, I, Arnesen, H. Effect of diet intervention on long-term mortality in healthy middle-aged men with combined hyperlipidaemia. Journal of Internal Medicine. 2004. 255:68-73. doi:10.1046/j.0954-6820.2003.01248.x

984 Hjerpsted, J, Leedo, E, Tholstrup, T. Cheese intake in large amounts lowers LDL-cholesterol concentrations compared with butter intake of equal fat content. Am J Clin Nutr. 2011. 94:1479-84. doi:10.3945/ajcn.111.022426

985 Hjorth, MF, Astrup, A, Zohar, Y, Urban, LE, Sayer, RD, Patterson, BW, Herring, SJ, Klein, S, Zemel, BS, Foster, GD, Wyatt, HR, Hill, JO. Personalized nutrition: pretreatment glucose metabolism determines individual long-term weight loss responsiveness in individuals with obesity on low-carbohydrate versus low-fat diet. Int J Obes (Lond). 2019. 43:2037-2044. doi:10.1038/s41366-018-0298-4

986 Hlebowicz, J, Persson, M, Gullberg, B, Sonestedt, E, Wallstrom, P, Drake, I, Nilsson, J, Hedblad, B, Wirfalt, E. Food patterns, inflammation markers and incidence of cardiovascular disease: the Malmo Diet and Cancer study. J Intern Med. 2011. 270:365-76. doi:10.1111/j.1365-2796.2011.02382.x

987 Ho, CY, Huang, YC, Lo, YT, Wahlqvist, ML, Lee, MS. Breakfast is associated with the metabolic syndrome and school performance among Taiwanese children. Res Dev Disabil. 2015. 43-44:179-88. doi:10.1016/j.ridd.2015.07.003

988 Hobbs, M, Green, M, Roberts, K, Griffiths, C, McKenna, J. Reconsidering the relationship between fast-food outlets, arealevel deprivation, diet quality and body mass index: an exploratory structural equation modelling approach. J Epidemiol Community Health. 2019. 73:861-866. doi:10.1136/jech-2018-211798

989 Hodge, AM, English, DR, O'Dea, K, Giles, GG. Dietary patterns and diabetes incidence in the Melbourne collaborative cohort study. American Journal of Epidemiology. 2007. 165:603-610. doi:10.1093/aje/kwk061

Intervention/Exposure; Comparator

Intervention/Exposure

Intervention/Exposure

Intervention/Exposure

Intervention/Exposure; Outcome

Intervention/Exposure

Intervention/Exposure

Publication Date

Overlaps with Existing Review

Study Design;

Intervention/Exposure; Country

Study Design

Intervention/Exposure; Publication Date

Overlaps with Existing Review 


\begin{tabular}{|c|c|c|}
\hline No. & Citation & Rationale \\
\hline 990 & $\begin{array}{l}\text { Hodge, AM, English, DR, O'Dea, K, Giles, GG. Glycemic index and dietary fiber and the risk of type } 2 \text { diabetes. Diabetes } \\
\text { Care. 2004. 27:2701-6. doi:10.2337/diacare.27.11.2701 }\end{array}$ & $\begin{array}{l}\text { Intervention/Exposure; } \\
\text { Outcome }\end{array}$ \\
\hline 991 & $\begin{array}{l}\text { Hodge, AM, O'Dea, K, English, DR, Giles, GG, Flicker, L. Dietary patterns as predictors of successful ageing. J Nutr Health } \\
\text { Aging. 2014. 18:221-7. doi:10.1007/s12603-013-0405-0 }\end{array}$ & ne \\
\hline 992 & $\begin{array}{l}\text { Hodgson, JM, Burke, V, Beilin, LJ, Puddey, IB. Partial substitution of carbohydrate intake with protein intake from lean red } \\
\text { meat lowers blood pressure in hypertensive persons. Am J Clin Nutr. 2006. 83:780-7. doi:10.1093/ajcn/83.4.780 }\end{array}$ & Study duration \\
\hline 993 & $\begin{array}{l}\text { Hodson, L, Harnden, KE, Roberts, R, Dennis, AL, Frayn, KN. Does the DASH diet lower blood pressure by altering } \\
\text { peripheral vascular function?. J Hum Hypertens. 2010. 24:312-9. doi:10.1038/jhh.2009.65 }\end{array}$ & $\begin{array}{l}\text { Intervention/Exposure; } \\
\text { Publication Date } \\
\text { Overlaps with Existing } \\
\text { Review }\end{array}$ \\
\hline 994 & $\begin{array}{l}\text { Hodson, L, Skeaff, CM, Chisholm, WA. The effect of replacing dietary saturated fat with polyunsaturated or monounsaturated } \\
\text { fat on plasma lipids in free-living young adults. Eur J Clin Nutr. 2001. 55:908-15. doi:10.1038/sj.ejcn.1601234 }\end{array}$ & $\begin{array}{l}\text { Intervention/Exposure; } \\
\text { Study duration }\end{array}$ \\
\hline 995 & $\begin{array}{l}\text { Hodson, L, Skeaff, CM, McKenzie, JE. Maximal response to a plasma cholesterol-lowering diet is achieved within two weeks. } \\
\text { Nutr Metab Cardiovasc Dis. 2002. 12:291-5. doi:unavailable }\end{array}$ & Study duration \\
\hline 996 & $\begin{array}{l}\text { Hoevenaar-Blom, MP, Nooyens, ACJ, Kromhout, D, Spijkerman, AMW, Beulens, JWJ, van der Schouw, YT, Bueno-de- } \\
\text { Mesquita, B, Verschuren, WMM. Mediterranean Style Diet and 12-Year Incidence of Cardiovascular Diseases: The EPIC-NL } \\
\text { Cohort Study. PLoS ONE. 2012. 7:. doi:10.1371/journal.pone.0045458 }\end{array}$ & $\begin{array}{l}\text { Publication Date } \\
\text { Overlaps with Existing } \\
\text { Review }\end{array}$ \\
\hline 997 & $\begin{array}{l}\text { Hoffman, JR, Ratamess, NA, Kang, J, Falvo, MJ, Faigenbaum, AD. Effect of protein intake on strength, body composition } \\
\text { and endocrine changes in strength/power athletes. J Int Soc Sports Nutr. 2006. 3:12-8. doi:10.1186/1550-2783-3-2-12 }\end{array}$ & Power/Size \\
\hline 998 & $\begin{array}{l}\text { Hoffmann, K, Schulze, MB, Schienkiewitz, A, Nothlings, U, Boeing, H. Application of a new statistical method to derive } \\
\text { dietary patterns in nutritional epidemiology. Am J Epidemiol. 2004. 159:935-44. doi:10.1093/aje/kwh134 }\end{array}$ & $\begin{array}{l}\text { Intervention/Exposure; } \\
\text { Publication Date } \\
\text { Overlaps with Existing } \\
\text { Review }\end{array}$ \\
\hline 999 & $\begin{array}{l}\text { Holloway, CJ, Cochlin, LE, Emmanuel, Y, Murray, A, Codreanu, I, Edwards, LM, Szmigielski, C, Tyler, DJ, Knight, NS, } \\
\text { Saxby, BK, Lambert, B, Thompson, C, Neubauer, S, Clarke, K. A high-fat diet impairs cardiac high-energy phosphate } \\
\text { metabolism and cognitive function in healthy human subjects. Am J Clin Nutr. 2011. 93:748-55. doi:10.3945/ajcn.110.002758 }\end{array}$ & Study duration \\
\hline 1000 & $\begin{array}{l}\text { Holmberg, S, Thelin, A, Stiernstrom, EL. Food choices and coronary heart disease: a population based cohort study of rural } \\
\text { Swedish men with } 12 \text { years of follow-up. Int J Environ Res Public Health. 2009. 6:2626-38. doi:10.3390/ijerph6102626 }\end{array}$ & Intervention/Exposure \\
\hline 1001 & $\begin{array}{l}\text { Holme, I. Long-term survival in pre-specified groups at risk in the Oslo Study, 1972-1973. Scand J Public Health. } 2015 . \\
\text { 43:117-22. doi:10.1177/1403494814558157 }\end{array}$ & $\begin{array}{l}\text { Intervention/Exposure; } \\
\text { Comparator }\end{array}$ \\
\hline 1002 & $\begin{array}{l}\text { Holt, S. Cochrane corner: Mediterranean diet for the prevention of cardiovascular disease. Advances in Integrative Medicine. } \\
\text { 2014. 1:61. doi:10.1016/j.aimed.2013.08.009 }\end{array}$ & $\begin{array}{l}\text { Study Design; } \\
\text { Publication Status }\end{array}$ \\
\hline
\end{tabular}


1003 Honemann, I, Ranke, C, Austel, A, Jarzemski, S, Deeken, I, Pleyer, I, Renziehausen, F, Pudel, V, Ellrott, T. Changes of cardiovascular risk factors while following one of three currently discussed dietetic weight-reducing strategies. Aktuelle ernahrungsmedizin. 2010. 35:227-235. doi:10.1055/s-0030-1248497

1004 Honerlaw, JP, Ho, YL, Nguyen, XT, Cho, K, Vassy, JL, Gagnon, DR, O'Donnell, CJ, Gaziano, JM, Wilson, PWF, Djousse, L. Fried food consumption and risk of coronary artery disease: The Million Veteran Program. Clin Nutr. 2019. :. doi:10.1016/j.clnu.2019.05.008

1005 Hong, K, Kim, K, Lee, S. High percentage of fat intakes, not low fat oxidation, may induce overweight cyclists. J Sports Med Phys Fitness. 2012. 52:405-12. doi:unavailable

1006 Hong, X, Xu, F, Wang, Z, Liang, Y, Li, J. Dietary patterns and the incidence of hyperglyacemia in China. Public Health Nutr. 2016. 19:131-41. doi:10.1017/s1368980015000774

1007 Hooshmand, F, Asghari, G, Yuzbashian, E, Mahdavi, M, Mirmiran, P, Azizi, F. Modified Healthy Eating Index and Incidence of Metabolic Syndrome in Children and Adolescents: Tehran Lipid and Glucose Study. J Pediatr. 2018. 197:134-139.e2. doi:10.1016/j.jpeds.2018.01.080

1008 Hoppu, U, Isolauri, E, Koskinen, P, Laitinen, K. Diet and blood lipids in 1-4 year-old children. Nutr Metab Cardiovasc Dis. 2013. 23:980-6. doi:10.1016/j.numecd.2012.10.007

1009 Horan, MK, McGowan, CA, Gibney, ER, Donnelly, JM, McAuliffe, FM. Maternal diet and weight at 3 months postpartum following a pregnancy intervention with a low glycaemic index diet: results from the ROLO randomised control trial. Nutrients 2014. 6:2946-55. doi:10.3390/nu6072946

1010 Hoscan, Y, Yigit, F, Muderrisoglu, H. Adherence to Mediterranean diet and its relation with cardiovascular diseases in Turkish population. Int J Clin Exp Med. 2015. 8:2860-6. doi:unavailable

1011 Hosking, D, Danthiir, V. Retrospective lifetime dietary patterns are associated with demographic and cardiovascular health variables in an older community-dwelling Australian population. Br J Nutr. 2013. 110:2069-83. doi:10.1017/s000711451300144x

1012 Hosseini, N, Talaei, M, Dianatkhah, M, Sadeghi, M, Oveisgharan, S, Sarrafzadegan, N. Determinants of Incident Metabolic Syndrome in a Middle Eastern Population: Isfahan Cohort Study. Metab Syndr Relat Disord. 2017. 15:354-362. doi:10.1089/met.2016.0156

1013 Hosseini, Z, Whiting, SJ, Vatanparast, H. Type 2 diabetes prevalence among Canadian adults - dietary habits and sociodemographic risk factors. Appl Physiol Nutr Metab. 2019. 44:1099-1104. doi:10.1139/apnm-2018-0567

1014 Hosseini-Esfahani, F, Esfandiar, Z, Mirmiran, P, Daneshpour, MS, Ghanbarian, A, Azizi, F. The interaction of cholestery ester transfer protein gene variations and diet on changes in serum lipid profiles. Eur J Clin Nutr. 2019. 73:1291-1298. doi:10.1038/s41430-019-0397-x

1015 Hosseini-Esfahani, F, Koochakpoor, G, Daneshpour, MS, Sedaghati-Khayat, B, Mirmiran, P, Azizi, F. Mediterranean Dietary Country Pattern Adherence Modify the Association between FTO Genetic Variations and Obesity Phenotypes. Nutrients. 2017. 9:. doi:10.3390/nu9101064 
1016 Hosseini-Esfahani, F, Koochakpoor, G, Mirmiran, P, Daneshpour, MS, Azizi, F. Dietary patterns modify the association between fat mass and obesity-associated genetic variants and changes in obesity phenotypes. Br J Nutr. 2019. 121:1247-1254. doi:10.1017/s0007114519000643

1017 Hosseini-Esfahani, F, Mirmiran, P, Daneshpour, MS, Mehrabi, Y, Hedayati, M, Soheilian-Khorzoghi, M, Azizi, F. Dietary

Intervention/Exposure;

Comparator

Comparator; Outcome Study. Br J Nutr. 2015. 113:644-53. doi:10.1017/s0007114514003687

1018 Hosseini-Esfahani, F, Mirmiran, P, Daneshpour, MS, Mehrabi, Y, Hedayati, M, Zarkesh, M, Azizi, F. Western dietary pattern interaction with APOC3 polymorphism in the risk of metabolic syndrome: Tehran Lipid and Glucose Study. J Nutrigenet Nutrigenomics. 2014. 7:105-17. doi:10.1159/000365445

1019 Houston, DK, Nicklas, BJ, Ding, J, Harris, TB, Tylavsky, FA, Newman, AB, Lee, JS, Sahyoun, NR, Visser, M, Kritchevsky, SB. Dietary protein intake is associated with lean mass change in older, community-dwelling adults: the Health, Aging, and Body Composition (Health ABC) Study. Am J Clin Nutr. 2008. 87:150-5. doi:10.1093/ajcn/87.1.150

1020 Howard, BV, Manson, JE, Stefanick, ML, Beresford, SA, Frank, G, Jones, B, Rodabough, RJ, Snetselaar, L, Thomson, C, Tinker, L, Vitolins, M, Prentice, R. Low-fat dietary pattern and weight change over 7 years: the Women's Health Initiative Dietary Modification Trial. Jama. 2006. 295:39-49. doi:10.1001/jama.295.1.39

1021 Howard, BV, Van Horn, L, Hsia, J, Manson, JE, Stefanick, MI, Wassertheil-Smoller, S, Kuller, LH, LaCroix, AZ, Langer, $\mathrm{RD}$, Lasser, NI, etal, . Low-fat dietary pattern and risk of cardiovascular disease: the women's health initiative randomized controlled dietary modification trial. Obstetrical \& gynecological survey. 2006. 61:451-453. doi:10.1097/01.ogx.0000224659.41638.7d

1022 Howard, BV, Van Horn, L, Hsia, J, Manson, JE, Stefanick, ML, Wassertheil-Smoller, S, Kuller, LH, LaCroix, AZ, Langer, RD, Lasser, NL, Lewis, CE, Limacher, MC, Margolis, KL, Mysiw, WJ, Ockene, JK, Parker, LM, Perri, MG, Phillips, L, Prentice, RL, Robbins, J, Rossouw, JE, Sarto, GE, Schatz, IJ, Snetselaar, LG, Stevens, VJ, Tinker, LF, Trevisan, M, Vitolins, MZ, Anderson, GL, Assaf, AR, Bassford, T, Beresford, SA, Black, HR, Brunner, RL, Brzyski, RG, Caan, B, Chlebowski, RT, Gass, M, Granek, I, Greenland, P, Hays, J, Heber, D, Heiss, G, Hendrix, SL, Hubbell, FA, Johnson, KC, Kotchen, JM. Low-fat dietary pattern and risk of cardiovascular disease: the Women's Health Initiative Randomized Controlled Dietary Modification Trial. Jama. 2006. 295:655-66. doi:10.1001/jama.295.6.655

1023 Howe, AS, Skidmore, PM, Parnell, WR, Wong, JE, Lubransky, AC, Black, KE. Cardiorespiratory fitness is positively associated with a healthy dietary pattern in New Zealand adolescents. Public Health Nutr. 2016. 19:1279-87. doi:10.1017/s1368980015002566

1024 Howe, HR, 3rd, Heidal, K, Choi, MD, Kraus, RM, Boyle, K, Hickner, RC. Increased adipose tissue lipolysis after a 2-week high-fat diet in sedentary overweight/obese men. Metabolism. 2011. 60:976-81. doi:10.1016/j.metabol.2010.09.007

1025 Hron, BM, Ebbeling, CB, Feldman, HA, Ludwig, DS. Relationship of insulin dynamics to body composition and resting energy expenditure following weight loss. Obesity (Silver Spring). 2015. 23:2216-22. doi:10.1002/oby.21213

1026 Hsiao, PY, Mitchell, DC, Wood, GC, Jensen, GL, Still, CD, Hartman, TJ. The association of dietary patterns and weight change in rural older adults 75 years and older. J Nutr Gerontol Geriatr. 2014. 33:357-75. doi:10.1080/21551197.2014.959681

Study Design; Country

Intervention/Exposure

Intervention/Exposure Publication Status

Intervention/Exposure 
1027 Hsu, WC, Lau, KH, Matsumoto, M, Moghazy, D, Keenan, H, King, GL. Improvement of insulin sensitivity by isoenergy high carbohydrate traditional Asian diet: a randomized controlled pilot feasibility study. PLoS One. 2014. 9:e106851. doi:10.1371/journal.pone.0106851

$1028 \mathrm{Hu}, \mathrm{FB}, \mathrm{Rimm}$, EB, Stampfer, MJ, Ascherio, A, Spiegelman, D, Willett, WC. Prospective study of major dietary patterns and risk of coronary heart disease in men. Am J Clin Nutr. 2000. 72:912-21. doi:10.1093/ajcn/72.4.912

$1029 \mathrm{Hu}, \mathrm{M}, \mathrm{Li}, \mathrm{Z}$, Fang, DZ. The C-514T polymorphism of hepatic lipase gene modulates the impact of a high carbohydrate diet on lipid profile in healthy Chinese young adults. Asian Biomedicine. 2012. 6:675-682. doi:10.5372/1905-7415.0605.107

$1030 \mathrm{Hu}, \mathrm{T}$, Reynolds, K, Yao, L, Bunol, C, Liu, Y, Chen, CS, He, J, Bazzano, L. The long-term effect of a low-carbohydrate diet on endothelial dysfunction and insulin resistance: a randomized controlled trial. Circulation. 2013. 127:. doi:unavailable

$1031 \mathrm{Hu}, \mathrm{T}$, Stuchlik, P, Yao, L, Reynolds, K, Whelton, P, He, J, Bazzano, L. Adherence to low carbohydrate and low fat diets in relation to weight loss and cardiovascular risk factor reduction. Circulation. 2015. 131:. doi:unavailable

$1032 \mathrm{Hu}, \mathrm{T}, \mathrm{Yao}, \mathrm{L}$, Reynolds, K, Niu, T, Li, S, Whelton, PK, He, J, Steffen, LM, Bazzano, LA. Adherence to low-carbohydrate and low-fat diets in relation to weight loss and cardiovascular risk factors. Obes Sci Pract. 2016. 2:24-31. doi:10.1002/osp4.23

$1033 \mathrm{Hu}, \mathrm{T}$, Yao, L, Reynolds, K, Whelton, PK, Niu, T, Li, S, He, J, Bazzano, LA. The Effects of a Low-Carbohydrate Diet vs. a Low-Fat Diet on Novel Cardiovascular Risk Factors: A Randomized Controlled Trial. Nutrients. 2015. 7:7978-94. doi:10.3390/nu7095377

$1034 \mathrm{Hu}$, TY, Chen, YC, Lin, P, Shih, CK, Bai, CH, Yuan, KC, Lee, SY, Chang, JS. Testosterone-Associated Dietary Pattern Predicts Low Testosterone Levels and Hypogonadism. Nutrients. 2018. 10:. doi:10.3390/nu10111786

$1035 \mathrm{Hu}, \mathrm{TY}$, Lee, SY, Shih, CK, Chou, MJ, Wu, MC, Teng, IC, Bai, CH, Sabrina, N, Tinkov, AA, Skalny, AV, Chang, JS. Soluble CD163-Associated Dietary Patterns and the Risk of Metabolic Syndrome. Nutrients. 2019. 11: doi:10.3390/nu11040940

1036 Huang, JH, Li, RH, Huang, SL, Sia, HK, Hsu, WT, Tang, FC. Health-associated nutrition and exercise behaviors in relation to metabolic risk factors stratified by body mass index. International Journal of Environmental Research and Public Health. 2019. 16:. doi:10.3390/ijerph16050869

1037 Huang, T, Beaty, T, Li, J, Liu, H, Zhao, W, Wang, Y. Association between dietary fat intake and insulin resistance in Chinese Country child twins. Br J Nutr. 2017. 117:230-236. doi:10.1017/s0007114516004542

1038 Huang, T, Huang, J, Qi, Q, Li, Y, Bray, GA, Rood, J, Sacks, FM, Qi, L. PCSK7 genotype modifies effect of a weight-loss diet on 2-year changes of insulin resistance: the POUNDS LOST trial. Diabetes Care. 2015. 38:439-44. doi:10.2337/dc14-0473

1039 Huang, T, Shen, Y, Zheng, Y, Smith, S, Bray, G, Sacks, F, Qi, L. Dietary fat intake, MC4R genotype and 2-year changes in body composition: the pounds lost trial. Circulation. 2015. 131:. doi:unavailable

1040 Huang, T, Wang, T, Heianza, Y, Sun, D, Ivey, K, Durst, R, Schwarzfuchs, D, Stampfer, MJ, Bray, GA, Sacks, FM, Shai, I, Qi, L. HNF1A variant, energy-reduced diets and insulin resistance improvement during weight loss: The POUNDS Lost trial and DIRECT. Diabetes Obes Metab. 2018. 20:1445-1452. doi:10.1111/dom.13250 
1041 Huang, T, Wang, T, Heianza, Y, Sun, D, Zheng, Y, Bray, G, Sacks, FM, Qi, L. Genetic susceptibility to obesity, weight-loss diets, and improvement of insulin resistance and beta-cell function: the pounds lost trial. Diabetes. 2016. 65:A391-. doi:10.2337/db16-1375-1656

1042 Huang, T, Xu, M, Lee, A, Cho, S, Qi, L. Consumption of whole grains and cereal fiber and total and cause-specific mortality: prospective analysis of 367,442 individuals. BMC Med. 2015. 13:59. doi:10.1186/s12916-015-0294-7

1043 Huang, T, Zheng, Y, Bray, GA, Sacks, FM, Qi, L. Diabetes genetic score, 2-year changes in insulin sensitivity, and insulin resistance in response to weight loss diets: the pounds lost trial. Diabetes. 2015. 64:A423-A424. doi:10.2337/db1514721800

1044 Huang, X, Gong, R, Lin, J, Li, R, Xiao, L, Duan, W, Fang, D. Effects of lipoprotein lipase gene variations, a highcarbohydrate low-fat diet, and gender on serum lipid profiles in healthy Chinese Han youth. Biosci Trends. 2011. 5:198-204. doi:unavailable

1045 Huang, Z, Li, N, Hu, YM. Dietary patterns and their effects on postpartum weight retention of lactating women in south central China. Nutrition. 2019. 67-68:110555. doi:10.1016/j.nut.2019.110555

1046 Hudson, JL, Kim, JE, Paddon-Jones, D, Campbell, WW. Evenly re-distributing daily dietary protein intake does not augment changes in body composition and cardio-metabolic health indexes. FASEB journal. 2017. 31:. doi:unavailable

1047 Huete, L, Manzano-Lista, FJ, Aranguez, I, Fernandez-Alfonso, MS. Impact of pharmacist's intervention on reducing cardiovascular risk in obese patients. Int J Clin Pharm. 2019. 41:1099-1109. doi:10.1007/s11096-019-00856-w

1048 Huffman, KM, Hawk, VH, Henes, ST, Ocampo, Cl, Orenduff, MC, Slentz, CA, Johnson, JL, Houmard, JA, Samsa, GP Kraus, WE, Bales, CW. Exercise effects on lipids in persons with varying dietary patterns - Does diet matter if they exercise? Responses in Studies of a Targeted Risk Reduction Intervention through Defined Exercise I. American Heart Journal. 2012. 164:117-124. doi:10.1016/j.ahj.2012.04.014

1049 Huisman, MJ, Soedamah-Muthu, SS, Vermeulen, E, Muilwijk, M, Snijder, MB, Nicolaou, MN, van Valkengoed, IGM. Does a High Sugar High Fat Dietary Pattern Explain the Unequal Burden in Prevalence of Type 2 Diabetes in a Multi-Ethnic Population in The Netherlands? The HELIUS Study. Nutrients. 2018. 10:. doi:10.3390/nu10010092

1050 Hulmi, JJ, Isola, V, Suonpaa, M, Jarvinen, NJ, Kokkonen, M, Wennerstrom, A, Nyman, K, Perola, M, Ahtiainen, JP, Hakkinen, K. The Effects of Intensive Weight Reduction on Body Composition and Serum Hormones in Female Fitness Competitors. Front Physiol. 2016. 7:689. doi:10.3389/fphys.2016.00689

1051 Hulsegge, G, van der Schouw, YT, Daviglus, ML, Smit, HA, Verschuren, WM. Determinants of attaining and maintaining a low cardiovascular risk profile--the Doetinchem Cohort Study. Eur J Public Health. 2016. 26:135-40. doi:10.1093/eurpub/ckv125

1052 Hume, DJ, Kroff, J, Clamp, LD, Lambert, EV. Compensations for Weight Loss in Successful and Unsuccessful Dieters. Am J Health Behav. 2015. 39:589-600. doi:10.5993/ajhb.39.5.1

1053 Hung, CJ, Huang, PC, Li, YH, Lu, SC, Ho, LT, Chou, HF. Taiwanese vegetarians have higher insulin sensitivity than omnivores. British Journal of Nutrition. 2006. 95:129-135. doi:10.1079/BJN20051588

Intervention/Exposure

Publication Status

Study duration ;

Country

Study Design;

Participants

Publication Status

Study Design;

Intervention/Exposure

Intervention/Exposure

Study Design

Intervention/Exposure

Outcome

Intervention/Exposure; Outcome

Study Design; Country 
1054 Hung, JD, Murray, SW, Shaw, MA, Unwin, D. Impact of a low carbohydrate diet on traditional CVD risk factors in people with features of the metabolic syndrome and type 2 diabetes. European journal of preventive cardiology. 2018. 25:S14-S15. doi:unavailable

1055 Hwalla, N, Torbay, N, Andari, N, Adra, N, Azar, ST, Habbal, Z. Restoration of normal insulinemia and insulin sensitivity in hyperinsulinemic normoglycemic men by a hypoenergetic high monounsaturated fat diet. Journal of Nutritional and Environmental Medicine. 2004. 14:29-38. doi:10.1080/13590840410001695211

1056 Hwang, JY, Lee, H, Ko, A, Han, CJ, Chung, HW, Chang, N. Dietary changes in Vietnamese marriage immigrant women: The KoGES follow-up study. Nutr Res Pract. 2014. 8:319-26. doi:10.4162/nrp.2014.8.3.319

1057 Hyde, PN, Sapper, TN, Crabtree, CD, LaFountain, RA, Bowling, ML, Buga, A, Fell, B, McSwiney, FT, Dickerson, RM, Miller, VJ, Scandling, D, Simonetti, OP, Phinney, SD, Kraemer, WJ, King, SA, Krauss, RM, Volek, JS. Dietary carbohydrate restriction improves metabolic syndrome independent of weight loss. JCI Insight. 2019. 4: doi:10.1172/jci.insight.128308

1058 lacovides, S, Goble, D, Paterson, B, Meiring, RM. Three consecutive weeks of nutritional ketosis has no effect on cognitive function, sleep, and mood compared with a high-carbohydrate, low-fat diet in healthy individuals: a randomized, crossover, controlled trial. Am J Clin Nutr. 2019. 110:349-357. doi:10.1093/ajcn/nqz073

1059 Ibarra-Reynoso Ldel, R, Pisarchyk, L, Perez-Luque, EL, Garay-Sevilla, ME, Malacara, JM. Dietary restriction in obese children and its relation with eating behavior, fibroblast growth factor 21 and leptin: a prospective clinical intervention study. Nutr Metab (Lond). 2015. 12:31. doi:10.1186/s12986-015-0027-0

1060 Ibe, Y, Takahashi, Y, Sone, H. Food groups and weight gain in Japanese men. Clin Obes. 2014. 4:157-64. doi:10.1111/cob.12056

1061 Ibrahim, M, Bonfiglio, S, Schlogl, M, Vinales, KL, Piaggi, P, Venti, C, Walter, M, Krakoff, J, Thearle, MS. Energy Expenditure and Hormone Responses in Humans After Overeating High-Fructose Corn Syrup Versus Whole-Wheat Foods Obesity (Silver Spring). 2018. 26:141-149. doi:10.1002/oby.22068

1062 Ibsen, DB, Laursen, ASD, Lauritzen, L, Tjonneland, A, Overvad, K, Jakobsen, MU. Substitutions between dairy product subgroups and risk of type 2 diabetes: the Danish Diet, Cancer and Health cohort. Br J Nutr. 2017. 118:989-997. doi:10.1017/s0007114517002896

1063 Iglay, HB, Thyfault, JP, Apolzan, JW, Campbell, WW. Resistance training and dietary protein: effects on glucose tolerance and contents of skeletal muscle insulin signaling proteins in older persons. Am J Clin Nutr. 2007. 85:1005-13. doi:10.1093/ajcn/85.4.1005

1064 Ilich, JZ, Kelly, OJ, Liu, PY, Shin, H, Kim, Y, Chi, Y, Wickrama, Kkas, Colic-Baric, I. Role of Calcium and Low-Fat Dairy Foods in Weight-Loss Outcomes Revisited: Results from the Randomized Trial of Effects on Bone and Body Composition in Overweight/Obese Postmenopausal Women. Nutrients. 2019. 11:. doi:10.3390/nu11051157

1065 Imamura, F, Lemaitre, RN, King, IB, Song, X, Steffen, LM, Folsom, AR, Siscovick, DS, Mozaffarian, D. Long-chain monounsaturated Fatty acids and incidence of congestive heart failure in 2 prospective cohorts. Circulation. 2013. 127:1512-21 1521e1-18. doi:10.1161/circulationaha.112.001197

Intervention/Exposure; Comparator

Study duration

Study duration

Intervention/Exposure

Intervention/Exposure; Comparator

Intervention/Exposure; Study duration

Intervention/Exposure

Intervention/Exposure

Intervention/Exposure

Intervention/Exposure 
1066 Inelmen, EM, Toffanello, ED, Enzi, G, Sergi, G, Coin, A, Busetto, L, Manzato, E. Differences in dietary patterns between older and younger obese and overweight outpatients. J Nutr Health Aging. 2008. 12:3-8. doi:unavailable
Publication Date

Overlaps with Existing Review

Intervention/Exposure

Intervention/Exposure Nutr. 2017. 117:1523-1530. doi:10.1017/s0007114517001568

1068 Isanejad, M, Mursu, J, Sirola, J, Kroger, H, Rikkonen, T, Tuppurainen, M, Erkkila, AT. Association of protein intake with the change of lean mass among elderly women: The Osteoporosis Risk Factor and Prevention - Fracture Prevention Study (OSTPRE-FPS). J Nutr Sci. 2015. 4:e41. doi:10.1017/jns.2015.31

1069 Isanejad, M, Mursu, J, Sirola, J, Kroger, H, Rikkonen, T, Tuppurainen, M, Erkkila, AT. Dietary protein intake is associated with better physical function and muscle strength among elderly women. $\mathrm{Br} \mathrm{J}$ Nutr. 2016. 115:1281-91. doi:10.1017/s000711451600012x

1070 Isanejad, M, Sirola, J, Mursu, J, Rikkonen, T, Kroger, H, Tuppurainen, M, Erkkila, AT. Association of the Baltic Sea and Mediterranean diets with indices of sarcopenia in elderly women, OSPTRE-FPS study. Eur J Nutr. 2018. 57:1435-1448. doi:10.1007/s00394-017-1422-2

1071 Itoh, K, Moriguchi, R, Yamada, Y, Fujita, M, Yamato, T, Oumi, M, Holst, JJ, Seino, Y. High saturated fatty acid intake induces insulin secretion by elevating gastric inhibitory polypeptide levels in healthy individuals. Nutr Res. 2014. 34:653-60. doi:10.1016/j.nutres.2014.07.013

1072 Izadi, V, Esmaillzadeh, A, Hashemipour, M, Surkan, PJ, Azadbakht, L, Kelishadi, R. High protein diets do not affect anthropometric indexes and cardiometabolic risk factors among children with excess weight: A randomized controlled trial. J Cardiovasc Thorac Res. 2018. 10:95-103. doi:10.15171/jcvtr.2018.15

1073 Izadpanah, A, Barnard, RJ, Almeda, AJ, Baldwin, GC, Bridges, SA, Shellman, ER, Burant, CF, Roberts, CK. A short-term diet and exercise intervention ameliorates inflammation and markers of metabolic health in overweight/obese children. Am J Physiol Endocrinol Metab. 2012. 303:E542-50. doi:10.1152/ajpendo.00190.2012

1074 Izaola, O, Primo, D, Gomez Hoyos, E, Lopez Gomez, JJ, Ortola, A, de Luis, D. Association of rs670 variant of APOA1 gene with lipid profile and insulin resistance after 9 months of a high protein/low carbohydrate vs a standard hypocaloric diet. Clinical nutrition (Edinburgh, Scotland). 2019. .. doi:10.1016/j.clnu.2019.04.030

1075 Jaacks, LM, Sher, S, Staercke, C, Porkert, M, Alexander, WR, Jones, DP, Vaccarino, V, Ziegler, TR, Quyyumi, AA. Pilot randomized controlled trial of a Mediterranean diet or diet supplemented with fish oil, walnuts, and grape juice in overweight or obese US adults. BMC Nutr. 2018. 4:26. doi:10.1186/s40795-018-0234-y

1076 Jaaskelainen, A, Kaila-Kangas, L, Leino-Arjas, P, Lindbohm, ML, Nevanpera, N, Remes, J, Jarvelin, MR, Laitinen, J. Association between occupational psychosocial factors and waist circumference is modified by diet among men. Eur J Clin Nutr. 2015. 69:1053-9. doi:10.1038/ejcn.2015.59

1077 Jabekk, PT, Moe, IA, Meen, HD, Tomten, SE, Hostmark, AT. Resistance training in overweight women on a ketogenic diet conserved lean body mass while reducing body fat. Nutr Metab (Lond). 2010. 7:17. doi:10.1186/1743-7075-7-17
Intervention/Exposure; Outcome

Power/Size

Intervention/Exposure; Study duration

Intervention/Exposure

Study duration

Study Design;

Intervention/Exposure

Power/Size

Intervention/Exposure

Study duration 
1078 Jaceldo-Siegl, K, Haddad, E, Knutsen, S, Fan, J, Lloren, J, Bellinger, D, Fraser, GE. Lower C-reactive protein and IL-6 associated with vegetarian diets are mediated by BMI. Nutr Metab Cardiovasc Dis. 2018. 28:787-794. doi:10.1016/j.numecd.2018.03.003

1079 Jaceldo-Siegl, K, Lutjohann, D, Sirirat, R, Mashchak, A, Fraser, GE, Haddad, E. Variations in dietary intake and plasma concentrations of plant sterols across plant-based diets among North American adults. Mol Nutr Food Res. 2017. 61 : doi:10.1002/mnfr.201600828

1080 Jacka, FN, Cherbuin, N, Anstey, KJ, Butterworth, P. Dietary patterns and depressive symptoms over time: examining the relationships with socioeconomic position, health behaviours and cardiovascular risk. PLoS One. 2014. 9:e87657. doi:10.1371/journal.pone.0087657

1081 Jackson, SL, Cunningham, SA. The stability of children's weight status over time, and the role of television, physical activity, and diet. Prev Med. 2017. 100:229-234. doi:10.1016/j.ypmed.2017.04.026

1082 Jacobo-Albavera, L, Posadas-Romero, C, Vargas-Alarcon, G, Romero-Hidalgo, S, Posadas-Sanchez, R, Gonzalez-Salazar Mdel, C, Carnevale, A, Canizales-Quinteros, S, Medina-Urrutia, A, Antunez-Arguelles, E, Villarreal-Molina, T. Dietary fat and carbohydrate modulate the effect of the ATP-binding cassette A1 (ABCA1) R230C variant on metabolic risk parameters in premenopausal women from the Genetics of Atherosclerotic Disease (GEA) Study. Nutr Metab (Lond). 2015. 12:45. doi:10.1186/s12986-015-0040-3

1083 Jacobs, B, De Angelis-Schierbaum, G, Egert, S, Assmann, G, Kratz, M. Individual serum triglyceride responses to high-fat and low-fat diets differ in men with modest and severe hypertriglyceridemia. J Nutr. 2004. 134:1400-5. doi:10.1093/jn/134.6.1400

1084 Jacques, PF, Cassidy, A, Rogers, G, Peterson, JJ, Dwyer, JT. Dietary flavonoid intakes and CVD incidence in the Framingham Offspring Cohort. Br J Nutr. 2015. 114:1496-503. doi:10.1017/s0007114515003141

1085 Jagim, A, Byrd, M, Lockard, B, Baetge, C, Levers, K, Galvan, E, Simbo, S, Jung, YP, Oliver, J, Koozehchian, M, etal, Adherence to a high protein and low fat energy-restricted diet while participating in a circuit resistance-exercise program promotes positive changes in blood glucose and lipids in postmenopausal women. FASEB journal. 2013. 27:. doi:unavailable

1086 Jago, R, Baranowski, T, Baranowski, JC, Thompson, D, Greaves, KA. BMI from 3-6y of age is predicted by TV viewing and physical activity, not diet. International Journal of Obesity. 2005. 29:557-564. doi:10.1038/sj.ijo.0802969

1087 Jakicic, JM, King, WC, Marcus, MD, Davis, KK, Helsel, D, Rickman, AD, Gibbs, BB, Rogers, RJ, Wahed, A, Belle, SH. Short-term weight loss with diet and physical activity in young adults: The IDEA study. Obesity (Silver Spring). 2015. 23:238597. doi:10.1002/oby. 21241

Study Design

Outcome

Study Design;

Outcome

Intervention/Exposure

Study Design;

Intervention/Exposure

Study duration

Intervention/Exposure

Publication Status

Intervention/Exposure

Intervention/Exposure 
1088 Jakobsen, MU, Dethlefsen, C, Due, KM, May, AM, Romaguera, D, Vergnaud, AC, Norat, T, Sorensen, TI, Halkjaer, J, Tjonneland, A, Boutron-Ruault, MC, Clavel-Chapelon, F, Fagherazzi, G, Teucher, B, Kuhn, T, Bergmann, MM, Boeing, H, Naska, A, Orfanos, P, Trichopoulou, A, Palli, D, Santucci De Magistris, M, Sieri, S, Bueno-de-Mesquita, HB, van der, DI A, Engeset, D, Hjartaker, A, Rodriguez, L, Agudo, A, Molina-Montes, E, Huerta, JM, Barricarte, A, Amiano, P, Manjer, J, Wirfalt, E, Hallmans, G, Johansson, I, Khaw, KT, Wareham, NJ, Key, TJ, Chajes, V, Slimani, N, Riboli, E, Peeters, PH, Overvad, K. Fish consumption and subsequent change in body weight in European women and men. Br J Nutr. 2013. 109:353-62. doi:10.1017/s0007114512001079

1089 Jakobsen, MU, Dethlefsen, C, Joensen, AM, Stegger, J, Tjonneland, A, Schmidt, EB, Overvad, K. Intake of carbohydrates compared with intake of saturated fatty acids and risk of myocardial infarction: importance of the glycemic index. Am $\mathrm{J}$ Clin Nutr. 2010. 91:1764-8. doi:10.3945/ajcn.2009.29099

1090 Jakobsen, MU, Madsen, L, Dethlefsen, C, Due, KM, Halkjaer, J, Sorensen, TI, Kristiansen, K, Overvad, K. Dietary n-6 PUFA, carbohydrate:protein ratio and change in body weight and waist circumference: a follow-up study. Public Health Nutr. 2015. 18:1317-23. doi:10.1017/s1368980014001578

1091 Jakobsen, MU, Madsen, L, Skjoth, F, Berentzen, TL, Halkjaer, J, Tjonneland, A, Schmidt, EB, Sorensen, TI, Kristiansen, K, Overvad, K. Dietary intake and adipose tissue content of long-chain n-3 PUFAs and subsequent 5-y change in body weight and waist circumference. Am J Clin Nutr. 2017. 105:1148-1157. doi:10.3945/ajcn.116.140079

1092 Jakobsen, MU, Overvad, K, Dyerberg, J, Schroll, M, Heitmann, BL. Dietary fat and risk of coronary heart disease: possible effect modification by gender and age. Am J Epidemiol. 2004. 160:141-9. doi:10.1093/aje/kwh193

1093 Jakse, B, Jakse, B, Pajek, J, Pajek, M. Effects of ad libitum consumed, low-fat, high-fiber plant-based diet supplemented with plant-based meal replacements on cardiovascular risk factors. Food Nutr Res. 2019. 63:. doi:10.29219/fnr.v63.1560

1094 Jalali-Farahani, S, Amiri, P, Akbar, HM, Cheraghi, L, Karimi, M, Azizi, F. Effects of a Healthy Lifestyle Education on the Incidence of Metabolic Syndrome in Children during a 13-Year Follow-up. Int J Behav Med. 2018. 25:131-140. doi:10.1007/s12529-017-9680-1

1095 Jamar, G, Pisani, LP, Medeiros, A, Oyama, LM, Masquio, DC, Colantonio, E, Garcia, S, Sanches, RB, dos Santos Moraes, A, Belote, C, Caranti, DA. Effect of Fat Intake on the Inflammatory Process and Cardiometabolic Risk in Obesity After Interdisciplinary Therapy. Horm Metab Res. 2016. 48:106-11. doi:10.1055/s-0035-1548871

1096 James, EL, Stacey, FG, Chapman, K, Boyes, AW, Burrows, T, Girgis, A, Asprey, G, Bisquera, A, Lubans, DR. Impact of a nutrition and physical activity intervention (ENRICH: Exercise and Nutrition Routine Improving Cancer Health) on health behaviors of cancer survivors and carers: a pragmatic randomized controlled trial. BMC Cancer. 2015. $15: 710$. doi:10.1186/s12885-015-1775-y

1097 Jancey, JM, Dos Remedios Monteiro, SM, Dhaliwal, SS, Howat, PA, Burns, S, Hills, AP, Anderson, AS. Dietary outcomes of a community based intervention for mothers of young children: a randomised controlled trial. Int J Behav Nutr Phys Act. 2014 11:120. doi:10.1186/s12966-014-0120-1 
1098 Janda, J, Veleminsky, M, Sulakova, T, Prochazka, B, Eliasek, J, Stransky, P, Rokyta, R. Effect of the DASH-diet and salt Kardisal $\circledast$ on blood pressure in adolescents with prehypertension (cooperative multicentre interventional study). Neuroendocrinology Letters. 2017. 38:544-548. doi:unavailable

1099 Janicke, DM, Lim, CS, Perri, MG, Mathews, AE, Bobroff, LB, Gurka, MJ, Parish, A, Brumback, BA, Dumont-Driscoll, M, Silverstein, JH. Featured Article: Behavior Interventions Addressing Obesity in Rural Settings: The E-FLIP for Kids Trial. J Pediatr Psychol. 2019. 44:889-901. doi:10.1093/jpepsy/jsz029

1100 Jankovic, N, Geelen, A, Streppel, MT, de Groot, LC, Kiefte-de Jong, JC, Orfanos, P, Bamia, C, Trichopoulou, A, Boffetta, P, Bobak, M, Pikhart, H, Kee, F, O'Doherty, MG, Buckland, G, Woodside, J, Franco, OH, Ikram, MA, Struijk, EA, Pajak, A Malyutina, S, Kubinova, R, Wennberg, M, Park, Y, Bueno-de-Mesquita, HB, Kampman, E, Feskens, EJ. WHO guidelines for a healthy diet and mortality from cardiovascular disease in European and American elderly: the CHANCES project. Am J Clin Nutr. 2015. 102:745-56. doi:10.3945/ajcn.114.095117

1101 Jankovic, N, Steppel, MT, Kampman, E, de Groot, LC, Boshuizen, HC, Soedamah-Muthu, SS, Kromhout, D, Feskens, EJ. Stability of dietary patterns assessed with reduced rank regression; the Zutphen Elderly Study. Nutr J. 2014. 13:30. doi:10.1186/1475-2891-13-30

1102 Jansen, EC, Kasper, N, Lumeng, JC, Brophy Herb, HE, Horodynski, MA, Miller, AL, Contreras, D, Peterson, KE. Changes in household food insecurity are related to changes in BMI and diet quality among Michigan Head Start preschoolers in a sexspecific manner. Soc Sci Med. 2017. 181:168-176. doi:10.1016/j.socscimed.2017.04.003

1103 Jansen, S, Lopez-Miranda, J, Castro, P, Lopez-Segura, F, Marin, C, Ordovas, JM, Paz, E, Jimenez-Pereperez, J, Fuentes, F, Perez-Jimenez, F. Low-fat and high-monounsaturated fatty acid diets decrease plasma cholesterol ester transfer protein concentrations in young, healthy, normolipemic men. Am J Clin Nutr. 2000. 72:36-41. doi:10.1093/ajcn/72.1.36

1104 Japas, C, Knutsen, S, Dehom, S, Dos Santos, H, Tonstad, S. Body mass index gain between ages 20 and 40 years and lifestyle characteristics of men at ages 40-60 years: the Adventist Health Study-2. Obes Res Clin Pract. 2014. 8:e549-57. doi:10.1016/j.orcp.2013.11.007

1105 Jarvis, M, McNaughton, L, Seddon, A, Thompson, D. The acute 1-week effects of the Zone diet on body composition, blood lipid levels, and performance in recreational endurance athletes. J Strength Cond Res. 2002. 16:50-7. doi:unavailable

1106 Jayasinghe, SN, Breier, BH, McNaughton, SA, Russell, AP, Della Gatta, PA, Mason, S, Stonehouse, W, Walsh, DCI, Kruger, R. Dietary Patterns in New Zealand Women: Evaluating Differences in Body Composition and Metabolic Biomarkers. Nutrients. 2019. 11:. doi:10.3390/nu11071643

1107 Jebb, SA, Lovegrove, JA, Griffin, BA, Frost, GS, Moore, CS, Chatfield, MD, Bluck, LJ, Williams, CM, Sanders, TA. Effect of changing the amount and type of fat and carbohydrate on insulin sensitivity and cardiovascular risk: the RISCK (Reading, Imperial, Surrey, Cambridge, and Kings) trial. Am J Clin Nutr. 2010. 92:748-58. doi:10.3945/ajen.2009.29096

1108 Jen, V, Karagounis, LG, Jaddoe, VWV, Franco, OH, Voortman, T. Dietary protein intake in school-age children and detailed measures of body composition: the Generation R Study. Int J Obes (Lond). 2018. 42:1715-1723. doi:10.1038/s41366-018-0098- 
1109 Jenkins, DJ, Jones, PJ, Frohlich, J, Lamarche, B, Ireland, C, Nishi, SK, Srichaikul, K, Galange, P, Pellini, C, Faulkner, D, de Souza, RJ, Sievenpiper, JL, Mirrahimi, A, Jayalath, VH, Augustin, LS, Bashyam, B, Leiter, LA, Josse, R, Couture, P, Ramprasath, V, Kendall, CW. The effect of a dietary portfolio compared to a DASH-type diet on blood pressure. Nutr Metab Cardiovasc Dis. 2015. 25:1132-9. doi:10.1016/j.numecd.2015.08.006

1110 Jenkins, DJ, Jones, PJ, Lamarche, B, Kendall, CW, Faulkner, D, Cermakova, L, Gigleux, I, Ramprasath, V, de Souza, R, Ireland, C, Patel, D, Srichaikul, K, Abdulnour, S, Bashyam, B, Collier, C, Hoshizaki, S, Josse, RG, Leiter, LA, Connelly, PW, Frohlich, J. Effect of a dietary portfolio of cholesterol-lowering foods given at 2 levels of intensity of dietary advice on serum lipids in hyperlipidemia: a randomized controlled trial. Jama. 2011. 306:831-9. doi:10.1001/jama.2011.1202

1111 Jenkins, DJ, Kendall, CW, Marchie, A, Faulkner, DA, Wong, JM, de Souza, R, Emam, A, Parker, TL, Vidgen, E, Lapsley, KG, Trautwein, EA, Josse, RG, Leiter, LA, Connelly, PW. Effects of a dietary portfolio of cholesterol-lowering foods vs lovastatin on serum lipids and C-reactive protein. Jama. 2003. 290:502-10. doi:10.1001/jama.290.4.502

Intervention/Exposure; Publication Date Overlaps with Existing Review

1112 , Kendall, CW, Marchie, A, Faulkner, DA, Wong, JM, de Souza, R, Emam, A, Parker, TL, Vidgen, E, Trautwein, EA, Lapsley, KG, Josse, RG, Leiter, LA, Singer, W, Connelly, PW. Direct comparison of a dietary portfolio of cholesterollowering foods with a statin in hypercholesterolemic participants. Am J Clin Nutr. 2005. 81:380-7. doi:10.1093/ajcn.81.2.380

1113 Jenkins, DJ, Kendall, CW, Nguyen, TH, Teitel, J, Marchie, A, Chiu, M, Taha, AY, Faulkner, DA, Kemp, T, Wong, JM, de Souza, R, Emam, A, Trautwein, EA, Lapsley, KG, Holmes, C, Josse, RG, Leiter, LA, Singer, W. Effect on hematologic risk factors for coronary heart disease of a cholesterol reducing diet. Eur J Clin Nutr. 2007. 61:483-92. doi:10.1038/sj.ejcn.1602551

1114 Jenkins, DJ, Kendall, CW, Popovich, DG, Vidgen, E, Mehling, CC, Vuksan, V, Ransom, TP, Rao, AV, Rosenberg-Zand, R, Tariq, N, Corey, P, Jones, PJ, Raeini, M, Story, JA, Furumoto, EJ, Illingworth, DR, Pappu, AS, Connelly, PW. Effect of a very-high-fiber vegetable, fruit, and nut diet on serum lipids and colonic function. Metabolism. 2001. 50:494-503. doi:10.1053/meta.2001.21037

1115 Jenkins, DJ, Kendall, CW, Vidgen, E, Mehling, CC, Parker, T, Seyler, H, Faulkner, D, Garsetti, M, Griffin, LC, Agarwal, S, Rao, AV, Cunnane, SC, Ryan, MA, Connelly, PW, Leiter, LA, Vuksan, V, Josse, R. The effect on serum lipids and oxidized low-density lipoprotein of supplementing self-selected low-fat diets with soluble-fiber, soy, and vegetable protein foods. Metabolism. 2000. 49:67-72. doi:10.1016/s0026-0495(00)90738-8

1116 Jenkins, DJ, Wong, JM, Kendall, CW, Esfahani, A, Ng, VW, Leong, TC, Faulkner, DA, Vidgen, E, Paul, G, Mukherjea, R, Krul, ES, Singer, W. Effect of a 6-month vegan low-carbohydrate ('Eco-Atkins') diet on cardiovascular risk factors and body weight in hyperlipidaemic adults: a randomised controlled trial. BMJ Open. 2014. 4:e003505. doi:10.1136/bmjopen-2013003505

1117 Jessri, M, Ng, AP, L'Abbe, MR. Adapting the Healthy Eating Index 2010 for the Canadian Population: Evidence from the Canadian National Nutrition Survey. Nutrients. 2017. 9:. doi:10.3390/nu9080910

1118 Jessri, M, Nishi, SK, L'Abbe, MR. Assessing the Nutritional Quality of Diets of Canadian Adults Using the 2014 Health Canada Surveillance Tool Tier System. Nutrients. 2015. 7:10447-68. doi:10.3390/nu7125543

1119 Jiang, J, Liu, M, Troy, LM, Bangalore, S, Hayes, RB, Parekh, N. Concordance with DASH diet and blood pressure change: results from the Framingham Offspring Study (1991-2008). J Hypertens. 2015. 33:2223-30. doi:10.1097/hjh.0000000000000710

Publication Date

Overlaps with Existing Review

Intervention/Exposure

Intervention/Exposure

Intervention/Exposure; Study duration

Intervention/Exposure

Power/Size

Study Design

Study Design;

Outcome

Outcome 
1120 Jiang, L, Audouze, K, Romero Herrera, JA, Angquist, LH, Kjaerulff, SK, Izarzugaza, JMG, Tjonneland, A, Halkjaer, J, Overvad, K, Sorensen, TIA, Brunak, S. Conflicting associations between dietary patterns and changes of anthropometric traits across subgroups of middle-aged women and men. Clin Nutr. 2019. : doi:10.1016/j.clnu.2019.02.003

1121 Jiang, L, Huang, H, Johnson, A, Dill, EJ, Beals, J, Manson, SM, Roubideaux, Y. Socioeconomic Disparities in Weight and Behavioral Outcomes Among American Indian and Alaska Native Participants of a Translational Lifestyle Intervention Project. Diabetes Care. 2015. 38:2090-9. doi:10.2337/dc15-0394

1122 Jimenez Jaime, T, Leiva Balich, L, Barrera Acevedo, G, de la Maza Cave, MP, Hirsch Birn, S, Henriquez Parada, S, Rodriguez Silva, J, Bunout Barnett, D. Effect of calorie restriction on energy expenditure in overweight and obese adult women. Nutr Hosp. 2015. 31:2428-36. doi:10.3305/nh.2015.31.6.8782

1123 Jimenez, AM, Oliva, SL, Vilar, EG, De Cuevillas, B, Morais Moreno, MDC, Gabella De Prado, J, Diaz, EA, Mauro Martin, IS. The Mediterranean diet pattern with intermittent semi-fasting may facilitate weight loss: Randomised controlled trial. Mediterranean Journal of Nutrition and Metabolism. 2019. 12:153-161. doi:10.3233/MNM-180257

1124 Jimenez-Gomez, Y, Cruz-Teno, C, Rangel-Zuniga, OA, Peinado, JR, Perez-Martinez, P, Delgado-Lista, J, Garcia-Rios, A, Camargo, A, Vazquez-Martinez, R, Ortega-Bellido, M, Perez-Jimenez, F, Roche, HM, Malagon, MM, Lopez-Miranda, J. Effect of dietary fat modification on subcutaneous white adipose tissue insulin sensitivity in patients with metabolic syndrome. Mol Nutr Food Res. 2014. 58:2177-88. doi:10.1002/mnfr.201300901

1125 Jin, Y, Kanaya, AM, Kandula, NR, Rodriguez, LA, Talegawkar, SA. Vegetarian Diets Are Associated with Selected Cardiometabolic Risk Factors among Middle-Older Aged South Asians in the United States. J Nutr. 2018. 148:1954-1960. doi:10.1093/jn/nxy217

1126 Jitnarin, N, Kosulwat, V, Rojroongwasinkul, N, Boonpraderm, A, Haddock, CK, Poston, WS. Risk factors for overweight and obesity among Thai adults: results of the National Thai Food Consumption Survey. Nutrients. 2010. 2:60-74. doi:10.3390/nu20100060

1127 Jobs, E, Adamsson, V, Larsson, A, Jobs, M, Nerpin, E, Ingelsson, E, Arnlov, J, Riserus, U. Influence of a prudent diet on circulating cathepsin S in humans. Nutr J. 2014. 13:84. doi:10.1186/1475-2891-13-84

1128 Johansson-Persson, A, Ulmius, M, Cloetens, L, Karhu, T, Herzig, KH, Onning, G. A high intake of dietary fiber influences Creactive protein and fibrinogen, but not glucose and lipid metabolism, in mildly hypercholesterolemic subjects. Eur $\mathrm{J}$ Nutr. 2014. 53:39-48. doi:10.1007/s00394-013-0496-8

1129 Johns, DJ, Lindroos, AK, Jebb, SA, Sjostrom, L, Carlsson, LM, Ambrosini, GL. Tracking of a dietary pattern and its components over 10-years in the severely obese. PLoS One. 2014. 9:e97457. doi:10.1371/journal.pone.0097457

1130 Johnson, C, Chaput, JP, Rioux, F, Diasparra, M, Richard, C, Dubois, L. An exploration of reported food intake among inmates who gained body weight during incarceration in Canadian federal penitentiaries. PLoS One. 2018. 13:e0208768. doi:10.1371/journal.pone.0208768

1131 Johnson, L, Mander, AP, Jones, LR, Emmett, PM, Jebb, SA. A prospective analysis of dietary energy density at age 5 and 7 years and fatness at 9 years among UK children. Int J Obes (Lond). 2008. 32:586-93. doi:10.1038/sj.ijo.0803746

Study Design;

Intervention/Exposure

tervention/Exposure: Study duration

Intervention/Exposure; Health Status

Study Design

Study Design

Comparator; Outcome

Intervention/Exposure Outcome

Study Design; Intervention/Exposure

Power/Size 
1132 Johnson, L, Mander, AP, Jones, LR, Emmett, PM, Jebb, SA. Energy-dense, low-fiber, high-fat dietary pattern is associated with increased fatness in childhood. Am J Clin Nutr. 2008. 87:846-54. doi:10.1093/ajcn/87.4.846

1133 Johnson, NA, Stannard, SR, Mehalski, K, Trenell, MI, Sachinwalla, T, Thompson, CH, Thompson, MW. Intramyocellular triacylglycerol in prolonged cycling with high- and low-carbohydrate availability. J Appl Physiol (1985). 2003. 94:1365-72. doi:10.1152/japplphysiol.00833.2002

1134 Johnson, NA, Stannard, SR, Rowlands, DS, Chapman, PG, Thompson, CH, O'Connor, H, Sachinwalla, T, Thompson, MW. Effect of short-term starvation versus high-fat diet on intramyocellular triglyceride accumulation and insulin resistance in physically fit men. Exp Physiol. 2006. 91:693-703. doi:10.1113/expphysiol.2006.033399

1135 Johnson, NA, Stannard, SR, Rowlands, DS, Chapman, PG, Thompson, CH, Sachinwalla, T, Thompson, MW. Short-term suppression of plasma free fatty acids fails to improve insulin sensitivity when intramyocellular lipid is elevated. Diabet Med. 2006. 23:1061-8. doi:10.1111/j.1464-5491.2006.01952.x

1136 Johnston, C, Cosgrove, K. Consistency of adherence to a vegan diet on acid-base balance: a randomized controlled trial in healthy omnivore college students. FASEB journal. 2017. 31:. doi:unavailable

1137 Johnston, CS, Tjonn, SL, Swan, PD, White, A, Hutchins, H, Sears, B. Ketogenic low-carbohydrate diets have no metabolic advantage over nonketogenic low-carbohydrate diets. Am J Clin Nutr. 2006. 83:1055-61. doi:10.1093/ajcn/83.5.1055

1138 Johnston, CS, Tjonn, SL, Swan, PD. High-protein, low-fat diets are effective for weight loss and favorably alter biomarkers in healthy adults. J Nutr. 2004. 134:586-91. doi:10.1093/jn/134.3.586

1139 Johnstone, AM, Horgan, GW, Murison, SD, Bremner, DM, Lobley, GE. Effects of a high-protein ketogenic diet on hunger, appetite, and weight loss in obese men feeding ad libitum. Am J Clin Nutr. 2008. 87:44-55. doi:10.1093/ajcn/87.1.44

1140 Johnstone, AM, Lobley, GE, Horgan, GW, Bremner, DM, Fyfe, CL, Morrice, PC, Duthie, GG. Effects of a high-protein, lowcarbohydrate v. high-protein, moderate-carbohydrate weight-loss diet on antioxidant status, endothelial markers and plasma indices of the cardiometabolic profile. Br J Nutr. 2011. 106:282-91. doi:10.1017/s0007114511000092

1141 Jones, JL, Comperatore, M, Barona, J, Calle, MC, Andersen, C, Mclntosh, M, Najm, W, Lerman, RH, Fernandez, ML. A Mediterranean-style, low-glycemic-load diet decreases atherogenic lipoproteins and reduces lipoprotein (a) and oxidized lowdensity lipoprotein in women with metabolic syndrome. Metabolism. 2012. 61:366-72. doi:10.1016/j.metabol.2011.07.013

1142 Jones, JL, Fernandez, ML, Mclntosh, MS, Najm, W, Calle, MC, Kalynych, C, Vukich, C, Barona, J, Ackermann, D, Kim, JE, Kumar, V, Lott, M, Volek, JS, Lerman, RH. A Mediterranean-style low-glycemic-load diet improves variables of metabolic syndrome in women, and addition of a phytochemical-rich medical food enhances benefits on lipoprotein metabolism. J Clin Lipidol. 2011. 5:188-196. doi:10.1016/j.jacl.2011.03.002

1143 Jones, RB, Alderete, TL, Kim, JS, Millstein, J, Gilliland, FD, Goran, MI. High intake of dietary fructose in overweight/obese teenagers associated with depletion of Eubacterium and Streptococcus in gut microbiome. Gut Microbes. 2019. :1-8. doi:10.1080/19490976.2019.1592420

1144 Joo, NS, Park, YW, Park, KH, Kim, CW, Kim, BT. Application of Protein-Rich Oriental Diet in a community-based obesity control program. Yonsei Med J. 2011. 52:249-56. doi:10.3349/ymj.2011.52.2.249

Study duration

Study duration

Study duration

Publication Status

Study duration

Study duration

Study duration

Study duration

Intervention/Exposure; Comparator

Intervention/Exposure; Comparator

Study Design;

Intervention/Exposure

Study duration 
1145 Joost, U, Villa, I, Comasco, E, Oreland, L, Veidebaum, T, Harro, J. Association between Transcription Factor AP-2B genotype, obesity, insulin resistance and dietary intake in a longitudinal birth cohort study. Int J Obes (Lond). 2019. 43:20952106. doi:10.1038/s41366-019-0396-y

1146 Josse, AR, Atkinson, SA, Tarnopolsky, MA, Phillips, SM. Consumption of higher dairy and dietary protein during diet- and exercise-induced weight loss promotes a metabolically favourable body composition change in overweight and obese young women. FASEB journal. 2011. 25:. doi:unavailable

1147 Josse, AR, Atkinson, SA, Tarnopolsky, MA, Phillips, SM. Increased consumption of dairy foods and protein during diet- and exercise-induced weight loss promotes fat mass loss and lean mass gain in overweight and obese premenopausal women. $\mathrm{J}$ Nutr. 2011. 141:1626-34. doi:10.3945/jn.111.141028

1148 Juanola-Falgarona, M, Salas-Salvado, J, Ibarrola-Jurado, N, Rabassa-Soler, A, Bullo, M. Effect of dietary glycemic index and glycemic load on body weight and cardiovascular risk factors: the GLYNDIET Study. Obesity facts. $2013.6: 111$. doi:unavailable

1149 Juanola-Falgarona, M, Salas-Salvado, J, Ibarrola-Jurado, N, Rabassa-Soler, A, Diaz-Lopez, A, Guasch-Ferre, M, Hernandez-Alonso, P, Balanza, R, Bullo, M. Effect of the glycemic index of the diet on weight loss, modulation of satiety, inflammation, and other metabolic risk factors: a randomized controlled trial. Am J Clin Nutr. 2014. 100:27-35. doi:10.3945/ajcn.113.081216

1150 Julia, C, Ducrot, P, Lassale, C, Fezeu, L, Mejean, C, Peneau, S, Touvier, M, Hercberg, S, Kesse-Guyot, E. Prospective associations between a dietary index based on the British Food Standard Agency nutrient profiling system and 13-year weight gain in the SU.VI.MAX cohort. Prev Med. 2015. 81:189-94. doi:10.1016/j.ypmed.2015.08.022

1151 Julia, C, Fezeu, LK, Ducrot, P, Mejean, C, Peneau, S, Touvier, M, Hercberg, S, Kesse-Guyot, E. The Nutrient Profile of Foods Consumed Using the British Food Standards Agency Nutrient Profiling System Is Associated with Metabolic Syndrome in the SU.VI.MAX Cohort. J Nutr. 2015. 145:2355-61. doi:10.3945/jn.115.213629

1152 Julibert, A, Bibiloni, MDM, Bouzas, C, Martinez-Gonzalez, MA, Salas-Salvado, J, Corella, D, Zomeno, MD, Romaguera, D, Vioque, J, Alonso-Gomez, AM, Warnberg, J, Martinez, JA, Serra-Majem, L, Estruch, R, Tinahones, FJ, Lapetra, J, Pinto, X, Lopez-Miranda, J, Garcia-Molina, L, Gaforio, JJ, Matia-Martin, P, Daimiel, L, Martin-Sanchez, V, Vidal, J, Vazquez, C, Ros, E, Toledo, E, Becerra-Tomas, N, Portoles, O, Perez-Vega, KA, Fiol, M, Torres-Collado, L, Tojal-Sierra, L, CarabanoMoral, R, Abete, I, Sanchez-Villegas, A, Casas, R, Bernal-Lopez, MR, Santos-Lozano, JM, Galera, A, Ugarriza, L, RuizCanela, M, Babio, N, Coltell, O, Schroder, H, Konieczna, J, Orozco-Beltran, D, Sorto-Sanchez, C, Eguaras, S, Barrubes, L, Fito, M, Tur, JA, Investigators, PP. Total and Subtypes of Dietary Fat Intake and Its Association with Components of the Metabolic Syndrome in a Mediterranean Population at High Cardiovascular Risk. Nutrients. 2019. 11:. doi:10.3390/nu11071493

1153 Jung, YP, Byrd, M, Baetge, C, Lockard, B, Levers, K, Galvan, E, Jagim, A, Simbo, S, Oliver, JM, Koozehchian, M, etal, . Adherence to a high protein and low fat energy-restricted diet while participating in a circuit resistance-exercise program promotes fat loss with no loss in fat free mass in postmenopausal women. FASEB journal. 2013. 27:. doi:unavailable

1154 Jurado-Fasoli, L, De-la, OA, Castillo, MJ, Amaro-Gahete, FJ. Dietary differences between metabolically healthy overweightobese and metabolically unhealthy overweight-obese adults. Br J Nutr. 2019. :1-21. doi:10.1017/s0007114519002071

Study Design

Intervention/Exposure

Publication Status

Intervention/Exposure

Intervention/Exposure

Weight

loss/Hypocaloric

Intervention/Exposure

Intervention/Exposure

\section{Study Design;}

Intervention/Exposure

Publication Status

Study Design;

Intervention/Exposure 
1155 Juraschek, SP, Appel, LJ, Anderson, CA, Miller, ER, 3rd. Effect of a high-protein diet on kidney function in healthy adults: results from the OmniHeart trial. Am J Kidney Dis. 2013. 61:547-54. doi:10.1053/j.ajkd.2012.10.017

1156 Juraschek, SP, Gelber, AC, Choi, HK, Appel, LJ, Miller, ER, 3rd. Effects of the Dietary Approaches to Stop Hypertension (DASH) Diet and Sodium Intake on Serum Uric Acid. Arthritis Rheumatol. 2016. 68:3002-3009. doi:10.1002/art.39813

Study duration

Outcome

1157 Juraschek, SP, Miller, ER, 3rd, Selvin, E, Carey, VJ, Appel, LJ, Christenson, RH, Sacks, FM. Effect of type and amount of dietary carbohydrate on biomarkers of glucose homeostasis and $C$ reactive protein in overweight or obese adults: results from the OmniCarb trial. BMJ Open Diabetes Res Care. 2016. 4:e000276. doi:10.1136/bmjdrc-2016-000276

1158 Juraschek, SP, Miller, ER, 3rd, Weaver, CM, Appel, LJ. Effects of Sodium Reduction and the DASH Diet in Relation to Baseline Blood Pressure. J Am Coll Cardiol. 2017. 70:2841-2848. doi:10.1016/j.jacc.2017.10.011

1159 Juraschek, SP, Woodward, M, Sacks, FM, Carey, VJ, Miller, ER, 3rd, Appel, LJ. Time Course of Change in Blood Pressure From Sodium Reduction and the DASH Diet. Hypertension. 2017. 70:923-929. doi:10.1161/hypertensionaha.117.10017

1160 Kahleova, H, Fleeman, R, Hlozkova, A, Holubkov, R, Barnard, ND. A plant-based diet in overweight individuals in a 16-week randomized clinical trial: metabolic benefits of plant protein. Nutr Diabetes. 2018. 8:58. doi:10.1038/s41387-018-0067-4

1161 Kahleova, H, Hlozkova, A, Fleeman, R, Fletcher, K, Holubkov, R, Barnard, ND. Fat Quantity and Quality, as Part of a LowFat, Vegan Diet, Are Associated with Changes in Body Composition, Insulin Resistance, and Insulin Secretion. A 16-Week Randomized Controlled Trial. Nutrients. 2019. 11:. doi:10.3390/nu11030615

1162 Kahleova, H, Tura, A, Hill, M, Holubkov, R, Barnard, N. A plant-based diet improves beta-cell function and insulin resistance in overweight adults-a 16-week randomized clinical trial. Diabetes. 2018. 67:A78-. doi:unavailable

1163 Kahleova, H, Tura, A, Hill, M, Holubkov, R, Barnard, ND. A Plant-Based Dietary Intervention Improves Beta-Cell Function and Insulin Resistance in Overweight Adults: A 16-Week Randomized Clinical Trial. Nutrients. 2018. 10. doi:10.3390/nu10020189

1164 Kaippert, VC, Dos Santos Lopes, MCO, De Carvalho, PD, Rosado, EL. Effects of unsaturated fatty acids on weight loss, body composition and obesity related biomarkers. Diabetology \& metabolic syndrome. 2015. 7:59-. doi:unavailable

1165 Kaitosaari, T, Ronnemaa, T, Viikari, J, Raitakari, O, Arffman, M, Marniemi, J, Kallio, K, Pahkala, K, Jokinen, E, Simell, O. Low-saturated fat dietary counseling starting in infancy improves insulin sensitivity in 9-year-old healthy children: the Special Turku Coronary Risk Factor Intervention Project for Children (STRIP) study. Diabetes Care. 2006. 29:781-5. doi:10.2337/diacare.29.04.06.dc05-1523

1166 Kalantarian, S, Rimm, EB, Herrington, DM, Mozaffarian, D. Dietary macronutrients, genetic variation, and progression of coronary atherosclerosis among women. Am Heart J. 2014. 167:627-635.e1. doi:10.1016/j.ahj.2014.01.001

1167 Kanamori, K, Ihana-Sugiyama, N, Yamamoto-Honda, R, Nakamura, T, Sobe, C, Kamiya, S, Kishimoto, M, Kajio, H, Kawano, K, Noda, M. Postprandial Glucose Surges after Extremely Low Carbohydrate Diet in Healthy Adults. Tohoku J Exp Med. 2017. 243:35-39. doi:10.1620/tjem.243.35

1168 Kang, HJ, Jun, DW, Lee, SM, Jang, EC, Cho, YK. Low salt and low calorie diet does not reduce more body fat than same calorie diet: a randomized controlled study. Oncotarget. 2018. 9:8521-8530. doi:10.18632/oncotarget.23959
Study duration

Study duration

Study duration

Data overlap with included article

Data overlap with included article

Publication Status

Data overlap with

included article

Publication Status

Intervention/Exposure

Intervention/Exposure

Study duration

Intervention/Exposure 
1169 Kang, SH, Cho, KH, Do, JY. Association Between the Modified Dietary Approaches to Stop Hypertension and Metabolic

Study Design; Syndrome in Postmenopausal Women Without Diabetes. Metab Syndr Relat Disord. 2018. 16:282-289.

Intervention/Exposure doi:10.1089/met.2018.0007

1170 Kanikowska, D, Sikorska, D, Kuczynska, B, Grzymislawski, M, Breborowicz, A, Witowski, J. Do medical students adhere to advice regarding a healthy lifestyle? A pilot study of BMI and some aspects of lifestyle in medical students in Poland. Adv Clin Exp Med. 2017. 26:1391-1398. doi:10.17219/acem/65783

1171 Kant, AK, Graubard, BI. Energy density of diets reported by American adults: association with food group intake, nutrient intake, and body weight. Int J Obes (Lond). 2005. 29:950-6. doi:10.1038/sj.ijo.0802980

1172 Kant, AK, Leitzmann, MF, Park, Y, Hollenbeck, A, Schatzkin, A. Patterns of recommended dietary behaviors predict subsequent risk of mortality in a large cohort of men and women in the United States. J Nutr. 2009. 139:1374-80. doi:10.3945/jn.109.104505

$1173 \mathrm{Kao}, \mathrm{HN}$, Yang, JH, Hsia, CH, Chen, CY, Lim, SY, Chien, YW. The effect of high protein diet on weight reduction and maintenance in overweight adults. FASEB journal. 2013. 27:. doi:unavailable

1174 Kapelios, CJ, Kyriazis, I, loannidis, I, Dimosthenopoulos, C, Hatziagelaki, E, Liatis, S. Diet, life-style and cardiovascular morbidity in the rural, free living population of Elafonisos island. BMC Public Health. 2017. 17:147. doi:10.1186/s12889-0174053-x

1175 Kapetanakis, M, Liuba, P, Odermarsky, M, Lundgren, J, Hallbook, T. Effects of ketogenic diet on vascular function. Eur J Paediatr Neurol. 2014. 18:489-94. doi:10.1016/j.ejpn.2014.03.006

1176 Kaplan, RJ, Greenwood, CE, Winocur, G, Wolever, TM. Cognitive performance is associated with glucose regulation in healthy elderly persons and can be enhanced with glucose and dietary carbohydrates. Am J Clin Nutr. 2000. 72:825-36. doi:10.1093/ajcn/72.3.825

1177 Kapoor, D, Iqbal, R, Singh, K, Jaacks, LM, Shivashankar, R, Sudha, V, Anjana, RM, Kadir, M, Mohan, V, Ali, MK, Narayan, KM, Tandon, N, Prabhakaran, D, Merchant, AT. Association of dietary patterns and dietary diversity with cardiometabolic disease risk factors among adults in South Asia: The CARRS study. Asia Pac J Clin Nutr. 2018. 27:1332-1343. doi:10.6133/apjen.201811_27(6).0021

1178 Karanja, N, Erlinger, TP, Pao-Hwa, L, Miller, liiER, Bray, GA. The DASH diet for high blood pressure: From clinical trial to dinner table. Cleveland Clinic Journal of Medicine. 2004. 71:745-753. doi:10.3949/ccjm.71.9.745

1179 Karfopoulou, E, Brikou, D, Mamalaki, E, Bersimis, F, Anastasiou, CA, Hill, JO, Yannakoulia, M. Dietary patterns in weight loss maintenance: results from the MedWeight study. Eur J Nutr. 2017. 56:991-1002. doi:10.1007/s00394-015-1147-z

1180 Karl, JP, Roberts, SB, Schaefer, EJ, Gleason, JA, Fuss, P, Rasmussen, H, Saltzman, E, Das, SK. Effects of carbohydrate quantity and glycemic index on resting metabolic rate and body composition during weight loss. Obesity (Silver Spring). 2015. 23:2190-8. doi:10.1002/oby.21268

Study Design;

Intervention/Exposure

Study Design

Publication Date

Overlaps with Existing Review

Publication Status

Study Design

Health Status

Study duration

Country

Study Design

Study Design Intervention/Exposure 
1181 Karwacki-Marugg, C, Huddy, K, Bernstein, B, Whitaker, M, Pranitis, L, Morse, B, Colangelo, R, Stuart, M, O'Donnell, M, Kelsey, AM. Support for Women Achieving Cardiovascular Health Through Exercise And Nutrition (SWAN) Study Pilot. Conn Med. 2016. 80:69-74. doi:unavailable

1182 Kasai, M, Maki, H, Nosaka, N, Aoyama, T, Ooyama, K, Uto, H, Okazaki, M, Igarashi, O, Kondo, K. Effect of medium-chain triglycerides on the postprandial triglyceride concentration in healthy men. Biosci Biotechnol Biochem. 2003. 67:46-53. doi:10.1271/bbb.67.46

1183 Kasim-Karakas, SE, Almario, RU, Mueller, WM, Peerson, J. Changes in plasma lipoproteins during low-fat, highcarbohydrate diets: effects of energy intake. Am J Clin Nutr. 2000. 71:1439-47. doi:10.1093/ajcn/71.6.1439

Kasim-Karakas, SE, Tsodikov, A, Singh, U, Jialal, I. Responses of inflammatory markers to a low-fat, high-carbohydrate diet: effects of energy intake. Am J Clin Nutr. 2006. 83:774-9. doi:10.1093/ajcn/83.4.774

1185 Katsa, ME, loannidis, A, Zyga, S, Tsironi, M, Koutsovitis, P, Chatzipanagiotou, S, Panagiotakos, D, Sachlas, A, Kolovos, P, Routsi, K, Pistikou, AM, Kougioumtzi Dimoliani, DE, Rojas Gil, AP. The Effect of Nutrition and Sleep Habits on Predisposition for Metabolic Syndrome in Greek Children. J Pediatr Nurs. 2018. 40:e2-e8. doi:10.1016/j.pedn.2018.01.012

1186 Katsiki, N, Mikhailidis, DP. The Mediterranean diet in the primary prevention of CVD. Cardiology Review. 2013. 29:. doi:unavailable

1187 Katzmarzyk, PT, Barreira, TV, Broyles, ST, Champagne, CM, Chaput, JP, Fogelholm, M, Hu, G, Johnson, WD, Kuriyan, R, Study Design Kurpad, A, Lambert, EV, Maher, C, Maia, J, Matsudo, V, Olds, T, Onywera, V, Sarmiento, OL, Standage, M, Tremblay, MS, Tudor-Locke, C, Zhao, P, Church, TS. Relationship between lifestyle behaviors and obesity in children ages 9-11: Results from a 12-country study. Obesity (Silver Spring). 2015. 23:1696-702. doi:10.1002/oby.21152

1188 Kaur, B, Ranawana, V, Teh, AL, Henry, CJK. The impact of a low glycemic index (GI) breakfast and snack on daily blood glucose profiles and food intake in young Chinese adult males. J Clin Transl Endocrinol. 2015. 2:92-98. doi:10.1016/j.jcte.2015.05.002

1189 Kawamura, A, Kajiya, K, Kishi, H, Inagaki, J, Mitarai, M, Oda, H, Umemoto, S, Kobayashi, S. Effects of the DASH-JUMP dietary intervention in Japanese participants with high-normal blood pressure and stage 1 hypertension: an open-label singlearm trial. Hypertens Res. 2016. 39:777-785. doi:10.1038/hr.2016.76

1190 Kawamura, A, Kajiya, K, Kishi, H, Inagaki, J, Mitarai, M, Oda, H, Umemoto, S, Kobayashi, S. The Nutritional Characteristics of the Hypotensive WASHOKU-modified DASH Diet: A Sub-analysis of the DASH-JUMP Study. Curr Hypertens Rev. 2018. 14:56-65. doi:10.2174/1573402114666180405100430

1191 Ke, Q, Chen, C, He, F, Ye, Y, Bai, X, Cai, L, Xia, M. Association between dietary protein intake and type 2 diabetes varies by dietary pattern. Diabetol Metab Syndr. 2018. 10:48. doi:10.1186/s13098-018-0350-5

1192 Kell, KP, Judd, SE, Pearson, KE, Shikany, JM, Fernandez, JR. Associations between socio-economic status and dietary patterns in US black and white adults. Br J Nutr. 2015. 113:1792-9. doi:10.1017/s0007114515000938

Study Design; Intervention/Exposure

Study Design;

Intervention/Exposure

Study Design;

Intervention/Exposure

Study Design

Intervention/Exposur

Study Design;

Intervention/Exposure

Study Design

Study Design; Country

Study Design;

Outcome 
1193 Kent, L, Morton, D, Rankin, P, Ward, E, Grant, R, Gobble, J, Diehl, H. The effect of a low-fat, plant-based lifestyle intervention (CHIP) on serum HDL levels and the implications for metabolic syndrome status - a cohort study. Nutr Metab (Lond). 2013. 10:58. doi:10.1186/1743-7075-10-58

1194 Kent, LM, Grant, RS, Watts, G, Morton, DP, Rankin, PM, Ward, EJ. HDL subfraction changes with a low-fat, plant-based Complete Health Improvement Program (CHIP). Asia Pac J Clin Nutr. 2018. 27:1002-1009. doi:10.6133/apjcn.052018.05

1195 Keogh, JB, Brinkworth, GD, Clifton, PM. Effects of weight loss on a low-carbohydrate diet on flow-mediated dilatation, adhesion molecules and adiponectin. Br J Nutr. 2007. 98:852-9. doi:10.1017/s0007114507747815

1196 Keogh, JB, Brinkworth, GD, Noakes, M, Belobrajdic, DP, Buckley, JD, Clifton, PM. Effects of weight loss from a very-lowcarbohydrate diet on endothelial function and markers of cardiovascular disease risk in subjects with abdominal obesity. Am $\mathrm{J}$ Clin Nutr. 2008. 87:567-76. doi:10.1093/ajcn/87.3.567

1197 Keogh, JB, Grieger, JA, Noakes, M, Clifton, PM. Flow-mediated dilatation is impaired by a high-saturated fat diet but not by a high-carbohydrate diet. Arterioscler Thromb Vasc Biol. 2005. 25:1274-9. doi:10.1161/01.ATV.0000163185.28245.a1

1198 Keogh, JB, Luscombe-Marsh, ND, Noakes, M, Wittert, GA, Clifton, PM. Long-term weight maintenance and cardiovascular risk factors are not different following weight loss on carbohydrate-restricted diets high in either monounsaturated fat or protein in obese hyperinsulinaemic men and women. Br J Nutr. 2007. 97:405-10. doi:10.1017/s0007114507252687

1199 Kephart, WC, Pledge, CD, Roberson, PA, Mumford, PW, Romero, MA, Mobley, CB, Martin, JS, Young, KC, Lowery, RP, Wilson, JM, Huggins, KW, Roberts, MD. The Three-Month Effects of a Ketogenic Diet on Body Composition, Blood Parameters, and Performance Metrics in CrossFit Trainees: A Pilot Study. Sports (Basel). 2018. 6:. doi:10.3390/sports6010001

1200 Kerksick, C, Thomas, A, Campbell, B, Taylor, L, Wilborn, C, Marcello, B, Roberts, M, Pfau, E, Grimstvedt, M, Opusunju, J, Magrans-Courtney, T, Rasmussen, C, Wilson, R, Kreider, RB. Effects of a popular exercise and weight loss program on weight loss, body composition, energy expenditure and health in obese women. Nutr Metab (Lond). 2009. 6:23. doi:10.1186/1743-7075-6-23

1201 Kerksick, CM, Wismann-Bunn, J, Fogt, D, Thomas, AR, Taylor, L, Campbell, BI, Wilborn, CD, Harvey, T, Roberts, MD, La Bounty, P, Galbreath, M, Marcello, B, Rasmussen, CJ, Kreider, RB. Changes in weight loss, body composition and cardiovascular disease risk after altering macronutrient distributions during a regular exercise program in obese women. Nutr $\mathrm{J}$. 2010. 9:59. doi:10.1186/1475-2891-9-59

1202 Kesse-Guyot, E, Bertrais, S, Péneau, S, Estaquio, C, Dauchet, L, Vergnaud, AC, Czernichow, S, Galan, P, Hercberg, S, Bellisle, F. Dietary patterns and their sociodemographic and behavioural correlates in French middle-aged adults from the SU.VI.MAX cohort. European Journal of Clinical Nutrition. 2009. 63:521-528. doi:10.1038/sj.ejcn.1602978

1203 Kesse-Guyot, E, Fezeu, L, Galan, P, Hercberg, S, Czernichow, S, Castetbon, K. Adherence to French nutritional guidelines is associated with lower risk of metabolic syndrome. Journal of Nutrition. 2011. 141:1134-1139. doi:10.3945/jn.110.136317

1204 Kesse-Guyot, E, Touvier, M, Henegar, A, Czernichow, S, Galan, P, Hercberg, S, Castetbon, K. Higher adherence to French dietary guidelines and chronic diseases in the prospective SU.VI.MAX cohort. European Journal of Clinical Nutrition. 2011. 65:887-894. doi:10.1038/ejcn.2011.61
Study Design;

Intervention/Exposure

Study Design;

Comparator

Weight

oss/Hypocaloric

Study duration

Study duration

Weight

oss/Hypocaloric

Intervention/Exposure; Comparator

Intervention/Exposure; Publication Status

Study duration

Study Design

Outcome; Publication Date Overlaps with Existing Review

Publication Date Overlaps with Existing Review 
1205 Kessler, K, Hornemann, S, Petzke, KJ, Kemper, M, Kramer, A, Pfeiffer, AF, Pivovarova, O, Rudovich, N. The effect of diurnal distribution of carbohydrates and fat on glycaemic control in humans: a randomized controlled trial. Sci Rep. 2017. 7:44170. doi:10.1038/srep44170

1206 Keyserling, TC, Samuel-Hodge, CD, Pitts, SJ, Garcia, BA, Johnston, LF, Gizlice, Z, Miller, CL, Braxton, DF, Evenson, KR, Smith, JC, Davis, GB, Quenum, EL, Elliott, NT, Gross, MD, Donahue, KE, Halladay, JR, Ammerman, AS. A communitybased lifestyle and weight loss intervention promoting a Mediterranean-style diet pattern evaluated in the stroke belt of North Carolina: the Heart Healthy Lenoir Project. BMC Public Health. 2016. 16:732. doi:10.1186/s12889-016-3370-9

1207 Khakimov, B, Poulsen, SK, Savorani, F, Acar, E, Gurdeniz, G, Larsen, TM, Astrup, A, Dragsted, LO, Engelsen, SB. New Nordic Diet versus Average Danish Diet: A Randomized Controlled Trial Revealed Healthy Long-Term Effects of the New Nordic Diet by GC-MS Blood Plasma Metabolomics. J Proteome Res. 2016. 15:1939-54. doi:10.1021/acs.jproteome.6b00109

1208 Khalil, H, Murrin, C, O'Reilly, M, Viljoen, K, Segurado, R, O'Brien, J, Somerville, R, McGillicuddy, F, Kelleher, CC. Total HDL cholesterol efflux capacity in healthy children - Associations with adiposity and dietary intakes of mother and child. Nutr Metab Cardiovasc Dis. 2017. 27:70-77. doi:10.1016/j.numecd.2016.10.002

1209 Kheirat, F, Merzouk, H, Saidi Merzouk, A, Merzouk, SA, Belarbi, B. One year changes in biochemical and redox markers in training menopausal women with adherence to Mediterranean diet. Science and Sports. 2018. 33:e25-e32. doi:10.1016/j.scispo.2017.07.013

1210 Khera, AV, Plutzky, J. Management of low levels of high-density lipoprotein-cholesterol. Circulation. 2013. 128:72-78. doi:10.1161/CIRCULATIONAHA.112.000443

1211 Kilinç, FN, Çağdaş, D. Evaluation of body compositions, dietary habits and nutritional knowledge of health college students. Turk Pediatri Arsivi. 2012. 47:181-188. doi:10.4274/tpa.842

1212 Kim, HS, Demyen, MF, Mathew, J, Kothari, N, Feurdean, M, Ahlawat, SK. Obesity, Metabolic Syndrome, and Cardiovascular Risk in Gluten-Free Followers Without Celiac Disease in the United States: Results from the National Health and Nutrition Examination Survey 2009-2014. Dig Dis Sci. 2017. 62:2440-2448. doi:10.1007/s10620-017-4583-1

1213 Kim, IY, Schutzler, SE, Azhar, G, Wolfe, RR, Ferrando, AA, Coker, RH. Short term elevation in dietary protein intake does not worsen insulin resistance or lipids in older adults with metabolic syndrome: a randomized-controlled trial. BMC Nutr. 2017. 3:. doi:10.1186/s40795-017-0152-4

$1214 \mathrm{Kim}, \mathrm{J}, \mathrm{Kim}$, J. Association between Fruit and Vegetable Consumption and Risk of Hypertension in Middle-Aged and Older Korean Adults. J Acad Nutr Diet. 2018. 118:1438-1449.e5. doi:10.1016/j.jand.2017.08.122

1215 Kim, M, Tanaka, K. Non-high-density lipoprotein cholesterol changes in middle-aged obese men with and without metabolic syndrome during weight loss. Metabolic Syndrome and Related Disorders. 2014. 12:464-471. doi:10.1089/met.2014.0032

$1216 \mathrm{Kim}, \mathrm{MH}$, Bae, YJ. Comparative Study of Serum Leptin and Insulin Resistance Levels Between Korean Postmenopausal Vegetarian and Non-vegetarian Women. Clin Nutr Res. 2015. 4:175-81. doi:10.7762/cnr.2015.4.3.175

$1217 \mathrm{Kim}, \mathrm{MH}, \mathrm{Bae}, \mathrm{YJ}$. Postmenopausal vegetarians' low serum ferritin level may reduce the risk for metabolic syndrome. Biological Trace Element Research. 2012. 149:34-41. doi:10.1007/s12011-012-9405-X

Intervention/Exposure

Intervention/Exposure; Outcome

Intervention/Exposure; Outcome

Study Design; Intervention/Exposure

Study Design

Study Design;

Language

Study Design

Intervention/Exposure

Intervention/Exposure; Outcome

Study Design

Study Design; Intervention/Exposure Study Design 
$1218 \mathrm{Kim}$, MK. Effects of low calorie diet-induced weight loss on post-exercise heart rate recovery in obese men. J Exerc Nutrition Biochem. 2014. 18:181-8. doi:10.5717/jenb.2014.18.2.181

Intervention/Exposure; Outcome

$1219 \mathrm{Kim}, \mathrm{N}, \mathrm{Seo}$, DC, King, MH, Lederer, AM, Sovinski, D. Long-term predictors of blood pressure among adolescents during an 18-month school-based obesity prevention intervention. Journal of Adolescent Health. 2014. 55:521-527. doi:10.1016/j.jadohealth.2014.04.011

$1220 \mathrm{Kim}, \mathrm{SA}, \mathrm{Ha}, \mathrm{K}$, Ahn, S, Ham, D, Kim, S, Joung, H. A randomized clinical trial for the effects of rice-based Korean diet on cardiovascular risk factors in Korean adults. FASEB journal. 2017. 31:. doi:unavailable

1221 Kim, Y, Keogh, JB, Clifton, PM. Consumption of red and processed meat and refined grains for 4weeks decreases insulin sensitivity in insulin-resistant adults: A randomized crossover study. Metabolism. 2017. 68:173-183. doi:10.1016/j.metabol.2016.12.011

1222 Kim, Y, Keogh, JB, Clifton, PM. Differential Effects of Red Meat/Refined Grain Diet and Dairy/Chicken/Nuts/Whole Grain Diet on Glucose, Insulin and Triglyceride in a Randomized Crossover Study. Nutrients. 2016. 8:. doi:10.3390/nu8110687

$1223 \mathrm{Kim}$, Y, Keogh, JB, Clifton, PM. Effects of Two Different Dietary Patterns on Inflammatory Markers, Advanced Glycation End Products and Lipids in Subjects without Type 2 Diabetes: A Randomised Crossover Study. Nutrients. 2017. 9:. doi:10.3390/nu9040336

1224 Kimokoti, RW, Brown, LS. Dietary management of the metabolic syndrome. Clinical Pharmacology and Therapeutics. 2011. 90:184-187. doi:10.1038/clpt.2011.92

1225 Kimokoti, RW, Gona, P, Zhu, L, Newby, PK, Millen, BE, Brown, LS, D'Agostino, RB, Fung, TT. Dietary patterns of women are associated with incident abdominal obesity but not metabolic syndrome. Journal of Nutrition. 2012. 142:1720-1727. doi:10.3945/jn.112.162479

1226 Kimokoti, RW, Newby, PK, Gona, P, Zhu, L, Campbell, WR, D'Agostino, RB, Millen, BE. Stability of the Framingham Nutritional Risk Score and its component nutrients over 8 years: The Framingham Nutrition Studies. European Journal of Clinical Nutrition. 2012. 66:336-344. doi:10.1038/ejcn.2011.167

1227 Kimokoti, RW, Newby, PK, Gona, P, Zhu, L, Jasuja, GK, Pencina, MJ, McKeon-O'Malley, C, Fox, CS, D'Agostino, RB, Millen, BE. Diet quality, physical activity, smoking status, and weight fluctuation are associated with weight change in women and men. Journal of Nutrition. 2010. 140:1287-1293. doi:10.3945/jn.109.120808

1228 Kineish, O, Stentz, FB, Kitabchi, AE. Effect of high protein vs high carbohydrate diets on satiety and cardiovascular factors. Journal of investigative medicine. 2013. 61:496. doi:10.231/JIM.0b013e3182820c55

1229 Kirk, E, Reeds, DN, Finck, BN, Mayurranjan, SM, Patterson, BW, Klein, S. Dietary fat and carbohydrates differentially alter insulin sensitivity during caloric restriction. Gastroenterology. 2009. 136:1552-60. doi:10.1053/j.gastro.2009.01.048

1230 Kirk, S, Brehm, B, Saelens, BE, Woo, JG, Kissel, E, D'Alessio, D, Bolling, C, Daniels, SR. Role of carbohydrate modification in weight management among obese children: a randomized clinical trial. J Pediatr. 2012. 161:320-7.e1. doi:10.1016/j.jpeds.2012.01.041

Intervention/Exposure

Publication Status

Study duration

Study duration

Study duration

Study Design

Intervention/Exposure; Publication Date

Overlaps with Existing Review

Study Design;

Intervention/Exposure

Intervention/Exposure

Publication Status

Study duration

Weight

loss/Hypocaloric 
1231 Kirk, S, Woo, JG, Brehm, B, Daniels, SR, Saelens, BE. Changes in Eating Behaviors of Children with Obesity in Response to Carbohydrate-Modified and Portion-Controlled Diets. Child Obes. 2017. 13:377-383. doi:10.1089/chi.2017.0020

Intervention/Exposure; Outcome

1232 Kirk, S, Woo, JG, Jones, MN, Siegel, RM. Increased frequency of dietitian visits is associated with improved body mass index outcomes in obese youth participating in a comprehensive pediatric weight management program. Child Obes. 2015. 11:202-8. doi:10.1089/chi.2014.0079

1233 Kirkegaard, H, Stovring, H, Rasmussen, KM, Abrams, B, Sørensen, TIA, Nohr, EA. Maternal weight change from prepregnancy to 7 years postpartum - The influence of behavioral factors. Obesity. 2015. 23:870-878. doi:10.1002/oby.21022

1234 Kirkwood, L, Aldujaili, E, Drummond, S. Effects of advice on dietary intake and/or physical activity on body composition, blood lipids and insulin resistance following a low-fat, sucrose-containing, high-carbohydrate, energy-restricted diet. Int J Food Sci Nutr. 2007. 58:383-97. doi:10.1080/09637480701252336

1235 Kistorp, C, Bliddal, H, Goetze, JP, Christensen, R, Faber, J. Cardiac natriuretic peptides in plasma increase after dietary induced weight loss in obesity. BMC Obes. 2014. 1:24. doi:10.1186/s40608-014-0024-2

1236 Kitabchi, AE, Brewer, A, Wan, J, Sands, C, Stentz, FB. Remission of impaired glucose tolerance (IGT) to normal glucose tolerance (NGT) in obese adults with high protein vs. High carbohydrate diet. Diabetes. 2015. 64:A23-A24 doi:10.2337/db151385

1237 Kitabchi, AE, Stentz, FB, Nyenwe, EA, McDaniel, KA, Tylavsky, FA, Wan, JY, Sands, CW. Effect of macronutrients on markers of oxidative stress, proinflammatory cytokines, adipokines, CV risk factors and lipid peroxidation in obese non-diabetic women. Diabetes. 2012. 61:A11. doi:10.2337/db12-1-377

1238 Klass, DM, Buhrmann, K, Sauter, G, Del Puppo, M, Scheibner, J, Fuchs, M, Stange, EF. Biliary lipids, cholesterol and bile synthesis: different adaptive mechanisms to dietary cholesterol in lean and obese subjects. Aliment Pharmacol Ther. 2006. 23:895-905. doi:10.1111/j.1365-2036.2006.02836.x

1239 Kleiner, RE, Hutchins, AM, Johnston, CS, Swan, PD. Effects of an 8-week high-protein or high-carbohydrate diet in adults with hyperinsulinemia. MedGenMed. 2006. 8:39. doi:unavailable

1240 Klempel, MC, Kroeger, CM, Norkeviciute, E, Goslawski, M, Phillips, SA, Varady, KA. Benefit of a low-fat over high-fat diet on vascular health during alternate day fasting. Nutr Diabetes. 2013. 3:e71. doi:10.1038/nutd.2013.14

1241 Klempel, MC, Kroeger, CM, Varady, KA. Alternate day fasting (ADF) with a high-fat diet produces similar weight loss and cardio-protection as ADF with a low-fat diet. Metabolism. 2013. 62:137-43. doi:10.1016/j.metabol.2012.07.002

1242 Klemsdal, TO, Holme, I, Nerland, H, Pedersen, TR, Tonstad, S. Effects of a low glycemic load diet versus a low-fat diet in subjects with and without the metabolic syndrome. Nutr Metab Cardiovasc Dis. 2010. 20:195-201. doi:10.1016/j.numecd.2009.03.010

1243 Klonizakis, M, Grammatikopoulou, MG, Theodoridis, X, Milner, M, Liu, Y, Chourdakis, M. Effects of Long-Versus Short-Term Exposure to the Mediterranean Diet on Skin Microvascular Function and Quality of Life of Healthy Adults in Greece and the UK. Nutrients. 2019. 11:. doi:10.3390/nu11102487

Participants

Weight

loss/Hypocaloric

Intervention/Exposure

Publication Status

Intervention/Exposure

Study duration

Intervention/Exposure

Intervention/Exposure; Outcome

Intervention/Exposure

Weight

loss/Hypocaloric

Study duration 
1244 Knopp, RH, Paramsothy, P, Retzlaff, BM, Dowdy, A, Fish, B. Undesirable effects of extreme dietary carbohydrate and saturated fat intakes: the search for the middle ground. Curr Atheroscler Rep. 2005. 7:409-11. doi:unavailable

1245 Knopp, RH, Retzlaff, B, Walden, C, Fish, B, Buck, B, McCann, B. One-year effects of increasingly fat-restricted, carbohydrate-enriched diets on lipoprotein levels in free-living subjects. Proc Soc Exp Biol Med. 2000. 225:191-9. doi:10.1046/j.1525-1373.2000.22524.x

1246 Koebnick, C, Plank-Habibi, S, Wirsam, B, Gruendel, S, Hahn, A, Meyer-Kleine, C, Leitzmann, C, Zunft, HJ. Double-blind, randomized feedback control fails to improve the hypocholesterolemic effect of a plant-based low-fat diet in patients with moderately elevated total cholesterol levels. Eur J Clin Nutr. 2004. 58:1402-9. doi:10.1038/sj.ejcn.1601984

1247 Koebnick, C, Plank-Habibi, S, Wirsam, B, Gruendel, S, Hahn, A, Meyer-Kleine, C, Leitzmann, C, Zunft, HJF. Double-blind, randomized feedback controls fails to improve the hypocholesterolemic effect of a plant-based low-fat diet in patients with moderately elevated total cholesterol levels. European Journal of Clinical Nutrition. 2004. 58:1402-1409. doi:10.1038/sj.ejcn.1601984

1248 Kohler, B, Andreen, I. Body weight maintenance and levels of mutans streptococci and lactobacilli in a group of Swedish women seven years after completion of a Weight Watchers' diet. Swed Dent J. 2011. 35:101-9. doi:unavailable

1249 Kokanovic, A, Mandic ML, Banjari, I. Does individual dietary intervention have any impact on adolescents with cardiovascular health risks?. Med Glas (Zenica). 2014. 11:234-7. doi:unavailable

1250 Kokkinos, P, Moutsatsos, G. Obesity and cardiovascular disease: The role of diet and physical activity. Journal of Cardiopulmonary Rehabilitation. 2004. 24:197-203. doi:10.1097/00008483-200405000-00011

1251 Kolahdooz, F, Nader, F, Daemi, M, Jang, SL, Johnston, N, Sharma, S. Prevalence of Known Risk Factors for Type 2 Diabetes Mellitus in Multiethnic Urban Youth in Edmonton: Findings From the WHY ACT NOW Project. Can J Diabetes. 2019. 43:207-214. doi:10.1016/j.jcjd.2018.10.002

1252 Kolehmainen, M, Ulven, SM, Paananen, J, de Mello, V, Schwab, U, Carlberg, C, Myhrstad, M, Pihlajamaki, J, Dungner, E, Sjolin, E, Gunnarsdottir, I, Cloetens, L, Landin-Olsson, M, Akesson, B, Rosqvist, F, Hukkanen, J, Herzig, KH, Dragsted, LO, Savolainen, MJ, Brader, L, Hermansen, K, Riserus, U, Thorsdottir, I, Poutanen, KS, Uusitupa, M, Arner, P, Dahlman, I. Healthy Nordic diet downregulates the expression of genes involved in inflammation in subcutaneous adipose tissue in individuals with features of the metabolic syndrome. Am J Clin Nutr. 2015. 101:228-39. doi:10.3945/ajcn.114.092783

1253 Komiyama, N, Saito, T, Hosaka, Y, Aida, K, Kaneko, T, Sato, A, Onaya, T, Kobayashi, T, Tawata, M. Effects of a 4-week $70 \%$ high carbohydrate/15\% low fat diet on glucose tolerance and on lipid profiles. Diabetes Res Clin Pract. 2004. 64:11-8. doi:10.1016/j.diabres.2003.10.002

1254 Kong, AP, Choi, KC, Chan, RS, Lok, K, Ozaki, R, Li, AM, Ho, CS, Chan, MH, Sea, M, Henry, CJ, Chan, JC, Woo, J. A randomized controlled trial to investigate the impact of a low glycemic index (GI) diet on body mass index in obese adolescents. BMC Public Health. 2014. 14:180. doi:10.1186/1471-2458-14-180
Study Design;

Publication Status

Study Design;

Intervention/Exposure

Study duration

Study duration

Intervention/Exposure

Study Design

Study Design

Intervention/Exposure

Outcome

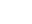

\section{Study duration}

Weight loss/Hypocaloric 
1255 Kong, LC, Holmes, BA, Cotillard, A, Habi-Rachedi, F, Brazeilles, R, Gougis, S, Gausseres, N, Cani, PD, Fellahi, S, Bastard, JP, Kennedy, SP, Dore, J, Ehrlich, SD, Zucker, JD, Rizkalla, SW, Clement, K. Dietary patterns differently associate with inflammation and gut microbiota in overweight and obese subjects. PLoS One. 2014. 9:e109434. doi:10.1371/journal.pone.0109434

1256 Koopman, KE, Booij, J, Fliers, E, La Fleur, SE, Serlie, MJ. A hypercaloric high fat high sugar diet decreases diencephalic serotonin transporters in humans. Clinical nutrition, supplement. 2012. 7:30. doi:10.1016/S1744-1161\%2812\%2970065-0

1257 Kouris-Blazos, A, Itsiopoulos, C. Low all-cause mortality despite high cardiovascular risk in elderly Greek-born Australians: attenuating potential of diet?. Asia Pacific journal of clinical nutrition. 2014. 23:532-544. doi:10.6133/apjcn.2014.23.4.16

1258 Koutsari, C, Malkova, D, Hardman, AE. Postprandial lipemia after short-term variation in dietary fat and carbohydrate. Metabolism. 2000. 49:1150-5. doi:10.1053/meta.2000.8612

1259 Koutsari, C, Sidossis, LS. Effect of isoenergetic low- and high-carbohydrate diets on substrate kinetics and oxidation in healthy men. Br J Nutr. 2003. 90:413-8. doi:10.1079/bjn2003894

1260 Kouvari, M, Notara, V, Panagiotakos, DB, Michalopoulou, M, Vassileiou, N, Papataxiarchis, E, Tzanoglou, D, Mantas, Y, Kogias, Y, Stravopodis, P, Papanagnou, G, Zombolos, S, Pitsavos, C. Exclusive olive oil consumption and 10-year (20042014) acute coronary syndrome incidence among cardiac patients: the GREECS observational study. J Hum Nutr Diet. 2016. 29:354-62. doi:10.1111/jhn.12324

1261 Kovell, LC, Yeung, EH, Miller, ER, 3rd, Appel, LJ, Christenson, RH, Rebuck, H, Schulman, SP, Juraschek, SP. Healthy diet reduces markers of cardiac injury and inflammation regardless of macronutrients: Results from the OmniHeart trial. Int $\mathrm{J}$ Cardiol. 2019. :. doi:10.1016/j.ijcard.2019.07.102

1262 Krajcovicova-Kudlackova, M, Blazicek, P, Babinska, K, Kopcova, J, Klvanova, J, Bederova, A, Magalova, T. Traditional and alternative nutrition--levels of homocysteine and lipid parameters in adults. Scand J Clin Lab Invest. 2000. 60:657-64. doi:10.1080/00365510050216385

1263 Kralova Lesna, I, Suchanek, P, Brabcova, E, Kovar, J, Malinska, H, Poledne, R. Effect of different types of dietary fatty acids on subclinical inflammation in humans. Physiol Res. 2013. 62:145-52. doi:unavailable

1264 Kramer, CS, Szmidt, MK, Sicinska, E, Brzozowska, A, Santoro, A, Franceschi, C, de Groot, Lcpgm, Berendsen, AAM. The Elderly-Nutrient Rich Food Score Is Associated With Biochemical Markers of Nutritional Status in European Older Adults. Front Nutr. 2019. 6:150. doi:10.3389/fnut.2019.00150

1265 Krasevec, J, An, X, Kumapley, R, Bégin, F, Frongillo, EA. Diet quality and risk of stunting among infants and young children in low- and middle-income countries. Maternal and Child Nutrition. 2017. 13:. doi:10.1111/mcn.12430

1266 Kratz, M, Weigle, DS, Breen, PA, Meeuws, KE, Burden, VR, Callahan, HS, Matthys, CC, Purnell, JQ. Exchanging carbohydrate or protein for fat improves lipid-related cardiovascular risk profile in overweight men and women when consumed ad libitum. J Investig Med. 2010. 58:711-9. doi:10.231/JIM.0b013e3181da4d37

1267 Krauss, RM, Blanche, PJ, Rawlings, RS, Fernstrom, HS, Williams, PT. Separate effects of reduced carbohydrate intake and weight loss on atherogenic dyslipidemia. Am J Clin Nutr. 2006. 83:1025-31; quiz 1205. doi:10.1093/ajcn/83.5.1025

Publication Status

Study Design

Study duration

Study duration

Health Status

Study duration

Study Design

ntervention/Exposure; Study duration

Study Design;

Outcome

Country; Age at Intervention/Exposure

Study Design;

Intervention/Exposure

Weight

oss/Hypocaloric 
1268 Krebs, NF, Gao, D, Gralla, J, Collins, JS, Johnson, SL. Efficacy and safety of a high protein, low carbohydrate diet for weight loss in severely obese adolescents. J Pediatr. 2010. 157:252-8. doi:10.1016/j.jpeds.2010.02.010

1269 Kreider, RB, Rasmussen, C, Kerksick, CM, Wilborn, C, Taylor, th L, Campbell, B, Magrans-Courtney, T, Fogt, D, Ferreira, M, Li, R, Galbreath, M, losia, M, Cooke, M, Serra, M, Gutierrez, J, Byrd, M, Kresta, JY, Simbo, S, Oliver, J, Greenwood, M. A carbohydrate-restricted diet during resistance training promotes more favorable changes in body composition and markers of health in obese women with and without insulin resistance. Phys Sportsmed. 2011. 39:27-40. doi:10.3810/psm.2011.05.1893

1270 Krieger, JP, Pestoni, G, Cabaset, S, Brombach, C, Sych, J, Schader, C, Faeh, D, Rohrmann, S. Dietary Patterns and Their Sociodemographic and Lifestyle Determinants in Switzerland: Results from the National Nutrition Survey menuCH. Nutrients. 2018. 11:. doi:10.3390/nu11010062

1271 Krishnan, S, Adams, SH, Allen, LH, Laugero, KD, Newman, JW, Stephensen, CB, Burnett, DJ, Witbracht, M, Welch, LC, Que, ES, Keim, NL. A randomized controlled-feeding trial based on the Dietary Guidelines for Americans on cardiometabolic health indexes. Am J Clin Nutr. 2018. 108:266-278. doi:10.1093/ajcn/nqy113

1272 Krishnan, S, Steffen, LM, Paton, CM, Cooper, JA. Impact of dietary fat composition on prediabetes: a 12-year follow-up study. Public Health Nutr. 2017. 20:1617-1626. doi:10.1017/s1368980016003669

1273 Krokstad, S, Ding, D, Grunseit, AC, Sund, ER, Holmen, TL, Rangul, V, Bauman, A. Multiple lifestyle behaviours and mortality, findings from a large population-based Norwegian cohort study - The HUNT Study. BMC Public Health. 2017. 17:58. doi:10.1186/s12889-016-3993-x

1274 Kromhout, D, Menotti, A, Alberti-Fidanza, A, Puddu, PE, Hollman, P, Kafatos, A, Tolonen, H, Adachi, H, Jacobs, DR, Jr. Comparative ecologic relationships of saturated fat, sucrose, food groups, and a Mediterranean food pattern score to 50 -year coronary heart disease mortality rates among 16 cohorts of the Seven Countries Study. Eur J Clin Nutr. 2018. 72:1103-1110. doi:10.1038/s41430-018-0183-1

1275 Krzyminiewski, R, Mrozinska, G, Dobosz, B. The impact of Fruit-Vegetable Diet on High Signal Resolution Pulse Wave (HSRPW) Parameters. J Nutr Health Aging. 2018. 22:420-424. doi:10.1007/s12603-017-0944-x

1276 Kucharska, A, Gajewska, D, Kiedrowski, M, Sinska, B, Juszczyk, G, Czerw, A, Augustynowicz, A, Bobinski, K, Deptala, A, Niegowska, J. The impact of individualised nutritional therapy according to DASH diet on blood pressure, body mass, and selected biochemical parameters in overweight/obese patients with primary arterial hypertension: a prospective randomised study. Kardiol Pol. 2018. 76:158-165. doi:10.5603/KP.a2017.0184

1277 Kuchta, A, Lebiedzinska, A, Fijalkowski, M, Galaska, R, Kreft, E, Toton, M, Czaja, K, Kozlowska, A, Cwiklinska, A, KortasStempak, B, Strzelecki, A, Gliwinska, A, Dabkowski, K, Jankowski, M. Impact of plant-based diet on lipid risk factors for atherosclerosis. Cardiol J. 2016. 23:141-8. doi:10.5603/CJ.a2016.0002

1278 Kunesova, M, Braunerova, R, Hlavaty, P, Tvrzicka, E, Stankova, B, Skrha, J, Hilgertova, J, Hill, M, Kopecky, J, Wagenknecht, M, Hainer, V, Matoulek, M, Parizkova, J, Zak, A, Svacina, S. The influence of n-3 polyunsaturated fatty acids and very low calorie diet during a short-term weight reducing regimen on weight loss and serum fatty acid composition in severely obese women. Physiol Res. 2006. 55:63-72. doi:unavailable

\section{Weight}

loss/Hypocaloric

Study duration

Study Design

Study duration

Intervention/Exposure Outcome

Intervention/Exposure

Study Design Study duration

Weight

loss/Hypocaloric

Study Design

Study duration 
1279 Kurihara, A, Okamura, T, Sugiyama, D, Higashiyama, A, Watanabe, M, Okuda, N, Kadota, A, Miyagawa, N, Fujiyoshi, A, Yoshita, K, Ohkubo, T, Okayama, A, Miura, K, Ueshima, H. Vegetable Protein Intake was Inversely Associated with Cardiovascular Mortality in a 15-Year Follow-Up Study of the General Japanese Population. J Atheroscler Thromb. 2019. 26:198-206. doi:10.5551/jat.44172

1280 Kurka, JM, Buman, MP, Ainsworth, BE. Validity of the Rapid Eating Assessment for Patients for assessing dietary patterns in NCAA athletes. J Int Soc Sports Nutr. 2014. 11:42. doi:10.1186/s12970-014-0042-y

1281 Kurotani, K, Nanri, A, Goto, A, Mizoue, T, Noda, M, Oba, S, Sawada, N, Tsugane, S. Cholesterol and egg intakes and the risk of type 2 diabetes: the Japan Public Health Center-based Prospective Study. Br J Nutr. 2014. 112:1636-43. doi:10.1017/s000711451400258x

1282 Kuzawa, CW, Adair, LS, Avila, JL, Cadungog, JH, Le, NA. Atherogenic lipid profiles in Filipino adolescents with low body mass index and low dietary fat intake. Am J Hum Biol. 2003. 15:688-96. doi:10.1002/ajhb.10200

1283 Kwagyan, J, Retta, TM, Ketete, M, Bettencourt, CN, Maqbool, AR, Xu, S, Randall, OS. Obesity and Cardiovascular Diseases in a High-Risk Population: Evidence-Based Approach to CHD Risk Reduction. Ethn Dis. 2015. 25:208-13. doi:unavailable

1284 Kwasniewska, M, Pikala, M, Bielecki, W, Aranowska, A, Waskiewicz, A, Kozakiewicz, K, Tykarski, A, Pajak, A, Zdrojewski, T, Drygas, W. Ten-year changes in the prevalence of healthy lifestyle behaviors and factors among Polish Adults. Results of the National Multicenter Health Surveys WOBASZ (2003-2005) and WOBASZ II (2013-2014). European journal of preventive cardiology. 2017. 24:S12-. doi:unavailable

1285 Kwon, HN, Nam, SS, Park, YK. Effect on 12-week Intensive Dietary and Exercise Program on Weight Reduction and Maintenance in Obese Women with Weight Cycling History. Clin Nutr Res. 2017. 6:183-197. doi:10.7762/cnr.2017.6.3.183

1286 Kynde, I, Johnsen, NF, Wedderkopp, N, Bygbjerg, IB, Helge, JW, Heitmann, BL. Intake of total dietary sugar and fibre is associated with insulin resistance among Danish 8-10- and 14-16-year-old girls but not boys. European Youth Heart Studies I and II. Public Health Nutr. 2010. 13:1669-74. doi:10.1017/s1368980010000285

1287 La Spina, P, Savica, R, Ciacciarelli, A, Cotroneo, M, Dell'Aera, C, Grillo, F, Casella, C, Fazio, MC, Trimarchi, G, Musolino, RF. Eating habits in the population of the Aeolian Islands: an observational study. Public Health Nutr. 2018. :1-7. doi:10.1017/s1368980018003397

1288 La Verde, M, Mule, S, Zappala, G, Privitera, G, Maugeri, G, Pecora, F, Marranzano, M. Higher adherence to the Mediterranean diet is inversely associated with having hypertension: is low salt intake a mediating factor?. Int J Food Sci Nutr. 2018. 69:235-244. doi:10.1080/09637486.2017.1350941

1289 Labayen Goni, I, Arenaza, L, Medrano, M, Garcia, N, Cadenas-Sanchez, C, Ortega, FB. Associations between the adherence to the Mediterranean diet and cardiorespiratory fitness with total and central obesity in preschool children: the PREFIT project. Eur J Nutr. 2018. 57:2975-2983. doi:10.1007/s00394-017-1571-3

1290 Labayen, I, Diez, N, Gonzalez, A, Parra, D, Martinez, JA. Effects of protein vs. carbohydrate-rich diets on fuel utilisation in obese women during weight loss. Forum Nutr. 2003. 56:168-70. doi:unavailable

Study Design;

Outcome

Intervention/Exposure

Study Design

Intervention/Exposure

Publication Status

Intervention/Exposure

Intervention/Exposure

Study Design

Study Design;

Outcome

Study Design

Weight

loss/Hypocaloric;

Study duration 
1291 Labayen, I, Margareto, J, Maldonado-Martin, S, Gorostegi, I, Illera, M, Medrano, M, Barrenechea, L, Larrarte, E. Independent and combined influence of the FTO rs9939609 and MC4Rrs17782313 polymorphisms on hypocaloric diet induced changes in body mass and composition and energy metabolism in non-morbid obese premenopausal women. Nutr Hosp. 2015. 31:2025-32. doi:10.3305/nh.2015.31.5.8666

1292 Labayen, I, Ruiz, JR, Ortega, FB, Huybrechts, I, Rodriguez, G, Jimenez-Pavon, D, Roccaldo, R, Nova, E, Widhalm, K, Kafatos, A, Molnar, D, Androutsos, O, Moreno, LA. High fat diets are associated with higher abdominal adiposity regardless of physical activity in adolescents; the HELENA study. Clin Nutr. 2014. 33:859-66. doi:10.1016/j.clnu.2013.10.008

1293 LaFountain, RA, Miller, VJ, Barnhart, EC, Hyde, PN, Crabtree, CD, McSwiney, FT, Beeler, MK, Buga, A, Sapper, TN, Short, JA, Bowling, ML, Kraemer, WJ, Simonetti, OP, Maresh, CM, Volek, JS. Extended Ketogenic Diet and Physical Training Intervention in Military Personnel. Mil Med. 2019. :. doi:10.1093/milmed/usz046

1294 Lafrenière, J, Carbonneau, É, Laramée, C, Corneau, L, Robitaille, J, Labonté, M, Lamarche, B, Lemieux, S. Is the Canadian Healthy Eating Index 2007 an Appropriate Diet Indicator of Metabolic Health? Insights from Dietary Pattern Analysis in the PREDISE Study. Nutrients. 2019. 11:. doi:10.3390/nu11071597

1295 Lagerpusch, M, Enderle, J, Eggeling, B, Braun, W, Johannsen, M, Pape, D, Muller, MJ, Bosy-Westphal, A. Carbohydrate quality and quantity affect glucose and lipid metabolism during weight regain in healthy men. J Nutr. 2013. 143:1593-601. doi:10.3945/jn.113.179390

1296 Lagerpusch, M, Enderle, J, Later, W, Eggeling, B, Pape, D, Muller, MJ, Bosy-Westphal, A. Impact of glycaemic index and dietary fibre on insulin sensitivity during the refeeding phase of a weight cycle in young healthy men. Br J Nutr. 2013. 109:160616. doi:10.1017/s000711451200462x

1297 Lagiou, P, Sandin, S, Lof, M, Trichopoulos, D, Adami, HO, Weiderpass, E. Low carbohydrate-high protein diet and incidence of cardiovascular diseases in Swedish women: prospective cohort study. Bmj. 2012. 344:e4026. doi:10.1136/bmj.e4026

1298 Lagiou, P, Sandin, S, Weiderpass, E, Lagiou, A, Mucci, L, Trichopoulos, D, Adami, HO. Low carbohydrate-high protein diet and mortality in a cohort of Swedish women. J Intern Med. 2007. 261:366-74. doi:10.1111/j.1365-2796.2007.01774.x

1299 Lai, HT, Threapleton, DE, Day, AJ, Williamson, G, Cade, JE, Burley, VJ. Fruit intake and cardiovascular disease mortality in the UK Women's Cohort Study. Eur J Epidemiol. 2015. 30:1035-48. doi:10.1007/s10654-015-0050-5

1300 Laitinen, TT, Nuotio, J, Juonala, M, Niinikoski, H, Rovio, S, Viikari, JSA, Ronnemaa, T, Magnussen, CG, Jokinen, E, Lagstrom, H, Jula, A, Simell, O, Raitakari, OT, Pahkala, K. Success in Achieving the Targets of the 20-Year Infancy-Onset Dietary Intervention: Association With Insulin Sensitivity and Serum Lipids. Diabetes Care. 2018. 41:2236-2244. doi:10.2337/dc18-0869

1301 Lamarche, B. Dietary sources of saturated fat may influence cardiovascular disease risk. Can Nurse. 2013. 109:19. doi:unavailable

1302 Lambert, EA, Phillips, S, Belski, R, Tursunalieva, A, Eikelis, N, Sari, Cl, Dixon, JB, Straznicky, N, Grima, M, Head, GA, Schlaich, M, Lambert, GW. Endothelial Function in Healthy Young Individuals Is Associated with Dietary Consumption of Saturated Fat. Front Physiol. 2017. 8:876. doi:10.3389/fphys.2017.00876 
1303 Lammert, O, Grunnet, N, Faber, P, Bjornsbo, KS, Dich, J, Larsen, LO, Neese, RA, Hellerstein, MK, Quistorff, B. Effects of isoenergetic overfeeding of either carbohydrate or fat in young men. Br J Nutr. 2000. 84:233-45. doi:unavailable

1304 Lamri, A, Bonnefond, A, Meyre, D, Balkau, B, Roussel, R, Marre, M, Froguel, P, Fumeron, F. Interaction between GPR120 p.R270H loss-of-function variant and dietary fat intake on incident type 2 diabetes risk in the D.E.S.I.R. study. Nutr Metab Cardiovasc Dis. 2016. 26:931-6. doi:10.1016/j.numecd.2016.04.010

1305 Lamri-Senhadji, MY, El Kebir, B, Belleville, J, Bouchenak, M. Assessment of dietary consumption and time-course of changes in serum lipids and lipoproteins before, during and after Ramadan in young Algerian adults. Singapore Med J. 2009. 50:288-94. doi:unavailable

1306 Landers, P, Wolfe, MM, Glore, S, Guild, R, Phillips, L. Effect of weight loss plans on body composition and diet duration. J Okla State Med Assoc. 2002. 95:329-31. doi:unavailable

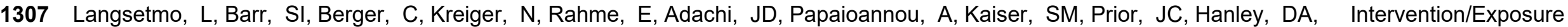
Kovacs, CS, Josse, RG, Goltzman, D. Associations of Protein Intake and Protein Source with Bone Mineral Density and Fracture Risk: A Population-Based Cohort Study. J Nutr Health Aging. 2015. 19:861-8. doi:10.1007/s12603-015-0544-6

1308 Langsetmo, L, Barr, SI, Dasgupta, K, Berger, C, Kovacs, CS, Josse, RG, Adachi, JD, Hanley, DA, Prior, JC, Brown, JP, Morin, SN, Davison, KS, Goltzman, D, Kreiger, N. Dietary patterns in men and women are simultaneously determinants of altered glucose metabolism and bone metabolism. Nutr Res. 2016. 36:328-336. doi:10.1016/j.nutres.2015.12.010

1309 Lankinen, M, Schwab, U, Kolehmainen, M, Paananen, J, Nygren, H, Seppanen-Laakso, T, Poutanen, K, Hyotylainen, T, Riserus, U, Savolainen, MJ, Hukkanen, J, Brader, L, Marklund, M, Rosqvist, F, Hermansen, K, Cloetens, L, Onning, G, Thorsdottir, I, Gunnarsdottir, I, Akesson, B, Dragsted, LO, Uusitupa, M, Oresic, M. A Healthy Nordic Diet Alters the Plasma Lipidomic Profile in Adults with Features of Metabolic Syndrome in a Multicenter Randomized Dietary Intervention. J Nutr. 2016. doi:10.3945/jn.115.220459

1310 Lapetra, J, Lozano-Rodriguez, JM, Miro-Moriano, L, Ortega-Calvo, M, Santos-Lozano, JM, Garcia-Corte, FJ, CaballeroValderrama, MR, Dominguez-Espinaco, C, Vaquero-Diaz, S, Santos-Calonge, A. Effect of a mediterranean diet on the primary prevention of atrial fibrillation and major cardiovascular events in hypertensive patients with high cardiovascular risk: results of ICFAMED randomized trial. European journal of clinical investigation. 2018. 48:182-183. doi:10.1111/eci.12926

1311 Lapointe, A, Weisnagel, SJ, Provencher, V, Begin, C, Dufour-Bouchard, AA, Trudeau, C, Lemieux, S. Comparison of a dietary intervention promoting high intakes of fruits and vegetables with a low-fat approach: long-term effects on dietary intakes, eating behaviours and body weight in postmenopausal women. Br J Nutr. 2010. 104:1080-90. doi:10.1017/s0007114510001716

1312 Lappalainen, T, Lindstrom, J, Paananen, J, Eriksson, JG, Karhunen, L, Tuomilehto, J, Uusitupa, M. Association of the fat mass and obesity-associated (FTO) gene variant (rs9939609) with dietary intake in the Finnish Diabetes Prevention Study. Br J Nutr. 2012. 108:1859-65. doi:10.1017/s0007114511007410

1313 Larsen, TM. Nordic diet in obese subjects: results from the SHOPUS study. Annals of nutrition and metabolism. Conference: 12th european nutrition conference, FENS 2015. Berlin germany. Conference start: 20151020. Conference end: 20151023 Conference publication: (var.pagings). 2015. 67:52. doi:10.1159/000440895

1314 Larsen, TM. The nordic diet. Obesity facts. 2014. 7:13-14. doi:10.1159/000363668

Outcome

Intervention/Exposure

Intervention/Exposure

Intervention/Exposure

Outcome

Publication Status

Intervention/Exposure;

Comparator

Intervention/Exposure

Publication Status

Publication Status 
1315 Larson-Meyer, DE, Borkhsenious, ON, Gullett, JC, Russell, RR, Devries, MC, Smith, SR, Ravussin, E. Effect of dietary fat on serum and intramyocellular lipids and running performance. Med Sci Sports Exerc. 2008. 40:892-902. doi:10.1249/MSS.0b013e318164cb33

1316 Larsson, SC, Akesson, A, Wolk, A. Primary prevention of stroke by a healthy lifestyle in a high-risk group. Neurology. 2015. 84:2224-8. doi:10.1212/wnl.0000000000001637

1317 Larsson, SC, Wolk, A. Dietary fiber intake is inversely associated with stroke incidence in healthy Swedish adults. J Nutr. 2014. 144:1952-5. doi:10.3945/jn.114.200634

1318 Lasker, DA, Evans, EM, Layman, DK. Moderate carbohydrate, moderate protein weight loss diet reduces cardiovascular disease risk compared to high carbohydrate, low protein diet in obese adults: A randomized clinical trial. Nutr Metab (Lond). 2008. 5:30. doi:10.1186/1743-7075-5-30

1319 Lau, C, Toft, U, Tetens, I, Carstensen, B, Jorgensen, T, Pedersen, O, Borch-Johnsen, K. Dietary patterns predict changes in two-hour post-oral glucose tolerance test plasma glucose concentrations in middle-aged adults. J Nutr. 2009. 139:588-93. doi:10.3945/jn.108.100339

1320 Laupsa Borge, J, Veum, VL, Eng, Ø, Rostrup, E, Larsen, TH, Nordrehaug, J, Nygård, OK, Sagen, JV, Gudbrandsen, OA Dankel, SN, etal, . Very-high-fat and low-fat isocaloric diets exert similar metabolic benefits but different temporal effects on cardiometabolic risk markers. Obesity facts. 2016. 9:185-186. doi:10.1159/000446744

1321 Lauria, F, Siani, A, Picó, C, Ahrens, W, Bammann, K, De Henauw, S, Foraita, R, lacoviello, L, Kourides, Y, Marild, S, Molnar, D, Moreno, LA, Pitsiladis, Y, Sánchez, J, Veidebaum, T, Wang, G, Russo, P. A common variant and the transcript levels of MC4R gene are associated with adiposity in children: The IDEFICS study. Journal of Clinical Endocrinology and Metabolism. 2016. 101:4229-4236. doi:10.1210/jc.2016-1992

1322 Layman, DK, Boileau, RA, Erickson, DJ, Painter, JE, Shiue, H, Sather, C, Christou, DD. A reduced ratio of dietary carbohydrate to protein improves body composition and blood lipid profiles during weight loss in adult women. J Nutr. 2003. 133:411-7. doi:10.1093/jn/133.2.411

1323 Layman, DK, Evans, E, Baum, JI, Seyler, J, Erickson, DJ, Boileau, RA. Dietary protein and exercise have additive effects on Power/Size body composition during weight loss in adult women. J Nutr. 2005. 135:1903-10. doi:10.1093/jn/135.8.1903

1324 Layman, DK, Evans, EM, Erickson, D, Seyler, J, Weber, J, Bagshaw, D, Griel, A, Psota, T, Kris-Etherton, P. A moderateprotein diet produces sustained weight loss and long-term changes in body composition and blood lipids in obese adults. J Nutr. 2009. 139:514-21. doi:10.3945/jn.108.099440

1325 Layman, DK, Shiue, H, Sather, C, Erickson, DJ, Baum, J. Increased dietary protein modifies glucose and insulin homeostasis in adult women during weight loss. J Nutr. 2003. 133:405-10. doi:10.1093/jn/133.2.405

1326 Lazarou, C, Karaolis, M, Matalas, AL, Panagiotakos, DB. Dietary patterns analysis using data mining method. An application to data from the CYKIDS study. Computer Methods and Programs in Biomedicine. 2012. 108:706-714. doi:10.1016/j.cmpb.2011.12.011

Intervention/Exposure

Intervention/Exposure

Weight

loss/Hypocaloric

Outcome

Publication Status

Intervention/Exposure

\section{Power/Size}

Weight

loss/Hypocaloric

Study duration

Study Design;

Outcome 
1327 Le, T, Flatt, SW, Natarajan, L, Pakiz, B, Quintana, EL, Heath, DD, Rana, BK, Rock, CL. Effects of Diet Composition and Insulin Resistance Status on Plasma Lipid Levels in a Weight Loss Intervention in Women. J Am Heart Assoc. 2016. 5:. doi:10.1161/jaha.115.002771

1328 Leblanc, V, Begin, C, Hudon, AM, Royer, MM, Corneau, L, Dodin, S, Lemieux, S. Gender differences in the long-term effects of a nutritional intervention program promoting the Mediterranean diet: changes in dietary intakes, eating behaviors, anthropometric and metabolic variables. Nutr J. 2014. 13:107. doi:10.1186/1475-2891-13-107

1329 Leblanc, V, Hudon, AM, Royer, MM, Corneau, L, Dodin, S, Begin, C, Lemieux, S. Differences between men and women in dietary intakes and metabolic profile in response to a 12-week nutritional intervention promoting the Mediterranean diet. $\mathrm{J}$ Nutr Sci. 2015. 4:e13. doi:10.1017/jns.2015.2

1330 Lecheminant, JD, Gibson, CA, Sullivan, DK, Hall, S, Washburn, R, Vernon, MC, Curry, C, Stewart, E, Westman, EC, Donnelly, JE. Comparison of a low carbohydrate and low fat diet for weight maintenance in overweight or obese adults enrolled in a clinical weight management program. Nutr J. 2007. 6:36. doi:10.1186/1475-2891-6-36

1331 LeCheminant, JD, Smith, BK, Westman, EC, Vernon, MC, Donnelly, JE. Comparison of a reduced carbohydrate and reduced fat diet for LDL, HDL, and VLDL subclasses during 9-months of weight maintenance subsequent to weight loss. Lipids Health Dis. 2010. 9:54. doi:10.1186/1476-511x-9-54

1332 Lee, A, Cheung, CK, Lo, K, Keung, VM, Mui, LW, Tam, WWS. Studying Impact of Nutrition on Growth (SING): a prospective cohort for comparing the health outcomes of young children with the dietary quality score. BMJ Open. 2017. 7:e018380. doi:10.1136/bmjopen-2017-018380

1333 Lee, A, Jeon, KJ, Kim, HK, Han, SN. Effect of a 12-week weight management program on the clinical characteristics and dietary intake of the young obese and the contributing factors to the successful weight loss. Nutr Res Pract. 2014. 8:571-9. doi:10.4162/nrp.2014.8.5.571

1334 Lee, BR, Ko, YM, Cho, MH, Yoon, YR, Kye, SH, Park, YK. Effects of 12-week Vegetarian Diet on the Nutritional Status, Stress Status and Bowel Habits in Middle School Students and Teachers. Clin Nutr Res. 2016. 5:102-11. doi:10.7762/cnr.2016.5.2.102

1335 Lee, CJ, Kim, JY, Shim, E, Hong, SH, Lee, M, Jeon, JY, Park, S. The Effects of Diet Alone or in Combination with Exercise in Patients with Prehypertension and Hypertension: a Randomized Controlled Trial. Korean Circ J. 2018. 48:637-651. doi:10.4070/kcj.2017.0349

1336 Lee, CJ, Youn, JC, Lee, SH, Park, S, Kang, SM, Choi, D. Randomized study to determine the effect of unmonitored diet and exercise education on blood pressure (the LSM study). European heart journal. 2015. 36:1184. doi:10.1093/eurheartj/ehv401

1337 Lee, E, Choi, J, Ahn, A, Oh, E, Kweon, H, Cho, D. Acceptable macronutrient distribution ranges and hypertension. Clin Exp Hypertens. 2015. 37:463-7. doi:10.3109/10641963.2015.1013116

1338 Lee, HA, Hwang, HJ, Oh, SY, Park, EA, Cho, SJ, Kim, HS, Park, H. The differential effects of changes in individual macronutrient intake on changes in lipid concentrations during childhood: From the Ewha Birth \& Growth Cohort. Clin Nutr. 2018. 37:1027-1033. doi:10.1016/j.clnu.2017.04.017

Study Design;

Intervention/Exposure

Power/Size

Power/Size

Study Design; Age at Intervention/Exposure

Intervention/Exposure

Study Design;

Intervention/Exposure

Intervention/Exposure

Publication Status

Study Design

Intervention/Exposure 
1339 Lee, HA, Hwang, HJ, Oh, SY, Park, EA, Cho, SJ, Kim, HS, Park, H. Which Diet-Related Behaviors in Childhood Influence a

1340 Lee, HH, Park, HA, Kang, JH, Cho, YG, Park, JK, Lee, R, Yoon, JY, Kim, OH. Factors related to body mass index and body mass index change in korean children: preliminary results from the obesity and metabolic disorders cohort in childhood. Korean J Fam Med. 2012. 33:134-43. doi:10.4082/kjfm.2012.33.3.134

1341 Lee, J, Kim, J. Association between Dietary Pattern and Incidence of Cholesterolemia in Korean Adults: The Korean Genome and Epidemiology Study. Nutrients. 2018. 10:. doi:10.3390/nu10010053

1342 Lee, J, Pase, M, Pipingas, A, Raubenheimer, J, Thurgood, M, Villalon, L, Macpherson, H, Gibbs, A, Scholey, A. Switching to a 10-day Mediterranean-style diet improves mood and cardiovascular function in a controlled crossover study. Nutrition. 2015. 31:647-52. doi:10.1016/j.nut.2014.10.008

1343 Lee, JK, Kim, H. Effects of the lifestyle modification program to reduce serum lipoprotein(a) and other cardiovascular risk factors in Korean college women. Gazzetta Medica Italiana Archivio per le Scienze Mediche. 2018. 177:468-474. doi:10.23736/S0393-3660.17.03592-6

1344 Lee, KW, Lyu, J, Park, JK, Jo, C, Kim, SS. Dietary carbohydrate quality and quantity in relation to the incidence of type 2 diabetes: A prospective cohort study of middle-aged and older Korean adults. Nutrition. 2019. 57:245-251. doi:10.1016/j.nut.2018.04.011

1345 Lee, KW, Woo, HD, Cho, MJ, Park, JK, Kim, SS. Identification of Dietary Patterns Associated with Incidence of Hyperglycemia in Middle-Aged and Older Korean Adults. Nutrients. 2019. 11:. doi:10.3390/nu11081801

1346 Lee, SK, Novotny, R, Daida, YG, Vijayadeva, V, Gittelsohn, J. Dietary patterns of adolescent girls in Hawaii over a 2-year period. J Am Diet Assoc. 2007. 107:956-61. doi:10.1016/j.jada.2007.03.009

1347 Lee, TA, Li, Z, Zerlin, A, Heber, D. Effects of dihydrocapsiate on adaptive and diet-induced thermogenesis with a high protein very low calorie diet: a randomized control trial. Nutr Metab (Lond). 2010. 7:78. doi:10.1186/1743-7075-7-78

1348 Lee, Y, Mitchell, DC, Smiciklas-Wright, H, Birch, LL. Diet quality, nutrient intake, weight status, and feeding environments of girls meeting or exceeding recommendations for total dietary fat of the American Academy of Pediatrics. Pediatrics. 2001. 107:E95. doi:10.1542/peds.107.6.e95

1349 Lee, YY, Wan Muda, WAM. Dietary intakes and obesity of Malaysian adults. Nutr Res Pract. 2019. 13:159-168. doi:10.4162/nrp.2019.13.2.159

1350 Leech, RM, McNaughton, SA, Timperio, A. Clustering of diet, physical activity and sedentary behaviour among Australian children: cross-sectional and longitudinal associations with overweight and obesity. Int J Obes (Lond). 2015. 39:1079-85. doi:10.1038/ijo.2015.66

Study Design

Outcome

Study duration

Intervention/Exposure; Study duration

Intervention/Exposure Outcome

Study Design;

Publication Date

Overlaps with Existing

Review

Intervention/Exposure; Study duration

Intervention/Exposure; Outcome

Study Design Intervention/Exposure 
1351 Lefevre, M, Champagne, CM, Tulley, RT, Rood, JC, Most, MM. Individual variability in cardiovascular disease risk factor responses to low-fat and low-saturated-fat diets in men: body mass index, adiposity, and insulin resistance predict changes in LDL cholesterol. Am J Clin Nutr. 2005. 82:957-63; quiz 1145-6. doi:10.1093/ajcn/82.5.957

1352 Leichtle, AB, Helmschrodt, C, Ceglarek, U, Shai, I, Henkin, Y, Schwarzfuchs, D, Golan, R, Gepner, Y, Stampfer, MJ, Bluher, M, Stumvoll, M, Thiery, J, Fiedler, GM. Effects of a 2-y dietary weight-loss intervention on cholesterol metabolism in moderately obese men. Am J Clin Nutr. 2011. 94:1189-95. doi:10.3945/ajcn.111.018119

1353 Leidy, HJ, Carnell, NS, Mattes, RD, Campbell, WW. Higher protein intake preserves lean mass and satiety with weight loss in pre-obese and obese women. Obesity (Silver Spring). 2007. 15:421-9. doi:10.1038/oby.2007.531

1354 Leidy, HJ, Hoertel, HA, Douglas, SM, Higgins, KA, Shafer, RS. A high-protein breakfast prevents body fat gain, through reductions in daily intake and hunger, in "Breakfast skipping" adolescents. Obesity (Silver Spring). 2015. 23:1761-4. doi:10.1002/oby.21185

1355 Leidy, HJ, Mattes, RD, Campbell, WW. Effects of acute and chronic protein intake on metabolism, appetite, and ghrelin during weight loss. Obesity (Silver Spring). 2007. 15:1215-25. doi:10.1038/oby.2007.143

1356 Lelong, H, Blacher, J, Baudry, J, Adriouch, S, Galan, P, Fezeu, L, Hercberg, S, Kesse-Guyot, E. Individual and Combined Effects of Dietary Factors on Risk of Incident Hypertension: Prospective Analysis From the NutriNet-Sante Cohort. Hypertension 2017. 70:712-720. doi:10.1161/hypertensionaha.117.09622

1357 Leme, AC, Philippi, ST. The "Healthy Habits, Healthy Girls" randomized controlled trial for girls: study design, protocol, and baseline results. Cad Saude Publica. 2015. 31:1381-94. doi:10.1590/0102-311x00136014

1358 Leonetti, F, Liguori, A, Petti, F, Rughini, S, Silli, L, Liguori, S, Bangrazi, S. Effects of basic traditional Chinese diet on body mass index, lean body mass, and eating and hunger behaviours in overweight or obese individuals. J Tradit Chin Med. 2016. 36:456-63. doi:unavailable

1359 León-Muñoz, LM, Guallar-Castillón, P, López-García, E, Rodríguez-Artalejo, F. Mediterranean Diet and Risk of Frailty in Community-Dwelling Older Adults. Journal of the American Medical Directors Association. 2014. 15:899-903. doi:10.1016/j.jamda.2014.06.013

1360 Letois, F, Mura, T, Scali, J, Gutierrez, LA, Féart, C, Berr, C. Nutrition and mortality in the elderly over 10 years of follow-up: The Three-City study. British Journal of Nutrition. 2016. 116:882-889. doi:10.1017/S000711451600266X

1361 Levitan, EB, Mittleman, MA, Wolk, A. Dietary glycemic index, dietary glycemic load, and incidence of heart failure events: a prospective study of middle-aged and elderly women. J Am Coll Nutr. 2010. 29:65-71. doi:10.1080/07315724.2010.10719818

1362 Lewis, AS, McCourt, HJ, Ennis, CN, Bell, PM, Courtney, CH, McKinley, MC, Young, IS, Hunter, SJ. Comparison of 5\% versus $15 \%$ sucrose intakes as part of a eucaloric diet in overweight and obese subjects: effects on insulin sensitivity, glucose metabolism, vascular compliance, body composition and lipid profile. A randomised controlled trial. Metabolism. 2013. 62:694702. doi:10.1016/j.metabol.2012.11.008

Outcome

Weight oss/Hypocaloric

Intervention/Exposure

Study duration

Outcome

Study Design;

Intervention/Exposure

Study duration

Outcome

Intervention/Exposure; Outcome

Intervention/Exposure

Intervention/Exposure; Study duration 
1363 Ley, SJ, Metcalf, PA, Scragg, RK, Swinburn, BA. Long-term effects of a reduced fat diet intervention on cardiovascular disease risk factors in individuals with glucose intolerance. Diabetes Res Clin Pract. 2004. 63:103-12. doi:10.1016/j.diabres.2003.09.001

$1364 \mathrm{Li}, \mathrm{J}$, Armstrong, C, Campbell, W. Effects of dietary protein quantity and source in appetite responses in energy-restricted overweight and obese adults. FASEB journal. 2015. 29:. doi:unavailable

$1365 \mathrm{Li}, \mathrm{J}$, Armstrong, CL, Campbell, WW. Effects of Dietary Protein Source and Quantity during Weight Loss on Appetite, Energy Expenditure, and Cardio-Metabolic Responses. Nutrients. 2016. 8:63. doi:10.3390/nu8020063

$1366 \mathrm{Li}, \mathrm{J}$, Wang, Y. Tracking of dietary intake patterns is associated with baseline characteristics of urban low-income AfricanAmerican adolescents. J Nutr. 2008. 138:94-100. doi:10.1093/jn/138.1.94 Li, M, Shi, Z. Dietary Pattern during 1991-2011 and Its Association with Cardio Metabolic Risks in Chinese Adults: The China Health and Nutrition Survey. Nutrients. 2017. 9:. doi:10.3390/nu9111218

1368 Li, S, Flint, A, Pai, JK, Forman, JP, Hu, FB, Willett, WC, Rexrode, KM, Mukamal, KJ, Rimm, EB. Low carbohydrate diet from plant or animal sources and mortality among myocardial infarction survivors. J Am Heart Assoc. 2014. 3:e001169. doi:10.1161/jaha.114.001169

1369 Li, S, Zhu, Y, Chavarro, JE, Bao, W, Tobias, DK, Ley, SH, Forman, JP, Liu, A, Mills, J, Bowers, K, Strom, M, Hansen, S, $\mathrm{Hu}$, FB, Zhang, C. Healthful Dietary Patterns and the Risk of Hypertension Among Women With a History of Gestational Diabetes Mellitus: A Prospective Cohort Study. Hypertension. 2016. 67:1157-65. doi:10.1161/hypertensionaha.115.06747

1370 Li, SX, Imamura, F, Schulze, MB, Zheng, J, Ye, Z, Agudo, A, Ardanaz, E, Aune, D, Boeing, H, Dorronsoro, M, Dow, C, Fagherazzi, G, Grioni, S, Gunter, MJ, Huerta, JM, Ibsen, DB, Jakobsen, MU, Kaaks, R, Key, TJ, Khaw, KT, Kyro, C, Mancini, FR, Molina-Portillo, E, Murphy, N, Nilsson, PM, Onland-Moret, NC, Palli, D, Panico, S, Poveda, A, Quiros, JR, Ricceri, F, Sluijs, I, Spijkerman, AMW, Tjonneland, A, Tumino, R, Winkvist, A, Langenberg, C, Sharp, SJ, Riboli, E, Scott, RA, Forouhi, NG, Wareham, NJ. Interplay between genetic predisposition, macronutrient intake and type 2 diabetes incidence: analysis within EPIC-InterAct across eight European countries. Diabetologia. 2018. 61:1325-1332. doi:10.1007/s00125-0184586-2

1371 Li, XS, Pinto-Martin, JA, Thompson, A, Chittams, J, Kral, TVE. Weight status, diet quality, perceived stress, and functional health of caregivers of children with autism spectrum disorder. J Spec Pediatr Nurs. 2018. 23:. doi:10.1111/jspn.12205

$1372 \mathrm{Li}$, Y, Ley, SH, Tobias, DK, Chiuve, SE, VanderWeele, TJ, Rich-Edwards, JW, Curhan, GC, Willett, WC, Manson, JE, Hu, FB, Qi, L. Birth weight and later life adherence to unhealthy lifestyles in predicting type 2 diabetes: prospective cohort study. Bmj. 2015. 351:h3672. doi:10.1136/bmj.h3672

1373 Li, Y, Ley, SH, VanderWeele, TJ, Curhan, GC, Rich-Edwards, JW, Willett, WC, Forman, JP, Hu, FB, Qi, L. Joint association between birth weight at term and later life adherence to a healthy lifestyle with risk of hypertension: a prospective cohort study. BMC Med. 2015. 13:175. doi:10.1186/s12916-015-0409-1

Study Design;

Outcome
Study Design;

Intervention/Exposure
rvention/Exposure; Study duration

Publication Date Overlaps with Existing Review

Country

Health Status

\section{Outcome}

Intervention/Exposure Intervention/Exposure 
1374 Li, Z, Tseng, CH, Li, Q, Deng, ML, Wang, M, Heber, D. Clinical efficacy of a medically supervised outpatient high-protein, low-calorie diet program is equivalent in prediabetic, diabetic and normoglycemic obese patients. Nutrition and Diabetes. 2014. 4:. doi:10.1038/nutd.2014.1

1375 Li, ZK, Tang, H, Gong, RR, Lin, J, Gan, CF, Huang, X, Li, RH, Fang, DZ. No decrease of HDL cholesterol after 6 days of low fat and high carbohydrate diets in a young Chinese Han population. Sichuan Da Xue Xue Bao Yi Xue Ban. 2008. 39:595600. doi:unavailable

1376 Liao, CM, Lin, CM. Life Course Effects of Socioeconomic and Lifestyle Factors on Metabolic Syndrome and 10-Year Risk of Cardiovascular Disease: A Longitudinal Study in Taiwan Adults. Int J Environ Res Public Health. 2018. 15:. doi:10.3390/ijerph15102178

1377 Liao, D, Asberry, PJ, Shofer, JB, Callahan, H, Matthys, C, Boyko, EJ, Leonetti, D, Kahn, SE, Austin, M, Newell, L, Schwartz, RS, Fujimoto, WY. Improvement of BMI, body composition, and body fat distribution with lifestyle modification in Japanese Americans with impaired glucose tolerance. Diabetes Care. 2002. 25:1504-1510. doi:10.2337/diacare.25.9.1504

1378 Lichtenstein, AH, Ausman, LM, Jalbert, SM, Vilella-Bach, M, Jauhiainen, M, McGladdery, S, Erkkila, AT, Ehnholm, C, Frohlich, J, Schaefer, EJ. Efficacy of a Therapeutic Lifestyle Change/Step 2 diet in moderately hypercholesterolemic middleaged and elderly female and male subjects. J Lipid Res. 2002. 43:264-73. doi:unavailable

1379 Liese, AD, Nichols, M, Sun, X, D'Agostino, RB, Jr, Haffner, SM. Adherence to the DASH Diet is inversely associated with incidence of type 2 diabetes: the insulin resistance atherosclerosis study. Diabetes Care. 2009. 32:1434-6. doi:10.2337/dc090228

1380 Lim, CC, Hayes, RB, Ahn, J, Shao, Y, Silverman, DT, Jones, RR, Thurston, GD. Mediterranean Diet and the Association Between Air Pollution and Cardiovascular Disease Mortality Risk. Circulation. 2019. 139:1766-1775. doi:10.1161/circulationaha.118.035742

1381 Lim, GB. Hypertension: Low sodium and DASH diet to lower blood pressure. Nat Rev Cardiol. 2018. 15:68. doi:10.1038/nrcardio.2017.214

1382 Lim, H, Kim, SY, Wang, Y, Lee, SJ, Oh, K, Sohn, CY, Moon, YM, Jee, SH. Preservation of a traditional Korean dietary pattern and emergence of a fruit and dairy dietary pattern among adults in South Korea: secular transitions in dietary patterns of a prospective study from 1998 to 2010 . Nutr Res. 2014. 34:760-70. doi:10.1016/j.nutres.2014.08.002

1383 Lim, J, Lee, Y, Shin, S, Lee, HW, Kim, CE, Lee, JK, Lee, SA, Kang, D. An association between diet quality index for Koreans (DQI-K) and total mortality in Health Examinees Gem (HEXA-G) study. Nutr Res Pract. 2018. 12:258-264. doi:10.4162/nrp.2018.12.3.258

1384 Lim, S, Kim, SS. Impact of repeated overnutrition intake: randomized controlled trial. Free radical biology \& medicine. 2010. 49:S222-. doi:10.1016/j.freeradbiomed.2010.10.647

1385 Lim, SS, Noakes, M, Keogh, JB, Clifton, PM. Long-term effects of a low carbohydrate, low fat or high unsaturated fat diet compared to a no-intervention control. Nutr Metab Cardiovasc Dis. 2010. 20:599-607. doi:10.1016/j.numecd.2009.05.003

Intervention/Exposure; Country

Intervention/Exposure; Comparator

\section{Study duration}

Publication Date

Overlaps with Existing Review

Intervention/Exposure; Outcome

Publication Status

Study Design;

Outcome

Outcome

Publication Status

Power/Size 
1386 Lima, ST, Souza, BS, Franca, AK, Salgado, JV, Salgado-Filho, N, Sichieri, R. Reductions in glycemic and lipid profiles in hypertensive patients undergoing the Brazilian Dietary Approach to Break Hypertension: a randomized clinical trial. Nutr Res. 2014. 34:682-7. doi:10.1016/j.nutres.2014.07.009

1387 Lin, J, Fang, DZ, Du, J, Shigdar, S, Xiao, LY, Zhou, XD, Duan, W. Elevated levels of triglyceride and triglyceride-rich lipoprotein triglyceride induced by a high-carbohydrate diet is associated with polymorphisms of APOA5-1131T>C and APOC3482C > T in Chinese healthy young adults. Ann Nutr Metab. 2011. 58:150-7. doi:10.1159/000327913

1388 Lin, P, Chang, CC, Yuan, KC, Yeh, HJ, Fang, SU, Cheng, T, Teng, KT, Chao, KC, Tang, JH, Kao, WY, Lin, PY, Liu, JS, Chang, JS. Red Blood Cell Aggregation-Associated Dietary Pattern Predicts Hyperlipidemia and Metabolic Syndrome. Nutrients. 2018. 10:. doi:10.3390/nu10081127

1389 Lin, PH, Wang, Y, Grambow, SC, Goggins, W, Almirall, D. Dietary saturated fat intake is negatively associated with weight maintenance among the PREMIER participants. Obesity (Silver Spring). 2012. 20:571-5. doi:10.1038/oby.2011.17

1390 Lin, TJ, Tang, SC, Liao, PY, Dongoran, RA, Yang, JH, Liu, CH. A comparison of L-carnitine and several cardiovascularrelated biomarkers between healthy vegetarians and omnivores. Nutrition. 2019. 66:29-37. doi:10.1016/j.nut.2019.03.019

1391 Lin, TY, Liao, PJ, Ting, MK, Hsu, KH. Lifestyle characteristics as moderators of the effectiveness of weight control interventions among semiconductor workers. Biomed J. 2018. 41:376-384. doi:10.1016/j.bj.2018.09.002

1392 Lin, X, Racette, SB, Lefevre, M, Ma, L, Spearie, CA, Steger-May, K, Ostlund, RE, Jr. Combined effects of ezetimibe and phytosterols on cholesterol metabolism: a randomized, controlled feeding study in humans. Circulation. 2011. 124:596-601. doi:10.1161/circulationaha.110.006692

1393 Linde, JA, Utter, J, Jeffery, RW, Sherwood, NE, Pronk, NP, Boyle, RG. Specific food intake, fat and fiber intake, and behavioral correlates of BMl among overweight and obese members of a managed care organization. Int $\mathrm{J}$ Behav Nutr Phys Act 2006. 3:42. doi:10.1186/1479-5868-3-42

1394 Lindquist, $\mathrm{CH}$, Gower, BA, Goran, MI. Role of dietary factors in ethnic differences in early risk of cardiovascular disease and type 2 diabetes. Am J Clin Nutr. 2000. 71:725-32. doi:10.1093/ajcn/71.3.725

1395 Lindqvist, HM, Radjursoga, M, Malmodin, D, Winkvist, A, Ellegard, L. Serum metabolite profiles of habitual diet: evaluation by $1 \mathrm{H}$-nuclear magnetic resonance analysis. Am J Clin Nutr. 2019. .. doi:10.1093/ajcn/nqz032

1396 Lindstrom, J, Peltonen, M, Eriksson, JG, Louheranta, A, Fogelholm, M, Uusitupa, M, Tuomilehto, J. High-fibre, low-fat diet predicts long-term weight loss and decreased type 2 diabetes risk: the Finnish Diabetes Prevention Study. Diabetologia. 2006. 49:912-20. doi:10.1007/s00125-006-0198-3

1397 Lingenhel, A, Eder, C, Zwiauer, K, Stangl, H, Kronenberg, F, Patsch, W, Strobl, W. Decrease of plasma apolipoprotein A-IV during weight reduction in obese adolescents on a low fat diet. Int J Obes Relat Metab Disord. 2004. 28:1509-13. doi:10.1038/sj.ijo.0802789

1398 Lingfors, H, Persson, LG. All-cause mortality among young men 24-26 years after a lifestyle health dialogue in a Swedish primary care setting: A longitudinal follow-up register study. BMJ Open. 2019. 9:. doi:10.1136/bmjopen-2018-022474

Study duration ;

Country

Intervention/Exposure

Intervention/Exposure

Intervention/Exposure

Study duration

Intervention/Exposure

Study Design;

Intervention/Exposure

Study Design

Weight

loss/Hypocaloric

Study duration

Intervention/Exposure; Outcome 
1399 Lioret, S, McNaughton, SA, Cameron, AJ, Crawford, D, Campbell, KJ, Cleland, VJ, Ball, K. Three-year change in diet quality and associated changes in BMI among schoolchildren living in socio-economically disadvantaged neighbourhoods. Br $\mathrm{J}$ Nutr. 2014. 112:260-8. doi:10.1017/s0007114514000749

1400 Lipscomb, ER, Caffrey, HM, Hays, LM, Finch, EA, Saha, CK, Ackermann, RT. Lower fat intake is associated with lower weight and cholesterol at 4 months in adults with impaired glucose metabolism. Diabetes Spectrum. 2012. 25:49-55. doi:10.2337/diaspect.25.1.49

1401 Lipsky, LM, Haynie, DL, Liu, D, Chaurasia, A, Gee, B, Li, K, lannotti, RJ, Simons-Morton, B. Trajectories of eating behaviors in a nationally representative cohort of U.S. adolescents during the transition to young adulthood. Int J Behav Nutr Phys Act. 2015. 12:138. doi:10.1186/s12966-015-0298-x

1402 Lipsky, LM, Nansel, TR, Haynie, DL, Liu, D, Li, K, Pratt, CA, lannotti, RJ, Dempster, KW, Simons-Morton, B. Diet quality of Outcome US adolescents during the transition to adulthood: changes and predictors. Am J Clin Nutr. 2017. 105:1424-1432. doi:10.3945/ajcn.116.150029

1403 Liu, CW. Healthy dietary pattern with daily egg consumption might be the true factor associated with decreased risks of cardiovascular diseases and mortality. Heart. 2018. 104:1804. doi:10.1136/heartjnl-2018-313774

1404 Liu, H, Lin, J, Zhu, X, Li, Y, Fan, M, Zhang, R, Fang, D. Effects of R219K polymorphism of ATP-binding cassette transporter 1 gene on serum lipids ratios induced by a high-carbohydrate and low-fat diet in healthy youth. Biol Res. 2014 . 47:4. doi:10.1186/0717-6287-47-4

1405 Liu, X, Li, Y, Tobias, DK, Wang, DD, Manson, JE, Willett, WC, Hu, FB. Changes in Types of Dietary Fats Influence Longterm Weight Change in US Women and Men. J Nutr. 2018. 148:1821-1829. doi:10.1093/jn/nxy183

1406 Liu, X, Zhang, G, Ye, X, Li, H, Chen, X, Tang, L, Feng, Y, Shai, I, Stampfer, MJ, Hu, FB, Lin, X. Effects of a low carbohydrate diet on weight loss and cardiometabolic profile in Chinese women: a randomised controlled feeding trial. Br $\mathrm{J}$ Nutr. 2013. 110:1444-53. doi:10.1017/s0007114513000640

1407 Liu, Y, Milner, M, Klonizakis, M. Physiological effects of a short-term lifestyle intervention based on the Mediterranean diet: comparison between older and younger healthy, sedentary adults. Nutrition. 2018. 55-56:185-191. doi:10.1016/j.nut.2018.05.006

1408 Liu, Z, Li, Q, Maddison, R, Ni Mhurchu, C, Jiang, Y, Wei, DM, Cheng, L, Cheng, Y, Wang, D, Wang, HJ. A School-Based Comprehensive Intervention for Childhood Obesity in China: A Cluster Randomized Controlled Trial. Child Obes. 2019. 15:105115. doi:10.1089/chi.2018.0251

1409 Livingstone, KM, Celis-Morales, C, Lara, J, Woolhead, C, O'Donovan, CB, Forster, H, Marsaux, CF, Macready, AL, Fallaize, R, Navas-Carretero, S, San-Cristobal, R, Kolossa, S, Tsirigoti, L, Lambrinou, CP, Moschonis, G, Surwillo, A, Drevon, CA, Manios, Y, Traczyk, I, Gibney, ER, Brennan, L, Walsh, MC, Lovegrove, JA, Martinez, JA, Saris, WH, Daniel, $\mathrm{H}$, Gibney, M, Mathers, JC. Clustering of adherence to personalised dietary recommendations and changes in healthy eating index within the Food4Me study. Public Health Nutr. 2016. 19:3296-3305. doi:10.1017/s1368980016001932

Publication Status

Study duration

Intervention/Exposure Weight

oss/Hypocaloric

Study Design;

Outcome

Intervention/Exposure

\section{Outcome}


1410 Livingstone, KM, Celis-Morales, C, Navas-Carretero, S, San-Cristobal, R, Macready, AL, Fallaize, R, Forster, H, Woolhead, C, O'Donovan, CB, Marsaux, CF, Kolossa, S, Tsirigoti, L, Lambrinou, CP, Moschonis, G, Godlewska, M, Surwillo, A, Drevon, CA, Manios, Y, Traczyk, I, Gibney, ER, Brennan, L, Walsh, MC, Lovegrove, JA, Saris, WH, Daniel, H, Gibney, M, Martinez, JA, Mathers, JC. Effect of an Internet-based, personalized nutrition randomized trial on dietary changes associated with the Mediterranean diet: the Food4Me Study. Am J Clin Nutr. 2016. 104:288-97. doi:10.3945/ajcn.115.129049

1411 Livingstone, KM, Givens, DI, Cockcroft, JR, Pickering, JE, Lovegrove, JA. Is fatty acid intake a predictor of arterial stiffness and blood pressure in men? Evidence from the Caerphilly Prospective Study. Nutr Metab Cardiovasc Dis. 2013. 23:1079-85. doi:10.1016/j.numecd.2012.12.002

1412 Llanos, AA, Krok, JL, Peng, J, Pennell, ML, Olivo-Marston, S, Vitolins, MZ, Degraffinreid, CR, Paskett, ED. Favorable effects of low-fat and low-carbohydrate dietary patterns on serum leptin, but not adiponectin, among overweight and obese premenopausal women: a randomized trial. Springerplus. 2014. 3:175. doi:10.1186/2193-1801-3-175

1413 Llanos, AAM, Krok, JL, Peng, J, Pennell, ML, Olivo-Marston, SE, Vitolins, MZ, Degraffinreid, CR, Paskett, ED. Effects of low-fat and low-carbohydrate dietary patterns combined with physical activity on serum adipokine concentrations among premenopausal women: a randomized trial. Cancer prevention research (philadelphia, pa.). 2013. 6:. doi:unavailable

1414 Lockyer, S, Tzanetou, M, Carvalho-Wells, AL, Jackson, KG, Minihane, AM, Lovegrove, JA. SATgene dietary model to implement diets of differing fat composition in prospectively genotyped groups (apoE) using commercially available foods. British Journal of Nutrition. 2012. 108:1705-1713. doi:10.1017/S0007114511007082

1415 Lofley, AC, Root, MM. Macronutrients Association with Change in Waist and Hip Circumference Over 9 Years. J Am Coll Nutr. 2017. 36:57-63. doi:10.1080/07315724.2016.1183241

1416 Lojko, D, Stelmach-Mardas, M, Suwalska, A. Diet quality and eating patterns in euthymic bipolar patients. Eur Rev Med Pharmacol Sci. 2019. 23:1221-1238. doi:10.26355/eurrev_201902_17016

1417 Loktionov, A, Scollen, S, McKeown, N, Bingham, SA. Gene-nutrient interactions: dietary behaviour associated with high coronary heart disease risk particularly affects serum LDL cholesterol in apolipoprotein E epsilon4-carrying free-living individuals. Br J Nutr. 2000. 84:885-90. doi:unavailable

1418 Longland, TM, Oikawa, SY, Mitchell, CJ, Devries, MC, Phillips, SM. Higher compared with lower dietary protein during an energy deficit combined with intense exercise promotes greater lean mass gain and fat mass loss: a randomized trial. Am $\mathrm{J}$ Clin Nutr. 2016. 103:738-46. doi:10.3945/ajcn.115.119339

1419 Loo, RL, Zou, X, Appel, LJ, Nicholson, JK, Holmes, E. Characterization of metabolic responses to healthy diets and association with blood pressure: application to the Optimal Macronutrient Intake Trial for Heart Health (OmniHeart), a randomized controlled study. Am J Clin Nutr. 2018. 107:323-334. doi:10.1093/ajcn/nqx072

1420 Looman, M, Feskens, EJ, de Rijk, M, Meijboom, S, Biesbroek, S, Temme, EH, de Vries, J, Geelen, A. Development and evaluation of the Dutch Healthy Diet index 2015. Public Health Nutr. 2017. 20:2289-2299. doi:10.1017/s136898001700091x

1421 Looney, S, Raynor, H. Are changes in consumption of "healthy" foods related to changes in consumption of "unhealthy" foods during pediatric obesity treatment?. Obesity. 2011. 19:S104-S105. doi:10.1038/oby.2011.226

Intervention/Exposure; Outcome

Outcome

Outcome; Publication Status

Outcome

Intervention/Exposure

Study Design; Comparator

Study Design; Intervention/Exposure

Study duration

Study duration

Outcome

Publication Status 
1422 López, CPR, Ramos-Terrones, I, Lazarevich, I, García-López, S, Arriaga, RV, Valverde, LF, Cervera, EG, Nájera-Medina,

Study Design;

O. Metabolic syndrome, physical activity and eating habits in school children of the south of Mexico city. Investigacion Clinica

Intervention/Exposure (Venezuela). 2019. 60:7-19. doi:10.22209/IC.v60n1a01

1423 Lopez-Garcia, E, Rodriguez-Artalejo, F, Li, TY, Fung, TT, Li, S, Willett, WC, Rimm, EB, Hu, FB. The Mediterranean-style dietary pattern and mortality among men and women with cardiovascular disease. Am J Clin Nutr. 2014. 99:172-80. doi:10.3945/ajcn.113.068106

1424 Lopez-Legarrea, P, De La Iglesia, R, Abete, I, Bondia-Pons, I, Navas-Carretero, S, Forga, L, Martinez, JA, Zulet, MA. Short-term role of the dietary total antioxidant capacity in two hypocaloric regimes on obese with metabolic syndrome symptoms: The RESMENA randomized controlled trial. Nutrition and Metabolism. 2013. 10:. doi:10.1186/1743-7075-10-22

1425 Lopez-Legarrea, P, De la Iglesia, R, Abete, I, Ibanez-Melo, A, Navas-Carretero, S, Bondia-Pons, I, Forga, L, Zulet, M, Martinez, A. The resmena diet: a new effective dietary strategy for reducing metabolic syndrome. Obesity facts. 2012. 5:230231. doi: $10.1159 / 000258190$

1426 Lopez-Legarrea, P, de la Iglesia, R, Abete, I, Navas-Carretero, S, Martinez, JA, Zulet, MA. The protein type within a hypocaloric diet affects obesity-related inflammation: the RESMENA project. Nutrition. 2014. 30:424-9. doi:10.1016/j.nut.2013.09.009

1427 Lopez-Moreno, J, Quintana-Navarro, GM, Camargo, A, Jimenez-Lucena, R, Delgado-Lista, J, Marin, C, Tinahones, FJ, Striker, GE, Roche, HM, Perez-Martinez, P, Lopez-Miranda, J, Yubero-Serrano, EM. Dietary fat quantity and quality modifies advanced glycation end products metabolism in patients with metabolic syndrome. Mol Nutr Food Res. 2017. 61: doi:10.1002/mnfr.201601029

1428 Lopez-Sanchez, GF, Radziminski, L, Skalska, M, Jastrzebska, J, Smith, L, Wakuluk, D, Jastrzebski, Z. Body Composition, Physical Fitness, Physical Activity and Nutrition in Polish and Spanish Male Students of Sports Sciences: Differences and Correlations. Int J Environ Res Public Health. 2019. 16:. doi:10.3390/ijerph16071148

1429 Loprinzi, PD, Nooe, A. Health characteristics and predicted 10-year risk for a first atherosclerotic cardiovascular disease (ASCVD) event using the Pooled Cohort Risk Equations among US adults who are free of cardiovascular disease. Physiol Behav. 2015. 151:591-5. doi:10.1016/j.physbeh.2015.08.031

1430 Lotrean, LM, Stan, O, Codruta, L, Laza, V. Dietary patterns, physical activity, body mass index, weight-related behaviours and their interrelationship among Romanian university students-trends from 2003 to 2016. Nutr Hosp. 2018. 35:375-383. doi:10.20960/nh.1296

1431 Louie, JC, Flood, VM, Burlutsky, G, Rangan, AM, Gill, TP, Mitchell, P. Dairy consumption and the risk of 15-year cardiovascular disease mortality in a cohort of older Australians. Nutrients. 2013. 5:441-54. doi:10.3390/nu5020441

Health Status

Study duration

Publication Status

Lovejoy, JC, Champagne, CM, Smith, SR, de Jonge, L, Xie, H. Ethnic differences in dietary intakes, physical activity, and energy expenditure in middle-aged, premenopausal women: the Healthy Transitions Study. Am J Clin Nutr. 2001. 74:90-5. doi:10.1093/ajcn/74.1.90 
1433 Lowry, R, Galuska, DA, Fulton, JE, Wechsler, H, Kann, L, Collins, JL. Physical activity, food choice, and weight management goals and practices among US college students. Am J Prev Med. 2000. 18:18-27. doi:10.1016/s07493797(99)00107-5

1434 Lowry, R, Michael, S, Demissie, Z, Kann, L, Galuska, DA. Associations of Physical Activity and Sedentary Behaviors with

Dietary Behaviors among US High School Students. J Obes. 2015. 2015:876524. doi:10.1155/2015/876524

Intervention/Exposure;

Outcome

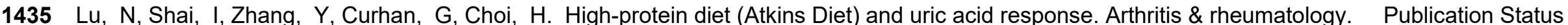
2014. 66:S71-S72. doi:10.1002/art.38914

$1436 \mathrm{Lu}, \mathrm{S}, \mathrm{Yu}, \mathrm{H}, \mathrm{Han}, \mathrm{R}, \mathrm{Su}, \mathrm{J}$, Pan, X, Zhang, Y, Zhou, J, Wu, M. Dietary patterns and the risk of cardiovascular diseases in Jiangsu Province, China: a nested case-control study. Lancet. 2016. 388:62-. doi:unavailable

1437 Ludy, MJ, Tan, SY, Leone, RJ, Morgan, AL, Tucker, RM. Weight gain in first-semester university students: Positive sleep and diet practices associated with protective effects. Physiol Behav. 2018. 194:132-136. doi:10.1016/j.physbeh.2018.05.009

1438 Luger, E, Aspalter, R, Luger, M, Longin, R, Rieder, A, Dorner, TE. Changes of dietary patterns during participation in a web- Study Design based weight-reduction programme. Public Health Nutr. 2016. 19:1211-21. doi:10.1017/s1368980015002852

1439 Luscombe, ND, Noakes, M, Wittert, G, Farnsworth, E, Argyiou, E, Clifton, PM. Effect of a high protein, energy restricted diet on body composition, insulin sensitivity and lipid levels in hyperinsulinemic subjects. Atherosclerosis. 2002. 3:92. doi:unavailable

1440 Luscombe-Marsh, ND, Noakes, M, Wittert, GA, Keogh, JB, Foster, P, Clifton, PM. Carbohydrate-restricted diets high in either monounsaturated fat or protein are equally effective at promoting fat loss and improving blood lipids. Am $\mathrm{J}$ Clin Nutr. 2005. 81:762-72. doi:10.1093/ajcn/81.4.762

1441 Lutjohann, D, Meyer, S, von Bergmann, K, Stellaard, F. Cholesterol Absorption and Synthesis in Vegetarians and Omnivores Mol Nutr Food Res. 2018. 62:e1700689. doi:10.1002/mnfr.201700689

1442 Lutze, J, Taylor, P, Brinkworth, GD, Wyld, B, Syrette, J, Wilson, CJ, Clifton, PM, Noakes, M. Psychological well-being response to high protein and high carbohydrate weight loss diets in overweight and obese men: Arandomised trial. e-SPEN Journal. 2013. 8:e235-e240. doi:10.1016/j.clnme.2013.08.002

1443 Lv, J, Yu, C, Guo, Y, Bian, Z, Yang, L, Chen, Y, Hu, X, Hou, W, Chen, J, Chen, Z, Qi, L, Li, L. Adherence to a healthy lifestyle and the risk of type 2 diabetes in Chinese adults. Int J Epidemiol. 2017. 46:1410-1420. doi:10.1093/ije/dyx074

Publication Status

Intervention/Exposure

Publication Status

Weight

loss/Hypocaloric

Study Design;

Outcome

Weight

loss/Hypocaloric

Country

1444 Lv, J, Yu, C, Guo, Y, Bian, Z, Yang, L, Chen, Y, Tang, X, Zhang, W, Qian, Y, Huang, Y, Wang, X, Chen, J, Chen, Z, Qi, L, Li, L. Adherence to Healthy Lifestyle and Cardiovascular Diseases in the Chinese Population. J Am Coll Cardiol. 2017. 69:1116-1125. doi:10.1016/j.jacc.2016.11.076

1445 Lynch, E, Emery-Tiburcio, E, Dugan, S, White, FS, Thomason, C, Jenkins, L, Feit, C, Avery-Mamer, E, Wang, Y, Mack, L, Ragland, A. Results of ALIVE: A Faith-Based Pilot Intervention to Improve Diet Among African American Church Members. Prog Community Health Partnersh. 2019. 13:19-30. doi:10.1353/cpr.2019.0005

1446 Ma, W, Hagan, KA, Heianza, Y, Sun, Q, Rimm, EB, Qi, L. Adult height, dietary patterns, and healthy aging. Am J Clin Nutr. $\quad$ Outcome 2017. 106:589-596. doi:10.3945/ajcn.116.147256 
1447 Ma, Y, Li, Y, Chiriboga, DE, Olendzki, BC, Hebert, JR, Li, W, Leung, K, Hafner, AR, Ockene, IS. Association between carbohydrate intake and serum lipids. J Am Coll Nutr. 2006. 25:155-63. doi:10.1080/07315724.2006.10719527

1448 Ma, Y, Olendzki, B, Chiriboga, D, Hebert, JR, Li, Y, Li, W, Campbell, M, Gendreau, K, Ockene, IS. Association between dietary carbohydrates and body weight. Am J Epidemiol. 2005. 161:359-67. doi:10.1093/aje/kwi051

1449 Ma, Y, Olendzki, BC, Li, W, Hafner, AR, Chiriboga, D, Hebert, JR, Campbell, M, Sarnie, M, Ockene, IS. Seasonal variation in food intake, physical activity, and body weight in a predominantly overweight population. Eur J Clin Nutr. 2006. 60:519-28. doi:10.1038/sj.ejcn.1602346

1450 Ma, Y, Olendzki, BC, Wang, J, Persuitte, GM, Li, W, Fang, H, Merriam, PA, Wedick, NM, Ockene, IS, Culver, AL, Schneider, KL, Olendzki, GF, Carmody, J, Ge, T, Zhang, Z, Pagoto, SL. Single-component versus multicomponent dietary goals for the metabolic syndrome: a randomized trial. Ann Intern Med. 2015. 162:248-57. doi:10.7326/m14-0611

1451 Machado-Rodrigues, AM, Fernandes, RA, Silva, MR, Gama, A, Mourao, I, Nogueira, H, Rosado-Marques, V, Padez, C. Overweight Risk and Food Habits in Portuguese Pre-school Children. J Epidemiol Glob Health. 2018. 8:106-109. doi:10.2991/j.jegh.2017.10.006

1452 Macias-Cervantes, MH, Rodriguez-Soto, JM, Uribarri, J, Diaz-Cisneros, FJ, Cai, W, Garay-Sevilla, ME. Effect of an advanced glycation end product-restricted diet and exercise on metabolic parameters in adult overweight men. Nutrition. 2015. 31:446-51. doi:10.1016/j.nut.2014.10.004

1453 Maciejewska, D, Michalczyk, M, Czerwinska-Rogowska, M, Banaszczak, M, Ryterska, K, Jakubczyk, K, Piotrwski, J, Holowko, J, Drozd, A, Wysokinki, P, Ficek, K, Wilk, K, Lubkowska, A, Cieszczyk, P, Bertrand, J, Stachowska, E. Seeking Optimal Nutrition for Healthy Body Mass Reduction among Former Athletes. J Hum Kinet. 2017. 60:63-75. doi:10.1515/hukin2017-0090

1454 Macknin, M, Kong, T, Weier, A, Worley, S, Tang, AS, Alkhouri, N, Golubic, M. Plant-based, no-added-fat or American Heart Association diets: impact on cardiovascular risk in obese children with hypercholesterolemia and their parents. J Pediatr. 2015. 166:953-9.e1-3. doi:10.1016/j.jpeds.2014.12.058

1455 Madero, M, Arriaga, JC, Jalal, D, Rivard, C, McFann, K, Perez-Mendez, O, Vazquez, A, Ruiz, A, Lanaspa, MA, Jimenez, CR, Johnson, RJ, Lozada, LG. The effect of two energy-restricted diets, a low-fructose diet versus a moderate natural fructose diet, on weight loss and metabolic syndrome parameters: a randomized controlled trial. Metabolism. 2011. 60:1551-9. doi:10.1016/j.metabol.2011.04.001

1456 Mady, MA, Kossoff, EH, McGregor, AL, Wheless, JW, Pyzik, PL, Freeman, JM. The ketogenic diet: adolescents can do it, too. Epilepsia. 2003. 44:847-51. doi:10.1046/j.1528-1157.2003.57002.x

1457 Maekawa, S, Kawahara, T, Nomura, R, Murase, T, Ann, Y, Oeholm, M, Harada, M. Retrospective study on the efficacy of a low-carbohydrate diet for impaired glucose tolerance. Diabetes Metab Syndr Obes. 2014. 7:195-201. doi:10.2147/dmso.S62681

1458 Maffeis, C, Maschio, M, Costanzi, S, Tommasi, M, Fasan, I, Morandi, A. Diet macronutrient composition reported before treatment predicts BMI change in obese children: the role of lipids. Eur J Clin Nutr. 2012. 66:1066-8. doi:10.1038/ejcn.2012.97
Intervention/Exposure

Intervention/Exposure

Intervention/Exposure

Intervention/Exposure

Study Design;

Intervention/Exposure

Intervention/Exposure

Intervention/Exposure

Study duration

Intervention/Exposure;

Comparator

Study Design; Health Status

Study Design;

Intervention/Exposure; Study duration

Intervention/Exposure 
1459 Maffiuletti, NA, Agosti, F, Marinone, PG, Silvestri, G, Lafortuna, CL, Sartorio, A. Changes in body composition, physical performance and cardiovascular risk factors after a 3-week integrated body weight reduction program and after 1-y follow-up in severely obese men and women. Eur J Clin Nutr. 2005. 59:685-94. doi:10.1038/sj.ejcn.1602130

1460 Magnusdottir, OK, Landberg, R, Gunnarsdottir, I, Cloetens, L, Akesson, B, Rosqvist, F, Schwab, U, Herzig, KH, Hukkanen, J, Savolainen, MJ, Brader, L, Hermansen, K, Kolehmainen, M, Poutanen, K, Uusitupa, M, Riserus, U, Thorsdottir, I. Whole grain rye intake, reflected by a biomarker, is associated with favorable blood lipid outcomes in subjects with the metabolic syndrome--a randomized study. PLoS One. 2014. 9:e110827. doi:10.1371/journal.pone.0110827

1461 Magriplis, E, Farajian, P, Risvas, G, Panagiotakos, D, Zampelas, A. Newly derived children-based food index. An index that may detect childhood overweight and obesity. Int J Food Sci Nutr. 2015. 66:623-32. doi:10.3109/09637486.2015.1056109

1462 Magriplis, E, Panagiotakos, D, Mitsopoulou, AV, Karageorgou, D, Bakogianni, I, Dimakopoulos, I, Micha, R, Michas, G, Chourdakis, M, Chrousos, GP, Roma, E, Zampelas, A. Prevalence of hyperlipidaemia in adults and its relation to the Mediterranean diet: the Hellenic National Nutrition and Health Survey (HNNHS). Eur J Prev Cardiol. 2019. :2047487319866023. doi:10.1177/2047487319866023

1463 Magriplis, E, Sialvera, TE, Papadopoulou, A, Efstathiou, SP, Trautwein, EA, Goumas, G, Dimakopoulos, I, Papavasiliou, K, Koutsouri, A, Zampelas, A. Effectiveness and easiness of adherence to behavioural guidelines for diet and lifestyle changes for cholesterol-lowering: the Increasing Adherence of Consumers to Diet \& Lifestyle Changes to Lower (LDL) Cholesterol (ACT) randomised controlled trial. J Hum Nutr Diet. 2019. 32:607-618. doi:10.1111/jhn.12667

1464 Mahmoud, AM, Hwang, CL, Szczurek, MR, Bian, JT, Ranieri, C, Gutterman, DD, Phillips, SA. Low-Fat Diet Designed for Weight Loss But Not Weight Maintenance Improves Nitric Oxide-Dependent Arteriolar Vasodilation in Obese Adults. Nutrients. 2019. 11: doi:10.3390/nu11061339

1465 Mahon, AK, Flynn, MG, Stewart, LK, McFarlin, BK, Iglay, HB, Mattes, RD, Lyle, RM, Considine, RV, Campbell, WW. Protein intake during energy restriction: effects on body composition and markers of metabolic and cardiovascular health in postmenopausal women. J Am Coll Nutr. 2007. 26:182-9. doi:10.1080/07315724.2007.10719600

1466 Maier, IB, Ozel, Y, Wagnerberger, S, Bischoff, SC, Bergheim, I. Dietary pattern and leisure time activity of overweight and normal weight children in Germany: sex-specific differences. Nutr J. 2013. 12:14. doi:10.1186/1475-2891-12-14

duration

Intervention/Exposure;

Outcome

Study Design

Study Design

Maier, JH, Barry, R. Associations among Physical Activity, Diet, and Obesity Measures Change during Adolescence. J Nutr Metab. 2015. 2015:805065. doi:10.1155/2015/805065

1468 Maijo, M, Ivory, K, Clements, SJ, Dainty, JR, Jennings, A, Gillings, R, Fairweather-Tait, S, Gulisano, M, Santoro, A, Franceschi, C, Carding, SR, Nicoletti, C. One-Year Consumption of a Mediterranean-Like Dietary Pattern With Vitamin D3 Supplements Induced Small Scale but Extensive Changes of Immune Cell Phenotype, Co-receptor Expression and Innate Immune Responses in Healthy Elderly Subjects: Results From the United Kingdom Arm of the NU-AGE Trial. Front Physiol. 2018. 9:997. doi:10.3389/fphys.2018.00997

1469 Makarem, N, Scott, M, Quatromoni, P, Jacques, P, Parekh, N. Trends in dietary carbohydrate consumption from 1991 to 2008 in the Framingham Heart Study Offspring Cohort. Br J Nutr. 2014. 111:2010-23. doi:10.1017/s0007114513004443

Intervention/Exposure; Outcome

Intervention/Exposure; Study duration

Intervention/Exposure

Study Design

Intervention/Exposure

Outcome 
1470 Maki, KC, Lawless, AL, Kelley, KM, Kaden, VN, Geiger, CJ, Dicklin, MR. Corn oil improves the plasma lipoprotein lipid profile compared with extra-virgin olive oil consumption in men and women with elevated cholesterol: results from a randomized controlled feeding trial. J Clin Lipidol. 2015. 9:49-57. doi:10.1016/j.jacl.2014.10.006

1471 Malin, SK, Kullman, EL, Scelsi, AR, Haus, JM, Filion, J, Pagadala, MR, Godin, JP, Kochhar, S, Ross, AB, Kirwan, JP. A whole-grain diet reduces peripheral insulin resistance and improves glucose kinetics in obese adults: A randomized-controlled trial. Metabolism. 2018. 82:111-117. doi:10.1016/j.metabol.2017.12.011

1472 Mamo, JC, James, AP, Soares, MJ, Griffiths, DG, Purcell, K, Schwenke, JL. A low-protein diet exacerbates postprandial chylomicron concentration in moderately dyslipidaemic subjects in comparison to a lean red meat protein-enriched diet. Eur $\mathrm{J}$ Clin Nutr. 2005. 59:1142-8. doi:10.1038/sj.ejcn.1602224

1473 Manco, M, Bertuzzi, A, Salinari, S, Scarfone, A, Calvani, M, Greco, AV, Mingrone, G. The ingestion of saturated fatty acid triacylglycerols acutely affects insulin secretion and insulin sensitivity in human subjects. Br J Nutr. 2004. 92:895-903. doi:10.1079/bjn20041268

1474 Manios, Y, Kourlaba, G, Grammatikaki, E, Androutsos, O, loannou, E, Roma-Giannikou, E. Comparison of two methods for identifying dietary patterns associated with obesity in preschool children: The GENESIS study. European Journal of Clinical Nutrition. 2010. 64:1407-1414. doi:10.1038/ejcn.2010.168

1475 Mann, J, Morenga, LT. Carbohydrates in the treatment and prevention of Type 2 diabetes. Diabet Med. 2015. 32:572-5. doi:10.1111/dme.12673

1476 Mann, J, Swinburn, B, Beaglehole, R, Ni Mhurchu, C, Jackson, R. Diverging global trends in heart disease and diabetes: implications for dietary guidelines. Lancet Diabetes Endocrinol. 2015. 3:584-5. doi:10.1016/s2213-8587(15)00206-5

1477 Mann, JI, De Leeuw, I, Hermansen, K, Karamanos, B, Karlstrom, B, Katsilambros, N, Riccardi, G, Rivellese, AA, Rizkalla, S, Slama, G, Toeller, M, Uusitupa, M, Vessby, B. Evidence-based nutritional approaches to the treatment and prevention of diabetes mellitus. Nutr Metab Cardiovasc Dis. 2004. 14:373-94. doi:unavailable

1478 Marckmann, P, Raben, A, Astrup, A. Ad libitum intake of low-fat diets rich in either starchy foods or sucrose: effects on blood lipids, factor VII coagulant activity, and fibrinogen. Metabolism. 2000. 49:731-5. doi:10.1053/meta.2000.6237

1479 Margolis, LM, Cao, JJ, Whigham, LD, McClung, JP, Combs, GF, Young, AJ, Pasiakos, SM. Dietary protein intake, energy deficit, and nitrogen balance in normal-weight adults: a randomized controlled trial. FASEB journal. 2013. 27:. doi:unavailable

1480 Marina, A, von Frankenberg, AD, Suvag, S, Callahan, HS, Kratz, M, Richards, TL, Utzschneider, KM. Effects of dietary fat and saturated fat content on liver fat and markers of oxidative stress in overweight/obese men and women under weight-stable conditions. Nutrients. 2014. 6:4678-90. doi:10.3390/nu6114678

1481 Marklund, M, Leander, K, Vikstrom, M, Laguzzi, F, Gigante, B, Sjogren, P, Cederholm, T, de Faire, U, Hellenius, ML,

Riserus, U. Polyunsaturated Fat Intake Estimated by Circulating Biomarkers and Risk of Cardiovascular Disease and All-Cause

Intervention/Exposure; Study duration

Intervention/Exposure; Comparator

Study duration

Intervention/Exposure Study Design; Publication Status

Study Design

Study Design;

Publication Status

Study duration

Publication Status

Study duration Mortality in a Population-Based Cohort of 60-Year-Old Men and Women. Circulation. 2015. 132:586-94. doi:10.1161/circulationaha.115.015607 
1482 Marklund, M, Magnusdottir, OK, Rosqvist, F, Cloetens, L, Landberg, R, Kolehmainen, M, Brader, L, Hermansen, K, Poutanen, KS, Herzig, KH, Hukkanen, J, Savolainen, MJ, Dragsted, LO, Schwab, U, Paananen, J, Uusitupa, M, Akesson, B, Thorsdottir, I, Riserus, U. A dietary biomarker approach captures compliance and cardiometabolic effects of a healthy Nordic diet in individuals with metabolic syndrome. J Nutr. 2014. 144:1642-9. doi:10.3945/jn.114.193771

1483 Marks, J, Barnett, LM, Allender, S. Change of School in Early Adolescence and Adverse Obesity-Related Dietary Behavior: A Longitudinal Cohort Study, Victoria, Australia, 2013-2014. Prev Chronic Dis. 2015. 12:E145. doi:10.5888/pcd12.150042

1484 Marques Rocha, JL, Cristine Anunciacao, P, Vaz Tostes, MG, Thomas Valdes, S, Cardoso Carraro, JC, Galdino Alves, NE, Bressan, J. Human ration does not alter weight and body composition, but improves the lipid profile of overweight woman. Nutr Hosp. 2012. 27:1460-8. doi:10.3305/nh.2012.27.5.5686

1485 Marshall, DA, Vernalis, MN, Remaley, AT, Walizer, EM, Scally, JP, Taylor, AJ. The role of exercise in modulating the impact of an ultralow-fat diet on serum lipids and apolipoproteins in patients with or at risk for coronary artery disease. Am Heart J. 2006. 151:484-91. doi:10.1016/j.ahj.2005.03.065

1486 Martens, EA, Gatta-Cherifi, B, Gonnissen, HK, Westerterp-Plantenga, MS. The potential of a high protein-low carbohydrate diet to preserve intrahepatic triglyceride content in healthy humans. PLoS One. 2014. 9:e109617. doi:10.1371/journal.pone.0109617

1487 Martens, EA, Lemmens, SG, Westerterp-Plantenga, MS. Protein leverage affects energy intake of high-protein diets in humans. Am J Clin Nutr. 2013. 97:86-93. doi:10.3945/ajcn.112.046540

1488 Martin, CK, Bray, GA, Ryan, DH, Carey, VJ, Champagne, CM, Williamson, DA, Anton, S, Laranjo, N, Sacks, FM. A high carbohydrate/average protein diet is less efficacious at promoting weight loss among women compared to men: subgroup analysis from POUNDS lost. Obesity (silver spring, md.). 2010. 18:S94-. doi:unavailable

1489 Martin, CL, Tate, DF, Schaffner, A, Brannen, A, Hatley, KE, Diamond, M, Munoz-Christian, K, Pomeroy, J, Sanchez, T, Mercado, A, Hagobian, T, Phelan, S. Acculturation Influences Postpartum Eating, Activity, and Weight Retention in LowIncome Hispanic Women. J Womens Health (Larchmt). 2017. 26:1333-1339. doi:10.1089/jwh.2016.6154

1490 Martin, JC, Moran, LJ, Teede, HJ, Ranasinha, S, Lombard, CB, Harrison, CL. Diet Quality in a Weight Gain Prevention Trial Outcome of Reproductive Aged Women: A Secondary Analysis of a Cluster Randomized Controlled Trial. Nutrients. 2018. 11:. doi:10.3390/nu11010049

1491 Martin, RM, Holly, JMP, Middleton, N, Davey Smith, G, Gunnell, D. Childhood diet and insulin-like growth factors in adulthood: 65-year follow-up of the Boyd Orr Cohort. European Journal of Clinical Nutrition. 2007. 61:1281-1292. doi:10.1038/sj.ejcn.1602616

1492 Martin-Diener, E, Meyer, J, Braun, J, Tarnutzer, S, Faeh, D, Rohrmann, S, Martin, BW. The combined effect on survival of four main behavioural risk factors for non-communicable diseases. Prev Med. 2014. 65:148-52. doi:10.1016/j.ypmed.2014.05.023

1493 Martínez-González, MA, Salas-Salvadó, J, Estruch, R, Corella, D, Fitó, M, Ros, E. Benefits of the Mediterranean Diet: Insights From the PREDIMED Study. Progress in Cardiovascular Diseases. 2015. 58:50-60. doi:10.1016/j.pcad.2015.04.003 
1494 Martínez-González, MA, Sánchez-Tainta, A, Corella, D, Salas-Salvadó, J, Ros, E, Arós, F, Gómez-Gracia, E, Fiol, M, Lamuela-Raventós, RM, Schröder, H, etal, . A provegetarian food pattern and reduction in total mortality in the Prevención con Dieta Mediterránea (PREDIMED) study. American journal of clinical nutrition. 2014. 100 Suppl 1:320S-8S. doi:10.3945/ajcn.113.071431

1495 Martinez-Gonzalez, MA, Sanchez-Villegas, A. The emerging role of Mediterranean diets in cardiovascular epidemiology: monounsaturated fats, olive oil, red wine or the whole pattern?. Eur J Epidemiol. 2004. 19:9-13. doi:10.1023/b:ejep.0000013351.60227.7b

1496 Martinez-Gonzalez, MA, Toledo, E, Aros, F, Fiol, M, Corella, D, Salas-Salvado, J, Ros, E, Covas, MI, Fernandez-Crehuet, J, Lapetra, J, Munoz, MA, Fito, M, Serra-Majem, L, Pinto, X, Lamuela-Raventos, RM, Sorli, JV, Babio, N, Buil-Cosiales, P, Ruiz-Gutierrez, V, Estruch, R, Alonso, A. Extravirgin olive oil consumption reduces risk of atrial fibrillation: the PREDIMED (Prevencion con Dieta Mediterranea) trial. Circulation. 2014. 130:18-26. doi:10.1161/circulationaha.113.006921

1497 Martinez-Lapiscina, EH, Clavero, P, Toledo, E, Estruch, R, Salas-Salvado, J, San Julian, B, Sanchez-Tainta, A, Ros, E, Valls-Pedret, C, Martinez-Gonzalez, MA. Mediterranean diet improves cognition: the PREDIMED-NAVARRA randomised trial. J Neurol Neurosurg Psychiatry. 2013. 84:1318-25. doi:10.1136/jnnp-2012-304792

1498 Martinez-Lopez, E, Garcia-Garcia, MR, Gonzalez-Avalos, JM, Maldonado-Gonzalez, M, Ruiz-Madrigal, B, Vizmanos, B, Hernandez-Nazara, Z, Roman, S, Panduro, A. Effect of Ala54Thr polymorphism of FABP2 on anthropometric and biochemical variables in response to a moderate-fat diet. Nutrition. 2013. 29:46-51. doi:10.1016/j.nut.2012.03.002

1499 Martins, MCT, Jaceldo-Siegl, K, Orlich, M, Fan, J, Mashchak, A, Fraser, GE. A New Approach to Assess Lifetime Dietary Patterns Finds Lower Consumption of Animal Foods with Aging in a Longitudinal Analysis of a Health-Oriented Adventist Population. Nutrients. 2017. 9:. doi:10.3390/nu9101118

1500 Marungruang, N, Tovar, J, Bjorck, I, Hallenius, FF. Improvement in cardiometabolic risk markers following a multifunctional diet is associated with gut microbial taxa in healthy overweight and obese subjects. Eur J Nutr. 2018. 57:2927-2936. doi:10.1007/s00394-017-1563-3

1501 Maruyama, C, Nakano, R, Shima, M, Mae, A, Shijo, Y, Nakamura, E, Okabe, Y, Park, S, Kameyama, N, Hirai, S, etal, Effects of a Japan Diet intake program on metabolic parameters in middle-aged men: a pilot study. Journal of atherosclerosis and thrombosis. 2017. 24:393-401. doi:10.5551/jat.36780

1502 Masa-Font, R, Fernandez-San-Martin, MI, Martin Lopez, LM, Alba Munoz, AM, Oller Canet, S, Martin Royo, J, San Emeterio Echevarria, L, Olona Tabuena, N, Ibarra Jato, M, Barroso Garcia, A, Gonzalez Tejon, S, Tajada Vitales, C, Diaz Mujica, B, Vinas Cabrera, L, Sanchis Catalan, R, Salvador Barbarroja, T. The effectiveness of a program of physical activity and diet to modify cardiovascular risk factors in patients with severe mental illness after 3-month follow-up: CAPiCOR randomized clinical trial. Eur Psychiatry. 2015. 30:1028-36. doi:10.1016/j.eurpsy.2015.09.006

1503 Masala, G, Bendinelli, B, Versari, D, Saieva, C, Ceroti, M, Santagiuliana, F, Caini, S, Salvini, S, Sera, F, Taddei, S, Ghiadoni, L, Palli, D. Anthropometric and dietary determinants of blood pressure in over 7000 Mediterranean women: The European Prospective Investigation into Cancer and Nutrition-Florence cohort. Journal of Hypertension. 2008. 26:2112-2120. doi:10.1097/HJH.0b013e32830ef75c
Outcome; Publication Date Overlaps with Existing Review

Study Design;

Publication Status

\section{Outcome}

Outcome

Intervention/Exposure

Outcome

Outcome

Study Design

Intervention/Exposure

Study Design; Health Status

Study Design 
1504 Mascherini, G, Petri, C, Cala, P, Bini, V, Galanti, G. Lifestyle and resulting body composition in young athletes. Minerva

Pediatr. 2016. .. doi:unavailable

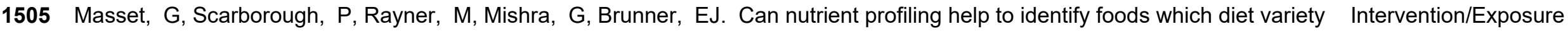
should be encouraged? Results from the Whitehall II cohort. Br J Nutr. 2015. 113:1800-9. doi:10.1017/s000711451500094x

1506 Mateo-Gallego, R, Lamiquiz-Moneo, I, Perez-Calahorra, S, Marco-Benedi, V, Bea, AM, Baila-Rueda, L, Laclaustra, M, Penalvo, JL, Civeira, F, Cenarro, A. Different protein composition of low-calorie diet differently impacts adipokine profile irrespective of weight loss in overweight and obese women. Nutr Metab Cardiovasc Dis. 2018. 28:133-142. doi:10.1016/j.numecd.2017.10.024

1507 Mateo-Gallego, R, Marco-Benedi, V, Perez-Calahorra, S, Bea, AM, Baila-Rueda, L, Lamiquiz-Moneo, I, de Castro-Oros, I, Cenarro, A, Civeira, F. Energy-restricted, high-protein diets more effectively impact cardiometabolic profile in overweight and obese women than lower-protein diets. Clin Nutr. 2017. 36:371-379. doi:10.1016/j.clnu.2016.01.018

1508 Mathers, JC. Impact of nutrition on the ageing process. Br J Nutr. 2015. 113 Suppl:S18-22. doi:10.1017/s0007114514003237

1509 Matsumoto, S, Beeson, WL, Shavlik, DJ, Siapco, G, Jaceldo-Siegl, K, Fraser, G, Knutsen, SF. Association between vegetarian diets and cardiovascular risk factors in non-Hispanic white participants of the Adventist Health Study-2. J Nutr Sci. 2019. 8:e6. doi:10.1017/jns.2019.1

1510 Mattei, J, Noel, SE, Tucker, KL. A meat, processed meat, and French fries dietary pattern is associated with high allostatic load in Puerto Rican older adults. J Am Diet Assoc. 2011. 111:1498-506. doi:10.1016/j.jada.2011.07.006

1511 Mattioli, AV, Coppi, F, Migaldi, M, Scicchitano, P, Ciccone, MM, Farinetti, A. Relationship between Mediterranean diet and asymptomatic peripheral arterial disease in a population of pre-menopausal women. Nutr Metab Cardiovasc Dis. 2017. 27:985990. doi:10.1016/j.numecd.2017.09.011

1512 Maukonen, M, Kanerva, N, Partonen, T, Kronholm, E, Konttinen, H, Wennman, H, Mannisto, S. The associations between chronotype, a healthy diet and obesity. Chronobiol Int. 2016. 33:972-81. doi:10.1080/07420528.2016.1183022

1513 May, AM, Struijk, EA, Fransen, HP, Onland-Moret, NC, de Wit, GA, Boer, JM, van der Schouw, YT, Hoekstra, J, Bueno-deMesquita, HB, Peeters, PH, Beulens, JW. The impact of a healthy lifestyle on Disability-Adjusted Life Years: a prospective cohort study. BMC Med. 2015. 13:39. doi:10.1186/s12916-015-0287-6

1514 Mayneris-Perxachs, J, Sala-Vila, A, Chisaguano, M, Castellote, Al, Estruch, R, Covas, MI, Fito, M, Salas-Salvado, J, Martinez-Gonzalez, MA, Lamuela-Raventos, R, Ros, E, Lopez-Sabater, MC. Effects of 1-year intervention with a Mediterranean diet on plasma fatty acid composition and metabolic syndrome in a population at high cardiovascular risk. PLoS One. 2014. 9:e85202. doi:10.1371/journal.pone.0085202

1515 Mayor, S. Low glycaemic index diet fails to reduce cardiovascular risk factors, study shows. BMJ (Online). 2014. 349:. doi:10.1136/bmj.g7719

Outcome

Power/Size

Study Design

Publication Status

Study Design

Study Design

Study Design;

Outcome

Intervention/Exposure; Comparator

Outcome

Outcome

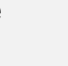


1516 Mazidi, M, Katsiki, N, Mikhailidis, DP, Bartlomiejczyk, MA, Banach, M. Association of Empirical Dietary Atherogenic Indices with All-Cause and Cause-Specific Mortality in a Multi-Ethnic Adult Population of the United States. Nutrients. 2019. 11: doi:10.3390/nu11102323

1517 Mazidi, M, Wong, ND, Katsiki, N, Mikhailidis, DP, Banach, M. Dietary patterns, plasma vitamins and Trans fatty acids are associated with peripheral artery disease. Lipids Health Dis. 2017. 16:254. doi:10.1186/s12944-017-0635-y

1518 Mc Clean, CM, Mc Laughlin, J, Burke, G, Murphy, MH, Trinick, T, Duly, E, Davison, GW. The effect of acute aerobic exercise on pulse wave velocity and oxidative stress following postprandial hypertriglyceridemia in healthy men. Eur $\mathrm{J}$ Appl Physiol. 2007. 100:225-34. doi:10.1007/s00421-007-0422-y

1519 McAuley, KA, Hopkins, CM, Smith, KJ, McLay, RT, Williams, SM, Taylor, RW, Mann, JI. Comparison of high-fat and highprotein diets with a high-carbohydrate diet in insulin-resistant obese women. Diabetologia. 2005. 48:8-16. doi:10.1007/s00125004-1603-4

1520 McAuley, KA, Smith, KJ, Taylor, RW, McLay, RT, Williams, SM, Mann, JI. Long-term effects of popular dietary approaches on weight loss and features of insulin resistance. Int J Obes (Lond). 2006. 30:342-9. doi:10.1038/sj.ijo.0803075

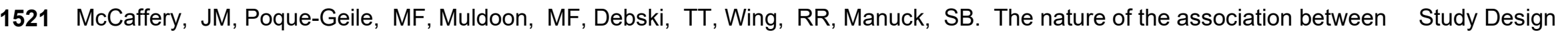
diet and serum lipids in the community: a twin study. Health Psychol. 2001. 20:341-50. doi:10.1037//0278-6133.20.5.341

1522 McCarter, DF. Low-carbohydrate diet effective for adults. Journal of family practice. 2003. 52:515-516. doi:unavailable

1523 McClain, AD, Otten, JJ, Hekler, EB, Gardner, CD. Adherence to a low-fat vs. low-carbohydrate diet differs by insulin resistance status. Diabetes, obesity \& metabolism. 2013. 15:87-90. doi:10.1111/j.1463-1326.2012.01668.x 1524 McCourt, HJ, Draffin, CR, Woodside, JV, Cardwell, CR, Young, IS, Hunter, SJ, Murray, LJ, Boreham, CA, Gallagher, AM,
Neville, CE, McKinley, MC. Dietary patterns and cardiovascular risk factors in adolescents and young adults: the Northern Ireland Young Hearts Project. Br J Nutr. 2014. 112:1685-98. doi:10.1017/s0007114514002682

1525 McCullough, D, Harrison, T, Lane, KE, Boddy, LM, Stewart, CE, Enright, KJ, Amirabdollahian, F, Schmidt, MA, Davies, IG. The effect of dietary carbohydrate manipulation on low-density lipoprotein-cholesterol and its associated cardiometabolic risk. Proceedings of the nutrition society. 2019. 78:. doi:10.1017/S002966511900020X

1526 McCullough, ML, Feskanich, D, Rimm, EB, Giovannucci, EL, Ascherio, A, Variyam, JN, Spiegelman, D, Stampfer, MJ, Willett, WC. Adherence to the Dietary Guidelines for Americans and risk of major chronic disease in men. Am $\mathrm{J}$ Clin Nutr. 2000. 72:1223-31. doi:10.1093/ajcn/72.5.1223

1527 McDougall, J, Thomas, LE, McDougall, C, Moloney, G, Saul, B, Finnell, JS, Richardson, K, Petersen, KM. Effects of 7 days on an ad libitum low-fat vegan diet: the McDougall Program cohort. Nutr J. 2014. 13:99. doi:10.1186/1475-2891-13-99

Study Design

Study duration

Study duration

Power/Size

Publication Status

Power/Size

Power/Size

Publication Status

Publication Date

Overlaps with Existing

Review

ntervention/Exposure Study duration fat influence the oxidation potential of VLDL, LDL and HDL subfractions?. Nutr Metab Cardiovasc Dis. 2013. 23:612-8. doi:10.1016/j.numecd.2011.12.007

1529 McKay, DL, Eliasziw, M, Chen, CYO, Blumberg, JB. A Pecan-Rich Diet Improves Cardiometabolic Risk Factors in Overweight and Obese Adults: A Randomized Controlled Trial. Nutrients. 2018. 10:. doi:10.3390/nu10030339

Outcome

Study duration 
1530 McLaughlin, T, Carter, S, Lamendola, C, Abbasi, F, Yee, G, Schaaf, P, Basina, M, Reaven, G. Effects of moderate variations in macronutrient composition on weight loss and reduction in cardiovascular disease risk in obese, insulin-resistant loss/Hypocaloric adults. Am J Clin Nutr. 2006. 84:813-21. doi:10.1093/ajcn/84.4.813

1531 McLaughlin, T, Liu, LF, Avery, E, Shen, WJ, Cohen, C, Kraemer, F, Cushman, S, Gardner, CD. Effect of low carbohydrate Publication Status weight loss diet on adipose cell size. Diabetes. 2016. 65:A443-A444. doi:10.2337/db16-1695-1770

1532 McManus, K, Antinoro, L, Sacks, F. A randomized controlled trial of a moderate-fat, low-energy diet compared with a low fat, low-energy diet for weight loss in overweight adults. Int J Obes Relat Metab Disord. 2001. 25:1503-11. doi:10.1038/sj.ijo.0801796

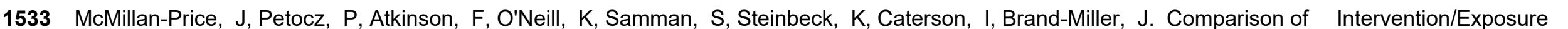
4 diets of varying glycemic load on weight loss and cardiovascular risk reduction in overweight and obese young adults: a randomized controlled trial. Arch Intern Med. 2006. 166:1466-75. doi:10.1001/archinte.166.14.1466

1534 McMorrow, AM, O'Reilly, ME, Connaughton, RM, Carolan, E, O'Shea, D, Lithander, FE, McGillicuddy, FC, Roche, HM. Weight status and dietary fat modulate high density lipoprotein particle function in adolescents: results from a cross-sectional analysis and a randomised, placebo-controlled, crossover trial. Proceedings of the nutrition society. 2016. 75:E70doi:10.1017/S0029665116000859

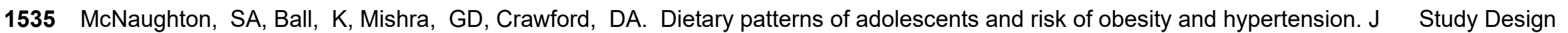
Nutr. 2008. 138:364-70. doi:10.1093/jn/138.2.364

1536 McNaughton, SA, Mishra, GD, Brunner, EJ. Dietary patterns, insulin resistance, and incidence of type 2 diabetes in the Whitehall II Study. Diabetes Care. 2008. 31:1343-8. doi:10.2337/dc07-1946

1537 McNeill, SH. Inclusion of red meat in healthful dietary patterns. Meat Sci. 2014. 98:452-60. doi:10.1016/j.meatsci.2014.06.028

1538 McSwiney, FT, Wardrop, B, Hyde, PN, Lafountain, RA, Volek, JS, Doyle, L. Keto-adaptation enhances exercise performance and body composition responses to training in endurance athletes. Metabolism. 2018. 81:25-34. doi:10.1016/j.metabol.2017.10.010

1539 McVay, MA, Jeffreys, AS, King, HA, Olsen, MK, Voils, Cl, Yancy, WS, Jr. The relationship between pretreatment dietary composition and weight loss during a randomised trial of different diet approaches. J Hum Nutr Diet. 2015.28 Suppl 2:16-23. doi:10.1111/jhn.12188

1540 McVay, MA, Voils, Cl, Geiselman, PJ, Smith, VA, Coffman, CJ, Mayer, S, Yancy, WS, Jr. Food preferences and weight change during low-fat and low-carbohydrate diets. Appetite. 2016. 103:336-343. doi:10.1016/j.appet.2016.04.035

Weight

loss/Hypocaloric

Publication Status

Mebonia, N, Trapaidze, D, Kvanchakhadze, R, Zhizhilashvili, S, Kasradze, N. DIETARY HABITS OF SCHOOL-AGE CHILDREN IN TBILISI. Georgian Med News. 2015. :68-73. doi:unavailable

1542 Meckling, KA, Gauthier, M, Grubb, R, Sanford, J. Effects of a hypocaloric, low-carbohydrate diet on weight loss, blood lipids, blood pressure, glucose tolerance, and body composition in free-living overweight women. Can J Physiol Pharmacol. 2002. 80:1095-105. doi:10.1139/y02-140 
1543 Meckling, KA, O'Sullivan, C, Saari, D. Comparison of a low-fat diet to a low-carbohydrate diet on weight loss, body composition, and risk factors for diabetes and cardiovascular disease in free-living, overweight men and women. $J$ Clin Endocrinol Metab. 2004. 89:2717-23. doi:10.1210/jc.2003-031606

1544 Meckling, KA, Sherfey, R. A randomized trial of a hypocaloric high-protein diet, with and without exercise, on weight loss, fitness, and markers of the Metabolic Syndrome in overweight and obese women. Appl Physiol Nutr Metab. 2007. 32:743-52. doi:10.1139/h07-059

1545 Medak, KD, Townsend, LK. Adding more fat to a high-fat diet only exacerbates hepatic insulin resistance. J Physiol. 2019. 597:1435-1436. doi:10.1113/jp277632

1546 Medenwald, D, Kluttig, A, Lacruz, ME, Schumann, J. Serum dietary fatty acids and coronary heart disease risk - A nested case-control-study within the CARLA cohort. Nutr Metab Cardiovasc Dis. 2019. 29:152-158. doi:10.1016/j.numecd.2018.10.006

1547 Medina-Remon, A, Casas, R, Tressserra-Rimbau, A, Ros, E, Martinez-Gonzalez, MA, Fito, M, Corella, D, Salas-Salvado, J, Lamuela-Raventos, RM, Estruch, R. Polyphenol intake from a Mediterranean diet decreases inflammatory biomarkers related to atherosclerosis: a substudy of the PREDIMED trial. British journal of clinical pharmacology. 2017. 83:114-128. doi:10.1111/bcp.12986

1548 Medina-Remon, A, Tresserra-Rimbau, A, Pons, A, Tur, JA, Martorell, M, Ros, E, Buil-Cosiales, P, Sacanella, E, Covas, MI Corella, D, Salas-Salvado, J, Gomez-Gracia, E, Ruiz-Gutierrez, V, Ortega-Calvo, M, Garcia-Valdueza, M, Aros, F, Saez, GT, Serra-Majem, L, Pinto, X, Vinyoles, E, Estruch, R, Lamuela-Raventos, RM. Effects of total dietary polyphenols on plasma nitric oxide and blood pressure in a high cardiovascular risk cohort. The PREDIMED randomized trial. Nutr Metab Cardiovasc Dis. 2015. 25:60-7. doi:10.1016/j.numecd.2014.09.001

1549 Meier, T, Grafe, K, Senn, F, Sur, P, Stangl, GI, Dawczynski, C, Marz, W, Kleber, ME, Lorkowski, S. Cardiovascular mortality attributable to dietary risk factors in 51 countries in the WHO European Region from 1990 to 2016 : a systematic analysis of the Global Burden of Disease Study. Eur J Epidemiol. 2019. 34:37-55. doi:10.1007/s10654-018-0473-x

1550 Meirelles, C, Candido, T, Gomes, PS. Effects of short-term very low-carbohydrate or conventional diet on strength performance. J Sports Med Phys Fitness. 2010. 50:189-95. doi:unavailable

1551 Meisinger, C, Rospleszcz, S, Wintermeyer, E, Lorbeer, R, Thorand, B, Bamberg, F, Peters, A, Schlett, CL, Linseisen, J. Isocaloric Substitution of Dietary Carbohydrate Intake with Fat Intake and MRI-Determined Total Volumes of Visceral, Subcutaneous and Hepatic Fat Content in Middle-Aged Adults. Nutrients. 2019. 11:. doi:10.3390/nu11051151

1552 Mekary, RA, Rimm, EB, Giovannucci, E, Stampfer, MJ, Willett, WC, Ludwig, DS, Hu, FB. Joint association of glycemic load and alcohol intake with type 2 diabetes incidence in women. Am J Clin Nutr. 2011. 94:1525-32. doi:10.3945/ajcn.111.023754

1553 Meksawan, K, Pendergast, DR, Leddy, JJ, Mason, M, Horvath, PJ, Awad, AB. Effect of low and high fat diets on nutrient intakes and selected cardiovascular risk factors in sedentary men and women. J Am Coll Nutr. 2004. 23:131-40. doi:10.1080/07315724.2004.10719353

1554 Meksawan, K, Venkatraman, JT, Awad, AB, Pendergast, DR. Effect of dietary fat intake and exercise on inflammatory mediators of the immune system in sedentary men and women. J Am Coll Nutr. 2004. 23:331-40. doi:10.1080/07315724.2004.10719376

Weight

loss/Hypocaloric

Study Design; Publication Status

Intervention/Exposure

Study Design;

Publication Status

Intervention/Exposure; Outcome

Study Design

Study duration

Study Design

ntervention/Exposure

Study duration

Outcome; Study duration 
1555 Mellberg, C, Sandberg, S, Ryberg, M, Eriksson, M, Brage, S, Larsson, C, Olsson, T, Lindahl, B. Long-term effects of a Palaeolithic-type diet in obese postmenopausal women: a 2-year randomized trial. Eur J Clin Nutr. 2014. 68:350-7. doi:10.1038/ejcn.2013.290

1556 Mellendick, K, Shanahan, L, Wideman, L, Calkins, S, Keane, S, Lovelady, C. Diets Rich in Fruits and Vegetables Are Associated with Lower Cardiovascular Disease Risk in Adolescents. Nutrients. 2018. 10:. doi:10.3390/nu10020136

Study Design;

Comparator

1557 Memelink, R, Verreijen, A, Engberink, M, Weijs, P. Higher than $1.2 \mathrm{G} / \mathrm{KG} / \mathrm{D}$ protein intake is associated with increased fat mass loss during weight loss in obese older adults. Clinical nutrition. 2014. 33:S110. doi:unavailable

1558 Mendonca, RD, Carvalho, NC, Martin-Moreno, JM, Pimenta, AM, Lopes, ACS, Gea, A, Martinez-Gonzalez, MA, BesRastrollo, M. Total polyphenol intake, polyphenol subtypes and incidence of cardiovascular disease: The SUN cohort study Nutr Metab Cardiovasc Dis. 2019. 29:69-78. doi:10.1016/j.numecd.2018.09.012

1559 Mendonca, RD, Lopes, AC, Pimenta, AM, Gea, A, Martinez-Gonzalez, MA, Bes-Rastrollo, M. Ultra-Processed Food Consumption and the Incidence of Hypertension in a Mediterranean Cohort: The Seguimiento Universidad de Navarra Project. Am J Hypertens. 2017. 30:358-366. doi:10.1093/ajh/hpw137

1560 Meneton, P, Kesse-Guyot, E, Fezeu, L, Galan, P, Hercberg, S, Menard, J. Distinctive unhealthy eating pattern in free-living middle-aged hypertensives when compared with dyslipidemic or overweight patients. J Hypertens. 2013. 31:1554-63. doi:10.1097/HJH.0b013e32836130f8

1561 Meng, H, Matthan, NR, Fried, SK, Berciano, S, Walker, ME, Galluccio, JM, Lichtenstein, AH. Effect of Dietary Carbohydrate Type on Serum Cardiometabolic Risk Indicators and Adipose Tissue Inflammatory Markers. J Clin Endocrinol Metab. 2018. 103:3430-3438. doi:10.1210/jc.2018-00667

1562 Meng, X, Zhu, K, Devine, A, Kerr, DA, Binns, CW, Prince, RL. A 5-year cohort study of the effects of high protein intake on lean mass and BMC in elderly postmenopausal women. J Bone Miner Res. 2009. 24:1827-34. doi:10.1359/jbmr.090513

1563 Mensink, M, Schutte, S, Chatindiara, I, Esser, D, Siebelink, E, Afman, L. Effect of caloric restriction and dietary composition on liver triglyceride content in subjects with abdominal obesity: the wageningen belly fat study. FASEB journal. Conference: experimental biology 2016, EB. San diego, CA united states. Conference start: 20160402. Conference end: 20160406. Conference publication: (var.pagings). 2016. 30:. doi:unavailable

1564 Mercanligil, SM, Arslan, P, Alasalvar, C, Okut, E, Akgul, E, Pinar, A, Geyik, PO, Tokgozoglu, L, Shahidi, F. Effects of hazelnut-enriched diet on plasma cholesterol and lipoprotein profiles in hypercholesterolemic adult men. Eur J Clin Nutr. 2007. 61:212-20. doi:10.1038/sj.ejcn.1602518

1565 Merino, J, Guasch-Ferre, M, Martinez-Gonzalez, MA, Corella, D, Estruch, R, Fito, M, Ros, E, Aros, F, Bullo, M, GomezGracia, E, Monino, M, Lapetra, J, Serra-Majem, L, Razquin, C, Buil-Cosiales, P, Sorli, JV, Munoz, MA, Pinto, X, Masana, L, Salas-Salvado, J. Is complying with the recommendations of sodium intake beneficial for health in individuals at high cardiovascular risk? Findings from the PREDIMED study. Am J Clin Nutr. 2015. 101:440-8. doi:10.3945/ajcn.114.096750

1566 Mero, AA, Huovinen, H, Matintupa, O, Hulmi, JJ, Puurtinen, R, Hohtari, H, Karila, TA. Moderate energy restriction with high protein diet results in healthier outcome in women. J Int Soc Sports Nutr. 2010. 7:4. doi:10.1186/1550-2783-7-4

Intervention/Exposure, data overlap with included article

Intervention/Exposure; Study duration 
1567 Merra, G, Gratteri, S, De Lorenzo, A, Barrucco, S, Perrone, MA, Avolio, E, Bernardini, S, Marchetti, M, Di Renzo, L.

Effects of very-low-calorie diet on body composition, metabolic state, and genes expression: a randomized double-blind placebocontrolled trial. Eur Rev Med Pharmacol Sci. 2017. 21:329-345. doi:unavailable

1568 Merra, G, Miranda, R, Barrucco, S, Gualtieri, P, Mazza, M, Moriconi, E, Marchetti, M, Chang, TF, De Lorenzo, A, Di Renzo, L. Very-low-calorie ketogenic diet with aminoacid supplement versus very low restricted-calorie diet for preserving muscle mass during weight loss: a pilot double-blind study. Eur Rev Med Pharmacol Sci. 2016. 20:2613-21. doi:unavailable

1569 Merrotsy, A, McCarthy, AL, Flack, J, Lacey, S, Coppinger, T. Project Spraoi: a two-year longitudinal study on the effectiveness of a school-based nutrition and physical activity intervention on dietary intake, nutritional knowledge and markers of health of Irish schoolchildren. Public Health Nutr. 2019. 22:2489-2499. doi:10.1017/s1368980019001368

1570 Mertens, E, Deforche, B, Mullie, P, Lefevre, J, Charlier, R, Knaeps, S, Huybrechts, I, Clarys, P. Longitudinal study on the association between three dietary indices, anthropometric parameters and blood lipids. Nutr Metab (Lond). 2015. 12:47. doi:10.1186/s12986-015-0042-1

1571 Mesquita de Carvalho, C, Dias Mendonca, D, Haas Piovesan, C, Edler Macagnan, F, Pandolfo Feoli, AM. Nutritional adequacy in subjects with metabolic syndrome. Nutr Hosp. 2014. 31:1147-53. doi:10.3305/nh.2015.31.3.8086

1572 Metkus, TS, Dobrosielski, D, Stewart, K. Effect of a low carbohydrate versus a low fat diet on the metabolic syndrome. Journal of the american college of cardiology. 2012. 59:E1753. doi:10.1016/S0735-1097\%2812\%2961754-9

1573 Metro, D, Tardugno, R, Papa, M, Bisignano, C, Manasseri, L, Calabrese, G, Gervasi, T, Dugo, G, Cicero, N. Adherence to the Mediterranean diet in a Sicilian student population. Nat Prod Res. 2018. 32:1775-1781. doi:10.1080/14786419.2017.1402317

1574 Mettler, S, Mitchell, N, Tipton, KD. Increased protein intake reduces lean body mass loss during weight loss in athletes. Med Sci Sports Exerc. 2010. 42:326-37. doi:10.1249/MSS.0b013e3181b2ef8e

1575 Mezzano, D, Leighton, F, Strobel, P, Martinez, C, Marshall, G, Cuevas, A, Castillo, O, Panes, O, Munoz, B, Rozowski, J, Pereira, J. Mediterranean diet, but not red wine, is associated with beneficial changes in primary haemostasis. Eur $\mathrm{J}$ Clin Nutr. 2003. 57:439-46. doi:10.1038/sj.ejcn.1601558

1576 Mia, FB, Vorster, HH. Coronary heart disease risk factors in Indian adolescents the role of diet. Cardiovasc J S Afr. 2000. 11:68-75. doi:unavailable

1577 Michalczyk, M, Zajac, A, Mikolajec, K, Zydek, G, Langfort, J. No Modification in Blood Lipoprotein Concentration but Changes in Body Composition after 4 Weeks of Low Carbohydrate Diet (LCD) Followed by 7 Days of Carbohydrate Loading in Basketball Players. J Hum Kinet. 2018. 65:125-137. doi:10.2478/hukin-2018-0102

1578 Michalczyk, MM, Chycki, J, Zajac, A, Maszczyk, A, Zydek, G, Langfort, J. Anaerobic performance after a low-carbohydrate diet (LCD) followed by 7 days of carbohydrate loading in male basketball players. Nutrients. 2019. 11:. doi:10.3390/nu11040778

1579 Michels, KB, Wolk, A. A prospective study of variety of healthy foods and mortality in women. International Journal of Epidemiology. 2002. 31:847-854. doi:10.1093/ije/31.4.847

Intervention/Exposure; Study duration

Intervention/Exposure

\section{Power/Size}

Outcome

Publication Status

Study Design;

Intervention/Exposure

Study duration

Outcome

Study Design; Country

Study Design; Study duration

Study duration

Publication Date Overlaps with Existing Review 
1580 Michielsen, Ccjr, Hangelbroek, RWJ, Feskens, EJM, Afman, LA. Disentangling the Effects of Monounsaturated Fatty Acids from Other Components of a Mediterranean Diet on Serum Metabolite Profiles: A Randomized Fully Controlled Dietary Intervention in Healthy Subjects at Risk of the Metabolic Syndrome. Mol Nutr Food Res. 2019. 63:e1801095. doi:10.1002/mnfr.201801095

1581 Mielgo Ayuso, J, Zourdos, MC, Urdampilleta, A, Calleja Gonzalez, J, Seco, J, Cordova, A. Relationship of long-term macronutrients intake on anabolic-catabolic hormones in female elite volleyball players. Nutr Hosp. 2017. 34:1155-1162. doi:10.20960/nh.763

1582 Mielgo-Ayuso, J, Zourdos, MC, Calleja-Gonzalez, J, Urdampilleta, A, Ostojic, SM. Dietary intake habits and controlled training on body composition and strength in elite female volleyball players during the season. Appl Physiol Nutr Metab. 2015. 40:827-34. doi:10.1139/apnm-2015-0100

1583 Miguet, M, Fillon, A, Masurier, J, Khammassi, M, Julian, V, Boirie, Y, Duclos, M, Blundell, J, Finlayson, G, Thivel, D. Energy intake, appetite and food reward responses to High Intensity Interval Exercise might depend on the degree of obesity in adolescents. Obesity facts. 2018. 11:33-. doi:10.1159/000489691

1584 Mihrshahi, S, Ding, D, Gale, J, Allman-Farinelli, M, Banks, E, Bauman, AE. Vegetarian diet and all-cause mortality: Evidence from a large population-based Australian cohort - the 45 and Up Study. Prev Med. 2017. 97:1-7. doi:10.1016/j.ypmed.2016.12.044

1585 Miksa, SA, Stadler, D, Karanja, N. Weight loss induced by low carbohydrate or high carbohydrate diets does not improve serum 25(OH)D concentrations. Obesity (silver spring, md.). 2010. 18:S95-. doi:unavailable

1586 Mildestvedt, T, Meland, E, Eide, GE. No difference in lifestyle changes by adding individual counselling to group-based rehabilitation RCT among coronary heart disease patients. Scandinavian Journal of Public Health. 2007. 35:591-598. doi:10.1080/14034940701349241

1587 Millar, L, Rowland, B, Nichols, M, Swinburn, B, Bennett, C, Skouteris, H, Allender, S. Relationship between raised BMI and sugar sweetened beverage and high fat food consumption among children. Obesity (Silver Spring). 2014. 22:E96-103. doi:10.1002/oby.20665

1588 Millen, BE, Quatromoni, PA, Nam, BH, O'Horo, CE, Polak, JF, D'Agostino, RB. Dietary patterns and the odds of carotid atherosclerosis in women: the Framingham Nutrition Studies. Prev Med. 2002. 35:540-7. doi:10.1006/pmed.2002.1116

1589 Millen, BE, Quatromoni, PA, Nam, BH, O'Horo, CE, Polak, JF, Wolf, PA, D'Agostino, RB. Dietary patterns, smoking, and subclinical heart disease in women: opportunities for primary prevention from the Framingham Nutrition Studies. J Am Diet Assoc. 2004. 104:208-14. doi:10.1016/j.jada.2003.11.007

1590 Miller, BV, Bertino, JS, Reed, RG, Burrington, CM, Davidson, LK, Green, A, Gartung, AM, Nafziger, AN. An evaluation of the atkins' diet. Metab Syndr Relat Disord. 2003. 1:299-309. doi:10.1089/1540419031361426

Study Design;

Comparator

Publication Status

\section{Outcome}

Publication Status

Health Status

Study Design Intervention/Exposure

Publication Date Overlaps with Existing Review

Study Design;

Intervention/Exposure; Outcome; Publication

Date Overlaps with Existing Review

Study duration 
1591 Miller, CK, Ulbrecht, JS, Lyons, J, Parker-Klees, L, Gutschall, MD, Smiciklas-Wright, H, Mitchell, DC, Covasa, M, Hayes,

M. A reduced-carbohydrate diet improves outcomes in patients with metabolic syndrome: A translational study. Topics in Clinical Nutrition. 2007. 22:82-91. doi:10.1097/00008486-200701000-00009

1592 Miller, ER, Cooper, LA, Carson, KA, Wang, NY, Appel, LJ, Gayles, D, Charleston, J, White, K, Dalcin, AT, Martin-Daniels $\mathrm{M}$, etal, . A randomized trial of a high potassium dietary intervention to lower blood pressure in urban African Americans with hypertension in the primary care setting: the "five plus nuts and beans" trial. Circulation. 2015. 131:. doi:unavailable

1593 Miller, GD, Beavers, DP, Hamm, D, Mihalko, SL, Messier, SP. Nutrient Intake During Diet-Induced Weight Loss and Exercise Interventions in a Randomized Trial in Older Overweight and Obese Adults. J Nutr Health Aging. 2017. 21:1216-1224. doi:10.1007/s12603-017-0892-5

1594 Miller, LE, Volpe, JJ, Coleman-Kelly, MD, Gwazdauskas, FC, Nickols-Richardson, SM. Anthropometric and leptin changes in women following different dietary approaches to weight loss. Obesity (Silver Spring). 2009. 17:199-201. doi:10.1038/oby.2008.498

1595 Miller, M, Beach, V, Sorkin, JD, Mangano, C, Dobmeier, C, Novacic, D, Rhyne, J, Vogel, RA. Comparative effects of three popular diets on lipids, endothelial function, and C-reactive protein during weight maintenance. J Am Diet Assoc. 2009. 109:713-7. doi:10.1016/j.jada.2008.12.023

1596 Minehira, K, Bettschart, V, Vidal, H, Vega, N, Di Vetta, V, Rey, V, Schneiter, P, Tappy, L. Effect of carbohydrate overfeeding on whole body and adipose tissue metabolism in humans. Obes Res. 2003. 11:1096-103. doi:10.1038/oby.2003.150

1597 Minehira, K, Vega, N, Vidal, H, Acheson, K, Tappy, L. Effect of carbohydrate overfeeding on whole body macronutrient metabolism and expression of lipogenic enzymes in adipose tissue of lean and overweight humans. Int $\mathrm{J}$ Obes Relat Metab Disord. 2004. 28:1291-8. doi:10.1038/sj.ijo.0802760

1598 Ministrini, S, Calzini, L, Nulli Migliola, E, Ricci, MA, Roscini, AR, Siepi, D, Tozzi, G, Daviddi, G, Martorelli, EE, Paganelli, MT, Lupattelli, G. Lysosomal Acid Lipase as a Molecular Target of the Very Low Carbohydrate Ketogenic Diet in Morbidly Obese Patients: The Potential Effects on Liver Steatosis and Cardiovascular Risk Factors. J Clin Med. 2019. 8:. doi:10.3390/jcm8050621

1599 Minoura, A, Wang, DH, Sato, Y, Zou, Y, Sakano, N, Kubo, M, Takemoto, K, Masatomi, C, Ogino, K. Association of dietary fat and carbohydrate consumption and predicted ten-year risk for developing coronary heart disease in a general Japanese population. Acta Med Okayama. 2014. 68:129-35. doi:10.18926/amo/52652

1600 Mirmiran, P, Asghari, G, Farhadnejad, H, Eslamian, G, Hosseini-Esfahani, F, Azizi, F. Low carbohydrate diet is associated with reduced risk of metabolic syndrome in Tehranian adults. Int J Food Sci Nutr. 2017. 68:358-365. doi:10.1080/09637486.2016.1242119

1601 Mirmiran, P, Bahadoran, Z, Esfandyari, S, Azizi, F. Dietary Protein and Amino Acid Profiles in Relation to Risk of Dysglycemia: Findings from a Prospective Population-Based Study. Nutrients. 2017. 9:. doi:10.3390/nu9090971

Publication Status

Study Design;

Intervention/Exposure

ntervention/Exposure Intervention/Exposure Study duration Study duration

Study Design; Study duration

Intervention/Exposure Intervention/Exposure; Outcome 
1602 Mirmiran, P, Bahadoran, Z, Mirzaei, S, Azizi, F. Dietary Intake, Changes in Lipid Parameters and the Risk of Hypertriglyceridemia: A Prospective Approach in the Tehran Lipid and Glucose Study. Int J Vitam Nutr Res. 2014. 84:269-76. doi:10.1024/0300-9831/a000213

1603 Mirmiran, P, Noori, N, Azizi, F. A prospective study of determinants of the metabolic syndrome in adults. Nutr Metab Cardiovasc Dis. 2008. 18:567-73. doi:10.1016/j.numecd.2007.06.002

1604 Mirmiran, P, Ramezankhani, A, Hekmatdoost, A, Azizi, F. Effect of nutrition intervention on non-communicable disease risk factors among Tehranian adults: Tehran Lipid and Glucose Study. Ann Nutr Metab. 2008. 52:91-5. doi:10.1159/000121364

1605 Mirza, NM, Palmer, MG, Sinclair, KB, McCarter, R, He, J, Ebbeling, CB, Ludwig, DS, Yanovski, JA. Effects of a low glycemic load or a low-fat dietary intervention on body weight in obese Hispanic American children and adolescents: a randomized controlled trial. Am J Clin Nutr. 2013. 97:276-85. doi:10.3945/ajcn.112.042630

1606 Mishra, S, Xu, J, Agarwal, U, Gonzales, J, Levin, S, Barnard, ND. A multicenter randomized controlled trial of a plant-based nutrition program to reduce body weight and cardiovascular risk in the corporate setting: the GEICO study. Eur $\mathrm{J}$ Clin Nutr. 2013. 67:718-24. doi:10.1038/ejcn.2013.92

1607 Misirli, G, Benetou, V, Lagiou, P, Bamia, C, Trichopoulos, D, Trichopoulou, A. Relation of the traditional Mediterranean diet to cerebrovascular disease in a Mediterranean population. Am J Epidemiol. 2012. 176:1185-92. doi:10.1093/aje/kws205

1608 Misra, A, Wasir, JS, Vikram, NK. Carbohydrate diets, postprandial hyperlipidaemia, abdominal obesity \& Asian Indians: A recipe for atherogenic disaster. Indian Journal of Medical Research. 2005. 121:5-8. doi:unavailable

1609 Mitchell, CJ, Milan, AM, Mitchell, SM, Zeng, N, Ramzan, F, Sharma, P, Knowles, S, Roy, N, Sjodin, A, Wagner, KH, etal, . Ten weeks of protein consumption at the RDA results: in a loss of appendicular lean mass in healthy older men, a randomized controlled trial. FASEB journal. 2017. 31:. doi:unavailable

1610 Mitchell, CJ, Milan, AM, Mitchell, SM, Zeng, N, Ramzan, F, Sharma, P, Knowles, SO, Roy, NC, Sjodin, A, Wagner, KH, Cameron-Smith, D. The effects of dietary protein intake on appendicular lean mass and muscle function in elderly men: a 10wk randomized controlled trial. Am J Clin Nutr. 2017. 106:1375-1383. doi:10.3945/ajcn.117.160325

1611 Mitchell, SM, Milan, AM, Mitchell, CJ, Gillies, NA, D'Souza, RF, Zeng, N, Ramzan, F, Sharma, P, Knowles, SO, Roy, NC Sjodin, A, Wagner, KH, Zeisel, SH, Cameron-Smith, D. Protein Intake at Twice the RDA in Older Men Increases Circulatory Concentrations of the Microbiome Metabolite Trimethylamine-N-Oxide (TMAO). Nutrients. 2019. 11:. doi:10.3390/nu11092207

1612 Mitra, SR, Tan, PY. Effect of an individualised high-protein, energy-restricted diet on anthropometric and cardio-metabolic parameters in overweight and obese Malaysian adults: a 6-month randomised controlled study. Br J Nutr. 2019. 121:1002-1017. doi:10.1017/s0007114519000345

1613 Mittendorfer, B, Sidossis, LS. Mechanism for the increase in plasma triacylglycerol concentrations after consumption of shortterm, high-carbohydrate diets. Am J Clin Nutr. 2001. 73:892-9. doi:10.1093/ajcn/73.5.892

Intervention/Exposure

Intervention/Exposure; Country

Intervention/Exposure; Publication Date Overlaps with Existing Review

Intervention/Exposure

Intervention/Exposure; Comparator

Publication Date

Overlaps with Existing Review

Publication Status

Publication Status

Intervention/Exposure

Intervention/Exposure

Weight

loss/Hypocaloric

Study duration 
1614 Miyagawa, N, Arima, H, Yoshita, K, Okuda, N, Ohkubo, T, Hisamatsu, T, Kondo, K, Miura, K. Feasibility, safety and efficacy of a modified dietary approaches to stop hypertension diet for japanese population. Circulation. 2018. 137: doi:unavailable

1615 Mogul, HR, Freeman, R, Scherer, PE, Frey, M, Hantash, FM, Greenbaum, R, Jozak, S, Klein, LA, Tanenbaum, K.

Publication Status Carbohydrate modified diet and insulin sensitizers reduce fasting insulin and body weight, modulate measures of the metabolic syndrome, and improve adipokines in EMPOWIR (enhance the metabolic profile of women with insulin resistance) a randomized clinical trial (NCT00618071) of normoglycemic, hyperinsulinemic women with midlife weight gain. Endocrine reviews. 2012. 33: doi:unavailable

1616 Mohammadifard, N, Talaei, M, Sadeghi, M, Oveisegharan, S, Golshahi, J, Esmaillzadeh, A, Sarrafzadegan, N. Dietary patterns and mortality from cardiovascular disease: Isfahan Cohort Study. Eur J Clin Nutr. 2017. 71:252-258. doi:10.1038/ejcn.2016.170

1617 Mohan, V, Spiegelman, D, Sudha, V, Gayathri, R, Hong, B, Praseena, K, Anjana, RM, Wedick, NM, Arumugam, K, Malik, V, Ramachandran, S, Bai, MR, Henry, JK, Hu, FB, Willett, W, Krishnaswamy, K. Effect of brown rice, white rice, and brown rice with legumes on blood glucose and insulin responses in overweight Asian Indians: a randomized controlled trial. Diabetes Technol Ther. 2014. 16:317-25. doi:10.1089/dia.2013.0259

1618 Mohler, ER, 3rd, Sibley, AA, Stein, R, Davila-Roman, V, Wyatt, H, Badellino, K, Rader, DJ, Klein, S, Foster, GD. Endothelial function and weight loss: comparison of low-carbohydrate and low-fat diets. Obesity (Silver Spring). 2013. 21:504-9. doi:10.1002/oby.20055

1619 Mohorko, N, Cernelic-Bizjak, M, Poklar-Vatovec, T, Grom, G, Kenig, S, Petelin, A, Jenko-Praznikar, Z. Weight loss, improved physical performance, cognitive function, eating behavior, and metabolic profile in a 12-week ketogenic diet in obese adults. Nutr Res. 2019. 62:64-77. doi:10.1016/j.nutres.2018.11.007

1620 Mojtahedi, MC, Thorpe, MP, Karampinos, DC, Johnson, CL, Layman, DK, Georgiadis, JG, Evans, EM. The effects of a higher protein intake during energy restriction on changes in body composition and physical function in older women. J Gerontol A Biol Sci Med Sci. 2011. 66:1218-25. doi:10.1093/gerona/glr120

1621 Mokhtari, Z, Sharafkhah, M, Poustchi, H, Sepanlou, SG, Khoshnia, M, Gharavi, A, Sohrabpour, AA, Sotoudeh, M, Dawsey, SM, Boffetta, P, Abnet, CC, Kamangar, F, Etemadi, A, Pourshams, A, FazeltabarMalekshah, A, Islami, F, Brennan, P, Malekzadeh, R, Hekmatdoost, A. Adherence to the Dietary Approaches to Stop Hypertension (DASH) diet and risk of total and cause-specific mortality: results from the Golestan Cohort Study. Int J Epidemiol. 2019. .. doi:10.1093/ije/dyz079

1622 Monge, A, Lajous, M, Ortiz-Panozo, E, Rodriguez, BL, Gongora, JJ, Lopez-Ridaura, R. Western and Modern Mexican dietary patterns are directly associated with incident hypertension in Mexican women: a prospective follow-up study. Nutr J. 2018. 17:21. doi:10.1186/s12937-018-0332-3

1623 Monica Dinu, M, Pagliai, G, Mangino, A, Cesari, F, Giusti, B, Marcucci, R, Casini, A, Sofi, F. Comparison between Mediterranean and Vegetarian diets for cardiovascular prevention: the CARDIVEG study. European journal of preventive cardiology. 2017. 24:S136-. doi:unavailable 
1624 Monjardino, T, Lucas, R, Ramos, E, Barros, H. Associations between a priori-defined dietary patterns and longitudinal changes in bone mineral density in adolescents. Public Health Nutr. 2014. 17:195-205. doi:10.1017/s1368980012004879

1625 Monteagudo, C, Mariscal-Arcas, M, Rivas, A, Lorenzo-Tovar, ML, Tur, JA, Olea-Serrano, F. Proposal of a Mediterranean Diet Serving Score. PLoS One. 2015. 10:e0128594. doi:10.1371/journal.pone.0128594

Outcome

Study Design

Intervention/Exposure; Outcome

1626 Moore, LL, Singer, MR, Bradlee, ML, Daniels, SR. Adolescent dietary intakes predict cardiometabolic risk clustering. Eur J Nutr. 2016. 55:461-468. doi:10.1007/s00394-015-0863-8

Intervention/Exposure; Outcome

1627 Moore, WJ, McGrievy, ME, Turner-McGrievy, GM. Dietary adherence and acceptability of five different diets, including vegan and vegetarian diets, for weight loss: The New DIETs study. Eat Behav. 2015. 19:33-8. doi:10.1016/j.eatbeh.2015.06.011

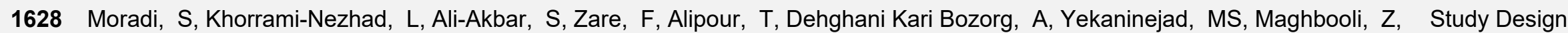
Mirzaei, K. The associations between dietary patterns and bone health, according to the TGF-beta1 T869-->C polymorphism, in postmenopausal Iranian women. Aging Clin Exp Res. 2018. 30:563-571. doi:10.1007/s40520-017-0828-2

1629 Moraleda, E, Martinez-Argudo, I, Rodriguez-Gomez, I, Ara, I, Aznar, S. Ketogenic diet and physical activity intervention to weight loss analyzing different FTO and AdipoQ polimorfisms. Annals of nutrition \& metabolism. 2019. 75:35-36. doi:10.1159/000501441

1630 Moran, LJ, Flynn, AC, Louise, J, Deussen, AR, Dodd, JM. The effect of a lifestyle intervention on pregnancy and postpartum dietary patterns determined by factor analysis. Obesity (Silver Spring). 2017. 25:1022-1032. doi:10.1002/oby.21848

1631 Moran, LJ, Noakes, M, Clifton, P, Buckley, J, Brinkworth, G, Thomson, R, Norman, RJ. Predictors of lifestyle intervention attrition or weight loss success in women with polycystic ovary syndrome who are overweight or obese. Nutrients. 2019. 11:. doi:10.3390/nu11030492

1632 Moreira, T, Severo, M, Oliveira, A, Ramos, E, Rodrigues, S, Lopes, C. Eating out of home and dietary adequacy in preschool children. Br J Nutr. 2015. 114:297-305. doi:10.1017/s0007114515001713

1633 Morell-Azanza, L, Ochotorena-Elicegui, A, Catalan-Lamban, A, Chueca, M, Marti, A, Julian, CAS. Assessment of adherence to mediterranean diet during a weight loss intervention in children with cardiometabolic risk. Hormone research in paediatrics. 2016. 86:310-. doi:10.1159/000449142

1634 Morenga, LT, Williams, S, Brown, R, Mann, J. Effect of a relatively high-protein, high-fiber diet on body composition and metabolic risk factors in overweight women. Eur J Clin Nutr. 2010. 64:1323-31. doi:10.1038/ejcn.2010.163

1635 Moreno Franco, B, Leon Latre, M, Andres Esteban, EM, Ordovas, JM, Casasnovas, JA, Penalvo, JL. Soluble and insoluble dietary fibre intake and risk factors for metabolic syndrome and cardiovascular disease in middle-aged adults: the AWHS cohort Nutr Hosp. 2014. 30:1279-88. doi:10.3305/nh.2014.30.6.7778

1636 Moreno, B, Bellido, D, Sajoux, I, Goday, A, Saavedra, D, Crujeiras, AB, Casanueva, FF. Comparison of a very low-calorieketogenic diet with a standard low-calorie diet in the treatment of obesity. Endocrine. 2014. 47:793-805. doi:10.1007/s12020014-0192-3 
1637 Moreno, LA. Effects of diet on growth of children with obesity. J Pediatr Gastroenterol Nutr. 2010. 51 Suppl 3:S147-8. doi:10.1097/MPG.0b013e3181efb1be

1638 Moretti, L, Canada, T. A Randomized Study Comparing the Effects of a Low-Carbohydrate Diet and a Conventional Diet on Lipoprotein Subfractions and C-reactive Protein Levels in Patients With Severe Obesity. Nutr Clin Pract. 2006. 21:187-188. doi:10.1177/0115426506021002187

1639 Morgan, LM, Griffin, BA, Millward, DJ, DeLooy, A, Fox, KR, Baic, S, Bonham, MP, Wallace, JM, MacDonald, I, Taylor, MA, Truby, H. Comparison of the effects of four commercially available weight-loss programmes on lipid-based cardiovascular risk factors. Public Health Nutr. 2009. 12:799-807. doi:10.1017/s1368980008003236

1640 Morgantini, C, Trifiro, S, Trico, D, Meriwether, D, Baldi, S, Mengozzi, A, Reddy, ST, Natali, A. A short-term increase in dietary cholesterol and fat intake affects high-density lipoprotein composition in healthy subjects. Nutr Metab Cardiovasc Dis 2018. 28:575-581. doi:10.1016/j.numecd.2018.03.005

1641 Morimoto, N, Kasuga, C, Tanaka, A, Kamachi, K, Ai, M, Urayama, KY, Tanaka, A. Association between dietary fibre:carbohydrate intake ratio and insulin resistance in Japanese adults without type 2 diabetes. Br J Nutr. 2018. 119:620-628. doi:10.1017/s0007114517003725

1642 Morin, E, Michaud-Letourneau, I, Couturier, Y, Roy, M. A whole-food, plant-based nutrition program: Evaluation of cardiovascular outcomes and exploration of food choices determinants. Nutrition. 2019. 66:54-61. doi:10.1016/j.nut.2019.03.020

1643 Morrison, JA, Glueck, CJ, Daniels, S, Wang, P. Determinants of persistent obesity and hyperinsulinemia in a biracial cohort: a 15-year prospective study of schoolgirls. J Pediatr. 2010. 157:559-65. doi:10.1016/j.jpeds.2010.04.030

1644 Morton, D, Rankin, P, Kent, L, Sokolies, R, Dysinger, W, Gobble, J, Diehl, H. The Complete Health Improvement Program (CHIP) and reduction of chronic disease risk factors in Canada. Can J Diet Pract Res. 2014. 75:72-7. doi:10.3148/75.2.2014.72

1645 Mosca, CL, Marshall, JA, Grunwald, GK, Cornier, MA, Baxter, J. Insulin resistance as a modifier of the relationship between dietary fat intake and weight gain. Int J Obes Relat Metab Disord. 2004. 28:803-12. doi:10.1038/sj.ijo.0802621

1646 Moslehi, N, Ehsani, B, Mirmiran, P, Hojjat, P, Azizi, F. Association of Dietary Proportions of Macronutrients with Visceral Adiposity Index: Non-Substitution and Iso-Energetic Substitution Models in a Prospective Study. Nutrients. 2015. 7:8859-70. doi:10.3390/nu7105436

1647 Motie, M, Evangelista, LS, Horwich, T, Hamilton, M, Lombardo, D, Cooper, DM, Galassetti, PR, Fonarow, GC. Pro-HEART - a randomized clinical trial to test the effectiveness of a high protein diet targeting obese individuals with heart failure: rationale, design and baseline characteristics. Contemp Clin Trials. 2013. 36:371-81. doi:10.1016/j.cct.2013.08.004

1648 Moyama, S, Minami, K, Yano, M, Okumura, M, Hayashi, S, Takayama, H, Yorimoto, A. Relationship between dietary patterns and brachial-ankle pulse wave velocity among middle-aged adults in Japan. Asia Pac J Clin Nutr. 2017. 26:539-544. doi:10.6133/apjen.032016.14

1649 Mozaffarian, D, Rimm, EB, Herrington, DM. Dietary fats, carbohydrate, and progression of coronary atherosclerosis in postmenopausal women. Am J Clin Nutr. 2004. 80:1175-84. doi:10.1093/ajcn/80.5.1175 
1650 Mozaffarian, D. The great fat debate: taking the focus off of saturated fat. J Am Diet Assoc. 2011. 111:665-6. doi:10.1016/j.jada.2011.03.030

1651 Mueller, C, Masri, B, Hogg, J, Mastrogiacomo, M, Chiu, YL. Carbohydrate- vs fat-controlled diet effect on weight loss and coronary artery disease risk: a pilot feeding study. Nutr Clin Pract. 2010. 25:542-7. doi:10.1177/0884533610379854

1652 Muhammad, HFL, Vink, RG, Roumans, NJT, Arkenbosch, LAJ, Mariman, EC, van Baak, MA. Dietary Intake after Weight Loss and the Risk of Weight Regain: Macronutrient Composition and Inflammatory Properties of the Diet. Nutrients. 2017. 9: doi:10.3390/nu9111205

1653 Mulla, UZ, Cooper, R, Mishra, GD, Kuh, D, Stephen, AM. Adult macronutrient intake and physical capability in the MRC national survey of health and development. Age and Ageing. 2013. 42:81-87. doi:10.1093/ageing/afs101

1654 Mullie, P, Clarys, P. Relation between dietary pattern analysis (principal component analysis) and body mass index: a 5-year follow-up study in a Belgian military population. J R Army Med Corps. 2016. 162:23-9. doi:10.1136/jramc-2014-000356

1655 Munsters, MJ, Geraedts, MC, Saris, WH. Effects of different protein and glycemic index diets on metabolic profiles and substrate partitioning in lean healthy males. Appl Physiol Nutr Metab. 2013. 38:1107-14. doi:10.1139/apnm-2012-0409

1656 Murakami, K, McCaffrey, TA, Gallagher, AM, Neville, CE, Boreham, CA, Livingstone, MB. Dietary glycemic index and glycemic load in relation to changes in body composition measures during adolescence: Northern Ireland Young Hearts Study. Int J Obes (Lond). 2014. 38:252-8. doi:10.1038/ijo.2013.63

1657 Muraki, I, Wu, H, Imamura, F, Laden, F, Rimm, EB, Hu, FB, Willett, WC, Sun, Q. Rice consumption and risk of cardiovascular disease: results from a pooled analysis of 3 U.S. cohorts. Am J Clin Nutr. 2015. 101:164-72. doi:10.3945/ajcn.114.087551

1658 Murphy, K, Davis, C, Knight, A, Bryan, J, Wilson, C, Hodgson, J, Woodman, R. Adherence to a mediterranean diet (MEDDIET) results in improved cardiometabolic health measures and maintained cognitive performance in older australians; Results from the mediterranean diet for cognition and cardiovascular health in the elderly (MEDLEY) trial. Revista espanola de nutricion humana y dietetica. 2016. 20:652-. doi:unavailable

1659 Murphy, KJ, Parker, B, Dyer, KA, Davis, CR, Coates, AM, Buckley, JD, Howe, PR. A comparison of regular consumption of fresh lean pork, beef and chicken on body composition: a randomized cross-over trial. Nutrients. 2014. 6:682-96. doi:10.3390/nu6020682

1660 Murrin, CM, Heinen, MM, Kelleher, CC. Are Dietary Patterns of Mothers during Pregnancy Related to Children's Weight Status? Evidence from the Lifeways Cross- Generational Cohort Study. AIMS Public Health. 2015. 2:274-296. doi:10.3934/publichealth.2015.3.274

1661 Mursu, J, Virtanen, JK, Rissanen, TH, Tuomainen, TP, Nykanen, I, Laukkanen, JA, Kortelainen, R, Voutilainen, S. Glycemic index, glycemic load, and the risk of acute myocardial infarction in Finnish men: the Kuopio Ischaemic Heart Disease Risk Factor Study. Nutr Metab Cardiovasc Dis. 2011. 21:144-9. doi:10.1016/j.numecd.2009.08.001

Outcome

Power/Size

Intervention/Exposure; Study duration

Intervention/Exposure

Intervention/Exposure

Publication Status

Intervention/Exposure

Study Design

Intervention/Exposure 
1662 Mursu, J, Virtanen, JK, Tuomainen, TP, Nurmi, T, Voutilainen, S. Intake of fruit, berries, and vegetables and risk of type 2 diabetes in Finnish men: the Kuopio Ischaemic Heart Disease Risk Factor Study. Am J Clin Nutr. 2014. 99:328-33. doi:10.3945/ajcn.113.069641

1663 Murtaugh, MA, Appel, LJ, Beasley, JM, Guenther, PM, Greene, T, McFadden, ML, Tooze, JA. Higher levels of sodium density (MG/KCAL) are associated with increased blood pressure independent of absolute sodium (MG): the dash sodium trial. Circulation. 2017. 135:. doi:unavailable

1664 Murtaza, N, Burke, LM, Vlahovich, N, Charlesson, B, O' Neill H, Ross, ML, Campbell, KL, Krause, L, Morrison, M. The effects of dietary pattern during intensified training on stool microbiota of elite race walkers. Nutrients. 2019. 11: doi:10.3390/nu11020261

1665 Muscogiuri, G, Barrea, L, Di Somma, C, Altieri, B, Vecchiarini, M, Orio, F, Spinosa, T, Colao, A, Savastano, S. Patient empowerment and the Mediterranean diet as a possible tool to tackle prediabetes associated with overweight or obesity: a pilot study. Hormones (Athens). 2019. 18:75-84. doi:10.1007/s42000-018-0090-9

1666 Mustafa, J, Ellison, RC, Singer, MR, Bradlee, ML, Kalesan, B, Holick, MF, Moore, LL. Dietary Protein and Preservation of Physical Functioning Among Middle-Aged and Older Adults in the Framingham Offspring Study. Am J Epidemiol. 2018. 187:1411-1419. doi:10.1093/aje/kwy014

1667 Muzio, F, Mondazzi, L, Harris, WS, Sommariva, D, Branchi, A. Effects of moderate variations in the macronutrient content of the diet on cardiovascular disease risk factors in obese patients with the metabolic syndrome. Am J Clin Nutr. 2007. 86:946-51. doi:10.1093/ajcn/86.4.946

1668 Muzio, F, Mondazzi, L, Sommariva, D, Branchi, A. Long-term effects of low-calorie diet on the metabolic syndrome in obese nondiabetic patients. Diabetes Care. 2005. 28:1485-6. doi:10.2337/diacare.28.6.1485

1669 Muzsik, A, Bajerska, J, Krzyzanowska, P, Walkowiak, J, Chmurzynska, A. The CED-MED project: the effect of a dietary intervention on inflammatory cytokines and lipid metabolism in women with metabolic syndrome: preliminary results. Annals of nutrition and metabolism. Conference: 12th european nutrition conference, FENS 2015. Berlin germany. Conference start: 20151020. Conference end: 20151023. Conference publication: (var.pagings). 2015. 67:231-232. doi:10.1159/000440895

1670 Mytton, OT, Forouhi, NG, Scarborough, P, Lentjes, M, Luben, R, Rayner, M, Khaw, KT, Wareham, NJ, Monsivais, P. Association between intake of less-healthy foods defined by the United Kingdom's nutrient profile model and cardiovascular disease: A population-based cohort study. PLoS Med. 2018. 15:e1002484. doi:10.1371/journal.pmed.1002484

$1671 \mathrm{Na}, \mathrm{L}, \mathrm{Han}, \mathrm{T}$, Zhang, W, Wu, X, Na, G, Du, S, Li, Y, Sun, C. A Snack Dietary Pattern Increases the Risk of Hypercholesterolemia in Northern Chinese Adults: A Prospective Cohort Study. PLoS One. 2015. 10:e0134294. doi:10.1371/journal.pone.0134294

$1672 \mathrm{Na}, \mathrm{W}, \mathrm{Yu}, \mathrm{TY}$, Sohn, C. Development of a food-based index of dietary inflammatory potential for Koreans and its relationship with metabolic syndrome. Nutr Res Pract. 2019. 13:150-158. doi:10.4162/nrp.2019.13.2.150 
1673 Nabuco, HC, Tomeleri, CM, Junior, PS, Fernandes, RR, Cavalcante, EF, Nunes, JP, Cunha, PF, Dos Santos, L, Cyrino, ES. Effects of higher habitual protein intake on resistance-training-induced changes in body composition and muscular strength in untrained older women: A clinical trial study. Nutr Health. 2019. 25:103-112. doi:10.1177/0260106019838365

1674 Nagai, Y, Kawanabe, S, Fukuda, H, Tanaka, Y. Changes of body composition after replacing dietary carbohydrate with a protein supplement in overweight Japanese subjects. Journal of diabetes investigation. 2018. 9:44-. doi:10.1111/jdi.12938

1675 Nagata, C, Wada, K, Tsuji, M, Kawachi, T, Nakamura, K. Dietary glycaemic index and glycaemic load in relation to all-cause and cause-specific mortality in a Japanese community: the Takayama study. Br J Nutr. 2014. 112:2010-7. doi:10.1017/s0007114514003109

1676 Nah, EH, Chu, J, Kim, S, Cho, S, Kwon, E. Efficacy of lifestyle interventions in the reversion to normoglycemia in Korean prediabetics: One-year results from a randomised controlled trial. Primary Care Diabetes. 2019. 13:212-220. doi:10.1016/j.pcd.2018.11.017

1677 Naja, F, Itani, L, Hwalla, N, Sibai, AM, Kharroubi, SA. Identification of dietary patterns associated with elevated blood pressure among Lebanese men: A comparison of principal component analysis with reduced rank regression and partial least square methods. PLoS One. 2019. 14:e0220942. doi:10.1371/journal.pone.0220942

1678 Naja, F, Shivappa, N, Nasreddine, L, Kharroubi, S, Itani, L, Hwalla, N, Mehio Sibai, A, Hebert, JR. Role of inflammation in the association between the western dietary pattern and metabolic syndrome among Lebanese adults. Int $\mathrm{J}$ Food Sci Nutr. 2017. 68:997-1004. doi:10.1080/09637486.2017.1312297

1679 Najjar, RS, Moore, CE, Montgomery, BD. A defined, plant-based diet utilized in an outpatient cardiovascular clinic effectively treats hypercholesterolemia and hypertension and reduces medications. Clinical Cardiology. 2018. 41:307-313. doi:10.1002/clc.22863

1680 Najjar, RS, Moore, CE, Montgomery, BD. Consumption of a defined, plant-based diet reduces lipoprotein(a), inflammation, and other atherogenic lipoproteins and particles within 4 weeks. Clin Cardiol. 2018. 41:1062-1068. doi:10.1002/clc.23027

Publication Status

Intervention/Exposure Intervention/Exposure Study Design;

Outcome

Study Design

Study Design

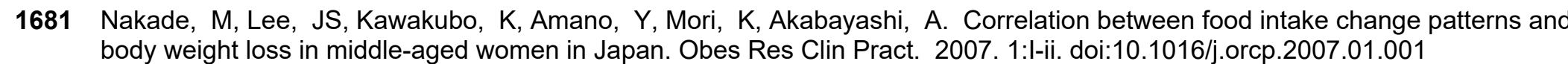

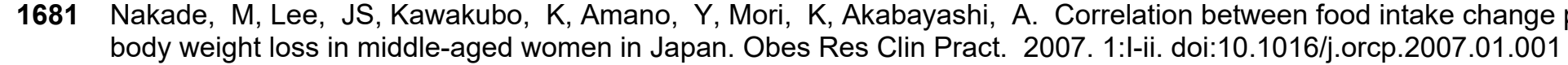

Study Design; Study duration

Publication Date Overlaps with Existing Review

1682 Nakamura, Y, Ueshima, H, Okuda, N, Higashiyama, A, Kita, Y, Kadowaki, T, Okamura, T, Murakami, Y, Okayama, A, Choudhury, SR, Rodriguez, B, Curb, JD, Stamler, J. Relation of dietary and other lifestyle traits to difference in serum adiponectin concentration of Japanese in Japan and Hawaii: the INTERLIPID Study. Am J Clin Nutr. 2008. 88:424-30. doi:10.1093/ajcn/88.2.424

1683 Nakamura, Y, Ueshima, H, Okuda, N, Miura, K, Kita, Y, Miyagawa, N, Yoshita, K, Nakagawa, H, Sakata, K, Saitoh, S, Okamura, T, Okayama, A, Choudhry, SR, Rodriguez, B, Masaki, KH, Chan, Q, Elliott, P, Stamler, J. Relationship of three different types of low-carbohydrate diet to cardiometabolic risk factors in a Japanese population: the INTERMAP/INTERLIPID Study. Eur J Nutr. 2016. 55:1515-24. doi:10.1007/s00394-015-0969-z

1684 Nanri, A, Mizoue, T, Yoshida, D, Takahashi, R, Takayanagi, R. Dietary patterns and A1C in Japanese men and women. Diabetes Care. 2008. 31:1568-73. doi:10.2337/dc08-0297

Study Design;

Intervention/Exposure

Outcome

Study Design 
1685 Nasreddine, L, Akika, R, Mailhac, A, Tamim, H, Zgheib, NK. The Interaction between Genetic Polymorphisms in FTO and TCF7L2 Genes and Dietary Intake with Regard to Body Mass and Composition: An Exploratory Study. J Pers Med. 2019. 9:. doi:10.3390/jpm9010011

1686 Nation, J, Humphrey, M, MacKay, M, Boneh, A. Linear growth of children on a ketogenic diet: does the protein-to-energy ratio matter?. J Child Neurol. 2014. 29:1496-501. doi:10.1177/0883073813508222

Intervention/Exposure; Health Status

1687 Naumann, E, Plat, J, Mensink, RP. Changes in serum concentrations of noncholesterol sterols and lipoproteins in healthy subjects do not depend on the ratio of plant sterols to stanols in the diet. J Nutr. 2003. 133:2741-7. doi:10.1093/jn/133.9.2741

1688 Navas-Carretero, S, Holst, C, Saris, WH, van Baak, MA, Jebb, SA, Kafatos, A, Papadaki, A, Pfeiffer, AF, HandjievaDarlenska, T, Hlavaty, P, Stender, S, Larsen, TM, Astrup, A, Martinez, JA. The Impact of Gender and Protein Intake on the Success of Weight Maintenance and Associated Cardiovascular Risk Benefits, Independent of the Mode of Food Provision: The DiOGenes Randomized Trial. J Am Coll Nutr. 2016. 35:20-30. doi:10.1080/07315724.2014.948642

1689 Navas-Carretero, S, Perez-Granados, AM, Schoppen, S, Vaquero, MP. An oily fish diet increases insulin sensitivity compared to a red meat diet in young iron-deficient women. Br J Nutr. 2009. 102:546-53. doi:10.1017/s0007114509220794

1690 Navas-Carretero, S, San-Cristobal, R, Livingstone, KM, Celis-Morales, C, Marsaux, CF, Macready, AL, Fallaize, R, O'Donovan, CB, Forster, H, Woolhead, C, Moschonis, G, Lambrinou, CP, Jarosz, M, Manios, Y, Daniel, H, Gibney, ER, Brennan, L, Walsh, MC, Drevon, CA, Gibney, M, Saris, WHM, Lovegrove, JA, Mathers, JC, Martinez, JA. Higher vegetable protein consumption, assessed by an isoenergetic macronutrient exchange model, is associated with a lower presence of overweight and obesity in the web-based Food4me European study. Int J Food Sci Nutr. 2019. 70:240-253. doi:10.1080/09637486.2018.1492524

1691 Nazare, JA, Smith, J, Borel, AL, Almeras, N, Tremblay, A, Bergeron, J, Poirier, P, Despres, JP. Changes in both global diet quality and physical activity level synergistically reduce visceral adiposity in men with features of metabolic syndrome. J Nutr. 2013. 143:1074-83. doi:10.3945/jn.113.175273

1692 Ndanuko, R, Tapsell, L, Charlton, K, Neale, E, Batterham, M. Dietary patterns associated with blood pressure in a clinical sample of overweight adults volunteering for a weight loss trial. Revista espanola de nutricion humana y dietetica. 2016. 20:460461. doi:unavailable

1693 Neacsu, M, Fyfe, C, Horgan, G, Johnstone, AM. Appetite control and biomarkers of satiety with vegetarian (soy) and meatbased high-protein diets for weight loss in obese men: a randomized crossover trial. Am J Clin Nutr. 2014. 100:548-58. doi:10.3945/ajcn.113.077503

1694 Negele, L, Schneider, B, Ristl, R, Stulnig, TM, Willfort-Ehringer, A, Helk, O, Widhalm, K. Effect of a low-fat diet enriched either with rapeseed oil or sunflower oil on plasma lipoproteins in children and adolescents with familial hypercholesterolaemia. Results of a pilot study. Eur J Clin Nutr. 2015. 69:337-43. doi:10.1038/ejen.2014.234

1695 Neri-Sanchez, M, Martinez-Carrillo, BE, Valdes-Ramos, R, Soto-Pina, AE, Vargas-Hernandez, JA, Benitez-Arciniega, AD. Dietary patterns, central obesity and serum lipids concentration in Mexican adults. Nutr Hosp. 2019. 36:109-117. doi:10.20960/nh.2002

Study duration

Intervention/Exposure

Study duration

Study Design

loss/Hypocaloric

Publication Status

Study duration

Intervention/Exposure

Power/Size 
1696 Ness, AR, Powles, JW. The role of diet, fruit and vegetables and antioxidants in the Aetiology of stroke. European Journal of Preventive Cardiology. 2014. 6:229-234. doi:10.1177/204748739900600407

1697 Nettleton, JA, Follis, JL, Ngwa, JS, Smith, CE, Ahmad, S, Tanaka, T, Wojczynski, MK, Voortman, T, Lemaitre, RN, Kristiansson, K, Nuotio, ML, Houston, DK, Perälä, MM, Qi, Q, Sonestedt, E, Manichaikul, A, Kanoni, S, Ganna, A, Mikkilä, V, North, KE, Siscovick, DS, Harald, K, McKeown, NM, Johansson, I, Rissanen, H, Liu, Y, Lahti, J, Hu, FB, Bandinelli, S, Rukh, G, Rich, S, Booij, L, Dmitriou, M, Ax, E, Raitakari, O, Mukamal, K, Männistö, S, Hallmans, G, Jula, A, Ericson, U, Jacobs, DR, J AVanRooijF, Deloukas, P, Sjögren, P, Kähönen, M, Djousse, L, Perola, M, Barroso, I, Hofman, A, Stirrups, K, Viikari, J, Uitterlinden, AG, Kalafati, IP, Franco, OH, Mozaffarian, D, Salomaa, V, Borecki, IB, Knekt, P, Kritchevsky, SB, Eriksson, JG, Dedoussis, GV, Qi, L, Ferrucci, L, Orho-Melander, M, Carola Zillikens, M, Ingelsson, E, Lehtimäki, T, Renström, F, Adrienne Cupples, L, Loos, RJ, Franks, PW. Gene $\times$ dietary pattern interactions in obesity: Analysis of up to 68 317 adults of European ancestry. Human Molecular Genetics. 2015. 24:4728-4738. doi:10.1093/hmg/ddv186

1698 Nettleton, JA, Polak, JF, Tracy, R, Burke, GL, Jacobs, DR, Jr. Dietary patterns and incident cardiovascular disease in the Multi-Ethnic Study of Atherosclerosis. Am J Clin Nutr. 2009. 90:647-54. doi:10.3945/ajcn.2009.27597

1699 Nettleton, JA, Rock, CL, Wang, Y, Jenny, NS, Jacobs, DR. Associations between dietary macronutrient intake and plasma lipids demonstrate criterion performance of the Multi-Ethnic Study of Atherosclerosis (MESA) food-frequency questionnaire. Br $\mathrm{J}$ Nutr. 2009. 102:1220-7. doi:10.1017/s0007114509382161

1700 Nettleton, JA, Schulze, MB, Jiang, R, Jenny, NS, Burke, GL, Jacobs Jr, DR. A priori-defined dietary patterns and markers of cardiovascular disease risk in the Multi-Ethnic Study of Atherosclerosis (MESA). American Journal of Clinical Nutrition. 2008. 88:185-194. doi:unavailable

1701 Nettleton, JA, Steffen, LM, Mayer-Davis, EJ, Jenny, NS, Jiang, R, Herrington, DM, Jacobs, DR, Jr. Dietary patterns are associated with biochemical markers of inflammation and endothelial activation in the Multi-Ethnic Study of Atherosclerosis (MESA). Am J Clin Nutr. 2006. 83:1369-79. doi:10.1093/ajcn/83.6.1369

1702 Nettleton, JA, Steffen, LM, Ni, H, Liu, K, Jacobs, DR, Jr. Dietary patterns and risk of incident type 2 diabetes in the MultiEthnic Study of Atherosclerosis (MESA). Diabetes Care. 2008. 31:1777-82. doi:10.2337/dc08-0760

1703 Neuhouser, ML, Howard, B, Lu, J, Tinker, LF, Van Horn, L, Caan, B, Rohan, T, Stefanick, ML, Thomson, CA. A low-fat dietary pattern and risk of metabolic syndrome in postmenopausal women: the Women's Health Initiative. Metabolism. 2012. 61:1572-81. doi:10.1016/j.metabol.2012.04.007

1704 Newton, AL, Hanks, LJ, Ashraf, AP, Williams, E, Davis, M, Casazza, K. Macronutrient intake influences the effect of 25hydroxy-vitamin d status on metabolic syndrome outcomes in african american girls. Cholesterol. 2012. 2012:581432. doi:10.1155/2012/581432

1705 Nguo, K, Bonham, MP, Truby, H, Barber, E, Brown, J, Huggins, CE. Effect of Macronutrient Composition on Appetite Hormone Responses in Adolescents with Obesity. Nutrients. 2019. 11:. doi:10.3390/nu11020340

Publication Date

Overlaps with Existing Review

Study Design

Publication Date

Overlaps with Existing Review

Outcome

Publication Date

Overlaps with Existing Review

Publication Date

Overlaps with Existing Review

Study duration

Study duration 
1706 Nguyen, BT, Shuval, K, Bertmann, F, Yaroch, AL. The Supplemental Nutrition Assistance Program, Food Insecurity, Dietary

1707 Nichols, SD, Francis, MP, Dalrymple, N. Sustainability of a Curriculum-based Intervention on Dietary Behaviours and Physical Activity among Primary School Children in Trinidad and Tobago. West Indian Med J. 2014. 63:68-77. doi:10.7727/wimj.2014.011

1708 Nicklas, J, Sacks, F, Smith, S, LeBoff, M, Rood, J, Bray, G, Ridker, P. Effect of macronutrient composition of weight loss diets on reduction of the inflammatory marker hsCRP. BMC complementary and alternative medicine. 2012. 12:. doi:unavailable

1709 Nicklas, JM, Sacks, FM, Smith, SR, LeBoff, MS, Rood, JC, Bray, GA, Ridker, PM. Effect of dietary composition of weight loss diets on high-sensitivity c-reactive protein: the Randomized POUNDS LOST trial. Obesity (Silver Spring). 2013. 21:681-9. doi:10.1002/oby.20072

1710 Nicklas, TA, Dwyer, J, Feldman, HA, Luepker, RV, Kelder, SH, Nader, PR. Serum cholesterol levels in children are associated with dietary fat and fatty acid intake. J Am Diet Assoc. 2002. 102:511-7. doi:10.1016/s0002-8223(02)90117-3

1711 Nickols-Richardson, SM, Coleman, MD, Volpe, JJ, Hosig, KW. Perceived hunger is lower and weight loss is greater in overweight premenopausal women consuming a low-carbohydrate/high-protein vs high-carbohydrate/low-fat diet. J Am Diet Assoc. 2005. 105:1433-7. doi:10.1016/j.jada.2005.06.025

1712 Nickols-Richardson, SM, Piehowski, KE, Metzgar, CJ, Miller, DL, Preston, AG. Changes in body weight, blood pressure and selected metabolic biomarkers with an energy-restricted diet including twice daily sweet snacks and once daily sugar-free beverage. Nutr Res Pract. 2014. 8:695-704. doi:10.4162/nrp.2014.8.6.695

1713 Niemczyk, NA. Low-carbohydrate versus low-fat diet: A randomized clinical trial. Journal of Midwifery and Women's Health. 2015. 60:104-105. doi:10.1111/jmwh.12281_1

1714 Niinikoski, H, Jula, A, Viikari, J, Ronnemaa, T, Heino, P, Lagstrom, H, Jokinen, E, Simell, O. Blood pressure is lower in children and adolescents with a low-saturated-fat diet since infancy: the special turku coronary risk factor intervention project. Hypertension. 2009. 53:918-24. doi:10.1161/hypertensionaha.109.130146

1715 Niinikoski, H, Pahkala, K, Viikari, J, Ronnemaa, T, Jula, A, Lagstrom, H, Simell, O, Raitakari, OT. The STRIP Study: longTerm Impact of a Low Saturated Fat/Low Cholesterol Diet. Current cardiovascular risk reports. 2014. 8:1-7. doi:10.1007/s12170-014-0410-9

1716 Nilholm, C, Roth, B, Hoglund, P, Blennow, K, Englund, E, Hansson, O, Zetterberg, H, Ohlsson, B. Dietary intervention with an Okinawan-based Nordic diet in type 2 diabetes renders decreased interleukin-18 concentrations and increased neurofilament light concentrations in plasma. Nutr Res. 2018. 60:13-25. doi:10.1016/j.nutres.2018.08.002

1717 Nilsson, A, Montiel Rojas, D, Kadi, F. Impact of Meeting Different Guidelines for Protein Intake on Muscle Mass and Physical Function in Physically Active Older Women. Nutrients. 2018. 10:. doi:10.3390/nu10091156

1718 Niswender, K, Piletic, M, Andersen, H, Conradsen Hiort, L, Hollander, P. Weight change upon once-daily initiation of insulin detemir with or without dietary intervention in overweight or obese insulin-naïve individuals with type 2 diabetes: results from the DIET trial. Diabetes, obesity \& metabolism. 2014. 16:186-192. doi:10.1111/dom.12218

Intervention/Exposure

Publication Status

Weight

loss/Hypocaloric

Intervention/Exposure

Outcome; Study duration

Intervention/Exposure

Study Design; Publication Status

Intervention/Exposure

Study Design Publication Status

Study Design; Study duration; Health Status

Study Design

Intervention/Exposure; Health Status 
1719 Niswender, KD, Fazio, S, Gower, BA, Silver, HJ. Balanced high fat diet reduces cardiovascular risk in obese women although changes in adipose tissue, lipoproteins, and insulin resistance differ by race. Metabolism. 2018. 82:125-134.

Study Design doi:10.1016/j.metabol.2018.01.020

1720 Niu, K, Momma, H, Kobayashi, Y, Guan, L, Chujo, M, Otomo, A, Ouchi, E, Nagatomi, R. The traditional Japanese dietary pattern and longitudinal changes in cardiovascular disease risk factors in apparently healthy Japanese adults. Eur J Nutr. 2016. 55:267-79. doi:10.1007/s00394-015-0844-y

1721 Nizamuddin, J, Turner, Z, Rubenstein, JE, Pyzik, PL, Kossoff, EH. Management and risk factors for dyslipidemia with the ketogenic diet. J Child Neurol. 2008. 23:758-61. doi:10.1177/0883073808318061

1722 Njike, VY, Ayettey, R, Petraro, P, Treu, JA, Katz, DL. Walnut ingestion in adults at risk for diabetes: effects on body composition, diet quality, and cardiac risk measures. BMJ Open Diabetes Res Care. 2015. 3:e000115. doi:10.1136/bmjdrc2015-000115

1723 Noakes, M, Foster, PR, Keogh, JB, James, AP, Mamo, JC, Clifton, PM. Comparison of isocaloric very low carbohydrate/high saturated fat and high carbohydrate/low saturated fat diets on body composition and cardiovascular risk. Nutr Metab (Lond). 2006. 3:7. doi:10.1186/1743-7075-3-7

1724 Noakes, M, Keogh, JB, Foster, PR, Clifton, PM. Effect of an energy-restricted, high-protein, low-fat diet relative to a conventional high-carbohydrate, low-fat diet on weight loss, body composition, nutritional status, and markers of cardiovascular health in obese women. Am J Clin Nutr. 2005. 81:1298-306. doi:10.1093/ajcn/81.6.1298

1725 Noakes, TD. Low-carbohydrate and high-fat intake can manage obesity and associated conditions: occasional survey. S Afr Med J. 2013. 103:826-30. doi:10.7196/samj.7302

1726 Noakes, TD. The Women's Health Initiative Randomized Controlled Dietary Modification Trial: an inconvenient finding and the diet-heart hypothesis. S Afr Med J. 2013. 103:824-5. doi:10.7196/samj.7343

Comparator

Power/Size

Health Status

Intervention/Exposure

Study duration

Weight

loss/Hypocaloric

Study Design;

Publication Status

Study Design;

Publication Status

1727 Nobili, V, Liccardo, D, Bedogni, G, Salvatori, G, Gnani, D, Bersani, I, Alisi, A, Valenti, L, Raponi, M. Influence of dietary pattern, physical activity, and I148M PNPLA3 on steatosis severity in at-risk adolescents. Genes Nutr. $2014.9: 392$. doi:10.1007/s12263-014-0392-8

1728 Noel, SE, Newby, PK, Ordovas, JM, Tucker, KL. Adherence to an (n-3) fatty acid/fish intake pattern is inversely associated with metabolic syndrome among Puerto Rican adults in the Greater Boston area. J Nutr. 2010. 140:1846-54. doi:10.3945/jn.110.124297

1729 Noll, C, Kunach, M, Frisch, F, Bouffard, L, Dubreuil, S, Jean-Denis, F, Phoenix, S, Cunnane, SC, Guerin, B, Turcotte, EE, Carpentier, AC. Seven-Day Caloric and Saturated Fat Restriction Increases Myocardial Dietary Fatty Acid Partitioning in Impaired Glucose-Tolerant Subjects. Diabetes. 2015. 64:3690-9. doi:10.2337/db15-0337

1730 Norman, A, Zeebari, Z, Nyberg, G, Elinder, LS. Parental support in promoting children's health behaviours and preventing overweight and obesity - a long-term follow-up of the cluster-randomised healthy school start study II trial. BMC Pediatr. 2019 19:104. doi:10.1186/s12887-019-1467-x

Study Design;

Intervention/Exposure; Outcome

Intervention/Exposure

Study duration 
1731 Norouzy, A, Salehi, M, Philippou, E, Arabi, H, Shiva, F, Mehrnoosh, S, Mohajeri, SM, Mohajeri, SA, Motaghedi Larijani, A, Nematy, M. Effect of fasting in Ramadan on body composition and nutritional intake: a prospective study. J Hum Nutr Diet.

Intervention/Exposure; 2013. 26 Suppl 1:97-104. doi:10.1111/jhn.12042

1732 Nosova, EV, Bartel, K, Chong, KC, Alley, HF, Conte, MS, Owens, CD, Grenon, SM. Analysis of nutritional habits and intake of polyunsaturated fatty acids in veterans with peripheral arterial disease. Vasc Med. 2015. 20:432-8.

Comparator doi:10.1177/1358863x15591088

1733 Novotny, R, Nigg, CR, Li, F, Wilkens, LR. Pacific kids DASH for health (PacDASH) randomized, controlled trial with DASH eating plan plus physical activity improves fruit and vegetable intake and diastolic blood pressure in children. Child Obes. 2015. 11:177-86. doi:10.1089/chi.2014.0141

1734 Nowlin, SY, Cleland, CM, Vadiveloo, M, D'Eramo Melkus, G, Parekh, N, Hagan, H. Explaining Racial/Ethnic Dietary Patterns in Relation to Type 2 Diabetes: An Analysis of NHANES 2007-2012. Ethn Dis. 2016. 26:529-536. doi:10.18865/ed.26.4.529

1735 Nowson, CA, Wattanapenpaiboon, N, Pachett, A. Low-sodium Dietary Approaches to Stop Hypertension-type diet including lean red meat lowers blood pressure in postmenopausal women. Nutr Res. 2009. 29:8-18. doi:10.1016/j.nutres.2008.12.002

1736 Nowson, CA, Worsley, A, Margerison, C, Jorna, MK, Frame, AG, Torres, SJ, Godfrey, SJ. Blood pressure response to dietary modifications in free-living individuals. J Nutr. 2004. 134:2322-9. doi:10.1093/jn/134.9.2322

1737 Nowson, CA, Worsley, A, Margerison, C, Jorna, MK, Godfrey, SJ, Booth, A. Blood pressure change with weight loss is affected by diet type in men. Am J Clin Nutr. 2005. 81:983-9. doi:10.1093/ajcn/81.5.983

1738 Numao, S, Kawano, H, Endo, N, Yamada, Y, Konishi, M, Takahashi, M, Sakamoto, S. Short-term low carbohydrate/high-fat diet intake increases postprandial plasma glucose and glucagon-like peptide-1 levels during an oral glucose tolerance test in healthy men. Eur J Clin Nutr. 2012. 66:926-31. doi:10.1038/ejcn.2012.58

1739 Nurkkala, M, Kaikkonen, K, Vanhala, ML, Karhunen, L, Keranen, AM, Korpelainen, R. Lifestyle intervention has a beneficia effect on eating behavior and long-term weight loss in obese adults. Eat Behav. 2015. 18:179-85. doi:10.1016/j.eatbeh.2015.05.009

1740 Nykanen, T, Pihlainen, K, Santtila, M, Vasankari, T, Fogelholm, M, Kyrolainen, H. Diet Macronutrient Composition, Physica Activity, and Body Composition in Soldiers During 6 Months Deployment. Mil Med. 2019. 184:e231-e237. doi:10.1093/milmed/usy232

1741 Nymo, S, Coutinho, SR, Jorgensen, J, Rehfeld, JF, Truby, H, Kulseng, B, Martins, C. Timeline of changes in appetite during Study Design weight loss with a ketogenic diet. Int J Obes (Lond). 2017. 41:1224-1231. doi:10.1038/ijo.2017.96

1742 Oba, S, Nagata, C, Nakamura, K, Fujii, K, Kawachi, T, Takatsuka, N, Shimizu, H. Dietary glycemic index, glycemic load and intake of carbohydrate and rice in relation to risk of mortality from stroke and its subtypes in Japanese men and women. Metabolism. 2010. 59:1574-82. doi:10.1016/j.metabol.2010.02.004

Intervention/Exposure;

Intervention/Exposure; Publication Date Overlaps with Existing Review

Intervention/Exposure

Intervention/Exposure

Study duration

Intervention/Exposure

Intervention/Exposure Comparator 
1743 Oba, S, Nanri, A, Kurotani, K, Goto, A, Kato, M, Mizoue, T, Noda, M, Inoue, M, Tsugane, S. Dietary glycemic index, glycemic load and incidence of type 2 diabetes in Japanese men and women: the Japan Public Health Center-based Prospective Study. Nutr J. 2013. 12:165. doi:10.1186/1475-2891-12-165

1744 Obarzanek, E, Sacks, FM, Vollmer, WM, Bray, GA, Miller, ER, 3rd, Lin, PH, Karanja, NM, Most-Windhauser, MM, Moore TJ, Swain, JF, Bales, CW, Proschan, MA. Effects on blood lipids of a blood pressure-lowering diet: the Dietary Approaches to Stop Hypertension (DASH) Trial. Am J Clin Nutr. 2001. 74:80-9. doi:10.1093/ajcn/74.1.80

Publication Date

Overlaps with Existing Review

1745 O'Brien, KD, Brehm, BJ, Seeley, RJ, Bean, J, Wener, MH, Daniels, S, D'Alessio, DA. Diet-induced weight loss is associated with decreases in plasma serum amyloid a and C-reactive protein independent of dietary macronutrient composition in obese subjects. J Clin Endocrinol Metab. 2005. 90:2244-9. doi:10.1210/jc.2004-1011

1746 O'Brien, KM, Hutchesson, MJ, Jensen, M, Morgan, P, Callister, R, Collins, CE. Participants in an online weight loss program can improve diet quality during weight loss: a randomized controlled trial. Nutr J. 2014. 13:82. doi:10.1186/1475-2891-13-82

1747 O'Connor, LE, Biberstine, SL, Paddon-Jones, D, Schwichtenberg, AJ, Campbell, WW. Adopting a Mediterranean-Style Eating Pattern with Different Amounts of Lean Unprocessed Red Meat Does Not Influence Short-Term Subjective Personal WellBeing in Adults with Overweight or Obesity. J Nutr. 2018. 148:1917-1923. doi:10.1093/jn/nxy235

1748 O'Connor, LE, Li, J, Sayer, RD, Hennessy, JE, Campbell, WW. Short-Term Effects of Healthy Eating Pattern Cycling on Cardiovascular Disease Risk Factors: Pooled Results from Two Randomized Controlled Trials. Nutrients. 2018. 10:. doi:10.3390/nu10111725

1749 O'Connor, LE, Paddon-Jones, D, Wright, AJ, Campbell, WW. A Mediterranean-style eating pattern with lean, unprocessed red meat has cardiometabolic benefits for adults who are overweight or obese in a randomized, crossover, controlled feeding trial. Am J Clin Nutr. 2018. 108:33-40. doi:10.1093/ajcn/nqy075

1750 O'Connor, LE, Wright, AJ, Paddon-Jones, D, Campbell, WW. Daily red meat intake does not affect improvements in cardiovascular disease risk factors induced by consuming the USDA's Healthy Mediterranean-Style Eating Pattern. FASEB journal. 2017. 31:. doi:unavailable

1751 Oddy, WH, Allen, KL, Trapp, GSA, Ambrosini, GL, Black, LJ, Huang, RC, Rzehak, P, Runions, KC, Pan, F, Beilin, LJ, Mori, TA. Dietary patterns, body mass index and inflammation: Pathways to depression and mental health problems in adolescents. Brain Behav Immun. 2018. 69:428-439. doi:10.1016/j.bbi.2018.01.002

1752 O'Doherty, MG, Skidmore, PM, Young, IS, McKinley, MC, Cardwell, C, Yarnell, JW, Gey, FK, Evans, A, Woodside, JV. Dietary patterns and smoking in Northern Irish men: a population at high risk of coronary heart disease. Int $\mathrm{J}$ Vitam Nutr Res. 2011. 81:21-33. doi:10.1024/0300-9831/a000047

1753 Oellingrath, IM, Svendsen, MV. BMI-specific associations between health-related behaviours and overweight - a longitudinal study among Norwegian adolescents. Public Health Nutr. 2017. 20:481-491. doi:10.1017/s1368980016002536

1754 Oh, C, No, JK, Kim, HS. Dietary pattern classifications with nutrient intake and body composition changes in Korean elderly. Nutr Res Pract. 2014. 8:192-7. doi:10.4162/nrp.2014.8.2.192
Intervention/Exposure

Intervention/Exposure; Outcome

Outcome; Study

duration

Study duration

Intervention/Exposure; Outcome

Publication Status

Power/Size

Study Design

Power/Size

Study Design 
1755 Oh, SM, Kim, HC, Rhee, Y, Park, SJ, Lee, HJ, Suh, I, Feskanich, D. Dietary protein in relation to bone stiffness index and fat-free mass in a population consuming relatively low protein diets. J Bone Miner Metab. 2013. 31:433-41. doi:10.1007/s00774013-0427-z

1756 Oh, SY, Ahn, H, Chang, N, Kang, MH, Oh J V. Dietary patterns and weight status associated with behavioural problems in young children. Public Health Nutr. 2014. 17:2563-9. doi:10.1017/s1368980013002917

1757 Ojeda Rodriguez, A, Zazpe, I, Morell Azanza, L, Chueca, M, Azcona Sanjulian, M, Marti, A. Improved vitamin D adequacy and its associations with diet quality indices in a lifestyle intervention of children and adolescents with abdominal obesity. Obesity facts. 2019. 12:112-. doi:10.1159/000489691

1758 Ojeda-Brito, R, Brito-Ojeda, M, Ruano, C, Nissensonh, M, Ruiz-Caballero, J, Serra-Majem, L. Drinking habits in a sample of Study Design university students. Relationship between the adherence to the Mediterranean Diet and BMI. Nutr Hosp. 2015.32 Suppl 2:10326. doi:10.3305/nh.2015.32.sup2.10326

1759 Ojeda-Rodriguez, A, Zazpe, I, Morell-Azanza, L, Chueca, MJ, Azcona-Sanjulian, MC, Marti, A. Improved Diet Quality and Nutrient Adequacy in Children and Adolescents with Abdominal Obesity after a Lifestyle Intervention. Nutrients. 2018. 10:. doi:10.3390/nu10101500

1760 Okekunle, AP, Wu, X, Feng, R, Li, Y, Sun, C. Higher intakes of energy-adjusted dietary amino acids are inversely associated with obesity risk. Amino Acids. 2019. 51:373-382. doi:10.1007/s00726-018-2672-x

1761 Okita, K, Takada, S, Kinugawa, S. Very low-carbohydrate diet can effectively reduce weight without deterioration in physical fitness. Journal of the hong kong college of cardiology. 2016. 24:A32-. doi:unavailable

1762 Okuda, N, Miura, K, Okayama, A, Okamura, T, Abbott, RD, Nishi, N, Fujiyoshi, A, Kita, Y, Nakamura, Y, Miyagawa, N Hayakawa, T, Ohkubo, T, Kiyohara, Y, Ueshima, H. Fruit and vegetable intake and mortality from cardiovascular disease in Japan: a 24-year follow-up of the NIPPON DATA80 Study. Eur J Clin Nutr. 2015. 69:482-8. doi:10.1038/ejcn.2014.276

1763 Oliveira, A, Jones, L, de Lauzon-Guillain, B, Emmett, P, Moreira, P, Charles, MA, Lopes, C. Early problematic eating behaviours are associated with lower fruit and vegetable intake and less dietary variety at 4-5 years of age. A prospective analysis of three European birth cohorts. Br J Nutr. 2015. 114:763-71. doi:10.1017/s0007114515002287

1764 Oliveira, C, Boule, N, Sharma, AM, Elliott, S, Ghosh, S, Siervo, M, Prado, C. The impact of a high-protein diet on energy expenditure and substrate oxidation: preliminary findings of a randomized, controlled, cross-over trial. Obesity facts. 2018. 11:308-. doi:10.1159/000489691

1765 O'Neal, EK, Smith, AF, Heatherly, AJ, Killen, LG, Waldman, HS, Hollingsworth, A, Koh, Y. Effects of a 3-week High-FatLow-Carbohydrate Diet on Lipid and Glucose Profiles in Experienced, Middle-age Male Runners. Int J Exerc Sci. 2019. 12:786799. doi:unavailable

1766 O'Neil, A, Shivappa, N, Jacka, FN, Kotowicz, MA, Kibbey, K, Hebert, JR, Pasco, JA. Pro-inflammatory dietary intake as a risk factor for CVD in men: a 5-year longitudinal study. Br J Nutr. 2015. 114:2074-82. doi:10.1017/s0007114515003815 
1767 O'Neil, CE, Nicklas, TA, Fulgoni, VL, 3rd. Nutrient Intake, Diet Quality, and Weight Measures in Breakfast Patterns Consumed by Children Compared with Breakfast Skippers: NHANES 2001-2008. AIMS Public Health. 2015. 2:441-468. doi:10.3934/publichealth.2015.3.441

1768 Ooi, EM, Adams, LA, Zhu, K, Lewis, JR, Kerr, DA, Meng, X, Solah, V, Devine, A, Binns, CW, Prince, RL. Consumption of a whey protein-enriched diet may prevent hepatic steatosis associated with weight gain in elderly women. Nutr Metab Cardiovasc Dis. 2015. 25:388-95. doi:10.1016/j.numecd.2014.11.005

1769 Ooi, EM, Lichtenstein, AH, Millar, JS, Diffenderfer, MR, Lamon-Fava, S, Rasmussen, H, Welty, FK, Barrett, PH, Schaefer, EJ. Effects of Therapeutic Lifestyle Change diets high and low in dietary fish-derived FAs on lipoprotein metabolism in middleaged and elderly subjects. J Lipid Res. 2012. 53:1958-67. doi:10.1194/jlr.P024315

1770 Oomen, CM, Feskens, EJ, Rasanen, L, Fidanza, F, Nissinen, AM, Menotti, A, Kok, FJ, Kromhout, D. Fish consumption and coronary heart disease mortality in Finland, Italy, and The Netherlands. Am J Epidemiol. 2000. 151:999-1006. doi:10.1093/oxfordjournals.aje.a010144

1771 Oppitz, IN, Cesar, JA, Neumann, NA. Overweight among children under five years of age in municipalities of the semiarid region. Rev Bras Epidemiol. 2014. 17:860-72. doi:unavailable

1772 Oranta, O, Pahkala, K, Ruottinen, S, Niinikoski, H, Lagstrom, H, Viikari, JS, Jula, A, Loo, BM, Simell, O, Ronnemaa, T, Raitakari, OT. Infancy-onset dietary counseling of low-saturated-fat diet improves insulin sensitivity in healthy adolescents 1520 years of age: the Special Turku Coronary Risk Factor Intervention Project (STRIP) study. Diabetes Care. 2013. 36:2952-9. doi:10.2337/dc13-0361

1773 Ortega, JF, Fernandez-Elias, VE, Hamouti, N, Mora-Rodriguez, R. Increased blood cholesterol after a high saturated fat diet is prevented by aerobic exercise training. Appl Physiol Nutr Metab. 2013. 38:42-8. doi:10.1139/apnm-2012-0123

1774 Ortega, RM, Rodríguez-Rodríguez, E, Aparicio, A, Marín-Arias, LI, López-Sobaler, AM. Responses to two weight-loss programs based on approximating the diet to the ideal: Differences associated with increased cereal or vegetable consumption. International Journal for Vitamin and Nutrition Research. 2006. 76:367-376. doi:10.1024/0300-9831.76.6.367

1775 Ortega-Azorin, C, Sorli, JV, Estruch, R, Asensio, EM, Coltell, O, Gonzalez, JI, Martinez-Gonzalez, MA, Ros, E, SalasSalvado, J, Fito, M, Aros, F, Lapetra, J, Serra-Majem, L, Ruiz-Gutierrez, V, Gomez-Gracia, E, Fiol, M, Flores, G, Pinto, X, Saiz, C, Ordovas, JM, Corella, D. Amino acid change in the carbohydrate response element binding protein is associated with lower triglycerides and myocardial infarction incidence depending on level of adherence to the Mediterranean diet in the PREDIMED trial. Circ Cardiovasc Genet. 2014. 7:49-58. doi:10.1161/circgenetics.113.000301

1776 Oshakbayev, K, Dukenbayeva, B, Otarbayev, N, Togizbayeva, G, Tabynbayev, N, Gazaliyeva, M, Idrisov, A, Oshakbayev $P$. Weight loss therapy for clinical management of patients with some atherosclerotic diseases: a randomized clinical trial. Nutr J. 2015. 14:120. doi:10.1186/s12937-015-0108-y

1777 Osterberg, KL, Boutagy, NE, McMillan, RP, Stevens, JR, Frisard, MI, Kavanaugh, JW, Davy, BM, Davy, KP, Hulver, MW. Probiotic supplementation attenuates increases in body mass and fat mass during high-fat diet in healthy young adults. Obesity (Silver Spring). 2015. 23:2364-70. doi:10.1002/oby.21230
Study Design;

Intervention/Exposure

Intervention/Exposure

ntervention/Exposure; Outcome

Intervention/Exposure

\section{Study Design;}

Intervention/Exposure

Intervention/Exposure

Study duration

Intervention/Exposure

Intervention/Exposure

Health Status

Study duration 
1778 Osterdahl, M, Kocturk, T, Koochek, A, Wandell, PE. Effects of a short-term intervention with a paleolithic diet in healthy volunteers. Eur J Clin Nutr. 2008. 62:682-5. doi:10.1038/sj.ejcn.1602790

1779 Otten, J, Mellberg, C, Ryberg, M, Sandberg, S, Kullberg, J, Lindahl, B, Larsson, C, Hauksson, J, Olsson, T. Strong and persistent effect on liver fat with a Paleolithic diet during a two-year intervention. Int J Obes (Lond). 2016. 40:747-53. doi:10.1038/ijo.2016.4

1780 Otten, J, Ryberg, M, Mellberg, C, Lindahl, B, Larsson, C, Juul Holst, J, Olsson, T. Weight loss by two different diets increases the postprandial response of GLP-1 but only the Paleolithic diet increases the postprandial response of GIP. Diabetologia. 2017. 60:S233-. doi:10.1007/s00125-017-4350-z

1781 Ozawa, M, Yoshida, D, Hata, J, Ohara, T, Mukai, N, Shibata, M, Uchida, K, Nagata, M, Kitazono, T, Kiyohara, Y, Ninomiya, T. Dietary Protein Intake and Stroke Risk in a General Japanese Population: The Hisayama Study. Stroke. 2017. 48:1478-1486. doi:10.1161/strokeaha.116.016059

1782 Padin, AC, Hebert, JR, Woody, A, Wilson, SJ, Shivappa, N, Belury, MA, Malarkey, WB, Sheridan, JF, Kiecolt-Glaser, JK. A proinflammatory diet is associated with inflammatory gene expression among healthy, non-obese adults: Can social ties protect against the risks?. Brain Behav Immun. 2019. 82:36-44. doi:10.1016/j.bbi.2019.07.031

1783 Padwal, R. The Atkins diet led to more weight loss than the Zone diet in overweight and obese premenopausal women at 12 months. Evidence-based medicine. 2007. 12:138-. doi:10.1136/ebm.12.5.138

1784 Pagliai, G, Dinu, M, Cesari, F, Gori, AM, Giusti, B, Marcucci, R, Casini, A, Sofi, F. Randomized controlled dietary intervention trial comparing mediterranean and vegetarian diets for cardiovascular prevention: preliminary results. European heart journal. 2016. 37:340-. doi:10.1093/eurheartj/ehw432

1785 Pagliai, G, Dinu, M, Mangino, A, Cesari, F, Giusti, B, Gori, AM, Marcucci, R, Casini, A, Sofi, F. Comparison between mediterranean and vegetarian diets for cardiovascular prevention: the cardiveg study. Nutrition, metabolism and cardiovascular diseases. 2017. 27:e30-e31. doi:10.1016/j.numecd.2016.11.084

1786 Pagliai, G, Russo, E, Baldi, S, Dinu, M, Bartolucci, G, Niccolai, E, Nannini, G, Casini, A, Amedei, A, Sofi, F. Impact of mediterranean vs vegetarian diets on gut microbiota and short chain fatty acids: the CARDIVEG study. Nutrition, metabolism and cardiovascular diseases. 2019. 29:879-. doi:10.1016/j.numecd.2019.05.032

1787 Paineau, D, Beaufils, F, Boulier, A, Cassuto, DA, Chwalow, J, Combris, P, Couet, C, Jouret, B, Lafay, L, Laville, M, Mahe, S, Ricour, C, Romon, M, Simon, C, Tauber, M, Valensi, P, Chapalain, V, Zourabichvili, O, Bornet, FR. The cumulative effect of small dietary changes may significantly improve nutritional intakes in free-living children and adults. Eur $\mathrm{J}$ Clin Nutr. 2010. 64:782-91. doi:10.1038/ejcn.2010.78

1788 Palacios, C, Torres, M, Lopez, D, Trak-Fellermeier, MA, Coccia, C, Perez, CM. Effectiveness of the Nutritional App "MyNutriCart" on Food Choices Related to Purchase and Dietary Behavior: A Pilot Randomized Controlled Trial. Nutrients. 2018. 10:. doi:10.3390/nu10121967

1789 Palacios, OM, Maki, KC, Nieman, KM, Lindner, E, Huebner, M, Sorce, J. Replacement of refined carbohydrates with a combination of egg protein and unsaturated fatty acids improves the lipoprotein lipid profile in adults with elevated triglycerides FASEB journal. 2017. 31:. doi:unavailable 
1790 Paletas, K, Athanasiadou, E, Sarigianni, M, Paschos, P, Kalogirou, A, Hassapidou, M, Tsapas, A. The protective role of the Mediterranean diet on the prevalence of metabolic syndrome in a population of Greek obese subjects. J Am Coll Nutr. 2010. 29:41-5. doi:10.1080/07315724.2010.10719815

1791 Pałkowska, E, Bartnikowska, E, Owsiak, D. The use of low-caloric diet with modified fatty acids pool in the therapy of the metabolic syndrome. Roczniki panstwowego zakladu higieny. 2012. 63:163-169. doi:unavailable

1792 Pallister, T, Jackson, MA, Martin, TC, Glastonbury, CA, Jennings, A, Beaumont, M, Mohney, RP, Small, KS, MacGregor, A, Steves, CJ, Cassidy, A, Spector, TD, Menni, C, Valdes, AM. Untangling the relationship between diet and visceral fat mass through blood metabolomics and gut microbiome profiling. Int J Obes (Lond). 2017. 41:1106-1113. doi:10.1038/ijo.2017.70

1793 Panagiotakos, D, Bountziouka, V, Zeimbekis, A, Vlachou, I, Polychronopoulos, E. Food pattern analysis and prevalence of cardiovascular disease risk factors among elderly people from Mediterranean islands. J Med Food. 2007. 10:615-21. doi:10.1089/jmf.2007.414

1794 Panagiotakos, DB, Georgousopoulou, EN, Pitsavos, C, Chrysohoou, C, Metaxa, V, Georgiopoulos, GA, Kalogeropoulou, K, Tousoulis, D, Stefanadis, C. Ten-year (2002-2012) cardiovascular disease incidence and all-cause mortality, in urban Greek population: the ATTICA Study. Int J Cardiol. 2015. 180:178-84. doi:10.1016/j.ijcard.2014.11.206

1795 Pandey, A, Clarus, S. The impact of dietary probiotics on estimated global cardiovascular risk in essential hypertensives. Canadian journal of cardiology. 2017. 33:S198-. doi:10.1016/j.cjca.2017.07.389

1796 Paniagua, JA, Gallego de la Sacristana, A, Romero, I, Vidal-Puig, A, Latre, JM, Sanchez, E, Perez-Martinez, P, LopezMiranda, J, Perez-Jimenez, F. Monounsaturated fat-rich diet prevents central body fat distribution and decreases postprandia adiponectin expression induced by a carbohydrate-rich diet in insulin-resistant subjects. Diabetes Care. 2007. 30:1717-23. doi:10.2337/dc06-2220

1797 Paniagua, JA, Perez-Martinez, P, Gjelstad, IM, Tierney, AC, Delgado-Lista, J, Defoort, C, Blaak, EE, Riserus, U, Drevon, CA, Kiec-Wilk, B, Lovegrove, JA, Roche, HM, Lopez-Miranda, J. A low-fat high-carbohydrate diet supplemented with longchain n-3 PUFA reduces the risk of the metabolic syndrome. Atherosclerosis. 2011. 218:443-50. doi:10.1016/j.atherosclerosis.2011.07.003

1798 Paniagua, JA, Romero, MI, Sanchez, ME, Valverde-Estepa, A, Ruano, J, Fuentes, F, Perez-Jimenez, F. Effect of 3 model diets on the glucose and insulin response, lipid profile and endothelial function in insulin-resistant subjects. Clinica e investigacion en arteriosclerosis. 2008. 20:55-63. doi:10.1016/S0214-9168\%2808\%2972584-1

1799 Panizza, CE, Lim, U, Yonemori, KM, Cassel, KD, Wilkens, LR, Harvie, MN, Maskarinec, G, Delp, EJ, Lampe, JW, Shepherd, JA, Le Marchand, L, Boushey, CJ. Effects of Intermittent Energy Restriction Combined with a Mediterranean Diet on Reducing Visceral Adiposity: A Randomized Active Comparator Pilot Study. Nutrients. 2019. 11:. doi:10.3390/nu11061386

1800 Panunzio, MF, Caporizzi, R, Antoniciello, A, Cela, EP, Ferguson, LR, D'Ambrosio, P. Randomized, controlled nutrition education trial promotes a Mediterranean diet and improves anthropometric, dietary, and metabolic parameters in adults. Ann Ig. 2011. 23:13-25. doi:unavailable

Language

Study Design;

Intervention/Exposure; Outcome

Study Design

Study Design;

Publication Status

Publication Status

Outcome; Study duration

Data overlap with included article

Language

Intervention/Exposure

Intervention/Exposure; Publication Date

Overlaps with Existing Review 
1801 Paoli, A, Bianco, A, Grimaldi, KA, Lodi, A, Bosco, G. Long term successful weight loss with a combination biphasic ketogenic Mediterranean diet and Mediterranean diet maintenance protocol. Nutrients. 2013. 5:5205-17. doi:10.3390/nu5125205

1802 Paoli, A, Cenci, L, Grimaldi, KA. Effect of ketogenic Mediterranean diet with phytoextracts and low carbohydrates/high-protein meals on weight, cardiovascular risk factors, body composition and diet compliance in Italian council employees. Nutr J. 2011. 10:112. doi:10.1186/1475-2891-10-112

1803 Paoli, A, Grimaldi, K, Bianco, A, Lodi, A, Cenci, L, Parmagnani, A. Medium term effects of a ketogenic diet and a Mediterranean diet on resting energy expenditure and respiratory ratio. BMC proceedings. 2012. 6:. doi:unavailable

1804 Paoli, A, Grimaldi, K, D'Agostino, D, Cenci, L, Moro, T, Bianco, A, Palma, A. Ketogenic diet does not affect strength performance in elite artistic gymnasts. J Int Soc Sports Nutr. 2012. 9:34. doi:10.1186/1550-2783-9-34

1805 Papadaki, A, Linardakis, M, Larsen, TM, van Baak, MA, Lindroos, AK, Pfeiffer, AF, Martinez, JA, Handjieva-Darlenska, T, Kunesova, M, Holst, C, Astrup, A, Saris, WH, Kafatos, A. The effect of protein and glycemic index on children's body composition: the DiOGenes randomized study. Pediatrics. 2010. 126:e1143-52. doi:10.1542/peds.2009-3633

1806 Papadaki, A, Linardakis, M, Plada, M, Larsen, T, Damsgaard, C, Van Baak, M, Jebb, S, Pfeiffer, A, Martinez, J, HandjievaDarlenska, T, etal, . Effect of dietary protein and glycemic index on metabolic syndrome status: the diogenes randomized controlled trial. Annals of nutrition \& metabolism. 2013. 63:1189-. doi:10.1159/000354245

1807 Papadaki, A, Linardakis, M, Plada, M, Larsen, TM, Damsgaard, CT, van Baak, MA, Jebb, S, Pfeiffer, AF, Martinez, JA, Handjieva-Darlenska, T, Kunesova, M, Holst, C, Saris, WH, Astrup, A, Kafatos, A. Impact of weight loss and maintenance with ad libitum diets varying in protein and glycemic index content on metabolic syndrome. Nutrition. 2014. 30:410-7. doi:10.1016/j.nut.2013.09.001

1808 Papadaki, A, Martinez-Gonzalez, MA, Alonso-Gomez, A, Rekondo, J, Salas-Salvado, J, Corella, D, Ros, E, Fito, M, Estruch, R, Lapetra, J, etal, . Mediterranean diet and risk of heart failure: results from the PREDIMED randomized controlled trial. European journal of heart failure. 2017. (no pagination):. doi:10.1002/ejhf.750

1809 Papakonstantinou, E, Zampelas, A. The effect of dietary protein intake on coronary heart disease risk. Nutrition bulletin. $2008 . \quad$ Study Design 33:287-297. doi:unavailable

1810 Papandreou, C, Hatzis, CM. Adherence to the Mediterranean Diet in Relation to Obesity Indices before and after a Weight Reduction Program in OSAS Patients. Iran J Public Health. 2014. 43:1454-5. doi:unavailable

1811 Paradis, AM, Godin, G, Perusse, L, Vohl, MC. Interaction between familial history of obesity and fat intakes on obesity phenotypes. J Nutrigenet Nutrigenomics. 2009. 2:37-42. doi:10.1159/000191281

1812 Parcina, M, Brune, M, Kaese, V, Zorn, M, Spiegel, R, Vojvoda, V, Fleming, T, Rudofsky, G, Paul Nawroth, P. No shortterm effects of calorie-controlled Mediterranean or fast food dietary interventions on established biomarkers of vascular or metabolic risk in healthy individuals. Nutr Res Pract. 2015. 9:165-73. doi:10.4162/nrp.2015.9.2.165

1813 Parillo, M, Licenziati, MR, Vacca, M, De Marco, D, lannuzzi, A. Metabolic changes after a hypocaloric, low-glycemic-index diet in obese children. J Endocrinol Invest. 2012. 35:629-33. doi:10.3275/7909

Study Design; Study duration

Study duration

Study duration

Intervention/Exposure

Intervention/Exposure; Publication Status

Intervention/Exposure

Publication Status

Study Design

Study Design

Power/Size

Intervention/Exposure 
1814 Park, H, Tserendejid, Z, Song, KH, Lee, J, Lee, Y. Dietary patterns and the association with dietary quality among Mongolian immigrants in South Korea. J Immigr Minor Health. 2015. 17:422-31. doi:10.1007/s10903-014-0047-2

Study Design;

Outcome

1815 Park, HA, Lee, JS, Kuller, LH. Relationship between premenopausal dietary intake and postmenopausal subclinical atherosclerosis. Atherosclerosis. 2006. 186:420-7. doi:10.1016/j.atherosclerosis.2005.08.002

1816 Park, JE, Miller, M, Rhyne, J, Wang, Z, Hazen, SL. Differential effect of short-term popular diets on TMAO and other cardiometabolic risk markers. Nutr Metab Cardiovasc Dis. 2019. 29:513-517. doi:10.1016/j.numecd.2019.02.003

1817 Park, SY, Kang, M, Wilkens, LR, Shvetsov, YB, Harmon, BE, Shivappa, N, Wirth, MD, Hebert, JR, Haiman, CA, Le Marchand, L, Boushey, CJ. The Dietary Inflammatory Index and All-Cause, Cardiovascular Disease, and Cancer Mortality in the Multiethnic Cohort Study. Nutrients. 2018. 10:. doi:10.3390/nu10121844

1818 Park, YM, Choi, MK, Lee, SS, Shivappa, N, Han, K, Steck, SE, Hebert, JR, Merchant, AT, Sandler, DP. Dietary inflammatory potential and risk of mortality in metabolically healthy and unhealthy phenotypes among overweight and obese adults. Clin Nutr. 2019. 38:682-688. doi:10.1016/j.clnu.2018.04.002

1819 Park, YM, Steck, SE, Fung, TT, Zhang, J, Hazlett, LJ, Han, K, Lee, SH, Kwon, HS, Merchant, AT. Mediterranean diet, Dietary Approaches to Stop Hypertension (DASH) style diet, and metabolic health in U.S. adults. Clin Nutr. 2017. 36:1301-1309. doi:10.1016/j.clnu.2016.08.018

1820 Parker, HM, O'Connor, HT, Keating, SE, Cohn, JS, Garg, ML, Caterson, ID, George, J, Johnson, NA. Efficacy of the Omega-3 Index in predicting non-alcoholic fatty liver disease in overweight and obese adults: a pilot study. Br J Nutr. 2015. 114:780-7. doi:10.1017/s0007114515002305

1821 Parr, EB, Coffey, VG, Cato, LE, Phillips, SM, Burke, LM, Hawley, JA. A randomized trial of high-dairy-protein, variablecarbohydrate diets and exercise on body composition in adults with obesity. Obesity (Silver Spring). 2016. 24:1035-45. doi:10.1002/oby.21451

1822 Partsalaki, I, Karvela, A, Spiliotis, BE. Metabolic impact of a ketogenic diet compared to a hypocaloric diet in obese children and adolescents. J Pediatr Endocrinol Metab. 2012. 25:697-704. doi:10.1515/jpem-2012-0131

1823 Pasiakos, SM, Cao, JJ, Margolis, LM, Sauter, ER, Whigham, LD, McClung, JP, Rood, JC, Carbone, JW, Combs, GF, Jr, Young, AJ. Effects of high-protein diets on fat-free mass and muscle protein synthesis following weight loss: a randomized controlled trial. Faseb j. 2013. 27:3837-47. doi:10.1096/fj.13-230227

1824 Pasiakos, SM, Lieberman, HR, Fulgoni, VL, 3rd. Higher-protein diets are associated with higher HDL cholesterol and lower BMI and waist circumference in US adults. J Nutr. 2015. 145:605-14. doi:10.3945/jn.114.205203

1825 Pastore, RL, Brooks, JT, Carbone, JW. Paleolithic nutrition improves plasma lipid concentrations of hypercholesterolemic adults to a greater extent than traditional heart-healthy dietary recommendations. Nutr Res. 2015. 35:474-9. doi:10.1016/j.nutres.2015.05.002

1826 Pastori, D, Carnevale, R, Bartimoccia, S, Nocella, C, Tanzilli, G, Cangemi, R, Vicario, T, Catena, M, Violi, F, Pignatelli, P. Does Mediterranean Diet Reduce Cardiovascular Events and Oxidative Stress in Atrial Fibrillation?. Antioxid Redox Signal. 2015. 23:682-7. doi:10.1089/ars.2015.6326 
1827 Pate, RR, Taverno Ross, SE, Liese, AD, Dowda, M. Associations among physical activity, diet quality, and weight status in US adults. Med Sci Sports Exerc. 2015. 47:743-50. doi:10.1249/mss.0000000000000456

1828 Patel, L, Alicandro, G, La Vecchia, C. Low-Calorie Beverage Consumption, Diet Quality and Cardiometabolic Risk Factors in Study Design British Adults. Nutrients. 2018. 10:. doi:10.3390/nu10091261

1829 Patino-Alonso, MC, Recio-Rodriguez, JI, Magdalena-Belio, JF, Gine-Garriga, M, Martinez-Vizcaino, V, Fernandez-Alonso, C, Study Design Arietaleanizbeaskoa, MS, Galindo-Villardon, MP, Gomez-Marcos, MA, Garcia-Ortiz, L. Clustering of lifestyle characteristics and their association with cardio-metabolic health: the Lifestyles and Endothelial Dysfunction (EVIDENT) study. Br J Nutr. 2015. 114:943-51. doi:10.1017/s0007114515002500

1830 Pavic, E, Hadziabdic, MO, Mucalo, I, Martinis, I, Romic, Z, Bozikov, V, Rahelic, D. Effect of the Mediterranean diet in combination with exercise on metabolic syndrome parameters: 1-year randomized controlled trial. Int J Vitam Nutr Res. 2019. 89:132-143. doi:10.1024/0300-9831/a000462

1831 Pavicic Zezelj, S, Kendel Jovanovic, G, Dragas Zubalj, N, Micovic, V, Sesar, Z. Associations between Adherence to the Mediterranean Diet and Lifestyle Assessed with the MEDLIFE Index among the Working Population. Int J Environ Res Public Health. 2018. 15:. doi:10.3390/ijerph15102126

1832 Pawlak, R. Vegetarian Diets in the Prevention and Management of Diabetes and Its Complications. Diabetes Spectr. 2017. 30:82-88. doi:10.2337/ds16-0057

1833 Payab, M, Kelishadi, R, Qorbani, M, Motlagh, ME, Ranjbar, SH, Ardalan, G, Zahedi, H, Chinian, M, Asayesh, H, Larijani, $\mathrm{B}$, Heshmat, R. Association of junk food consumption with high blood pressure and obesity in Iranian children and adolescents: the CASPIAN-IV Study. J Pediatr (Rio J). 2015. 91:196-205. doi:10.1016/j.jped.2014.07.006

1834 Peairs, AD, Rankin, JW, Lee, YW. Effects of acute ingestion of different fats on oxidative stress and inflammation in overweight and obese adults. Nutr J. 2011. 10:122. doi:10.1186/1475-2891-10-122

1835 Pearce, K, Hatzinikolas, A, Moran, L, de Courten, MPJ, Forbes, J, Scheijen, Jljm, Schalkwijk, CG, Walker, K, de Courten, B. Disparity in the micronutrient content of diets high or low in advanced glycation end products (AGEs) does not explain changes in insulin sensitivity. Int J Food Sci Nutr. 2017. 68:1021-1026. doi:10.1080/09637486.2017.1319468

1836 Pehleman, TL, Peters, SJ, Heigenhauser, GJ, Spriet, LL. Enzymatic regulation of glucose disposal in human skeletal muscle after a high-fat, low-carbohydrate diet. J Appl Physiol (1985). 2005. 98:100-7. doi:10.1152/japplphysiol.00686.2004

1837 Pelkman, CL, Fishell, VK, Maddox, DH, Pearson, TA, Mauger, DT, Kris-Etherton, PM. Effects of moderate-fat (from monounsaturated fat) and low-fat weight-loss diets on the serum lipid profile in overweight and obese men and women. Am $J$ Clin Nutr. 2004. 79:204-12. doi:10.1093/ajcn/79.2.204

1838 Penesova, A, Bajer, B, Vlcek, M, Imrich, R. The effect of 8 weeks of weight loss intervention on cardiometabolic parameters (ongoing study). Vnitrni lekarstvi. 2017. 63:2S54-. doi:unavailable

1839 Pengpid, S, Peltzer, K. Overweight or obesity and related lifestyle and psychosocial factors among adolescents in Brunei Darussalam. Int J Adolesc Med Health. 2018. .. doi:10.1515/ijamh-2018-0019

Weight

loss/Hypocaloric

Study Design;

Intervention/Exposure

Study Design

Study Design

Study duration

Study duration

Study duration

Intervention/Exposure

Publication Status

Study Design 
1840 Pereira, EV, Costa Jde, A, Alfenas Rde, C. Effect of glycemic index on obesity control. Arch Endocrinol Metab. 2015. 59:24551. doi:10.1590/2359-3997000000045

1841 Pereira, MA, Swain, J, Goldfine, AB, Rifai, N, Ludwig, DS. Effects of a low-glycemic load diet on resting energy expenditure and heart disease risk factors during weight loss. Jama. 2004. 292:2482-90. doi:10.1001/jama.292.20.2482

Intervention/Exposure; Study duration

1842 Perera, MJ, Chirinos, DA, Brintz, CE, Schneiderman, N, Daviglus, M, Talavera, GA, Perreira, KM, Giacinto, RAE, Qi, Q, Llabre, MM. Body Mass of U.S. Hispanics/Latinos From the Hispanic Community Health Study/Study of Latinos (HCHS/SOL): How Do Diet Quality and Sedentary Time Relate?. Hisp Health Care Int. 2019. :1540415319874809. doi:10.1177/1540415319874809

1843 Perez, LF, Miller, CK, Groner, JA. Adolescents with at-risk eating and lifestyle behaviors are affected by after school schedules across the clinical weight spectrum. Patient Educ Couns. 2017. 100:1511-1518. doi:10.1016/j.pec.2017.03.008

1844 Perez-Ferre, N, Del Valle, L, Torrejon, MJ, Barca, I, Calvo, MI, Matia, P, Rubio, MA, Calle-Pascual, AL. Diabetes mellitus and abnormal glucose tolerance development after gestational diabetes: A three-year, prospective, randomized, clinical-based Mediterranean lifestyle interventional study with parallel groups. Clin Nutr. 2015. 34:579-85. doi:10.1016/j.clnu.2014.09.005

1845 Perez-Guisado, J, Munoz-Serrano, A, Alonso-Moraga, A. Spanish Ketogenic Mediterranean Diet: a healthy cardiovascular diet for weight loss. Nutr J. 2008. 7:30. doi:10.1186/1475-2891-7-30

1846 Perez-Guisado, J, Munoz-Serrano, A. A pilot study of the Spanish Ketogenic Mediterranean Diet: an effective therapy for the metabolic syndrome. J Med Food. 2011. 14:681-7. doi:10.1089/jmf.2010.0137

1847 Perez-Guisado, J, Munoz-Serrano, A. The effect of the Spanish Ketogenic Mediterranean Diet on nonalcoholic fatty liver disease: a pilot study. J Med Food. 2011. 14:677-80. doi:10.1089/jmf.2011.0075

1848 Perez-Jimenez, F, Lopez-Miranda, J, Pinillos, MD, Gomez, P, Paz-Rojas, E, Montilla, P, Marin, C, Velasco, MJ, BlancoMolina, A, Jimenez Pereperez, JA, Ordovas, JM. A Mediterranean and a high-carbohydrate diet improve glucose metabolism in healthy young persons. Diabetologia. 2001. 44:2038-43. doi:10.1007/s001250100009

1849 Perona, JS, Covas, MI, Fito, M, Cabello-Moruno, R, Aros, F, Corella, D, Ros, E, Garcia, M, Estruch, R, Martinez-Gonzalez, MA, Ruiz-Gutierrez, V. Reduction in systemic and VLDL triacylglycerol concentration after a 3-month Mediterranean-style diet in high-cardiovascular-risk subjects. J Nutr Biochem. 2010. 21:892-8. doi:10.1016/j.jnutbio.2009.07.005

1850 Perry, ML. Nutrient distribution for type 2 diabetes: what's a dietetics professional to do?. J Am Diet Assoc. 2005. 105:581-2. doi:10.1016/j.jada.2005.02.036

1851 Persson, M, Winkvist, A, Mogren, I. Lifestyle and health status in a sample of Swedish women four years after pregnancy: a comparison of women with a history of normal pregnancy and women with a history of gestational diabetes mellitus. BMC Pregnancy Childbirth. 2015. 15:57. doi:10.1186/s12884-015-0487-2

1852 Petersen, M, Taylor, MA, Saris, WH, Verdich, C, Toubro, S, Macdonald, I, Rossner, S, Stich, V, Guy-Grand, B, Langin, D, Martinez, JA, Pedersen, O, Holst, C, Sorensen, TI, Astrup, A. Randomized, multi-center trial of two hypo-energetic diets in obese subjects: high- versus low-fat content. Int J Obes (Lond). 2006. 30:552-60. doi:10.1038/sj.ijo.0803186

Study Design

Study Design

Intervention/Exposure; Health Status

Study Design

Study Design

Study Design; Intervention/Exposure

Study duration

Study Design;

Outcome

Study Design;

Publication Status

Study Design;

Intervention/Exposure

Study duration 
1853 Pettitt, C, Liu, J, Kwasnicki, RM, Yang, GZ, Preston, T, Frost, G. A pilot study to determine whether using a lightweight, wearable micro-camera improves dietary assessment accuracy and offers information on macronutrients and eating rate. British Journal of Nutrition. 2016. 115:160-167. doi:10.1017/S0007114515004262

Outcome; Study duration

Study duration

1854 Petzke, KJ, Lemke, S, Klaus, S. Increased fat-free body mass and no adverse effects on blood lipid concentrations 4 weeks after additional meat consumption in comparison with an exclusion of meat in the diet of young healthy women. $\mathrm{J}$ Nutr Metab. 2011. 2011:210930. doi:10.1155/2011/210930

1855 Pfister, R, Michels, G, Sharp, SJ, Luben, R, Wareham, NJ, Khaw, KT. Estimated urinary sodium excretion and risk of heart failure in men and women in the EPIC-Norfolk study. Eur J Heart Fail. 2014. 16:394-402. doi:10.1002/ejhf.56

1856 Philippou, E, Bovill-Taylor, C, Rajkumar, C, Vampa, ML, Ntatsaki, E, Brynes, AE, Hickson, M, Frost, GS. Preliminary report: the effect of a 6-month dietary glycemic index manipulation in addition to healthy eating advice and weight loss on arterial compliance and 24-hour ambulatory blood pressure in men: a pilot study. Metabolism. 2009. 58:1703-8 doi:10.1016/j.metabol.2009.05.026

1857 Philippou, E, McGowan, BM, Brynes, AE, Dornhorst, A, Leeds, AR, Frost, GS. The effect of a 12-week low glycaemic index diet on heart disease risk factors and $24 \mathrm{~h}$ glycaemic response in healthy middle-aged volunteers at risk of heart disease: a pilot study. Eur J Clin Nutr. 2008. 62:145-9. doi:10.1038/sj.ejcn.1602688

1858 Phillips, SA, Jurva, JW, Syed, AQ, Syed, AQ, Kulinski, JP, Pleuss, J, Hoffmann, RG, Gutterman, DD. Benefit of low-fat over low-carbohydrate diet on endothelial health in obesity. Hypertension. 2008. 51:376-82. doi:10.1161/hypertensionaha.107.101824

1859 Pieke, B, von Eckardstein, A, Gulbahce, E, Chirazi, A, Schulte, H, Assmann, G, Wahrburg, U. Treatment of hypertriglyceridemia by two diets rich either in unsaturated fatty acids or in carbohydrates: effects on lipoprotein subclasses, lipolytic enzymes, lipid transfer proteins, insulin and leptin. Int J Obes Relat Metab Disord. 2000. 24:1286-96. doi:10.1038/sj.ijo.0801440

1860 Piernas, C, Wang, D, Du, S, Zhang, B, Wang, Z, Su, C, Popkin, BM. The double burden of under- and overnutrition and nutrient adequacy among Chinese preschool and school-aged children in 2009-2011. Eur J Clin Nutr. 2015. 69:1323-9. doi:10.1038/ejcn.2015.106

1861 Piers, LS, Walker, KZ, Stoney, RM, Soares, MJ, O'Dea, K. Substitution of saturated with monounsaturated fat in a 4-week diet affects body weight and composition of overweight and obese men. Br J Nutr. 2003. 90:717-27. doi:10.1079/bjn2003948

1862 Pierucci, P, Misciagna, G, Ventura, MT, Inguaggiato, R, Cisternino, AM, Guerra, VM, Suppressa, P, Resta, F, Sabba, C. Diet and myocardial infarction: a nested case-control study in a cohort of elderly subjects in a Mediterranean area of southern Italy. Nutr Metab Cardiovasc Dis. 2012. 22:727-33. doi:10.1016/j.numecd.2010.12.002

1863 Pignone, M. High-protein and low-glycemic diets improve weight maintenance among overweight adults. Clinical Diabetes. 2011. 29:73-74. doi:10.2337/diaclin.29.2.73

1864 Pikija, S, Trkulja, V, Malojcic, B, Mutzenbach, JS, Sellner, J. A High Burden of Ischemic Stroke in Regions of Eastern/Central Europe is Largely Due to Modifiable Risk Factors. Curr Neurovasc Res. 2015. 12:341-52. doi:unavailable
Intervention/Exposure

Intervention/Exposure

Intervention/Exposure

Study duration

Study duration

Study Design; Country

Intervention/Exposure; Comparator

Intervention/Exposure

Publication Status

Study Design; Intervention/Exposure 
1865 Pilis, K, Pilis, A, Stec, K, Pilis, W, Langfort, J, Letkiewicz, S, Michalski, C, Czuba, M, Zych, M, Chalimoniuk, M. ThreeYear Chronic Consumption of Low-Carbohydrate Diet Impairs Exercise Performance and Has a Small Unfavorable Effect on Lipid Profile in Middle-Aged Men. Nutrients. 2018. 10:. doi:10.3390/nu10121914

1866 Pilleron, S, Ajana, S, Jutand, MA, Helmer, C, Dartigues, JF, Samieri, C, Féart, C. Dietary Patterns and 12-Year Risk of Frailty: Results From the Three-City Bordeaux Study. Journal of the American Medical Directors Association. 2017. 18:169-175. doi:10.1016/j.jamda.2016.09.014

1867 Pimenta, AM, Toledo, E, Rodriguez-Diez, MC, Gea, A, Lopez-Iracheta, R, Shivappa, N, Hebert, JR, Martinez-Gonzalez, MA. Dietary indexes, food patterns and incidence of metabolic syndrome in a Mediterranean cohort: The SUN project. Clin Nutr 2015. 34:508-14. doi:10.1016/j.clnu.2014.06.002

1868 Pimpin, L, Jebb, S, Johnson, L, Wardle, J, Ambrosini, GL. Dietary protein intake is associated with body mass index and weight up to $5 \mathrm{y}$ of age in a prospective cohort of twins. Am J Clin Nutr. 2016. 103:389-97. doi:10.3945/ajcn.115.118612

1869 Pimpin, L, Jebb, SA, Johnson, L, Llewellyn, C, Ambrosini, GL. Sources and pattern of protein intake and risk of overweight or obesity in young UK twins. Br J Nutr. 2018. 120:820-829. doi:10.1017/s0007114518002052

1870 Piotrowicz, K, Palkowska, E, Bartnikowska, E, Krzesinski, P, Stanczyk, A, Biecek, P, Skrobowski, A, Gielerak, G. Selfreported health-related behaviors and dietary habits in patients with metabolic syndrome. Cardiol J. 2015. 22:413-20. doi:10.5603/CJ.a2015.0020

1871 Piovesan, CH, Macagnan, FE, Bodanese, LC, Feoli, AM. Dietary quality improvement after a short-term nutritional counseling program in individuals with metabolic syndrome. Arch Latinoam Nutr. 2014. 64:91-8. doi:unavailable

1872 Pisa, PT, Pedro, TM, Kahn, K, Tollman, SM, Pettifor, JM, Norris, SA. Nutrient patterns and their association with sociodemographic, lifestyle factors and obesity risk in rural South African adolescents. Nutrients. 2015. 7:3464-82. doi:10.3390/nu7053464

1873 Pivovarova, O, Kessler, K, Hornemann, S, Markova, M, Petzke, KJ, Kemper, M, Rudovich, N, Kramer, A, Pfeiffer, AFH. Time of fat and carbohydrate intake affects substrate oxidation and adipokine secretion in subjects with impaired glucose metabolism. Diabetologia. 2018. 61:S330-. doi:10.1007/s00125-018-4693-0

1874 Playdon, MC, Moore, SC, Derkach, A, Reedy, J, Subar, AF, Sampson, JN, Albanes, D, Gu, F, Kontto, J, Lassale, C, Liao, Outcome LM, Mannisto, S, Mondul, AM, Weinstein, SJ, Irwin, ML, Mayne, ST, Stolzenberg-Solomon, R. Identifying biomarkers of dietary patterns by using metabolomics. Am J Clin Nutr. 2017. 105:450-465. doi:10.3945/ajcn.116.144501

1875 Poirier, P, Hernandez, TL, Weil, KM, Shepard, TJ, Eckel, RH. Impact of diet-induced weight loss on the cardiac autonomic nervous system in severe obesity. Obes Res. 2003. 11:1040-7. doi:10.1038/oby.2003.143

1876 Poli, VFS, Sanches, RB, Moraes, ADS, Fidalgo, JPN, Nascimento, MA, Bresciani, P, Andrade-Silva, SG, Cipullo, MAT, Clemente, JC, Caranti, DA. The excessive caloric intake and micronutrient deficiencies related to obesity after a long-term interdisciplinary therapy. Nutrition. 2017. 38:113-119. doi:10.1016/j.nut.2017.01.012

1877 Polley, KR, Oswell, NJ, Pegg, RB, Paton, CM, Cooper, JA. A 5-day high-fat diet rich in cottonseed oil improves cholesterol profiles and triglycerides compared to olive oil in healthy men. Nutr Res. 2018. 60:43-53. doi:10.1016/j.nutres.2018.09.001

Study Design Outcome; Study duration

Power/Size

Outcome

Age at Intervention/Exposure

Age at Intervention/Exposure

Study Design;

Intervention/Exposure

Study Design;

Comparator

Study Design

Publication Status

Intervention/Exposure

Study Design;

Intervention/Exposure

Study duration 
1878 Pontes Torrado, Y, Garcia-Villaraco Velasco, A, Hernandez Galiot, A, Goni Cambrodon, I. A strategy for weight loss based on healthy dietary habits and control of emotional response to food. Nutr Hosp. 2015. 31:2392-9. doi:10.3305/nh.2015.31.6.8736

1879 Ponzo, V, Goitre, I, Fadda, M, Gambino, R, De Francesco, A, Soldati, L, Gentile, L, Magistroni, P, Cassader, M, Bo, S Dietary flavonoid intake and cardiovascular risk: a population-based cohort study. J Transl Med. 2015. 13:218. doi:10.1186/s12967-015-0573-2

1880 Poppitt, SD, Keogh, GF, Mulvey, TB, Phillips, A, McArdle, BH, MacGibbon, AK, Cooper, GJ. Effect of moderate changes in dietary fatty acid profile on postprandial lipaemia, haemostatic and related CVD risk factors in healthy men. Eur $\mathrm{J}$ Clin Nutr. 2004. 58:819-27. doi:10.1038/sj.ejcn.1601882

1881 Poppitt, SD, Keogh, GF, Prentice, AM, Williams, DE, Sonnemans, HM, Valk, EE, Robinson, E, Wareham, NJ. Long-term effects of ad libitum low-fat, high-carbohydrate diets on body weight and serum lipids in overweight subjects with metabolic syndrome. Am J Clin Nutr. 2002. 75:11-20. doi:10.1093/ajcn/75.1.11

1882 Pou, SA, Del Pilar Diaz, M, De La Quintana, AG, Forte, CA, Aballay, LR. Identification of dietary patterns in urban population of Argentina: study on diet-obesity relation in population-based prevalence study. Nutr Res Pract. 2016. 10:616-622. doi:10.4162/nrp.2016.10.6.616

1883 Pounis, GD, Tyrovolas, S, Antonopoulou, M, Zeimbekis, A, Anastasiou, F, Bountztiouka, V, Metallinos, G, Gotsis, E, Lioliou, E, Polychronopoulos, E, Lionis, C, Panagiotakos, DB. Long-term animal-protein consumption is associated with an increased prevalence of diabetes among the elderly: the Mediterranean Islands (MEDIS) study. Diabetes Metab. 2010. 36:484-90. doi:10.1016/j.diabet.2010.06.007

1884 Praagman, J, Beulens, JW, Alssema, M, Zock, PL, Wanders, AJ, Sluijs, I, van der Schouw, YT. The association between dietary saturated fatty acids and ischemic heart disease depends on the type and source of fatty acid in the European Prospective Investigation into Cancer and Nutrition-Netherlands cohort. Am J Clin Nutr. 2016. 103:356-65. doi:10.3945/ajcn.115.122671

1885 Praagman, J, Franco, OH, Ikram, MA, Soedamah-Muthu, SS, Engberink, MF, van Rooij, FJ, Hofman, A, Geleijnse, JM. Dairy products and the risk of stroke and coronary heart disease: the Rotterdam Study. Eur J Nutr. 2015. 54:981-90. doi:10.1007/s00394-014-0774-0

1886 Prentice, RL, Aragaki, AK, Howard, BV, Chlebowski, RT, Thomson, CA, Van Horn, L, Tinker, LF, Manson, JE, Anderson, GL, Kuller, LE, Neuhouser, ML, Johnson, KC, Snetselaar, L, Rossouw, JE. Low-Fat Dietary Pattern among Postmenopausal Women Influences Long-Term Cancer, Cardiovascular Disease, and Diabetes Outcomes. J Nutr. 2019. 149:1565-1574. doi:10.1093/jn/nxz107

1887 Prentice, RL, Aragaki, AK, Van Horn, L, Thomson, CA, Beresford, SA, Robinson, J, Snetselaar, L, Anderson, GL, Manson, JE, Allison, MA, Rossouw, JE, Howard, BV. Low-fat dietary pattern and cardiovascular disease: results from the Women's Health Initiative randomized controlled trial. Am J Clin Nutr. 2017. 106:35-43. doi:10.3945/ajcn.117.153270

\section{Study Design;}

Comparator

Intervention/Exposure Intervention/Exposure

Intervention/Exposure

Intervention/Exposure; Comparator 
1888 Prinelli, F, Adorni, F, Leite, MLC, Pettenati, C, Russo, A, Di Santo, S, Musicco, M. Different Exposures to Risk Factors Do Not Explain the Inverse Relationship of Occurrence Between Cancer and Neurodegenerative Diseases: An Italian Nested Casecontrol Study. Alzheimer Dis Assoc Disord. 2018. 32:76-82. doi:10.1097/wad.0000000000000204

1889 Prinelli, F, Yannakoulia, M, Anastasiou, CA, Adorni, F, Di Santo, SG, Musicco, M, Scarmeas, N, Correa Leite, ML. Mediterranean diet and other lifestyle factors in relation to 20-year all-cause mortality: A cohort study in an Italian population. British Journal of Nutrition. 2015. 113:1003-1011. doi:10.1017/S0007114515000318

1890 Properzi, C, O'Sullivan, TA, Sherriff, JL, Ching, HL, Jeffrey, GP, Buckley, RF, Tibballs, J, MacQuillan, GC, Garas, G, Adams, LA. Ad Libitum Mediterranean and Low-Fat Diets Both Significantly Reduce Hepatic Steatosis: A Randomized Controlled Trial. Hepatology. 2018. 68:1741-1754. doi:10.1002/hep.30076

1891 Psaltopoulou, T, Naska, A, Orfanos, P, Trichopoulos, D, Mountokalakis, T, Trichopoulou, A. Olive oil, the Mediterranean diet, and arterial blood pressure: the Greek European Prospective Investigation into Cancer and Nutrition (EPIC) study. Am J Clin Nutr. 2004. 80:1012-8. doi:10.1093/ajcn/80.4.1012

1892 Ptomey, LT, Saunders, RR, Saunders, M, Washburn, RA, Mayo, MS, Sullivan, DK, Gibson, CA, Goetz, JR, Honas, JJ, Willis, EA, Danon, JC, Krebill, R, Donnelly, JE. Weight management in adults with intellectual and developmental disabilities: A randomized controlled trial of two dietary approaches. J Appl Res Intellect Disabil. 2018. 31 Suppl 1:82-96. doi:10.1111/jar.12348

1893 Ptomey, LT, Steger, FL, Lee, J, Sullivan, DK, Goetz, JR, Honas, JJ, Washburn, RA, Gibson, CA, Donnelly, JE. Changes in Energy Intake and Diet Quality during an 18-Month Weight-Management Randomized Controlled Trial in Adults with Intellectual and Developmental Disabilities. J Acad Nutr Diet. 2018. 118:1087-1096. doi:10.1016/j.jand.2017.11.003

1894 Ptomey, LT, Willis, EA, Goetz, JR, Lee, J, Szabo-Reed, AN, Sullivan, DK, Donnelly, JE. Portion-controlled meals provide increases in diet quality during weight loss and maintenance. J Hum Nutr Diet. 2016. 29:209-16. doi:10.1111/jhn.12296

1895 Puga, GM, Meyer, C, Everman, S, Mandarino, LJ, Katsanos, CS. Postprandial lipemia in the elderly involves increased incorporation of ingested fat in plasma free fatty acids and small (Sf 20-400) triglyceride-rich lipoproteins. Am $\mathrm{J}$ Physiol Endocrinol Metab. 2011. 301:E356-61. doi:10.1152/ajpendo.00670.2010

1896 Puga, GM, Meyer, C, Mandarino, LJ, Katsanos, CS. Postprandial spillover of dietary lipid into plasma is increased with moderate amounts of ingested fat and is inversely related to adiposity in healthy older men. J Nutr. 2012. 142:1806-11. doi:10.3945/jn.112.162008

1897 Purcell, K, Sumithran, P, Prendergast, LA, Bouniu, CJ, Delbridge, E, Proietto, J. The effect of rate of weight loss on longterm weight management: a randomised controlled trial. Lancet Diabetes Endocrinol. 2014. 2:954-62. doi:10.1016/s2213$8587(14) 70200-1$

1898 Pysz, M, Leszczynska, T, Cieslik, E, Kopec, A, Wielgos, B, Piatkowska, E. Relationship between the intake of energy and basic nutrients and the BMI values in group of children aged 10-12. Rocz Panstw Zakl Hig. 2014. 65:345-52. doi:unavailable 
1899 Qi, Q, Downer, MK, Kilpelainen, TO, Taal, HR, Barton, SJ, Ntalla, I, Standl, M, Boraska, V, Huikari, V, Kiefte-de Jong, JC, Korner, A, Lakka, TA, Liu, G, Magnusson, J, Okuda, M, Raitakari, O, Richmond, R, Scott, RA, Bailey, ME, Scheuermann, K, Holloway, JW, Inskip, H, Isasi, CR, Mossavar-Rahmani, Y, Jaddoe, VW, Laitinen, J, Lindi, V, Melen, E, Pitsiladis, Y, Pitkanen, N, Snieder, H, Heinrich, J, Timpson, NJ, Wang, T, Yuji, H, Zeggini, E, Dedoussis, GV, Kaplan, RC, Wylie-Rosett, J, Loos, RJ, Hu, FB, Qi, L. Dietary Intake, FTO Genetic Variants, and Adiposity: A Combined Analysis of Over 16,000 Children and Adolescents. Diabetes. 2015. 64:2467-76. doi:10.2337/db14-1629

1900 Qi, Q, Durst, R, Schwarzfuchs, D, Leitersdorf, E, Shpitzen, S, Li, Y, Wu, H, Champagne, CM, Hu, FB, Stampfer, MJ, Bray, GA, Sacks, FM, Shai, I, Qi, L. CETP genotype and changes in lipid levels in response to weight-loss diet intervention in the POUNDS LOST and DIRECT randomized trials. J Lipid Res. 2015. 56:713-21. doi:10.1194/jlr.P055715

1901 Qi, Q, Zheng, Y, Huang, T, Rood, J, Bray, GA, Sacks, FM, Qi, L. Vitamin D metabolism-related genetic variants, dietary protein intake and improvement of insulin resistance in a 2 year weight-loss trial: POUNDS Lost. Diabetologia. 2015. 58:2791-9. doi:10.1007/s00125-015-3750-1

1902 Qin, Y, Melse-Boonstra, A, Pan, X, Zhao, J, Yuan, B, Dai, Y, Zhou, M, Geleijnse, JM, Kok, FJ, Shi, Z. Association of dietary pattern and body weight with blood pressure in Jiangsu Province, China. BMC Public Health. 2014. 14:948. doi:10.1186/1471-2458-14-948

1903 Quatromoni, PA, Copenhafer, DL, D'Agostino, RB, Millen, BE. Dietary patterns predict the development of overweight in women: The Framingham Nutrition Studies. J Am Diet Assoc. 2002. 102:1239-46. doi:10.1016/s0002-8223(02)90275-0

1904 Quatromoni, PA, Copenhafer, DL, Demissie, S, D'Agostino, RB, O'Horo, CE, Nam, BH, Millen, BE. The internal validity of a dietary pattern analysis. The Framingham Nutrition Studies. J Epidemiol Community Health. 2002. 56:381-8. doi:10.1136/jech.56.5.381

Study Design; Age a Intervention/Exposure Comparator

Study Design;

Intervention/Exposure

Country

Power/Size

Study Design;

Publication Date

Overlaps with Existing Review

Outcome

1905 Quinteiros Fidalgo, AS, Vollenweider, P, Marques-Vidal, P. No association between dietary markers and incident hypertension in a population-based sample. Clin Nutr ESPEN. 2018. 28:208-213. doi:10.1016/j.clnesp.2018.07.013

1906 Raad, N, Rehder, D, Sherma, N, Beezhold, B, Johnston, C, Borges, C, Sweazea, K. Replacing dietary meat with fish increases plasma glucose without affecting protein glycation. FASEB journal. 2015. 29:. doi:unavailable

1907 Raatz, SK, Young, LR, Picklo, MJ, Sr, Sauter, ER, Qin, W, Kurzer, MS. Total dietary fat and fatty acid content modifies plasma phospholipid fatty acids, desaturase activity indices, and urinary prostaglandin E in women. Nutr Res. 2012. 32:1-7. doi:10.1016/j.nutres.2011.12.006

1908 Rabani, Z, Feizi, A, Najafi, M, Askari, G. Comparison of dietary intake and anthropometric indicators of autistic and nonAutistic children. Annals of Tropical Medicine and Public Health. 2018. :SP30. doi:unavailable

1909 Raben, A, Astrup, A, Vasilaras, TH, Prentice, AM, Zunft, HJ, Formiguera, X, Verboeket-van de Venne, WP, Poppitt, SD Seppelt, B, Johnston, S, etal, . The CARMEN trial: increased intake of carbohydrates--simple or complex--and unchanged blood lipids in overweight subjects. Ugeskrift for laeger. 2002. 164:627-631. doi:unavailable

1910 Raben, A, Holst, JJ, Madsen, J, Astrup, A. Diurnal metabolic profiles after $14 \mathrm{~d}$ of an ad libitum high-starch, high-sucrose, or high-fat diet in normal-weight never-obese and postobese women. Am J Clin Nutr. 2001. 73:177-89. doi:10.1093/ajcn/73.2.177
Publication Status

Intervention/Exposure; Outcome

Study Design; Outcome

Language

Study duration 
1911 Racette, SB, Deusinger, SS, Strube, MJ, Highstein, GR, Deusinger, RH. Weight changes, exercise, and dietary patterns during freshman and sophomore years of college. J Am Coll Health. 2005. 53:245-51. doi:10.3200/jach.53.6.245-251

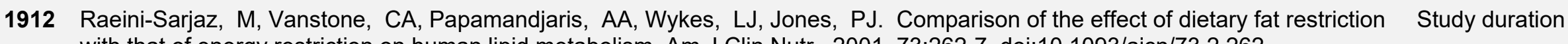
with that of energy restriction on human lipid metabolism. Am J Clin Nutr. 2001. 73:262-7. doi:10.1093/ajcn/73.2.262

1913 Rahi, B, Ajana, S, Tabue-Teguo, M, Dartigues, JF, Peres, K, Feart, C. High adherence to a Mediterranean diet and lower risk of frailty among French older adults community-dwellers: Results from the Three-City-Bordeaux Study. Clinical Nutrition. 2018. 37:1293-1298. doi:10.1016/j.clnu.2017.05.020

1914 Rajaie, S, Azadbakht, L, Khazaei, M, Esmaillzadeh, A. Effects of a moderately-restricted carbohydrate diet on cardiovascula risk factors among women with metabolic syndrome. Journal of isfahan medical school. 2012. 29:. doi:unavailable

1915 Rajaie, S, Azadbakht, L, Khazaei, M, Sherbafchi, M, Esmaillzadeh, A. Moderate replacement of carbohydrates by dietary fats affects features of metabolic syndrome: a randomized crossover clinical trial. Nutrition. 2014. 30:61-8. doi:10.1016/j.nut.2013.06.011

1916 Rajaie, S, Azadbakht, L, Saneei, P, Khazaei, M, Esmaillzadeh, A. The effect of moderate substitution of dietary carbohydrates by fats on serum levels of adipocytokines, inflammatory indices, and biomarkers of endothelial function amongwomen with metabolic syndrome. Journal of zanjan university of medical sciences and health services. 2012 . 20: doi:unavailable

1917 Rajaobelina, K, Dow, C, Romana Mancini, F, Dartois, L, Boutron-Ruault, MC, Balkau, B, Bonnet, F, Fagherazzi, G. Population attributable fractions of the main type 2 diabetes mellitus risk factors in women: Findings from the French E3N cohort. J Diabetes. 2019. 11:242-253. doi:10.1111/1753-0407.12839

1918 Rallidis, LS, Lekakis, J, Kolomvotsou, A, Zampelas, A, Vamvakou, G, Efstathiou, S, Dimitriadis, G, Raptis, SA, Kremastinos, DT. Close adherence to a Mediterranean diet improves endothelial function in subjects with abdominal obesity. Am J Clin Nutr. 2009. 90:263-8. doi:10.3945/ajcn.2008.27290

1919 Ralston, PA, Lemacks, JL, Wickrama, KK, Young-Clark, I, Coccia, C, Ilich, JZ, Harris, CM, Hart, CB, Battle, AM, O'Neal, CW. Reducing cardiovascular disease risk in mid-life and older African Americans: a church-based longitudinal intervention project at baseline. Contemp Clin Trials. 2014. 38:69-81. doi:10.1016/j.cct.2014.03.003

1920 Ramallal, R, Toledo, E, Martinez-Gonzalez, MA, Hernandez-Hernandez, A, Garcia-Arellano, A, Shivappa, N, Hebert, JR, Ruiz-Canela, M. Dietary Inflammatory Index and Incidence of Cardiovascular Disease in the SUN Cohort. PLoS One. 2015. 10:e0135221. doi:10.1371/journal.pone.0135221

1921 Ramon-Krauel, M, Salsberg, SL, Ebbeling, CB, Voss, SD, Mulkern, RV, Apura, MM, Cooke, EA, Sarao, K, Jonas, MM, Ludwig, DS. A low-glycemic-load versus low-fat diet in the treatment of fatty liver in obese children. Child Obes. 2013. 9:25260. doi:10.1089/chi.2013.0022

1922 Ramos-Lopez, O, Riezu-Boj, JI, Milagro, FI, Cuervo, M, Goni, L, Alfredo Martinez, J. Models integrating genetic and lifestyle interactions on two adiposity phenotypes for personalized prescription of energy-restricted diets with different macronutrient distribution. Frontiers in Genetics. 2019. 10:. doi:10.3389/fgene.2019.00686 
1923 Ramos-Lopez, O, Riezu-Boj, JI, Milagro, FI, Goni, L, Cuervo, M, Martinez, JA. Association of the Gly482Ser PPARGC1A gene variant with different cholesterol outcomes in response to two energy-restricted diets in subjects with excessive weight. Nutrition. 2018. 47:83-89. doi:10.1016/j.nut.2017.10.008

1924 Rampelli, S, Guenther, K, Turroni, S, Wolters, M, Veidebaum, T, Kourides, Y, Molnar, D, Lissner, L, Benitez-Paez, A, Sanz, Y, Fraterman, A, Michels, N, Brigidi, P, Candela, M, Ahrens, W. Pre-obese children's dysbiotic gut microbiome and unhealthy diets may predict the development of obesity. Commun Biol. 2018. 1:222. doi:10.1038/s42003-018-0221-5

1925 Ramprasath, VR, Jenkins, DJ, Lamarche, B, Kendall, CW, Faulkner, D, Cermakova, L, Couture, P, Ireland, C, Abdulnour, S, Patel, D, Bashyam, B, Srichaikul, K, de Souza, RJ, Vidgen, E, Josse, RG, Leiter, LA, Connelly, PW, Frohlich, J, Jones PJ. Consumption of a dietary portfolio of cholesterol lowering foods improves blood lipids without affecting concentrations of fat soluble compounds. Nutr J. 2014. 13:101. doi:10.1186/1475-2891-13-101

1926 Ramprasath, VR, Jones, PJH, Buckley, DD, Woollett, LA, Heubi, JE. Decreased plasma cholesterol concentrations after pufa-rich diets are not due to reduced cholesterol absorption/synthesis. Lipids. 2012. 47:1063-1071. doi:10.1007/s11745-0123708-8

1927 Ramsden, CE, Zamora, D, Leelarthaepin, B, Majchrzak-Hong, SF, Faurot, KR, Suchindran, CM, Ringel, A, Davis, JM, Hibbeln, JR. Use of dietary linoleic acid for secondary prevention of coronary heart disease and death: evaluation of recovered data from the Sydney Diet Heart Study and updated meta-analysis. BMJ (clinical research ed.). 2013. 346:e8707. doi:10.1136/bmj.e8707

1928 Ramya Bai, RM, Anjana, RM, Unnikrishnan, R, Mohan, V. The dietary advance glycation end products (dAGE) of high carbohydrate Indian diets and its effect on inflammatory markers in overweight adults. International journal of diabetes in developing countries. 2018. 38:S122-. doi:10.1007/s13410-018-0702-6

1929 Randolph, JM, Edirisinghe, I, Masoni, AM, Kappagoda, T, Burton-Freeman, B. Potatoes, glycemic index, and weight loss in free-living individuals: practical implications. J Am Coll Nutr. 2014. 33:375-84. doi:10.1080/07315724.2013.875441

1930 Rangan, AM, Randall, D, Hector, DJ, Gill, TP, Webb, KL. Consumption of 'extra' foods by Australian children: types, quantities and contribution to energy and nutrient intakes. Eur J Clin Nutr. 2008. 62:356-64. doi:10.1038/sj.ejcn.1602720

1931 Rangel-Zuniga, OA, Camargo, A, Marin, C, Pena-Orihuela, P, Perez-Martinez, P, Delgado-Lista, J, Gonzalez-Guardia, L, Yubero-Serrano, EM, Tinahones, FJ, Malagon, MM, Perez-Jimenez, F, Roche, HM, Lopez-Miranda, J. Proteome from patients with metabolic syndrome is regulated by quantity and quality of dietary lipids. BMC Genomics. 2015. 16:509. doi:10.1186/s12864-015-1725-8

1932 Ranjit, N, Wilkinson, AV, Lytle, LM, Evans, AE, Saxton, D, Hoelscher, DM. Socioeconomic inequalities in children's diet: the role of the home food environment. Int J Behav Nutr Phys Act. 2015. 12 Suppl 1:S4. doi:10.1186/1479-5868-12-s1-s4

1933 Rankin, JW, Turpyn, AD. Low carbohydrate, high fat diet increases C-reactive protein during weight loss. J Am Coll Nutr. 2007. 26:163-9. doi:10.1080/07315724.2007.10719598

1934 Rantala, M, Silaste, ML, Tuominen, A, Kaikkonen, J, Salonen, JT, Alfthan, G, Aro, A, Kesaniemi, YA. Dietary modifications and gene polymorphisms alter serum paraoxonase activity in healthy women. J Nutr. 2002. 132:3012-7. doi:10.1093/jn/131.10.3012

Study Design;

Publication Status

Intervention/Exposure

Study Design;

Intervention/Exposure

Intervention/Exposure; Outcome

Study Design

Outcome; Study duration Intervention/Exposure 
1935 Ranucci, C, Pippi, R, Buratta, L, Aiello, C, Gianfredi, V, Piana, N, Reginato, E, Tirimagni, A, Chiodini, E, Sbroma Tomaro,

Intervention/ E, Gili, A, De Feo, P, Fanelli, C, Mazzeschi, C. Effects of an Intensive Lifestyle Intervention to Treat Overweight/Obese Outcome Children and Adolescents. Biomed Res Int. 2017. 2017:8573725. doi:10.1155/2017/8573725

1936 Rašeta, N, Simović, S, Đurić, S, Suzić, N, Prtina, A, Zeljković, N. Eating habits and standard body parameters among students at university of banja luka. Serbian Journal of Experimental and Clinical Research. 2018. 19:41-49. doi:10.1515/SJECR-2017-0014

1937 Ratliff, J, Mutungi, G, Puglisi, MJ, Volek, JS, Fernandez, ML. Carbohydrate restriction (with or without additional dietary cholesterol provided by eggs) reduces insulin resistance and plasma leptin without modifying appetite hormones in adult men. Nutr Res. 2009. 29:262-8. doi:10.1016/j.nutres.2009.03.007

1938 Ratliff, JC, Mutungi, G, Puglisi, MJ, Volek, JS, Fernandez, ML. Eggs modulate the inflammatory response to carbohydrate restricted diets in overweight men. Nutr Metab (Lond). 2008. 5:6. doi:10.1186/1743-7075-5-6

1939 Rautiainen, S, Levitan, EB, Mittleman, MA, Wolk, A. Fruit and vegetable intake and rate of heart failure: a population-based prospective cohort of women. Eur J Heart Fail. 2015. 17:20-6. doi:10.1002/ejhf.191

1940 Rautiainen, S, Wang, L, Lee, IM, Manson, JE, Buring, JE, Sesso, HD. Higher Intake of Fruit, but Not Vegetables or Fiber, at Baseline Is Associated with Lower Risk of Becoming Overweight or Obese in Middle-Aged and Older Women of Normal BMI at Baseline. J Nutr. 2015. 145:960-8. doi:10.3945/jn.114.199158

1941 Rautio, N, Jokelainen, J, Polonen, A, Oksa, H, Peltonen, M, Vanhala, M, Puolijoki, H, Moilanen, L, Tuomilehto, J, Uusitupa, M, Keinanen-Kiukaanniemi, S, Saaristo, T. Changes in lifestyle modestly reduce the estimated cardiovascular disease risk in one-year follow-up of the Finnish diabetes prevention program (FIN-D2D). Eur J Cardiovasc Nurs. 2015. 14:145-52. doi:10.1177/1474515114521713

1942 Raynor, H, Looney, S, Steeves, EA, Spence, M, Gorin, A. The effect of a dietary energy density prescription on dietary quality, restriction, and weight loss. Obesity. 2011. 19:S113. doi:10.1038/oby.2011.226

1943 Raynor, HA, Anderson, AM, Miller, GD, Reeves, R, Delahanty, LM, Vitolins, MZ, Harper, P, Mobley, C, Konersman, K, Mayer-Davis, E. Partial Meal Replacement Plan and Quality of the Diet at 1 Year: Action for Health in Diabetes (Look AHEAD) Trial. J Acad Nutr Diet. 2015. 115:731-42. doi:10.1016/j.jand.2014.11.003

1944 Raynor, HA, Looney, SM, Steeves, EA, Spence, M, Gorin, AA. The effects of an energy density prescription on diet quality and weight loss: a pilot randomized controlled trial. J Acad Nutr Diet. 2012. 112:1397-1402. doi:10.1016/j.jand.2012.02.020

1945 Raynor, HA, Osterholt, KM, Hart, CN, Jelalian, E, Vivier, P, Wing, RR. Efficacy of U.S. paediatric obesity primary care guidelines: two randomized trials. Pediatr Obes. 2012. 7:28-38. doi:10.1111/j.2047-6310.2011.00005.X

1946 Razquin, C, Alfredo Martinez, J, Martinez-Gonzalez, MA, Corella, D, Santos, JM, Marti, A. The Mediterranean diet protects against waist circumference enlargement in 12Ala carriers for the PPARgamma gene: 2 years' follow-up of 774 subjects at high cardiovascular risk. Br J Nutr. 2009. 102:672-9. doi:10.1017/s0007114509289008

Intervention/Exposure; Comparator

Intervention/Exposure

Intervention/Exposure

Intervention/Exposure

Intervention/Exposure Publication Status

Intervention/Exposure; Outcome

Intervention/Exposure Intervention/Exposure

Intervention/Exposure 
1947 Razquin, C, Martinez, JA, Martinez-Gonzalez, MA, Bes-Rastrollo, M, Fernandez-Crehuet, J, Marti, A. A 3-year intervention with a Mediterranean diet modified the association between the rs9939609 gene variant in FTO and body weight changes. Int $J$ Obes (Lond). 2010. 34:266-72. doi:10.1038/ijo.2009.233

1948 Razquin, C, Martinez, JA, Martinez-Gonzalez, MA, Fernandez-Crehuet, J, Santos, JM, Marti, A. A Mediterranean diet rich in virgin olive oil may reverse the effects of the $-174 \mathrm{G} / \mathrm{C}$ IL6 gene variant on 3-year body weight change. Mol Nutr Food Res. 2010. 54 Suppl 1:S75-82. doi:10.1002/mnfr.200900257

1949 Razquin, C, Martinez, JA, Martinez-Gonzalez, MA, Fernández-Crehuet, J, Santos, JM, Marti, A. A mediterranean diet rich in virgin olive oil may reverse the effects of the-174g/c il6 gene variant on 3-year body weight change. Molecular Nutrition and Food Research. 2010. 54:S75-S82. doi:10.1002/mnfr.200900257

1950 Razquin, C, Martinez, JA, Martinez-Gonzalez, MA, Salas-Salvado, J, Estruch, R, Marti, A. A 3-year Mediterranean-style dietary intervention may modulate the association between adiponectin gene variants and body weight change. Eur $\mathrm{J}$ Nutr. 2010. 49:311-9. doi:10.1007/s00394-009-0090-2

1951 Razquin, C, Sanchez-Tainta, A, Salas-Salvado, J, Buil-Cosiales, P, Corella, D, Fito, M, Ros, E, Estruch, R, Aros, F, Gomez-Gracia, E, Fiol, M, Lapetra, J, Serra-Majem, L, Pinto, X, Schroder, H, Tur, J, Sorli, JV, Lamuela-Raventos, RM, Bullo, M, Bes-Rastrollo, M, Martinez-Gonzalez, MA. Dietary energy density and body weight changes after 3 years in the PREDIMED study. Int J Food Sci Nutr. 2017. 68:865-872. doi:10.1080/09637486.2017.1295028

1952 Rebollo-Ramos, M, Velazquez-Diaz, D, Corral-Perez, J, Barany-Ruiz, A, Perez-Bey, A, Fernandez-Ponce, C, Garcia-Cozar FJ, Ponce-Gonzalez, JG, Cuenca-Garcia, M. Aerobic fitness, Mediterranean diet and cardiometabolic risk factors in adults. Endocrinol Diabetes Nutr. 2019. :. doi:10.1016/j.endinu.2019.04.004

1953 Receveur, O, Morou, K, Gray-Donald, K, Macaulay, AC. Consumption of key food items is associated with excess weight among elementary-school-aged children in a Canadian first nations community. J Am Diet Assoc. 2008. 108:362-6. doi:10.1016/j.jada.2007.09.002

1954 Recio-Rodriguez, JI, Garcia-Yu, IA, Alonso-Dominguez, R, Maderuelo-Fernandez, JA, Patino-Alonso, MC, Agudo-Conde, C, Study Design Sanchez-Aguadero, N, Ramos, R, Marti, R, Rodriguez-Sanchez, E, Gomez-Marcos, MA, Garcia-Ortiz, L. Diet quality and carotid atherosclerosis in intermediate cardiovascular risk individuals. Nutr J. 2017. 16:40. doi:10.1186/s12937-017-0266-1

1955 Reeds, J, Mansuri, S, Mamakeesick, M, Harris, SB, Zinman, B, Gittelsohn, J, Wolever, TM, Connelly, PW, Hanley, A. Dietary Patterns and Type 2 Diabetes Mellitus in a First Nations Community. Can J Diabetes. 2016. 40:304-10. doi:10.1016/j.jcjd.2016.05.001

1956 Reichard, A, Saunders, MD, Saunders, RR, Donnelly, JE, Lauer, E, Sullivan, DK, Ptomey, L. A comparison of two weight management programs for adults with mobility impairments. Disabil Health J. 2015. 8:61-9. doi:10.1016/j.dhjo.2014.08.002

Intervention/Exposure Study Design; Intervention/Exposure

Intervention/Exposure Intervention/Exposure

Language

Study Design;

Intervention/Exposure

Reig Garcia-Galbis, M, Rizo Baeza, M, Cortes Castell, E. INDICATORS OF SUCCESS IN THE DIETARY MANAGEMENT OF OVERWEIGHT AND OBESITY: WEIGHT, BODY FAT LOSS AND QUALITY. Nutr Hosp. 2015. 32:1009-16. doi: $10.3305 /$ nh.2015.32.3.9248 
1958 Reinehr, T, Schaefer, A, Winkel, K, Finne, E, Toschke, AM, Kolip, P. An effective lifestyle intervention in overweight children: Findings from a randomized controlled trial on " Obeldicks light". Clinical Nutrition. 2010. 29:331-336. doi:10.1016/j.clnu.2009.12.010

1959 Renault, KM, Carlsen, EM, Norgaard, K, Nilas, L, Pryds, O, Secher, NJ, Cortes, D, Jensen, JE, Olsen, SF, Halldorsson, TI. Intake of carbohydrates during pregnancy in obese women is associated with fat mass in the newborn offspring. Am $\mathrm{J}$ Clin Nutr. 2015. 102:1475-81. doi:10.3945/ajcn.115.110551

1960 Resch, KL. Dietary Intervention Randomized Controlled Trial (DIRECT) group: weight loss with a low-carbohydrate, Mediterranean, or low-fat diet. Forschende komplementarmedizin (2006). 2008. 15:351-352. doi:unavailable

1961 Reseland, JE, Anderssen, SA, Solvoll, K, Hjermann, I, Urdal, P, Holme, I, Drevon, CA. Effect of long-term changes in diet and exercise on plasma leptin concentrations. Am J Clin Nutr. 2001. 73:240-5. doi:10.1093/ajcn/73.2.240

1962 Retterstol, K, Svendsen, M, Narverud, I, Holven, KB. Effect of low carbohydrate high fat diet on LDL cholesterol and gene expression in normal-weight, young adults: A randomized controlled study. Atherosclerosis. 2018. 279:52-61. doi:10.1016/j.atherosclerosis.2018.10.013

1963 Reyes-Castillo, P, Gonzalez-Vazquez, R, Gutierrez-Nava, A, Mendoza-Perez, F, Navarro Gonzalez, MDC, Salgado-Sguayo, A, Torres-Maravilla, E, Mayorga Reyes, L, Azaola-Espinosa, A. Anthropometric measure and adipokine levels of a young undergraduate population with a usual diet. Nutr Hosp. 2019. 36:80-86. doi:10.20960/nh.1894

1964 Rhyu, HS, Cho, SY, Roh, HT. The effects of ketogenic diet on oxidative stress and antioxidative capacity markers of Taekwondo athletes. J Exerc Rehabil. 2014. 10:362-6. doi:10.12965/jer.140178

1965 Rhyu, HS, Cho, SY. The effect of weight loss by ketogenic diet on the body composition, performance-related physical fitness factors and cytokines of Taekwondo athletes. J Exerc Rehabil. 2014. 10:326-31. doi:10.12965/jer.140160

1966 Ricci, C, Baumgartner, J, Zec, M, Kruger, HS, Smuts, CM. Type of dietary fat intakes in relation to all-cause and causespecific mortality in US adults: an iso-energetic substitution analysis from the American National Health and Nutrition Examination Survey linked to the US mortality registry. Br J Nutr. 2018. 119:456-463. doi:10.1017/s0007114517003889

1967 Ricci, G, Canducci, E, Guida, A, Frascari, A, Rossi, A, Bersani, G, Ravani, B, Alvisi, V. The gender-related differences of nutrient intakes in a group of Italian obese patients display the ongoing transition from Mediterranean to western dietary patterns. Obes Surg. 2014. 24:965-7. doi:10.1007/s11695-014-1238-6

1968 Richard, C, Couture, P, Desroches, S, Lichtenstein, AH, Lamarche, B. Effect of an isoenergetic traditional Mediterranean diet on apolipoprotein A-I kinetic in men with metabolic syndrome. Nutr J. 2013. 12:76. doi:10.1186/1475-2891-12-76

1969 Richard, C, Couture, P, Desroches, S, Nehme, B, Bourassa, S, Droit, A, Lamarche, B. Effect of an isoenergetic traditional Mediterranean diet on the high-density lipoprotein proteome in men with the metabolic syndrome. J Nutrigenet Nutrigenomics. 2014. 7:48-60. doi:10.1159/000363137

1970 Richard, C, Couture, P, Ooi, EM, Tremblay, AJ, Desroches, S, Charest, A, Lichtenstein, AH, Lamarche, B. Effect of Mediterranean diet with and without weight loss on apolipoprotein B100 metabolism in men with metabolic syndrome. Arterioscler Thromb Vasc Biol. 2014. 34:433-8. doi:10.1161/atvbaha.113.302185
Intervention/Exposure; Comparator

Participants

Language

Intervention/Exposure

Study duration

Study Design

Study duration

Intervention/Exposure: Study duration

Intervention/Exposure

Study Design

Comparator

Study duration

Study Design;

Intervention/Exposure

Intervention/Exposure; Comparator 
1971 Ricordi, C, Garcia-Contreras, M, Farnetti, S. Diet and Inflammation: Possible Effects on Immunity, Chronic Diseases, and Life Span. J Am Coll Nutr. 2015. 34 Suppl 1:10-3. doi:10.1080/07315724.2015.1080101

1972 Rietman, A, Schwarz, J, Blokker, BA, Siebelink, E, Kok, FJ, Afman, LA, Tome, D, Mensink, M. Increasing protein intake modulates lipid metabolism in healthy young men and women consuming a high-fat hypercaloric diet. J Nutr. 2014. 144:117480. doi:10.3945/jn.114.191072

1973 Rietman, A, Schwarz, J, Siebelink, E, Kok, F, Tome, D, Mensink, M. High dietary protein intake results in lower intra hepatic lipid content in healthy humans on a hypercaloric high-fat diet. FASEB journal. 2013. 27:. doi:unavailable

1974 Riggs, AJ, White, BD, Gropper, SS. Changes in energy expenditure associated with ingestion of high protein, high fat versus high protein, low fat meals among underweight, normal weight, and overweight females. Nutr J. 2007. 6:40. doi:10.1186/14752891-6-40

1975 Rist, PM, Buring, JE, Kase, CS, Kurth, T. Healthy Lifestyle and Functional Outcomes from Stroke in Women. Am J Med. 2016. 129:715-724.e2. doi:10.1016/j.amjmed.2016.02.002

1976 Rito, Al, Dinis, A, Rascôa, C, Maia, A, Mendes, S, Stein-Novais, C, Lima, J. Mediterranean Diet Index (KIDMED) Adherence, Socioeconomic Determinants, and Nutritional Status of Portuguese Children: The Eat Mediterranean Program. Portuguese Journal of Public Health. 2018. 36:. doi:10.1159/000495803

1977 Rizkalla, SW, Prifti, E, Cotillard, A, Pelloux, V, Rouault, C, Allouche, R, Laromiguiere, M, Kong, L, Darakhshan, F, Massiera, F, Clement, K. Differential effects of macronutrient content in 2 energy-restricted diets on cardiovascular risk factors and adipose tissue cell size in moderately obese individuals: a randomized controlled trial. Am J Clin Nutr. 2012. 95:49-63. doi:10.3945/ajcn.111.017277

1978 Ro, A, Osborn, B. Exploring Dietary Factors in the Food Insecurity and Obesity Relationship Among Latinos in California. J Health Care Poor Underserved. 2018. 29:1108-1122. doi:10.1353/hpu.2018.0082

1979 Roberge, JB, Van Hulst, A, Barnett, TA, Drapeau, V, Benedetti, A, Tremblay, A, Henderson, M. Lifestyle Habits, Dietary Factors, and the Metabolically Unhealthy Obese Phenotype in Youth. J Pediatr. 2019. 204:46-52.e1. doi:10.1016/j.jpeds.2018.08.063

1980 Roberts, CK, Chen, AK, Barnard, RJ. Effect of a short-term diet and exercise intervention in youth on atherosclerotic risk factors. Atherosclerosis. 2007. 191:98-106. doi:10.1016/j.atherosclerosis.2006.09.011

1981 Roberts, CK, Izadpanah, A, Angadi, SS, Barnard, RJ. Effects of an intensive short-term diet and exercise intervention: comparison between normal-weight and obese children. Am J Physiol Regul Integr Comp Physiol. 2013. 305:R552-7. doi:10.1152/ajpregu.00131.2013

1982 Roberts, CK, Liu, S. Carbohydrate Intake and Obesity: An Association that Needs "Refining". Journal of the American Dietetic Association. 2009. 109:1163-1164. doi:10.1016/j.jada.2009.04.016

1983 Roberts, CK, Ng, C, Hama, S, Eliseo, AJ, Barnard, RJ. Effect of a short-term diet and exercise intervention on inflammatory/anti-inflammatory properties of HDL in overweight/obese men with cardiovascular risk factors. J Appl Physiol (1985). 2006. 101:1727-32. doi:10.1152/japplphysiol.00345.2006

Intervention/Exposure; Study duration

Intervention/Exposure

Study Design;

Intervention/Exposure

Study duration

Study Design; Intervention/Exposure

Intervention/Exposure

Study duration

Study duration

Study Design; Publication Status Study duration 
1984 Roberts, CK, Ng, C, Hama, S, Eliseo, AJ, Barnard, RJ. Effect of a short-term diet and exercise intervention on inflammatory/antiinflammatory properties of HDL in overweight/obese men with cardiovascular risk factors. Journal of Applied Physiology. 2006. 101:1727-1732. doi:10.1152/japplphysiol.00345.2006

1985 Roberts, CK, Vaziri, ND, Barnard, RJ. Effect of diet and exercise intervention on blood pressure, insulin, oxidative stress, and nitric oxide availability. Circulation. 2002. 106:2530-2. doi:10.1161/01.cir.0000040584.91836.0d

1986 Roberts, J, Zinchenko, A, Mahbubani, K, Johnstone, J, Smith, L, Merzbach, V, Blacutt, M, Banderas, O, Villasenor, L, Varvik, FT, Henselmans, M. Satiating Effect of High Protein Diets on Resistance-Trained Subjects in Energy Deficit. Nutrients. 2018. 11: doi:10.3390/nu11010056

1987 Roberts, K, Cade, J, Dawson, J, Holdsworth, M. Empirically Derived Dietary Patterns in UK Adults Are Associated with Sociodemographic Characteristics, Lifestyle, and Diet Quality. Nutrients. 2018. 10:. doi:10.3390/nu10020177

1988 Roberts, R, Bickerton, AS, Fielding, BA, Blaak, EE, Wagenmakers, AJ, Chong, MF, Gilbert, M, Karpe, F, Frayn, KN Reduced oxidation of dietary fat after a short term high-carbohydrate diet. Am J Clin Nutr. 2008. 87:824-31. doi:10.1093/ajcn/87.4.824

1989 Roberts, SB, Das, SK. One strike against low-carbohydrate diets. Cell Metabolism. 2015. 22:357-358. doi:10.1016/j.cmet.2015.07.022

1990 Roberts, WC. The cause of atherosclerosis. Nutr Clin Pract. 2008. 23:464-7. doi:10.1177/0884533608324586

1991 Robinson, F, Hackett, AF, Billington, D, Stratton, G. Changing from a mixed to self-selected vegetarian diet--influence on blood lipids. J Hum Nutr Diet. 2002. 15:323-9. doi:10.1046/j.1365-277x.2002.00383.x

Study duration

Outcome; Study

duration

Outcome

Study duration

Study Design;

Publication Status

Study Design; Publication Status

Study Design; Intervention/Exposure

1992 Roblin, DW, Robinson, BE, Benjamin, SA. Evaluation of a worksite wellness program designed to reduce cardiovascular risks. J Ambul Care Manage. 2013. 36:272-9. doi:10.1097/JAC.0b013e3182a3e728

1993 Rock, CL, Flatt, SW, Pakiz, B, Quintana, EL, Heath, DD, Rana, BK, Natarajan, L. Effects of diet composition on weight loss, metabolic factors and biomarkers in a 1-year weight loss intervention in obese women examined by baseline insulin resistance status. Metabolism. 2016. 65:1605-1613. doi:10.1016/j.metabol.2016.07.008

1994 Rodrigues, SSP, Trichopoulou, A, De Almeida, MDV. Household diet quality in relation to mortality in Portuguese regions: An ecological study. Journal of Public Health. 2008. 16:43-51. doi:10.1007/s10389-007-0113-5

1995 Rodriguez, JM, Leiva Balich, L, Concha, MJ, Mizon, C, Bunout Barnett, D, Barrera Acevedo, G, Hirsch Birn, S, Jimenez Jaime, T, Henriquez, S, Uribarri, J, de la Maza Cave, MP. Reduction of serum advanced glycation end-products with a low calorie Mediterranean diet. Nutr Hosp. 2015. 31:2511-7. doi:10.3305/nh.2015.31.6.8936

1996 Rodriguez-Cano, A, Mier-Cabrera, J, Balas-Nakash, M, Munoz-Manrique, C, Legorreta-Legorreta, J, Perichart-Perera, O. Dietary changes associated with improvement of metabolic syndrome components in postmenopausal women receiving two

Intervention/Exposure

Intervention/Exposure Study Design; Intervention/Exposure; Outcome

Study Design different nutrition interventions. Menopause. 2015. 22:758-64. doi:10.1097/gme.0000000000000400 
1997 Rodriguez-Garcia, E, Ruiz-Nava, J, Santamaria-Fernandez, S, Fernandez-Garcia, JC, Vargas-Candela, A, Yahyaoui, R, Tinahones, FJ, Bernal-Lopez, MR, Gomez-Huelgas, R. Implications of the Mediterranean diet and physical exercise on the lipid profile of metabolically healthy obese women as measured by nuclear magnetic resonance spectroscopy ((1)H NMR).

Intervention/Exposure; Chem Phys Lipids. 2018. 213:68-75. doi:10.1016/j.chemphyslip.2018.03.007

1998 Rodriguez-Martin, C, Alonso-Dominguez, R, Patino-Alonso, MC, Gomez-Marcos, MA, Maderuelo-Fernandez, JA, MartinCantera, C, Garcia-Ortiz, L, Recio-Rodriguez, JI. The EVIDENT diet quality index is associated with cardiovascular risk and arterial stiffness in adults. BMC Public Health. 2017. 17:305. doi:10.1186/s12889-017-4194-y

1999 Rodriguez-Martin, C, Garcia-Ortiz, L, Rodriguez-Sanchez, E, Maderuelo-Fernandez, C, Lugones-Sanchez, A, MartinCantera, MS, Soriano-Cano, JF, Arietaleanizbeaskoa, M, Magdalena-Belio, JA, Menendez-Suarez, C, Gomez-Marcos, MA Recio-Rodriguez, JI, Evident Investigators Group, Obotei. The Relationship of the Atlantic Diet with Cardiovascular Risk Factors and Markers of Arterial Stiffness in Adults without Cardiovascular Disease. Nutrients. 2019. 11: doi:10.3390/nu11040742

2000 Rodriguez-Moran, M, Guerrero-Romero, F, Rascon-Pacheco, RA. Dietary factors related to the increase of cardiovascular risk factors in traditional Tepehuanos communities from Mexico. A 10 year follow-up study. Nutr Metab Cardiovasc Dis. 2009. 19:409-16. doi:10.1016/j.numecd.2008.08.005

2001 Rodriguez-Rejon, Al, Castro-Quezada, I, Ruano-Rodriguez, C, Ruiz-Lopez, MD, Sanchez-Villegas, A, Toledo, E, Artacho, R, Estruch, R, Salas-Salvado, J, Covas, MI, Corella, D, Gomez-Gracia, E, Lapetra, J, Pinto, X, Aros, F, Fiol, M, LamuelaRaventos, RM, Ruiz-Gutierrez, V, Schroder, H, Ros, E, Martinez-Gonzalez, MA, Serra-Majem, L. Effect of a Mediterranean Diet Intervention on Dietary Glycemic Load and Dietary Glycemic Index: The PREDIMED Study. J Nutr Metab. 2014. 2014:985373. doi:10.1155/2014/985373

2002 Rogerson, D, Macas, D, Milner, M, Liu, Y, Klonizakis, M. Contrasting Effects of Short-Term Mediterranean and Vegan Diets on Microvascular Function and Cholesterol in Younger Adults: A Comparative Pilot Study. Nutrients. 2018. 10:. doi:10.3390/nu10121897

2003 Rogerson, D, McNeill, S, Kononen, H, Klonizakis, M. Encouraging effects of a short-term, adapted Nordic diet intervention on skin microvascular function and skin oxygen tension in younger and older adults. Nutrition. 2018. 49:96-101. doi:10.1016/j.nut.2017.11.001

2004 Rohde, JF, Handel, MN, Stougaard, M, Olsen, NJ, Traerup, M, Mortensen, EL, Heitmann, BL. Relationship between pickiness and subsequent development in body mass index and diet intake in obesity prone normal weight preschool children. PLoS One. 2017. 12:e0172772. doi:10.1371/journal.pone.0172772

2005 Rohde, JF, Larsen, SC, Angquist, L, Olsen, NJ, Stougaard, M, Mortensen, EL, Heitmann, BL. Effects of the Healthy Start randomized intervention on dietary intake among obesity-prone normal-weight children. Public Health Nutr. 2017. 20:29882997. doi:10.1017/s1368980017002026

2006 Rolland, C, Broom, J. A randomised controlled trial of a low-carbohydrate vs. a very low calorie diet - A two year follow up. Obesity reviews. 2010. 11:245-246. doi:10.1111/j.1467-789X.2010.00763-7.x 
2007 Rolland, C, Hession, M, Murray, S, Wise, A, Broom, I. Randomized clinical trial of standard dietary treatment versus a lowcarbohydrate/high-protein diet or the LighterLife Programme in the management of obesity*. J Diabetes. 2009. 1:207-17. doi:10.1111/j.1753-0407.2009.00033.x

2008 Rolland-Cachera, MF, Thibault, H, Souberbielle, JC, Soulie, D, Carbonel, P, Deheeger, M, Roinsol, D, Longueville, E, Bellisle, F, Serog, P. Massive obesity in adolescents: dietary interventions and behaviours associated with weight regain at 2 y follow-up. Int J Obes Relat Metab Disord. 2004. 28:514-9. doi:10.1038/sj.ijo.0802605

2009 Romero-Moraleda, B, Peinado Lozano, AB, Morencos Martinez, E, Lopez-Plaza, B, Gomez Candela, C, Calderon Montero, FJ. Lipid profile response to weight loss program in overweight and obese patient is related with gender and age. Nutr Hosp. 2015. 31:2455-64. doi:10.3305/nh.2015.31.6.8926

2010 Roncero-Ramos, I, Alcala-Diaz, JF, Rangel-Zuniga, OA, Gomez-Delgado, F, Jimenez-Lucena, R, Garcia-Rios, A, ValsDelgado, C, Romero-Baldonado, C, Luque, RM, Ordovas, JM, Perez-Martinez, P, Camargo, A, Lopez-Miranda, J. Prediabetes diagnosis criteria, type 2 diabetes risk and dietary modulation: The CORDIOPREV study. Clin Nutr. 2019. : doi:10.1016/j.clnu.2019.02.027

2011 Rondanelli, M, Klersy, C, Perna, S, Faliva, MA, Montorfano, G, Roderi, P, Colombo, I, Corsetto, PA, Fioravanti, M, Solerte, SB, Rizzo, AM. Effects of two-months balanced diet in metabolically healthy obesity: lipid correlations with gender and BMIrelated differences. Lipids Health Dis. 2015. 14:139. doi:10.1186/s12944-015-0131-1

2012 Root, MM, Dawson, HR. DASH-like diets high in protein or monounsaturated fats improve metabolic syndrome and calculated vascular risk. International Journal for Vitamin and Nutrition Research. 2014. 83:224-231. doi:10.1024/0300-9831/a000164

2013 Rosamond, WD, Ammerman, AS, Holliday, JL, Tawney, KW, Hunt, KJ, Keyserling, TC, Will, JC, Mokdad, AH. Cardiovascular disease risk factor intervention in low-income women: The North Carolina WISEWOMAN project. Preventive Medicine. 2000. 31:370-379. doi:10.1006/pmed.2000.0726

2014 Rosi, A, Calestani, MV, Parrino, L, Milioli, G, Palla, L, Volta, E, Brighenti, F, Scazzina, F. Weight Status Is Related with Gender and Sleep Duration but Not with Dietary Habits and Physical Activity in Primary School Italian Children. Nutrients. 2017. 9:. doi:10.3390/nu9060579

2015 Ross, SM. Cardiovascular disease mortality: the deleterious effects of excess dietary sugar intake. Holist Nurs Pract. 2015. 29:53-7. doi:10.1097/hnp.0000000000000066

2016 Ross, SM. Effects of extra virgin olive oil phenolic compounds and the Mediterranean diet on cardiovascular health. Holist Nurs Pract. 2013. 27:303-7. doi:10.1097/HNP.0b013e3182a0c668

2017 Rosvall, M, Persson, M, Ostling, G, Nilsson, PM, Melander, O, Hedblad, B, Engstrom, G. Risk factors for the progression of carotid intima-media thickness over a 16-year follow-up period: the Malmo Diet and Cancer Study. Atherosclerosis. 2015. 239:615-21. doi:10.1016/j.atherosclerosis.2015.01.030

2018 Rothacker, DQ, Ellis, PK. Elevated intakes of calcium and vitamin D without added calories and fat in overweight adults: A crossover study in Wisconsin. Current Therapeutic Research - Clinical and Experimental. 2002. 63:507-512. doi:10.1016/S0011-393X(02)80056-X

Intervention/Exposure; Health Status

Intervention/Exposure

Intervention/Exposure; Health Status

Study Design

Publication Status

Intervention/Exposure

Study Design

Intervention/Exposure; Publication Status

Study Design

Intervention/Exposure Study duration 
2019 Rouhani, MH, Kelishadi, R, Hashemipour, M, Esmaillzadeh, A, Azadbakht, L. The effect of an energy restricted low glycemic index diet on blood lipids, apolipoproteins and lipoprotein (a) among adolescent girls with excess weight: a randomized clinical

Intervention/Exposure; trial. Lipids. 2013. 48:1197-205. doi:10.1007/s11745-013-3834-y

2020 Rouhani, MH, Kelishadi, R, Hashemipour, M, Esmaillzadeh, A, Azadbakht, L. The effect of low glycemic index diet on body weight status and blood pressure in overweight adolescent girls: a randomized clinical trial. Nutr Res Pract. 2013. 7:385-92. doi:10.4162/nrp.2013.7.5.385

2021 Rouillier, MA, David-Riel, S, Brazeau, AS, St-Pierre, DH, Karelis, AD. Effect of an Acute High Carbohydrate Diet on Body Composition Using DXA in Young Men. Ann Nutr Metab. 2015. 66:233-6. doi:10.1159/000435840

2022 Roush, K. Diets vs. combinations of fat, protein, and carbohydrates. Am J Nurs. 2009. 109:64. doi:10.1097/01.NAJ.0000363356.61507.fC

Comparator

Intervention/Exposure Roussell, MA, Hill, AM, Gaugler, TL, West, SG, Ulbrecht, JS, Vanden Heuvel, JP, Gillies, PJ, Kris-Etherton, PM. Effects of a DASH-like diet containing lean beef on vascular health. J Hum Hypertens. 2014. 28:600-5. doi:10.1038/jhh.2014.34

2024 Rousset, S, Patureau Mirand, P, Brandolini, M, Martin, JF, Boirie, Y. Daily protein intakes and eating patterns in young and elderly French. Br J Nutr. 2003. 90:1107-15. doi:10.1079/bjn20031004

2025 Rowlands, DS, Hopkins, WG. Effects of high-fat and high-carbohydrate diets on metabolism and performance in cycling. Metabolism. 2002. 51:678-90. doi:10.1053/meta.2002.32723

2026 Roy, BD, Luttmer, K, Bosman, MJ, Tarnopolsky, MA. The influence of post-exercise macronutrient intake on energy balance and protein metabolism in active females participating in endurance training. Int J Sport Nutr Exerc Metab. 2002. 12:172-88. doi:10.1123/ijsnem.12.2.172

2027 Roy, HJ, Most, MM, Sparti, A, Lovejoy, JC, Volaufova, J, Peters, JC, Bray, GA. Effect on body weight of replacing dietary fat with olestra for two or ten weeks in healthy men and women. J Am Coll Nutr. 2002. 21:259-67. doi:10.1080/07315724.2002.10719219

2028 Ruan, Y, Huang, Y, Zhang, Q, Qin, S, Du, X, Sun, Y. Association between dietary patterns and hypertension among Han and multi-ethnic population in southwest China. BMC Public Health. 2018. 18:1106. doi:10.1186/s12889-018-6003-7

2029 Rubini, A, Bosco, G, Lodi, A, Cenci, L, Parmagnani, A, Grimaldi, K, Zhongjin, Y, Paoli, A. Effects of Twenty Days of the Ketogenic Diet on Metabolic and Respiratory Parameters in Healthy Subjects. Lung. 2015. 193:939-45. doi:10.1007/s00408015-9806-7

2030 Ruiz-Cabello Turmo, P, Aparicio Garcia-Molina, V, Fernandez Martinez Mdel, M, Moratalla Cecilia, N, Gregorio Arenas, E, Aranda Ramirez, P. Mediterranean countries facing the Mediterranean Diet, are we still on track? The example of southern Spain midlife women. Nutr Hosp. 2015. 31:2523-32. doi:10.3305/nh.2015.31.6.8862

Study duration

Study Design;

Publication Status

Study duration

Study Design

Study duration

Study duration

Study duration

Study Design

Study duration

Study Design 
2031 Ruiz-Canela, M, Guasch-Ferre, M, Toledo, E, Clish, CB, Razquin, C, Liang, L, Wang, DD, Corella, D, Estruch, R, Hernaez, A, Yu, E, Gomez-Gracia, E, Zheng, Y, Aros, F, Romaguera, D, Dennis, C, Ros, E, Lapetra, J, Serra-Majem, L,

Intervention/Exposure; Papandreou, C, Portoles, O, Fito, M, Salas-Salvado, J, Hu, FB, Martinez-Gonzalez, MA. Plasma branched chain/aromatic Comparator amino acids, enriched Mediterranean diet and risk of type 2 diabetes: case-cohort study within the PREDIMED Trial. Diabetologia. 2018. 61:1560-1571. doi:10.1007/s00125-018-4611-5

2032 Ruottinen, S, Ronnemaa, T, Niinikoski, H, Lagstrom, H, Saarinen, M, Pahkala, K, Kaitosaari, T, Viikari, J, Simell, O. Carbohydrate intake, serum lipids and apolipoprotein E phenotype show association in children. Acta Paediatr. 2009. 98:166773. doi:10.1111/j.1651-2227.2009.01399.x

2033 Rush, E, Paterson, J, Obolonkin, V. Food frequency information-relationships to body composition and apparent growth in 4year-old children in the Pacific Island Family Study. New Zealand Medical Journal. 2008. 121:63-71. doi:unavailable

2034 Rushing, K, Stenhouse, A, Evans, M, Huffman, L, Bowie, V. A low-carbohydrate diet versus a low-calorie diet: Poor retention. Topics in Clinical Nutrition. 2006. 21:251-259. doi:10.1097/00008486-200610000-00002

2035 Russell, JC, Flood, VM, Sadeghpour, A, Gopinath, B, Mitchell, P. Total Diet Score as a valid method of measuring diet quality among older adults. Asia Pac J Clin Nutr. 2017. 26:212-219. doi:10.6133/apjcn.122015.08

2036 Ruth, MR, Port, AM, Shah, M, Bourland, AC, Istfan, NW, Nelson, KP, Gokce, N, Apovian, CM. Consuming a hypocaloric high fat low carbohydrate diet for 12 weeks lowers C-reactive protein, and raises serum adiponectin and high density lipoproteincholesterol in obese subjects. Metabolism. 2013. 62:1779-87. doi:10.1016/j.metabol.2013.07.006

2037 Ruth, MR, Shah, M, Bourland, A, Port, AM, Gokce, N, Apovian, C. A 12-week hypocaloric low-carbohydrate diet improves inflammation and cardiovascular risk factors in obese adults. Obesity. 2011. 19:S114. doi:10.1038/oby.2011.226

2038 Ryberg, M, Sandberg, S, Mellberg, C, Stegle, O, Lindahl, B, Larsson, C, Hauksson, J, Olsson, T. A Palaeolithic-type diet causes strong tissue-specific effects on ectopic fat deposition in obese postmenopausal women. J Intern Med. 2013. 274:67-76. doi:10.1111/joim.12048

2039 Ryman, TK, Boyer, BB, Hopkins, S, Philip, J, Beresford, SA, Thompson, B, Heagerty, PJ, Pomeroy, JJ, Thummel, KE, Austin, MA. Associations between diet and cardiometabolic risk among Yup'ik Alaska Native people using food frequency questionnaire dietary patterns. Nutr Metab Cardiovasc Dis. 2015. 25:1140-5. doi:10.1016/j.numecd.2015.08.003

2040 Sabrina, N, Bai, CH, Chang, CC, Chien, YW, Chen, JR, Chang, JS. Serum Iron:Ferritin Ratio Predicts Healthy Body Composition and Reduced Risk of Severe Fatty Liver in Young Adult Women. Nutrients. 2017. 9:. doi:10.3390/nu9080833

2041 Sacks, FM, Bray, GA, Carey, VJ, Smith, SR, Ryan, DH, Anton, SD, McManus, K, Champagne, CM, Bishop, LM, Laranjo, N, Leboff, MS, Rood, JC, de Jonge, L, Greenway, FL, Loria, CM, Obarzanek, E, Williamson, DA. Comparison of weightloss diets with different compositions of fat, protein, and carbohydrates. N Engl J Med. 2009. 360:859-73. doi:10.1056/NEJMoa0804748

2042 Sacks, FM, Carey, VJ, Anderson, CA, Miller, ER, 3rd, Copeland, T, Charleston, J, Harshfield, BJ, Laranjo, N, McCarron, $\mathrm{P}$, Swain, J, White, K, Yee, K, Appel, LJ. Effects of high vs low glycemic index of dietary carbohydrate on cardiovascular disease risk factors and insulin sensitivity: the OmniCarb randomized clinical trial. Jama. 2014. 312:2531-41. doi:10.1001/jama.2014.16658

Study Design; Intervention/Exposure

Study Design

Weight

loss/Hypocaloric

Study duration 
2043 Sacks, FM, Svetkey, LP, Vollmer, WM, Appel, LJ, Bray, GA, Harsha, D, Obarzanek, E, Conlin, PR, Miller, ER, 3rd,

Intervention/Exposure; Simons-Morton, DG, Karanja, N, Lin, PH. Effects on blood pressure of reduced dietary sodium and the Dietary Approaches to Stop Hypertension (DASH) diet. DASH-Sodium Collaborative Research Group. N Engl J Med. 2001. 344:3-10. doi:10.1056/nejm200101043440101

2044 Sadeghi, M, Talaei, M, Parvaresh Rizi, E, Dianatkhah, M, Oveisgharan, S, Sarrafzadegan, N. Determinants of incident prediabetes and type 2 diabetes in a 7-year cohort in a developing country: The Isfahan Cohort Study. J Diabetes. 2015. 7:63341. doi:10.1111/1753-0407.12236

2045 Sadov, S, Virtanen, HE, Main, KM, Andersson, AM, Juul, A, Jula, A, Raitakari, OT, Pahkala, K, Niinikoski, H, Toppari, J. Low-saturated-fat and low-cholesterol diet does not alter pubertal development and hormonal status in adolescents. Acta Paediatrica, International Journal of Paediatrics. 2019. 108:321-327. doi:10.1111/apa.14480

2046 Safaeiyan, A, Pourghassem-Gargari, B, Zarrin, R, Fereidooni, J, Alizadeh, M. Randomized controlled trial on the effects of legumes on cardiovascular risk factors in women with abdominal obesity. ARYA Atheroscler. 2015. 11:117-25. doi:unavailable

2047 Saffari, M, Pakpour, AH, Mohammadi-Zeidi, I, Samadi, M, Chen, H. Long-term effect of motivational interviewing on dietary intake and weight loss in Iranian obese/overweight women. Health Promot Perspect. 2014. 4:206-13. doi:10.5681/hpp.2014.027

2048 Saha, S, Nordstrom, J, Gerdtham, UG, Mattisson, I, Nilsson, PM, Scarborough, P. Prevention of Cardiovascular Disease and Cancer Mortality by Achieving Healthy Dietary Goals for the Swedish Population: A Macro-Simulation Modelling Study. Int J Environ Res Public Health. 2019. 16:. doi:10.3390/ijerph16050890

2049 Saha, S, Nordstrom, J, Mattisson, I, Nilsson, PM, Gerdtham, UG. Modelling the Effect of Compliance with Nordic Nutrition Recommendations on Cardiovascular Disease and Cancer Mortality in the Nordic Countries. Nutrients. 2019. 11: doi:10.3390/nu11061434

2050 Said, N, Nor, NM, Sharoni, SKA. A Preliminary study of the evaluation on the dietary pattern among obese school children. Indian Journal of Public Health Research and Development. 2019. 10:1416-1421. doi:10.5958/0976-5506.2019.00912.4

2051 Saint-Maurice, PF, Coughlan, D, Kelly, SP, Keadle, SK, Cook, MB, Carlson, SA, Fulton, JE, Matthews, CE. Association of Leisure-Time Physical Activity Across the Adult Life Course With All-Cause and Cause-Specific Mortality. JAMA Netw Open. 2019. 2:e190355. doi:10.1001/jamanetworkopen.2019.0355

2052 Sakae, PN, Bianco, HT, Camargo, LM, Carvalho, JG, Izar, MCO, Ihara, SSM, Fonseca, FAH. Effect of high protein/very low carbohydrate diet and standard hypocaloric diet in obese subjects: nutritional, biochemical and endothelial function evaluations. BBA clinical. Conference: 15th brazilian congress of atherosclerosis. Brazil. Conference start: 20150814. Conference end: 20150815. 2015. 3:S7. doi:10.1016/j.bbacli.2015.05.020

2053 Sakurai, Y, Tamura, Y, Takeno, K, Kumashiro, N, Sato, F, Kakehi, S, Ikeda, S, Ogura, Y, Saga, N, Naito, H, Katamoto, S Fujitani, Y, Hirose, T, Kawamori, R, Watada, H. Determinants of intramyocellular lipid accumulation after dietary fat loading in non-obese men. J Diabetes Investig. 2011. 2:310-7. doi:10.1111/j.2040-1124.2010.00091.x 
2054 Salas-Salvado, J, Diaz-Lopez, A, Ruiz-Canela, M, Basora, J, Fito, M, Corella, D, Serra-Majem, L, Warnberg, J, Romaguera, D, Estruch, R, Vidal, J, Martinez, JA, Aros, F, Vazquez, C, Ros, E, Vioque, J, Lopez-Miranda, J, BuenoCavanillas, A, Tur, JA, Tinahones, FJ, Martin, V, Lapetra, J, Pinto, X, Daimiel, L, Delgado-Rodriguez, M, Matia, P, GomezGracia, E, Diez-Espino, J, Babio, N, Castaner, O, Sorli, JV, Fiol, M, Zulet, MA, Bullo, M, Goday, A, Martinez-Gonzalez, MA. Effect of a Lifestyle Intervention Program With Energy-Restricted Mediterranean Diet and Exercise on Weight Loss and Cardiovascular Risk Factors: One-Year Results of the PREDIMED-Plus Trial. Diabetes Care. 2019. 42:777-788. doi:10.2337/dc18-0836

2055 Salas-Salvado, J, Fernandez-Ballart, J, Ros, E, Martinez-Gonzalez, MA, Fito, M, Estruch, R, Corella, D, Fiol, M, GomezGracia, E, Aros, F, Flores, G, Lapetra, J, Lamuela-Raventos, R, Ruiz-Gutierrez, V, Bullo, M, Basora, J, Covas, MI. Effect of a Mediterranean diet supplemented with nuts on metabolic syndrome status: one-year results of the PREDIMED randomized trial. Arch Intern Med. 2008. 168:2449-2458. doi:10.1001/archinte.168.22.2449

2056 Salas-Salvado, J. Weight loss using a healthy diet and exercise promotion to prevent cardiovascular disease: the predimedplus trial. Annals of nutrition \& metabolism. 2017. 71:24-. doi:10.1159/000480486

2057 Saldanha-Gomes, C, Heude, B, Charles, MA, de Lauzon-Guillain, B, Botton, J, Carles, S, Forhan, A, Dargent-Molina, P, Lioret, S. Prospective associations between energy balance-related behaviors at 2 years of age and subsequent adiposity: the EDEN mother-child cohort. Int J Obes (Lond). 2017. 41:38-45. doi:10.1038/ijo.2016.138

2058 Sallinen, J, Pakarinen, A, Fogelholm, M, Alen, M, Volek, JS, Kraemer, WJ, Häkkinen, K. Dietary intake, serum hormones, muscle mass and strength during strength training in 49-73-year-old men. International Journal of Sports Medicine. 2007. 28:1070-1076. doi:10.1055/s-2007-965003

2059 Salmeron, J, Hu, FB, Manson, JE, Stampfer, MJ, Colditz, GA, Rimm, EB, Willett, WC. Dietary fat intake and risk of type 2 diabetes in women. Am J Clin Nutr. 2001. 73:1019-26. doi:10.1093/ajcn/73.6.1019

2060 Salo, P, Viikari, J, Hamalainen, M, Lapinleimu, H, Routi, T, Niinikoski, H, Rask-Nissila, L, Tammi, A, Ronnemaa, T, Seppanen, R, Jokinen, E, Valimaki, I, Simell, O. Fatty acid composition of serum cholesterol esters as a reflector of lowsaturated-fat, low-cholesterol diet in young children: the STRIP project. The Special Turku coronary Risk factor Intervention Project. Acta Paediatr. 2000. 89:399-405. doi:10.1080/080352500750028087

2061 Salvia, R, D'Amore, S, Graziano, G, Capobianco, C, Sangineto, M, Paparella, D, de Bonfils, P, Palasciano, G, Vacca, M. Short-term benefits of an unrestricted-calorie traditional Mediterranean diet, modified with a reduced consumption of carbohydrates at evening, in overweight-obese patients. Int J Food Sci Nutr. 2017. 68:234-248 doi:10.1080/09637486.2016.1228100

2062 Samaha, FF, lqbal, N, Seshadri, P, Chicano, KL, Daily, DA, McGrory, J, Williams, T, Williams, M, Gracely, EJ, Stern, L. A low-carbohydrate as compared with a low-fat diet in severe obesity. N Engl J Med. 2003. 348:2074-81. doi:10.1056/NEJMoa022637

2063 Samkani, A, Skytte, MJ, Thomsen, MN, Astrup, A, Deacon, CF, Holst, JJ, Madsbad, S, Rehfeld, JF, Krarup, T, Haugaard, SB. Acute Effects of Dietary Carbohydrate Restriction on Glycemia, Lipemia and Appetite Regulating Hormones in NormalWeight to Obese Subjects. Nutrients. 2018. 10:. doi:10.3390/nu10091285
Intervention/Exposure; Comparator

Study Design

Publication Date

Overlaps with Existing Review

Publication Status

Power/Size

Intervention/Exposure

Intervention/Exposure

Intervention/Exposure

Intervention/Exposure

Weight

loss/Hypocaloric

Study Design;

Intervention/Exposure; Study duration 
2064 Samman, S, Lai, NT, Sullivan, DR. The effect of a lipid-lowering diet on plasma lipids and lipoproteins in mildly hypercholesterolaemic subjects: A potential role for occasional treats. Journal of Nutritional Biochemistry. 2000. 11:250-254. doi:10.1016/S0955-2863(00)00072-3

2065 San Mauro Martin, I, Sanz Rojo, S, Becerra, X, Garicano Vilar, E. Successful Implementation of a Mediterranean Weight Loss Program to Prevent Overweight and Obesity in the Workplace. Journal of Occupational and Environmental Medicine. 2019. 61:E329-E332. doi:10.1097/JOM.0000000000001628

2066 Sanchez-Bayle, M, Soriano-Guillen, L. Influence of dietary inte Paediatr. 2003. 92:1043-6. doi:10.1080/08035250310004775

2067 Sanchez-Benito, JL, Sanchez Soriano, E. The excesive intake of macronutrients: does it influence the sports performances of young cyclists?. Nutr Hosp. 2007. 22:461-70. doi:unavailable

2068 Sanchez-Oliva, D, Grao-Cruces, A, Carbonell-Baeza, A, Cabanas-Sanchez, V, Veiga, OL, Castro-Pinero, J. Lifestyle Clusters in School-Aged Youth and Longitudinal Associations with Fatness: The UP\&DOWN Study. J Pediatr. 2018. 203:317324.e1. doi:10.1016/j.jpeds.2018.07.092

2069 Sanchez-Villegas, A, Bes-Rastrollo, M, Martinez-Gonzalez, MA, Serra-Majem, L. Adherence to a Mediterranean dietary pattern and weight gain in a follow-up study: the SUN cohort. Int J Obes (Lond). 2006. 30:350-8 https://www.ncbi.nlm.nih.gov/pubmed/16231028

2070 Sanchez-Villegas, A, Ruiz-Canela, M, de la Fuente-Arrillaga, C, Gea, A, Shivappa, N, Hebert, JR, Martinez-Gonzalez, MA Dietary inflammatory index, cardiometabolic conditions and depression in the Seguimiento Universidad de Navarra cohort study. Br J Nutr. 2015. 114:1471-9. doi:10.1017/s0007114515003074

2071 San-Cristobal, R, Navas-Carretero, S, Celis-Morales, C, Brennan, L, Walsh, M, Lovegrove, JA, Daniel, H, Saris, WH, Traczyk, I, Manios, Y, Gibney, ER, Gibney, MJ, Mathers, JC, Martinez, JA. Analysis of Dietary Pattern Impact on Weight Status for Personalised Nutrition through On-Line Advice: The Food4Me Spanish Cohort. Nutrients. 2015. 7:9523-37. doi:10.3390/nu7115482

2072 , Celis-Morales, $C$, Livingstone, KM, Stewart-Knox, $B$, Rankin, $A$, Macready, $A$, Fallaize, R, O'Donovan, CB, Forster, H, Woolhead, C, Walsh, MC, Lambrinou, CP, Moschonis, G, Manios, Y, Jarosz, M, Daniel, H, Gibney, ER, Brennan, L, Gundersen, TE, Drevon, CA, Gibney, M, Marsaux, CFM, Saris, WHM, Lovegrove, JA, Frewer, LJ, Mathers, JC, Martinez, JA. Capturing health and eating status through a nutritional perception screening questionnaire (NPSQ9) in a randomised internet-based personalised nutrition intervention: the Food4Me study. Int J Behav Nutr Phys Act. 2017. 14:168. doi:10.1186/s12966-017-0624-6

2073 Sanderlin, AH, Hoscheidt, SM, Hanson, AJ, Baker, LD, Sink, KM, Wittmer, P, Craft, S. THE EFFECTS OF DIET INTERVENTION ON METABOLIC HEALTH AND CEREBRAL SPINAL FLUID ALZHEIMER'S DISEASE BIOMARKERS: a RANDOMIZED TRIAL. Alzheimer's \& dementia. 2018. 14:P1069-P1070. doi:10.1016/j.jalz.2018.06.1374

2074 Saneei, P, Hashemipour, M, Kelishadi, R, Esmaillzadeh, A. The Dietary Approaches to Stop Hypertension (DASH) diet affects inflammation in childhood metabolic syndrome: a randomized cross-over clinical trial. Ann Nutr Metab. 2014. 64:20-7. doi:10.1159/000358341
Study Design;

Intervention/Exposure

Study Design;

Intervention/Exposure

Study Design

Study Design

Intervention/Exposure; Comparator

Data overlap with another included article

Intervention/Exposure

Power/Size

Study Design

Publication Status

Outcome 
2075 Saneei, P, Hashemipour, M, Kelishadi, R, Rajaei, S, Esmaillzadeh, A. Effects of recommendations to follow the Dietary Approaches to Stop Hypertension (DASH) diet v. usual dietary advice on childhood metabolic syndrome: a randomised cross-

Intervention/Exposure; over clinical trial. Br J Nutr. 2013. 110:2250-9. doi:10.1017/s0007114513001724

2076 Santiago, S, Zazpe, I, Bes-Rastrollo, M, Sanchez-Tainta, A, Sayon-Orea, C, de la Fuente-Arrillaga, C, Benito, S, Martinez, JA, Martinez-Gonzalez, MA. Carbohydrate quality, weight change and incident obesity in a Mediterranean cohort: the SUN Project. Eur J Clin Nutr. 2015. 69:297-302. doi:10.1038/ejcn.2014.187

2077 Santiago-Torres, M, De Dieu Tapsoba, J, Kratz, M, Lampe, JW, Breymeyer, KL, Levy, L, Song, X, Villasenor, A, Wang, CY, Fejerman, L, Neuhouser, ML, Carlson, CS. Genetic ancestry in relation to the metabolic response to a US versus traditional Mexican diet: a randomized crossover feeding trial among women of Mexican descent. Eur J Clin Nutr. 2017. 71:395401. doi:10.1038/ejcn.2016.211

2078 Santiago-Torres, M, Kratz, M, Lampe, JW, Tapsoba Jde, D, Breymeyer, KL, Levy, L, Villasenor, A, Wang, CY, Song, X, Neuhouser, ML. Metabolic responses to a traditional Mexican diet compared with a commonly consumed US diet in women of Mexican descent: a randomized crossover feeding trial. Am J Clin Nutr. 2016. 103:366-74. doi:10.3945/ajcn.115.119016

2079 Santomauro, F, Lorini, C, Tanini, T, Indiani, L, Lastrucci, V, Comodo, N, Bonaccorsi, G. Adherence to Mediterranean diet in a sample of Tuscan adolescents. Nutrition. 2014. 30:1379-83. doi:10.1016/j.nut.2014.04.008

2080 Santos, LP, Assuncao, MCF, Matijasevich, A, Santos, IS, Barros, AJD. Dietary intake patterns of children aged 6 years and their association with socioeconomic and demographic characteristics, early feeding practices and body mass index. BMC Public Health. 2016. 16:1055. doi:10.1186/s12889-016-3725-2

2081 Santos, LP, Ong, KK, Santos, IS, Matijasevich, A, Barros, AJD. Effects of dietary intake patterns from 1 to 4 years on BMI Z score and body shape at age of 6 years: a prospective birth cohort study from Brazil. Eur J Nutr. 2019. 58:1723-1734 doi:10.1007/s00394-018-1720-3

2082 Santos, RD, Suen, VM, lannetta, O, Marchini, JS. Climacteric, physically active women ingesting their routine diet oxidize more carbohydrates than lipids. Climacteric. 2008. 11:454-60. doi:10.1080/13697130802398491

2083 Saquib, N, Natarajan, L, Rock, CL, Flatt, SW, Madlensky, L, Kealey, S, Pierce, JP. The impact of a long-term reduction in dietary energy density on body weight within a randomized diet trial. Nutr Cancer. 2008. 60:31-8. doi:10.1080/01635580701621320

2084 Saracci, R. The dietary prevention of ischaemic heart disease. IARC Sci Publ. 2002. 156:531-3. doi:unavailable

Comparator

Intervention/Exposure

Study duration

Study duration

Study Design; Outcome

Study Design;

Intervention/Exposure

Country

Study Design

Intervention/Exposure; Publication Date

Overlaps with Existing Review

Study Design; Publication Status

2085 Saraf-Bank, S, Esmaillzadeh, A, Faghihimani, E, Azadbakht, L. Effects of Legume-Enriched Diet on Cardiometabolic Risk Factors among Individuals at Risk for Diabetes: A Crossover Study. J Am Coll Nutr. 2016. 35:31-40. doi:10.1080/07315724.2014.931262

2086 Sarebanhassanabadi, M, Mirhosseini, SJ, Mirzaei, M, Namayandeh, SM, Soltani, MH, Pakseresht, M, Pedarzadeh, A, Baramesipour, Z, Faraji, R, Salehi-Abargouei, A. Effect of dietary habits on the risk of metabolic syndrome: Yazd Healthy Heart Project. Public Health Nutr. 2018. 21:1139-1146. doi:10.1017/s1368980017003627

Intervention/Exposure; Outcome 
2087 Sarebanhassanabadi, M, Mirhosseini, SJ, Mirzaei, M, Namayandeh, SM, Soltani, MH, Salehi-Abargouei, A. The association between a dietary habits score and the risk of metabolic syndrome: A cohort study. Clin Nutr. 2019. :

Intervention/Exposure; doi:10.1016/j.clnu.2019.02.005

2088 Sares-Jaske, L, Knekt, P, Lundqvist, A, Heliovaara, M, Mannisto, S. Dieting attempts modify the association between quality of diet and obesity. Nutr Res. 2017. 45:63-72. doi:10.1016/j.nutres.2017.08.001

2089 Saris, WH. The fat/carbohydrate ratio in the diet--implications for a healthy diet. Int Dent J. 2001. 51:407. doi:10.1111/j.1875595x.2001.tb00587.x

2090 Saris, WHM, Astrup, A, Prentice, AM, Zunft, HJF, Formiguera, X, Verboeket-Van De Venne, WPHG, Raben, A, Poppitt, SD, Seppelt, B, Johnston, S, Vasilaras, TH, Keogh, GF. Randomized controlled trial of changes in dietary carbohydrate/fat ratio and simple vs complex carbohydrates on body weight and blood lipids: The CARMEN study. International Journal of Obesity. 2000. 24:1310-1318. doi:10.1038/sj.ijo.0801451

2091 Sarter, B, Campbell, TC, Fuhrman, J. Effect of a high nutrient density diet on long-term weight loss: a retrospective chart review. Altern Ther Health Med. 2008. 14:48-53. doi:unavailable

2092 Saslow, LR, Daubenmier, JJ, Moskowitz, JT, Kim, S, Murphy, EJ, Phinney, SD, Ploutz-Snyder, R, Goldman, V, Cox, RM, Mason, AE, Moran, P, Hecht, FM. Twelve-month outcomes of a randomized trial of a moderate-carbohydrate versus very lowcarbohydrate diet in overweight adults with type 2 diabetes mellitus or prediabetes. Nutr Diabetes. 2017. 7:304. doi:10.1038/s41387-017-0006-9

2093 Sauvaget, C, Nagano, J, Allen, N, Grant, EJ, Beral, V. Intake of animal products and stroke mortality in the Hiroshima/Nagasaki Life Span Study. Int J Epidemiol. 2003. 32:536-43. doi:10.1093/ije/dyg151

2094 Sauvaget, C, Nagano, J, Hayashi, M, Yamada, M. Animal protein, animal fat, and cholesterol intakes and risk of cerebral infarction mortality in the adult health study. Stroke. 2004. 35:1531-7. doi:10.1161/01.Str.0000130426.52064.09

2095 Sayer, R, Chen, N, Wright, A, Campbell, W. The effects of pork vs. Chicken/fish in a DASH diet on blood pressure control. FASEB journal. Conference: experimental biology 2014, EB. San diego, CA united states. 2014. 28:. doi:unavailable

2096 Sayer, RD, Speaker, KJ, Pan, Z, Peters, JC, Wyatt, HJ, Hill, JO. Body fat changes in the beef WISE study: beef's role in weight improvement, satisfaction, and energy. FASEB journal. 2017. 31:. doi:unavailable

2097 Sayer, RD, Speaker, KJ, Pan, Z, Peters, JC, Wyatt, HR, Hill, JO. Equivalent reductions in body weight during the Beef WISE Study: beef's role in weight improvement, satisfaction and energy. Obesity Science and Practice. 2017. 3:298-310.

Country

Study Design

Publication Status

Intervention/Exposure doi:10.1002/osp4.118

2098 Sayer, RD, Wright, AJ, Chen, N, Campbell, WW. Dietary Approaches to Stop Hypertension diet retains effectiveness to reduce blood pressure when lean pork is substituted for chicken and fish as the predominant source of protein. Am $\mathrm{J}$ Clin Nutr. 2015. 102:302-8. doi:10.3945/ajcn.115.111757

2099 Scaglioni, S, Agostoni, C, Notaris, RD, Radaelli, G, Radice, N, Valenti, M, Giovannini, M, Riva, E. Early macronutrient intake and overweight at five years of age. Int J Obes Relat Metab Disord. 2000. 24:777-81. doi:10.1038/sj.ijo.0801225

Study Design;

Intervention/Exposure

Intervention/Exposure

Intervention/Exposure

Intervention/Exposure

Publication Status

Publication Status

Weight

loss/Hypocaloric

Study duration

Intervention/Exposure 
2100 Scanlan, B, McTigue, KM, Wang, L, Winger, D, Conroy, MB. Association of eating habits with weight loss and diet self monitoring in an online weight loss trial: results from the OCELOT-PC study. Journal of general internal medicine. 2015. 30:S105. doi:unavailable

2101 Scarapicchia, TM, Sabiston, CM, Faulkner, G. Exploring the prevalence and correlates of meeting health behaviour guidelines among university students. Can J Public Health. 2015. 106:e109-14. doi:10.17269/cjph.106.4784

2102 Scarborough, P, Nnoaham, KE, Clarke, D, Capewell, S, Rayner, M. Modelling the impact of a healthy diet on cardiovascular disease and cancer mortality. J Epidemiol Community Health. 2012. 66:420-6. doi:10.1136/jech.2010.114520

2103 Scherr, R, Linnell, J, Smith, M, Nicholson, Y, Spezzano, T, Bergman, J, Brian, K, Briggs, M, Feenstra, G, Hillhouse, C, etal, . The Shaping Healthy Choices Program results in improved nutrition and health-related outcomes. FASEB journal. 2014. 28:. doi:unavailable

2104 Schioldan, AG, Gregersen, S, Hald, S, Bjornshave, A, Bohl, M, Hartmann, B, Holst, JJ, Stodkilde-Jorgensen, H, Hermansen, K. Effects of a diet rich in arabinoxylan and resistant starch compared with a diet rich in refined carbohydrates on postprandial metabolism and features of the metabolic syndrome. Eur J Nutr. 2018. 57:795-807. doi:10.1007/s00394-016-13698

2105 Schmedes, M, Balderas, C, Aadland, EK, Jacques, H, Lavigne, C, Graff, IE, Eng, O, Holthe, A, Mellgren, G, Young, JF, Sundekilde, UK, Liaset, B, Bertram, HC. The Effect of Lean-Seafood and Non-Seafood Diets on Fasting and Postprandial Serum Metabolites and Lipid Species: Results from a Randomized Crossover Intervention Study in Healthy Adults. Nutrients. 2018. 10:. doi:10.3390/nu10050598

2106 Schnabel, L, Kesse-Guyot, E, Alles, B, Touvier, M, Srour, B, Hercberg, S, Buscail, C, Julia, C. Association Between Ultraprocessed Food Consumption and Risk of Mortality Among Middle-aged Adults in France. JAMA Intern Med. 2019. 179:490-498. doi:10.1001/jamainternmed.2018.7289

2107 Schneider, BC, Dumith Sde, C, Lopes, C, Severo, M, Assuncao, MC. How Do Tracking and Changes in Dietary Pattern during Adolescence Relate to the Amount of Body Fat in Early Adulthood?. PLoS One. 2016. 11:e0149299. doi:10.1371/journal.pone.0149299

2108 Schooff, M. Are low-fat diets better than other weight-reducing diets in achieving long-term weight loss?. Am Fam Physician. 2003. 67:507-8. doi:unavailable

2109 Schrauwen, P, Wagenmakers, AJ, van Marken Lichtenbelt, WD, Saris, WH, Westerterp, KR. Increase in fat oxidation on a high-fat diet is accompanied by an increase in triglyceride-derived fatty acid oxidation. Diabetes. 2000. 49:640-6. doi:10.2337/diabetes.49.4.640

2110 Schrauwen-Hinderling, VB, Kooi, ME, Hesselink, MK, Moonen-Kornips, E, Schaart, G, Mustard, KJ, Hardie, DG, Saris, WH, Nicolay, K, Schrauwen, P. Intramyocellular lipid content and molecular adaptations in response to a 1-week high-fat diet. Obes Res. 2005. 13:2088-94. doi:10.1038/oby.2005.259

2111 Schrempft, S, van Jaarsveld, CH, Fisher, A, Wardle, J. The Obesogenic Quality of the Home Environment: Associations with Diet, Physical Activity, TV Viewing, and BMI in Preschool Children. PLoS One. 2015. 10:e0134490 doi:10.1371/journal.pone.0134490

Study Design;

Intervention/Exposure

Study Design

Publication Status

Intervention/Exposure

Intervention/Exposure; Outcome

Outcome

Country

Study Design

Study duration

Study duration

Intervention/Exposure 
2112 Schrijvers, JK, McNaughton, SA, Beck, KL, Kruger, R. Exploring the Dietary Patterns of Young New Zealand Women and Associations with BMI and Body Fat. Nutrients. 2016. 8:. doi:10.3390/nu8080450

2113 Schröder, H, Mendez, MA, Gomez, SF, Fíto, M, Ribas, L, Aranceta, J, Serra-Majem, L. Energy density, diet quality, and central body fat in a nationwide survey of young Spaniards. Nutrition. 2013. 29:1350-1355. doi:10.1016/j.nut.2013.05.019

2114 Schroder, H, Salas-Salvado, J, Martinez-Gonzalez, MA, Fito, M, Corella, D, Estruch, R, Ros, E. Baseline adherence to the Mediterranean diet and major cardiovascular events: Prevencion con Dieta Mediterranea trial. JAMA Intern Med. 2014. 174:1690-2. doi:10.1001/jamainternmed.2014.3463

2115 Schröder, H, Salas-Salvadó, J, Martínez-González, MA, Fíto, M, Corella, D, Estruch, R, Ros, E. Baseline adherence to the Mediterranean diet and major cardiovascular events: prevención con Dieta Mediterránea trial. JAMA internal medicine. 2014. 174:1690-1692. doi:10.1001/jamainternmed.2014.3463

2116 Schroeder, N, Park, YH, Kang, MS, Kim, Y, Ha, GK, Kim, HR, Yates, AA, Caballero, B. A randomized trial on the effects of 2010 Dietary Guidelines for Americans and Korean diet patterns on cardiovascular risk factors in overweight and obese adults. J Acad Nutr Diet. 2015. 115:1083-92. doi:10.1016/j.jand.2015.03.023

2117 Schulz, M, Nothlings, U, Hoffmann, K, Bergmann, MM, Boeing, H. Identification of a food pattern characterized by high-fiber and low-fat food choices associated with low prospective weight change in the EPIC-Potsdam cohort. J Nutr. 2005. 135:1183-9. doi:10.1093/jn/135.5.1183

2118 Schulze, MB, Hoffmann, K, Manson, JE, Willett, WC, Meigs, JB, Weikert, C, Heidemann, C, Colditz, GA, Hu, FB. Dietary pattern, inflammation, and incidence of type 2 diabetes in women. Am J Clin Nutr. 2005. 82:675-84; quiz 714-5. doi:10.1093/ajen.82.3.675

2119 Schumacher, TL, Burrows, TL, Cliff, DP, Jones, RA, Okely, AD, Baur, LA, Morgan, PJ, Callister, R, Boggess, MM, Collins, CE. Dietary Intake Is Related to Multifactor Cardiovascular Risk Score in Obese Boys. Healthcare (Basel). 2014. 2:282-98. doi:10.3390/healthcare2030282

2120 Schwarz, JM, Linfoot, P, Dare, D, Aghajanian, K. Hepatic de novo lipogenesis in normoinsulinemic and hyperinsulinemic subjects consuming high-fat, low-carbohydrate and low-fat, high-carbohydrate isoenergetic diets. Am J Clin Nutr. 2003. 77:4350. doi:10.1093/ajcn/77.1.43

2121 Schwenke, DC. Dietary patterns to mitigate genetic risk of obesity. Curr Opin Lipidol. 2019. 30:351-352. doi:10.1097/mol.0000000000000617

2122 Schwingshackl, L, Hoffmann, G. Monounsaturated fatty acids in the primary prevention of cardiovascular disease in overweight / obese patients and diabetics. Aktuelle ernahrungsmedizin. 2011. 36:44-49. doi:10.1055/s-0030-1265978

2123 Schwingshackl, L, Knuppel, S, Michels, N, Schwedhelm, C, Hoffmann, G, lqbal, K, De Henauw, S, Boeing, H, Devleesschauwer, B. Intake of 12 food groups and disability-adjusted life years from coronary heart disease, stroke, type 2 diabetes, and colorectal cancer in 16 European countries. Eur J Epidemiol. 2019. 34:765-775. doi:10.1007/s10654-019-00523-4

Study Design

Study Design;

Publication Status

Publication Status

Study duration

Publication Date

Overlaps with Existing Review

Publication Date

Overlaps with Existing Review

Intervention/Exposure

Study duration

Study Design; Publication Status

Language

Intervention/Exposure 
2124 Sciacqua, A, Perticone, M, Falbo, T, Grillo, N, Tassone, EJ, Sinopoli, F, Lo Russo, C, Succurro, E, Andreozzi, F, Sesti, G, Perticone, F. Dietary patterns and 1-h post-load glucose in essential hypertension. Nutr Metab Cardiovasc Dis. 2014. 24:54753. doi:10.1016/j.numecd.2013.11.005

2125 Scott, D, Blizzard, L, Fell, J, Giles, G, Jones, G. Associations between dietary nutrient intake and muscle mass and strength in community-dwelling older adults: The Tasmanian older adult cohort study. Journal of the American Geriatrics Society. 2010. 58:2129-2134. doi:10.1111/j.1532-5415.2010.03147.x

2126 Scott, LW, Balasubramanyam, A, Kimball, KT, Aherns, AK, Fordis, CM, Ballantyne, CM. Long-term, randomized clinical trial of two diets in the metabolic syndrome and type 2 diabetes. Diabetes care. 2003. 26:2481-2482. doi:10.2337/diacare.26.8.2481

2127 Scully, A, Neacsu, M, Russell, W, Vaughan, N, Fyfe, C, Hudson, M, Taylor, K, Johnstone, AM. Plant protein influence on appetite and food intake in healthy subjects. Proceedings of the nutrition society. 2017. 76:E44-. doi:10.1017/S0029665117001008

2128 Seangpraw, K, Auttama, N, Tonchoy, P, Panta, P. The effect of the behavior modification program Dietary Approaches to Stop Hypertension (DASH) on reducing the risk of hypertension among elderly patients in the rural community of Phayao, Thailand. Journal of Multidisciplinary Healthcare. 2019. 12:109-118. doi:10.2147/JMDH.S185569

2129 Sedaghat, F, Naja, F, Darand, M, Beyzai, B, Rashidkhani, B. Adherence to a Mediterranean dietary pattern and overweight and obesity among female adolescents in Iran. Int J Adolesc Med Health. 2017. 31:. doi:10.1515/ijamh-2016-0160

2130 Segal-Isaacson, CJ, Johnson, S, Tomuta, V, Cowell, B, Stein, DT. A randomized trial comparing low-fat and lowcarbohydrate diets matched for energy and protein. Obes Res. 2004. 12 Suppl 2:130s-40s. doi:10.1038/oby.2004.278

2131 Segovia-Siapco, G, Sabaté, J. Health and sustainability outcomes of vegetarian dietary patterns: a revisit of the EPIC-Oxford and the Adventist Health Study-2 cohorts. European Journal of Clinical Nutrition. 2018. :. doi:10.1038/s41430-018-0310-z

2132 Seidelmann, SB, Claggett, B, Cheng, S, Henglin, M, Shah, A, Steffen, LM, Folsom, AR, Rimm, EB, Willett, WC, Solomon, SD. Dietary carbohydrate intake and mortality: a prospective cohort study and meta-analysis. The Lancet Public Health. 2018. 3:e419-e428. doi:10.1016/S2468-2667(18)30135-X

2133 Seip, RL, Volek, JS, Windemuth, A, Kocherla, M, Fernandez, ML, Kraemer, WJ, Ruano, G. Physiogenomic comparison of human fat loss in response to diets restrictive of carbohydrate or fat. Nutr Metab (Lond). 2008. 5:4. doi:10.1186/1743-7075-5-4

2134 Sejda, T, Kovar, J, Pitha, J, Cifkova, R, Svandova, E, Poledne, R. The effect of an acute fat load on endothelial function after different dietary regimens in young healthy volunteers. Physiol Res. 2002. 51:99-105. doi:unavailable

2135 Selby, LM, Tobin, BS, Conner, BT, Gomez, M, Busch, G, Hauser, J. A quantitative, retrospective inquiry of the impact of a provider-guided low-carbohydrate, high-fat diet on adults in a wellness clinic setting. Diabetes Metab Syndr. 2019. 13:23142319. doi:10.1016/j.dsx.2019.05.031

2136 Serra, MC, Beavers, DP, Henderson, RM, Kelleher, JL, Kiel, JR, Beavers, KM. Effects of a hypocaloric, nutritionally complete, higher protein meal plan on regional body fat and cardiometabolic biomarkers in older adults with obesity. Annals of Nutrition and Metabolism. 2019. 74:149-155. doi:10.1159/000497066

\section{Study Design}

Intervention/Exposure

Intervention/Exposure

Publication Status

Publication Status

Intervention/Exposure; Comparator

Study Design

Study duration

Study Design

Intervention/Exposure; Outcome

Power/Size

Study duration

Study Design;

Intervention/Exposure

Intervention/Exposure 
2137 Serra, MC, Treuth, MS, Ryan, AS. Dietary prescription adherence and non-structured physical activity following weight loss with and without aerobic exercise. J Nutr Health Aging. 2014. 18:888-93. doi:10.1007/s12603-014-0481-9

Intervention/Exposure; Comparator

2138 Seshadri, P, Iqbal, N, Stern, L, Williams, M, Chicano, KL, Daily, DA, McGrory, J, Gracely, EJ, Rader, DJ, Samaha, FF. A randomized study comparing the effects of a low-carbohydrate diet and a conventional diet on lipoprotein subfractions and Creactive protein levels in patients with severe obesity. Am J Med. 2004. 117:398-405. doi:10.1016/j.amjmed.2004.04.009

2139 Seshadri, P, Samaha, FF, Stern, L, Ahima, RS, Daily, D, Iqbal, N. Adipocytokine changes caused by low-carbohydrate compared to conventional diets in obesity. Metab Syndr Relat Disord. 2005. 3:66-74. doi:10.1089/met.2005.3.66

2140 Setayeshgar, S, Ekwaru, JP, Maximova, K, Majumdar, SR, Storey, KE, McGavock, J, Veugelers, PJ. Dietary intake and prospective changes in cardiometabolic risk factors in children and youth. Appl Physiol Nutr Metab. 2017. 42:39-45. doi:10.1139/apnm-2016-0215

2141 Setayeshgar, S, Maximova, K, Ekwaru, JP, Gray-Donald, K, Henderson, M, Paradis, G, Tremblay, A, Veugelers, P. Diet quality as measured by the Diet Quality Index-International is associated with prospective changes in body fat among Canadian children. Public Health Nutr. 2017. 20:456-463. doi:10.1017/s1368980016002500

2142 Setayeshgar, S, Whiting, SJ, Pahwa, P, Vatanparast, H. Predicted 10-year risk of cardiovascular disease among Canadian adults using modified Framingham Risk Score in association with dietary intake. Appl Physiol Nutr Metab. 2015. 40:1068-74. doi:10.1139/apnm-2015-0074

2143 Sezaki, A, Imai, T, Miyamoto, K, Kawase, F, Shimokata, H. Mediterranean diet score and incidence of IHD: a global comparative study. Public Health Nutr. 2019. 22:1444-1450. doi:10.1017/s1368980018003877

2144 Shah, B, Newman, JD, Woolf, K, Ganguzza, L, Guo, Y, Allen, N, Zhong, J, Fisher, EA, Slater, J. Anti-Inflammatory Effects of a Vegan Diet Versus the American Heart Association-Recommended Diet in Coronary Artery Disease Trial. J Am Heart Assoc. 2018. 7:e011367. doi:10.1161/jaha.118.011367

2145 Shah, M, Adams-Huet, B, Grundy, SM, Garg, A. Effect of a high-carbohydrate vs a high-cis-monounsaturated fat diet on lipid and lipoproteins in individuals with and without type 2 diabetes. Nutrition research (new york, N.Y.). 2004. 24:969-979. doi:10.1016/j.nutres.2004.08.001

2146 Shahnazari, M, Ceresa, C, Foley, S, Fong, A, Zidaru, E, Moody, S. Nutrition-focused wellness coaching promotes a reduction in body weight in overweight US veterans. J Acad Nutr Diet. 2013. 113:928-35. doi:10.1016/j.jand.2013.04.001

2147 Shai, I, Gepner, Y, Shelef, I, Schwarzfuchs, D, Zelicha, H, Tene, L, Yaskolka Meir, A, Tsaban, G, Wolak, A, Stumvoll, M, etal, . Precision nutrition. Obesity facts. 2019. 12:1-. doi:10.1159/000489691

2148 Shai, I, Schwarzfuchs, D, Henkin, Y, Shahar, DR, Witkow, S, Greenberg, I, Golan, R, Fraser, D, Bolotin, A, Vardi, H, Tangi-Rozental, O, Zuk-Ramot, R, Sarusi, B, Brickner, D, Schwartz, Z, Sheiner, E, Marko, R, Katorza, E, Thiery, J, Fiedler, GM, Bluher, M, Stumvoll, M, Stampfer, MJ. Weight loss with a low-carbohydrate, Mediterranean, or low-fat diet. N Engl J Med. 2008. 359:229-41. doi:10.1056/NEJMoa0708681

2149 Shai, I. The effect of low-carb, mediterranean and low-fat diets on renal function; a 2-year dietary intervention randomized controlled trial (direct). Obesity facts. 2012. 5:19-. doi:10.1159/000171026
Power/Size; Weight

loss/Hypocaloric

Power/Size; Weight loss/Hypocaloric

Intervention/Exposure

Power/Size

Study Design

Study Design

Intervention/Exposure

Health Status

Study duration

Intervention/Exposure

Publication Status

Study duration ;

Health Status

Publication Status 
2150 Shang, X, Scott, D, Hodge, A, English, DR, Giles, GG, Ebeling, PR, Sanders, KM. Dietary protein from different food sources, incident metabolic syndrome and changes in its components: An 11-year longitudinal study in healthy communitydwelling adults. Clin Nutr. 2017. 36:1540-1548. doi:10.1016/j.clnu.2016.09.024

2151 Sharafi, M, Duffy, VB, Miller, RJ, Winchester, SB, Huedo-Medina, TB, Sullivan, MC. Dietary behaviors of adults born prematurely may explain future risk for cardiovascular disease. Appetite. 2016. 99:157-167. doi:10.1016/j.appet.2016.01.007

2152 Shariff, ZM, Lin, KG, Sariman, S, Siew, CY, Yusof, BN, Mun, CY, Lee, HS, Mohamad, M. Higher Dietary Energy Density is Associated with Stunting but not Overweight and Obesity in a Sample of Urban Malaysian Children. Ecol Food Nutr. 2016. 55:378-89. doi:10.1080/03670244.2016.1181065

2153 Sharman, MJ, Gomez, AL, Kraemer, WJ, Volek, JS. Very low-carbohydrate and low-fat diets affect fasting lipids and postprandial lipemia differently in overweight men. J Nutr. 2004. 134:880-5. doi:10.1093/jn/134.4.880

2154 Sharman, MJ, Kraemer, WJ, Love, DM, Avery, NG, Gomez, AL, Scheett, TP, Volek, JS. A ketogenic diet favorably affects serum biomarkers for cardiovascular disease in normal-weight men. J Nutr. 2002. 132:1879-85. doi:10.1093/jn/132.7.1879

2155 Sharman, MJ, Volek, JS. Weight loss leads to reductions in inflammatory biomarkers after a very-low-carbohydrate diet and a low-fat diet in overweight men. Clin Sci (Lond). 2004. 107:365-9. doi:10.1042/cs20040111

2156 Sharrett, AR, Heiss, G, Chambless, LE, Boerwinkle, E, Coady, SA, Folsom, AR, Patsch, W. Metabolic and lifestyle determinants of postprandial lipemia differ from those of fasting triglycerides: The Atherosclerosis Risk In Communities (ARIC) study. Arterioscler Thromb Vasc Biol. 2001. 21:275-81. doi:10.1161/01.atv.21.2.275

2157 Shay, CM, Stamler, J, Dyer, AR, Brown, IJ, Chan, Q, Elliott, P, Zhao, L, Okuda, N, Miura, K, Daviglus, ML, Van Horn, L. Nutrient and food intakes of middle-aged adults at low risk of cardiovascular disease: the international study of macro/micronutrients and blood pressure (INTERMAP). Eur J Nutr. 2012. 51:917-26. doi:10.1007/s00394-011-0268-2

2158 Sheikh, VK, Raynor, HA. Decreases in High-Fat and/or High-Added-Sugar Food Group Intake Occur when a Hypocaloric, Low-Fat Diet Is Prescribed Within a Lifestyle Intervention: A Secondary Cohort Analysis. J Acad Nutr Diet. 2016. 116:1599-605. doi:10.1016/j.jand.2016.06.007

2159 Shepard, TY, Weil, KM, Sharp, TA, Grunwald, GK, Bell, ML, Hill, JO, Eckel, RH. Occasional physical inactivity combined with a high-fat diet may be important in the development and maintenance of obesity in human subjects. Am $\mathrm{J}$ Clin Nutr. 2001. 73:703-8. doi:10.1093/ajcn/73.4.703

2160 Sherafat-Kazemzadeh, R, Egtesadi, S, Mirmiran, P, Gohari, M, Farahani, SJ, Esfahani, FH, Vafa, MR, Hedayati, M, Azizi, F. Dietary patterns by reduced rank regression predicting changes in obesity indices in a cohort study: Tehran Lipid and Glucose Study. Asia Pac J Clin Nutr. 2010. 19:22-32. doi:unavailable

2161 Sherwood, NE, Jeffery, RW, French, SA, Hannan, PJ, Murray, DM. Predictors of weight gain in the Pound of Prevention study. Int J Obes Relat Metab Disord. 2000. 24:395-403. doi:10.1038/sj.ijo.0801169

2162 Shi, L, Brunius, C, Johansson, I, Bergdahl, IA, Lindahl, B, Hanhineva, K, Landberg, R. Plasma metabolites associated with healthy Nordic dietary indexes and risk of type 2 diabetes-a nested case-control study in a Swedish population. Am J Clin Nutr. 2018. 108:564-575. doi:10.1093/ajcn/nqy145

Study Design;

Intervention/Exposure

Study duration

Study duration

Outcome

Intervention/Exposure

Study Design

Outcome

Intervention/Exposure

Study duration

Country

Intervention/Exposure

Intervention/Exposure 
2163 Shi, Z, Papier, K, Yiengprugsawan, V, Kelly, M, Seubsman, SA, Sleigh, AC. Dietary patterns associated with hypertension risk among adults in Thailand: 8-year findings from the Thai Cohort Study. Public Health Nutr. 2019. 22:307-313. doi:10.1017/s1368980018002203

2164 Shi, Z, Zhen, S, Zimmet, PZ, Zhou, Y, Zhou, Y, Magliano, DJ, Taylor, AW. Association of impaired fasting glucose, diabetes Country and dietary patterns with mortality: a 10-year follow-up cohort in Eastern China. Acta Diabetol. 2016. 53:799-806. doi:10.1007/s00592-016-0875-8

2165 Shih, CW, Hauser, ME, Aronica, L, Rigdon, J, Gardner, CD. Changes in blood lipid concentrations associated with changes in intake of dietary saturated fat in the context of a healthy low-carbohydrate weight-loss diet: a secondary analysis of the Diet Intervention Examining The Factors Interacting with Treatment Success (DIETFITS) trial. Am J Clin Nutr. 2019. 109:433-441. doi:10.1093/ajcn/nqy305

2166 Shikany, JM, Margolis, KL, Pettinger, M, Jackson, RD, Limacher, MC, Liu, S, Phillips, LS, Tinker, LF. Effects of a low-fat dietary intervention on glucose, insulin, and insulin resistance in the Women's Health Initiative (WHI) Dietary Modification trial. Am J Clin Nutr. 2011. 94:75-85. doi:10.3945/ajcn.110.010843

2167 Shikany, JM, Phadke, RP, Redden, DT, Gower, BA. Effects of low- and high-glycemic index/glycemic load diets on coronary heart disease risk factors in overweight/obese men. Metabolism. 2009. 58:1793-801. doi:10.1016/j.metabol.2009.06.006

2168 Shikany, JM, Safford, MM, Bryan, J, Newby, PK, Richman, JS, Durant, RW, Brown, TM, Judd, SE. Dietary Patterns and Mediterranean Diet Score and Hazard of Recurrent Coronary Heart Disease Events and All-Cause Mortality in the REGARDS Study. J Am Heart Assoc. 2018. 7:. doi:10.1161/jaha.117.008078

2169 Shimabukuro, M, Chinen, I, Higa, N, Takasu, N, Yamakawa, K, Ueda, S. Effects of dietary composition on postprandial endothelial function and adiponectin concentrations in healthy humans: a crossover controlled study. Am J Clin Nutr. 2007. 86:923-8. doi:10.1093/ajcn/86.4.923

2170 Shimoda, T, Suzuki, T, Takahashi, N, Tsutsumi, K, Samukawa, M, Yoshimachi, S, Goto, T, Enomoto, H, Kise, N, Ogasawara, K, Yoshimura, S. Nutritional Status and Body Composition of Independently Living Older Adults in a Snowy Region of Japan. Gerontol Geriatr Med. 2017. 3:2333721417706854. doi:10.1177/2333721417706854

2171 Shin, D, Lee, KW, Song, WO. Dietary Patterns during Pregnancy Are Associated with Risk of Gestational Diabetes Mellitus. Nutrients. 2015. 7:9369-82. doi:10.3390/nu7115472

2172 Shin, JH, Jung, S, Kim, SA, Kang, MS, Kim, MS, Joung, H, Hwang, GS, Shin, DM. Differential Effects of Typical Korean Versus American-Style Diets on Gut Microbial Composition and Metabolic Profile in Healthy Overweight Koreans: A Randomized Crossover Trial. Nutrients. 2019. 11:. doi:10.3390/nu11102450

2173 Shin, KO, Oh, SY, Park, HS. Empirically derived major dietary patterns and their associations with overweight in Korean preschool children. Br J Nutr. 2007. 98:416-21. doi:10.1017/s0007114507720226

Intervention/Exposure; Outcome

Intervention/Exposure; Publication Date

Overlaps with Existing Review

Health Status

Study duration

Study Design;

Intervention/Exposure

Outcome; Participants

Outcome

Overlaps with Existing

Review 
2174 Shin, PK, Kim, MS, Lee, HJ, Park, SJ, Kwon, DY, Choi, SW. A traditional Korean diet reduced total blood cholesterol and increased telomere length of peripheral blood cells in perimenopausal Korean women. FASEB journal. 2017. 31: doi:unavailable

2175 Shin, S, Lee, S. Association between Total Diet Quality and Metabolic Syndrome Incidence Risk in a Prospective Cohort of Korean Adults. Clin Nutr Res. 2019. 8:46-54. doi:10.7762/cnr.2019.8.1.46

2176 Shinozaki, K, Okuda, M, Sasaki, S, Kunitsugu, I, Shigeta, M. Dietary Fiber Consumption Decreases the Risks of Overweight and Hypercholesterolemia in Japanese Children. Ann Nutr Metab. 2015. 67:58-64. doi:10.1159/000434634

2177 Shintani, TT, Beckham, S, Brown, AC, O'Connor, HK. The Hawaii Diet: ad libitum high carbohydrate, low fat multi-cultural diet for the reduction of chronic disease risk factors: obesity, hypertension, hypercholesterolemia, and hyperglycemia. Hawaii Med J. 2001. 60:69-73. doi:unavailable

2178 Shirai, K. Ideal body mass index determined by mortality in Europe, and adequate high protein and low carbohydrate diet to maintain bodyweight. J Diabetes Investig. 2011. 2:421-2. doi:10.1111/j.2040-1124.2011.00145.x

2179 Shivappa, N, Steck, SE, Hussey, JR, Ma, Y, Hebert, JR. Inflammatory potential of diet and all-cause, cardiovascular, and cancer mortality in National Health and Nutrition Examination Survey III Study. Eur J Nutr. 2017. 56:683-692. doi:10.1007/s00394-015-1112-x

2180 Shlisky, JD, Durward, CM, Zack, MK, Gugger, CK, Campbell, JK, Nickols-Richardson, SM. An energy-reduced dietary pattern, including moderate protein and increased nonfat dairy intake combined with walking promotes beneficial body composition and metabolic changes in women with excess adiposity: a randomized comparative trial. Food Sci Nutr. 2015 3:376-93. doi:10.1002/fsn3.231

2181 Shmaya, Y, Eilat-Adar, S, Leitner, Y, Reif, S, Gabis, L. Nutritional deficiencies and overweight prevalence among children with autism spectrum disorder. Res Dev Disabil. 2015. 38:1-6. doi:10.1016/j.ridd.2014.11.020

2182 Shroff, MR, Perng, W, Baylin, A, Mora-Plazas, M, Marin, C, Villamor, E. Adherence to a snacking dietary pattern and soda intake are related to the development of adiposity: a prospective study in school-age children. Public Health Nutr. 2014. 17:1507-13. doi:10.1017/s136898001300133x

2183 Shu, L, Shen, XM, Li, C, Zhang, XY, Zheng, PF. Dietary patterns are associated with type 2 diabetes mellitus among middle- Study Design aged adults in Zhejiang Province, China. Nutr J. 2017. 16:81. doi:10.1186/s12937-017-0303-0

2184 Shyam, S, Arshad, F, Abdul Ghani, R, Wahab, NA, Safii, NS, Nisak, MY, Chinna, K, Kamaruddin, NA. Low glycaemic index diets improve glucose tolerance and body weight in women with previous history of gestational diabetes: a six months randomized trial. Nutr J. 2013. 12:68. doi:10.1186/1475-2891-12-68

2185 Shyam, S, Arshad, F, Ghani, RA, Wahab, NA, Mohd Yusof, BN, Safii, NS, Chinna, K, Kamaruddin, NA. Low glycaemic index diet aids management of fasting blood sugar and body weight in asian women with previous history of gestational diabetes mellitus. Journal of diabetes. 2013. 5:27. doi:10.1111/1753-0407.12032

2186 Sichieri, R, Moura, AS, Genelhu, V, Hu, F, Willett, WC. An 18-mo randomized trial of a low-glycemic-index diet and weight change in Brazilian women. Am J Clin Nutr. 2007. 86:707-13. doi:10.1093/ajcn/86.3.707

Outcome

Study Design; Intervention/Exposure

Study Design

Study Design

Intervention/Exposure

Intervention/Exposure

Study Design

Country

Intervention/Exposure

Publication Status

Intervention/Exposure 


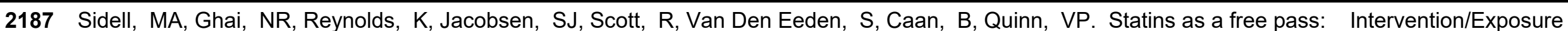
Body mass index and other cardiovascular risk factors among lipid-lowering medication users and nonusers in the California Men's Health Study. Prev Med. 2019. 129:105822. doi:10.1016/j.ypmed.2019.105822

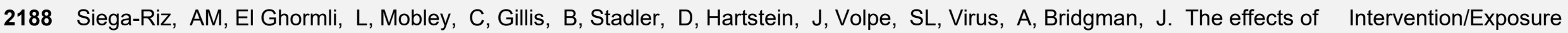
the HEALTHY study intervention on middle school student dietary intakes. Int J Behav Nutr Phys Act. 2011. 8:7. doi:10.1186/1479-5868-8-7 diet intervention in obese teens. Clin Pediatr (Phila). 2009. 48:745-9. doi:10.1177/0009922809332585

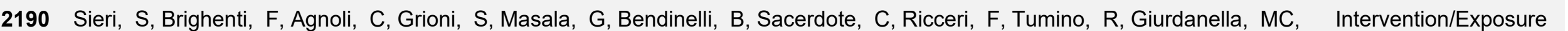
Pala, V, Berrino, F, Mattiello, A, Chiodini, P, Panico, S, Krogh, V. Dietary glycemic load and glycemic index and risk of cerebrovascular disease in the EPICOR cohort. PLoS One. 2013. 8:e62625. doi:10.1371/journal.pone.0062625

2191 Sieri, S, Krogh, V, Berrino, F, Evangelista, A, Agnoli, C, Brighenti, F, Pellegrini, N, Palli, D, Masala, G, Sacerdote, C, Veglia, F, Tumino, R, Frasca, G, Grioni, S, Pala, V, Mattiello, A, Chiodini, P, Panico, S. Dietary glycemic load and index and risk of coronary heart disease in a large italian cohort: the EPICOR study. Arch Intern Med. 2010. 170:640-7. doi:10.1001/archinternmed.2010.15

2192 Sijtsma, FP, Soedamah-Muthu, SS, de Hoon, SE, Jacobs, DR, Jr, Kromhout, D. Healthy eating and survival among elderly men with and without cardiovascular-metabolic diseases. Nutr Metab Cardiovasc Dis. 2015. 25:1117-24. doi:10.1016/j.numecd.2015.08.008

2193 Silaste, ML, Rantala, M, Alfthan, G, Aro, A, Witztum, JL, Kesaniemi, YA, Horkko, S. Changes in dietary fat intake alter plasma levels of oxidized low-density lipoprotein and lipoprotein(a). Arterioscler Thromb Vasc Biol. 2004. 24:498-503. doi:10.1161/01.ATV.0000118012.64932.f4

2194 Silbernagel, G, Kovarova, M, Cegan, A, Machann, J, Schick, F, Lehmann, R, Haring, HU, Stefan, N, Schleicher, E, Fritsche, A, Peter, A. High hepatic SCD1 activity is associated with low liver fat content in healthy subjects under a lipogenic diet. J Clin Endocrinol Metab. 2012. 97:E2288-92. doi:10.1210/jc.2012-2152

2195 Silva, FM, Giatti, L, de Figueiredo, RC, Molina, Mdcb, de Oliveira Cardoso, L, Duncan, BB, Barreto, SM. Consumption of ultra-processed food and obesity: cross sectional results from the Brazilian Longitudinal Study of Adult Health (ELSA-Brasil) cohort (2008-2010). Public Health Nutr. 2018. 21:2271-2279. doi:10.1017/s1368980018000861

2196 Silva, KF, Prata, A, da Cunha, DF. Frequency of metabolic syndrome and the food intake patterns in adults living in a rural area of Brazil. Revista da Sociedade Brasileira de Medicina Tropical. 2011. 44:425-429. doi:10.1590/S003786822011000400005

2197 Silveira, BKS, de Novaes, JF, Reis, NA, Lourenco, LP, Capobiango, AHM, Vieira, SA, Hermsdorff, HHM. "Traditional" and "Healthy" Dietary Patterns Are Associated with Low Cardiometabolic Risk in Brazilian Subjects. Cardiol Res Pract. 2018. 2018:4585412. doi:10.1155/2018/4585412

Power/Size

Intervention/Exposure; Comparator

Study Design; Intervention/Exposure; Outcome

Study Design

Study Design

Study Design 
2198 Silventoinen, K, Hasselbalch, AL, Lallukka, T, Bogl, L, Pietilainen, KH, Heitmann, BL, Schousboe, K, Rissanen, A, Kyvik,

$\mathrm{KO}$, Sorensen, TI, Kaprio, J. Modification effects of physical activity and protein intake on heritability of body size and composition. Am J Clin Nutr. 2009. 90:1096-103. doi:10.3945/ajcn.2009.27689

2199 Silver, HJ, Kang, H, Keil, CD, Muldowney, JA, 3rd, Kocalis, H, Fazio, S, Vaughan, DE, Niswender, KD. Consuming a balanced high fat diet for 16 weeks improves body composition, inflammation and vascular function parameters in obese premenopausal women. Metabolism. 2014. 63:562-73. doi:10.1016/j.metabol.2014.01.004

2200 Simila, ME, Kontto, JP, Mannisto, S, Valsta, LM, Virtamo, J. Glycaemic index, carbohydrate substitution for fat and risk of CHD in men. Br J Nutr. 2013. 110:1704-11. doi:10.1017/s0007114513000858

2201 Simila, ME, Valsta, LM, Kontto, JP, Albanes, D, Virtamo, J. Low-, medium- and high-glycaemic index carbohydrates and risk of type 2 diabetes in men. Br J Nutr. 2011. 105:1258-64. doi:10.1017/s000711451000485x

2202 Simpson, EJ, Clark, M, Razak, A, Salter, A. Reduced meat consumption and its effect on serum lipid profile; An intervention trial in healthy volunteers. Proceedings of the nutrition society. 2017. 76:E54-. doi:10.1017/S0029665117001276

2203 Simpson, EJ, Holdsworth, M, Macdonald, IA. Interstitial glucose profile associated with symptoms attributed to hypoglycemia by otherwise healthy women. Am J Clin Nutr. 2008. 87:354-61. doi:10.1093/ajcn/87.2.354

2204 Singh, PN, Clark, RW, Herring, P, Sabate, J, Shavlik, D, Fraser, GE. Obesity and life expectancy among long-lived Black adults. J Gerontol A Biol Sci Med Sci. 2014. 69:63-72. doi:10.1093/gerona/glt049

2205 Singh-Manoux, A, Fayosse, A, Sabia, S, Tabak, A, Shipley, M, Dugravot, A, Kivimaki, M. Clinical, socioeconomic, and behavioural factors at age 50 years and risk of cardiometabolic multimorbidity and mortality: A cohort study. PLoS Med. 2018. 15:e1002571. doi:10.1371/journal.pmed.1002571

2206 Siriwoen, R, Chongsuwat, R, Tansakul, S, Siri, S. Effectiveness of a Weight Management Program Applying Mobile Health Technology as a Supporting Tool for Overweight and Obese Working Women. Asia Pac J Public Health. 2018. 30:572-581. doi:10.1177/1010539518800367

2207 Sjaarda, LA, Schisterman, EF, Schliep, KC, Plowden, T, Zarek, SM, Yeung, E, Wactawski-Wende, J, Mumford, SL. Dietary Carbohydrate Intake Does Not Impact Insulin Resistance or Androgens in Healthy, Eumenorrheic Women. J Clin Endocrinol Metab. 2015. 100:2979-86. doi:10.1210/jc.2015-1957

2208 Sjogren, P, Becker, W, Warensjo, E, Olsson, E, Byberg, L, Gustafsson, IB, Karlstrom, B, Cederholm, T. Mediterranean and carbohydrate-restricted diets and mortality among elderly men: a cohort study in Sweden. Am J Clin Nutr. 2010. 92:967-74. doi:10.3945/ajcn.2010.29345

2209 Sjostrom, L, Rissanen, A, Andersen, T, Boldrin, M, Golay, A, Koppeschaar, H, Krempf, M. Randomized placebo-controlled trial of orlistat for weight loss and prevention of weight regain in obese patients. Terapevticheskii arkhiv. 2000. 72:50-54. doi:unavailable

2210 Skeaff, CM, Hodson, L, McKenzie, JE. Dietary-induced changes in fatty acid composition of human plasma, platelet, and erythrocyte lipids follow a similar time course. J Nutr. 2006. 136:565-9. doi:10.1093/jn/136.3.565

Intervention/Exposure; Comparator

Intervention/Exposure

Intervention/Exposure

Publication Status

Study duration

Intervention/Exposure

Intervention/Exposure

Study Design

\section{Study Design;}

Outcome

Intervention/Exposure; Publication Date

Overlaps with Existing Review

Intervention/Exposure Study duration 
2211 Skeaff, CM, Thoma, C, Mann, J, Chisholm, A, Williams, S, Richmond, K. Isocaloric substitution of plant sterol-enriched fat spread for carbohydrate-rich foods in a low-fat, fibre-rich diet decreases plasma low-density lipoprotein cholesterol and increases high-density lipoprotein concentrations. Nutr Metab Cardiovasc Dis. 2005. 15:337-44. doi:10.1016/j.numecd.2005.03.002

2212 Skinner, JD, Bounds, W, Carruth, BR, Morris, M, Ziegler, P. Predictors of children's body mass index: a longitudinal study of diet and growth in children aged 2-8 y. Int J Obes Relat Metab Disord. 2004. 28:476-82. doi:10.1038/sj.ijo.0802405

2213 Skoog, M, Xu, N, Berggren-Soderlund, M, Lovegrove, JA, Nilsson-Ehle, P. ACTH reduces the rise in ApoB-48 levels after fat intake. Atherosclerosis. 2007. 191:433-9. doi:10.1016/j.atherosclerosis.2006.05.012

2214 Skov, AR, Haulrik, N, Toubro, S, Molgaard, C, Astrup, A. Effect of protein intake on bone mineralization during weight loss: a 6-month trial. Obes Res. 2002. 10:432-8. doi:10.1038/oby.2002.60

2215 Skreden, M, Hillesund, ER, Wills, AK, Brantsaeter, AL, Bere, E, Overby, NC. Adherence to the New Nordic Diet during pregnancy and subsequent maternal weight development: a study conducted in the Norwegian Mother and Child Cohort Study (MoBa). Br J Nutr. 2018. 119:1286-1294. doi:10.1017/s0007114518000776

2216 Slavicek, J, Kittnar, O, Fraser, GE, Medova, E, Konecna, J, Zizka, R, Dohnalova, A, Novak, V. Lifestyle decreases risk factors for cardiovascular diseases. Cent Eur J Public Health. 2008. 16:161-4. doi:unavailable

2217 Sloth, B, Due, A, Larsen, TM, Holst, JJ, Heding, A, Astrup, A. The effect of a high-MUFA, low-glycaemic index diet and a low-fat diet on appetite and glucose metabolism during a 6-month weight maintenance period. Br J Nutr. 2009. 101:1846-58. doi:10.1017/s0007114508137710

2218 Sluik, D, Brouwer-Brolsma, EM, Berendsen, AAM, Mikkila, V, Poppitt, SD, Silvestre, MP, Tremblay, A, Perusse, L, Bouchard, C, Raben, A, Feskens, EJM. Protein intake and the incidence of pre-diabetes and diabetes in 4 population-based studies: the PREVIEW project. Am J Clin Nutr. 2019. 109:1310-1318. doi:10.1093/ajcn/nqy388

2219 Smith, CE, Tucker, KL, Lee, YC, Lai, CQ, Parnell, LD, Ordovas, JM. Low-density lipoprotein receptor-related protein 1 variant interacts with saturated fatty acids in Puerto Ricans. Obesity (Silver Spring). 2013. 21:602-8. doi:10.1002/oby.20001

2220 Smith, GI, Yoshino, J, Kelly, SC, Reeds, DN, Okunade, A, Patterson, BW, Klein, S, Mittendorfer, B. High-Protein Intake during Weight Loss Therapy Eliminates the Weight-Loss-Induced Improvement in Insulin Action in Obese Postmenopausal Women. Cell Rep. 2016. 17:849-861. doi:10.1016/j.celrep.2016.09.047

2221 Smith, JD, Hou, T, Ludwig, DS, Rimm, EB, Willett, W, Hu, FB, Mozaffarian, D. Changes in intake of protein foods, carbohydrate amount and quality, and long-term weight change: results from 3 prospective cohorts. Am J Clin Nutr. 2015. 101:1216-24. doi:10.3945/ajcn.114.100867

2222 Smith, R. Are some diets "mass murder"?. Bmj. 2014. 349:g7654. doi:10.1136/bmj.g7654

2223 Smith, SR, De Jonge, L, Zachwieja, JJ, Roy, H, Nguyen, T, Rood, JC, Windhauser, MM, Bray, GA. Fat and carbohydrate balances during adaptation to a high-fat diet. American Journal of Clinical Nutrition. 2000. 71:450-457. doi:unavailable

Study Design; Publication Status

Intervention/Exposure; Comparator

Study duration

Outcome; Weight loss/Hypocaloric Study duration

Age at

Intervention/Exposure; Participants

Study duration

Power/Size

Intervention/Exposure

Intervention/Exposure

Intervention/Exposure; Outcome

Intervention/Exposure Study duration 
2224 Smith, SR, de Jonge, L, Zachwieja, JJ, Roy, H, Nguyen, T, Rood, JC, Windhauser, MM, Bray, GA. Fat and carbohydrate balances during adaptation to a high-fat. Am J Clin Nutr. 2000. 71:450-7. doi:10.1093/ajcn/71.2.450

2225 Snetselaar, L, Stumbo, P, Chenard, C, Ahrens, L, Smith, K, Zimmerman, B. Adolescents eating diets rich in either lean beef or lean poultry and fish reduced fat and saturated fat intake and those eating beef maintained serum ferritin status. $\mathrm{J}$ Am Diet Assoc. 2004. 104:424-8. doi:10.1016/j.jada.2003.12.016

2226 Soare, A, Khazrai, YM, Fontana, L, Del Toro, R, Lazzaro, MC, Di Rosa, C, Buldo, A, Fioriti, E, Maddaloni, E, Angeletti, S, Di Mauro, A, Gesuita, R, Skrami, E, Tuccinardi, D, Fallucca, S, Pianesi, M, Pozzilli, P. Treatment of reactive hypoglycemia with the macrobiotic Ma-pi 2 diet as assessed by continuous glucose monitoring: The MAHYP randomized crossover trial. Metabolism. 2017. 69:148-156. doi:10.1016/j.metabol.2017.01.023

2227 Soares, TS, Piovesan, CH, Gustavo Ada, S, Macagnan, FE, Bodanese, LC, Feoli, AM. Alimentary habits, physical activity, and Framingham global risk score in metabolic syndrome. Arq Bras Cardiol. 2014. 102:374-82. doi:10.5935/abc.20140029

2228 Soderstrom, L, Rosenblad, A, Adolfsson, ET, Wolk, A, Hakansson, N, Bergkvist, L. A high energy intake from dietary fat among middle-aged and older adults is associated with increased risk of malnutrition 10 years later. Br J Nutr. 2015. 114:91523. doi:10.1017/s0007114515002317

2229 Soenen, S, Bonomi, AG, Lemmens, SG, Scholte, J, Thijssen, MA, van Berkum, F, Westerterp-Plantenga, MS. Relatively high-protein or 'low-carb' energy-restricted diets for body weight loss and body weight maintenance?. Physiol Behav. 2012. 107:374-80. doi:10.1016/j.physbeh.2012.08.004

2230 Soenen, S, Martens, EA, Hochstenbach-Waelen, A, Lemmens, SG, Westerterp-Plantenga, MS. Normal protein intake is required for body weight loss and weight maintenance, and elevated protein intake for additional preservation of resting energy expenditure and fat free mass. J Nutr. 2013. 143:591-6. doi:10.3945/jn.112.167593

2231 Soenen, S, Westerterp-Plantenga, MS. Changes in body fat percentage during body weight stable conditions of increased daily protein intake vs. control. Physiol Behav. 2010. 101:635-8. doi:10.1016/j.physbeh.2010.09.014

2232 Sofi, F, Dinu, M, Pagliai, G, Cesari, F, Gori, AM, Sereni, A, Becatti, M, Fiorillo, C, Marcucci, R, Casini, A. Low-Calorie Vegetarian Versus Mediterranean Diets for Reducing Body Weight and Improving Cardiovascular Risk Profile: CARDIVEG Study (Cardiovascular Prevention With Vegetarian Diet). Circulation. 2018. 137:1103-1113. doi:10.1161/circulationaha.117.030088

2233 Sofi, F, Giorgi, G, Cesari, F, Gori, AM, Mannini, L, Parisi, G, Casini, A, Abbate, R, Gensini, GF, Poli, BM. The atherosclerotic risk profile is affected differently by fish flesh with a similar EPA and DHA content but different $n-6 / n-3$ ratio. Asia Pac J Clin Nutr. 2013. 22:32-40. doi:10.6133/apjen.2013.22.1.12

2234 Somaraki, M, Eli, K, Ek, A, Sandvik, P, Sorjonen, K, Nowicka, P. Changes in parental feeding practices and children's food intake: a randomized control trial of obesity treatment in preschoolers. Obesity research \& clinical practice. 2019. 13:323-. doi:10.1016/j.orcp.2018.11.236

2235 Sondike, SB, Copperman, N, Jacobson, MS. Effects of a low-carbohydrate diet on weight loss and cardiovascular risk factor in overweight adolescents. J Pediatr. 2003. 142:253-8. doi:10.1067/mpd.2003.4

Intervention/Exposure

Study duration

Intervention/Exposure

Intervention/Exposure; Outcome

Intervention/Exposure

Intervention/Exposure

Intervention/Exposure

Weight

loss/Hypocaloric

Intervention/Exposure

Publication Status

Power/Size 
2236 Sonestedt, E, Lyssenko, V, Ericson, U, Gullberg, B, Wirfalt, E, Groop, L, Orho-Melander, M. Genetic variation in the glucose-dependent insulinotropic polypeptide receptor modifies the association between carbohydrate and fat intake and risk of type 2 diabetes in the Malmo Diet and Cancer cohort. J Clin Endocrinol Metab. 2012. 97:E810-8. doi:10.1210/jc.2011-2444

2237 Song, S, Kim, J, Kim, J. Gender Differences in the Association between Dietary Pattern and the Incidence of Hypertension in Middle-Aged and Older Adults. Nutrients. 2018. 10:. doi:10.3390/nu10020252

2238 Song, S, Song, WO, Song, Y. Dietary carbohydrate and fat intakes are differentially associated with lipid abnormalities in Korean adults. J Clin Lipidol. 2017. 11:338-347.e3. doi:10.1016/j.jacl.2017.01.016

2239 Soto Rodríguez, A, García Soidán, JL, de Toro Santos, M, Lagoa Labrador, F, Failde Garrido, JM, Pérez Fernández, MR. Benefits of an educational intervention on diet and anthropometric profile of women with one cardiovascular risk factor. Medicina clinica. 2016. 146:436-439. doi:10.1016/j.medcli.2015.12.013

2240 Soto-Estrada, G, Moreno Altamirano, L, Garcia-Garcia, JJ, Ochoa Moreno, I, Silberman, M. Trends in frequency of type 2 diabetes in Mexico and its relationship to dietary patterns and contextual factors. Gac Sanit. 2018. 32:283-290. doi:10.1016/j.gaceta.2017.08.001

2241 Sotos-Prieto, M, Luben, R, Khaw, KT, Wareham, NJ, Forouhi, NG. The association between Mediterranean Diet Score and glucokinase regulatory protein gene variation on the markers of cardiometabolic risk: an analysis in the European Prospective Investigation into Cancer (EPIC)-Norfolk study. Br J Nutr. 2014. 112:122-31. doi:10.1017/s0007114514000580

2242 Spengler, S, Mess, F, Schmocker, E, Woll, A. Longitudinal associations of health-related behavior patterns in adolescence with change of weight status and self-rated health over a period of 6 years: results of the MoMo longitudinal study. BMC Pediatr. 2014. 14:242. doi:10.1186/1471-2431-14-242

2243 Spieth, LE, Harnish, JD, Lenders, CM, Raezer, LB, Pereira, MA, Hangen, SJ, Ludwig, DS. A low-glycemic index diet in the treatment of pediatric obesity. Arch Pediatr Adolesc Med. 2000. 154:947-51. doi:10.1001/archpedi.154.9.947

2244 Spiller, GA, Miller, A, Olivera, K, Reynolds, J, Miller, B, Morse, SJ, Dewell, A, Farquhar, JW. Effects of plant-based diets high in raw or roasted almonds, or roasted almond butter on serum lipoproteins in humans. J Am Coll Nutr. 2003. 22:195-200. doi:10.1080/07315724.2003.10719293

2245 Spring, B, Moller, AC, Colangelo, LA, Siddique, J, Roehrig, M, Daviglus, ML, Polak, JF, Reis, JP, Sidney, S, Liu, K. Healthy lifestyle change and subclinical atherosclerosis in young adults: Coronary Artery Risk Development in Young Adults (CARDIA) study. Circulation. 2014. 130:10-7. doi:10.1161/circulationaha.113.005445

2246 Sta hl, M, Hulthen, L, Manousou, S, Larsson, C, Lindahl, B, Mellberg, C, Olsson, T, Ryberg, M, Sandberg, S, Filipsson Nystrom, H. A modified paleolithic diet results in iodine deficiency-a study of 24 hour urinary iodine excretion in postmenopausal overweight and obese women in Sweden. Thyroid. 2013. 23:A23-A24. doi:10.1089/thy.2013.2310.abs

2247 Stang, J, Zephier, EM, Story, M, Himes, JH, Yeh, JL, Welty, T, Howard, BV. Dietary intakes of nutrients thought to modify cardiovascular risk from three groups of American Indians: The Strong Heart Dietary Study, Phase II. J Am Diet Assoc. 2005. 105:1895-903. doi:10.1016/j.jada.2005.09.003 
2248 Stanhope, KL, Bremer, AA, Medici, V, Nakajima, K, Ito, Y, Nakano, T, Chen, G, Fong, TH, Lee, V, Menorca, RI, Keim,

Intervention/Exposure; NL, Havel, PJ. Consumption of fructose and high fructose corn syrup increase postprandial triglycerides, LDL-cholesterol, and Study duration apolipoprotein-B in young men and women. J Clin Endocrinol Metab. 2011. 96:E1596-605. doi:10.1210/jc.2011-1251

2249 Steer, CD, Lattka, E, Koletzko, B, Golding, J, Hibbeln, JR. Maternal fatty acids in pregnancy, FADS polymorphisms, and child intelligence quotient at 8 y of age. Am J Clin Nutr. 2013. 98:1575-82. doi:10.3945/ajcn.112.051524

2250 Steffen, LM, Hootman, KC. A posteriori data-derived dietary patterns and incident coronary heart disease: Making sense of inconsistent findings. Curr Nutr Rep. 2016. 5:168-179. doi:10.1007/s13668-016-0176-4

2251 Steffen, LM, Jacobs, DR, Jr, Murtaugh, MA, Moran, A, Steinberger, J, Hong, CP, Sinaiko, AR. Whole grain intake is associated with lower body mass and greater insulin sensitivity among adolescents. Am J Epidemiol. 2003. 158:243-50. doi:10.1093/aje/kwg146

2252 Steffen, LM. A variety of food and drink improves CVD profile. Br J Nutr. 2009. 101:305-6. doi:10.1017/s0007114508076319

2253 Stefler, D, Pikhart, H, Jankovic, N, Kubinova, R, Pajak, A, Malyutina, S, Simonova, G, Feskens, EJM, Peasey, A, Bobak, M. Healthy diet indicator and mortality in Eastern European populations: prospective evidence from the HAPIEE cohort. Eur J Clin Nutr. 2014. 68:1346-1352. doi:10.1038/ejcn.2014.134

2254 Steinberg, D, Kay, M, Burroughs, J, Svetkey, LP, Bennett, GG. The Effect of a Digital Behavioral Weight Loss Intervention on Adherence to the Dietary Approaches to Stop Hypertension (DASH) Dietary Pattern in Medically Vulnerable Primary Care Patients: Results from a Randomized Controlled Trial. J Acad Nutr Diet. 2019. 119:574-584. doi:10.1016/j.jand.2018.12.011

2255 Stelmach-Mardas, M, Mardas, M, Warchol, W, Jamka, M, Walkowiak, J. Successful maintenance of body weight reduction after individualized dietary counseling in obese subjects. Sci Rep. 2014. 4:6620. doi:10.1038/srep06620

2256 Stentz, FB, Brewer, A, Wan, J, Garber, C, Daniels, B, Sands, C, Kitabchi, AE. Remission of pre-diabetes to normal glucose tolerance in obese adults with high protein versus high carbohydrate diet: randomized control trial. BMJ Open Diabetes Res Care. 2016. 4:e000258. doi:10.1136/bmjdrc-2016-000258

2257 Stentz, FB, Garber, C, Kitabchi, A. Efficacy of high protein vs. High carbohydrate diet on remission of impaired glucose tolerance (IGT) to normal glucose tolerance (NGT) and physiological parameters in obese adults. Diabetes. 2015 . 64:A512. doi:10.2337/db1519292253

2258 Sterling, S, Judd, S, Bertrand, B, Carson, TL, Chandler-Laney, P, Baskin, ML. Dietary Patterns Among Overweight and Obese African-American Women Living in the Rural South. J Racial Ethn Health Disparities. 2018. 5:141-150. doi:10.1007/s40615-017-0351-3

2259 Stern, L, Iqbal, N, Seshadri, P, Chicano, KL, Daily, DA, McGrory, J, Williams, M, Gracely, EJ, Samaha, FF. The effects of low-carbohydrate versus conventional weight loss diets in severely obese adults: one-year follow-up of a randomized trial. Ann Intern Med. 2004. 140:778-85. doi:10.7326/0003-4819-140-10-200405180-00007

Study Design; Age a Intervention/Exposure

Study Design

Study Design

Study Design; Publication Status

Intervention/Exposure

Study Design

Intervention/Exposure

Intervention/Exposure

Intervention/Exposure

Publication Status

Study Design;

Outcome

Power/Size; Weight loss/Hypocaloric 
2260 Stettler, R, Ith, M, Acheson, KJ, Decombaz, J, Boesch, C, Tappy, L, Binnert, C. Interaction between dietary lipids and physical inactivity on insulin sensitivity and on intramyocellular lipids in healthy men. Diabetes Care. 2005. 28:1404-9. doi:10.2337/diacare.28.6.1404

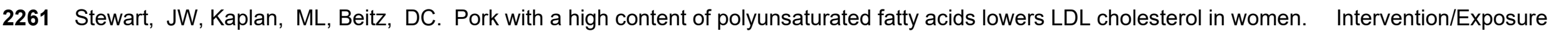
Am J Clin Nutr. 2001. 74:179-87. doi:10.1093/ajcn/74.2.179

2262 Stewart, KJ, Ouyang, P, Silber, HA, Zakaria, S, Desai, D, Shapiro, EP, Dobrosielski, DA. Effect of a low-carbohydrate versus a low-fat weight loss program on endothelial function. Circulation. 2012. 125:. doi:unavailable

2263 Stewart, KJ, Ouyang, P, Vaidya, D, Dobrosielski, DA. Reductions in systematic inflammation after a low-carbohydrate diet versus a low-fat diet each combined with exercise. Circulation. 2012. 126:. doi:unavailable

2264 Stinson, EJ, Piaggi, P, Ibrahim, M, Venti, C, Krakoff, J, Votruba, SB. High Fat and Sugar Consumption During Ad Libitum Intake Predicts Weight Gain. Obesity (Silver Spring). 2018. 26:689-695. doi:10.1002/oby.22124

2265 Stoa, EM, Nyhus, LK, Borresen, SC, Nygaard, C, Hovet, AM, Bratland-Sanda, S, Helgerud, J, Storen, O. Day to day variability in fat oxidation and the effect after only 1 day of change in diet composition. Appl Physiol Nutr Metab. 2016. 41:397404. doi:10.1139/apnm-2015-0334

2266 Stocks, T, Angquist, L, Banasik, K, Harder, MN, Taylor, MA, Hager, J, Arner, P, Oppert, JM, Martinez, JA, Polak, J, Rousseau, F, Langin, D, Rossner, S, Holst, C, MacDonald, IA, Kamatani, Y, Pfeiffer, AF, Kunesova, M, Saris, WH, Hansen, T, Pedersen, O, Astrup, A, Sorensen, TI. TFAP2B influences the effect of dietary fat on weight loss under energy restriction. PLoS One. 2012. 7:e43212. doi:10.1371/journal.pone.0043212

2267 Stocks, T, Taylor, MA, Angquist, L, Macdonald, IA, Arner, P, Holst, C, Oppert, JM, Martinez, JA, Rossner, S, Polak, J, Langin, D, Saris, WH, Astrup, A, Sorensen, TI. Change in proportional protein intake in a 10-week energy-restricted low- or high-fat diet, in relation to changes in body size and metabolic factors. Obes Facts. 2013. 6:217-27. doi:10.1159/000351726

2268 Stoernell, CK, Tangney, CC, Rockway, SW. Short-term changes in lipoprotein subclasses and C-reactive protein levels of hypertriglyceridemic adults on low-carbohydrate and low-fat diets. Nutr Res. 2008. 28:443-9. doi:10.1016/j.nutres.2008.03.013

2269 Stomby, A, Simonyte, K, Mellberg, C, Ryberg, M, Stimson, RH, Larsson, C, Lindahl, B, Andrew, R, Walker, BR, Olsson, T. Diet-induced weight loss has chronic tissue-specific effects on glucocorticoid metabolism in overweight postmenopausal women. Int J Obes (Lond). 2015. 39:814-9. doi:10.1038/ijo.2014.188

2270 Stonehouse, W, Benassi-Evans, B, James-Martin, G, Abeywardena, M. Fatty acid regio-specificity of triacylglycerol molecules may affect plasma lipid responses to dietary fats-a randomised controlled cross-over trial. Eur J Clin Nutr. 2019. : doi:10.1038/s41430-019-0452-7

2271 St-Onge, MP, Newcomer, BR, Buchthal, S, Aban, I, Allison, DB, Bosarge, A, Gower, B. Intramyocellular lipid content is lower with a low-fat diet than with high-fat diets, but that may not be relevant for health. Am J Clin Nutr. 2007. 86:1316-22. doi:10.1093/ajcn/86.5.1316

2272 St-Onge, MP, Zhang, S, Darnell, B, Allison, DB. Baseline serum C-reactive protein is associated with lipid responses to lowfat and high-polyunsaturated fat diets. J Nutr. 2009. 139:680-3. doi:10.3945/jn.108.098251

Intervention/Exposure; Publication Status

Publication Status

Study Design; Study duration

Outcome; Study duration

Study Design

Intervention/Exposure

Intervention/Exposure

Study duration

Power/Size

Intervention/Exposure

Study duration

Study duration 
2273 Storniolo, CE, Casillas, R, Bullo, M, Castaner, O, Ros, E, Saez, GT, Toledo, E, Estruch, R, Ruiz-Gutierrez, V, Fito, M, Martinez-Gonzalez, MA, Salas-Salvado, J, Mitjavila, MT, Moreno, JJ. A Mediterranean diet supplemented with extra virgin olive oil or nuts improves endothelial markers involved in blood pressure control in hypertensive women. Eur J Nutr. 2017. 56:89-97. doi:10.1007/s00394-015-1060-5

2274 Stow, R, Ives, N, Smith, C, Rick, C, Rushton, A. A cluster randomised feasibility trial evaluating nutritional interventions in the treatment of malnutrition in care home adult residents. Trials. 2015. 16:433. doi:10.1186/s13063-015-0952-2

2275 Strandberg, E, Edholm, P, Ponsot, E, Wahlin-Larsson, B, Hellmen, E, Nilsson, A, Engfeldt, P, Cederholm, T, Riserus, U, Kadi, F. Influence of combined resistance training and healthy diet on muscle mass in healthy elderly women: a randomized controlled trial. J Appl Physiol (1985). 2015. 119:918-25. doi:10.1152/japplphysiol.00066.2015

2276 Straznicky, NE, Grima, MT, Sari, Cl, Lambert, EA, Phillips, SE, Eikelis, N, Kobayashi, D, Hering, D, Mariani, JA, Dixon, JB, Nestel, PJ, Karapanagiotidis, S, Schlaich, MP, Lambert, GW. Reduction in peripheral vascular resistance predicts improvement in insulin clearance following weight loss. Cardiovasc Diabetol. 2015. 14:113. doi:10.1186/s12933-015-0276-2

2277 Streppel, MT, Sluik, D, van Yperen, JF, Geelen, A, Hofman, A, Franco, OH, Witteman, JC, Feskens, EJ. Nutrient-rich foods, cardiovascular diseases and all-cause mortality: the Rotterdam study. Eur J Clin Nutr. 2014. 68:741-7. doi:10.1038/ejcn.2014.35

2278 Hoekstra, J, Bueno-De-Mesquita, HB, Peeters, PHM. Dietary patterns in relation to disease burden expressed in DisabilityAdjusted Life Years. American Journal of Clinical Nutrition. 2014. 100:1158-1165. doi:10.3945/ajcn.113.082032

2279 Stulnig, TM. The ZONE Diet and Metabolic Control in Type 2 Diabetes. J Am Coll Nutr. 2015. 34 Suppl 1:39-41. doi:10.1080/07315724.2015.1080110

2280 Su, HY, Lee, HC, Cheng, WY, Huang, SY. A calorie-restriction diet supplemented with fish oil and high-protein powder is associated with reduced severity of metabolic syndrome in obese women. Eur J Clin Nutr. 2015. 69:322-8. doi:10.1038/ejcn.2014.196

2281 Su, M, Qiu, L, Wang, Q, Jiang, Z, Liu, XJ, Lin, J, Fang, DZ. Associations of Leu72Met Polymorphism of Preproghrelin with Ratios of Plasma Lipids Are Diversified by a High-Carbohydrate Diet in Healthy Chinese Adolescents. Ann Nutr Metab. 2015. 67:236-42. doi:10.1159/000440777

2282 Suchanek, P, Lanska, V, Hubacek, JA. Body Composition Changes in Adult Females after Lifestyle Intervention Are Influenced by the NYD-SP18 Variant. Cent Eur J Public Health. 2015. 23 Suppl:S19-22. doi:10.21101/cejph.a4105

2283 Suckling, RJ, Swift, PA. The health impacts of dietary sodium and a low-salt diet. Clin Med (Lond). 2015. 15:585-8. doi:10.7861/clinmedicine.15-6-585

2284 Sugawara, S, Kushida, M, Iwagaki, Y, Asano, M, Yamamoto, K, Tomata, Y, Tsuji, I, Tsuduki, T. The 1975 Type Japanese Diet Improves Lipid Metabolic Parameters in Younger Adults: A Randomized Controlled Trial. J Oleo Sci. 2018. 67:599-607. doi:10.5650/jos.ess 17259
Intervention/Exposure; Outcome

Intervention/Exposure Intervention/Exposure

Power/Size

Intervention/Exposure

Outcome

Study Design

Intervention/Exposure

Study Design

Study Design; Intervention/Exposure

Study Design

Study duration 
2285 Sugihiro, T, Yoneda, M, Ohno, H, Oki, K, Hattori, N. Associations of nutrient intakes with obesity and diabetes mellitus in the

Intervention/Exposure longitudinal medical surveys of Japanese Americans. J Diabetes Investig. 2019. 10:1229-1236. doi:10.1111/jdi.13010

2286 Suh, Y, Kang, H, Kim, M, Kim, S. Effect of weight reduction on Korean high protein diet in obese women. Obesity reviews. 2014. 15:229. doi:10.1111/obr.12153

2287 Suljkovicova, H, Marion-Latard, F, Hejnova, J, Majercik, M, Crampes, F, De Glisezinski, I, Berlan, M, Riviere, D, Stich, V. Effect of macronutrient composition of the diet on the regulation of lipolysis in adipose tissue at rest and during exercise: microdialysis study. Metabolism. 2002. 51:1291-7. doi:10.1053/meta.2002.35190

2288 Sumithran, P, Prendergast, LA, Delbridge, E, Purcell, K, Shulkes, A, Kriketos, A, Proietto, J. Ketosis and appetite-mediating nutrients and hormones after weight loss. Eur J Clin Nutr. 2013. 67:759-64. doi:10.1038/ejcn.2013.90

2289 Summer, SS, Brehm, BJ, Benoit, SC, D'Alessio, DA. Adiponectin changes in relation to the macronutrient composition of a weight-loss diet. Obesity (Silver Spring). 2011. 19:2198-204. doi:10.1038/oby.2011.60

2290 Sun, D, Heianza, Y, Huang, T, Wang, T, Han, L, Bray, GA, Sacks, FM, Qi, L. Genetic susceptibility and changes of blood pressure in response to weight-loss diets: the pounds lost trial. Diabetes. 2017. 66:A401-A402. doi:unavailable

2291 Sun, J, Buys, NJ, Hills, AP. Dietary pattern and its association with the prevalence of obesity, hypertension and other cardiovascular risk factors among Chinese older adults. Int J Environ Res Public Health. 2014. 11:3956-71. doi:10.3390/ijerph110403956

2292 Sundararajan, K, Campbell, MK, Choi, YH, Sarma, S. The relationship between diet quality and adult obesity: evidence from Canada. J Am Coll Nutr. 2014. 33:1-17. doi:10.1080/07315724.2013.848157

2293 Sunehag, AL, Toffolo, G, Campioni, M, Bier, DM, Haymond, MW. Effects of dietary macronutrient intake on insulin sensitivity and secretion and glucose and lipid metabolism in healthy, obese adolescents. J Clin Endocrinol Metab. 2005. 90:4496-502. doi:10.1210/jc.2005-0626

2294 Sung, KC, Sung, E, Byrne, CD, Kim, YB, Ahn, CW, Chung, HK. Composition of dietary macronutrient intake is not associated with prevalence of coronary artery calcification in healthy Korean adults. Ann Nutr Metab. 2015. 66:36-43. doi:10.1159/000369563

2295 Sureda, A, Bibiloni, MDM, Julibert, A, Bouzas, C, Argelich, E, Llompart, I, Pons, A, Tur, JA. Adherence to the Mediterranean Diet and Inflammatory Markers. Nutrients. 2018. 10:. doi:10.3390/nu10010062

2296 Surowska, A, Jegatheesan, P, Campos, V, Marques, AS, Egli, L, Cros, J, Rosset, R, Lecoultre, V, Kreis, R, Boesch, C, Pouymayou, B, Schneiter, P, Tappy, L. Effects of Dietary Protein and Fat Content on Intrahepatocellular and Intramyocellular Lipids during a 6-Day Hypercaloric, High Sucrose Diet: A Randomized Controlled Trial in Normal Weight Healthy Subjects. Nutrients. 2019. 11:. doi:10.3390/nu11010209

2297 Suyardi, MA, Johanes, W, Harahap, IP. The effects of balanced low calorie diet on body composition and serum leptin of obese women. Medical Journal of Indonesia. 2005. 14:220-224. doi:10.13181/mji.v14i4.202

Publication Status

Study duration

Study Design; Intervention/Exposure Health Status

Publication Status

Study Design

Study Design

Study duration

Study Design;

Outcome

Study Design

\section{Study duration}

Study duration 
2298 Suzuki, R, Tamura, Y, Takeno, K, Kakehi, S, Funayama, T, Furukawa, Y, Kaga, H, Sugimoto, D, Kadowaki, S, Someya, Y, Kanazawa, A, Kawamori, R, Watada, H. Three days of a eucaloric, low-carbohydrate/high-fat diet increases insulin clearance in healthy non-obese Japanese men. Sci Rep. 2019. 9:3857. doi:10.1038/s41598-019-40498-6

2299 Svetkey, LP, Moore, TJ, Simons-Morton, DG, Appel, LJ, Bray, GA, Sacks, FM, Ard, JD, Mortensen, RM, Mitchell, SR, Conlin, PR, Kesari, M. Angiotensinogen genotype and blood pressure response in the Dietary Approaches to Stop Hypertension (DASH) study. J Hypertens. 2001. 19:1949-56. doi:10.1097/00004872-200111000-00004

2300 Swinburn, BA, Metcalf, PA, Ley, SJ. Long-term (5-year) effects of a reduced-fat diet intervention in individuals with glucose intolerance. Diabetes Care. 2001. 24:619-24. doi:10.2337/diacare.24.4.619

2301 Sylvetsky, AC, Edelstein, SL, Walford, G, Boyko, EJ, Horton, ES, lbebuogu, UN, Knowler, WC, Montez, MG, Temprosa, M, Hoskin, M, Rother, KI, Delahanty, LM. A High-Carbohydrate, High-Fiber, Low-Fat Diet Results in Weight Loss among Adults at High Risk of Type 2 Diabetes. J Nutr. 2017. 147:2060-2066. doi:10.3945/jn.117.252395

2302 Szczuko, M, Gutowska, I, Seidler, T. Nutrition and nourishment status of Polish students in comparison with students from other countries. Rocz Panstw Zakl Hig. 2015. 66:261-8. doi:unavailable

2303 Tabung, FK, Fung, TT, Chavarro, JE, Smith-Warner, SA, Willett, WC, Giovannucci, EL. Associations between adherence to the World Cancer Research Fund/American Institute for Cancer Research cancer prevention recommendations and biomarkers of inflammation, hormonal, and insulin response. Int J Cancer. 2017. 140:764-776. doi:10.1002/ijc.30494

2304 Takagi, H, Kobayashi, Y, Taguchi, O, Takei, Y, Sumida, Y. Influence of dietary intake of fish oil, magnesium, and zinc on metabolic parameters among individuals tested for diabetes. Nutrition. 2015. 31:988-93. doi:10.1016/j.nut.2015.02.019

2305 Talaei, M, Koh, WP, Yuan, JM, van Dam, RM. DASH Dietary Pattern, Mediation by Mineral Intakes, and the Risk of Coronary Artery Disease and Stroke Mortality. J Am Heart Assoc. 2019. 8:e011054. doi:10.1161/jaha.118.011054

2306 Tam, CH, Wang, Y, Lee, HM, Luk, AO, Tong, PC, Chan, MH, Ozaki, R, Kong, AP, So, WY, Chan, JC, Ma, RC. Early gene-diet interaction between glucokinase regulatory protein (GCKR) polymorphism, vegetable and fish intakes in modulating triglyceride levels in healthy adolescents. Nutr Metab Cardiovasc Dis. 2015. 25:951-8. doi:10.1016/j.numecd.2015.06.011

2307 Tambalis, KD, Panagiotakos, DB, Psarra, G, Sidossis, LS. Association of cardiorespiratory fitness levels with dietary habits and lifestyle factors in schoolchildren. Appl Physiol Nutr Metab. 2019. 44:539-545. doi:10.1139/apnm-2018-0407

Intervention/Exposure

Weight

loss/Hypocaloric

Intervention/Exposure

Study Design

Study Design;

Outcome

Study Design

Country

Study Design

Study Design

Tamura, T, Yatabe, T, Kitagawa, H, Yamashita, K, Hanazaki, K, Yokoyama, M. Oral carbohydrate loading with 18\% carbohydrate beverage alleviates insulin resistance. Asia Pac J Clin Nutr. 2013. 22:48-53. doi:10.6133/apjcn.2013.22.1.20

2309 Tamura, Y, Watada, H, Igarashi, Y, Nomiyama, T, Onishi, T, Takahashi, K, Doi, S, Katamoto, S, Hirose, T, Tanaka, Y, Kawamori, R. Short-term effects of dietary fat on intramyocellular lipid in sprinters and endurance runners. Metabolism. 2008. 57:373-9. doi:10.1016/j.metabol.2007.10.013

2310 Tang, H, Zhang, Z, Li, Z, Lin, J, Fang, DZ. High-Carbohydrate/Low-Fat Diet-Induced Gender-Specific Serum Lipid Profile Changes Are Associated with LEPR Polymorphisms in Chinese Youth. Ann Nutr Metab. 2017. 70:1-8. doi:10.1159/000455165

2311 Tang, M, Armstrong, CL, Leidy, HJ, Campbell, WW. Normal vs. high-protein weight loss diets in men: effects on body composition and indices of metabolic syndrome. Obesity (Silver Spring). 2013. 21:E204-10. doi:10.1002/oby.20078 
2312 Tang, M, Leidy, HJ, Campbell, WW. Regional, but not total, body composition changes in overweight and obese adults consuming a higher protein, energy-restricted diet are sex specific. Nutr Res. 2013. 33:629-35.

Intervention/Exposure doi:10.1016/j.nutres.2013.05.012

2313 Tang, Z, Zhou, T, Luo, Y, Xie, C, Huo, D, Tao, L, Pan, L, Sun, F, Zhu, H, Yang, X, Wang, W, Yan, A, Li, X, Guo, X. Risk factors for cerebrovascular disease mortality among the elderly in Beijing: A competing risk analysis. PLoS ONE. 2014. 9: doi:10.1371/journal.pone.0087884

2314 Tangney, C, Sarkar, D, Staffileno, BA. Comparison of three DASH scoring paradigms and prevalent hypertension among older Hispanics. J Hum Hypertens. 2016. 30:210-5. doi:10.1038/jhh.2015.50

2315 Tanisawa, K, Ito, T, Sun, X, Ise, R, Oshima, S, Cao, ZB, Sakamoto, S, Tanaka, M, Higuchi, M. Strong influence of dietary intake and physical activity on body fatness in elderly Japanese men: age-associated loss of polygenic resistance against obesity. Genes Nutr. 2014. 9:416. doi:10.1007/s12263-014-0416-4

2316 Tanskey, LA, Goldberg, JP, Chui, K, Must, A, Sacheck, JM. Accelerated Summer Weight Gain in a Low-Income, Ethnically Diverse Sample of Elementary School Children in Massachusetts. Child Obes. 2019. 15:244-253. doi:10.1089/chi.2017.0228

2317 Tapsell, L, Batterham, M, Huang, XF, Tan, SY, Teuss, G, Charlton, K, Oshea, J, Warensjo, E. Short term effects of energy restriction and dietary fat sub-type on weight loss and disease risk factors. Nutr Metab Cardiovasc Dis. 2010. 20:317-25. doi:10.1016/j.numecd.2009.04.007

2318 Tapsell, LC, Batterham, MJ, Charlton, KE, Neale, EP, Probst, YC, O'Shea, JE, Thorne, RL, Zhang, Q, Louie, JC. Foods, nutrients or whole diets: effects of targeting fish and LCn3PUFA consumption in a 12mo weight loss trial. BMC Public Health. 2013. 13:1231. doi:10.1186/1471-2458-13-1231

2319 Tardivo, AP, Nahas-Neto, J, Orsatti, CL, Dias, FB, Poloni, PF, Schmitt, EB, Nahas, EA. Effects of omega-3 on metabolic markers in postmenopausal women with metabolic syndrome. Climacteric. 2015. 18:290-8. doi:10.3109/13697137.2014.981521

2320 Tay, J, Brinkworth, GD, Noakes, M, Keogh, J, Clifton, PM. Metabolic effects of weight loss on a very-low-carbohydrate diet compared with an isocaloric high-carbohydrate diet in abdominally obese subjects. J Am Coll Cardiol. 2008. 51:59-67. doi:10.1016/j.jacc.2007.08.050

2321 Tayyem, RF, Al-Radaideh, AM, Hammad, SS, Al-Hajaj, S, Allehdan, SS, Agraib, LM, Al-Fayomi, KI, Malkawi, AA, Hijjawi, NS. Subcutaneous and visceral fat volumes measured by MRI and their relationships with nutrient intakes among adults. Asia Pac J Clin Nutr. 2019. 28:300-309. doi:10.6133/apjcn.201906_28(2).0012

2322 Te Morenga, L, Docherty, P, Williams, S, Mann, J. The Effect of a Diet Moderately High in Protein and Fiber on Insulin Sensitivity Measured Using the Dynamic Insulin Sensitivity and Secretion Test (DISST). Nutrients. 2017. 9:. doi:10.3390/nu9121291

2323 Te Morenga, LA, Levers, MT, Williams, SM, Brown, RC, Mann, J. Comparison of high protein and high fiber weight-loss diets in women with risk factors for the metabolic syndrome: a randomized trial. Nutr J. 2011. 10:40. doi:10.1186/1475-2891-10-40

Intervention/Exposure; Country

Study Design

Study Design;

Intervention/Exposure

Intervention/Exposure

Intervention/Exposure; Comparator

Intervention/Exposure; Comparator

Intervention/Exposure

Weight

loss/Hypocaloric

Study Design

Study Design;

Intervention/Exposure

Study duration 
2324 Te Morenga, LA, McAuley, KA, Docherty, PD, Williams, SM, Mann, J. The effect of a high protein, high fibre diet on insulin sensitivity measured using the Dynamic Insulin Sensitivity and Secretion Test (DISST). Australasian medical journal. 2011. 4:780. doi:unavailable

2325 Teff, KL, Elliott, SS, Tschop, M, Kieffer, TJ, Rader, D, Heiman, M, Townsend, RR, Keim, NL, D'Alessio, D, Havel, PJ. Dietary fructose reduces circulating insulin and leptin, attenuates postprandial suppression of ghrelin, and increases triglycerides in women. J Clin Endocrinol Metab. 2004. 89:2963-72. doi:10.1210/jc.2003-031855

2326 Teixeira, JA, Castro, TG, Grant, CC, Wall, CR, Castro, Alds, Francisco, RPV, Vieira, SE, Saldiva, Srdm, Marchioni, DM. Dietary patterns are influenced by socio-demographic conditions of women in childbearing age: a cohort study of pregnant women. BMC Public Health. 2018. 18:301. doi:10.1186/s12889-018-5184-4

2327 Telford, RD, Cunningham, RB, Telford, RM, Riley, M, Abhayaratna, WP. Determinants of Childhood Adiposity: Evidence from the Australian LOOK Study. PLoS ONE. 2012. 7:. doi:10.1371/journal.pone.0050014

2328 Telford, RD, Telford, RM, Martin, MK, Welvaert, M. Drivers of adolescent adiposity: Evidence from the Australian LOOK study. J Sci Med Sport. 2019. .. doi:10.1016/j.jsams.2019.07.013

2329 Teunissen-Beekman, KF, Dopheide, J, Geleijnse, JM, Bakker, SJ, Brink, EJ, de Leeuw, PW, Schalkwijk, CG, van Baak, MA. Dietary proteins improve endothelial function under fasting conditions but not in the postprandial state, with no effects on markers of low-grade inflammation. Br J Nutr. 2015. 114:1819-28. doi:10.1017/s0007114515003530

2330 Thalacker-Mercer, AE, Fleet, JC, Craig, BA, Carnell, NS, Campbell, WW. Inadequate protein intake affects skeletal muscle transcript profiles in older humans. Am J Clin Nutr. 2007. 85:1344-52. doi:10.1093/ajcn/85.5.1344

2331 Tharrey, M, Mariotti, F, Mashchak, A, Barbillon, P, Delattre, M, Huneau, JF, Fraser, GE. Patterns of amino acids intake are strongly associated with cardiovascular mortality, independently of the sources of protein. Int J Epidemiol. 2019. : doi:10.1093/ije/dyz194

2332 The Editors Of The Lancet Diabetes, Endocrinology. Expression of concern-Effect of a high-fat Mediterranean diet on bodyweight and waist circumference: a prespecified secondary outcomes analysis of the PREDIMED randomised controlled trial. Lancet Diabetes Endocrinol. 2018. 6:763. doi:10.1016/s2213-8587(18)30236-5

2333 Thomas, SJ, Booth, JN, 3rd, Dai, C, Li, X, Allen, N, Calhoun, D, Carson, AP, Gidding, S, Lewis, CE, Shikany, JM, Shimbo, D, Sidney, S, Muntner, P. Cumulative Incidence of Hypertension by 55 Years of Age in Blacks and Whites: The CARDIA Study. J Am Heart Assoc. 2018. 7:. doi:10.1161/jaha.117.007988

2334 Thorning, TK, Raziani, F, Bendsen, NT, Astrup, A, Tholstrup, T, Raben, A. Diets with high-fat cheese, high-fat meat, or carbohydrate on cardiovascular risk markers in overweight postmenopausal women: a randomized crossover trial. Am $\mathrm{J}$ Clin Nutr. 2015. 102:573-81. doi:10.3945/ajcn.115.109116

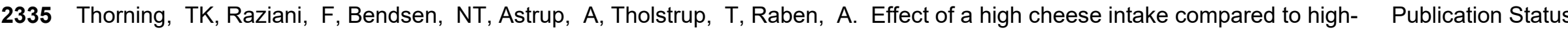
fat meat or carbohydrate on cardiovascular risk markers in overweight postmenopausal women. Atherosclerosis. 2015. 241:e143. doi:unavailable 
2336 Thorpe, MG, Milte, CM, Crawford, D, McNaughton, SA. A comparison of the dietary patterns derived by principal component analysis and cluster analysis in older Australians. Int J Behav Nutr Phys Act. 2016. 13:30. doi:10.1186/s12966-016-0353-2

2337 Thorpe, MP, Jacobson, EH, Layman, DK, He, X, Kris-Etherton, PM, Evans, EM. A diet high in protein, dairy, and calcium attenuates bone loss over twelve months of weight loss and maintenance relative to a conventional high-carbohydrate diet in adults. J Nutr. 2008. 138:1096-100. doi:10.1093/jn/138.6.1096

2338 Threapleton, DE, Burley, VJ, Greenwood, DC, Cade, JE. Dietary fibre intake and risk of ischaemic and haemorrhagic stroke in the UK Women's Cohort Study. Eur J Clin Nutr. 2015. 69:467-74. doi:10.1038/ejcn.2014.260

2339 Tian, X, Huang, Y, Wang, H. Deviation of Chinese Adults' Diet from the Chinese Food Pagoda 2016 and Its Association with Adiposity. Nutrients. 2017. 9:. doi:10.3390/nu9090995

2340 Tielemans, MJ, Erler, NS, Leermakers, ET, van den Broek, M, Jaddoe, VW, Steegers, EA, Kiefte-de Jong, JC, Franco, OH. Participants A Priori and a Posteriori Dietary Patterns during Pregnancy and Gestational Weight Gain: The Generation R Study. Nutrients. 2015. 7:9383-99. doi:10.3390/nu7115476

2341 Tielemans, SM, Kromhout, D, Altorf-van der Kuil, W, Geleijnse, JM. Associations of plant and animal protein intake with 5year changes in blood pressure: the Zutphen Elderly Study. Nutr Metab Cardiovasc Dis. 2014. 24:1228-33. doi:10.1016/j.numecd.2014.05.013

2342 Tinker, LF, Bonds, DE, Margolis, KL, Manson, JE, Howard, BV, Larson, J, Perri, MG, Beresford, SA, Robinson, JG, Rodriguez, B, Safford, MM, Wenger, NK, Stevens, VJ, Parker, LM. Low-fat dietary pattern and risk of treated diabetes mellitus in postmenopausal women: the Women's Health Initiative randomized controlled dietary modification trial. Arch Intern Med. 2008. 168:1500-11. doi:10.1001/archinte.168.14.1500

2343 Tinker, LF, Sarto, GE, Howard, BV, Huang, Y, Neuhouser, ML, Mossavar-Rahmani, Y, Beasley, JM, Margolis, KL, Eaton, CB, Phillips, LS, Prentice, RL. Biomarker-calibrated dietary energy and protein intake associations with diabetes risk among postmenopausal women from the Women's Health Initiative. Am J Clin Nutr. 2011. 94:1600-6. doi:10.3945/ajcn.111.018648

2344 Tinsley, GM, Morales, E, Forsse, JS, Grandjean, PW. Impact of Acute Dietary Manipulations on DXA and BIA Body Composition Estimates. Med Sci Sports Exerc. 2017. 49:823-832. doi:10.1249/mss.0000000000001148

2345 Tippens, KM, Erlandsen, A, Hanes, DA, Graybill, R, Jackson, C, Briley, J, Zwickey, H. Impact of a Short-Term Naturopathic Whole-Foods-Based Nutrition Education Intervention on Dietary Behavior and Diabetes Risk Markers: A Pilot Study. J Altern Complement Med. 2019. 25:234-240. doi:10.1089/acm.2018.0025

2346 Todd, SA, Wright, C. Weight loss intervention trial comparing intermittent low carbohydrate versus continuous Mediterranean diet. Obesity facts. Conference: european obesity summit (EOS): 1st joint congress of EASO and IFSO-EC. Gothenburg sweden. Conference start: 20160601. Conference end: 20160604. Conference publication: (var.pagings). 2016. 9:252. doi:10.1159/000446744

2347 Tognon, G, Lissner, L, Saebye, D, Walker, KZ, Heitmann, BL. The Mediterranean diet in relation to mortality and CVD: a Danish cohort study. Br J Nutr. 2014. 111:151-9. doi:10.1017/s0007114513001931 
2348 Tognon, G, Rothenberg, E, Eiben, G, Sundh, V, Winkvist, A, Lissner, L. Does the Mediterranean diet predict longevity in the elderly? A Swedish perspective. Age (Dordr). 2011. 33:439-50. doi:10.1007/s11357-010-9193-1

2349 Toledo, E, Hu, FB, Estruch, R, Salas-Salvado, J, Corella, D, Covas, MI, Aros, F, Gomez-Gracia, E, Fiol, M, Ruiz-Gutierrez, $\mathrm{V}$, etal, . Association between adherence to the Mediterranean diet and blood pressure: 4-year follow-up in the PREDIMED trial. European journal of epidemiology. 2012. 27:S46-S47. doi:10.1007/s10654-012-9722-6

2350 Toledo, E, Wang, DD, Ruiz-Canela, M, Clish, CB, Razquin, C, Zheng, Y, Guasch-Ferre, M, Hruby, A, Corella, D, GomezGracia, E, Fiol, M, Estruch, R, Ros, E, Lapetra, J, Fito, M, Aros, F, Serra-Majem, L, Liang, L, Salas-Salvado, J, Hu, FB, Martinez-Gonzalez, MA. Plasma lipidomic profiles and cardiovascular events in a randomized intervention trial with the Mediterranean diet. Am J Clin Nutr. 2017. 106:973-983. doi:10.3945/ajcn.116.151159

2351 Tometich, DB, Mosher, CE, Winger, JG, Badr, HJ, Snyder, DC, Sloane, RJ, Demark-Wahnefried, W. Effects of diet and exercise on weight-related outcomes for breast cancer survivors and their adult daughters: an analysis of the DAMES trial. Support Care Cancer. 2017. 25:2559-2568. doi:10.1007/s00520-017-3665-0

2352 Tomlinson, DJ, Erskine, RM, Morse, Cl, Onambele, GL. Impact of Above-Average Proanabolic Nutrients Is Overridden by High Protein and Energy Intake in the Muscle-Tendon Unit Characteristics of Middle- to Older-Aged Adults. J Nutr. 2018. 148:1776-1785. doi:10.1093/jn/nxy185

2353 Tonstad, S, Malik, N, Haddad, E. A high-fibre bean-rich diet versus a low-carbohydrate diet for obesity. J Hum Nutr Diet. 2014. 27 Suppl 2:109-16. doi:10.1111/jhn.12118

2354 Torbay, N, Hwalla Baba, N, Sawaya, S, Bajjani, R, Habbal, Z, Azar, S, Hashim, SA. High protein vs high carbohydrate hypoenergetic diet in treatment of obese normoinsulinemic and hyperinsulinemic subjects. Nutrition Research. 2002. 22:587598. doi:10.1016/S0271-5317(02)00359-7

2355 Torres, SJ, Nowson, CA. Effect of a weight-loss program on mental stress-induced cardiovascular responses and recovery. Nutrition. 2007. 23:521-8. doi:10.1016/j.nut.2007.04.016

2356 Torres, SJ, Robinson, S, Orellana, L, O'Connell, SL, Grimes, CA, Mundell, NL, Dunstan, DW, Nowson, CA, Daly, RM. Effects of progressive resistance training combined with a protein-enriched lean red meat diet on health-related quality of life in elderly women: Secondary analysis of a 4-month cluster randomised controlled trial. British Journal of Nutrition. 2017. 117:1550-1559. doi:10.1017/S0007114517001507

2357 Torres-Pena, JD, Garcia-Rios, A, Delgado-Casado, N, Gomez-Luna, P, Alcala-Diaz, JF, Yubero-Serrano, EM, GomezDelgado, F, Leon-Acuna, A, Lopez-Moreno, J, Camargo, A, Tinahones, FJ, Delgado-Lista, J, Ordovas, JM, Perez-Martinez, $\mathrm{P}$, Lopez-Miranda, J. Mediterranean diet improves endothelial function in patients with diabetes and prediabetes: A report from the CORDIOPREV study. Atherosclerosis. 2018. 269:50-56. doi:10.1016/j.atherosclerosis.2017.12.012

2358 Tovar, A, Choumenkovitch, SF, Hennessy, E, Boulos, R, Must, A, Hughes, SO, Gute, DM, Vikre, EK, Economos, CD. Low demanding parental feeding style is associated with low consumption of whole grains among children of recent immigrants.

Publication Status

Intervention/Exposure;

Outcome

Intervention/Exposure; Outcome

Study Design;

Intervention/Exposure Appetite. 2015. 95:211-8. doi:10.1016/j.appet.2015.06.006

Intervention/Exposure; Health Status

Weight

loss/Hypocaloric

Study duration

Intervention/Exposure

Outcome

Study Design

Intervention/Exposure 
2359 Tovar, J, Johansson, M, Bjorck, I. A multifunctional diet improves cardiometabolic-related biomarkers independently of weight changes: an 8-week randomized controlled intervention in healthy overweight and obese subjects. Eur J Nutr. 2016. 55:2295306. doi:10.1007/s00394-015-1039-2

2360 Towner, EK, Robson, SM, Stark, LJ. Secondary impact of a behavioral intervention on dietary quality in preschoolers with obesity. Children's Health Care. 2019. 48:75-89. doi:10.1080/02739615.2018.1463532

2361 Trapp, CM, Burke, G, Gorin, AA, Wiley, JF, Hernandez, D, Crowell, RE, Grant, A, Beaulieu, A, Cloutier, MM. The relationship between dietary patterns, body mass index percentile, and household food security in young urban children. Child Obes. 2015. 11:148-55. doi:10.1089/chi.2014.0105

2362 Trepanowski, JF. Improved insulin sensitivity with a healthy low fat or a healthy low carbohydrate weight loss diet: a twelve month randomized trial. Circulation. 2017. 135:. doi:unavailable

2363 Trichopoulou, A, Psaltopoulou, T, Orfanos, P, Hsieh, CC, Trichopoulos, D. Low-carbohydrate-high-protein diet and long-term survival in a general population cohort. Eur J Clin Nutr. 2007. 61:575-81. doi:10.1038/sj.ejcn.1602557

2364 Trico, D, Trifiro, S, Mengozzi, A, Morgantini, C, Baldi, S, Mari, A, Natali, A. Reducing Cholesterol and Fat Intake Improves Glucose Tolerance by Enhancing beta Cell Function in Nondiabetic Subjects. J Clin Endocrinol Metab. 2018. 103:622-631. doi:10.1210/jc.2017-02089

2365 Tricò, D, Trifirò, S, Mengozzi, A, Morgantini, C, Baldi, S, Mari, A, Natali, A. Reducing Cholesterol and Fat Intake Improves Glucose Tolerance by Enhancing $\beta$ Cell Function in Nondiabetic Subjects. Journal of clinical endocrinology and metabolism. 2018. 103:622-631. doi:10.1210/jc.2017-02089

2366 Tripp, ML, Dahlberg, CJ, Eliason, S, Lamb, JJ, Ou, JJ, Gao, W, Bhandari, J, Graham, D, Dudleenamjil, E, Babish, JG. A Low-Glycemic, Mediterranean Diet and Lifestyle Modification Program with Targeted Nutraceuticals Reduces Body Weight, Improves Cardiometabolic Variables and Longevity Biomarkers in Overweight Subjects: A 13-Week Observational Trial. J Med Food. 2019. 22:479-489. doi:10.1089/jmf.2018.0063

2367 Truby, H, Baxter, K, Ware, RS, Jensen, DE, Cardinal, JW, Warren, JM, Daniels, L, Davies, PS, Barrett, P, Blumfield, ML, Batch, JA. A Randomized Controlled Trial of Two Different Macronutrient Profiles on Weight, Body Composition and Metabolic Parameters in Obese Adolescents Seeking Weight Loss. PLoS One. 2016. 11:e0151787. doi:10.1371/journal.pone.0151787

2368 Trude, AC, Kharmats, A, Jock, B, Liu, D, Lee, K, Martins, PA, Pardilla, M, Swartz, J, Gittelsohn, J. Patterns of Food Consumption are Associated with Obesity, Self-Reported Diabetes and Cardiovascular Disease in Five American Indian Communities. Ecol Food Nutr. 2015. 54:437-54. doi:10.1080/03670244.2014.922070

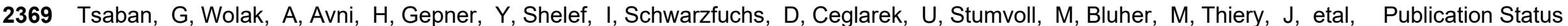
. Pericardial adiposity trajectory during long-term dietary intervention. European heart journal. 2016. 37:547-. doi:10.1093/eurheartj/ehw432

2370 Tsigga, M, Filis, V, Hatzopoulou, K, Kotzamanidis, C, Grammatikopoulou, MG. Healthy Eating Index during pregnancy according to pre-gravid and gravid weight status. Public Health Nutr. 2011. 14:290-6. doi:10.1017/s1368980010001989

Outcome

Study Design

Publication Status

Intervention/Exposure; Outcome

Intervention/Exposure; Study duration

Study duration

Study Design;

Intervention/Exposure

Weight

loss/Hypocaloric

Study Design

Study Design;

Outcome 
2371 Tsilingiris, D, Dellis, D, Eleftheriadou, I, Tentolouris, A, Karanasiou, M, Meimari, A, Dellis, G, Dimosthenopoulos, C, Lazarou, S, Kokkinos, A, etal, . Integration of half-day carbohydrate restriction into a hypocaloric Mediterranean-type diet in overweight and obese subjects: an open label, randomised, controlled trial. Diabetologia. 2018. 61:S98-. doi:10.1007/s00125018-4693-0

2372 Tsioufis, C. The Mediterranean and the DASH dietary patterns: Insights into their role in cardiovascular disease prevention. Hellenic J Cardiol. 2018. 59:134-135. doi:10.1016/j.hjc.2017.04.006

2373 Tsugane, S, Sawada, N. The JPHC study: design and some findings on the typical Japanese diet. Jpn J Clin Oncol. 2014. 44:777-82. doi:10.1093/jjco/hyu096

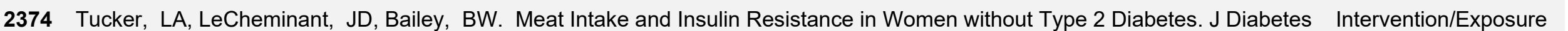
Res. 2015. 2015:174742. doi:10.1155/2015/174742

2375 Tulk, HM, Robinson, LE. Modifying the $n-6 / n-3$ polyunsaturated fatty acid ratio of a high-saturated fat challenge does not acutely attenuate postprandial changes in inflammatory markers in men with metabolic syndrome. Metabolism. 2009. 58:170916. doi:10.1016/j.metabol.2009.05.031

2376 Tuomilehto, J, Lindström, J, Eriksson, JG. Changes in diet and physical activity prevented type 2 diabetes mellitus in people with impaired glucose tolerance. Evidence-Based Medicine. 2001. 6:176. doi:10.1136/ebm.6.6.176

2377 Tura, A, Conte, B, Caparrotto, C, Spinella, P, Maestrelli, P, Valerio, A, Pacini, G, Avogaro, A. Insulin sensitivity and secretion in young, healthy subjects are not changed by Zone and Mediterranean diets. Mediterranean Journal of Nutrition and Metabolism. 2010. 3:233-237. doi:10.1007/s12349-010-0026-7

2378 Turner, KM, Keogh, JB, Clifton, PM. Red meat, dairy, and insulin sensitivity: a randomized crossover intervention study. Am J Clin Nutr. 2015. 101:1173-9. doi:10.3945/ajcn.114.104976

2379 Turner, KM, Keogh, JB, Meikle, PJ, Clifton, PM. Changes in Lipids and Inflammatory Markers after Consuming Diets High in Red Meat or Dairy for Four Weeks. Nutrients. 2017. 9:. doi:10.3390/nu9080886

2380 Turner-McGrievy, GM, Barnard, ND, Scialli, AR. A two-year randomized weight loss trial comparing a vegan diet to a more moderate low-fat diet. Obesity (Silver Spring). 2007. 15:2276-81. doi:10.1038/oby.2007.270

2381 Turner-McGrievy, GM, Davidson, CR, Wilcox, S. Does the type of weight loss diet affect who participates in a behavioral weight loss intervention? A comparison of participants for a plant-based diet versus a standard diet trial. Appetite. 2014. 73:15662. doi:10.1016/j.appet.2013.11.008

2382 Turner-McGrievy, GM, Wirth, MD, Shivappa, N, Wingard, EE, Fayad, R, Wilcox, S, Frongillo, EA, Hebert, JR. Randomization to plant-based dietary approaches leads to larger short-term improvements in Dietary Inflammatory Index scores and macronutrient intake compared with diets that contain meat. Nutr Res. 2015. 35:97-106. doi:10.1016/j.nutres.2014.11.007

2383 Tuttolomondo, A, Casuccio, A, Butta, C, Pecoraro, R, Di Raimondo, D, Della Corte, V, Arnao, V, Clemente, G, Maida, C, Simonetta, I, Miceli, G, Lucifora, B, Cirrincione, A, Di Bona, D, Corpora, F, Maugeri, R, lacopino, DG, Pinto, A. Mediterranean Diet in patients with acute ischemic stroke: Relationships between Mediterranean Diet score, diagnostic subtype, and stroke severity index. Atherosclerosis. 2015. 243:260-7. doi:10.1016/j.atherosclerosis.2015.09.017

Study Design; Publication Status

Study Design

Study duration

Study Design; Publication Status

Study duration

ntervention/Exposure; Study duration

Study duration

Intervention/Exposure

\section{Outcome}

Outcome

Health Status 
2384 Tykhorskyi, O, Dzhym, V, Slavitiak, O, Galashko, N, Zhadan, A, Piven, O, Melnyk, A. Influence of hypo-caloric diet on absolute and relative strength of elite male bodybuilders` while preparing for the competition. Research Journal of

Intervention/Exposure; Pharmaceutical, Biological and Chemical Sciences. 2019. 10:1434-1440. doi:unavailable

2385 Tyrovolas, S, Haro, JM, Polychronopoulos, E, Mariolis, A, Piscopo, S, Valacchi, G, Makri, K, Zeimbekis, A, Tyrovola, D, Bountziouka, V, Gotsis, E, Metallinos, G, Katsoulis, Y, Tur, JA, Matalas, A, Lionis, C, Panagiotakos, D. Factors associated with components of arterial pressure among older individuals (the multinational MEDIS study): the role of the Mediterranean diet and alcohol consumption. J Clin Hypertens (Greenwich). 2014. 16:645-51. doi:10.1111/jch.12370

2386 Tyrovolas, S, Psaltopoulou, T, Pounis, G, Papairakleous, N, Bountziouka, V, Zeimbekis, A, Gotsis, E, Antonopoulou, M, Metallinos, G, Polychronopoulos, E, Lionis, C, Panagiotakos, DB. Nutrient intake in relation to central and overall obesity status among elderly people living in the Mediterranean islands: the MEDIS study. Nutr Metab Cardiovasc Dis. 2011. 21:438-45. doi:10.1016/j.numecd.2009.10.012

2387 Tyson, CC, Barnhart, H, Sapp, S, Poon, V, Lin, PH, Svetkey, LP. Ambulatory blood pressure in the dash diet trial: Effects of race and albuminuria. J Clin Hypertens (Greenwich). 2018. 20:308-314. doi:10.1111/jch.13170

2388 Tyson, CC, Davenport, CA, Lin, PH, Scialla, JJ, Hall, R, Diamantidis, CJ, Lunyera, J, Bhavsar, N, Rebholz, CM, Pendergast, J, Boulware, LE, Svetkey, LP. DASH Diet and Blood Pressure Among Black Americans With and Without CKD: The Jackson Heart Study. Am J Hypertens. 2019. 32:975-982. doi:10.1093/ajh/hpz090

2389 Tyson, CC, Kuchibhatla, M, Patel, UD, Pun, PH, Chang, A, Nwankwo, C, Joseph, MA, Svetkey, LP. Impact of Kidney Function on Effects of the Dietary Approaches to Stop Hypertension (Dash) Diet. J Hypertens (Los Angel). 2014. 3: doi:10.4172/2167-1095.1000168

2390 Uauy, R, Mize, CE, Castillo-Duran, C. Fat intake during childhood: metabolic responses and effects on growth. Am J Clin Nutr. 2000. 72:1354s-1360s. doi:10.1093/ajcn/72.5.1354s

2391 Um, YJ, Oh, SW, Lee, CM, Kwon, HT, Joh, HK, Kim, YJ, Kim, HJ, Ahn, SH. Dietary Fat Intake and the Risk of Metabolic Syndrome in Korean Adults. Korean J Fam Med. 2015. 36:245-52. doi:10.4082/kjfm.2015.36.5.245

2392 Umemoto, S, Kawano, R, Kawamura, A, Onaka, U, Motoi, S, Mitarai, M. Effects of Japan diet-based antihypertensive diet on blood pressure and its variability in subjects with untreated normal high blood pressure and stage I hypertension: a randomized controlled trial. Circulation. 2017. 136:. doi:unavailable

2393 Umpleby, AM, Shojaee-Moradie, F, Fielding, B, Li, X, Marino, A, Alsini, N, Isherwood, C, Jackson, N, Ahmad, A, Stolinski, M, Lovegrove, JA, Johnsen, S, Jeewaka, RMendisAS, Wright, J, Wilinska, ME, Hovorka, R, Bell, JD, Thomas, EL, Frost, GS, Griffin, BA. Impact of liver fat on the differential partitioning of hepatic triacylglycerol into VLDL subclasses on high and low sugar diets. Clin Sci (Lond). 2017. 131:2561-2573. doi:10.1042/cs20171208

2394 Unwin, D, Unwin, J. Low carbohydrate diet to achieve weight loss and improve HbA1c in type 2 diabetes and pre-diabetes: Experience from one general practice. Practical Diabetes. 2014. 31:76-79. doi:10.1002/pdi.1835

2395 Unwin, DJ, Tobin, SD, Murray, SW, Delon, C, Brady, AJ. Substantial and Sustained Improvements in Blood Pressure, Weight and Lipid Profiles from a Carbohydrate Restricted Diet: An Observational Study of Insulin Resistant Patients in Primary Care. Int J Environ Res Public Health. 2019. 16:. doi:10.3390/ijerph16152680

Study Design

Study Design

Intervention/Exposure

Intervention/Exposure; Outcome

Intervention/Exposure

Intervention/Exposure

Intervention/Exposure; Outcome

Publication Status

Intervention/Exposure

Study Design;

Intervention/Exposure

Intervention/Exposure; Health Status 
2396 Urbain, P, Strom, L, Morawski, L, Wehrle, A, Deibert, P, Bertz, H. Impact of a 6-week non-energy-restricted ketogenic diet on physical fitness, body composition and biochemical parameters in healthy adults. Nutr Metab (Lond). 2017. 14:17. doi:10.1186/s12986-017-0175-5

2397 Utzschneider, KM, Arbuckle, MD, Tidwell, JM, Craft, S. An isocaloric low-fat diet decreases liver fat in older subjects. \#journal\#. 2010. .. doi:unavailable

2398 Utzschneider, KM, Bayer-Carter, JL, Arbuckle, MD, Tidwell, JM, Richards, TL, Craft, S. Beneficial effect of a weight-stable, low-fat/low-saturated fat/low-glycaemic index diet to reduce liver fat in older subjects. Br J Nutr. 2013. 109:1096-104. doi:10.1017/s0007114512002966

2399 Uusitupa, M. Healthy nordic diet and metabolic syndrome-the sysdiet study. Annals of nutrition and metabolism. Conference: 12th european nutrition conference, FENS 2015. Berlin germany. Conference start: 20151020. Conference end: 20151023. Conference publication: (var.pagings). 2015. 67:52. doi:10.1159/000440895

2400 Vadiveloo, M, Sacks, FM, Champagne, CM, Bray, GA, Mattei, J. Greater Healthful Dietary Variety Is Associated with Greater 2-Year Changes in Weight and Adiposity in the Preventing Overweight Using Novel Dietary Strategies (POUNDS Lost) Trial. J Nutr. 2016. 146:1552-9. doi:10.3945/jn.115.224683

2401 Vakili, R, Nematy, M. Comparison of the effect of ketogenic diet and low caloric diet on weight loss in Iranian obese and overweight children. Hormone research in paediatrics. 2011. 76:180. doi:10.1159/000334327

2402 Valente-Dos-Santos, J, Tavares, OM, Duarte, JP, Sousa, ESilvaPM, Rama, LM, Casanova, JM, Fontes-Ribeiro, CA, Marques, EA, Courteix, D, Ronque, ERV, Cyrino, ES, Conde, J, Coelho, ESilvaMJ. Total and regional bone mineral and tissue composition in female adolescent athletes: comparison between volleyball players and swimmers. BMC Pediatr. 2018. 18:212. doi:10.1186/s12887-018-1182-z

2403 Van Baak, M, Dopheide, J, Geleijnse, J, Bakker, S, Brink, E. Blood pressure and postprandial hemodynamic changes on a high protein versus high carbohydrate diet. Annals of nutrition and metabolism. 2011. 58:188. doi:10.1159/000334393

2404 van Baak, MA, Larsen, TM, Jebb, SA, Martinez, A, Saris, WHM, Handjieva-Darlenska, T, Kafatos, A, Pfeiffer, AFH, Kunesova, M, Astrup, A. Dietary Intake of Protein from Different Sources and Weight Regain, Changes in Body Composition and Cardiometabolic Risk Factors after Weight Loss: The DIOGenes Study. Nutrients. 2017. 9:. doi:10.3390/nu9121326

2405 van Bakel, MM, Kaaks, R, Feskens, EJ, Rohrmann, S, Welch, AA, Pala, V, Avloniti, K, van der Schouw, YT, van der, DI A, Du, H, Halkjaer, J, Tormo, MJ, Cust, AE, Brighenti, F, Beulens, JW, Ferrari, P, Biessy, C, Lentjes, M, Spencer, EA, Panico, S, Masala, G, Bueno-de-Mesquita, HB, Peeters, PH, Trichopoulou, A, Psaltopoulou, T, Clavel-Chapelon, F, Touvier, M, Skeie, G, Rinaldi, S, Sonestedt, E, Johansson, I, Schulze, M, Ardanaz, E, Buckland, G, Tjonneland, A, Overvad, K, Bingham, S, Riboli, E, Slimani, N. Dietary glycaemic index and glycaemic load in the European Prospective Investigation into Cancer and Nutrition. Eur J Clin Nutr. 2009. 63 Suppl 4:S188-205. doi:10.1038/ejcn.2009.81

2406 van Bussel, BC, Henry, RM, Ferreira, I, van Greevenbroek, MM, van der Kallen, CJ, Twisk, JW, Feskens, EJ, Schalkwijk CG, Stehouwer, CD. A healthy diet is associated with less endothelial dysfunction and less low-grade inflammation over a 7year period in adults at risk of cardiovascular disease. J Nutr. 2015. 145:532-40. doi:10.3945/jn.114.201236

Publication Status

Study duration

Publication Status

Intervention/Exposure Publication Status

Study Design;

Intervention/Exposure

Publication Status

Study Design

Study Design;

Intervention/Exposure;

Outcome

Intervention/Exposure 
Publication Date

Overlaps with Existing Review

2408 van Dam, RM, Willett, WC, Rimm, EB, Stampfer, MJ, Hu, FB. Dietary fat and meat intake in relation to risk of type 2 diabetes in men. Diabetes Care. 2002. 25:417-24. doi:10.2337/diacare.25.3.417

2409 van der Gaag, EJ, Wieffer, R, van der Kraats, J. Advising Consumption of Green Vegetables, Beef, and Full-Fat Dairy Products Has No Adverse Effects on the Lipid Profiles in Children. Nutrients. 2017. 9:. doi:10.3390/nu9050518

2410 Van Elten, TM, Karsten, MDA, Geelen, A, Gemke, RJBJ, Groen, H, Hoek, A, Van Poppel, MNM, Roseboom, TJ. Preconception lifestyle intervention reduces long term energy intake in women with obesity and infertility: A randomised controlled trial 11 Medical and Health Sciences 1117 Public Health and Health Services 11 Medical and Health Sciences 1111 Nutrition and Dietetics. International Journal of Behavioral Nutrition and Physical Activity. 2019. 16:. doi:10.1186/s12966-0180761-6

2411 van Elten, TM, Karsten, MDA, Geelen, A, Gemke, Rjbj, Groen, H, Hoek, A, van Poppel, MNM, Roseboom, TJ. Preconception lifestyle intervention reduces long term energy intake in women with obesity and infertility: a randomised controlled trial. Int J Behav Nutr Phys Act. 2019. 16:3. doi:10.1186/s12966-018-0761-6

2412 Van Elten, TM, Van Poppel, MNM, Gemke, Rjbj, Groen, H, Hoek, A, Mol, BW, Roseboom, TJ. Cardiometabolic Health in Relation to Lifestyle and Body Weight Changes 3(-)8 Years Earlier. Nutrients. 2018. 10:. doi:10.3390/nu10121953

2413 Van Elten, TM, Van Poppel, MNM, Gemke, RJBJ, Groen, H, Hoek, A, Mol, BW, Roseboom, TJ. Cardiometabolic health in relation to lifestyle and body weight changes 3-8 years earlier. Nutrients. 2018. 10:. doi:10.3390/nu10121953

2414 van Gemert, WA, Schuit, AJ, van der Palen, J, May, AM, lestra, JA, Wittink, H, Peeters, PH, Monninkhof, EM. Effect of weight loss, with or without exercise, on body composition and sex hormones in postmenopausal women: the SHAPE-2 trial. Breast Cancer Res. 2015. 17:120. doi:10.1186/s13058-015-0633-9

2415 van Hees, AM, Jocken, JW, Essers, Y, Roche, HM, Saris, WH, Blaak, EE. Adipose triglyceride lipase and hormonesensitive lipase protein expression in subcutaneous adipose tissue is decreased after an isoenergetic low-fat high-complex carbohydrate diet in the metabolic syndrome. Metabolism. 2012. 61:1404-12. doi:10.1016/j.metabol.2012.03.017

2416 van Herpen, NA, Schrauwen-Hinderling, VB, Schaart, G, Mensink, RP, Schrauwen, P. Three weeks on a high-fat diet increases intrahepatic lipid accumulation and decreases metabolic flexibility in healthy overweight men. $\mathrm{J}$ Clin Endocrinol Metab. 2011. 96:E691-5. doi:10.1210/jc.2010-2243

2417 Van Horn, L, Peaceman, A, Kwasny, M, Vincent, E, Fought, A, Josefson, J, Spring, B, Neff, LM, Gernhofer, N. Dietary Approaches to Stop Hypertension Diet and Activity to Limit Gestational Weight: Maternal Offspring Metabolics Family Intervention Trial, a Technology Enhanced Randomized Trial. Am J Prev Med. 2018. 55:603-614. doi:10.1016/j.amepre.2018.06.015

2418 van Nassau, F, Singh, AS, Cerin, E, Salmon, J, van Mechelen, W, Brug, J, Chinapaw, MJ. The Dutch Obesity Intervention in Teenagers (DOiT) cluster controlled implementation trial: intervention effects and mediators and moderators of adiposity and energy balance-related behaviours. Int J Behav Nutr Phys Act. 2014. 11:158. doi:10.1186/s12966-014-0158-0
Intervention/Exposure

Intervention/Exposure

Intervention/Exposure; Comparator

Intervention/Exposure; Outcome

Intervention/Exposure

Intervention/Exposure

Intervention/Exposure

Power/Size

Intervention/Exposure

Study duration

Participants

Intervention/Exposure 
2419 van Nielen, M, Feskens, EJ, Rietman, A, Siebelink, E, Mensink, M. Partly replacing meat protein with soy protein alters insulin resistance and blood lipids in postmenopausal women with abdominal obesity. J Nutr. 2014. 144:1423-9. doi:10.3945/jn.114.193706

Intervention/Exposure; Outcome; Study duration

2420 van Oostrom, AJ, Castro Cabezas, M, Ribalta, J, Masana, L, Twickler, TB, Remijnse, TA, Erkelens, DW. Diurnal triglyceride profiles in healthy normolipidemic male subjects are associated to insulin sensitivity, body composition and diet. Eur $\mathrm{J}$ Clin Invest. 2000. 30:964-71. doi:10.1046/j.1365-2362.2000.00732.x

2421 van Vught, AJ, Dagnelie, PC, Arts, IC, Froberg, K, Andersen, LB, El-Naaman, B, Bugge, A, Nielsen, BM, Heitman, BL. Dietary arginine and linear growth: the Copenhagen School Child Intervention Study. Br J Nutr. 2013. 109:1031-9. doi:10.1017/s0007114512002942

2422 van Vught, AJ, Heitmann, BL, Nieuwenhuizen, AG, Veldhorst, MA, Brummer, RJ, Westerterp-Plantenga, MS. Association between dietary protein and change in body composition among children (EYHS). Clin Nutr. 2009. 28:684-8. doi:10.1016/j.clnu.2009.05.001

2423 Vanacore, D, Messina, G, Lama, S, Bitti, G, Ambrosio, P, Tenore, G, Messina, A, Monda, V, Zappavigna, S, Boccellino, M, Novellino, E, Monda, M, Stiuso, P. Effect of restriction vegan diet's on muscle mass, oxidative status, and myocytes differentiation: A pilot study. J Cell Physiol. 2018. 233:9345-9353. doi:10.1002/jcp.26427

2424 Varady, KA, Bhutani, S, Klempel, MC, Phillips, SA. Improvements in vascular health by a low-fat diet, but not a high-fat diet, are mediated by changes in adipocyte biology. Nutr J. 2011. 10:8. doi:10.1186/1475-2891-10-8

2425 Varady, KA, Dam, VT, Klempel, MC, Horne, M, Cruz, R, Kroeger, CM, Santosa, S. Effects of weight loss via high fat vs. low fat alternate day fasting diets on free fatty acid profiles. Sci Rep. 2015. 5:7561. doi:10.1038/srep07561

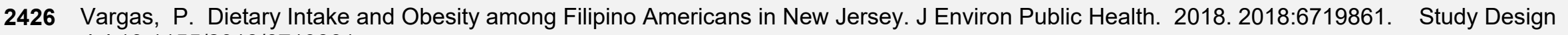
doi:10.1155/2018/6719861

2427 Vargas, S, Romance, R, Petro, JL, Bonilla, DA, Galancho, I, Espinar, S, Kreider, RB, Benitez-Porres, J. Efficacy of ketogenic diet on body composition during resistance training in trained men: a randomized controlled trial. J Int Soc Sports Nutr 2018. 15:31. doi:10.1186/s12970-018-0236-9

2428 Vasilaras, TH, Raben, A, Astrup, A. Twenty-four hour energy expenditure and substrate oxidation before and after 6 months' ad libitum intake of a diet rich in simple or complex carbohydrates or a habitual diet. Int J Obes Relat Metab Disord. 2001. 25:954-65. doi:10.1038/sj.ijo.0801630

2429 Velazquez-Lopez, L, Gonzalez-Figueroa, E, Medina-Bravo, P, Pineda-Del Aguila, I, Avila-Jimenez, L, Ramos-Hernandez, R, Klunder-Klunder, M, Escobedo-de la Pena, J. Low calorie and carbohydrate diet: to improve the cardiovascular risk indicators in overweight or obese adults with prediabetes. Endocrine. 2013. 43:593-602. doi:10.1007/s12020-012-9775-Z

2430 Velazquez-Lopez, L, Santiago-Diaz, G, Nava-Hernandez, J, Munoz-Torres, AV, Medina-Bravo, P, Torres-Tamayo, M. Mediterranean-style diet reduces metabolic syndrome components in obese children and adolescents with obesity. BMC Pediatr. 2014. 14:175. doi:10.1186/1471-2431-14-175 
2431 Veldhorst, MA, Westerterp, KR, van Vught, AJ, Westerterp-Plantenga, MS. Presence or absence of carbohydrates and the proportion of fat in a high-protein diet affect appetite suppression but not energy expenditure in normal-weight human subjects fed in energy balance. Br J Nutr. 2010. 104:1395-405. doi:10.1017/s0007114510002060

2432 Veldhorst, MA, Westerterp-Plantenga, MS, Westerterp, KR. Gluconeogenesis and energy expenditure after a high-protein carbohydrate-free diet. Am J Clin Nutr. 2009. 90:519-26. doi:10.3945/ajcn.2009.27834

2433 Veldhorst, MAB, Verbruggen, Scat, van Harskamp, D, Vermes, A, Schierbeek, H, van Goudoever, JB, van den Akker, ELT. Effects of a high-protein intake on metabolic targets for weight loss in children with obesity: a randomized trial. Obes Sci Pract. 2018. 4:347-356. doi:10.1002/osp4.277

2434 Venn, BJ, Perry, T, Green, TJ, Skeaff, CM, Aitken, W, Moore, NJ, Mann, JI, Wallace, AJ, Monro, J, Bradshaw, A, Brown, RC, Skidmore, PM, Doel, K, O'Brien, K, Frampton, C, Williams, S. The effect of increasing consumption of pulses and wholegrains in obese people: a randomized controlled trial. J Am Coll Nutr. 2010. 29:365-72. doi:10.1080/07315724.2010.10719853

2435 Veno, SK, Schmidt, EB, Jakobsen, MU, Lundbye-Christensen, S, Bach, FW, Overvad, K. Substitution of Linoleic Acid for Other Macronutrients and the Risk of Ischemic Stroke. Stroke. 2017. 48:3190-3195. doi:10.1161/strokeaha.117.017935

2436 Ventura, E, Davis, J, Byrd-Williams, C, Alexander, K, McClain, A, Lane, CJ, Spruijt-Metz, D, Weigensberg, M, Goran, M. Reduction in risk factors for type 2 diabetes mellitus in response to a low-sugar, high-fiber dietary intervention in overweight Latino adolescents. Arch Pediatr Adolesc Med. 2009. 163:320-7. doi:10.1001/archpediatrics.2009.11

2437 Verges, B, Fumeron, F. Potential risks associated with increased plasma plant-sterol levels. Diabetes Metab. $2015.41: 76-81$. doi:10.1016/j.diabet.2014.11.003

2438 Vergnaud, AC, Norat, T, Mouw, T, Romaguera, D, May, AM, Bueno-de-Mesquita, HB, van der, AD, Agudo, A, Wareham, N, Khaw, KT, Romieu, I, Freisling, H, Slimani, N, Perquier, F, Boutron-Ruault, MC, Clavel-Chapelon, F, Palli, D, Berrino, F, Mattiello, A, Tumino, R, Ricceri, F, Rodriguez, L, Molina-Montes, E, Amiano, P, Barricarte, A, Chirlaque, MD, Crowe, FL, Orfanos, P, Naska, A, Trichopoulou, A, Teucher, B, Kaaks, R, Boeing, H, Buijsse, B, Johansson, I, Hallmans, G, Drake, I, Sonestedt, E, Jakobsen, MU, Overvad, K, Tjonneland, A, Halkjaer, J, Skeie, G, Braaten, T, Lund, E, Riboli, E, Peeters, $\mathrm{PH}$. Macronutrient composition of the diet and prospective weight change in participants of the EPIC-PANACEA study. PLoS One. 2013. 8:e57300. doi:10.1371/journal.pone.0057300

2439 Verhoef, P, van Vliet, T, Olthof, MR, Katan, MB. A high-protein diet increases postprandial but not fasting plasma total homocysteine concentrations: a dietary controlled, crossover trial in healthy volunteers. Am J Clin Nutr. 2005. 82:553-8. doi:10.1093/ajcn.82.3.553

2440 Vernon, MC, Kueser, B, Transue, M, Yates, HE, Yancy, WS, Westman, EC. Clinical experience of a carbohydrate-restricted diet for the metabolic syndrome. Metab Syndr Relat Disord. 2004. 2:180-6. doi:10.1089/met.2004.2.180

2441 Veronese, N, Li, Y, Manson, JE, Willett, WC, Fontana, L, Hu, FB. Combined associations of body weight and lifestyle factors with all cause and cause specific mortality in men and women: prospective cohort study. Bmj. 2016. 355:i5855. doi:10.1136/bmj.i5855

Publication Date

Overlaps with Existing

Review

Intervention/Exposure

Intervention/Exposure

Study Design

Intervention/Exposure

Intervention/Exposure

Study duration

Intervention/Exposure

Intervention/Exposure 
2442 Verreijen, AM, Engberink, MF, Houston, DK, Brouwer, IA, Cawthon, PM, Newman, AB, Tylavsky, FA, Harris, TB, Weijs, PJM, Visser, M. Dietary protein intake is not associated with 5-y change in mid-thigh muscle cross-sectional area by computed tomography in older adults: the Health, Aging, and Body Composition (Health ABC) Study. Am J Clin Nutr. 2019. 109:535-543. doi:10.1093/ajcn/nqy341

2443 Verreijen, AM, Engberink, MF, Memelink, RG, van der Plas, SE, Visser, M, Weijs, PJ. Effect of a high protein diet and/or resistance exercise on the preservation of fat free mass during weight loss in overweight and obese older adults: a randomized controlled trial. Nutr J. 2017. 16:10. doi:10.1186/s12937-017-0229-6

2444 Vetter, ML, Iqbal, N, Dalton-Bakes, C, Volger, S, Wadden, TA. Long-term effects of low-carbohydrate versus low-fat diets in obese persons. Annals of Internal Medicine. 2010. 152:334-335. doi:10.7326/0003-4819-152-5-201003020-00020

2445 Veum, V, Laupsa-Borge, J, Eng, O, Rostrup, E, Solsvik, M, Larsen, T, Nordrehaug, JE, Nygaard, O, Sagen, J, Gudbrandsen, O, etal, . Very-high-fat and isocaloric low-fat diet interventions in overweight middle-aged men-results from a randomized trial. Obesity facts. 2014. 7:68. doi:10.1159/000363668

2446 Veum, VL, Laupsa-Borge, J, Eng, O, Rostrup, E, Larsen, TH, Nordrehaug, JE, Nygard, OK, Sagen, JV, Gudbrandsen, OA, Dankel, SN, Mellgren, G. Visceral adiposity and metabolic syndrome after very high-fat and low-fat isocaloric diets: a randomized controlled trial. Am J Clin Nutr. 2017. 105:85-99. doi:10.3945/ajcn.115.123463

2447 Vicari, RM, Bramlet, D, Olivera, B, Barber, L, Alexander, L, Parker, L, Howard, M. Atherosclerosis and teen eating study. J Clin Lipidol. 2007. 1:194-7. doi:10.1016/j.jacl.2007.04.005

2448 Vidal-Peracho, C, Tricas-Moreno, JM, Lucha-Lopez, AC, Lucha-Lopez, MO, Camunas-Pescador, AC, Caverni-Munoz, A, Fanlo-Mazas, P. Adherence to Mediterranean Diet Pattern among Spanish Adults Attending a Medical Centre: Nondiabetic Subjects and Type 1 and 2 Diabetic Patients. J Diabetes Res. 2017. 2017:5957821. doi:10.1155/2017/5957821

2449 Vidon, C, Boucher, P, Cachefo, A, Peroni, O, Diraison, F, Beylot, M. Effects of isoenergetic high-carbohydrate compared with high-fat diets on human cholesterol synthesis and expression of key regulatory genes of cholesterol metabolism. Am $\mathrm{J}$ Clin Nutr. 2001. 73:878-84. doi:10.1093/ajcn/73.5.878

2450 Vilela, S, Oliveira, A, Severo, M, Lopes, C. Chrono-Nutrition: The Relationship between Time-of-Day Energy and Macronutrient Intake and Children's Body Weight Status. J Biol Rhythms. 2019. 34:332-342. doi:10.1177/0748730419838908

2451 Viljakainen, HT, Valta, H, Lipsanen-Nyman, M, Saukkonen, T, Kajantie, E, Andersson, S, Makitie, O. Bone Characteristics and Their Determinants in Adolescents and Young Adults with Early-Onset Severe Obesity. Calcif Tissue Int. 2015. 97:364-75. doi:10.1007/s00223-015-0031-4

2452 Viljanen, AP, Karmi, A, Borra, R, Parkka, JP, Lepomaki, V, Parkkola, R, Lautamaki, R, Jarvisalo, M, Taittonen, M, Ronnemaa, T, lozzo, P, Knuuti, J, Nuutila, P, Raitakari, OT. Effect of caloric restriction on myocardial fatty acid uptake, left ventricular mass, and cardiac work in obese adults. Am J Cardiol. 2009. 103:1721-6. doi:10.1016/j.amjcard.2009.02.025

Intervention/Exposure; Comparator

Publication Status

Publication Status

Weight

loss/Hypocaloric

Intervention/Exposure; Publication Date

Overlaps with Existing

Review

Study Design

Study duration

Intervention/Exposure

Study Design;

Outcome

Study Design 
2453 Viner, RT, Harris, M, Berning, JR, Meyer, NL. Energy Availability and Dietary Patterns of Adult Male and Female Competitive

Comparator; Outcome Cyclists With Lower Than Expected Bone Mineral Density. Int J Sport Nutr Exerc Metab. 2015. 25:594-602. doi:10.1123/ijsnem.2015-0073

2454 Virtanen, HEK, Voutilainen, S, Koskinen, TT, Mursu, J, Kokko, P, Ylilauri, MPT, Tuomainen, TP, Salonen, JT, Virtanen, JK. Dietary proteins and protein sources and risk of death: the Kuopio Ischaemic Heart Disease Risk Factor Study. Am J Clin Nutr. 2019. 109:1462-1471. doi:10.1093/ajcn/nqz025

2455 Virtanen, JK, Mursu, J, Tuomainen, TP, Voutilainen, S. Dietary fatty acids and risk of coronary heart disease in men: the Kuopio Ischemic Heart Disease Risk Factor Study. Arterioscler Thromb Vasc Biol. 2014. 34:2679-87. doi:10.1161/atvbaha.114.304082

2456 Viscogliosi, G, Cipriani, E, Liguori, ML, Marigliano, B, Saliola, M, Ettorre, E, Andreozzi, P. Mediterranean dietary pattern adherence: Associations with prediabetes, metabolic syndrome, and related microinflammation. Metabolic Syndrome and Related Disorders. 2013. 11:210-216. doi:10.1089/met.2012.0168

2457 Vissers, LET, Waller, M, van der Schouw, YT, Hebert, JR, Shivappa, N, Schoenaker, Dajm, Mishra, GD. A proinflammatory diet is associated with increased risk of developing hypertension among middle-aged women. Nutr Metab Cardiovasc Dis. 2017. 27:564-570. doi:10.1016/j.numecd.2017.03.005

2458 Visuthranukul, C, Sirimongkol, P, Prachansuwan, A, Pruksananonda, C, Chomtho, S. Low-glycemic index diet may improve insulin sensitivity in obese children. Pediatr Res. 2015. 78:567-73. doi:10.1038/pr.2015.142

2459 Vogel, RA, Corretti, MC, Plotnick, GD. The postprandial effect of components of the Mediterranean diet on endothelial function. Journal of the American College of Cardiology. 2000. 36:1455-1460. doi:10.1016/S0735-1097(00)00896-2

2460 Volek, J, Sharman, M, Gomez, A, Judelson, D, Rubin, M, Watson, G, Sokmen, B, Silvestre, R, French, D, Kraemer, W Comparison of energy-restricted very low-carbohydrate and low-fat diets on weight loss and body composition in overweight men and women. Nutr Metab (Lond). 2004. 1:13. doi:10.1186/1743-7075-1-13

2461 Volek, JS, Ballard, KD, Silvestre, R, Judelson, DA, Quann, EE, Forsythe, CE, Fernandez, ML, Kraemer, WJ. Effects of dietary carbohydrate restriction versus low-fat diet on flow-mediated dilation. Metabolism. 2009. 58:1769-77. doi:10.1016/j.metabol.2009.06.005

2462 Volek, JS, Phinney, SD, Forsythe, CE, Quann, EE, Wood, RJ, Puglisi, MJ, Kraemer, WJ, Bibus, DM, Fernandez, ML, Feinman, RD. Carbohydrate restriction has a more favorable impact on the metabolic syndrome than a low fat diet. Lipids. 2009. 44:297-309. doi:10.1007/s11745-008-3274-2

2463 Volek, JS, Sharman, MJ, Gomez, AL, DiPasquale, C, Roti, M, Pumerantz, A, Kraemer, WJ. Comparison of a very lowcarbohydrate and low-fat diet on fasting lipids, LDL subclasses, insulin resistance, and postprandial lipemic responses in overweight women. J Am Coll Nutr. 2004. 23:177-84. doi:10.1080/07315724.2004.10719359

2464 Volek, JS, Sharman, MJ, Gomez, AL, Scheett, TP, Kraemer, WJ. An isoenergetic very low carbohydrate diet improves serum HDL cholesterol and triacylglycerol concentrations, the total cholesterol to HDL cholesterol ratio and postprandial pipemic responses compared with a low fat diet in normal weight, normolipidemic women. J Nutr. 2003. 133:2756-61. doi:10.1093/jn/133.9.2756 
2465 Volek, JS, Sharman, MJ, Love, DM, Avery, NG, Gomez, AL, Scheett, TP, Kraemer, WJ. Body composition and hormonal responses to a carbohydrate-restricted diet. Metabolism. 2002. 51:864-70. doi:10.1053/meta.2002.32037

2466 Volger, S, Sheng, X, Tong, LM, Zhao, D, Fan, T, Zhang, F, Ge, J, Ho, WM, Hays, NP, Yao, MP. Nutrient intake and dietary patterns in children $2.5-5$ years of age with picky eating behaviours and low weight-for-height. Asia Pac $\mathrm{J}$ Clin Nutr. 2017. 26:104-109. doi:10.6133/apjcn.102015.02

2467 Vollmer, WM, Sacks, FM, Svetkey, LP. New insights into the effects on blood pressure of diets low in salt and high in fruits and vegetables and low-fat dairy products. Curr Control Trials Cardiovasc Med. 2001. 2:71-74. doi:10.1186/cvm-2-2-071

2468 Volp, AC, Hermsdorff, HH, Bressan, J. Glycemia and insulinemia evaluation after high-sucrose and high-fat diets in lean and overweight/obese women. J Physiol Biochem. 2008. 64:103-13. doi:unavailable

2469 Volp, AC, Hermsdorff, HM, Bressan, J. Effect of high sucrose- and high-fat diets ingested under free-living conditions in insulin Language resistance in normal weight and overweight women. Nutricion hospitalaria. 2007. 22:46-60. doi:unavailable

2470 Voltas, N, Arija, V, Aparicio, E, Canals, J. Longitudinal study of psychopathological, anthropometric and sociodemographic factors related to the level of Mediterranean diet adherence in a community sample of Spanish adolescents. Public Health Nutr. 2016. 19:1812-22. doi:10.1017/s1368980015003560

2471 von Frankenberg, AD, Marina, A, Song, X, Callahan, HS, Kratz, M, Utzschneider, KM. A high-fat, high-saturated fat diet decreases insulin sensitivity without changing intra-abdominal fat in weight-stable overweight and obese adults. Eur $\mathrm{J}$ Nutr. 2017. 56:431-443. doi:10.1007/s00394-015-1108-6

2472 von Ruesten, A, Feller, S, Bergmann, MM, Boeing, H. Diet and risk of chronic diseases: results from the first 8 years of follow-up in the EPIC-Potsdam study. Eur J Clin Nutr. 2013. 67:412-9. doi:10.1038/ejcn.2013.7

2473 Voortman, T, Braun, KV, Kiefte-de Jong, JC, Jaddoe, VW, Franco, OH, van den Hooven, EH. Protein intake in early childhood and body composition at the age of 6 years: The Generation R Study. Int J Obes (Lond). 2016. 40:1018-25. doi:10.1038/ijo.2016.29

2474 Voortman, T, Leermakers, ET, Franco, OH, Jaddoe, VW, Moll, HA, Hofman, A, van den Hooven, EH, Kiefte-de Jong, JC. A priori and a posteriori dietary patterns at the age of 1 year and body composition at the age of 6 years: the Generation $\mathrm{R}$ Study Eur J Epidemiol. 2016. 31:775-83. doi:10.1007/s10654-016-0179-X

2475 Wade, AT, Davis, CR, Dyer, KA, Hodgson, JM, Woodman, RJ, Keage, HAD, Murphy, KJ. A Mediterranean diet supplemented with dairy foods improves mood and processing speed in an Australian sample: results from the MedDairy randomized controlled trial. Nutr Neurosci. 2018. :1-13. doi:10.1080/1028415x.2018.1543148

2476 Wade, AT, Davis, CR, Dyer, KA, Hodgson, JM, Woodman, RJ, Murphy, KJ. A Mediterranean diet supplemented with dairy foods improves markers of cardiovascular risk: results from the MedDairy randomized controlled trial. Am J Clin Nutr. 2018. 108:1166-1182. doi:10.1093/ajcn/nqy207

2477 Wade, AT, Davis, CR, Dyer, KA, Hodgson, JM, Woodman, RJ, Murphy, KJ. Effects of Mediterranean diet supplemented with lean pork on blood pressure and markers of cardiovascular risk: findings from the MedPork trial. Br J Nutr. 2019. 122:873883. doi:10.1017/s0007114519001168

duration Outcome

Outcome Age at

Age at

Outcome

Outcome; Study

Intervention/Exposure;

Study Design

Study duration

Intervention/Exposure

Intervention/Exposure; Intervention/Exposure

Intervention/Exposure

Study duration

Study duration 
2478 Wadolowska, L, Hamulka, J, Kowalkowska, J, Ulewicz, N, Hoffmann, M, Gornicka, M, Bronkowska, M, Leszczynska, T, Glibowski, P, Korzeniowska-Ginter, R. Changes in Sedentary and Active Lifestyle, Diet Quality and Body Composition Nine Months after an Education Program in Polish Students Aged 11(-)12 Years: Report from the ABC of Healthy Eating Study. Nutrients. 2019. 11:. doi:10.3390/nu11020331

2479 Waern, RV, Cumming, RG, Blyth, F, Naganathan, V, Allman-Farinelli, M, Le Couteur, D, Simpson, SJ, Kendig, H, Hirani, V. Adequacy of nutritional intake among older men living in Sydney, Australia: findings from the Concord Health and Ageing in Men Project (CHAMP). Br J Nutr. 2015. 114:812-21. doi:10.1017/s0007114515002421

2480 Waijers, PM, Ocke, MC, van Rossum, CT, Peeters, PH, Bamia, C, Chloptsios, Y, van der Schouw, YT, Slimani, N, Buenode-Mesquita, HB. Dietary patterns and survival in older Dutch women. Am J Clin Nutr. 2006. 83:1170-6. doi:10.1093/ajcn/83.5.1170

2481 , Buetz, J, Ruebsamen, N, Zyriax, BC, Blaum, C, Kroeger, F, Kohsiack, R, Schrage, B, Sinning, C, Becher, PM, Karakas, M, Zeller, T, Westermann, D, Sydow, K, Blankenberg, S, Seiffert, M, Schnabel, RB. Adherence to Mediterranean diet, high-sensitive C-reactive protein, and severity of coronary artery disease: Contemporary data from the INTERCATH cohort. Atherosclerosis. 2018. 275:256-261. doi:10.1016/j.atherosclerosis.2018.06.877

2482 Waldman, HS, Krings, BM, Basham, SA, Smith, JEW, Fountain, BJ, McAllister, MJ. Effects of a 15-Day Low Carbohydrate, High-Fat Diet in Resistance-Trained Men. J Strength Cond Res. 2018. 32:3103-3111. doi:10.1519/jsc.0000000000002282

2483 Waldman, HS, Smith, JW, Lamberth, J, Fountain, BJ, McAllister, MJ. A 28-Day Carbohydrate-Restricted Diet Improves Markers of Cardiometabolic Health and Performance in Professional Firefighters. J Strength Cond Res. 2019. :. doi:10.1519/jsc.0000000000003354

2484 Walilko, E, Napierala, MU, Bryskiewicz, ME, Majkowska, L. Influence of short-term high-protein diet and low glycemic index diet on body mass and composition in overweight and obese subjects. Diabetes. 2013. 62:A195-A196. doi:10.2337/db13-680858

2485 Walker, CG, Loos, RJ, Mander, AP, Jebb, SA, Frost, GS, Griffin, BA, Lovegrove, JA, Sanders, TA, Bluck, LJ. Genetic predisposition to type 2 diabetes is associated with impaired insulin secretion but does not modify insulin resistance or secretion in response to an intervention to lower dietary saturated fat. Genes Nutr. 2012. 7:529-36. doi:10.1007/s12263-012-0284-8

2486 Walker, CG, Loos, RJ, Olson, AD, Frost, GS, Griffin, BA, Lovegrove, JA, Sanders, TA, Jebb, SA. Genetic predisposition influences plasma lipids of participants on habitual diet, but not the response to reductions in dietary intake of saturated fatty acids. Atherosclerosis. 2011. 215:421-7. doi:10.1016/j.atherosclerosis.2010.12.039

2487 Walker, CG, Solis-Trapala, I, Holzapfel, C, Ambrosini, GL, Fuller, NR, Loos, RJ, Hauner, H, Caterson, ID, Jebb, SA. Modelling the Interplay between Lifestyle Factors and Genetic Predisposition on Markers of Type 2 Diabetes Mellitus Risk. PLoS One. 2015. 10:e0131681. doi:10.1371/journal.pone.0131681

2488 Wall, CR, Gammon, CS, Bandara, DK, Grant, CC, Atatoa Carr, PE, Morton, SM. Dietary Patterns in Pregnancy in New Zealand-Influence of Maternal Socio-Demographic, Health and Lifestyle Factors. Nutrients. 2016. 8:. doi:10.3390/nu8050300
Intervention/Exposure; Outcome

Outcome

Outcome

Outcome

Study duration

Study Design

Publication Status;

Study duration

Intervention/Exposure

Study Design;

Intervention/Exposure

Intervention/Exposure

Study Design; Outcome; Participants 
2489 Wallström, P, Sonestedt, E, Hlebowicz, J, Ericson, U, Drake, I, Persson, M, Gullberg, B, Hedblad, B, Wirfält, E. Dietary fiber and saturated fat intake associations with cardiovascular disease differ by sex in the Malmö diet and cancer cohort: A prospective study. PLoS ONE. 2012. 7:. doi:10.1371/journal.pone.0031637

2490 Walsh, El, Jacka, FN, Butterworth, P, Anstey, KJ, Cherbuin, N. The association between Western and Prudent dietary patterns and fasting blood glucose levels in type 2 diabetes and normal glucose metabolism in older Australian adults. Heliyon. 2017. 3:e00315. doi:10.1016/j.heliyon.2017.e00315

2491 Walthouwer, MJ, Oenema, A, Lechner, L, de Vries, H. Comparing a Video and Text Version of a Web-Based ComputerTailored Intervention for Obesity Prevention: A Randomized Controlled Trial. J Med Internet Res. 2015. 17:e236. doi:10.2196/jmir.4083

2492 Wang, C, Almoosawi, S, Palla, L. Day-Time Patterns of Carbohydrate Intake in Adults by Non-Parametric Multi-Level Latent Class Analysis-Results from the UK National Diet and Nutrition Survey (2008/09-2015/16). Nutrients. 2019. 11: doi:10.3390/nu11102476

2493 Wang, DD, Li, Y, Chiuve, SE, Hu, FB, Willett, WC. Improvements In US Diet Helped Reduce Disease Burden And Lower Premature Deaths, 1999-2012; Overall Diet Remains Poor. Health Aff (Millwood). 2015. 34:1916-22. doi:10.1377/hlthaff.2015.0640

2494 Wang, DD, Li, Y, Chiuve, SE, Stampfer, MJ, Manson, JE, Rimm, EB, Willett, WC, Hu, FB. Association of Specific Dietary Fats With Total and Cause-Specific Mortality. JAMA Intern Med. 2016. 176:1134-45. doi:10.1001/jamainternmed.2016.2417

2495 Wang, H, Fox, CS, Troy, LM, McKeown, NM, Jacques, PF. Longitudinal association of dairy consumption with the changes in blood pressure and the risk of incident hypertension: the Framingham Heart Study. Br J Nutr. 2015. 114:1887-99. doi:10.1017/s0007114515003578

2496 Wang, HH, Wong, MC, Mok, RY, Kwan, MW, Chan, WM, Fan, CK, Lee, CL, Griffiths, SM. Factors associated with grade 1 hypertension: implications for hypertension care based on the Dietary Approaches to Stop Hypertension (DASH) in primary care settings. BMC Fam Pract. 2015. 16:26. doi:10.1186/s12875-015-0239-4

2497 Wang, J, Garcia-Bailo, B, Nielsen, DE, El-Sohemy, A. ABO genotype, 'blood-type' diet and cardiometabolic risk factors. PLoS Study Design One. 2014. 9:e84749. doi:10.1371/journal.pone.0084749

2498 Wang, L, Bordi, PL, Fleming, JA, Hill, AM, Kris-Etherton, PM. Effect of a moderate fat diet with and without avocados on lipoprotein particle number, size and subclasses in overweight and obese adults: a randomized, controlled trial. J Am Heart Assoc. 2015. 4:e001355. doi:10.1161/jaha.114.001355

2499 Wang, L, Dalton, WT, Schetzina, KE, Fulton-Robinson, H, Holt, N, Ho, AL, Tudiver, F, Wu, T. Home food environment, dietary intake, and weight among overweight and obese children in southern appalachia. Southern Medical Journal. 2013. 106:550-557. doi:10.1097/SMJ.0000000000000008

2500 Wang, P, Holst, C, Andersen, MR, Astrup, A, Bouwman, FG, van Otterdijk, S, Wodzig, WK, van Baak, MA, Larsen, TM Jebb, SA, Kafatos, A, Pfeiffer, AF, Martinez, JA, Handjieva-Darlenska, T, Kunesova, M, Saris, WH, Mariman, EC. Blood profile of proteins and steroid hormones predicts weight change after weight loss with interactions of dietary protein level and glycemic index. PLoS One. 2011. 6:e16773. doi:10.1371/journal.pone.0016773

Study Design

Intervention/Exposure

Study Design

Intervention/Exposure

Study Design;

Intervention/Exposure

Intervention/Exposure

Intervention/Exposure

Study Design

Study duration

Study Design;

Intervention/Exposure

Intervention/Exposure 
2501 Wang, T, Heianza, Y, Sun, D, Rood, J, Bray, GA, Sacks, FM, Qi, L. Dietary protein intake modifies the genetic risk on insulin resistance in the pounds lost trial. Circulation. 2016. 134:. doi:unavailable

2502 Wang, X, Li, X, Vaartjes, I, Neal, B, Bots, ML, Hoes, AW, Wu, Y. Does education level affect the efficacy of a community based salt reduction program? - A post-hoc analysis of the China Rural Health Initiative Sodium Reduction Study (CRHI-SRS). BMC Public Health. 2016. 16:759. doi:10.1186/s12889-016-3454-6

2503 Wang, Y, Kaneko, T, Wang, PY, Sato, A. Decreased carbohydrate intake is more important than increased fat intake in the glucose intolerance by a low-carbohydrate/high-fat diet. Diabetes Research and Clinical Practice. 2002. 55:61-63. doi:10.1016/S0168-8227(01)00291-1

2504 Wang, YF, Yancy, WS, Jr, Yu, D, Champagne, C, Appel, LJ, Lin, PH. The relationship between dietary protein intake and blood pressure: results from the PREMIER study. J Hum Hypertens. 2008. 22:745-54. doi:10.1038/jhh.2008.64

2505 Wang, Z, Adair, LS, Cai, J, Gordon-Larsen, P, Siega-Riz, AM, Zhang, B, Popkin, BM. Diet Quality Is Linked to Insulin Resistance among Adults in China. J Nutr. 2017. 147:2102-2108. doi:10.3945/jn.117.256180

2506 Wang, Z, Siega-Riz, AM, Gordon-Larsen, P, Cai, J, Adair, LS, Zhang, B, Popkin, BM. Diet quality and its association with type 2 diabetes and major cardiometabolic risk factors among adults in China. Nutr Metab Cardiovasc Dis. 2018. 28:987-1001. doi:10.1016/j.numecd.2018.06.012

2507 Waśkiewicz, A, Piotrowski, W, Sygnowska, E, Rywik, S, Jasiński, B. Did favourable trends in food consumption observed in the 1984-2001 period contribute to the decrease in cardiovascular mortality? - Pol-MONICA Warsaw Project. Kardiologia Polska. 2006. 64:16-23. doi:unavailable

2508 Waskiewicz, A, Piotrowski, W, Szostak-Wegierek, D, Cicha-Mikolajczyk, A. Relationship between 28-year food consumption trends and the 10-year global risk of death due to cardiovascular diseases in the adult Warsaw population. Kardiol Pol. 2015. 73:650-5. doi:10.5603/KP.a2015.0070

2509 Waterplas, J, Versele, V, D'Hondt, E, Lefevre, J, Mertens, E, Charlier, R, Knaeps, S, Clarys, P. A 10-year longitudinal study on the associations between changes in plant-based diet indices, anthropometric parameters and blood lipids in a Flemish adult population. Nutr Diet. 2019. .. doi:10.1111/1747-0080.12578 
2510 Weber, B, Bersch-Ferreira, AC, Torreglosa, CR, Marcadenti, A, Lara, ES, da Silva, JT, Costa, RP, Santos, RHN, Berwanger, O, Bosquetti, R, Pagano, R, Mota, LGS, de Oliveira, JD, Soares, RM, Galante, AP, da Silva, SA, Zampieri, FG, Status Kovacs, C, Amparo, FC, Moreira, P, da Silva, RA, Dos Santos, KG, Monteiro, AS, Paiva, CCJ, Magnoni, CD, Moreira, ASB, Pecanha, DO, Missias, KCS, de Paula, LS, Marotto, D, Souza, P, Martins, PRT, Dos Santos, EM, Santos, MR, Silva, LP, Torres, RS, Barbosa, Snaa, de Pinho, PM, de Araujo, SHA, Verissimo, AOL, Guterres, AS, Cardoso, AFR, Palmeira, MM, de Ataide, BRB, Costa, LPS, Marinho, HA, de Araujo, CBP, Carvalho, HMS, Maquine, RO, Caiado, AC, de Matos, CH, Barretta, C, Specht, CM, Onofrei, M, Bertacco, RTA, Borges, LR, Bertoldi, EG, Longo, A, Ribas, BLP, Dobke, F, Pretto, ADB, Bachettini, NP, Gastaud, A, Necchi, R, Souza, GC, Zuchinali, P, Fracasso, BM, Bobadra, S, Sangali, TD, Salamoni, J, Garlini, LM, Shirmann, GS, de Los Santos, MLP, Bortonili, VMS, Dos Santos, CP, Braganca, GCM, Ambrozio, CL, Lima SB E, Schiavini, J, Napparo, AS, Boemo, JL, Nagano, FEZ, Modanese, PVG, Cunha, NM, Frehner, C, da Silva, LF, Formentini, FS, Ramos, MEM, Ramos, SS, Lucas, MCS, Machado, BG, Ruschel, KB, Beiersdorf, JR, Nunes, CE, Rech, RL, Damiani, M, Berbigier, M, Poloni, S, Vian, I, Russo, DS, Rodrigues, JA, de Moraes, MAP, da Costa, LM, Boklis, M, EI Kik, RM, Adorne, EF, Teixeira, JM, Trescastro, EP, Chiesa, FL, Telles, CT, Pellegrini, LA, Reis, LF, Cardoso, RGM, Closs, VE, Feres, NH, da Silva, NF, Silva, NE, Dutra, ES, Ito, MK, Lima, MEP, Carvalho, Appf, Taboada, MIS, Machado, MMA, David, MM, Junior, DGS, Dourado, C, Fagundes, Vcfo, Uehara, RM, Sasso, S, Vieira, JSO, de Oliveira, BAS, Pereira, JL, Rodrigues, IG, Pinho, CPS, Sousa, ACS, Almeida, AS, de Jesus, MT, da Silva, GB, Alves, LVS, Nascimento, VOG, Vieira, SA, Coura, AGL, Dantas, CF, Leda, Nmfs, Medeiros, AL, Andrade, ACL, Pinheiro, JMF, de Lima, LRM, Sabino, LS, de Souza, CVS, Vasconcelos, SML, Costa, FA, Ferreira, RC, Cardoso, IB, Navarro, LNP, Ferreira, RB, Junior, AES, Silva, MBG, Almeida, KMM, Penafort, AM, de Queiros, APO, Farias, GMN, Carlos, DMO, Cordeiro, Cgnc, Vasconcelos, VB, de Araujo, Emvmc, Sahade, V, Ribeiro, CSA, Araujo, GA, Goncalves, LB, Teixeira, CS, Silva, Lmaj, da Costa, LB, Souza, TS, de Jesus, SO, Luna, AB, da Rocha, BRS, Santos, MA, Neto, JAF, Dias, LPP, Cantanhede, RCA, Morais, JM, Duarte, RCL, Barbosa, ECB, Barbosa, JMA, de Sousa, RML, Dos Santos, AF, Teixeira, AF, Moriguchi, EH, Bruscato, NM, Kesties, J, Vivian, L, de Carli, W, Shumacher, M, Izar, MCO, Asoo, MT, Kato, JT, Martins, CM, Machado, VA, Bittencourt, CRO, de Freitas, TT, Sant'Anna, VAR, Lopes, JD, Fischer, Scpm, Pinto, SL, Silva, KC, Gratao, LHA, Holzbach, LC, Backes, LM, Rodrigues, MP, Deucher, Klal, Cantarelli, M, Bertoni, VM, Rampazzo, D, Bressan, J, Hermsdorff, HHM, Caldas, APS, Felicio, MB, Honorio, CR, da Silva, A, Souza, SR, Rodrigues, PA, de Meneses, TMX, Kumbier, MCC, Barreto, AL, Cavalcanti, AB. Implementation of a Brazilian Cardioprotective Nutritional (BALANCE) Program for improvement on quality of diet and secondary prevention of cardiovascular events: A randomized, multicenter trial. Am Heart J. 2019. 215:187-197. doi:10.1016/j.ahj.2019.06.010

2511 Weder, S, Hoffmann, M, Becker, K, Alexy, U, Keller, M. Energy, Macronutrient Intake, and Anthropometrics of Vegetarian, Vegan, and Omnivorous Children (1(-)3 Years) in Germany (VeChi Diet Study). Nutrients. 2019. 11:. doi:10.3390/nu11040832

2512 Weech, M, Vafeiadou, K, Mihaylova, R, Hasaj, M, Yaqoob, P, Todd, S, Jackson, KG, Lovegrove, JA. Substitution of dietary saturated fatty acids with either monounsaturated or $n-6$ polyunsaturated fatty acids improves the plasma lipid profile in those at risk from CVD: results from the DIVAS study. Proceedings of the nutrition society. 2013. 72:E182. doi:10.1017/S0029665113002073 
2513 Wei, M, Brandhorst, S, Shelehchi, M, Mirzaei, H, Cheng, CW, Budniak, J, Groshen, S, Mack, WJ, Guen, E, Di Biase, S, Cohen, P, Morgan, TE, Dorff, T, Hong, K, Michalsen, A, Laviano, A, Longo, VD. Fasting-mimicking diet and markers/risk factors for aging, diabetes, cancer, and cardiovascular disease. Science Translational Medicine. 2017. 9:. doi:10.1126/scitranslmed.aai8700

2514 Weickert, MO, Roden, M, Isken, F, Hoffmann, D, Nowotny, P, Osterhoff, M, Blaut, M, Alpert, C, Gogebakan, O, BumkeVogt, C, Mueller, F, Machann, J, Barber, TM, Petzke, KJ, Hierholzer, J, Hornemann, S, Kruse, M, Illner, AK, Kohl, A, Loeffelholz, CV, Arafat, AM, Mohlig, M, Pfeiffer, AF. Effects of supplemented isoenergetic diets differing in cereal fiber and protein content on insulin sensitivity in overweight humans. Am J Clin Nutr. 2011. 94:459-71. doi:10.3945/ajcn.110.004374

2515 Weigle, DS, Breen, PA, Matthys, CC, Callahan, HS, Meeuws, KE, Burden, VR, Purnell, JQ. A high-protein diet induces sustained reductions in appetite, ad libitum caloric intake, and body weight despite compensatory changes in diurnal plasma leptin and ghrelin concentrations. Am J Clin Nutr. 2005. 82:41-8. doi:10.1093/ajcn.82.1.41

2516 Weigle, DS, Cummings, DE, Newby, PD, Breen, PA, Frayo, RS, Matthys, CC, Callahan, HS, Purnell, JQ. Roles of leptin and ghrelin in the loss of body weight caused by a low fat, high carbohydrate diet. J Clin Endocrinol Metab. 2003. 88:1577-86. doi:10.1210/jc.2002-021262

2517 Weihua Mmed, L, Yougang, W, Jing, W. Reduced or modified dietary fat for preventing cardiovascular disease. J Cardiovasc Nurs. 2013. 28:204-5. doi:10.1097/JCN.0b013e3182816005

2518 Weijs, PJM, Wolfe, RR. Exploration of the protein requirement during weight loss in obese older adults. Clin Nutr. 2016. 35:394-398. doi:10.1016/j.clnu.2015.02.016

2519 Weise, CM, Hohenadel, MG, Krakoff, J, Votruba, SB. Body composition and energy expenditure predict ad-libitum food and macronutrient intake in humans. Int J Obes (Lond). 2014. 38:243-51. doi:10.1038/ijo.2013.85

2520 Weker, H, Baranska, M, Riahi, A, Strucinska, M, Wiech, M, Rowicka, G, Dylag, H, Klemarczyk, W, Bzikowska, A, Socha, $P$. Dietary patterns in toddlers with excess weight. The 2016 pitnuts study. Dev Period Med. 2017. 21:272-285. doi:unavailable

2521 Wekesa, AL, Doyle, LM, Fitzmaurice, D, O'Donovan, O, Phelan, JP, Ross, MD, Cross, KS, Harrison, M. Influence of a lowcarbohydrate diet on endothelial microvesicles in overweight women. Appl Physiol Nutr Metab. 2016. 41:522-7. doi:10.1139/apnm-2015-0507

2522 Wellington, N, Shanmuganathan, M, de Souza, RJ, Zulyniak, MA, Azab, S, Bloomfield, J, Mell, A, Ly, R, Desai, D, Anand, SS, Britz-McKibbin, P. Metabolic Trajectories Following Contrasting Prudent and Western Diets from Food Provisions: Identifying Robust Biomarkers of Short-Term Changes in Habitual Diet. Nutrients. 2019. 11:. doi:10.3390/nu11102407

2523 Wennberg, M, Soderberg, S, Uusitalo, U, Tuomilehto, J, Shaw, JE, Zimmet, PZ, Kowlessur, S, Pauvaday, V, Magliano, DJ. High consumption of pulses is associated with lower risk of abnormal glucose metabolism in women in Mauritius. Diabet Med. 2015. 32:513-20. doi:10.1111/dme.12618

2524 West, JA, de Looy, AE. Weight loss in overweight subjects following low-sucrose or sucrose-containing diets. Int $\mathrm{J}$ Obes Relat Metab Disord. 2001. 25:1122-8. doi:10.1038/sj.ijo.0801652

Power/Size

Study Design

Intervention/Exposure;

Study duration

Power/Size

Study Design;

Publication Status

Intervention/Exposure

Outcome; Study duration

Study Design

Power/Size

Study Design; Study duration

Intervention/Exposure: Country Intervention/Exposure 
2525 West, NR, Dorling, J, Thackray, AE, Hanson, NC, Decombel, SE, Stensel, DJ, Grice, SJ. Effect of Obesity-Linked FTO rs9939609 Variant on Physical Activity and Dietary Patterns in Physically Active Men and Women. J Obes. 2018. 2018:7560707. doi:10.1155/2018/7560707

2526 Westcott, WL, Apovian, CM, Puhala, K, Corina, L, Larosa Loud, R, Whitehead, S, Blum, K, DiNubile, N. Nutrition programs enhance exercise effects on body composition and resting blood pressure. Phys Sportsmed. 2013. 41:85-91. doi:10.3810/psm.2013.09.2027

2527 Westerbacka, J, Lammi, K, Hakkinen, AM, Rissanen, A, Salminen, I, Aro, A, Yki-Jarvinen, H. Dietary fat content modifies liver fat in overweight nondiabetic subjects. J Clin Endocrinol Metab. 2005. 90:2804-9. doi:10.1210/jc.2004-1983

2528 Westergren, HU, Gan, LM, Månsson, M, Svedlund, S. Randomized clinical trial studying effects of a personalized supervised lifestyle intervention program on cardiovascular status in physically inactive healthy volunteers. Oncotarget. 2018. 9:9498-9511. doi:10.18632/oncotarget.23958

2529 Westerterp-Plantenga, MS, Lejeune, MP, Nijs, I, van Ooijen, M, Kovacs, EM. High protein intake sustains weight maintenance after body weight loss in humans. Int J Obes Relat Metab Disord. 2004. 28:57-64. doi:10.1038/sj.ijo.0802461

2530 Westerterp-Plantenga, MS, Lejeune, MP, Smeets, AJ, Luscombe-Marsh, ND. Sex differences in energy homeostatis following a diet relatively high in protein exchanged with carbohydrate, assessed in a respiration chamber in humans. Physiol Behav. 2009. 97:414-9. doi:10.1016/j.physbeh.2009.03.010

2531 Westman, EC, Yancy, WS, Haub, MD, Volek, JS. Insulin resistance from a low carbohydrate, high fat diet perspective. Metab Syndr Relat Disord. 2005. 3:14-8. doi:10.1089/met.2005.3.14

2532 Wheaton, N, Millar, L, Allender, S, Nichols, M. The stability of weight status through the early to middle childhood years in Australia: a longitudinal study. BMJ Open. 2015. 5:e006963. doi:10.1136/bmjopen-2014-006963

2533 Wheeler, DH, Stentz, FB, Kitabchi, AE. Effect of macronutrient diet compositions on weight change and metabolic parameters. Journal of investigative medicine. 2012. 60:352. doi:10.231/JIM.0b013e3182820c2e

2534 Whelton, SP, Silverman, MG, McEvoy, JW, Budoff, MJ, Blankstein, R, Eng, J, Blumenthal, RS, Szklo, M, Nasir, K, Blaha, MJ. Predictors of Long-Term Healthy Arterial Aging: Coronary Artery Calcium Nondevelopment in the MESA Study. JACC Cardiovasc Imaging. 2015. 8:1393-1400. doi:10.1016/j.jcmg.2015.06.019

2535 White, AM, Johnston, CS, Swan, PD, Tjonn, SL, Sears, B. Blood ketones are directly related to fatigue and perceived effort during exercise in overweight adults adhering to low-carbohydrate diets for weight loss: a pilot study. J Am Diet Assoc. 2007 107:1792-6. doi:10.1016/j.jada.2007.07.009

2536 White, C, Drummond, S, De Looy, A. Comparing advice to decrease both dietary fat and sucrose, or dietary fat only, on weight loss, weight maintenance and perceived quality of life. Int J Food Sci Nutr. 2010. 61:282-94. doi:10.3109/09637480903397355

2537 White, J, Jago, R, Thompson, JL. Dietary risk factors for the development of insulin resistance in adolescent girls: a 3-year prospective study. Public Health Nutr. 2014. 17:361-8. doi:10.1017/s1368980012004983
Study Design;

Intervention/Exposure

Intervention/Exposure

Study Design; Study duration

Intervention/Exposure

Intervention/Exposure

Study duration

Study Design

Intervention/Exposure

Publication Status

Outcome

Study duration

Intervention/Exposure

Study Design; Intervention/Exposure 
2538 Widya, RL, Hammer, S, Boon, MR, van der Meer, RW, Smit, JW, de Roos, A, Rensen, PC, Lamb, HJ. Effects of short-term nutritional interventions on right ventricular function in healthy men. PLoS One. 2013. 8:e76406. doi:10.1371/journal.pone.0076406

2539 Wien, MA, Sabate, JM, Ikle, DN, Cole, SE, Kandeel, FR. Almonds vs complex carbohydrates in a weight reduction program. Int J Obes Relat Metab Disord. 2003. 27:1365-72. doi:10.1038/sj.ijo.0802411

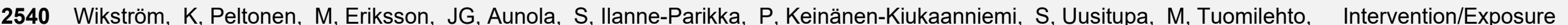
J, Lindström, J. Educational attainment and effectiveness of lifestyle intervention in the Finnish Diabetes Prevention Study. Diabetes Research and Clinical Practice. 2009. 86:e1-e5. doi:10.1016/j.diabres.2009.06.014

2541 Wilke, MS, French, MA, Goh, YK, Ryan, EA, Jones, PJ, Clandinin, MT. Synthesis of specific fatty acids contributes to VLDLtriacylglycerol composition in humans with and without type 2 diabetes. Diabetologia. 2009. 52:1628-37. doi:10.1007/s00125009-1405-9

2542 Williams, A, de Vlieger, N, Young, M, Jensen, ME, Burrows, TL, Morgan, PJ, Collins, CE. Dietary outcomes of overweight fathers and their children in the Healthy Dads, Healthy Kids community randomised controlled trial. J Hum Nutr Diet. 2018. 31:523-532. doi:10.1111/jhn. 12543

2543 Williams, CL, Strobino, BA. Childhood diet, overweight, and CVD risk factors: The healthy start project. Preventive Cardiology. 2008. 11:11-20. doi:10.1111/j.1520-037X.2007.06677.x

2544 Williams, PT, Bergeron, N, Chiu, S, Krauss, RM. A randomized, controlled trial on the effects of almonds on lipoprotein response to a higher carbohydrate, lower fat diet in men and women with abdominal adiposity. Lipids Health Dis. $2019.18: 83$. doi:10.1186/s12944-019-1025-4

2545 Willows, ND, Barbarich, BN, Wang, LC, Olstad, DL, Clandinin, MT. Dietary inadequacy is associated with anemia and suboptimal growth among preschool-aged children in Yunnan Province, China. Nutr Res. 2011. 31:88-96. doi:10.1016/j.nutres.2011.01.003

2546 Wilson, G, Pritchard, PP, Papageorgiou, C, Phillips, S, Kumar, P, Langan-Evans, C, Routledge, H, Owens, DJ, Morton, JP, Study Design Close, GL. Fasted Exercise and Increased Dietary Protein Reduces Body Fat and Improves Strength in Jockeys. Int J Sports Med. 2015. 36:1008-14. doi:10.1055/s-0035-1549920

2547 Wilson, HK, Armstrong, CLH, Hogan, JA, Campbell, WW. Effects of protein quantity and source (animal versus plant) on appetite and plasma amino acid responses in energy-restricted subjects. FASEB journal. 2012. 26:. doi:unavailable

2548 Wilson, JM, Lowery, RP, Roberts, MD, Sharp, MH, Joy, JM, Shields, KA, Partl, J, Volek, JS, D'Agostino, D. The Effects of Ketogenic Dieting on Body Composition, Strength, Power, and Hormonal Profiles in Resistance Training Males. J Strength Cond Res. 2017. :. doi:10.1519/jsc.0000000000001935

2549 Windler, E, Nitschmann, S. Effects of glycemic index on cardiovascular risk factors: the OmniCarb randomized clinical trial. Der internist. 2015. 56:949-952. doi:10.1007/s00108-015-3765-1 
2550 Witjaksono, F, Jutamulia, J, Annisa, NG, Prasetya, SI, Nurwidya, F. Comparison of low calorie high protein and low calorie standard protein diet on waist circumference of adults with visceral obesity and weight cycling. BMC Res Notes. 2018 . 11:674. doi:10.1186/s13104-018-3781-z

2551 Wittenbecher, C, Muhlenbruch, K, Kroger, J, Jacobs, S, Kuxhaus, O, Floegel, A, Fritsche, A, Pischon, T, Prehn, C, Adamski, J, Joost, HG, Boeing, H, Schulze, MB. Amino acids, lipid metabolites, and ferritin as potential mediators linking red meat consumption to type 2 diabetes. Am J Clin Nutr. 2015. 101:1241-50. doi:10.3945/ajcn.114.099150

2552 Wolever, TM, Gibbs, AL, Mehling, C, Chiasson, JL, Connelly, PW, Josse, RG, Leiter, LA, Maheux, P, Rabasa-Lhoret, R, Rodger, NW, Ryan, EA. The Canadian Trial of Carbohydrates in Diabetes (CCD), a 1-y controlled trial of low-glycemic-index dietary carbohydrate in type 2 diabetes: no effect on glycated hemoglobin but reduction in C-reactive protein. Am $\mathrm{J}$ Clin Nutr. 2008. 87:114-25. doi:10.1093/ajcn/87.1.114

2553 Wolever, TM, Isaacs, RL, Ramdath, DD. Lower diet glycaemic index in African than South Asian men in Trinidad and Tobago. Int J Food Sci Nutr. 2002. 53:297-303. doi:10.1080/09637480220138142

2554 Wolever, TM, Mehling, C. Long-term effect of varying the source or amount of dietary carbohydrate on postprandial plasma glucose, insulin, triacylglycerol, and free fatty acid concentrations in subjects with impaired glucose tolerance. Am $\mathrm{J}$ Clin Nutr 2003. 77:612-21. doi:10.1093/ajcn/77.3.612

2555 Wolff, E, Vergnes, MF, Portugal, H, Defoort, C, Amiot-Carlin, MJ, Lairon, D, Nicolay, A. Cholesterol-absorber status modifies the LDL cholesterol-lowering effect of a Mediterranean-type diet in adults with moderate cardiovascular risk factors. $\mathrm{J}$ Nutr. 2011. 141:1791-8. doi:10.3945/jn.111.141333

2556 Wolfram, G, Bechthold, A, Boeing, H, Ellinger, S, Hauner, H, Kroke, A, Leschik-Bonnet, E, Linseisen, J, Lorkowski, S, Schulze, M, Stehle, P, Dinter, J. Evidence-Based Guideline of the German Nutrition Society: Fat Intake and Prevention of Selected Nutrition-Related Diseases. Ann Nutr Metab. 2015. 67:141-204. doi:10.1159/000437243

2557 Wolters, M, Joslowski, G, Plachta-Danielzik, S, Standl, M, Muller, MJ, Ahrens, W, Buyken, AE. Dietary Patterns in Primary School are of Prospective Relevance for the Development of Body Composition in Two German Pediatric Populations. Nutrients. 2018. 10:. doi:10.3390/nu10101442

2558 Woo, J, Cheung, B, Ho, S, Sham, A, Lam, TH. Influence of dietary pattern on the development of overweight in a Chinese population. Eur J Clin Nutr. 2008. 62:480-7. doi:10.1038/sj.ejcn.1602702

Intervention/Exposure; Country

Intervention/Exposure

Health Status

Study Design;

Outcome

Intervention/Exposure

Intervention/Exposure

Study Design

Power/Size

Intervention/Exposure; Country

Outcome

2559 Wood, AD, Strachan, AA, Thies, F, Aucott, LS, Reid, DM, Hardcastle, AC, Mavroeidi, A, Simpson, WG, Duthie, GG, Macdonald, HM. Patterns of dietary intake and serum carotenoid and tocopherol status are associated with biomarkers of chronic low-grade systemic inflammation and cardiovascular risk. Br J Nutr. 2014. 112:1341-52. doi:10.1017/s0007114514001962

2560 Wood, RJ, Volek, JS, Liu, Y, Shachter, NS, Contois, JH, Fernandez, ML. Carbohydrate restriction alters lipoprotein metabolism by modifying VLDL, LDL, and HDL subfraction distribution and size in overweight men. J Nutr. 2006. 136:384-9. doi:10.1093/jn/136.2.384

2561 Woodhall-Melnik, J, Cooke, M, Bigelow, PL. Serving the food nation: Exploring Body Mass Index in food service workers. Work. 2015. 52:901-9. doi:10.3233/wor-152101

Study Design;

Intervention/Exposure

Study Design;

Intervention/Exposure 
2562 Woolhead, C, Walsh, MC, Gibney, MJ, Daniel, H, Drevon, CA, Lovegrove, JA, Manios, Y, Martinez, JA, Mathers, JC, Traczyk, I, etal, . Dietary patterns in Europe: the Food4Me proof of principle study. Proceedings of the nutrition society. 2015. 74:. doi:10.1017/S002966511500275X

2563 Wosje, KS, Khoury, PR, Claytor, RP, Copeland, KA, Hornung, RW, Daniels, SR, Kalkwarf, HJ. Dietary patterns associated with fat and bone mass in young children. Am J Clin Nutr. 2010. 92:294-303. doi:10.3945/ajcn.2009.28925

2564 Wright, C, Zhou, J, Sayer, R, Kim, JE, Campbell, W. Effect of a high protein, high egg diet on muscle composition, metabolic health and systemic inflammation in overweight and obese, older adults. FASEB journal. 2015. 29:. doi:unavailable

2565 Wright, CS, Zhou, J, Sayer, RD, Kim, JE, Campbell, WW. Effects of a High-Protein Diet Including Whole Eggs on Muscle Composition and Indices of Cardiometabolic Health and Systemic Inflammation in Older Adults with Overweight or Obesity: A Randomized Controlled Trial. Nutrients. 2018. 10:. doi:10.3390/nu10070946

2566 Wright, M, Sotres-Alvarez, D, Mendez, MA, Adair, L. The association of trajectories of protein intake and age-specific protein intakes from 2 to 22 years with BMI in early adulthood. Br J Nutr. 2017. 117:750-758. doi:10.1017/s0007114517000502

2567 Wroble, KA, Trott, MN, Schweitzer, GG, Rahman, RS, Kelly, PV, Weiss, EP. Low-carbohydrate, ketogenic diet impairs anaerobic exercise performance in exercise-trained women and men: a randomized-sequence crossover trial. J Sports Med Phys Fitness. 2019. 59:600-607. doi:10.23736/s0022-4707.18.08318-4

2568 Wu, H, Flint, AJ, Qi, Q, van Dam, RM, Sampson, LA, Rimm, EB, Holmes, MD, Willett, WC, Hu, FB, Sun, Q. Association between dietary whole grain intake and risk of mortality: two large prospective studies in US men and women. JAMA Intern Med. 2015. 175:373-84. doi:10.1001/jamainternmed.2014.6283

2569 Wu, S, An, S, Li, W, Lichtenstein, AH, Gao, J, Kris-Etherton, PM, Wu, Y, Jin, C, Huang, S, Hu, FB, Gao, X. Association of Trajectory of Cardiovascular Health Score and Incident Cardiovascular Disease. JAMA Netw Open. 2019. 2:e194758. doi:10.1001/jamanetworkopen.2019.4758

2570 Wulan, SN, Bouwman, FG, Westerterp, KR, Mariman, ECM, Plasqui, G. Molecular adaptation in adipose tissue in response to overfeeding with a high-fat diet under sedentary conditions in South Asian and Caucasian men. Br J Nutr. 2019. 122:241251. doi:10.1017/s0007114519001260

2571 Wulan, SN, Schrauwen-Hinderling, VB, Westerterp, KR, Plasqui, G. Substrate utilization and metabolic profile in response to overfeeding with a high-fat diet in South Asian and white men: a sedentary lifestyle study. Int J Obes (Lond). 2019. :. doi:10.1038/s41366-019-0368-2

2572 Wulan, SN, Westerterp, KR, Plasqui, G. Dietary and 24-h fat oxidation in Asians and whites who differ in body composition. Am J Clin Nutr. 2012. 95:1335-41. doi:10.3945/ajcn.111.031369

2573 Wulan, SN, Westerterp, KR, Plasqui, G. Metabolic profile before and after short-term overfeeding with a high-fat diet: a comparison between South Asian and White men. Br J Nutr. 2014. 111:1853-61. doi:10.1017/s0007114514000014

2574 Wyatt, CJ, Triana Tejas, MA. Nutrient intake and growth of preschool children from different socioeconomic regions in the city Study Design of oaxaca, Mexico. Ann Nutr Metab. 2000. 44:14-20. doi:10.1159/000012816

Publication Date

Overlaps with Existing Review

ntervention/Exposure; Publication Status

Weight

loss/Hypocaloric

Intervention/Exposure; Country

Outcome; Study

duration

Intervention/Exposure

Country

Intervention/Exposure Study duration

Study duration

Study Design; Study duration

Study duration 
2575 Wycherley, T, Brinkworth, G, Clifton, P, Noakes, M. A one year high protein, low fat weight loss diet improves body composition and cardiometabolic risk factors in overweight males. FASEB journal. 2012. 26:. doi:unavailable

2576 Wycherley, TP, Brinkworth, GD, Clifton, PM, Noakes, M. A one year high protein, low fat weight loss diet improves body composition and cardiometabolic risk factors in overweight males with features of the metabolic syndrome. Australasian medical journal. 2011. 4:731. doi:unavailable

2577 Wycherley, TP, Brinkworth, GD, Clifton, PM, Noakes, M. Comparison of the effects of 52 weeks weight loss with either a high-protein or high-carbohydrate diet on body composition and cardiometabolic risk factors in overweight and obese males. Nutr Diabetes. 2012. 2:e40. doi:10.1038/nutd.2012.11

2578 Wycherley, TP, Brinkworth, GD, Keogh, JB, Noakes, M, Buckley, JD, Clifton, PM. Long-term effects of weight loss with a very low carbohydrate and low fat diet on vascular function in overweight and obese patients. J Intern Med. 2010. 267:452-61. doi:10.1111/j.1365-2796.2009.02174.x

2579 Wycherley, TP, Brinkworth, GD, Keogh, JB, Noakes, M, Buckley, JD, Clifton, PM. Long-term effects of weight loss with a very low carbohydrate and low fat diet on vascular function in overweight and obese patients: Original Article. Journal of Internal Medicine. 2010. 267:452-461. doi:10.1111/j.1365-2796.2009.02174.x

2580 Wycherley, TP, Buckley, JD, Noakes, M, Clifton, PM, Brinkworth, GD. Comparison of the effects of weight loss from a highprotein versus standard-protein energy-restricted diet on strength and aerobic capacity in overweight and obese men. Eur $\mathrm{J}$ Nutr. 2013. 52:317-25. doi:10.1007/s00394-012-0338-0

2581 Wycherley, TP, Buckley, JD, Noakes, M, Clifton, PM, Brinkworth, GD. Long-term effects of a very low-carbohydrate weight loss diet on exercise capacity and tolerance in overweight and obese adults. J Am Coll Nutr. 2014. 33:267-73. doi:10.1080/07315724.2014.911668

2582 Wyka, J, Malczyk, E, Misiarz, M, Zolotenka-Synowiec, M, Calyniuk, B, Baczynska, S. Assessment of food intakes for women adopting the high protein Dukan diet. Rocz Panstw Zakl Hig. 2015. 66:137-42. doi:unavailable

2583 Xiao, RS, Simas, TA, Person, SD, Goldberg, RJ, Waring, ME. Diet quality and history of gestational diabetes mellitus among childbearing women, United States, 2007-2010. Prev Chronic Dis. 2015. 12:E25. doi:10.5888/pcd12.140360

$2584 \mathrm{Xu}$, J, Eilat-Adar, S, Loria, C, Goldbourt, U, Howard, BV, Fabsitz, RR, Zephier, EM, Mattil, C, Lee, ET. Dietary fat intake and risk of coronary heart disease: the Strong Heart Study. Am J Clin Nutr. 2006. 84:894-902. doi:10.1093/ajcn/84.4.894

$2585 \mathrm{Xu}, \mathrm{M}, \mathrm{Ng}$, SS, Bray, GA, Ryan, DH, Sacks, FM, Ning, G, Qi, L. Dietary Fat Intake Modifies the Effect of a Common Variant in the LIPC Gene on Changes in Serum Lipid Concentrations during a Long-Term Weight-Loss Intervention Trial. J Nutr. 2015. 145:1289-94. doi:10.3945/jn.115.212514

$2586 \mathrm{Xu}, \mathrm{M}, \mathrm{Qi}, \mathrm{Q}$, Liang, J, Bray, GA, Hu, FB, Sacks, FM, Qi, L. Genetic determinant for amino acid metabolites and changes in body weight and insulin resistance in response to weight-loss diets: the Preventing Overweight Using Novel Dietary Strategies (POUNDS LOST) trial. Circulation. 2013. 127:1283-9. doi:10.1161/circulationaha.112.000586

2587 Xu, X, Hall, J, Byles, J, Shi, Z. Dietary Pattern Is Associated with Obesity in Older People in China: Data from China Health and Nutrition Survey (CHNS). Nutrients. 2015. 7:8170-88. doi:10.3390/nu7095386
Publication Status

Publication Status

Weight

oss/Hypocaloric

Power/Size

Study Design

Outcome

Power/Size

Power/Size

Study Design

Study Design; Outcome

Intervention/Exposure

Intervention/Exposure

Intervention/Exposure

Country 
2588 Xu, X, Hall, J, Byles, J, Shi, Z. Dietary pattern, serum magnesium, ferritin, C-reactive protein and anaemia among older Study Design; Country people. Clin Nutr. 2017. 36:444-451. doi:10.1016/j.clnu.2015.12.015

2589 Xu, X, Parker, D, Shi, Z, Byles, J, Hall, J, Hickman, L. Dietary Pattern, Hypertension and Cognitive Function in an Older Population: 10-Year Longitudinal Survey. Front Public Health. 2018. 6:201. doi:10.3389/fpubh.2018.00201

2590 Yackobovitch-Gavan, M, Nagelberg, N, Demol, S, Phillip, M, Shalitin, S. Influence of weight-loss diets with different macronutrient compositions on health-related quality of life in obese youth. Appetite. 2008. 51:697-703. doi:10.1016/j.appet.2008.06.010

2591 Yahia, DA. Insulinoresistance, inflammation, lecithin: cholesterol acyltransferase and HDL composition are improved by Mediterranean diet in metabolic syndrome patients. Annals of nutrition and metabolism. Conference: 12th european nutrition conference, FENS 2015. Berlin germany. Conference start: 20151020. Conference end: 20151023. Conference publication: (var.pagings). 2015. 67:343. doi:10.1159/000440895

2592 Yamagishi, K, Iso, H, Date, C, Fukui, M, Wakai, K, Kikuchi, S, Inaba, Y, Tanabe, N, Tamakoshi, A. Fish, omega-3 polyunsaturated fatty acids, and mortality from cardiovascular diseases in a nationwide community-based cohort of Japanese men and women the JACC (Japan Collaborative Cohort Study for Evaluation of Cancer Risk) Study. J Am Coll Cardiol. 2008. 52:988-96. doi:10.1016/j.jacc.2008.06.018

2593 Yancy, WS, Jr, Mayer, SB, Coffman, CJ, Smith, VA, Kolotkin, RL, Geiselman, PJ, McVay, MA, Oddone, EZ, Voils, Cl. Effect of Allowing Choice of Diet on Weight Loss: A Randomized Trial. Ann Intern Med. 2015. 162:805-14. doi:10.7326/m142358

2594 Yancy, WS, Jr, Olsen, MK, Guyton, JR, Bakst, RP, Westman, EC. A low-carbohydrate, ketogenic diet versus a low-fat diet to treat obesity and hyperlipidemia: a randomized, controlled trial. Ann Intern Med. 2004. 140:769-77. doi:10.7326/0003-4819140-10-200405180-00006

2595 Yang, H, Kim, H, Kim, JM, Chung, HW, Chang, N. Associations of dietary intake and metabolic syndrome risk parameters in Vietnamese female marriage immigrants in South Korea: The KoGES follow-up study. Nutr Res Pract. 2016. 10:313-20. doi:10.4162/nrp.2016.10.3.313

2596 Yanos, BR, Saules, KK, Schuh, LM, Sogg, S. Predictors of Lowest Weight and Long-Term Weight Regain Among Roux-en-Y Health Status Gastric Bypass Patients. Obes Surg. 2015. 25:1364-70. doi:10.1007/s11695-014-1536-z

2597 Yap, RWK, Shidoji, Y, Yap, WS, Masaki, M. Association and Interaction Effect of AGTR1 and AGTR2 Gene Polymorphisms with Dietary Pattern on Metabolic Risk Factors of Cardiovascular Disease in Malaysian Adults. Nutrients. 2017. 9:. doi:10.3390/nu9080853

2598 Ye, J, Lv, Y, Li, Z, Yao, Y, Jin, L. Associations of dietary patterns with hypertension among adults in Jilin Province, China: structural equation modelling approach. Public Health Nutr. 2018. :1-8. doi:10.1017/s1368980018003129

2599 Ye, X, Gao, X, Scott, T, Tucker, KL. Habitual sugar intake and cognitive function among middle-aged and older Puerto Ricans without diabetes. Br J Nutr. 2011. 106:1423-32. doi:10.1017/s0007114511001760

Country

Intervention/Exposure

Publication Status

Intervention/Exposure

Study Design;

Comparator

Weight

loss/Hypocaloric

Study Design;

Intervention/Exposure

Study Design

Study Design; Outcome

Study Design;

Intervention/Exposure; Outcome 
2600 Yeo, R, Yoon, SR, Kim, OY. The Association between Food Group Consumption Patterns and Early Metabolic Syndrome Risk in Non-Diabetic Healthy People. Clin Nutr Res. 2017. 6:172-182. doi:10.7762/cnr.2017.6.3.172

2601 Yeung, EH, Appel, LJ, Miller, ER, 3rd, Kao, WH. The effects of macronutrient intake on total and high-molecular weight adiponectin: results from the OMNI-Heart trial. Obesity (Silver Spring). 2010. 18:1632-7. doi:10.1038/oby.2009.402

2602 Yoon, C, Jacobs, DR, Jr, Duprez, DA, Neumark-Sztainer, D, Steffen, LM, Mason, SM. Problematic eating behaviors and attitudes predict long-term incident metabolic syndrome and diabetes: The Coronary Artery Risk Development in Young Adults Study. Int J Eat Disord. 2019. 52:304-308. doi:10.1002/eat.23020

2603 Yosaee, S, Esteghamati, A, Nazari Nasab, M, Khosravi, A, Alinavaz, M, Hosseini, B, Djafarian, K. Diet quality in obese/overweight individuals with/without metabolic syndrome compared to normal weight controls. Med J Islam Repub Iran 2016. 30:376. doi:unavailable

2604 Yoshimoto, Y, Muto, S, Fujikura, J, Sakuma, M, Kaneko, Y, Otto, CT, Nakamura, L. Obesity, bone status and dietary intake of Palauan elderly congregating in a Senior Citizen's Center. Pac Health Dialog. 2005. 12:22-32. doi:unavailable

Outcome

Intervention/Exposure

Yoshizaki, T, Ishihara, J, Kotemori, A, Yamamoto, J, Kokubo, Y, Saito, I, Yatsuya, H, Yamagishi, K, Sawada, N, Iwasaki, $\mathrm{M}$, Iso, H, Tsugane, S. Association of vegetable, fruit, and Okinawan vegetable consumption with incident stroke and coronary heart disease. J Epidemiol. 2019. .. doi:10.2188/jea.JE20180130

2606 Young, D, Camhi, S, Wu, T, Hagberg, J, Stefanick, M. Relationships among changes in C-reactive protein and cardiovascular disease risk factors with lifestyle interventions. Nutr Metab Cardiovasc Dis. 2013. 23:857-63. doi:10.1016/j.numecd.2012.05.003

2607 Young, DR, Coughlin, J, Jerome, GJ, Myers, V, Chae, SE, Brantley, PJ. Effects of the PREMIER interventions on healthrelated quality of life. Ann Behav Med. 2010. 40:302-12. doi:10.1007/s12160-010-9220-6

2608 Young, LR, Kurzer, MS, Thomas, W, Redmon, JB, Raatz, SK. Low-fat diet with omega-3 fatty acids increases plasma insulin-like growth factor concentration in healthy postmenopausal women. Nutr Res. 2013. 33:565-71. doi:10.1016/j.nutres.2013.04.011

2609 Young, M, Morgan, P, Hollis, J, Collins, C, Teixeira, P. Sitting time at work and marital status: novel pre-treatment predictors of weight loss success in overweight and obese men. Obesity reviews. 2014. 15:149. doi:10.1111/obr.12151

2610 Yu, C, Benhammou, JN, Goyal, D, Oh, D, Wang, L, Jacobs, J, Dixit, V, Tache, Y, Pisegna, J. High protein dietary intervention improves body mass index (BMI) and reduces the NAFLD fibrosis score (NFS) in veterans with obesity. American journal of gastroenterology. 2016. 111:S349-. doi:10.1038/ajg.2016.359

2611 Yu, D, Shu, XO, Li, H, Xiang, YB, Yang, G, Gao, YT, Zheng, W, Zhang, X. Dietary carbohydrates, refined grains, glycemic load, and risk of coronary heart disease in Chinese adults. Am J Epidemiol. 2013. 178:1542-9. doi:10.1093/aje/kwt178

2612 Yu, D, Shu, XO, Li, H, Yang, G, Cai, Q, Xiang, YB, Ji, BT, Franke, AA, Gao, YT, Zheng, W, Zhang, X. Dietary isoflavones, urinary isoflavonoids, and risk of ischemic stroke in women. Am J Clin Nutr. 2015. 102:680-6. doi:10.3945/ajcn.115.111591 
2613 Yu, D, Zhang, X, Xiang, YB, Yang, G, Li, H, Gao, YT, Zheng, W, Shu, XO. Adherence to dietary guidelines and mortality: A Country report from prospective cohort studies of 134,000 Chinese adults in urban Shanghai. American Journal of Clinical Nutrition. 2014. 100:693-700. doi:10.3945/ajcn.113.079194

2614 Yu, D, Zheng, W, Cai, H, Xiang, YB, Li, H, Gao, YT, Shu, XO. Long-term Diet Quality and Risk of Type 2 Diabetes Among Urban Chinese Adults. Diabetes Care. 2018. 41:723-730. doi:10.2337/dc17-1626

$2615 \mathrm{Yu}$, SH, Song, Y, Park, M, Kim, SH, Shin, S, Joung, H. Relationship between adhering to dietary guidelines and the risk obesity in Korean children. Nutr Res Pract. 2014. 8:705-12. doi:10.4162/nrp.2014.8.6.705

2616 Yuan, YQ, Li, F, Meng, P, You, J, Wu, M, Li, SG, Chen, B. Gender Difference on the Association between Dietary Patterns and Obesity in Chinese Middle-Aged and Elderly Populations. Nutrients. 2016. 8:. doi:10.3390/nu8080448

2617 Yubero-Serrano, EM, Delgado-Lista, J, Tierney, AC, Perez-Martinez, P, Garcia-Rios, A, Alcala-Diaz, JF, Castano, JP, Tinahones, FJ, Drevon, CA, Defoort, C, Blaak, EE, Dembinska-Kiec, A, Riserus, U, Lovegrove, JA, Perez-Jimenez, F, Roche, HM, Lopez-Miranda, J. Insulin resistance determines a differential response to changes in dietary fat modification on metabolic syndrome risk factors: the LIPGENE study. Am J Clin Nutr. 2015. 102:1509-17. doi:10.3945/ajcn.115.111286

2618 Yuzbashian, E, Asghari, G, Mirmiran, P, Amouzegar-Bahambari, P, Azizi, F. Adherence to low-sodium Dietary Approaches to Stop Hypertension-style diet may decrease the risk of incident chronic kidney disease among high-risk patients: a secondary prevention in prospective cohort study. Nephrol Dial Transplant. 2018. 33:1159-1168. doi:10.1093/ndt/gfx352

2619 Zajac, A, Poprzecki, S, Maszczyk, A, Czuba, M, Michalczyk, M, Zydek, G. The effects of a ketogenic diet on exercise metabolism and physical performance in off-road cyclists. Nutrients. 2014. 6:2493-508. doi:10.3390/nu6072493

2620 Zamora, D, Gordon-Larsen, P, Jacobs, DR, Jr, Popkin, BM. Diet quality and weight gain among black and white young adults: the Coronary Artery Risk Development in Young Adults (CARDIA) Study (1985-2005). Am J Clin Nutr. 2010. 92:784-93. doi:10.3945/ajcn.2010.29161

2621 Zarrazquin, I, Torres-Unda, J, Ruiz, F, Irazusta, J, Kortajarena, M, Hoyos Cillero, I, Gil, J, Irazusta, A. Longitudinal study: lifestyle and cardiovascular health in health science students. Nutr Hosp. 2014. 30:1144-51. doi:10.3305/nh.2014.30.5.7833

2622 Zaslavsky, O, Zelber-Sagi, S, Hebert, JR, Steck, SE, Shivappa, N, Tabung, FK, Wirth, MD, Bu, Y, Shikany, JM, Orchard, T, Wallace, RB, Snetselaar, L, Tinker, LF. Biomarker-calibrated nutrient intake and healthy diet index associations with mortality risks among older and frail women from the Women's Health Initiative. Am J Clin Nutr. 2017. 105:1399-1407. doi:10.3945/ajcn.116.151530

2623 Zatonski, WA, Willett, W. Changes in dietary fat and declining coronary heart disease in Poland: population based study. Bmj. 2005. 331:187-8. doi:10.1136/bmj.331.7510.187

2624 Zazpe, I, Sanchez-Tainta, A, Toledo, E, Sanchez-Villegas, A, Martinez-Gonzalez, MA. Dietary patterns and total mortality in a Mediterranean cohort: the SUN project. J Acad Nutr Diet. 2014. 114:37-47. doi:10.1016/j.jand.2013.07.024

2625 Zazpe, I, Santiago, S, Gea, A, Ruiz-Canela, M, Carlos, S, Bes-Rastrollo, M, Martinez-Gonzalez, MA. Association between a dietary carbohydrate index and cardiovascular disease in the SUN (Seguimiento Universidad de Navarra) Project. Nutr Metab Cardiovasc Dis. 2016. 26:1048-1056. doi:10.1016/j.numecd.2016.07.002

Country

Study Design

Study Design

Intervention/Exposure

Outcome

Intervention/Exposure

Publication Date

Overlaps with Existing Review

Intervention/Exposure

Outcome

Study Design; Publication Status

Outcome

Intervention/Exposure 
2626 Zeybek, C, Celebi, A, Aktuglu-Zeybek, C, Onal, H, Yalcin, Y, Erdem, A, Akdeniz, C, Imanov, E, Altay, S, Aydin, A. The effect of low-carbohydrate diet on left ventricular diastolic function in obese children. Pediatr Int. 2010. 52:218-23. doi:10.1111/j.1442-200X.2009.02940.x

2627 Zhang, F, Tapera, TM, Gou, J. Application of a new dietary pattern analysis method in nutritional epidemiology. BMC Med Res Methodol. 2018. 18:119. doi:10.1186/s12874-018-0585-8

2628 Zhang, J, Wang, C, Li, L, Man, Q, Song, P, Meng, L, Du, ZY, Froyland, L. Inclusion of Atlantic salmon in the Chinese diet reduces cardiovascular disease risk markers in dyslipidemic adult men. Nutr Res. 2010. 30:447-54. doi:10.1016/j.nutres.2010.06.010

2629 Zhang, J, Wang, H, Wang, Y, Xue, H, Wang, Z, Du, W, Su, C, Zhang, J, Jiang, H, Zhai, F, Zhang, B. Dietary patterns and their associations with childhood obesity in China. Br J Nutr. 2015. 113:1978-84. doi:10.1017/s0007114515001154

2630 Zhang, JG, Wang, ZH, Wang, HJ, Du, WW, Su, C, Zhang, J, Jiang, HR, Zhai, FY, Zhang, B. Dietary patterns and their associations with general obesity and abdominal obesity among young Chinese women. Eur J Clin Nutr. 2015. 69:1009-14. doi:10.1038/ejcn.2015.8

2631 Zhang, L, Pagoto, S, Olendzki, B, Persuitte, G, Churchill, L, Oleski, J, Ma, Y. A nonrestrictive, weight loss diet focused on fiber and lean protein increase. Nutrition. 2018. 54:12-18. doi:10.1016/j.nut.2018.02.006

2632 Zhang, Q, Chen, X, Liu, Z, Varma, DS, Wan, R, Wan, Q, Zhao, S. Dietary Patterns in Relation to General and Central Obesity among Adults in Southwest China. Int J Environ Res Public Health. 2016. 13:. doi:10.3390/ijerph13111080

2633 Zhang, S, Zhuang, X, Lin, X, Zhong, X, Zhou, H, Sun, X, Xiong, Z, Huang, Y, Fan, Y, Guo, Y, Du, Z, Liao, X. LowCarbohydrate Diets and Risk of Incident Atrial Fibrillation: A Prospective Cohort Study. J Am Heart Assoc. 2019. 8:e011955. doi:10.1161/jaha.119.011955

2634 Zhang, X, Qi, Q, Hu, FB, Sacks, FM, Qi, L. Apoa5 genotype modulates 2-year changes in lipid profile in response to weightloss diet intervention: the pounds lost trial. Diabetes. 2012. 61:A158. doi:10.2337/db12-378-655

2635 Zhang, Z, Lanza, E, Kris-Etherton, PM, Colburn, NH, Bagshaw, D, Rovine, MJ, Ulbrecht, JS, Bobe, G, Chapkin, RS Hartman, TJ. A high legume low glycemic index diet improves serum lipid profiles in men. Lipids. 2010. 45:765-75. doi:10.1007/s11745-010-3463-7

2636 Zhao, G, Etherton, TD, Martin, KR, West, SG, Gillies, PJ, Kris-Etherton, PM. Dietary alpha-linolenic acid reduces inflammatory and lipid cardiovascular risk factors in hypercholesterolemic men and women. J Nutr. 2004. 134:2991-7. doi:10.1093/jn/134.11.2991

2637 Zhen, S, Ma, Y, Zhao, Z, Yang, X, Wen, D. Dietary pattern is associated with obesity in Chinese children and adolescents: data from China Health and Nutrition Survey (CHNS). Nutr J. 2018. 17:68. doi:10.1186/s12937-018-0372-8

2638 Zheng, Y, Huang, T, Zhang, X, Rood, J, Bray, GA, Sacks, FM, Qi, L. Dietary Fat Modifies the Effects of FTO Genotype on Changes in Insulin Sensitivity. J Nutr. 2015. 145:977-82. doi:10.3945/jn.115.210005

Study Design

Country

Study Design

Study Design

Study Design

Study Design

Outcome

Publication Status

Intervention/Exposure; Publication Date

Overlaps with Existing Review

Intervention/Exposure: Comparator

\section{Country}

Intervention/Exposure Intervention/Exposure 
2639 Zhou, J, Kim, JE, Armstrong, CL, Chen, N, Campbell, WW. Higher-protein diets improve indexes of sleep in energyrestricted overweight and obese adults: results from 2 randomized controlled trials. Am J Clin Nutr. 2016. 103:766-74. doi:10.3945/ajcn.115.124669

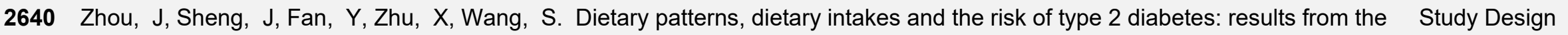
Hefei Nutrition and Health Study. Int J Food Sci Nutr. 2019. 70:412-420. doi:10.1080/09637486.2018.1515184

2641 Zhu, C, Sawrey-Kubicek, L, Beals, E, Hughes, RL, Rhodes, CH, Sacchi, R, Zivkovic, AM. Short term fast food and Mediterranean diet are able to change the HDL lipidome with different patterns. FASEB journal. 2017. 31:. doi:unavailable

2642 Zhu, C, Sawrey-Kubicek, L, Beals, E, Hughes, RL, Rhodes, CH, Sacchi, R, Zivkovic, AM. The HDL lipidome is widely remodeled by fast food versus Mediterranean diet in 4 days. Metabolomics. 2019. 15:. doi:10.1007/s11306-019-1579-1 Zhu, X, Lin, J, Song, Y, Liu, H, Zhang, R, Fan, M, Li, Y, Tian, R, Fang, D. A high-carbohydrate diet lowered blood pressure in healthy Chinese male adolescents. Biosci Trends. 2014. 8:132-7. doi:unavailable

2644 Zhuang, P, Zhang, Y, He, W, Chen, X, Chen, J, He, L, Mao, L, Wu, F, Jiao, J. Dietary Fats in Relation to Total and CauseSpecific Mortality in a Prospective Cohort of 521120 Individuals With 16 Years of Follow-Up. Circ Res. 2019. 124:757-768. doi:10.1161/circresaha.118.314038

2645 Ziegler, D, Strom, A, Nowotny, B, Zahiragic, L, Nowotny, PJ, Carstensen-Kirberg, M, Herder, C, Roden, M. Effect of LowEnergy Diets Differing in Fiber, Red Meat, and Coffee Intake on Cardiac Autonomic Function in Obese Individuals With Type 2 Diabetes. Diabetes Care. 2015. 38:1750-7. doi:10.2337/dc15-0466

2646 Zinn, C, McPhee, J, Harris, N, Williden, M, Prendergast, K, Schofield, G. A 12-week low-carbohydrate, high-fat diet improves metabolic health outcomes over a control diet in a randomised controlled trial with overweight defence force personnel. Appl Physiol Nutr Metab. 2017. 42:1158-1164. doi:10.1139/apnm-2017-0260

2647 Zinn, C, Wood, M, Williden, M, Chatterton, S, Maunder, E. Ketogenic diet benefits body composition and well-being but not performance in a pilot case study of New Zealand endurance athletes. J Int Soc Sports Nutr. 2017. 14:22. doi:10.1186/s12970017-0180-0

2648 Zoltick, ES, Sahni, S, McLean, RR, Quach, L, Casey, VA, Hannan, MT. Dietary protein intake and subsequent falls in older men and women: the Framingham Study. J Nutr Health Aging. 2011. 15:147-52. doi:unavailable

2649 Zou, P, Dennis, CL, Lee, R, Parry, M. Dietary approach to stop hypertension with sodium reduction for Chinese Canadians (Dashna-CC): A pilot randomized controlled trial. Journal of Nutrition, Health and Aging. 2017. 21:1225-1232. doi:10.1007/s12603-016-0861-4

2650 Zubair, N, Kuzawa, CW, Lee, NR, McDade, TW, Adair, LS. Clustering and determinants of cardiometabolic risk factors among Filipino young adults. Asia Pac J Clin Nutr. 2014. 23:148-58. doi:10.6133/apjcn.2014.23.1.06

\section{Study duration}

Study Design

ntervention/Exposure; Outcome

Health Status

Intervention/Exposure

Zubair, N, Kuzawa, CW, McDade, TW, Adair, LS. Cluster analysis reveals important determinants of cardiometabolic risk patterns in Filipino women. Asia Pac J Clin Nutr. 2012. 21:271-81. doi:unavailable

2652 Zuo, L, He, F, Tinsley, GM, Pannell, BK, Ward, E, Arciero, PJ. Comparison of high-protein, intermittent fasting low-calorie diet and heart healthy diet for vascular health of the obese. Frontiers in Physiology. 2016. 7:. doi:10.3389/fphys.2016.00350

Study Design

Intervention/Exposure; Outcome

Intervention/Exposure; Comparator

Intervention/Exposure; Outcome

Intervention/Exposure; Country

Intervention/Exposure 
